\title{
A venture into the epigenetics of aging and Alzheimer's Disease
}

Citation for published version (APA):

Lardenoije, R. (2017). A venture into the epigenetics of aging and Alzheimer's Disease. [Doctoral Thesis, Maastricht University]. Maastricht University. https://doi.org/10.26481/dis.20170907rl

Document status and date:

Published: 01/01/2017

DOI:

10.26481/dis.20170907rl

Document Version:

Publisher's PDF, also known as Version of record

\section{Please check the document version of this publication:}

- A submitted manuscript is the version of the article upon submission and before peer-review. There can be important differences between the submitted version and the official published version of record.

People interested in the research are advised to contact the author for the final version of the publication, or visit the DOI to the publisher's website.

- The final author version and the galley proof are versions of the publication after peer review.

- The final published version features the final layout of the paper including the volume, issue and page numbers.

Link to publication

\footnotetext{
General rights rights.

- You may freely distribute the URL identifying the publication in the public portal. please follow below link for the End User Agreement:

www.umlib.nl/taverne-license

Take down policy

If you believe that this document breaches copyright please contact us at:

repository@maastrichtuniversity.nl

providing details and we will investigate your claim.
}

Copyright and moral rights for the publications made accessible in the public portal are retained by the authors and/or other copyright owners and it is a condition of accessing publications that users recognise and abide by the legal requirements associated with these

- Users may download and print one copy of any publication from the public portal for the purpose of private study or research.

- You may not further distribute the material or use it for any profit-making activity or commercial gain

If the publication is distributed under the terms of Article $25 \mathrm{fa}$ of the Dutch Copyright Act, indicated by the "Taverne" license above, 


\section{A VENTURE INTO THE EPIGENETICS OF AGING AND ALZHEIMER'S DISEASE}

\section{Roy Lardenoije}


COPYRIGHT ๑ Roy Lardenoije 2017 GRAPHIC DESIGN: Eric Lardenoije PUBLISHED BY: Roy Lardenoije ISBN: 978-90-9030435-9

PRINTED BY: Drukkerij De Bink 


\section{A VENTURE INTO THE EPIGENETICS OF AGING AND ALZHEIMER'S DISEASE}

\section{Dissertation}

To obtain the degree of Doctor at Maastricht University, on authority of the Rector Magnificus Prof. dr. Rianne M. Letschert, in accordance with the decision of the Board of Deans, to be defended in public on: Thursday 7 September 2017, at 16:00 hrs.

by ROY LARDENOIJE 
PROMOTORS

Prof. dr. Bart P. F. Rutten

Prof. dr. Harry W. M. Steinbusch

CO-PROMOTORS

Dr. Daniël L. A. van den Hove

Dr. Cynthia A. Lemere (Harvard

Medical School, USA)

ASSESSMENT COMMITTEE

Prof. dr. Bert Smeets (Chair)

Dr. Alfredo Ramirez (University of

Cologne, Germany)

Dr. Bas Heijmans (Leiden University

Medical Center)

Prof. dr. Frans Verhey

Prof. dr. Klaus-Peter Lesch 

To mom and dad, for their unwavering faith in me. 


\section{Contents}

CHAPTER 1 GENERAL INTRODUCTION 15

Overview of thesis $\quad 19$

CHAPTER 2 The epigenetics of aging and neurodegeneration 25

Abstract $\quad 26$

2.1 Epigenetics $\quad 27$

2.1.1. DNA methylation 28

2.1.2. DNA demethylation 30

2.1.3. Chromatin remodeling 31

2.1.4. Non-coding RNAs 34

2.1.5. Additional epigenetic mechanisms 36

2.1.5.1. RNA editing 36

2.1.5.2. RNA methylation 38

2.1.5.3. Mitochondrial epigenetics 39

2.1.6. Epigenetic processes are interdependent 40

2.2. Aging 41

2.2.1. DNA (de)methylation in aging 4.2

2.2.2. Chromatin remodeling in aging 44

2.2.3. Non-coding RNAs in aging $\quad 46$

2.3. Neurodegeneration $\quad 50$

2.3.1. Alzheimer's disease $\quad 50$

2.3.2. Parkinson's disease 53

2.3.3. Huntington's disease 54

2.4. DNA (de)methylation in neurodegeneration 55

2.4.1. DNA (de)methylation in Alzheimer's disease 55

2.4.2. DNA (de)methylation in Parkinson's disease 60

2.4.3. DNA (de)methylation in Huntington's disease 62

2.5. Chromatin remodeling in neurodegeneration 63

2.5.1. Chromatin remodeling in Alzheimer's disease 63

2.5.2. Chromatin remodeling in Parkinson's disease 67

2.5.3. Chromatin remodeling in Huntington's disease 70

2.6. Non-coding RNAs in neurodegeneration 71

2.6.1. Non-coding RNAs and Alzheimer's disease 71

2.6.2. Non-coding RNAs and Parkinson's disease 75

2.6.3. Non-coding RNAs and Huntington's disease 78

2.7. Epigenetic-based diagnostics and therapies 78

2.7.1. Strategies targeting DNA methylation 78

2.7.1.1. Alzheimer's disease $\quad 79$

2.7.1.2. Parkinson's disease 80

2.7.2. Strategies targeting chromatin modifications 80

2.7.2.1. Alzheimer's disease $\quad 81$

2.7.2.3. Huntington's disease 82

2.7.3. Strategies targeting non-coding RNAs 84

2.7.3.1. Alzheimer's disease $\quad 85$ 
2.7.3.2. Huntington's disease

2.8. Discussion and future perspectives

CHAPTER 3 RODENT MODELS OF COGNITIVE

DISORDERS: IMPAIRMENT, AGING \& DEMENTIA

3.1. Introduction

3.2.1. Inhibition of energy/glucose metabolism

3.2.2. Cholinergic interventions

3.2.2.1. Cholinergic toxins

125

3.2.2.2. Cholinergic antagonists

126

3.2.3. Glutamatergic antagonists

3.2.4. Serotonergic intervention

3.3.1. Normal aging

130

3.3.2. Alzheimer's disease

131

3.3.2.1. APP

3.3.2.2. PS1, PS2, and PS1 $\times$ APP

131

3.3.2.3. MAPT

131

3.3.2.4. $\mathrm{PS} 1 \times \mathrm{APP} \times \mathrm{MAPT}$

132

3.3.2.5. APOE4

134

3.3.3. Parkinson's disease

134

3.3.3.1. a-Syn

3.3.3.2. DJ1(PARK7)KO

134

3.3.3.3. Parkin(PARK2)KO

135

3.3.4. Huntington's disease

135

3.3.4.1. R6/2

135

136

3.3.4.2. YAC128

136

3.3.4.3. tgHD Rats

136

3.3.5. Frontotemporal dementia

3.3.5.1. TDP43

3.3.6. Down syndrome

3.3.6.1. TgDyrk1A 137

3.4. Translation to clinics: limitations and difficulties

CHAPTER 4 EPIGENETIC MODIFICATIONS IN MOUSE

CEREBELLAR PURKINJE CELLS: EFFECTS OF AGING, CALORIC RESTRICTION, AND OVEREXPRESSION OF SUPEROXIDE DISMUTASE 1 ON 5-METHYLGYTOSINE AND 5-HYDROXYMETHYLGYTOSINE

Abstract

4.1. Introduction

4.2. Materials and methods

4.2.1. Animals

4.2.2. Tissue processing

4.2.3. Immunohistochemical detection of $5-\mathrm{mC}$ and $5-\mathrm{hmC}$

4.2.4. Analysis of 5-mC and 5-hmC immunoreactivity 
4.3.1. Qualitative analysis of 5-mC and 5-hmC

immunoreactivity

4.3.2. Semi-quantitative analysis of 5-mC and 5-hmC

immunoreactivity

156

4.4. Discussion

4.4.1. Nuclear staining pattern of $5-\mathrm{mC}$ and $5-\mathrm{hmC}$ immunoreactivity

4.4.2. 5-mC and 5-hmC levels increase with age

159

4.4.3. Diet affects age-related changes in 5-mC and 5-hmC

161

4.4.4. SOD1 does not affect age-related changes in 5-mC

and 5-hmC levels

162

4.4.5. Strengths, limitations, and future perspectives

163

4.5. Conclusion

CHAPTER 5 AGE-RELATED EPIGENETIC CHANGES IN HIPPOCAMPAL SUBREGIONS OF FOUR ANIMAL

MODELS OF ALZHEIMER'S DISEASE 171

Abstract

172

5.1. Introduction

173

5.2. Materials and methods 174

5.2.1. Animal models 174

5.2.2. Tissue preparation $\quad 175$

5.2.3. Immunohistochemistry 175

5.2.4. Analysis of 5-mC, 5-hmC and DNMT3A

immunoreactivity, and plaque load 176

5.2.5. Statistical analysis $\quad 177$

5.3. Results 178

5.3.1. Qualitative analysis of 5-mC, 5-hmC, and DNMT3A IR 178

5.3.2. Semiquantitative analysis of 5-mC, 5 -hmC, and

DNMT3A IR

5.3.3. Plaque load correlates with age-related changes in 5-mC IR

5.4. Discussion

5.4.1. Age-related decrease of DNA methylation levels in the DG and CA3 of J20 mice

5.4.2. Epigenetic markers remain stable with age in APP/PSldE9 mice

5.4.3. DNA methylation levels increase in the DG and

CA1-2 of 3xTg-AD mice

5.4.4. No age-related alterations of global epigenetic marks are detected in the hippocampus of Caribbean vervets 
CHAPTER 6 ACTIVE AMYLOID- $\beta$ IMMUNOTHERAPY

RESULTS IN EPIGENETIC CHANGES IN THE HIPPO-

CAMPUS OF AN ALZHEIMER'S DISEASE MOUSE MODEL

Abstract

6.1. Introduction

6.2. Materials and methods

6.2.1. Animals and treatment

6.2.2. Immunohistochemistry

6.2.3. Immunoreactivity of epigenetic markers and

plaque load

6.2.4. Additional characterizations

6.2.5. Data analysis

6.3.1. Fraction of hippocampal area covered by plaques

6.3.2. Integrated density measures of epigenetic markers

6.3.3. Correlation analysis of $\mathrm{AD}$ markers, epigenetic

markers, and behavior

6.4. Discussion

SUPPLEMENTARY DATA

CHAPTER 7 THE DNA METHYLOME AND

HYDROXYMETHYLOME IN ALZHEIMER'S DISEASE;

CORTICAL DYSREGULATION OF OXT, C3, ANK1

AND RHBDF2

Abstract

7.1. Introduction

7.2. Methods

7.3. Results and discussion

Supplementary methods

Subjects and tissue

preparation

Methylomic and hydroxymethylomic profiling

Quality control and data normalization

Data analysis

Expression data generation and analysis

Cross-region validation of DMRs

SUPPLEMENTARY DATA

CHAPTER 8 DETECTION OF BLOOD METHYLOMIC MARKERS ASSOCIATED WITH CLINICAL FEATURES OF ALZHEIMER'S DISEASE; AN EXPLORATORY

APPROACH

Abstract

8.1. Introduction

8.2. Methods

8.2.1. Subjects

8.2.2. CSF tau and amyloid beta

8.2.3. Hippocampal volume 
8.2.4. Methylomic profiling 269

8.2.5. Processing of array data 269

8.2.6. Data analysis $\quad 270$

8.3.1. Alzheimer's disease $\quad 271$

8.3.2. Mini-mental state examination scores 276

8.3.3. Cerebrospinal fluid phosphorylated tau

amyloid- $\beta$ ratio $\quad 280$

8.3.4. Hippocampal volume 281

8.4. Discussion 282

SUPPLEMENTARY DATA 290

CHAPTER 9 IDENTIFICATION OF PRECLINICAL

METHYLOMIC BLOOD MARKERS ASSOCIATED

WITH CONVERSION TO ALZHEIMER'S DISEASE 307

Abstract 308

9.1. Introduction 309

9.2. Methods 310

9.2.1. Subjects $\quad 310$

9.2.2. Processing of array data 310

9.2.3. Data analysis 311

9.3. Results 313

9.3.1. Methylomic differences before the development

of dementia 313

9.3.2. Methylomic differences during conversion to dementia 320

9.4. Discussion 325

SUPPLEMENTARY DATA 331

CHAPTER 10 GENERAL DISCUSSION 335

10.1. Summary of key findings 336

10.2. Limitations 339

10.3. Future perspectives $\quad 341$

CHAPTER 11 SUMMARY 34.5

CHAPTER 12 SAMENVATTING 351

CHAPTER 13 VALORIZATION 357

CHAPTER 14 CURRICULUM VITAE 363

CHAPTER 15 LIST OF PUBLICATIONS 367

Published 368

Submitted and in preparation 369

CHAPTER 16 ACKNOWLEDGMENTS 371

CHAPTER 17 ABBREVIATIONS 381 




\section{GENERAL INTRODUCTION}


Alzheimer's disease (AD) is devastating for mind and body, and aging, something all humans do, is the most important risk factor [1]. AD is mainly feared for the development of cognitive problems such as memory loss, of which it is the most common cause, although clinical symptoms may also include a wide array of aberrations in other functional abilities of the brain and other organs [2, 3]. Apart from the heavy burden on patients, caregivers and/or loved ones, it also presents a massive economic burden, which can be deduced from the estimated 36.5 million cases of dementia worldwide, most of which due to AD [4, 5]. As a consequence of the lack of treatment options and the progressive, yet relatively slow, course of disease, patients with $A D$ require long-time care. This results in high healthcare expenses; amounting to annual costs of $\$ 215$ billion in 2010 in the United States, which is expected to double by 2040 [6]. However, it is interesting to note that a study found that the prevalence of dementia actually has decreased in recent years, which is thought to be the result of increased education levels [7]. Nevertheless, while breakthroughs in other clinical fields have led to a decrease in deaths due to common age-related diseases such as heart failure, stroke, and cancer, death due to AD has risen to the sixth most common cause of death in the United States [8]. With the increasing geriatric population, $A D$ thus presents a titanic problem for modern society. To understand why no viable strategies to halt or slow disease progression have been developed since the discovery of the disease over a century ago, it is important to get an idea of the complexity of the disease.

$A D$ as it is currently defined, actually comprises two distinct forms of dementia, linked by the occurrence of protein aggregates, the extracellular senile or amyloid plaques, and the intracellular neurofibrillary tangles. The first form, which was originally described by Alzheimer in 1906 , is early onset $A D$, characterized by the development of dementia before the age of 65 years [9]. Although the clinical manifestations of $A D$ dementia had already been described by the ancient Greeks [10], and plaques were first observed by Redlich [11], Alzheimer is thought to be the first to describe tangles [12]. Alzheimer's description of the disease led his colleague Kraepelin to name the disease after him [13]. Interestingly, since this early onset variant of $A D$ is quite rare, it did not receive much scientific attention [14], as did late-onset senile dementia, which was thought to be caused by stiffening of the blood vessels [10]. The second type of $A D$, late-onset $A D$, was not defined until the late 1960 s, made possible by the discovery of AD pathology in most cases of senile dementia $[15,16]$. Thus, with the additional identification of lateonset $A D$ and the technical advances of the last decades, the brunt of $A D$ research was actually performed in the past 25-30 years [14]. 
Naturally, its diagnosis being based on the presence of plaques and tangles, initial research widely focused on these lesions, leading to the identification of amyloid- $\beta(A \beta)$ and phosphorylated tau as the main constituents of plaques and tangles, respectively [10]. In parallel, causative genes in families with familial, usually early-onset, $A D$ were investigated, leading to the identification of mutations in the gene encoding the precursor protein of $A \beta, A P P$, and in the presenilin genes PSEN1 and PSEN2, which are involved in $A \beta$ processing [10]. These early-onset familial variants of $A D$ can already start to manifest in the late 30s [17]. Although familial AD is much less common than sporadic, usually late-onset, $\mathrm{AD}$, the implication of the APP and PSEN genes in familial $A D$ has been pivotal for the development of transgenic animal models of $A D$ and mechanistic studies of $A \beta$ and tau neurotoxicity [10]. Of note, no mutations in MAPT, the gene encoding tau, have been associated with $A D$, although other forms of dementia are associated with MAPT mutations [10].

Sporadic AD has a much less obvious genetic origin, however, as no mutations in APP, PSEN1, or PSEN2 are associated with this form of AD. For some time $A P O E$ allele 4 was the only genetic risk factor associated with sporadic $A D$ [18], and remains the factor conveying the highest risk for developing sporadic AD [19]. The advent of large-scale genomewide association studies (GWAS) has led to the identification of several additional loci associated with sporadic $A D$ [20,21], the largest of which included 74,046 subjects and identified 20 AD-associated loci in genes involved in $A \beta$ and tau processing, immune system function, endocytosis, and lipid transport [22]. Even though early- and late-onset $A D$ are both associated with plaques and tangles, it remains largely unknown how most of the genetic risk factors associated with late-onset $A D$ lead to these pathological hallmarks [1].

Apart from genetic susceptibility, there are likely also other factors at play that affect the manifestation of AD. Indeed, a wide array of conditions and environmental exposures have been identified that may influence, either in a negative or positive sense, the development of $A D[1,23]$. Pre-existing conditions increasing the risk for developing $A D$ include obesity, hypertension, stroke, and diabetes [1]. In general, vascular risk factors, factors affecting the brain's glucose metabolism, and stress can also be considered as risk factors for AD [24-26]. The link between stress and $A D$ is thought to be mediated through the immune system [1,27]. In line with these risk factors, it was found that regular physical activity and a healthy diet, including fish (omega-3), but with limited sugar and saturated fat intake, may be protective against AD dementia [28-31]. Another protective lifestyle factor is being frequently engaged in mentally complex and demanding activities [32]. This may lead to more extensive 
dendritic connections than required for normal functioning. Such a 'cognitive reserve' may reinforce the threshold for cognitive impairment in the face of progressive neurodegeneration, and may explain why the presence of $A D$ pathological hallmarks in the brain is no guarantee for clinical dementia.

Interestingly, the exceedingly complex and possibly life-long etiopathogenesis of $A D$ is exemplified by a study showing a decrease in the prevalence of late-onset $A D$ due to an increase in education levels [7]. Even more perplexing may be the finding that having a high sense of purpose in life is associated with lower AD pathology and cognitive impairment [33]. Indeed, general psychological well-being is associated with lower AD pathology [34]. It has furthermore been found that symptoms of affective psychopathology precede cognitive problems in patients with $A D$ [35]. Affective issues may thus drive neuropathology, be an early sign of neuropathology, or both. The first possibility raises the critical question whether treatment strategies based solely on the neuropathology, neglecting the affective component of $A D$, are ineffective by design. Could, in case of late-onset $A D$, the pre-clinical identification of patients at risk of conversion to AD dementia, followed by psychological and behavioral therapy, possibly complemented with pharmacotherapeutic interventions focused on affective dysregulation, aid in forestalling the development of $A D$ dementia?

To investigate such questions and advance the field of $A D$ research, it must first be possible to identify people who will develop AD dementia, which in turn requires insight into the pre-clinical stages of the disease. From the brief history of $A D$ research above, it appears that sporadic $A D$ may arise due to the concerted effects of genetic susceptibility and exposure to environmental risk factors [36]. Recent years have seen an extensive deepening of our understanding of epigenetic regulation of gene expression, followed by a widespread invasion of epigenetics studies in complex afflictions such as depression, psychosis, and neurodegeneration [37-39]. Epigenetics has moved from being regarded as a programmed process of gene silencing during cell differentiation and development, to being identified as a delicate, crucial regulatory system involved in translating environmental exposures to changes in gene expression and even poses a means of transgenerational inheritance that transcends genetic mutations $[40,41]$. Such complex regulation is established through multiple layers of effector mechanisms, including DNA and chromatin modifications, and non-coding RNAs [39]. As a mediator between genetic and environmental factors, epigenetic markers may thus offer a unique window into the etiopathogenesis of sporadic $A D$ before the onset of clinical signs. With age being the most important risk factor for developing $A D$, it will also be critical to investigate how 
age-related epigenetic alterations may facilitate age-related diseases

and, thus, how healthy aging differs epigenetically from pathologic aging leading to diseases such as AD.

\section{Overview of thesis}

The research compiled in this thesis can be divided in three parts. The first two chapters comprise reviews providing a broad introduction to epigenetics, aging, $A D$, and the study thereof. The following three chapters involve investigations of global levels of epigenetic markers in relation to aging and $A D$, making use of animal models. Finally, three epigenome-wide association studies (EWAS) in relation to AD are presented, covering both the brain and the blood.

While this general introduction only briefly touches epigenetic regulation itself, CHAPTER 2 offers an in-depth discussion of the various layers of epigenetic control over transcription and translation. Additionally, it provides an overview of epigenetic dysregulation in aging and neurodegeneration, specifically in $A D$, Parkinson's disease, and Huntington's disease, as well as an outlook on therapeutic strategies based on epigenetic principles. It concludes with an exploration of the challenges the field of epigenetics research currently faces and how these may be tackled in future research.

As many critical insights into cognitive dysfunction, as observed in AD, and the exploration of therapeutic interventions can be attributed to animal model research, CHAPTER 3 constitutes a concise overview of available rodent animal models for the study of cognitive impairment and neurodegenerative diseases. The chapter covers both pharmacological and transgenic animal models and also presents options beyond AD.

With aging being the principal risk factor for AD, CHAPTER 4 investigates age-related changes in global DNA methylation and hydroxymethylation levels in mouse cerebellar Purkinje cells, cells that are particularly vulnerable to aging. It was also studied whether the known interference of caloric restriction and increased anti-oxidant levels with age-related deterioration might be mediated through epigenetic mechanisms, as reflected in the global DNA methylation and hydroxymethylation levels.

Global age-related epigenetic alterations in rodent animal models expressing mutant $A P P$, both mutant $A P P$ and $P S E N 1$, or a combination of mutant $A P P, P S E N 1$ and $M A P T$, and a nonhuman primate model of sporadic $A D$ are investigated in CHAPTER 5. This study focused on the hippocampus, one of the primary brain regions affected in $A D$. 
In CHAPTER 6 the epigenetic ramifications of active $A \beta$ immunotherapy in APPswe/PS1dE9 mice are investigated. Specifically, the effects of lowering $A \beta$ plaque pathology on global DNA methylation and hydroxymethylation levels, as well as DNA methyltransferase (DNMT) $3 \mathrm{~A}$, responsible for de novo DNA methylation, were determined in the hippocampus. Correlations between these epigenetic players and measures of cognition were also established.

CHAPTER 7 moves from animal models to the human brain, comparing the methylome and hydroxymethylome of AD patients and age-matched healthy controls. Differentially methylated, hydroxymethylated, and unmodified cytosine positions were investigated, as well as altered genomic regions.

CHAPTER 8 features an exploration of the blood methylome associated with $A D$, while also looking at the overlap with methylomic changes observed in subjects with mild cognitive impairment (MCl). The relationship of the $A D$ methylomic profile with other established measures of clinical $A D$, including cognitive performance, levels of $A \beta$ and tau in the cerebrospinal fluid, and hippocampal volume, was also investigated.

The final study included within this thesis, as described in CHAPTER 9, ventured into the pre-clinical stage of AD and identified the blood methylomic signature associated with conversion to $A D$ dementia. Additionally, methylomic markers showing differential timerelated changes in converters and non-converters were observed, which may represent signs of vulnerability or resilience to conversion to $A D$ dementia. The identified markers not only offer mechanistic insight into $A D$ etiopathogenesis, but may also act as potential biomarkers.

In its totality, the work gathered in this thesis covers a wide spectrum of studies, including multiple species, tissues, and techniques, geared towards advancing our understanding of the epigenetic involvement in $A D$ versus healthy aging, and in addition provide novel diagnostic candidates.

\section{REFERENCES}

[1] Robinson M, Lee BY, Hane FT. Recent Progress in Alzheimer's Disease Research, Part 2: Genetics and Epidemiology. J Alzheimers Dis 2017; 57: 317-330.

[2] Bediou B, Ryff I, Mercier $B$, et al. Impaired Social Cognition in Mild Alzheimer Disease.

$J$ Geriatr Psychiatry Neurol 2009; 22: $130-140$.

[3] Budson AE, Solomon PR. New criteria for Alzheimer disease and mild cognitive impairment: implications for the practicing clinician. Neurologist 2012; 18: 356-363.

[4] Ferri CP, Prince M, Brayne C, et al. Global prevalence of dementia: a Delphi consensus study. Lancet 2005; 366: 21122117.

\section{[5] Sosa-Ortiz AL,} Acosta-Castillo I, Prince MJ. Epidemiology of dementias and Alzheimer's disease. Arch Med Res 2012; 43: 600-608.

[6] Hurd MD, Martorell P, Delavande A, et al. Monetary Costs of Dementia in the United States. N Engl J Med 2013; 368: 1326-1334.

[7] Langa KM, Larson EB, Crimmins EM, et al. A Comparison of the Prevalence of Dementia in the United States in 2000 and 2012. JAMA Intern Med 2017; 177: 51-58.

[8] Alzheimer's Association. 2015 Alzheimer's disease facts and figures. Alzheimers Dement 2015; 11: 332-84.

[9] Alzheimer A. Über eine eigenartige Erkrankung der Hirnrinde. Allg Zeitschrift fur 
Psychiatr und Psych Medizin

1907; 64: 146-148.

[10] Hardy J, Saura CA, Choi SY, et al. A hundred years of Alzheimer's disease research. Neuron 2006; 52: 3-13.

[11] Redlich F. Uber miliare

Sklerose der Hirnrinde bei seniler Atrophie. Jarbucher fur Psychiatr und Neurol; 17.

[12] Perusini G. The nosographic value of some characteristic histopathologic findings in senility. Riv Ital di Neuropathol Psichiatr ed Elettroterapia 1991; 4: 193-213. [13] Kraepelin E. Das senile und prasenile Irreseine. Psychiatr ein Lehrb fur Stud und Arzte 1910; 593-632.

\section{[14] Altman J. Alzheimer:}

100 Years and Beyondhttp://www. alzforum.org/news/conferencecoverage/alzheimer-100-yearsand-beyond (2007, accessed 11 May 2017).

[15] Blessed G, Tomlinson

BE, Roth M. The association between quantitative measures of dementia and of senile change in the cerebral grey matter of elderly subjects. Br J Psychiatry 1968; 114: 797-811.

[16] Tomlinson BE, Blessed G, Roth M. Observations on the brains of demented old people. J Neurol Sci 1970; 11: 205-42. [17] Martin JJ, Gheuens J, Bruyland M, et al. Early-onset Alzheimer's disease in 2 large Belgian families. Neurology 1991; 41: 62-8.

[18] Corder EH, Saunders

AM, Strittmatter WJ, et al. Gene dose of apolipoprotein $\mathrm{E}$ type 4 allele and the risk of Alzheimer's disease in late onset families.

Science 1993; 261: 921-3.

[19] Farrer LA, Adrienne

Cupples L, Van Duijn CM, et

al. Apolipoprotein E genotype

in patients with alzheimer's

disease: Implications for the risk of dementia among relatives. Ann

Neurol 1995; 38: 797-808.

[20] Medway C, Morgan

K. Review: The genetics of Alzheimer's disease; putting flesh on the bones. Neuropathol Appl

Neurobiol 2014; 40: 97-105.

[21] Tosto G, Reitz C.

Genome-wide Association Studies in Alzheimer's Disease: A Review. Curr Neurol Neurosci Rep 2013; 13: 381.

[22] Lambert J-C, Ibrahim-

Verbaas CA, Harold D, et

al. Meta-analysis of 74,046

individuals identifies 11 new susceptibility loci for Alzheimer's disease. Nat Genet 2013; 45: 1452-1458.

[23] Chouliaras L, Rutten BPF, Kenis G, et al. Epigenetic regulation in the pathophysiology of Alzheimer's disease. Prog Neurobiol 2010; 90: 498-510. [24] Li J, Wang YJ, Zhang $\mathrm{M}$, et al. Vascular risk factors promote conversion from mild cognitive impairment to Alzheimer disease. Neurology 2011; 76 : 1485-1491.

[25] Baker LD, Cross DJ, Minoshima S, et al. Insulin Resistance and Alzheimerlike Reductions in Regional Cerebral Glucose Metabolism for Cognitively Normal Adults With Prediabetes or Early Type 2 Diabetes. Arch Neurol 2011; 68: 51-7.
[26] Sotiropoulos I, Catania C, Pinto LG, et al. Stress acts cumulatively to precipitate Alzheimer's disease-like tau pathology and cognitive deficits. J Neurosci 2011; 31: 7840-7. [27] Cohen S, JanickiDeverts D, Doyle WJ, et al. Chronic stress, glucocorticoid receptor resistance, inflammation, and disease risk. Proc Natl Acad Sci U S A 2012; 109: 5995-9. [28] Buchman AS, Boyle PA, Yu L, et al. Total daily physical activity and the risk of $A D$ and cognitive decline in older adults. Neurology 2012; 78: 1323-1329. [29] Hardman RJ, Kennedy $\mathrm{G}$, Macpherson $\mathrm{H}$, et al. Adherence to a MediterraneanStyle Diet and Effects on Cognition in Adults: A Qualitative Evaluation and Systematic Review of Longitudinal and Prospective Trials. Front Nutr 2016; 3: 22. [30] Francis $\mathrm{H}$, Stevenson $R$. The longer-term impacts of Western diet on human cognition and the brain. Appetite 2013; 63: 119-28.

[31] Huang TL. Omega-3 Fatty Acids, Cognitive Decline, and Alzheimer's Disease: A Critical Review and Evaluation of the Literature. J Alzheimer's Dis 2010; 21: 673-690.

\section{[32] Stern Y. Cognitive} reserve in ageing and Alzheimer's disease. Lancet Neurol 2012; 11 : 1006-1012.

[33] Boyle PA, Buchman AS, Wilson RS, et al. Effect of purpose in life on the relation between Alzheimer disease pathologic changes on cognitive function in advanced age. Arch Gen 
Psychiatry 2012; 69: 499-505.

[34] Chen ST, Siddarth P, Saito NY, et al. Psychological well-being and regional brain amyloid and tau in mild cognitive impairment. Am J Geriatr Psychiatry 2014; 22: 362-9.

[35] Babulal GM, Ghoshal N, Head D, et al. Mood Changes in Cognitively Normal Older Adults are Linked to Alzheimer Disease Biomarker Levels. Am J Geriatr Psychiatry 2016; 24: 1095-1104. [36] Lahiri DK, Maloney B, Zawia NH. The LEARn model: an epigenetic explanation for idiopathic neurobiological diseases. Mol Psychiatry 2009; 14: 992-1003.

[37] Januar V, Saffery R, Ryan J. Epigenetics and depressive disorders: a review of current progress and future directions. Int J Epidemiol 2015; 44: 1364-1387.

[38] Pishva E, Kenis G, van den Hove D, et al. The epigenome and postnatal environmental influences in psychotic disorders. Soc Psychiatry Psychiatr Epidemiol 2014; 49: 337-348. [39] Lardenoije $R$, latrou $A$, Kenis $\mathrm{G}$, et al. The epigenetics of aging and neurodegeneration.

Prog Neurobiol 2015; 131: 21-64. [40] Goldberg AD, Allis $C D$, Bernstein E. Epigenetics: A Landscape Takes Shape. Cell 2007; 128: 635-638.

[41] Nagy C, Turecki G. Transgenerational epigenetic inheritance: an open discussion. Epigenomics 2015; 7: 781-790. 




\section{THE EPIGENETICS OF AGING AND NEURODE- GENERATION}

ROY LARDENOIJEA ${ }^{A}$ ARTEMIS IATROUA ${ }^{\text {, GUNTER KENIS }}{ }^{\text {, }}$ KONSTANTINOS KOMPOTIS ${ }^{B}$, HARRY W. M. STEINBUSCH ${ }^{A}$, DIEGO MASTROENI ${ }^{A, ~}{ }^{\mathrm{C}}$, PAUL COLEMAN ${ }^{ }$, CYNTHIA A. LEMERE ${ }^{\mathrm{D}}$, PATRICK R. HOF $^{\ddagger}$, DANIËL L. A. VAN DEN HOVEA, F, *, BART P. F. RUTTEN ${ }^{A, *}$

A SCHOOL FOR MENTAL HEALTH AND NEUROSCIENCE (MHENS), DEPARTMENT OF PSYCHIATRY AND NEUROPSYCHOLOGY, MAASTRICHT UNIVERSITY, UNIVERSITEITSSINGEL 50, 6200 MD MAASTRICHT, THE NETHERLANDS ${ }^{\mathrm{B}}$ CENTER FOR INTEGRATIVE GENOMICS, UNIVERSITY OF LAUSANNE, GENOPODE BUILDING, 1015 LAUSANNE-DORIGNY, SWITZERLAND

' L.J. ROBERTS ALZHEIMER'S DISEASE CENTER, BANNER SUN HEALTH RESEARCH INSTITUTE, 10515 W. SANTA FE DRIVE, SUN CITY, AZ 85351, USA ${ }^{D}$ CENTER FOR NEUROLOGIC DISEASES, DEPARTMENT OF NEUROLOGY, BRIGHAM AND WOMEN'S HOSPITAL, HARVARD MEDICAL SCHOOL, 77 AVENUE LOUIS PASTEUR, BOSTON, MA 02115, USA

E FISHBERG DEPARTMENT OF NEUROSCIENCE AND FRIEDMAN BRAIN INSTITUTE, ICAHN SCHOOL OF MEDICINE AT MOUNT SINAI, ONE GUSTAVE L. LEVY PLACE, NEW YORK, NY 10029, USA F LABORATORY OF TRANSLATIONAL NEUROSCIENCE, DEPARTMENT OF PSYCHIATRY, PSYCHOSOMATICS AND PSYCHOTHERAPY, UNIVERSITY OF WUERZBURG, FUECHSLEINSTRASSE 15, 97080 WUERZBURG, GERMANY

* THESE AUTHORS CONTRIBUTED EQUALLY TO THIS WORK. 


\section{Abstract}

Epigenetics is a quickly growing field encompassing mechanisms regulating gene expression that do not involve changes in the genotype. Epigenetics is of increasing relevance to neuroscience, with epigenetic mechanisms being implicated in brain development and neuronal differentiation, as well as in more dynamic processes related to cognition. Epigenetic regulation covers multiple levels of gene expression; from direct modifications of the DNA and histone tails, regulating the level of transcription, to interactions with messenger RNAs, regulating the level of translation. Importantly, epigenetic dysregulation currently garners much attention as a pivotal player in aging and age-related neurodegenerative disorders, such as Alzheimer's disease, Parkinson's disease, and Huntington's disease, where it may mediate interactions between genetic and environmental risk factors, or directly interact with disease-specific pathological factors. We review current knowledge about the major epigenetic mechanisms, including DNA methylation and DNA demethylation, chromatin remodeling and noncoding RNAs, as well as the involvement of these mechanisms in normal aging and in the pathophysiology of the most common neurodegenerative diseases. Additionally, we examine the current state of epigenetics-based therapeutic strategies for these diseases, which either aim to restore the epigenetic homeostasis or skew it to a favorable direction to counter disease pathology. Finally, methodological challenges of epigenetic investigations and future perspectives are discussed.

KEYWORDS: Epigenetics; aging; neurodegeneration; Alzheimer's disease; Parkinson's disease; Huntington's 


\subsection{Epigenetics}

Conrad Hal Waddington coined the term "epigenetics" in 1942, an event commonly viewed as the birth of epigenetics as it developed from a phenomenon to an immensely studied branch of science [1]. A merger between the terms "genetics" and "epigenesis", the concept of epigenetics in a way represents the association of two views on development that have been clashing at least since the time of Hippocrates and Aristotle [2]. Hippocrates proposed what became known as the preformationist view of development; all parts of a mature organism are already present at the embryonic stage, albeit in a miniature stage, and they simply grow during development. Aristotle argued against this preformationist view, providing an alternative explanation that lies at the foundation of the epigenesis concept: embryonic development involves the formation of new parts. After numerous scientific discoveries, these views evolved over the centuries. A contemporary preformationist would hold that all that is needed to generate a mature organism is its genetic code, whereas a supporter of epigenesis would argue that the genome only holds the information of building blocks - but that how these are put together depends on environmental influences. The contemporary perspective of "epigenetics" is that of the field of science that studies how changes in gene expression occur without changes in the DNA sequence [1]. Such changes can be induced by environmental factors, while some are more programmed, as seen during cell differentiation. As such, these epigenetic alterations can be highly stable, such as those resulting from genetic imprinting, or dynamic such as the epigenetic changes associated with memory. Many epigenetic modifications can be inherited through mitosis and some have even found to be transgenerational [3-6]. Thus, whereas genetic alterations usually reflect permanent changes of the DNA sequence, epigenetic changes are mediated through processes that are in principle reversible [7]. While environmental influences can potentially alter the phenotype of an organism by interacting with and by acting on both the genome and epigenome [8], the reversible nature of epigenetic changes makes them more suitable as candidates for clinical interventions [9]. Over the past decade there have been ample studies investigating the contributions of epigenetic modifications to aging and age-related neurodegenerative diseases, including Alzheimer's disease (AD), Parkinson's disease (PD) and Huntington's disease (HD). The epigenetic machinery covers multiple levels of control, including DNA methylation and hydroxymethylation, chromatin remodeling, and noncoding RNA (ncRNA) regulation [10]. See Figure 1 for a general overview of the epigenetic mechanisms discussed below. 


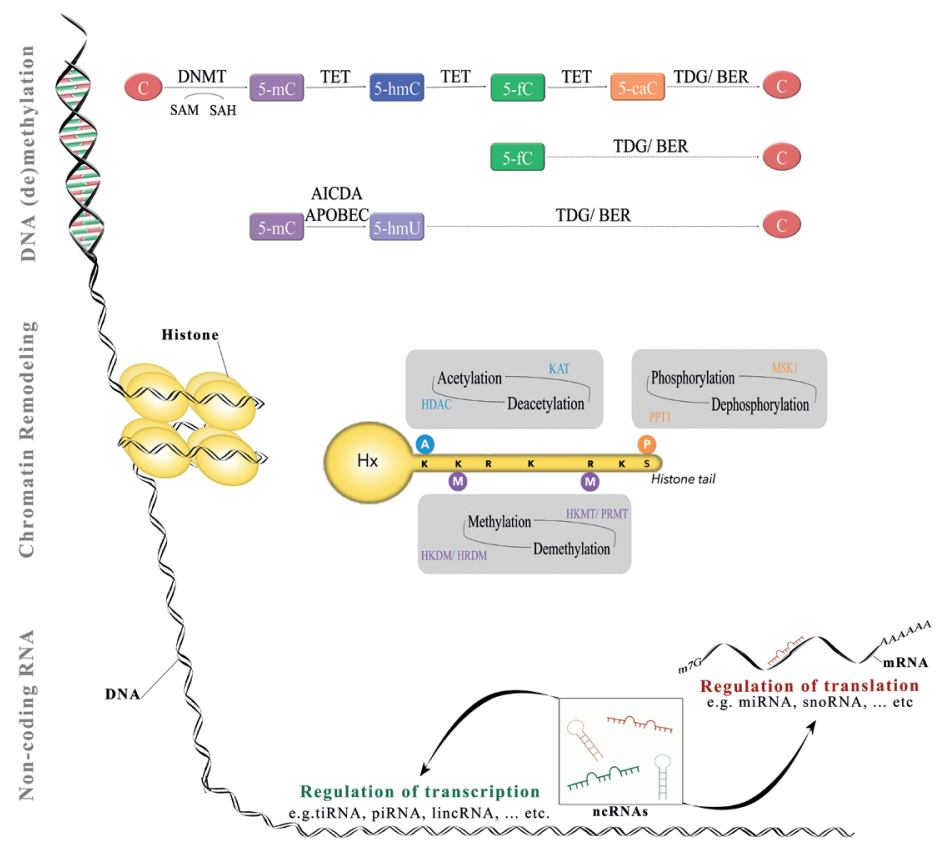

\subsubsection{DNA methylation}

The best characterized epigenetic modification, DNA methylation involves the addition of a methyl group at the 5 position on the pyrimidine ring of cytosines, creating 5-methylcytosine $(5-\mathrm{mC})$ [11]. These modifications primarily occur at cytosine-phosphate-guanine (CpG) islands. Recently, however, non-CpG methylation has received increased attention [12]. Apart from cytosines, there are also reports of guanine and adenine methylation, resulting in 7-methylguanine (7-mG) and 3-methyladenine, respectively [13]. In this review, however, DNA methylation refers exclusively to 5-mC, unless mentioned otherwise. Generally, DNA methylation is associated with transcriptional repression and is mostly found in heterochromatin [14], while the euchromatin typically contains low amounts of methylated DNA. Some genes, however, are suspected to show enhanced expression when hypermethylated [15]. Additionally, DNA methylation within gene bodies (the transcribed portion of a gene) has been implicated in alternative splicing $[16,17]$. How DNA methylation exactly affects gene transcription is highly dependent on the location in or around the gene [18]. In promoter regions, methylated DNA can directly disrupt the transcriptional process by interfering with the binding of transcription factors [19]. Additional repression can be established through the attraction of methyl-CpG-binding domain proteins (MBDs) and subsequent activation of the histone tail modifying machinery,
FIGURE 1. The three levels of epigenetic regulation. The upper section summarizes DNA methylation and demethylation processes, the middle section summarizes the most important chromatin remodeling processes, and the bottom section summarizes non-coding RNA regulation.

ABBREVIATIONS: 5-caC, 5-carboxylcytosine; 5 -fC, 5 -formylcytosine; 5-hmC, 5-hydroxymethylcytosine; 5-hmU, 5-hydroxymethyluracil; 5-mC, 5-methylcytosine; A, acetyl modification; AICDA, activation-induced cytidine deaminase; APOBEC, apolipoprotein B mRNA editing enzyme, catalytic polypeptide-like protein; BER, base excision repair; $\mathrm{C}$, cytosine; DNMT, DNA methyltransferase; $\mathrm{H}$, histone; HDAC, histone deacetylase; HKDM, histone lysine demethylase; HKMT, histone lysine methyltransferase; HRDM, histone arginine demethylase; K, lysine; KAT, lysine acetyltransferase; lincRNA, large intergenic non-coding RNA; M, methyl modification; miRNA, micro RNA; MSK1, mitogen- and stress-activated protein kinase 1; ncRNA, non-coding RNA; P, phosphate modification; piRNA, piwi-interacting RNA; PPT1, protein phosphatase 1; PRMT, protein arginine methyltransferase; R, arginine; SAH, S-adenosylhomocysteine; SAM, S-adenosylmethionine; snoRNA, small nucleolar RNA; TDG, thymine DNA glycosylase; TET, ten-eleven translocation; tiRNA, transcription initiation RNA; tRNA, transfer RNA. 
leading ultimately to chromatin compaction [20]. How gene expression is enhanced through methylation of gene bodies remains unclear.

Although DNA methylation is the most stable epigenetic modification, the DNA methylation profile, or 'methylome', is highly dynamic [21-23]. DNA methylation profiles are, at least partly, heritable, both after cell division, as well as in a transgenerational fashion [24, 25]. Heritable DNA methylation needs to be copied to the newly synthesized DNA strand, a process that is referred to as maintenance DNA methylation. The addition of completely new DNA methylation marks is called de novo DNA methylation. DNA methyltransferases (DNMT) are responsible for maintenance and de novo DNA methylation [26]. There are four known types of DNMTs; DNMT1, DNMT2, DNMT3A and DNMT3B, all of which use S-adenosylmethionine (SAM) as the methyl donor [19, 26]. Note, however, that DNMT2 was actually found to be a RNA methyltransferase [27]. Furthermore, there is another DNMT, DNMT3-like (DNMT3L), which exhibits no enzymatic activity, but detects unmethylated lysine 4 of histone $\mathrm{H} 3$ tails (H3K4) and recruits or activates DNMT3A [28]. Interestingly, the DNMT3B splice variants DNMT3B3 and DNMT3B4 are also inactive and play a regulatory role in de novo DNA methylation [29]. DNMT1 is the most common variant in somatic cells and primarily involved in maintenance DNA methylation, and DNMT3A and DNMT3B are responsible for de novo DNA methylation [30]. DNMT3A and DNMT3B isoforms are expressed in a more cell-type-specific manner [12, 30].

It is worth mentioning that the methyl donor of the DNMTs, SAM, is generated through a complex cycle and is the methyl donor of numerous additional transmethylation reactions [31, 32]. This cycle starts with the conversion of tetrahydrofolate (THF) to 5,10-methylenetetrahydrofolate (MTHF) by vitamin B6-dependent serine hydroxymethyltransferase (SHMT), and the subsequent conversion of 5,10-MTHF to 5-MTHF by B2dependent MTHF reductase (MTHFR). 5-MTHF acts as the methyl donor for the methylation of homocysteine (Hcy), producing methionine, by cobalamin-dependent methionine synthase (MetH). SAM is subsequently generated from methionine by methionine adenosyltransferase (MAT). During methyltransferase reactions SAM is converted to S-adenosylhomocysteine (SAH), which is further hydrolyzed to Hcy and adenosine by $\mathrm{SAH}$ hydrolase $(\mathrm{SAHH})$. Folate serves as cardinal input for this cycle and the proper elimination of Hcy and adenosine is important to maintain homeostasis. For instance, global DNA hypomethylation could be induced through folate deficiency and high levels of Hcy [31].

As DNA methylation can be relatively simple and robustly assessed using genomic DNA, it has been the primary focus of human epidemiological epigenetic research [33]. Early investigations into DNA methylation 
showed its cardinal importance in the proliferation and differentiation of neural stem cells [34]. More recently, it has been established that DNA methylation is pivotal for synaptic plasticity, neuronal repair, neuronal survival, and learning and memory [35-37]. Such dynamic processes are more dependent on de novo methylation, although the importance of maintenance DNA methylation should not be underestimated, as a loss of DNMT1 was shown to result in increased histone acetylation, a disruption of the nuclear organization and eventually cell death [35, 38-41]. Since these are factors disturbed in a neurodegenerative state, DNA methylation is a valid target when investigating neurodegeneration.

\subsubsection{DNA demethylation}

While DNA methylation is a well-established epigenetic mechanism, the existence of active DNA demethylation in animals has long been a point of controversy [42]. Observations such as high levels of DNMTs in nondividing cells [43] and a significant decrease in methylated DNA levels when DNA methylation is blocked [14, 22], despite the stability of the 5-mC mark, have led to an avid search for the players responsible for an active demethylation process. This search generated several mechanisms, including an RNA-dependent pathway by which the methyl group is removed from 5-mC, a pathway involving the nucleotide excision repair mechanism, and a base excision repair based pathway [21, 44-47].

Although still a point of discussion, the base excision repair pathway is a prime candidate as the primary road to demethylation in postmitotic neurons [45], which does not exclude the possibility of multiple overlapping demethylation pathways. DNA demethylation is thought to be initiated by the oxidation of 5-mC into 5-hydroxymethylcytosine (5$\mathrm{hmC}$ ) by the ten-eleven translocation (TET) proteins [48, 49]. There are 3 TET proteins, TET1, TET2 and TET3, which are differentially expressed and regulated throughout the body [50]. Interestingly, in the last few years 5 -hmC was shown to be an important epigenetic marker that is functionally different from 5-mC [51]. While DNA hydroxymethylation is generally associated with increased gene activity, work by Jin et al. [52] indicates that this correlation does not always hold and depends on the location of 5-hmC in the gene and the CpG content. 5-hmC is present in most tissues and cell types, but is especially enriched in the brain [53], with cerebellar Purkinje cells exhibiting some of the highest levels [54]. Furthermore, in the frontal cortex, 5 -hmC is selectively enriched in promoter and intragenic regions [52]. Interestingly, 5-hmC levels are particularly low in stem cell-rich areas $[53,55]$. In addition to the formation of 5 -hmC, it has recently been discovered that TET enzymes can further oxidize 5 -hmC to 5 -formylcytosine $(5-\mathrm{fC})$, and 5 -fC to 5 -carboxylcytosine 
(5-caC) [49]. Although it is generally accepted that $5-\mathrm{hmC}$ is a functional epigenetic marker, such a role remains to be established for 5 - $\mathrm{fC}$ and 5 -caC $[56,57]$. Apart from oxidation, $5-\mathrm{mC}$ and $5-\mathrm{hmC}$ can be deaminated instead, by either activation-induced cytidine deaminase (AICDA) or apolipoprotein $B$ mRNA editing enzyme, catalytic polypeptide-like protein (APOBEC), resulting in thymidine or 5-hydroxymethyluracil (5-hmU) $[48,58]$. 5-caC, thymidine or 5-hmU present a mismatch in the base pairing of the DNA sequence (5-caC:G, T:G or 5-hmU:G, respectively). Such a mismatch can be detected and mended through the removal of the transformed bases by thymidine or uracil glycosylases [48, 59-61]. Alternatively, 5-fC and 5-caC can be changed back to cytosine by removal of formic acid or decarboxylation, respectively [49]. In addition to the aforementioned enzymes, the growth arrest and DNA damage 45 (GADD45) proteins are crucially involved in directing the activity of these enzymes, thereby assisting in the localization of demethylation activity to specific gene promoters [44, 62-64]. Clearly, the exact mechanisms underlying DNA demethylation remain to be elucidated.

\subsubsection{Chromatin remodel- ing}

Chromatin can be seen as a string of nucleosomes, which mainly consist of DNA and the histones around which it is wrapped. There are five types of histone proteins; $\mathrm{H} 2 \mathrm{~A}, \mathrm{H} 2 \mathrm{~B}, \mathrm{H} 3$, and $\mathrm{H} 4$ forming the octameric core of the nucleosome, and $\mathrm{H} 1$ serving as a linker and stabilizer, binding to DNA among nucleosomes $[32,65]$. The conformation of these histones largely determines the accessibility of the DNA for transcription, and can be adjusted through reversible modifications of their $\mathrm{N}$-terminal tails. Such modifications include lysine $(\mathrm{K})$, arginine $(\mathrm{R})$ or histidine (His) methylation [66], K acetylation [67], serine (S), threonine (T) or tyrosine (Y) phosphorylation [68], ubiquitination [69], adenosine diphosphate (ADP)-ribosylation [70], crotonylation [71], hydroxylation [72], proline isomerization [73] and $\mathrm{K}$ SUMOylation [74], which together constitute the histone code. A specific state of the histone code may either lead to gene activation or silencing [75]. The endless possible combinations of the various modifications and target sites, allows the histone code for highly versatile fine tuning of gene expression, but is also critically involved in DNA repair and replication [76]. Owing to the attention that chromatinmodifying enzymes have received over the past years, many enzymes that had been identified as modifying histones, were later found to have many additional substrates. In view of this, Allis et al. [77] have proposed an updated nomenclature that better reflects the full spectrum of functions of these enzymes. For instance, histone (lysine) acetyltransferases 
(HATs) were renamed to lysine acetyltransferases (KATs). As, however, this new nomenclature has only sporadically been adopted, both old and new names will be stated to avoid confusion.

Acetyl coenzyme A serves as donor of the acetyl group, which is transferred to lysines of histone tails by KATs [78]. There are a multitude of proteins with KAT activity, which can be divided into five main groups, comprising KAT2A (or general control of amino acid synthesis [GCN] 5), KAT2B (or P300/CBP-associated factor [PCAF]), KAT6-8, cyclic adenosine monophosphate (CAMP) response element-binding protein binding protein (CREBBP or CBP) and adenovirus early region $1 \mathrm{~A}(\mathrm{E} 1 \mathrm{~A})$ binding protein P300 (EP300 or P300) [79].

Currently, there are 18 known histone deacetylases (HDACs) in humans, generally subdivided into four classes; class I (HDACs 1, 2, 3 and 8), class Ila (HDACs 4, 5, 7 and 9), class IIb (HDACs 6 and 10), class III (sirtuins [SIRTs] 1, 2, 3, 4, 5, 6 and 7) and class IV (HDAC11) [80]. Although their name suggests that histones are the primary targets of HDACs, phylogenetic analysis indicates that the evolutionary development of HDACs preceded that of histones [81]. Indeed, over 50 non-histone targets of HDACs have been identified, including proteins important for proliferation, migration, and cell death [82-84]. Thus, as for HATs, it was suggested that HDACs should be more appropriately referred to as lysine deacetylases [85]. The different HDACs fulfill many different roles, either by affecting gene expression or by directly regulating protein functioning. Class I HDACs for instance, are thought to play a general role in cell survival and proliferation, whereas class II HDACs have a more tissue-specific role [86-92]. The SIRTs further differ from the other HDACs in that their activity is nicotine adenine dinucleotide (NAD+)-dependent, whereas the other classes require the presence of zinc. Not all SIRTs are even primarily deacetylases. This has led to the suggestion that SIRTs should be classified as deacylases, as opposed to deacetylases [93]. Although class I and class IV HDACs are mainly nuclear, class Ila shuttles between the nucleus and cytoplasm, and class IIb is primarily cytoplasmic. The SIRTs of class III are most varied in their localization, and can be either expressed in the nucleus (SIRTs 1, 2, 6 and 7), cytoplasm (SIRTs 1 and 2) or mitochondria (SIRTs 3, 4 and 5) [94]. The expression of the different HDACs is also highly region- and cell-type-specific, for instance, while HDAC2 is expressed in most brain regions, it is predominantly active in mature neurons and weakly or not in progenitor and glial cells [95, 96].

Methylation of histone tails happens at $\mathrm{K}$ or $\mathrm{R}$ residues of $\mathrm{H} 3$ or $\mathrm{H} 4$, and is executed by histone lysine methyltransferases (HKMTs) and protein arginine methyltransferase (PRMT), respectively, whereas demethylation 
is performed by histone lysine demethylases (HKDMs) and histone arginine demethylases (HRDMs) [97, 98]. The other histone modifications are less well characterized. Phosphorylation and dephosphorylation of histones is executed by protein kinases, such as mitogen- and stress-activated protein kinase (MSK)-1, and protein phosphatases, such as protein phosphatase (PPT) 1, respectively [99, 100]. Histone phosphorylation is dynamic in function; $\mathrm{H} 3$ phosphorylation, for instance, marks open chromatin during active gene expression, whereas during mitosis this marker associates with condensed chromatin [101]. Ubiquitination can either enhance or inhibit gene expression, whereas SUMOylation is primarily thought to suppress transcription [98].

The actual effect and interplay between these modifications are complex, and depend on the type of histone protein and the specific amino acid that is modified, and a combination of certain modifications can even have a function that is different from that of these modifications in isolation [75, 101-104]. For instance, acetylation of K 9 (H3K9ac) and 14 (H3K14ac) of $\mathrm{H} 3$, or tri-methylation at $\mathrm{K} 4$ of $\mathrm{H} 3$ (H3K4me3), H3K36me, H3K79me, H3R2me, H3R17me, H3R26me and H4R3me are associated with gene activation, whereas H3K9me2/3, H3K27me3, H3R8me, H4K2Ome3 and H4R3me are generally associated with gene repression [98]. Interestingly, in case of histone methylation, the number of attached methyl groups also matters, as the previously mentioned H3K9me3, H3K27me3 and H4K20me3 marks that are associated with gene repression, all have monomethylated counterparts that are associated with gene activation [105]. Of note, recent studies mapping histone tail modifications to genomic regions found that many transposable elements (TEs) are enriched with certain histone marks [106-108] and it has therefore been suggested that TEs may attract certain histone marks to induce heterochromatic and euchromatic states, or serve as boundary elements that prevent the propagation of such states [109].

In addition to histone modifications, chromatin remodeling also occurs through adenosine triphosphate (ATP)-dependent multi-protein chromatin remodeling complexes. Four distinct remodeling complex families have been identified, including the $\mathrm{Brg} 1 / \mathrm{hBrm}$ associated factor (BAF; previously known as switching defective/sucrose nonfermenting [SWI/ SNF]), imitation SWI (ISWI), chromodomain, helicase, DNA-binding (CHD) and inositol requiring 80 (INO) families [110-112]. These complexes, or remodelers, can bind nucleosomes, disrupt nucleosomeDNA binding, and then move, destabilize, eject or restructure them, using ATP hydrolysis as energy source. In doing this, remodelers can either induce transcriptional activation or repression, through the recruitment of coactivator or corepressor complexes. The different remodeling complexes are defined by their ATPase subunits, but variation in their 
remaining subunit composition, possibly altering the DNA and protein binding properties of the complex, allows for great diversity, leading to cell-type specific roles $[113,114]$. Furthermore, multiple functionally different versions of a complex may be present within one cell [115]. The BAF complex, consisting of at least 15 subunits, is of particular interest, as it is the only chromatin remodeling complex with a neuron-specific subunit, BAF53b. The BAF complex is thought to play an important role in neuronal development and functioning, with unique subunit compositions in embryonic stem cells, neuronal progenitors and mature neurons [112]. The BAF53b subunit was shown to be important for dendritic development in vitro and long term memory in mice [116]. See the excellent review by Hargreaves and Crabtree [111] for a detailed discussion of the different chromatin remodeling complexes.

\subsubsection{Non-coding RNAs}

Until recently, it was widely believed that most of the human genome consisted of so-called 'junk', or nonfunctional DNA. It was later revealed that almost the whole genome is transcribed, but that only about $2 \%$ is actually translated into proteins [117]. Most of the 'junk' is functional and is primarily involved in the regulation of gene expression, usually in the form of ncRNAs. There are many types of ncRNAs, including microRNAs (miRNAs), small interfering RNAs (siRNAs), small nuclear RNAs (snRNAs), small nucleolar RNAs (snoRNAs), small Cajal body-specific RNAs (scaRNAs), piwi-interacting RNAs (piRNAs), splice junctionassociated RNAs (spliRNAs), small modulatory RNAs (smRNAs), repeatassociated small interfering RNAs (rasiRNAs), transcription initiation RNAs (tiRNAs), promoter-associated short RNAs (PASRs), transcription start site-associated RNAs (TSSa-RNAs), promoter upstream transcripts (PROMPTS), ribosomal RNAs (rRNAs), transfer RNAs (tRNAs), and small double-stranded RNAs (dsRNAs) [118-122]. These are small ncRNAs (sncRNAs), of $<200$ nucleotides, whereas there are also long ncRNAs (IncRNAs), which can exceed 100,000 nucleotides, often including TE-derived sequences that may confer specific protein or nucleic acid interacting properties [123]. Examples of IncRNAs are intergenic ncRNAs (lincRNAs), natural antisense transcripts (NATs), ncRNA expansion repeats, promoter-associated RNAs (PARs), and enhancer RNAs (eRNAs) [124-128]. The sncRNAs fulfill various functions, including infrastructural (rRNAs, tRNAs and snRNAs) and regulatory roles (miRNAs, siRNAs, snoRNAs, piRNAs and spliRNAs), whereas the IncRNAs are primarily regulatory. Interestingly, IncRNAs are expressed in a highly cell-specific manner, may undergo alternative splicing, and may even have protein-coding isoforms [129-132]. Alternatively, some mRNAs may function as trans-acting regulatory RNAs [133-135]. In terms of 
epigenetics, ncRNAs are cardinally involved in gene expression control, in the silencing of TEs, X-chromosome inactivation, alternative splicing, and DNA imprinting [136-138]. Additionally, some IncRNAs have been proposed to direct epigenetic enzymes to their target sites [125, 139, 140], while others are thought to bind and sequester other epigenetic players, such as DNMTs and miRNAs, thereby hampering their activity $[132,140,141]$.

The best characterized of the ncRNAs are miRNAs. These begin their lives as primary miRNAs, after which they are cleaved by ribonuclease type III Drosha to form precursor miRNAs (pre-miRNAs) [142]. These pre-miRNAs are then transported from the nucleus to the cytosol by Exportin-5, where Dicer makes the final adjustment to produce doublestranded mature miRNAs. Mature miRNAs span 21-25 nucleotides and regulate protein production through an RNA interference pathway, involving the association of one of the strands of the miRNA duplex with the RNA-induced silencing complex (RISC) [143, 144]. They interfere with gene expression through binding to messenger RNA (mRNA), usually to the 3' untranslated region (UTR), which hampers the initiation of translation and affects mRNA stability. MiRNAs can potentially regulate the translation of multiple genes, whereas genes can also be regulated by several miRNAs, as the sequences of the miRNA and its target are not required to be a perfect match for interference to take effect [145, 146]. While siRNAs are processed and function similarly, they require stricter matching with their target sequence when compared to miRNAs [147]. Apart from interacting with RISC, some miRNAs have been observed to promote mRNA translation and gene transcription, by binding to gene promoter regions [148, 149]. There are numerous miRNAs, many of which are expressed according to strict spatial and temporal patterns. Currently, there are 1881 precursors and 2588 mature human miRNAs registered in the fast growing miRBase [150]. While expressed throughout the whole human body, the brain is especially enriched in miRNAs, suggesting an important role for them in neuronal development, functioning, and aging $[151,152]$. Their biological role has been further characterized, and reviewed by Santosh and colleagues, as well as Qu and Adelson [153, 154]. Both the reviews extend beyond the aforementioned functions, and present the key role of ncRNA in RNA splicing, transcriptional, posttranscriptional, and translational regulation by either binding directly to transcriptional factors or by generating siRNAs, that consequently interact with the translational machinery. 


\subsubsection{Additional epigenetic mechanisms}

DNA methylation, chromatin remodeling, and ncRNAs represent the best-studied epigenetic mechanisms, especially in relation to aging and neurodegenerative diseases. Epigenetic regulation goes much deeper, however, and also includes the rising subfields of RNA editing, RNA methylation and mitochondrial epigenetics, which will be briefly touched upon in this section, but will not be further discussed in relation to aging and neurodegeneration due to the as of yet extremely limited findings in this respect. Clearly, more studies on the role of these additional layers of epigenetic regulation in aging and neurodegeneration are warranted.

\subsubsection{RNA editing}

The observation of discrepancies in genomic and cDNA sequences led to the discovery of RNA editing [155]. The finding that RNA can be edited, a process that seems particularly important in the brain, adds another layer to the transcriptional and post-transcriptional regulation of gene expression. It has even been proposed that a dramatically increased RNA editing capacity was crucial for the evolution of the mammalian brain as it may function as a mechanism driving phenotypic adaptability, which ultimately led to the superior cognitive abilities of humans [156, 157]. In support of this, roughly 35 times more RNA editing is observed in humans compared to mice. This surplus appears to be mainly directed to retrotransposed Alu elements that are primate-specific and constitute $10.5 \%$ of the human genome [158-162].

In contrast to RNA and DNA modifications, RNA editing involves a change in RNA sequence by deamination of either adenosine $(A)$ or cytosine (C), to inosine (I) $[155,163]$ or uracil $(U)[164,165]$, respectively. A to I editing is performed by adenosine deaminases that act on RNA (ADARs), while $C$ to $U$ editing is carried out by APOBECs. APOBECs are related to AICDAs, which targets DNA and is pivotal in the generation of the immunoglobulin repertoire [166]. Although not much is known about the targeting, regulation and functional impact of ADARs and APOBECs, they are thought to be evolved from adenosine deaminases that act on tRNAs (ADATs) and thus bind double-stranded RNA regions, such as those seen in hairpin formations that are also present in tRNAs [155, 164].

ADAR1 and ADAR2 are ubiquitously expressed and they appear to be enriched in the brain, while the expression of ADAR3 seems to be restricted to the brain. A to I RNA editing has been observed in coding 
transcripts, for instance leading to changes in the amino acid sequence of glutamate and serotonin receptors. Most editing, however, happens to noncoding sequences, such as miRNAs [167-169] and transposonderived repetitive sequences [170], suggesting that RNA editing not only directly affects gene expression, but also indirectly by regulating other epigenetic players [156]. APOBECs, together with overall RNA editing, appear to have undergone a substantial expansion over the course of evolution, with APOBEC3 being especially favored in humans with eight orthologs, compared to one in mice $[165,171,172]$. Although it has been suggested that these enzymes have evolved to combat retrotransposons and endogenous retroviruses [173, 174], there is also evidence indicating that these elements actually have been harnessed as epigenetic regulators involved in growth and differentiation, including the generation of neuronal diversity [175-177].

Recently, the implication of RNA editing in the etiopathogenesis and progression of neurodegenerative disorders as well as normal aging processes has gained momentum and the few available studies begin to elucidate this connection. The majority of these studies are focusing on aging. Sebastiani et al. [178] observed that 5 single nucleotide polymorphisms (SNPs) in the ADAR encoding genes ADARB1 and $A D A R B 2$ are associated with extreme longevity in 4 independent human studies. The observation of the critical role of ADARs in aging was also verified in a CAENORHABDITIS ELEGANS (C. ELEGANS) model with loss of function of $A D R 1$ and $A D R 2$ ( $A D A R B 1$ and $A D A R B 2$ orthologues), which had a $50 \%$ decrease in lifespan. After this study a lot of RNA editing targets were discovered, such as Gabra2, Cyfip2, Kcnal, Flna, Blcap, Cog3, Neat1, Neat2, Malat1 and Pisd-ps1 that are differentially edited in the aging murine and human brain. Among them, Cyfip2 and Pisd-ps1 have gained considerable attention [179-181]. Nicholas et al. [180] demonstrated that $A$ to I editing declines with age in humans, in a gene-specific manner, resulting in downregulation of an ADAR2 target gene, Cyfip2, which is responsible for synaptic maintenance. In the hippocampal formation of aged mice Stilling et al. [181] showed that the altered RNA editing levels of Pisd-ps1 results in higher editing frequency with age and thus upregulation of gene expression.

In case of $A D$, Akbarian et al. [182] observed a decrease in the RNA editing levels of glutamate receptor (GluR) 2 in the prefrontal cortex of $A D$ human brains. Rechavi's team [183] following the aforementioned research line, examined the GluR2 Q/R RNA editing levels in the hippocampus of AD human brains, where they found a decrease in the aforementioned levels in $\mathrm{AD}$ samples in comparison to controls. Additionally, they showed lower GluR2 Q/R RNA editing in the hippocampi of APOE\&4 carriers. The mRNA expression of ADARs was 
also investigated in this specific study; unexpectedly no differences were found in the hippocampus but a 37\% decrease of ADAR2 mRNA expression was noticed in the caudate. Finally, Akbarian et al. [182] extended the study on HD where they also showed a decrease in GluR2 RNA editing levels in the striatum.

\subsubsection{RNA methylation}

Although the discovery of methylated RNA was done decades ago [184, 185], over a hundred RNA nucleotide modifications have been identified across different organisms [186, 187]. In eukaryotes the best-studied mRNA modifications are N6-methyladenosine (m6A) and 5-mC, which mainly occur at 3' UTRs and stop codon sites [188]. As in DNA, 5-hmC has also been observed in RNA [189]. m6A is the most prevalent mRNA modification in mammals and has also been observed in tRNAs, rRNAs and snoRNAs [190-197]. In humans the m6A modification shows high tissue specificity, with the highest levels occurring in the brain, in transcripts such as Bdnf, Dscam, Lis1 and Ube3a [198]. In mice and humans m6A methyltransferase-like protein (METTL) 3 is responsible for the post-transcriptional m6A RNA modification [199]. Additionally, METTL14 and Wilm's tumor-associated protein (WTAP) have been shown to interact with METTL3 and are thought to be additional components involved in RNA methylation [200, 201]. Just as DNA methylation, the identification of $\mathrm{m} 6 \mathrm{~A}$ demethylases fat mass and obesity-associated protein (FTO) and AlkB, alkylation repair homolog (ALKBH) 5 [202, 203], indicates that RNA methylation is a dynamic regulatory mechanism. FTO, a dioxygenase, demethylates RNA via a similar oxidation procedure as is employed by the TET enzymes that are thought to be involved in DNA demethylation, namely through the generation of intermediates N6hydroxymethyladenosine (hm6A) and N6-formyladenosine ( $6 \mathrm{~A})$, before being reversed to $A$ [204]. Although these intermediates remain stable for several hours, no separate regulatory roles for hm6A and $f 6 A$ have been reported yet. ALKBH5 is thought to remove m6A directly, without the generation of intermediates [203]. Although the exact regulatory functions of m6A RNA methylation remain to be elucidated, its main occurrence at 3' UTRs and stop codons has been suggested to indicate a role in switching genes on or off [205]. Alternatively, the observation that players involved in m6A RNA methylation were located at splice sites suggests that the m6A RNA modification may modulate splicing [188]. More recent findings show a relation between the m6A modification and mRNA degradation, as m6A selectively binds human YTH domain family (YTHDF) 2 proteins, which can bind and target mRNA to decay sites, such as processing bodies (P-bodies) [206]. Other members of the YTH domain family, YTHDF1 and YTHDF3 also selectively bind m6A modified 
RNA. Another study, however, indicated that m6A does not lead to RNA decay through the YTHDF2 pathway, but by interacting with miRNAs, and that the removal of $6 \mathrm{~A}$ promotes human antigen $\mathrm{R}(\mathrm{HuR})$ binding, a protein that protects against RNA decay [201]. These studies suggest that the m6A mark may dynamically regulate mRNA lifetime.

An alternative pathway of RNA methylation involves the versatile regulatory ncRNAs snoRNAs, which can guide 2'-O-methylation and pseudo-uridylation of RNA transcripts, including mRNAs [207]. 2'-O-methylation is important for the functioning of certain rRNAs [208], but also determines the guide strand and targeting specificity of siRNAs [209]. Apart from their role in RNA modifications, snoRNAs can be further processed into snoRNA-derived small RNAs (sdRNAS), which are similar to miRNAs [210-213].

The pathway that most closely resembles DNA methylation involves DNMT2, which, despite its name, transfers methyl groups to cytosines in RNA [27, 214]. Apart from tRNA, the exact substrates of DNMT2 still need to be identified. Nevertheless, DNMT2 has been implicated in brain development and retrotransposon silencing [215, 216]. Other known RNA methylation modifications include N1-methyladenosine (m1A) and N1methylguanine $(\mathrm{m} 1 \mathrm{G})$, which occur mainly in tRNAs and are thought to enhance tRNA stability, and m1G also decoding accuracy [217-220].

The only reported studies connecting RNA methylation to aging, as well as neurodegenerative disorders, were performed by Giordano et al. [221] and Thomas et al. [13], respectively. Bellizzi's team studied the methylated cytosine residues in two mitochondrial genes, 12S and $16 \mathrm{~S}$ rRNA and they showed that the methylation levels of 12S rRNA are decreased with age in males. Thomas et al. [13], while attempting to develop a novel method for detecting trace amounts of $7-\mathrm{mG}$ in biological samples, observed differential methylation patters in murine HD models and significantly increased levels of 7-mG in postmortem human HD brain samples.

\subsubsection{Mitochondrial epigenetics}

Apart from the nuclear genome, human cells can harbor thousands of copies of the mitochondrial genome. Both the nuclear and mitochondrial genome consist of DNA, but there are some striking differences [222]. The mitochondrial genome is only $16 \mathrm{~kb}$ long, contains 37 genes without introns and is much more prone to mutations than the nuclear genome. With respect to epigenetics, its regulation seems to be less complex, as mitochondrial DNA (mtDNA) is thought not to be wrapped around histones 
and not to contain $\mathrm{CpG}$ islands; the $435 \mathrm{CpG}$ sites in the mitochondrial genome are almost evenly dispersed.

Over 40 years ago, methylated mtDNA was discovered in loaches, and it was shown there is DNMT activity in mitochondria that is independent from DNMT activity outside mitochondria [223, 224]. Later, mtDNA methylation was also observed in humans [225], and a mitochondrial DNMT (mtDNMT) was discovered [226]. Of note, however, more recent studies cast doubt on the general notion that DNA methylation is the prime epigenetic mechanism at work in mitochondria. For instance, Hong et al. [227] were unable to detect CpG methylation in the genome of human mitochondria, whereas Choi et al. [228] report on the possible existence of mitochondrial histones, and Barrey et al. [229] found miRNAs in mitochondria. Nevertheless, there are also many recent reports supporting the presence of methylated mtDNA and even hydroxymethylated mtDNA [230-233], showing that it is not always located at CpG sites [234, 235], and that mtDNA methylation plays a role in mitochondrial gene regulation [236, 237]. Clearly, these rapid developments within the field of mitochondrial epigenetics warrant further attention. In the recent study of Dzitoyeva et al. [231] not only the hydroxymethylation of mtDNA was reported but they also demonstrated that solely the levels of hydroxymethylated mtDNA reduce with age in the frontal cortex of mice. This decrease in $5-\mathrm{hmC}$ is associated with an increase in complex I components (ND2, ND4, ND4L, ND5, ND6) in the same area. Furthermore, they observed region-specific differential expression of epigenetic players; the mRNA levels of TET2 and TET3, which are also responsible for the hydroxymethylation of mtDNA, are only increased in the cerebellum, whereas the mRNA levels of mtDNMT1 decrease solely in the frontal cortex.

\subsubsection{Epigenetic processes are interdependent}

The epigenetic processes of DNA (de)methylation, chromatin remodeling, and miRNAs do not act independently, but closely interact to form a complex, multilayered regulatory system that can dynamically fine-tune gene expression. DNA methylation stability in promoter regions, for instance, is enforced by methyl CpG binding protein (MeCP) 1 , which also binds the nucleosome remodeling and histone deacetylase (NuRD) core and cyclin-dependent kinase 2 associated protein (CDK2AP) 1; forming a protein complex not only able to stabilize DNA methylation, but also to modify the histone code [238, 239]. MeCP1 is attracted to methylated DNA through its affinity for MBD2, which directly binds to 
DNA methylated at $\mathrm{CpG}$ sites. As such, DNA methylation and histone modifications act in concert to regulate gene expression, through interference with transcription factor binding and chromatin compaction [19]. Another interesting interplay, between DNMT3A-dependent DNA methylation and Polycomb-group (PcG)-dependent H3K27me3 marks was discovered by Wu et al. [240]. They showed that DNMT3A activity at non-promoter regions correlated with increased expression of neurogenic genes, by interfering with PcG binding and H3K27me3mediated gene repression. In contrast, DNMT3A activity at the promoter regions inhibited gene expression. Alternatively, MBD1 can antagonize $\mathrm{H} 3 \mathrm{~K} 4 \mathrm{me}$, leading to chromatin compaction. DNA methylation can thus in a bottom-up fashion induce changes on the chromatin level [241]. The other way around is also possible, as exemplified by Detich et al. [242]. They showed that increases in $\mathrm{H} 3$ acetylation can induce DNA demethylation, and thereby gene expression in vitro. Conversely, HDAC activity is thought to inhibit gene expression through the induction of DNA methylation [243]. There are additional complex interactions between miRNAs and other components of the epigenetic machinery. Where some miRNAs regulate the expression of proteins involved in epigenetic regulation, the expression of various miRNAs themselves is also subject to factors such as DNA methylation and histone modifications [244]. For example, miRNA 184 (miR-184), involved in the regulation of proliferation and differentiation of neural stem cells, is surrounded by $\mathrm{CpG}$ islands, attracting MBD1, which can suppress its expression as described above [241].

\subsection{Aging}

Before delving into the aberrant epigenetic processes associated with neurodegeneration, it is important to consider the epigenetic changes associated with normal aging and related hallmarks, such as oxidative stress, as these can already be quite dramatic. Bocklandt et al. [245] for instance, devised a method to determine the age of an individual based on the methylation of specific sites in the EDARADD, TOM1L1 and NPTX2 genes. At these sites a linear correlation between methylation and age was observed, allowing for a prediction of age with an average accuracy of 5.2 years. Horvath later devised an even more accurate method to determine the 'DNAm age', based on the methylation status of $353 \mathrm{CpGs}$ [246]. The DNAm age of Horvath has a chronological age correlation of about 0.96 and an error of 3.6 years and is applicable in many different tissue and cell-types. Note, however, that epigenetic processes are not the only players involved in aging. According to the "free radical theory of aging", oxidative stress is thought to play an integral role in the aging process [247]. Oxidative stress refers to the 
generation of reactive oxygen species (ROS), which are damaging to proteins, nucleic acids and lipids and are known to also affect epigenetic players [248]. Furthermore, as aging is the prime risk-factor of most neurodegenerative diseases, it is possible that age-related processes, including epigenetic alterations and oxidative stress, facilitate the development of these illnesses.

\subsubsection{DNA (de)methylation in aging}

Early research established that DNA methylation plays a crucial role during development. Later studies identified aging to be a pivotal modulator of the epigenome. The DNAm age of Horvath offers some interesting insights in this respect [246]. Of the $353 \mathrm{CpG}$ sites used to predict the DNAm age, 193 got hypermethylated and 160 got hypomethylated with age, and most are associated with genes involved in cell death and survival, cell growth and proliferation, organismal and tissue development, and cancer. Additionally, DNAm age shows a logarithmic relationship with chronological age until adulthood, and a linear relationship later in life, indicating that the epigenetic clock 'ticks' faster during growth and development. While highly accurate in most tissues, Horvath found that the DNAm age was consistently lower in tissues which may be renewed through the presence of stem cells, such as skeletal and heart muscle. It was, however, also observed that DNAm age does not reflect cellular senescence, as it highly correlated with chronological age in short and long lived cells, as well as immortal cells. As could be expected, embryonic stem cells appeared to have a DNAm age close to zero. Interestingly, the DNAm age of induced pluripotent stem cells did not differ significantly from that of embryonic stem cells. While the clock CpGs used for Horvath's DNAm age are enriched in cancer genes, there are some important differences between normally aging and cancerous tissue. In general, cancer tissue exhibits an accelerated DNAm age. Due to the heterogeneity of cancer types, however, general statements about its use should be interpreted with caution, as for example thyroid cancer progression negatively correlates with age acceleration. Additionally, Horvath observed that an increased DNAm age may promote genomic stability, as he found in several cancer types a negative relation between DNAm age acceleration and somatic mutations. He proposes that cancer triggers a hypothetical epigenetic maintenance system that promotes genetic stability, a process that is dependent on P53, as mutations in the TP53 gene are associated with a lower DNAm age acceleration. Interestingly, in glioblastoma multiforme TP53 mutations appear to be associated with an increased DNAm age 
acceleration. While it thus seems that in general cancer is associated with an increased DNAm age profile and aging with global DNA hypermethylation, neurodegenerative diseases such as AD and PD are associated with global DNA hypomethylation [26, 249]. Note, however, that the DNAm age itself has not yet been assessed in neurodegenerative tissue. Nevertheless, despite having age as a common risk factor, cancer and age-related neurodegenerative diseases seem to involve (at least partly) different epigenetic dysregulation or compensatory mechanisms.

Taking a more specific approach, Siegmund et al. [250] investigated the DNA methylation status of $50 \mathrm{CpG}$ islands associated with genes involved in brain growth and development in subjects of various ages, and they observed a robust and progressive increase in DNA methylation of multiple genes with age (Table 1). They also confirmed that a rise in DNA methylation typically results in a decline of corresponding mRNA levels. In addition, it was observed that DNMT3A was expressed across all ages, supporting the notion that DNA methylation can be dynamically altered throughout the lifespan. Interestingly, in relation to $A D$, the promoter region of the amyloid- $\beta$ precursor protein (APP) gene becomes hypomethylated with age [251]. Additionally, binding sites for granulocyte chemotactic factor (GCF), known to repress CG-rich promoters, and interaction sites for specificity factor (SP) 1, which enhances gene expression in the tau promoter, became hypoand hypermethylated, respectively, with age, decreasing its overall expression. This finding suggests that certain age-related epigenetic changes might facilitate the development of AD. Although expressed across all ages, levels of DNMT3A and 5-mC actually increase with age in the dentate gyrus (DG), cornu ammonis (CA) 1-2, and CA3 regions of the mouse hippocampus [252, 253], which is in line with previous reports [254]. An interesting study by Oliveira et al. [255] showed that hippocampal levels of DNMT3A2, an isoform of DNMT3A, decrease with age and that this decrease correlated with age-related cognitive decline in mice. Importantly, experimental restoration of DNMT3A2 levels alleviated this age-related cognitive impairment. Additionally, Hernandez et al. [256] investigated 27,000 CpG sites in brain samples of varying ages and detected a general positive correlation between age and methylation levels. In contrast, it was found that expression levels of DNMT1 decrease with aging in human fetal lung fibroblasts, which would be in support of reports of global DNA hypomethylation with aging and cell senescence, including non-coding regions and repetitive sequences in the blood [254, 257, 258]. Mazin [259] put forward an interesting hypothesis, proposing a DNA methylation-dependent aging process. This model is based on the observation that methylation of cytosines may induce $\mathrm{C}>\mathrm{T}$ mutations, which is suggested to result in age-related genome disintegration, and eventually cell apoptosis, organism aging and 
death. Due to the age-related increase in $5-\mathrm{mC}>\mathrm{T}$ transitions, this model predicts an age-related depletion of $5-\mathrm{mC}$.

Note, however, that DNA methylation profiles are not only known to be different between different tissues, regions and cell-types, but that these also seem to be differentially affected by the aging process [260-262]. An interesting study by Fraga et al. [263], investigating DNA methylation and histone acetylation during the lifetime of monozygotic twins, illustrates that the epigenome not only changes with age, but also that differences in the epigenome might explain phenotypic disparity in genotypically identical individuals.

In addition to the age-related increases of DNMT3A and 5-mC levels, a significant age-related increase in 5-hmC levels was found in the DG, CA1-2, and CA3 regions of the mouse hippocampus [264], which is in line with previous investigations into the spatial and temporal distribution of $5-\mathrm{hmC}$ in the brain $[265,266]$. While some of the genes that exhibit age-related increases in 5-hmC levels are associated with age-related neurodegenerative diseases [266], further investigations are required to elucidate the functional consequences of these findings, taking into account the differential functions of the $5-\mathrm{mC}$ and $5-\mathrm{hmC}$ markers. Table 1 summarizes the age-related alterations regarding DNA (de)methylation.

\subsubsection{Chromatin remodel- ing in aging}

Apart from widespread changes in the neuronal DNA methylation profile throughout the lifespan, the neuronal histone code also undergoes age-related alterations (Table 2). An example is the observation of lower levels of histone acetylation with aging in vitro [270], and an age-related progressive decline of $\mathrm{H} 3$ and $\mathrm{H} 4$ methylation [271] and monoacetylated $\mathrm{H} 4$ levels, discovered in neurons from the rat cerebral cortex [272]. Apart from detecting decreased levels of $\mathrm{H} 3 \mathrm{~K} 9 \mathrm{ac}$ and increased levels of H3S10p [273], Nakamura et al. [274] detected decreased acetylation of extranuclear proteins. In senescence-accelerated prone mouse 8 (SAMP8) brains it was shown that many histone marks are altered with age [275] (Table 2). In rats, however, some of these markers were observed not to undergo significant age-related changes [276]. These not always compatible findings between species illustrate the necessity of translating results regarding epigenetic changes to the human situation. Apart from changes in specific histone methylation marks, the HKMTs polycomb repressive complex member Bmi1 (PRC1) and polycomb repressive complex member enhancer of zeste homolog
TABLE 1. Epigenetic dysregulation in aging: DNA (de)methylation. 个 indicates increased levels and $\Downarrow$ indicates decreased levels.

ABBREVIATIONS: 5 -hmC, 5 -hydroxymethylcytosine; 5-mC, 5 -methylcytosine; BS, bisulfite sequencing; CA, cornu ammonis; ChIP; chromatin immunoprecipitation; DG, dentate gyrus; DNMT, DNA methyltransferase; HPLC: high-performance liquid chromatography; IHC, immunohistochemistry; MS-PCR, methylation specific-PGR; PGR, polymerase chain reaction; RT-PCR, real time-PCR. 
(EZH) 2 (Drosophila) (PRC2) have been observed to decrease with cell senescence, a common, but limited, in vitro model of aging, while the HKDM jumonji domain containing (JMJD) 3 increased [277, 278]. The balance between PcG and JMJD3 gene expression is in turn thought to be regulated by HDAC activity.

The finding that HDAC2 expression increases with age in the mouse hippocampus is in line with findings of decreased acetylation levels [279]. Age-associated reduction in acetylated $\mathrm{H} 4$ is thought to reduce chromatin structural plasticity and may result in a decreased accessibility of the DNA for repairing enzymes and other regulatory factors [272, 280].

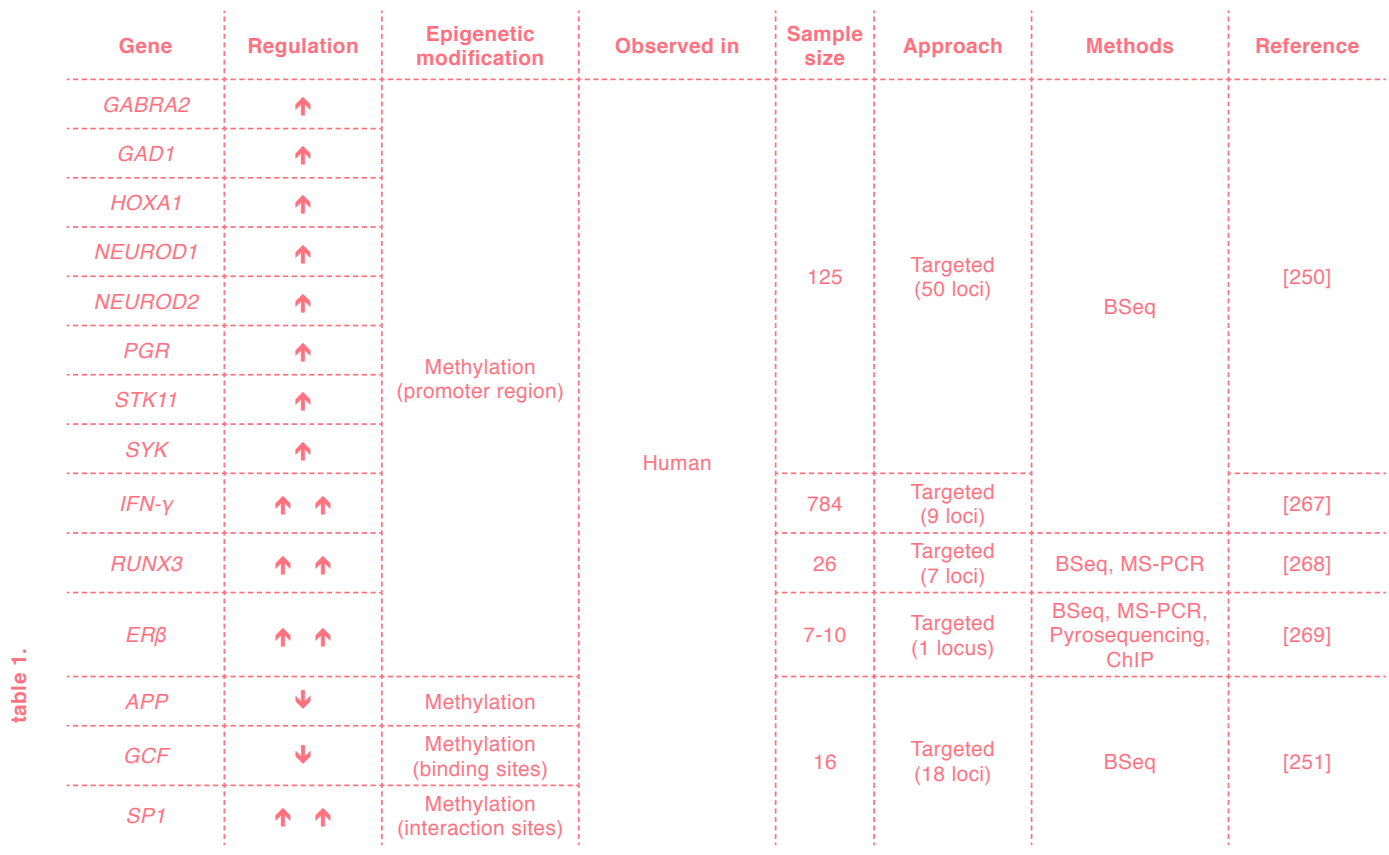

\begin{tabular}{|c|c|c|c|c|c|c|}
\hline $\begin{array}{c}\text { Protein/ } \\
\text { (Hydroxy) } \\
\text { Methylated } \\
\text { base }\end{array}$ & Regulation & Modification in & Observed in & $\begin{array}{l}\text { Sample } \\
\text { size }\end{array}$ & Methods & Reference \\
\hline DNMT1 & $\downarrow$ & & $\begin{array}{l}\text { Fibroblasts } \\
\text { (fetal lung) }\end{array}$ & - & $\begin{array}{l}\text { DNMT assay } \\
\text { ChIP, RT-PCR }\end{array}$ & {$[254,258]$} \\
\hline DNMT3A2 & $\downarrow$ & & $\begin{array}{c}\text { Mice } \\
\text { (12 month old) }\end{array}$ & 15 & $\mathrm{IHC}$ & [255] \\
\hline DNMT3A & $\uparrow$ & Expression & Mouse & 240 & $\mathrm{IHC}$ & [252] \\
\hline 5-mC & $\uparrow$ & & $\begin{array}{l}\text { hippocampus } \\
\text { (DG, CA1-2 }\end{array}$ & & & {$[253]$} \\
\hline 5-hmC & $\uparrow$ & & and 3) & 4 & HPLC & {$[265,266]$} \\
\hline
\end{tabular}


Other studies are more specific, pointing towards a role of deregulated H4K12ac in age-related memory impairment [281], a negative influence of HDAC2 on synaptic plasticity and memory formation through the suppression of neuronal gene transcription [95, 282], and a dependence of histone acetylation on citrate levels [283], which decline in the aging brain [284]. Furthermore, the KAT CREBBP is important for long-term memory formation and late-phase long-term potentiation in the hippocampus of mice $[285,286]$. Apart from histone acetylation, H3K4me3 [287] and H3 phosphorylation [288] are also involved in memory formation. The SIRT HDACs have also been implicated in aging. In contrast to HDAC2, SIRT1 levels were found to drop with age, a change not limited to the brain $[289,290]$ and also observed in senescent cells [291]. Reduced levels of SIRT1 have been associated with increased levels of H4K16ac in vitro [292]. SIRT1 can in addition directly deacetylate the HKMT suppressor of variegation 3-9 homologue (SUV39H) 1, which increases the activity of SUV39H1 [293]. This HKMT is responsible for $\mathrm{H} 3 \mathrm{~K} 9 \mathrm{me} 3$, which is important for the formation of facultative heterochromatin. Despite the association between senescence and decreased $\mathrm{H} 3 \mathrm{~K} 9 \mathrm{me} 3$ levels, $\mathrm{H} 3 \mathrm{~K} 9 \mathrm{me} 3$ is thought to accumulate in senescence-associated heterochromatin foci (SAHF), a form of facultative heterochromatin, which are thought to induce senescence through the repression of the pro-proliferation E2F transcription factor family [294, 295]. Alternatively, in C. elegans, it has been observed that sir-2.1, the ortholog of mammalian SIRT1, can extend lifespan through its product nicotinamide [296]. Nicotinamide can be methylated by nicotinamide-N-methyltransferase-1, producing 1-methylnicotinamide, and 1-methylnicotinamide in turn is processed by aldehyde oxidase gastrulation defective 3 (GAD-3) to generate hydrogen peroxide. This hydrogen peroxide is thought to play a mitohormetic role, inducing longevity [297]. Reinstating SIRT levels, for instance through caloric restriction, has in addition been reported to increase lifespan in yeast, invertebrates, and vertebrates [298, 299], and has been argued to facilitate healthy aging in humans, thereby slowing the development of age-related neurodegenerative diseases such as AD [300, 301].

\subsubsection{Non-coding RNAs in aging}

One of the first ncRNAs reported to affect the aging process was miRNA lin-4, whose expression was observed to modulate lifespan in $C$. elegans [302]. In neurons of $C$. elegans, miR-71 promotes longevity through the dauer 16/forkhead box O (DAF-16/FOXO) pathway, increasing resistance towards heat shock and oxidative stress [303]. Other studies
TABLE 2. Epigenetic dysregulation in aging: chromatin remodeling. $\uparrow$ indicates increased levels, $\Downarrow$ indicates decreased levels, and $\neq$ indicates altered, not further specified.

ABBREVIATIONS: ChIP-Seq, chromatin immunoprecipitation sequencing; H, histone; hAD-MSC, human adipose tissue-derived mesenchymal stem cells; HDAC, histone deacetylase; HPLC, high-performance liquid chromatography; hUCB-MSC, human umbilical cord blood-derived MSCs; ICC, immunocytochemistry; IHC, immunohistochemistry; K, lysine; MALDI-TOF, matrix assisted laser desorption/ionization time-of-flight; Nano-LC, nano liquid chromatography; NS, not specified; p, phosphorylation, PRC; polycomb repressive complex; RT-PGR, real-time PCR; S, serine; SAMP8, senescence-accelerated prone mouse 8; SIRT, sirtuin; SOD, copper-zinc superoxide dismutase 1 ; TOF MS, time-of-flight mass spectrometry; WB, western blot. 
found increased levels of some miRNAs with age, but did not detect any significantly downregulated miRNAs in mice [304] (Table 3). In human blood mononuclear cells, however, various miRNAs were significantly decreased in older participants [305] (Table 3). Altered expression of various miRNAs, has been linked to age-related cardiovascular problems [306-310] (Table 3). Moreover, various members of the miR-17-92 cluster were reported to be downregulated in humans [311] (Table 3). Another study in human endothelial cells detected additional miRNAs affected by age [310] (Table 3). Increased ROS levels in human endothelial cells were observed to induce miR-200c and concomitant initiation of apoptosis and senescence [312]. Several studies have recently shown the importance of certain miRNAs specific to the aging brain and their roles in the development of neurodegenerative diseases [313, 314]. In the cortex and cerebellum of humans, chimpanzees, and macaque monkeys, miR-144 was observed to be upregulated [315]. This miRNA targets the ataxin-1 gene, which is critically involved in the development

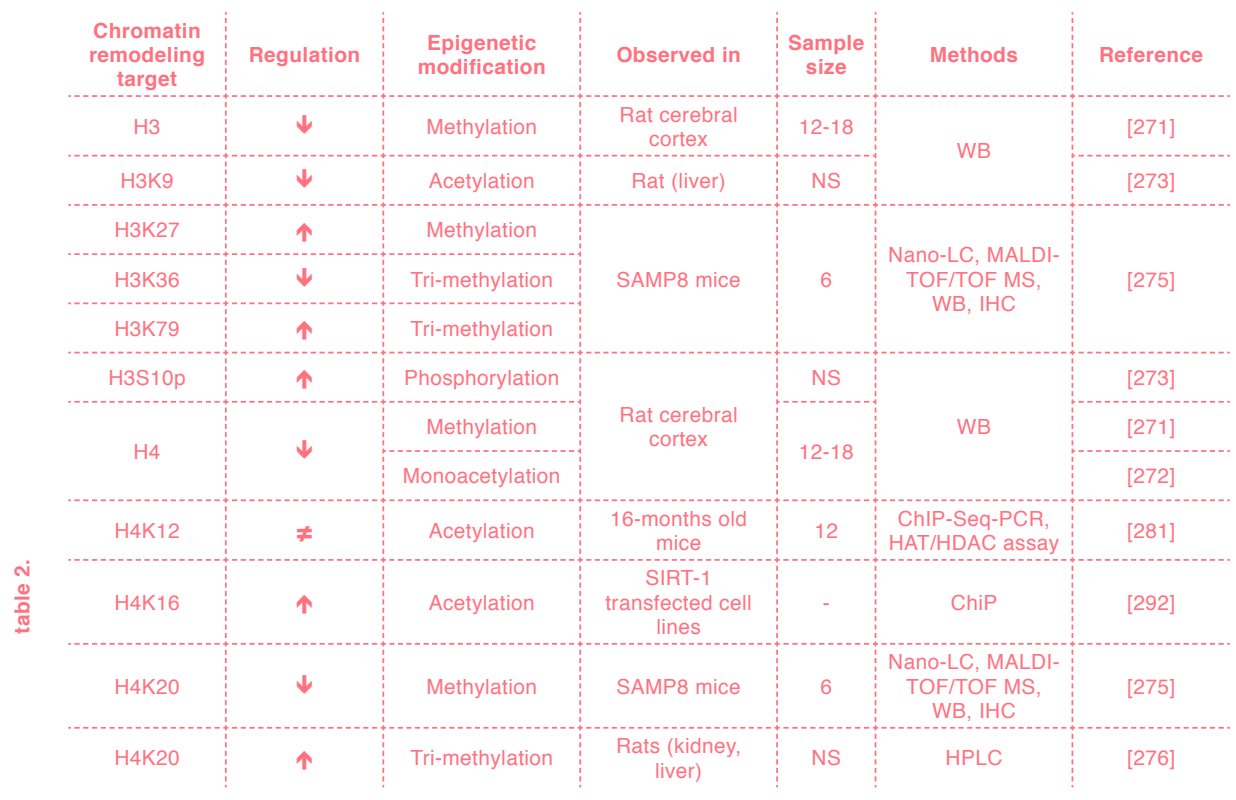

\begin{tabular}{|c|c|c|c|c|c|c|}
\hline Enzyme & Regulation & Modification in & Observed in & $\begin{array}{l}\text { Sample } \\
\text { size }\end{array}$ & Methods & Reference \\
\hline HDAC2 & $\uparrow$ & & SOD mice & 48 & IHC & [279] \\
\hline SIRT1 & $\downarrow$ & Expression & $\begin{array}{l}\text { Rat brain, } \\
\text { senescent cells }\end{array}$ & $24-45,-$ & WB, IHC, RT-PCR & [289-291] \\
\hline PRC1, PRC2 & $\uparrow$ & & $\begin{array}{l}\text { hAD-MSCs, } \\
\text { hUCB-MSCs }\end{array}$ & - & ICC, WB, RT-PCR & [278] \\
\hline
\end{tabular}


of spinocerebellar ataxia type 1 , and its age-related dysregulation could thus facilitate the development of this disease. Li et al. [316] forged a link between aberrant miRNA expression and age-related declines in mitochondrial respiration rates. They found 70 miRNAs to be upregulated in the aging mouse brain, 27 of which were implicated in the downregulation of mitochondrial complexes III, IV and F0F1-ATPase that are all pivotal to the oxidative phosphorylation process. Interestingly, in the SAMP8, a mouse model of accelerated aging, miR-16 was found to be dysregulated. This miRNA modulates AD-related APP protein expression and with age APP levels were shown to drastically increase in the hippocampus of SAMP8 mice, leading to the suggestion that this model might serve as a model for AD [317]. Table 3 provides an overview of some of the ncRNAs that undergo age-related changes.
TABLE 3. Epigenetic dysregulation in aging: non-coding RNAs.

$\uparrow$ indicates increased expression levels, $\Downarrow$ indicates decreased expression levels, and $\neq$ indicates altered expression, not further specified.

ABBREVIATIONS: C57BL/6J mice, C57 black 6 inbred mouse strain; $C$. elegans, Caenorhabditis elegans; HAEC, human aortic endothelial cells; HCAEC, human coronary artery endothelial cells; HUVEC, human umbilical vein endothelial cells; IHC, immunohistochemistry; ISH, in situ hybridization; miR(NA), micro RNA; NB, northern blot; NS, not specified; PCR, polymerase chain reaction; RFLP, restriction fragment length polymorphism; SAMP8 mouse, senescence-accelerated prone mouse 8 ; SB, Southern blot; WB, western blot. 


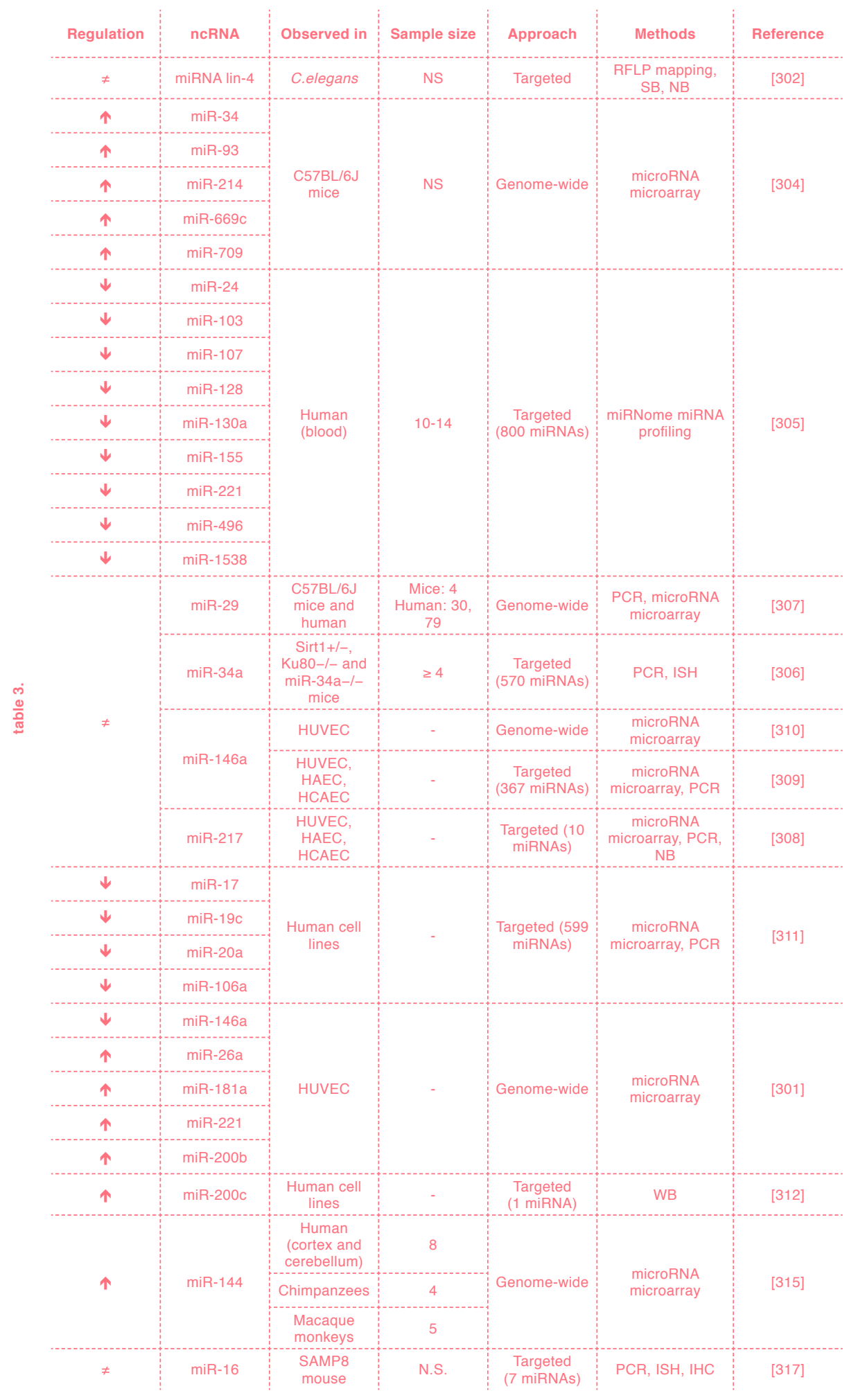




\subsection{Neurodegeneration}

Neurodegenerative diseases typically involve a progressive loss of neuronal integrity and function, followed by neuronal death. Depending on where in the brain the loss of integrity and neuronal loss occur, various functional disabilities may arise and which gradually worsen as the neurodegeneration spreads. The underlying cause and localization of the neurodegenerative processes, however, often vary between different neurodegenerative disorders. Some of the most common include AD, $\mathrm{PD}$ and $\mathrm{HD}$, but also amyotrophic lateral sclerosis and prion diseases are well studied forms of neurodegeneration [318]. Multiple sclerosis is more recently also being investigated as a neurodegenerative disease [319]. The exact etiology of most neurodegenerative diseases is far from clear, while in some cases, such as for HD [320], it is clear that the origin is largely genetic, for others, including sporadic AD and PD, the link between genetics and disease development is much more complex, possibly involving gene-gene and gene-environment interactions [321-323]. Numerous studies have, where genetics did not give simple answers, investigated other possible instigators, of which epigenetic mechanisms seem to be most promising. Although it remains to be elucidated whether dysfunctional epigenetic machinery plays a causal role, it has been critically implicated in various neurodegenerative processes. Additionally, environmental factors enjoy much attention as either direct modulators of disease development, or indirect via genetic or epigenetic pathways [321, 322, 324, 325].

\subsubsection{Alzheimer's disease}

The most ubiquitous neurodegenerative disorder and form of dementia is $A D$, with an estimated worldwide prevalence of over 35 million cases [326]. Mainly characterized by cognitive decline, late-stage AD concomitantly involves progressive motor aberrancies, mood instabilities and other behavioral and physical abnormalities [327, 328]. Although most of these symptoms arise as a result of cortical degeneration, others are due to degeneration of subcortical or autonomic functionrelated areas. It should be noted, however, that AD pathology does not equally affect the whole brain, as certain brain areas and cell types are specifically vulnerable to AD pathology [329]. Among the areas mainly affected by degeneration in $A D$ are the frontal cortex, temporal and parietal lobes, including the hippocampus and entorhinal cortex (EC), and the cingulate gyrus, whereas the cerebellum is largely spared [330]. Interestingly, there is some evidence indicating that, while the cerebellum is mostly spared, the Purkinje cells are specifically targeted by AD pathology [331]. Despite numerous pre-clinical and clinical 
trials for $A D$ treatments, only basic symptom management therapies are currently Food and Drug Administration (FDA)-approved (some acetylcholinesterase inhibitors and an $\mathrm{N}$-methyl-D-aspartic acid [NMDA] receptor antagonist), which cannot halt, or slow down the progressive neurodegeneration and the associated decline of memory, cognitive and executive functions. Apart from being a scourge among the elderly and the relatives of patients, dementia also incurs a tremendous socioeconomic burden; amounting to an estimated $\$ 200$ billion in 2013 in the United States of America alone [332].

$A D$ is a complex, multifaceted disorder, involving dysregulated homeostasis on various fronts, including energy metabolism, inflammation, and cell cycle control [26], likely resulting from a complex interplay between genetic, epigenetic and environmental factors [31, 318]. Despite much research into the pathophysiology of $A D$, including amyloid $(A B)$ and phosphorylated tau proteins [333], its exact etiology remains to be elucidated [334]. $A \beta$, which exists in monomeric, oligomeric, and aggregated forms (senile plaques), is the product of APP cleavage by the $\beta$ - and $\gamma$-secretases [335, 336]. APP cleavage by $Y$-secretases can result in either $A \beta 40$ or $A \beta 42$, of which $A \beta 42$ is thought to be especially neurotoxic. APP can also be cleaved by a-secretases such as a disintegrin and metalloproteases domain (ADAM) 10 and tumor necrosis factor alpha (TNF-a) converting enzyme (TACE), but this cleavage does not result in $A \beta$, but generates APPs- $a$, which is thought to be neuroprotective [337]. One of the most prominent theories of $A D$ pathology is the amyloid hypothesis, which states that $A \beta$ is responsible for initiating the pathogenic pathway that leads to neurodegeneration and dementia in AD. Generally, this theory proposes that neurodegeneration is the result of impaired $A \beta$ homeostasis, which leads to aberrant calcium homeostasis, triggering - and sensitizing cells to - damaging processes, including excitotoxicity and the formation of neurofibrillary tangles (NFTs) [338-340]. This hypothesis is applicable especially to early onset familial types of $A D$ ( $f A D$ ), which have a much more evident genetic component than the far more common late onset sporadic AD (SAD) [341]. Mutations in the APP gene and the presenilin (PS) genes PS1 and $P S 2$, have been observed in $\mathrm{ADD}$ cases [323, 338, 342-346]. PS1 and PS2 are $\gamma$-secretase-associated proteins involved in the generation of $A \beta$ from APP, and PS mutations are able to bias this process towards $A \beta 42$ production, the 42 amino acid-long $A \beta$ isoform that is more prone to aggregate then the shorter $A \beta 40$ isoform, by either increasing $A \beta 42$ production, or lowering $A \beta 40$ production [347]. This relation has been corroborated by the detection of elevated $A \beta 42$ levels in the blood and brains of $f A D$ cases with PS mutations [342]. Their major impact on disease development has led to the widespread use of mutant forms of the APP and PS genes to generate animal models of AD [348]. Although 
some mutations in the PS and APP genes seem to play a large role in disease development in $\mathrm{fAD}$ cases, most of the SAD susceptibility genes, including the risk factor with the highest population-attributable risk, the $\varepsilon 4$ allele of the apolipoprotein E (APOE) gene and those identified through genome-wide association studies ( $A B C A 7, C L U, C R 1, C D 33$, PICALM, MS4A6A, MS4A4E, CD2AP) have a relatively minor influence on AD progression when altered [349-351]. Moreover, despite the robust association with SAD of some of these common sequence variants, it remains largely unknown how they influence the development and course of SAD [352-355]. The same applies to the rare mutations recently discovered in the TREM2 gene, although they confer a much larger increase in risk to develop SAD than the common sequence variants [356-358]. Although these genetic risk factors may be informative in screening for populations at risk to develop $S A D$, it has not yet been discovered how they exactly affect AD development [351]. Most is known about the involvement of the major risk factor APOE\&4. For instance, increased levels of brain APOE 44 mRNA in AD cases, compared to controls with the same allele, were detected [359]. Interestingly, the APOE\&3 allele is thought to protect against $A \beta$ neurotoxicity [360]. Additionally, a study with a transgenic mouse model of AD expressing human APOE isoforms indicated that different APOE alleles might influence clearing soluble $A \beta$ from the brain [361]. This is in line with evidence indicating that $S A D$ is characterized by an inability to clear $A \beta$ from the brain and not an increase $A \beta$ production [362]. A similar effect is suggested for the $C L U$ gene, another important risk gene associated with $S A D$, implicating it in the aggregation and clearance of $A \beta$, thereby mainly influencing age of onset and progression [363]. Apart from the gene, clusterin (CLU) levels were shown to be elevated in the cerebrospinal fluid and brains of AD patients and CLU plasma levels were associated with several AD hallmarks [364, 365].

Besides an abnormal $A \beta$ homeostasis, dysfunctional tau has also been pointed out as a pivotal player in AD pathology. Tau, a microtubuleassociated protein that promotes microtubule assembly [366], becomes hyperphosphorylated in AD. This causes it to dissociate from microtubules and aggregate, which induces cytoskeletal disorganization, neuronal dysfunction and cell death [367, 368]. This pathological process of aggregation is thought to play a part in the neurodegeneration and memory deficits as seen in $\operatorname{AD}[369,370]$. Interestingly, while a similar process occurs in other tauopathies, diseases involving pathological tau aggregation, these generally involve mutations of the tau encoding MAPT gene, whereas such mutations are usually not found in AD cases [367, 371]. Mitochondrial abnormalities have also been investigated as contributors to $A D$ pathogenesis, mainly in relation to energy imbalances and increased ROS levels [372]. 
$A \beta$ and tau have long been the direct focus of treatment strategies, involving potential aggregation inhibitors, immunotherapy, and enzyme modulators [373]. More recently, however, while the epigenetic involvement in neurodegeneration is being explored, the epigenetic machinery has become an attractive target for novel intervention strategies. That minor aberrancies in the epigenetic machinery can have widespread consequences on gene expression, combined with the sporadic and complex nature of $A D$, has led to a recent interest in the role of epigenetic factors in the etiology of $A D[31,374]$.

\subsubsection{Parkinson's disease}

PD is the second most common progressive neurodegenerative disorder, affecting the dopaminergic neurons of the midbrain substantia nigra. Because the dopaminergic projections from the substantia nigra are crucially involved in the initiation of motor events, PD is mainly known for symptoms such as tremor, rigidity, bradykinesia, and gait disturbances [375]. These motor disturbances are, however, complemented by psychiatric symptoms, autonomic impairments, sleep disturbances, and cognitive dysfunctions, including dementia, that are intrinsic to the disease pathology and may even precede the motor symptoms [376-378]. These non-motor symptoms are related to imbalances in other neurotransmitter systems, including serotonergic, noradrenergic, and cholinergic malfunctions [379]. Furthermore, cognitive impairments in PD are generally accompanied by the occurrence of Lewy bodies in brain areas including the midbrain and cortex [380]. Lewy bodies are cytoplasmic protein aggregates, consisting mainly of a-synuclein, parkin, and ubiquitin [381]. Exactly what part Lewy bodies play in PD pathophysiology warrants additional investigation. As with $A D, P D$ exists as a familial (fPD) and a sporadic (SPD) variant, of which the former is again much rarer. SNCA, the gene encoding the presynaptic protein a-synuclein, is one of the cardinal risk genes for PD; increased expression of only this gene (through point mutations and multiplications) can already induce familial parkinsonian syndromes [382, 383]. In addition to SNCA, MAPT, PARK16 and LRRK2 are also indicated as risk genes, with SNCA and MAPT SNPs conferring the highest risk $[384,385]$. Although genetic predisposition remains a high risk factor for SPD, age and environmental variations are also thought to be highly influential [386-388], with factors such as a rural environment increasing the risk to develop PD and factors such as smoking and the consumption of coffee decreasing the risk [389]. Additionally, the development of SPD has often been linked with exposure to environmental toxins, of which 1-methyl-4-phenyl-1,2,3,6-tetrahydropyridine (MPTP) has the most prominent link to developing PD, leading to its widespread use to 
induce PD-like symptoms in animal models [390, 391]. A causal role in the development of PD of most other toxins, however, remains a point of controversy [392]. Nevertheless, evidence is accumulating pointing towards a cardinal role of the epigenetic machinery in mediating the effect of chronic environmental exposures on alterations in gene expression that can lead to the development of late-onset neurodegenerative diseases [393]. At least for some genes a mechanism of DNA methylation-induced allelic skewing is proposed as the underlying mechanism of how an epigenetic process can modulate the interaction between genotype and environment. DNA methylation-induced allelic skewing is the process by which the paternal and maternal alleles are differentially methylated, leading to the preferential expression of either one.

\subsubsection{Huntington's disease}

In contrast to $\mathrm{AD}$ and $\mathrm{PD}, \mathrm{HD}$ is primarily a genetic, autosomal-dominant neurologic disorder, with the sporadic variant being rarer. When symptoms start to occur progress can be fast and will result in death, with no treatment options currently available to change this devastating process [394]. The most characteristic symptom of HD is chorea, but other prominent symptoms include cognitive deterioration and psychiatric disturbances. It is known that HD pathology is ignited by an expansion of a cytosine-adenine-guanine (CAG) repeat section, coding for glutamine, in the coding region of the huntingtin (HTT) gene on chromosome $4 \mathrm{p} 16.3$ [395]. Note that the familial and sporadic variants have the same genetic origin. A CAG repeat number of 36 units leads to the development of $\mathrm{HD}$ and sporadic cases are caused by de novo mutations that increase the repeat number to above the critical number, with high repeat numbers leading to a younger age of onset [396, 397]. The primary risk factor for developing HD is thus having family members with HD, or members with a high CAG repeat number. The expansion results in a dysfunctional HTT protein, which has been shown to disrupt transcription via multiple pathways [398, 399]. It remains, however, unclear exactly how the production of mutant HTT leads to the lethal neurodegeneration associated with HD [400]. Curiously, HD neurodegeneration is very region and cell-type specific, mainly affecting the medium-sized spiny neurons of the neostriatal nuclei, caudate nucleus and putamen, explaining the grave motor symptoms [401-408]. Despite the specificity of neurodegeneration in HD, the HTT protein can be found in neurons throughout the whole brain [409]. Wildtype HTT is mainly situated in the cytoplasm, its exact function, however, remains elusive, with proposed roles in intracellular transport, autophagy, transcription, mitochondrial functioning and signal transduction [410-413]. Nevertheless, HTT is critical for survival, as complete deletion of the HTT gene results on nonviable offspring [414]. 
Mutant HTT was shown to impair fast axonal transport, destabilize microtubules, and through its interactions with a multitude of proteins it disrupts important cellular pathways leading to hampered proteolysis, mitochondrial dysfunction, oxidative damage, inflammatory reactions, excitotoxicity and induction of apoptosis [415-417]. Additionally, evidence indicates that mutant HTT has a widespread impact on gene expression, through interactions with specific transcription factors [418], interference with the core transcriptional machinery and posttranscriptional modifications of histones, skewing the chromatin towards a more condensed state [419].

\subsection{DNA (de)methylation in} neurodegeneration

\subsubsection{DNA (de)methylation in Alzheimer's disease}

Early epigenetic investigations related to AD by West et al. [420] focused on DNA methylation, reporting an AD-specific hypomethylation of the APP gene promoter region in a single patient. This was confirmed by another study and linked to elevated $A \beta$ levels [251]. A later study with a larger sample was, however, unable to replicate this finding, nor find any other significant AD-related abnormalities in MAPT, APP and $P S 1$ methylation [421]. Others also did not find significant AD-related methylation changes in the APP promoter. Barrachina et al. [421] did report the presence of low and high methylated $C p G$ sites in and close to the $A P P$ promoter region, as did Fuso et al. for the $A P P, P S$ and $B A C E$ genes [422]. Conversely, Brohede et al. [423] observed no methylation at the investigated $\mathrm{CpG}$ site of the APP gene in a small sample of fAD patients, in all brain areas investigated, including the frontal cortex, parietal cortex, temporal cortex and cerebellum, concluding that APP is not transcriptionally regulated by methylation. All in all, these studies provide inconclusive evidence of whether APP methylation is involved in $A D$, raising the need for studies clearly separating between $S A D$ and $f A D$, investigating multiple CpG sites and ideally also differentiate between cell types instead of using homogenates of whole regions. Wang et al. [424] observed a high interindividual variance in promoter methylation of $P S 1$, APOE, MTHFR, and DNMT1, and a particularly marked epigenetic drift in $\mathrm{AD}$ cases.

A finding relevant not only to global DNA methylation, but also for many other biochemical pathways, is a severe AD-associated reduction of 
SAM (up to $85 \%$ ) and its demethylated metabolite SAH (up to $79 \%$ ) in several neocortical areas, the hippocampus and putamen [425]. Additionally, cerebrospinal fluid levels of folate and SAM, and levels of SAM in the frontal cortex, occipital cortex, temporal cortex, putamen and hippocampus, were found to be decreased in AD cases [425-427], concomitant with an increase in brain SAH levels [428]. Accordingly, lower serum folate levels and increased plasma Hcy levels were observed in SAD patients versus controls [429]. Cell culture work has indicated that increased Hcy levels can be linked to enhanced tau hyperphosphorylation and subsequent NFT formation [430], which may be the result of the inhibitory effect of Hcy on methyltransferases, thereby preventing the methylation of protein phosphatase $2 \mathrm{~A}$ (PP2A), which is required for its proper activation. PP2A can dephosphorylate phosphorylated tau and its decreased activity thus promotes the hyperphosphorylation of tau. In both mouse Neuro-2a (N2a) cells expressing human mutant APP and transgenic mice expressing human mutant PS1 and APP, PP2A was also found to be hypomethylated, resulting in elevated tau phosphorylation [431]. Furthermore, antagonizing folate with methotrexate in rat primary neuron cultures heightened phosphorylated tau, APP and BACE levels [432]. Interestingly, hypomethylated PP2A, but not normally methylated PP2A colocalized with hyperphosphorylated tau in the hippocampus of rats and $A D$ cases [433].

The apparent importance of folate and vitamins B12 and B6 in maintaining SAM levels has stimulated investigations into the potentially protective effects of supplementing these vitamins to counteract cognitive decline and possibly the onset of dementia [434]. In vitro folate deprivation was able to induce global DNA hypomethylation, leading to an increased expression of BACE and PS1, but unaltered TACE, ADAM10 and APP expression [422]. SAM supplementation successfully restored the folate deficiency-induced abnormalities. In a follow-up study, mutant human APP transgenic mice deprived of folate, vitamin B12 and vitamin B6 [435], showed increased SAH to SAM ratios and increased PS1 and BACE levels, thus corroborating the in vitro findings. These increases in PS1 and BACE expression were paired with elevated $A \beta$ aggregation, early appearance of intraneuronal $A \beta$ and mild spatial learning and memory impairments. In a similar study, it was later shown that SAM supplementation was also able to remedy the B vitamin deficiencyinduced detrimental effects in mice, resulting in a reduction in PS1 and BACE1 expression, amyloid production, tau phosphorylation, and subsequent enhanced spatial memory [436]. Vitamin B deficiency induced hypomethylation of $\mathrm{CpG}$ sites near the PS1 promoter, indicating that PS1 expression is indeed regulated by methylation [437]. Another group also found beneficial effects of dietary SAM supplementation in the 3xTg$A D$ mouse model [438]. Additionally, a vitamin/nutriceutical formulation 
including folate and vitamin B was shown to delay the progression of dementia in a small sample of early stage [439], and moderate to late stage AD [440].

Observations of an overall reduction in DNA methylation in AD patients are in line with these findings and further stress the importance of DNA methylation in AD [26, 31, 424]. Interestingly, despite this AD-associated global DNA hypomethylation, specific loci of the MTHFR gene, which is crucial for SAM synthesis, were found to be hypermethylated, in both postmortem prefrontal cortex and peripheral lymphocyte samples of $A D$ patients [424].

Studies focusing on the hippocampus, one of the brain areas early affected by $A D$ and aging, have observed that levels of 5-mC [253] and DNMT3A [252] increase with age in mice, whereas these levels are significantly decreased in the hippocampus of AD patients [441].

Siegmund et al. [250] found an increase in the methylation of SORBS3 and a decrease in the methylation of $5100 A 2$ in AD subjects, compared to controls of 60 years and older. Interestingly, although a progressive increase in SORBS3 and decrease in S100A2 methylation is normal with aging, this process is accelerated in AD. SORBS3 encodes a cell adhesion molecule and the product of $S 100 A 2$ is observed in corpora amylacea, which are a hallmark of human brain aging and, in greater numbers, of neurodegenerative diseases [442]. A decline in SORBS3 expression might have a hand in the synaptic abnormalities associated with AD [443]. Intriguingly, recent epigenome-wide association studies identified another gene that encodes an adaptor protein and its methylation signature is highly associated with AD pathology. More specifically, a differential cortex-specific hypermethylated region of ANK1 was found to be associated with the early stages as well as the progression of AD neuropathology [444, 445].

Remarkably, $A \beta$ has also been implicated as a trigger of epigenetic changes. Chen et al. [446] found that $A \beta$ induces global DNA hypomethylation, while promoting hypermethylation of $N E P$, a gene that encodes neprilysin. Neprilysin is one of the enzymes involved in $A \beta$ degradation and its expression is known to decrease with aging and $A D$. This finding indicates that $A \beta$ is able to induce a vicious cycle that depends on epigenetic processes and favors $A \beta$ deposition. Other regulatory players may further enforce this cycle, for instance, TNF-a and cysteine-dependent aspartate-directed protease (caspase)-3 were found to increase $A \beta$ production, and they are increasingly expressed in response to hypomethylation [447-450]. 
Tau gene expression is also subject to complex epigenetic regulation, involving differentially methylated binding sites for transcription factors. It was found that with age, the activator-binding site for transcription factor SP1 became hypermethylated in the tau gene promoter region, whereas the repressor-binding site for GCF was hypomethylated in the human cerebral cortex [251], which might be relevant to AD and other agerelated tauopathies. This points toward an age-related decrease in tau expression, which has indeed been detected in the human frontal cortex and hippocampus, but this did not correlate with NFT pathology [451].

The APOE gene promoter has a low $\mathrm{CpG}$ count and generally exhibits low levels of DNA methylation. There is, however, a CpG island located at the 3' end that is usually heavily methylated, and which contains the sequence of the APOE \&4-haplotype, the prime genetic risk factor for $\mathrm{SAD}$ [424]. It has been suggested that the $\varepsilon 4$ allele might disturb the epigenetic regulation of the $A P O E$ gene, as this allele is associated with a $\mathrm{C}>\mathrm{T}$ transition, preventing this site from being methylated. The $C L U$ gene is more clearly regulated by epigenetic mechanisms, as its promoter regions contain a CpG island, the demethylation of which after 5-aza-2'deoxycytidine (decitabine; DAC) treatment was shown to enhance CLU expression in cancer cell lines [452]. A similar demethylating treatment in addition to the administration of HDAC inhibitors (HDACls) has also been observed to increase CLU expression and secretion in human neurons and retinal pigment epithelial cells [453, 454].

There is increasing evidence of disturbed cell-cycle control and subsequent induction of apoptosis in degenerating AD neurons and, although not directly investigated, many of the proteins involved in these processes that have been shown to be upregulated in these neurons and are also known to be regulated through DNA methylation [447, 455-458]. In addition to genes involved in cell-cycle control, the promoter regions of COX-2 and NF-KB were found to be hypomethylated, while the promoter regions of $B D N F$ and $C R E B$ were hypermethylated in the frontal cortex of AD patients [459].

Bollati et al. [460] specifically investigated blood for the methylation status of repetitive elements, including Arthrobacter luteus elements $(A / u)$, long interspersed element 1 (LINE-1) and satellite- $a$ (SAT- $a$ ), which comprise a large portion of the human genome and are known to contain large numbers of CpG sites. Interestingly, they found that LINE-1 methylation was increased in AD patients and that within the AD group enhanced LINE-1 methylation was associated with a better cognitive performance.

Although not as well studied in relation to AD as DNA methylation, the DNA demethylation process is receiving increased attention. As for 
5-mC, 5-hmC levels were also found to be greatly decreased in the hippocampus of AD patients [441]. This is in line with previous findings indicating a global DNA hypomethylation in EC NFT-bearing neurons of AD patients [26]. Additionally, it was found that global 5-hmC levels were decreased in the EC and cerebellum of AD subjects, while no significant disease-related changes in 5-mC, 5-fC and 5-caC were detected [461]. In contrast, levels of 5-mC and 5-hmC were immunohistochemically found to be increased in the middle frontal gyrus and middle temporal gyrus of $A D$ patients and positively correlated with $A \beta, N F T$, and ubiquitin load [462]. This study included a cell-type specific analysis and found that 5-hmC and 5-mC were mainly present in Neuronal Nuclei (NeuN; a neuronal marker)-positive cells, with glial fibrillary acidic protein (GFAP; an astrocyte marker)-positive cells and ionized calcium-binding adapter molecule 1 (IBA1; a microglial/macrophage maker)-positive cells only presenting with weak or no immunoreactivity. This latter study is in line with findings from Bradley-Whitman and Lovell [463], who observed increased levels of TET1, 5-mC and 5-hmC in the hippocampus and parahippocampal gyrus in subjects with preclinical and late-stage AD. In addition, it was found that 5-fC and 5-caC levels were significantly decreased. Another study detected global hypermethylation in the frontal cortex of AD patients [459]. Whether global 5-mC and 5-hmC levels are thus decreased or increased in AD remains to be conclusively determined. Possible factors contributing to the discordant findings have been suggested and include differences in the brain regions studied, tissue processing, and detection methods and protocols [462]. An additional factor that could influence readings is whether a cell type-specific analysis is conducted, or different cell types are grouped together. Considering the uncertainty regarding global $5-\mathrm{mC}$ and $5-\mathrm{hmC}$ changes in relation to $\mathrm{AD}$ it might be a bit too early to speculate about the consequences of such changes. Nevertheless, Coppieters et al. [462], who detected a global DNA hypermethylation and hyperhydroxymethylation argue that these changes may facilitate cell death, as the methylation of cytosines is thought to enhance the mutation rate of these cytosines and this increased mutation rate could facilitate the loss of neurons in AD. There are, however, also studies indicating that DNA hypomethylation leads to neuronal degeneration [35, 464], suggesting that no simple conclusions can be drawn from observations of globally increased or decreased DNA methylation levels.

Münzel et al. [265] observed an age-related increase in 5-hmC levels, which seemed to be especially prominent in genes associated with neurodegeneration. Another finding indicating DNA demethylation to play a role in the development of AD is a SNP in the TET1 gene that was associated with SAD [465]. See Table 4 for an overview of the aberrant DNA (de)methylation in AD. 


\subsubsection{DNA (de)methylation in Parkinson's disease}

Obeid et al. [249] explored the relation between the methylation potential, represented by the SAM/SAH ratio, and cognitive performance in PD patients, and found that a higher methylation potential correlated with better cognitive capabilities. In addition, it was found that a-synuclein can associate with DNMT1, sequestering it in the cytoplasm, resulting in global DNA hypomethylation. This property of a-synuclein was not only found in PD cases, but also in dementia with Lewy bodies and a transgenic mouse model expressing human a-synuclein [466]. Because a-synuclein can also be observed in AD [467], this mechanism might also contribute to the global DNA hypomethylation observed there. In vitro overexpression of DNMT1, as well as in transgenic mice, was able to normalize the nuclear localization of DNMT1. Jowaed et al. [468] specifically investigated methylation of human SNCA and showed that expression of this gene is regulated through methylation of the first intron.

Interestingly, a negative correlation between SNCA intron 1 methylation and SNCA expression has also been identified, and that SNCA methylation is decreased in the substantia nigra, putamen, and cortex of SPD patients [468, 469]. Another study, investigating the high-resolution methylome of Lewy body disease cases, including PD, found, however, no overall differences in SNCA intron 1 methylation [470]. Although this study reported some differences at the single $\mathrm{CpG}$ level, it signifies that the extent of erroneous DNA methylation in PD warrants additional research efforts. Apart from SNCA, however, additional genes, including PARK16, GPNMB and STX1B have also reported to be differentially methylated in $\mathrm{PD}$ [471]. A very recent EWAS in blood from PD patients, using a discovery and replication cohort, identified additional differentially methylated genes, with the most reliable differentially methylated CpGs being located in the FANCC and TNKS2 genes [472]. How this aberrant DNA methylation exactly affects gene expression and ultimately influences PD pathology remains to be unveiled.

Interestingly, although a mutation in PARK2, the gene encoding parkin, has been associated with a juvenile form of $P D$, deviant methylation patterns in the promoter of this gene have been observed in myelogenous leukemia and acute lymphoblastic leukemia, but not PD [473, 474]. Similar observations were made for $U C H L 1$, a gene associated with PD, and ATP13A2, which causes a recessive form of parkinsonism, failing to establish a relation between abnormal promoter methylation and PD, although the promoter of UCHL1 was found to be hypermethylated in cancer [383, 421, 475]. Table 5 summarizes the findings regarding dysregulated DNA (de)methylation in PD.
TABLE 4. Epigenetic dysregulation in Alzheimer's disease: DNA (de) methylation.

$\uparrow$ indicates increased levels, $\Downarrow$ indi-

cates decreased levels, and $\neq$ indi-

cates altered, not further specified.

ABBREVIATIONS: 450K BeadChip, Illumina Infinium HumanMethylation 450K BeadChip; 5-caC, 5 -carboxylcytosine; 5 -fC, 5 -formylcytosine; 5 -hmC, 5 -hydroxymethylcytosine; 5 -mC, 5 -methylcytosine; $A \beta$, amyloid- $\beta$; $\mathrm{AD}$, Alzheimer's disease; BSeq, bisulfite sequencing; CEC, cerebral endothelial cell; CiEC, circulating endothelial cells; CpG, cytosine-phosphate-guanine; EC, entorhinal cortex; FC, frontal cortex; GCF, granulocyte chemotactic factor; HPLC, high-performance liquid chromatography; IHC, immunohistochemistry; LINE-1, long interspersed element 1; MFG, middle frontal gyrus; MS-PCR, methylation, specific PCR; MTG, middle temporal gyrus; N2a, neuro-2a cell line; NS, not specified; PFC, prefrontal cortex; SB, Southern blot; SK-N-BE, SK-N-SH, human neuroblastoma cell lines; SP, specificity factor; STC, superior temporal cortex; TET1, ten-eleven translocation 1. 


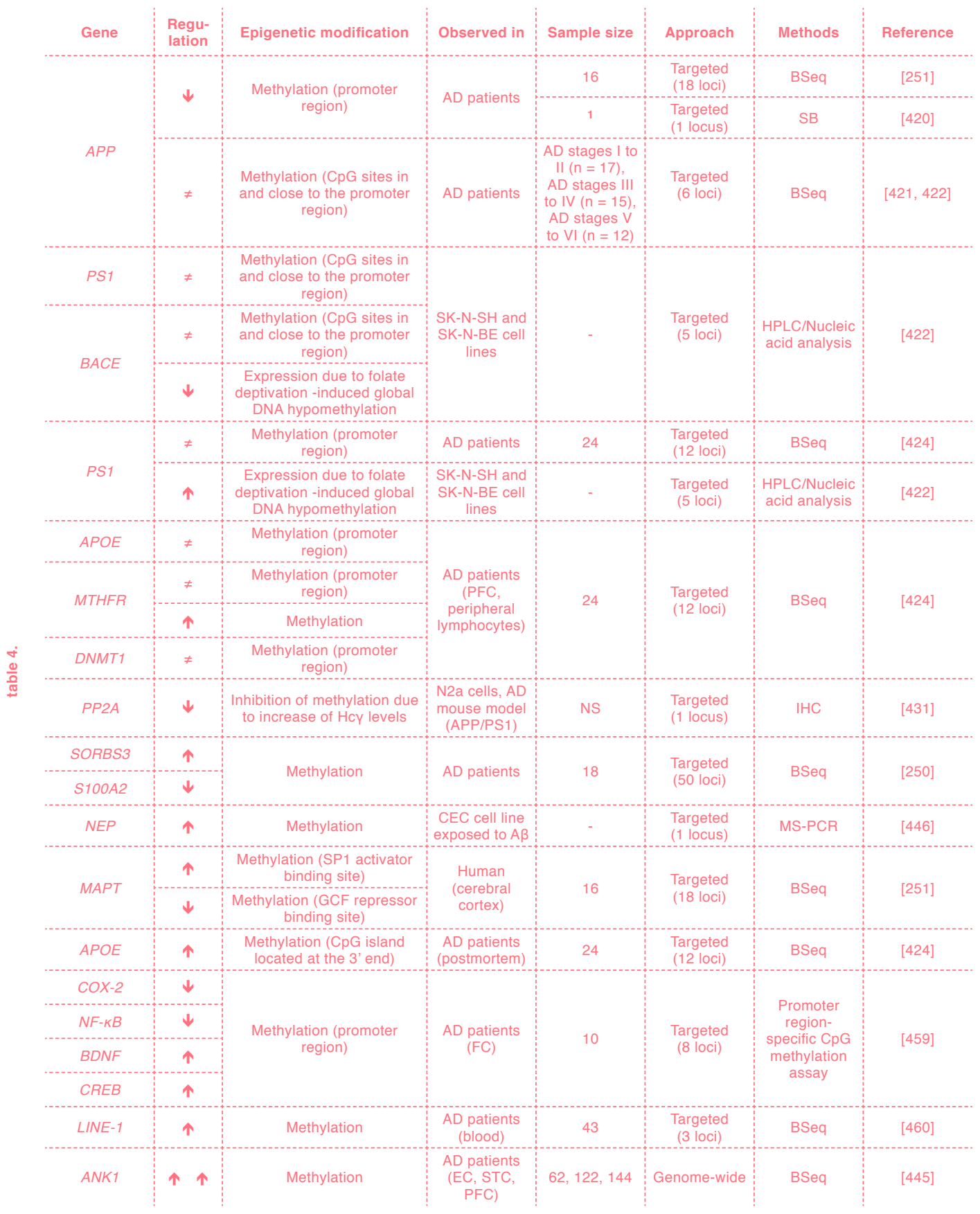




\begin{tabular}{|c|c|c|c|c|}
\hline $\begin{array}{c}\text { Protein/ } \\
\text { (Hydroxy) } \\
\text { Methylated } \\
\text { base }\end{array}$ & $\begin{array}{l}\text { Regu- } \\
\text { lation }\end{array}$ & Modification in & Observed in & Sample size \\
\hline 5-hmC & $\downarrow$ & & $\begin{array}{l}\text { AD patients } \\
\text { (EC, } \\
\text { cerebellum) }\end{array}$ & 10 \\
\hline 5-mC, 5-hmC & $\uparrow$ & & $\begin{array}{l}\text { AD patients } \\
\text { (MFG, MTG, }\end{array}$ & $\begin{array}{c}7 \text { late- } \\
\text { stage AD } 5 \\
\text { preclinical AD }\end{array}$ \\
\hline & & & hippocampus) & $\begin{array}{c}13 \text { MFG } 29 \\
\text { MTG }\end{array}$ \\
\hline 5-fC, 5-caC & $\downarrow$ & Expression & $\begin{array}{c}\text { AD patients } \\
\text { (hippocampus) }\end{array}$ & $\begin{array}{c}7 \text { late- } \\
\text { stage AD, } 5 \\
\text { preclinical } A D\end{array}$ \\
\hline DNMT3A & $\downarrow$ & & AD patients & 10 \\
\hline DNMT3A2 & $\downarrow$ & & $\begin{array}{c}\text { Mice (12 } \\
\text { months old) }\end{array}$ & 15 \\
\hline TET1 & $\uparrow$ & & $\begin{array}{c}\text { AD patients } \\
\text { (hippocampus) }\end{array}$ & $\begin{array}{c}7 \text { late- } \\
\text { stage AD, } 5 \\
\text { preclinical AD }\end{array}$ \\
\hline
\end{tabular}

\subsubsection{DNA (de)methylation in Huntington's disease}

DNA methylation states have been investigated in transgenic models, and to a lesser extend in HD patients [476-478] (Table 6). Promoter regions of genes important for neurogenesis were found to be hypermethylated in the presence of mutant HTT [476] (Table 6). Although these findings need to be replicated in HD patients, reduced hippocampal neurogenesis might partially underlie the cognitive impairments seen in HD [407]. Decreased expression of the adenosine A2a receptor in HD patients is also epigenetically regulated. In both HD patients and transgenic mice adenosine A2a receptor expression was observed to be downregulated [478]. However, in patients this was associated with increased 5' UTR DNA methylation, whereas in the mouse model with decreased 5' UTR DNA hydroxymethylation of the adenosine A2a receptor gene (ADORA2A). This finding indicates that epigenetic regulation might differ between species and illustrates the importance of replicating findings in human cases. The widely neglected 7-mG form of DNA methylation, which also occurs in RNA, was found to be disturbed in HD mouse models and patients, in both the nucleus and cytoplasm, the latter primarily reflecting methylated RNA [13].

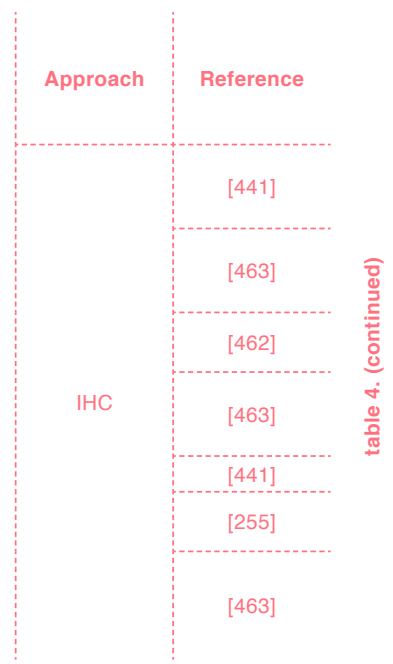

TABLE 5. Epigenetic dysregulation in Parkinson's disease: DNA (de) methylation.

$\Downarrow$ indicates decreased levels, and $\nRightarrow$ indicates altered, not further specified.

ABBREVIATIONS: $450 \mathrm{~K}$ BeadChip, Illumina Infinium HumanMethylation 450K BeadChip; (s)PD, (sporadic) Parkinson's disease; $\mathrm{SN}$, substantia nigra; BSeq, bisulfite sequencing; methQTL, methylation quantitative trait locus. 
TABLE 6. Epigenetic dysregulation in Huntington's disease: DNA (de) methylation.

$\uparrow$ indicates increased levels and $\neq$ indicates altered, not further specified.

ABBREVIATIONS: 7-mG, 7-methylguanine; CAG140 KI mice, transgenic mouse model overexpressing human HTT with 140 cytosine-adenine-guanine repeats; HD, Huntington's disease; HTT, Huntingtin; R6/2 mice, transgenic mouse model overexpressing exon 1 of human HTT with an expanded cytosine-adenine-guanine repeat length; NS, not specified; RRBS, reduced representation bisulfite sequencing; MeDIP-Seq, methylated DNA immunoprecipitation sequencing; ECD, electrochemical detection; HPLC, high-performance liquid chromatography; ChIP-Seq, chromatin immunoprecipitation sequencing.

\subsection{Chromatin remodeling in} neurodegeneration

\subsubsection{Chromatin remodel- ing in Alzheimer's disease}

Going from the DNA to the chromatin level, additional epigenetic dysregulation can be observed in AD (Table 7). Histone acetylation was found to be drastically decreased in the temporal lobe of AD patients when compared to aged controls [479], but also in animal models of $A D$ [480]. The importance of gene-specific investigations apart from global changes in epigenetic markers is exemplified by the observation of increased $\mathrm{H} 3$ acetylation at the promoter region of the BACE1 gene in $A D$ patients [481]. The increase in $\mathrm{H} 3$ acetylation enhanced promoter accessibility and subsequent gene expression. Importantly, it was found that indirectly enhancing histone acetylation through chronic treatment with HDACls was able to reverse cognitive deficits in double transgenic mice overexpressing human APP isoform 695 with the double KM670/671NL Swedish mutation (APPswe) and the human PS1 deleted in exon 9 mutation (PS1dE9) (APPswe/PS1dE9 mice) [482]. The mechanism of action of $\mathrm{HDACl}$ treatment might be related to the

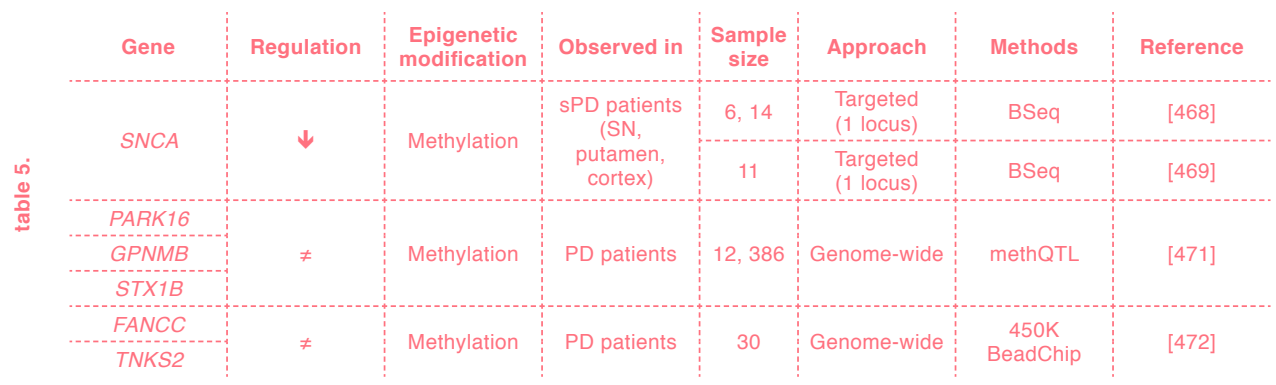

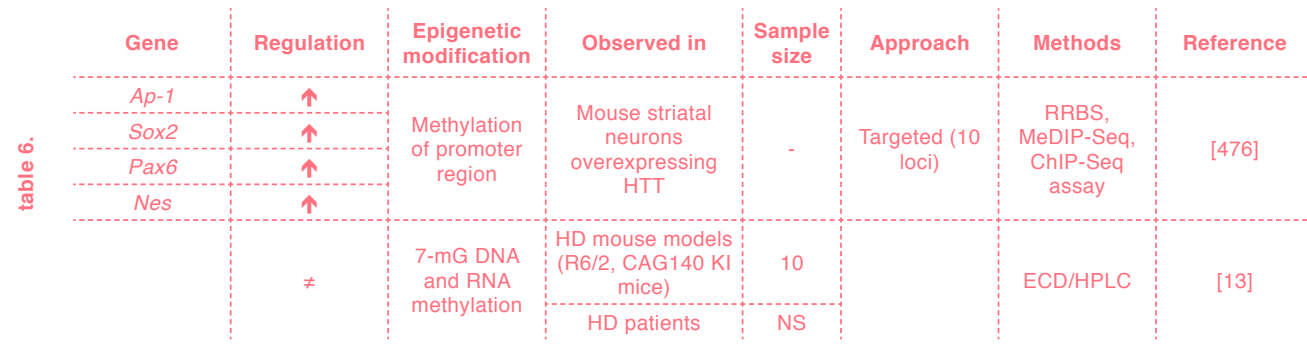


finding that dysregulation of $\mathrm{H} 4 \mathrm{~K} 12 \mathrm{ac}$ is implicated in mediating cognitive impairment seen in aged mice, impairments which were alleviated through $\mathrm{HDACl}$ administration [281]. Another study using transgenic APP/PS1 mice observed diminished acetylation of $\mathrm{H} 4$ and linked this to memory impairments, which could be alleviated through trichostatin $A$ (TSA), an $\mathrm{HDACl}$, administration [483]. Decreased histone acetylation is in line with the discovery of elevated nuclear translocation of EP300 interacting inhibitor of differentiation 1 (EID1) in cortical neurons of $A D$ subjects [484]. EID1 inhibits EP300 and CREBBP, important KATs, and the overexpression of EID1 in mice resulted in learning and memory impairments thought to be the result of this inhibition. In the triple transgenic mouse model of $A D$, expressing human mutant APPK670N/ M671L, PS1M146V, and TauP301L, (3xTg-AD mice) CREBBP expression was also decreased, while overexpression of CREBBP elevated brainderived neurotrophic factor (BDNF) levels and restored memory function in this AD model [485]. Additionally, expression of a truncated inhibitory form of EP300 impaired memory in transgenic mice [486, 487]. Curiously, while knock-out of KAT2B resulted in memory impairments in mice [488], such mice were resistant to the neurotoxic effects of $A \beta$ injected into the lateral ventricles in another study [489].

Conversely, cultured neurons from 3xTg-AD mice and non-transgenic controls, harvested at different ages, revealed increased $\mathrm{H} 3$ and $\mathrm{H} 4$ acetylation levels from an age of 4 months, which is before the onset of memory impairments in this model of AD [490]. With normal aging, H3 acetylation levels seem to remain unchanged, whereas $\mathrm{H} 4$ acetylation levels decreased, but administration of $A \beta$ to the non-transgenic neurons increased acetylation levels. The repressive H3K9 mark in these same neurons increased with age in both the transgenic and non-transgenic neurons, but was more prominent in the transgenic cells. This later finding has been corroborated in humans; comparing two monozygotic twins discordant for $A D$ it was found that the one with $A D$ exhibited higher levels of H3K9me3 in the temporal cortex and hippocampus [491]. Using transgenic mice overexpressing APPswe (Tg2576 mice), increased H3 acetylation levels were found in the prefrontal cortex, as well as increased $\mathrm{H} 4$ acetylation levels in the CA1 region of the hippocampus [492].

Additionally, they also reported elevated levels of $\mathrm{H} 3$ phosphorylation and methylation in the prefrontal cortex, but decreased $\mathrm{H} 3$ methylation in the striatum.

While the use of non-selective HDACls is a promising strategy for the treatment of cognitive problems, it might be even better to target the specific HDACs that induce the memory problems. Currently, HDAC2 is a prime suspect $[95,493]$. Especially the group of Gräff and Tsai has contributed significantly in this respect, starting with their detection of 
increased levels of HDAC2 in the hippocampus and prefrontal cortex of a mouse model of AD, while levels of the related HDAC1 and HDAC3 were not affected. Note, however, that recently it was reported that MS-275 treatment, an HDACI that favors HDAC1, was able to partially alleviate behavioral deficits, neuroinflammation and plaque load in transgenic mice overexpressing APPswe and human PS1 with the L166P mutation, line 21 (APP/PS1-21 mice) [494], and that HDAC3 inhibition enhanced longterm memory formation in the C57 black 6 inbred mouse strain (C57BL/6 mice) [495]. To study the effects of this HDAC2 dysregulation at the gene level, Gräff et al. [493] focused on genes involved in learning, memory and synaptic plasticity that were previously shown to be downregulated in the AD brain and found that HDAC2 was significantly enriched at the promoter and coding regions of these genes, in their mouse model. In addition, they found several acetylation marks, associated with neuroplasticity, to be hypoacetylated (Table 7). Subsequently, it was shown that increased localization of HDAC2 to the investigated genes and hypoacetylation negatively correlated with RNA polymerase (RNAP) II binding and mRNA expression. Interestingly, knock-down of HDAC2 ameliorated the cognitive problems and aberrant synaptic plasticity. It was then investigated how HDAC2 could be induced in AD, by testing in vitro the effect of the $A D$-associated neurotoxic stimuli hydrogen peroxide and $A \beta$ in primary hippocampal neurons. Both stimuli were found to enhance HDAC2 mRNA levels through activation of glucocorticoid receptor (GR) 1. Importantly, HDAC2 was also investigated in human postmortem brain samples from $A D$ patients revealing that in $A D$ cases HDAC2 was markedly increased in the hippocampus and EC. Already at Braak stages I and II HDAC2 levels were found to be significantly elevated in hippocampal area CA1 and the EC, indicating that increased HDAC2 activity might be involved in the early stages of $A D$. In addition to HDAC2, HDAC6 levels were found to be significantly higher in the hippocampus of AD cases when compared to controls [496]. Interestingly, HDAC6 is thought to interact with tau, affecting its phosphorylation and aggregation [497]. HDAC6 has been suggested to make tau vulnerable to phosphorylation through deacetylation, a finding relevant to tauopathies in general [498]. HDAC6 also indirectly affects tau clearance through deacetylation of chaperone protein heat shock protein (HSP) 90, which affects its drive towards refolding or degradation [499]. It has been reported that tau can actually act as a HDAC6 inhibitor [500]. Accordingly, in a mouse model for AD, reduction of HDAC6 levels mitigated learning and memory problems [501]. Studies on HDAC6 suggest that the role of HDACs in neurodegeneration might not solely depend on the deacetylation of histones, but also on the deacetylation of other targets, such as a-tubulin in the case of HDAC6 [497, 501, 502]. The same holds true for KATs, as the KAT human immunodeficiency virus type 1 transactivating protein interactive protein (TIP60/KAT5), the proposed 
counterpart of HDAC6 [503] that has been associated with microtubule acetylation [504], has been shown to guard against $A \beta$ toxicity [505]. KAT5 in addition regulates the expression of genes involved in apoptosis [505], axonal transport [506] and DNA damage control [507], and was found to interact with the APP intracellular domain [508].

Not all HDACs have a detrimental effect on learning and memory, as inhibition of the class Ila HDACs HDAC4 and HDAC5 impair these processes [509, 510]. Moreover, SIRT1, also an HDAC, was found to be decreased in the parietal cortex of AD patients [511]. SIRT1 has been linked to neurogenesis, DNA repair, apoptosis, cell stress responses, and various other vital signaling pathways [512]. SIRT1 expression is suggested to be beneficial in case of AD [513], as it induces ADAM10 expression, an a-secretase that can cleave APP without producing $A \beta$ [514]. Additionally, SIRT1 is able to deacetylate tau and its deficiency in $A D$ is thus thought to enhance tau expression and pathology [511, 515]. Note that this is in conflict with more recent findings regarding HDAC6, which is thought to increase tau pathology through deacetylation of tau, as stated above [498], although the SIRT1 study investigated global tau acetylation, whereas the HDAC6 study specifically investigated the acetylation of KXGS motifs. Nevertheless, enhancing SIRT1 expression attenuated axonal neurodegeneration and microglia-dependent $A \beta$ toxicity $[513,516,517]$. Interestingly, SIRT1 was found to be upregulated in AD mouse models, but which might be a defense mechanism [512, 513], although it was found to be decreased in AD patients [511]. Histone modification abnormalities in $\mathrm{AD}$ also include histone phosphorylation, as $\mathrm{H} 3$ phosphorylation was found to be increased in the frontal cortex of $A D$ patients [459]. Phosphorylation of histone protein H2A member X (H2AX) at $\mathrm{S139}$, a marker of DNA damage, was shown to be increased in the AD hippocampus, but specifically in astrocytes [518].

Accumulating evidence indicates that dysfunctional protein localization might be a chief player in the incapacitation of the epigenetic machinery in $A D$, and possibly in neurodegeneration in general. Ogawa et al. [519] made some fundamental observations in this respect. As some neurons in AD erroneously exhibit signs of cell cycle activation, they investigated H3S10 phosphorylation, a histone modification critical for chromosome compaction during cell division. Strikingly, it was not only found that $\mathrm{H} 3$ phosphorylation was increased in hippocampal AD neurons, but also that this epigenetic marker was abnormally restricted to the cytoplasm in these neurons. In addition, it has also been shown that the mitogenactivated protein kinase (MAPK) pathway involved in the phosphorylation of $\mathrm{H} 3$ is upregulated in degeneration vulnerable neurons in AD [520-522]. Furthermore, the presence of high levels of histones in the cytoplasm of neurons in the HD brain [523] suggests that incapacitated nuclear 
transport might be a common denominator for neurodegenerative processes. In support of this, Mastroeni et al. [524] found that $A \beta$ could reduce rat sarcoma (Ras)-related nuclear protein (RAN) expression, a pivotal player in nucleocytoplasmic transport. As an apparent result, they observed DNMT1 and RNAPII to be erroneously sequestered in the cytoplasm of neurons from AD patients.

Histone 1 ADP-ribosylation has not been directly investigated in relation to $A D$, but the observations that a loss of poly[ADP]-ribose polymerase (PARP) 1 induces memory problems in mice [525] and that a dysregulation of PARP-1 is associated with amyloid pathology and SAD [526-528] suggests that ADP-ribosylation might be a relevant target for future studies. Although various histone methylation marks and histone methylation and demethylation enzymes have been linked to cognitive functioning in mice and humans (shortly reviewed in [503]), no links with $A D$ have been firmly identified yet.

\subsubsection{Chromatin remodel- ing in Parkinson's disease}

a-Synuclein normally localizes to the nucleus and presynaptic nerve terminals, but increased nuclear targeting is neurotoxic, possibly contributing to PD-related neurodegeneration [529]. This nuclear toxicity of a-synuclein is supported by the finding that fPD a-synuclein mutations $\mathrm{A} 30 \mathrm{P}$ and $\mathrm{A} 53 \mathrm{~T}$ result in an increased nuclear targeting of a-synuclein. Kontopoulos et al. [529] found that nuclear toxicity of a-synuclein might be the result of direct binding of a-synuclein to histones, reducing the levels of acetylated histone $\mathrm{H} 3$ and acetylation in general in cultured cells through interactions with SIRT2. In cell cultures and transgenic flies, it was further shown that a rescue of a-synuclein toxicity could be achieved through HDACls [530, 531]. Similar findings were found after exposure to oxidative stress, which induces the relocation of a-synuclein to the nucleus, where it subsequently binds to the PGC1-a promoter element [532]. This binding of a-synuclein causes histone deacetylation, lowering peroxisome $\mathrm{p}$ roliferator receptor gamma coactivator-1 alpha (PGC1-a) expression, which is deleterious for mitochondrial functioning. Interestingly, levels of PGC1-a were significantly reduced in postmortem substantia nigra neurons of PD patients [533].

Curiously, not only does a-synuclein interact with the epigenetic machinery, the KAT EP300 interacts with protein aggregation in Lewy bodies. A specific domain of EP300, reminiscent of prion-like domains, was found to serve as a potential interaction site for misfolded proteins, 


\begin{tabular}{|c|c|c|c|c|c|c|}
\hline $\begin{array}{l}\text { Chromatin } \\
\text { remodelling } \\
\text { target }\end{array}$ & Regulation & $\begin{array}{l}\text { Epigenetic } \\
\text { modification }\end{array}$ & Observed in & $\begin{array}{c}\text { Sample } \\
\text { size }\end{array}$ & Methods & Reference \\
\hline $\begin{array}{l}\text { Histones } \\
\text { (globally) }\end{array}$ & $\downarrow$ & Acetylation & AD patients & $5-6$ & $\begin{array}{l}\text { Targeted } \\
\text { proteomics } \\
\text { assay }\end{array}$ & {$[479]$} \\
\hline \multirow{7}{*}{$\mathrm{H} 3$} & & $\begin{array}{c}\text { Acetylation (BACE1 } \\
\text { promoter region) }\end{array}$ & AD patients & 31 & FAIRE/ChIP & [481] \\
\hline & $\uparrow$ & & $\begin{array}{l}\text { AD mouse model } \\
\text { (3xTg-AD mice; } 4 \\
\text { months of age) }\end{array}$ & 6 & IF & {$[481]$} \\
\hline & & Acetyration & $\begin{array}{l}\text { AD mouse model } \\
\text { (Tg2576 mice; } \\
\text { PFC) }\end{array}$ & \multirow{5}{*}{ N.S. } & \multirow{5}{*}{ N.S. } & \multirow{5}{*}{ [492] } \\
\hline & $\uparrow$ & Phosphorylation & $\begin{array}{c}\text { AD mouse model } \\
\text { (Tg2576 mice; } \\
\text { PFC) }\end{array}$ & & & \\
\hline & $\downarrow$ & & $\begin{array}{l}\text { AD patients } \\
\text { (hippocampus) }\end{array}$ & & & \\
\hline & $\uparrow$ & Methylation & $\begin{array}{c}\text { AD mouse model } \\
\text { (Tg2576 mice; } \\
\text { PFC) }\end{array}$ & & & \\
\hline & $\downarrow$ & Methylation & $\begin{array}{l}\text { AD mouse model } \\
\text { (Tg2576 mice; } \\
\text { striatum) }\end{array}$ & & & \\
\hline Н3К9 & $\uparrow$ & Tri-methylation & $\begin{array}{l}\text { AD monozygotic } \\
\text { twin (temporal } \\
\text { cortex and } \\
\text { hippocampus) }\end{array}$ & 1 & $\mathrm{IHC}$ & {$[491]$} \\
\hline \multirow{3}{*}{$\mathrm{H} 4$} & $\downarrow$ & Acetylation & $\begin{array}{l}\text { AD mouse model } \\
\text { (APP/PS1 mice) }\end{array}$ & 4 & WB & {$[483]$} \\
\hline & $\uparrow$ & Acetylation & $\begin{array}{l}\text { AD mouse model } \\
\text { (3xTg-AD mice; } 4 \\
\text { months of age) }\end{array}$ & 6 & IF & [490] \\
\hline & $\uparrow$ & Acetylation & $\begin{array}{c}\text { AD mouse model } \\
\text { (Tg2576 mice; } \\
\text { CA1) }\end{array}$ & N.S. & N.S. & [492] \\
\hline H2AX (S139) & $\uparrow$ & Phoshorylation & $\begin{array}{l}\text { AD patients } \\
\text { (hippocampus } \\
\text { and astrocytes) }\end{array}$ & 13 & ICC & [518] \\
\hline HDAC2 & $\uparrow$ & Expression & $\begin{array}{l}\text { AD mouse models } \\
\text { (CK-p25, 5xFAD, } \\
\text { Cdk5cKO mice; } \\
\text { hippocampus and } \\
\text { PFC) }\end{array}$ & $6-9$ & & \multirow{5}{*}{ [493] } \\
\hline & & & $\begin{array}{l}\text { AD patients } \\
\text { (hippocampus } \\
\text { and EC) }\end{array}$ & $4-8$ & & \\
\hline $\mathrm{H} 2 \mathrm{BK} 5$ & $\downarrow$ & Acetylation & $\begin{array}{l}\text { AD mouse models } \\
\text { (CK-p25, 5xFAD, } \\
\text { Cdk5cKO mice; } \\
\text { hippocampus and } \\
\text { PFC) }\end{array}$ & $6-9$ & $\begin{array}{l}\text { IHC, WB, Co- } \\
\text { IP, ChIP, PCR }\end{array}$ & \\
\hline H3K 14 & $\downarrow$ & Acetylation & AD mouse models & & & \\
\hline $\mathrm{H} 4 \mathrm{~K} 5$ & $\downarrow$ & Acetylation & $\begin{array}{c}\text { Cdk5cKO mice; } \\
\text { hippocampus and } \\
\text { PFC) }\end{array}$ & $6-9$ & & \\
\hline $\mathrm{H} 4 \mathrm{~K} 12$ & $\downarrow$ & Acetylation & $\begin{array}{l}\text { Mice (16 months } \\
\text { old) }\end{array}$ & $4-5$ & $\begin{array}{l}\text { ChIP, PCR, } \\
\text { HAT/ HDAC } \\
\text { assay }\end{array}$ & [281] \\
\hline \multirow{2}{*}{ SIRT1 } & $\downarrow$ & Expression & $\begin{array}{l}\text { AD patients } \\
\text { (parietal cortex) }\end{array}$ & 19 & \multirow{2}{*}{ WB, ISH } & \multirow{2}{*}[511]{} \\
\hline & $\uparrow$ & Expression & $\begin{array}{l}\text { AD mouse model } \\
(3 \times T g-A D \text { mice })\end{array}$ & NS & & \\
\hline
\end{tabular}


TABLE 7. Epigenetic dysregulation in Alzheimer's disease: chromatin remodeling.

$\uparrow$ indicates increased levels and $\Downarrow$ indicates decreased levels.

ABBREVIATIONS: 3xTg-AD mice, triple transgenic mouse model of $A D$ expressing human mutant amyloid precursor protein (APPK670N/M671L), presenilin 1 (PSIM146V), and tau (TauP301L); 5xFAD mice, transgenic mouse model overexpressing mutant human APP (695) with the Swedish (K670N and M671L), Florida (I716V), and London (V717I) mutations, and mutant PS1 with the M146L and L286V familial AD mutations; AD, Alzheimer's disease; APP/PSl mice, transgenic mouse model expressing mutant human APPK670N/M671L and PSIMl46V; CA, cornu ammonis; Cdk5cKO mice, cyclin-dependent kinase 5 knock-out mouse model; ChIP, chromatin immunoprecipitation; CK-p25 mice, transgenic mouse model overexpressing p25 under control of an inducible calcium/calmodulin-dependent protein kinase II a promoter; Co-IP, protein complex immunoprecipitation; EC, entorhinal cortex; FAIRE, formaldehyde-assisted isolation of regulation; $\mathrm{H}$, histone; $\mathrm{HAT}$, histone acetyltransferase; HDAC, histone deacetylase; ICC, immunocytochemistry; IF, immunofluorescence; IHC, immunohistochemistry; ISH, in situ hybridization; K, lysine; NS, not specified; PCR, polymerase chain reaction; PFC, prefrontal cortex; S, serine; SIRT, sirtuin; Tg2576 mice, transgenic mice overexpressing human APP isoform 695 with the double KM670/671NL Swedish mutation; WB, western blot. such as a-synuclein found in Lewy bodies, and enhance their aggregation [534]. Conversely, a-synuclein was found to have neuroprotective actions via its interactions with EP300 and nuclear transcription factor kappa $\mathrm{B}(\mathrm{NF}-\mathrm{KB})$, downregulating the proapoptotic protein kinase $\mathrm{C} \delta$ (PKC $\delta$ ) [535].

In PD patients, most of the aforementioned findings regarding the involvement of histone modifications still need to be replicated, but there is a report of an fPD case with a heterozygous A53T SNCA mutation, in which the affected allele was epigenetically silenced through histone modifications and the normal allele displayed expression levels exceeding those of two normal alleles in controls [536].

Previously, the mechanism of DNA methylation-induced allelic skewing was described as a mediator between the genotype and environment. Histone modifications, however, are the most common epigenetic modality affected by environmental toxins such as pesticides, herbicides and industrial agents [386]. MPTP, for instance, has been shown to lower H3K4me3 levels in the striatum of mice and non-human primates [537]. Interestingly, $\mathrm{H} 3 \mathrm{~K} 4 \mathrm{me} 3$ levels could be restored through chronic L-3,4dihydroxy-phenylalanine (L-DOPA) treatment. Additionally, the herbicide paraquat and the insecticide dieldrin, which have both been associated with the development of PD, were found to affect histone acetylation, with exposure to paraquat increasing $\mathrm{H} 3$ acetylation and hampering overall HDAC activity, and exposure to dieldrin increasing $\mathrm{H} 3$ and $\mathrm{H} 4$ acetylation, in N27 dopaminergic cells [538, 539]. Dieldrin induces apoptosis in neurons and is thought to enhance histone acetylation through its inhibitory interaction with the proteasome system, leading to the buildup of CREBBP, an important KAT. Administration of the KAT inhibitor anacardic acid in a mouse model exposed to dieldrin, decreased histone acetylation and apoptosis, suggesting that the neurotoxic effect of dieldrin leading to apoptosis might be the result of detrimental histone acetylation [538]. See Table 8 for an overview of the aberrant chromatin remodeling seen in PD. 


\begin{tabular}{|c|c|c|c|c|c|c|}
\hline $\begin{array}{l}\text { Chromatin } \\
\text { remodeling } \\
\text { target }\end{array}$ & Regulation & $\begin{array}{l}\text { Epigenetic } \\
\text { modification }\end{array}$ & Observed in & $\begin{array}{l}\text { Sample } \\
\text { size }\end{array}$ & Methods & Reference \\
\hline $\begin{array}{l}\text { Histones } \\
\text { (globally) }\end{array}$ & $\uparrow$ & $\begin{array}{l}\text { Deacetylation } \\
\text { via binding of } \\
\text { a-synuclein to } \\
\text { PGC1-a promoter } \\
\text { element }\end{array}$ & PD patients (SN) & 16 & GWES & {$[533]$} \\
\hline \multirow[t]{2}{*}{ H3 } & $\downarrow$ & $\begin{array}{l}\text { Acetylation via } \\
\text { the interaction of } \\
\text { a-synuclein with } \\
\text { SIRT1 }\end{array}$ & $\begin{array}{c}\text { a-Synuclein- } \\
\text { transfected SH- } \\
\text { SY5Y cells } \\
\text { PD Drosophila } \\
\text { model }\end{array}$ & NS & WB & {$[529]$} \\
\hline & $\uparrow$ & $\begin{array}{l}\text { Acetylation due to } \\
\text { paraquat and/or } \\
\text { dieldrin exposure }\end{array}$ & $\begin{array}{c}\text { N27 dopaminergic } \\
\text { cells }\end{array}$ & - & WB & {$[538,539]$} \\
\hline $\mathrm{H} 3 \mathrm{~K} 4$ & $\downarrow$ & $\begin{array}{l}\text { Tri-methylation } \\
\text { due to MPTP- } \\
\text { induced toxicity }\end{array}$ & $\begin{array}{c}\text { Mice (striatum) } \\
\text { Non-human } \\
\text { primates (striatum) }\end{array}$ & 18 & WB & {$[537]$} \\
\hline $\mathrm{H} 4$ & $\uparrow$ & $\begin{array}{l}\text { Acetylation due to } \\
\text { dieldrin exposure }\end{array}$ & $\begin{array}{l}\text { N27 dopaminergic } \\
\text { cells }\end{array}$ & - & WB & {$[539]$} \\
\hline $\begin{array}{c}\text { Heterozygous } \\
\text { A53T SNCA } \\
\text { mutation }\end{array}$ & $x$ & $\begin{array}{l}\text { Epigenetically } \\
\text { silenced affected } \\
\text { allele via histone } \\
\text { modifications }\end{array}$ & fPD patient & 1 & PCR & {$[536]$} \\
\hline
\end{tabular}

\subsubsection{Chromatin remodel- ing in Huntington's disease}

In general, HD is associated with hypoacetylated and hypermethylated histones [75, 540, 541] (Table 9). The mechanism underlying histone hypoacetylation has been fairly well characterized and is thought to center around the deleterious interaction between CREBBP and mutant HTT [285, 286]. The polyglutamine section of mutant HTT is thought to physically interact and sequester CREBBP, hampering its KAT activity [407]. Besides its KAT activity, CREBBP has additional integral functions in the regulation of transcription, interacting with various transcription factors and the RNAPII complex. Sequestration of CREBBP by mutant HTT thus disrupts transcription at multiple levels [540, 542-545]. Interestingly, a study using transgenic mice expressing a form of CREBBP without KAT activity found that this modification specifically affected the consolidation of short-term memory into long-term memory, leaving short-term memory unaffected [286]. A similar study with inactive EP300, a homolog of CREBBP, found long-term recognition and contextual fear memory to be impaired [487].

It has been proposed that disruption of CREBBP functioning by mutant HTT is also indirectly responsible for the induction of histone hypermethylation and the subsequent formation of large abnormal heterochromatin domains [546]. CREBBP is normally thought to repress the expression of Drosophila Su(var)3-9 and enhancer of zeste proteins

TABLE 8. Epigenetic dysregulation in Parkinson's disease: chromatin remodeling.

$\uparrow$ indicates increased expression levels, $\Downarrow$ indicates decreased expression levels, and $\times$ indicates a genetic mutation affecting epigenetic regulation.

ABBREVIATIONS: fPD, familial

PD; $\mathrm{H}$, histone; K, lysine; MPTP, 1-methyl-4-phenyl-1,2,3,6-tetrahydropyridine; PD, Parkinson's disease; PGCl-a, peroxisome proliferator receptor gamma coactivator-1 alpha; SH-SY5Y cells, human neuroblastoma cell line; SIRT1, sirtuin 1; SN, substantia nigra; SNCA, synuclein alpha; N.S., not specified; GWES, genome-wide epistasis study; WB, Western blot; PCR, polymerase chain reaction. 
(SET) domain, bifurcated 1 (SETDB1), an HKMT that methylates H3K9. Due to the shutdown of CREBBP by mutant HTT, the repression of SETDB1 is released and SETDB1 levels increase, subsequently resulting in $\mathrm{H} 3 \mathrm{~K} 9$ hypermethylation. This mechanism is corroborated by observations of increased levels of SETDB1 and H3K9me3 in striatal neurons of both transgenic HD mice and HD cases [544]. Additionally, $\mathrm{H} 3 \mathrm{~K} 9 \mathrm{me} 3$ induced chromatin remodeling has been directly associated with altered gene expression profiles in HD [407, 546, 547]. Among the genes thought to be affected by this aberrant chromatin condensation is CHRM1 [548]. Decreased expression of muscarinic acetylcholine receptor 1 (CHRM1) has been proposed to induce synaptic dysfunction and CHRM1 levels are indeed lowered in the HD striatum [549, 550]. Deregulation of striatal cholinergic signaling has been identified as a pivotal factor in the pathophysiology of HD, especially affecting medium spiny neurons [551].

TABLE 9. Epigenetic dysregulation in Huntington's disease: chromatin remodeling.

$\uparrow$ indicates increased levels and $\neq$ indicates altered, not further specified.

ABBREVIATIONS: H, histone; HD, Huntington's disease; K, lysine; NS, not specified; R6/2 mice, transgenic mouse model overexpressing exon 1 of human HTT with an expanded cytosine-adenine-guanine repeat length; RT-PCR, real-time polymerase chain reaction; WB, western blot.

\begin{tabular}{|c|c|c|c|c|c|c|}
\hline $\begin{array}{c}\text { Chromatin } \\
\text { remodeling } \\
\text { target }\end{array}$ & Regulation & $\begin{array}{l}\text { Epigenetic } \\
\text { modification }\end{array}$ & Observed in & $\begin{array}{l}\text { Sample } \\
\text { size }\end{array}$ & Methods & Reference \\
\hline $\begin{array}{l}\text { histones } \\
\text { (Globally) }\end{array}$ & $\neq$ & Acetylation & $\begin{array}{c}\text { HD mouse } \\
\text { model ( } R 6 / 2 \\
\text { mice) }\end{array}$ & NS & WB & {$[540]$} \\
\hline H3К9 & $\uparrow$ & $\begin{array}{c}\text { Tri- } \\
\text { methylation }\end{array}$ & $\begin{array}{l}\text { HD mouse } \\
\text { model (R6/2 } \\
\text { mice; striatal } \\
\text { neurons) }\end{array}$ & 10 & $\begin{array}{l}\text { RT-PCR, } \\
\text { histone } \\
\text { methylation }\end{array}$ & {$[544]$} \\
\hline & & & $\begin{array}{l}\text { HD patients } \\
\text { (striatum) }\end{array}$ & 6 & assay & \\
\hline
\end{tabular}

\subsection{Non-coding $R N A$ s in neu- rodegeneration}

\subsubsection{Non-coding RNAs and Alzheimer's disease}

In addition to DNA methylation and chromatin remodeling, ncRNAs, and especially miRNAs, have more recently been identified as possible contributors to AD pathology [552] (Table 10). Interestingly, miRNA profiling studies have found several miRNAs to be upregulated in peripheral blood mononuclear cells of AD patients [553]. Apart from the blood, many brain region-specific imbalances in miRNA expression have been identified in relation to AD (for review see [554]), including those with candidate binding sites in the 3' UTRs of $\beta$-secretase (BACE), PS1 and APP mRNA. More specifically, miR-16, -17, -20a, -101, -106a, $-106 b,-107,-124,-137,-147,-153,-195,-323-3 p,-520 c,-644,-655$ and 
let-7 are thought to regulate APP metabolism and A $\beta$ production [313, 317, 555-561]. MiR-16 overexpression was found to reduce APP levels in SAMP8 mice [317]. In human neurons, miR-106a, -153 and -520c were found to target APP mRNA, downregulating APP and A $\beta$ levels [561, 562]. Others, however, could not corroborate the involvement of $\mathrm{miR}-106 \mathrm{a}$ and $\mathrm{miR}-520 \mathrm{c}$ in the regulation of APP expression $[313,556]$. Inhibiting miR-101 in hippocampal neurons proved to decrease APP expression and $A \beta$ load, indicating a possible detrimental role of the miRNA in AD [559]. Conversely, miR-124, a miRNA involved in adult neuronal differentiation [563], is reported to be downregulated in some AD patients [564]. MiR-124 is thought to, together with polypyrimidine tract binding protein (PTBP) 1, modulate the alternative splicing of APP exons 7 and 8 . Additionally, miR-124, but also miR-9, -29a/b-1, -29c, $-107,-195,-298,-328$ and $-485-5 p$, affect $A \beta$ indirectly by modulating BACE1 mRNA translation [313, 565-567]. In addition, in SAMP8 mice miR-195 expression was found to be decreased, whereas BACE1 levels were heightened [567]. The involvement of all these miRNAs in $A D$ might, however, not be a general phenomenon. For instance, the $\mathrm{miR}-29 \mathrm{a} / \mathrm{b}-1$ cluster was found to be lowered in the anterior temporal cortex of SAD patients, coupled with high BACE1 protein levels, but only in approximately $30 \%$ of the examined cases [566]. In a transgenic AD mouse model miR-29c was observed to be highly expressed and was found to hamper BACE1 expression [568]. Levels of miR-107 were found to be lowered in the temporal cortex of $A D$ cases, which was suggested to facilitate $A D$ progression as a result of diminished BACE1 repression [569-571]. MiR-195, -298, and -328 also reduce $A \beta$ production by inhibiting BACE1 mRNA translation [567, 572]. Interestingly, while most of the miRNAs affecting BACE1 expression repress translation by binding to the 3' UTR of its mRNA, miR-485-5p represses BACE1 by binding to the open reading frame in exon 6 [573]. The involvement of post-transcriptional regulation of BACE1 is further supported by the observation that in AD brains BACE1 protein levels are increased, whereas mRNA levels remain unchanged [313]. Serine palmitoyltransferase (SPT) is an enzyme crucial for ceramide synthesis, which is thought to facilitate $A \beta$ production. MiR-9, $-29 a / b-1,-137$ and $-181 \mathrm{c}$ negatively modulate SPT production and their levels were lowered in the frontal cortex of AD patients [574]. MiR-137 is known to additionally promote proliferation of neural stem cells through the inhibition of differentiation and dendrite formation $[575,576]$.

MiRNAs can thus affect $A \beta$ production, but $A \beta$ can also affect the expression of some miRNAs in vitro, for example inducing miR-106b expression [577] but repressing miR-9 and miR-181c [578]. Curiously, Hébert et al. [579] found miR-106b to be downregulated in the anterior temporal cortex of AD brains. Furthermore, miR-106 was reported to 
not only directly bind to and inhibit the translation of APP mRNA, but also affect APP trafficking and $A \beta$ clearance. Additionally, by regulating ATP binding cassette subfamily A member 1 (ABCA1), which transports cholesterol, it is thought to influence BACE and $\gamma$-secretase functioning. ABCA1 expression in the hippocampus has been positively correlated with cognitive impairments in AD [580]. Normally, miR-106b is thought to promote neurogenesis through its regulation of the insulin-like growth factor (IGF) 1 pathway [581]. MiR-9 has been reported to be a pivotal player in the differentiation and migration of neural stem cells [582, 583].

Furthermore, while there are generally no AD-associated mutations in tau, miR-15, $-16,-132$, and -497 are thought to regulate tau expression and might play a role in AD. In example, a decrease in miR-132 is suggested to mediate the alternative splicing of tau exon 10 , through a lowered repression of PTBP2, which hampers physiological phosphorylation of tau $[584,585]$. Alternative splicing of tau influences whether it contains 3 or 4 microtubule-binding repeats (3R-tau and $4 \mathrm{R}$-tau, respectively) [586]. Furthermore, changes in the $3 \mathrm{R}: 4 \mathrm{R}$ tau ratio are thought to be related to neurodegeneration [587]. Apart from miR-132, miR-9, -124 and -137 have also been reported to affect the 3R:4R tau ratio. MiR-212 and miR-454 have also been implicated in NFT pathology in AD [570, 588]. Note that dysregulated miRNA expression in relation to tau is probably not unique for $A D$ and likely also occurs in other tauopathies. For instance, miR-132 was found to be downregulated in progressive supranuclear palsy and frontotemporal lobar degeneration [585, 589].

Phosphorylation of tau is performed by extracellular signal-regulated kinase (ERK) 1, which in turn is regulated by members of the miR-16 family (miR-15, -16, -195 and -495), of which miR-15 was found to be downregulated in AD [584]. Tau can also be phosphorylated by glycogen synthase kinase GSK $3 \beta$, which has been implicated in A $\beta$ and NFT formation, and has been reported to be negatively regulated by miR$26 a$, a miRNA that is dysregulated in $\operatorname{AD}[588,590]$. As stated above, SIRT1 negatively regulates tau expression, while miR-9, $-34 \mathrm{c}$ and -181c, however, have been shown to inhibit SIRT1 production, thereby enhancing tau production in $\mathrm{AD}[578,591]$. MiR-128 has been suggested to affect tau clearance, through its regulation of cochaperone B-cell chronic lymphocytic leukemia/lymphoma 2-associated athanogene 2 (BAG2), and has been reported to be altered in AD [592, 593]. There is additional indirect evidence for the involvement of miRNAs in the regulation of tau metabolism, as studies knocking out Dicer, which is crucial for miRNA processing, observed increased hyperphosphorylation of tau, alternate splicing of tau and neurodegeneration [594, 595]. 
Next to miRNAs impacting on $A \beta$ and tau metabolism, various miRNAs that were found to be dysregulated in $A D$ also affect other pathological hallmarks of $A D$. MiR-146a, is for instance a regulator of inflammatory processes through its interaction with interleukin-1 receptor-associated kinase (IRAK) 1 that is upregulated in AD brains [596, 597]. In addition to IRAK1, miR-146a was reported to bind to the 3' UTR of complement factor $\mathrm{H}$, a suppressor of inflammation which is downregulated in $\mathrm{AD}$ [598]. Another regulator of inflammation is miR-101, which normally inhibits (COX)-2, but its levels were shown to be lowered in $A D$, whereas levels of COX-2 were increased [559]. MiR-132 and miR-125b have been linked to synaptic plasticity, and miR-132 was lower in the hippocampus, cerebellum and medial frontal gyrus of AD patients, whereas miR-125b levels were higher in these areas [599]. Brain cytoplasmic RNA 200 (BC200) was initially reported to be decreased in the temporal neocortex of $A D$ cases [600], but later studies reported increased BC200 levels in the hippocampus and superior frontal gyrus, but erroneously located in the neuronal soma [601]. BC200 is thought to enhance long-term synaptic plasticity by interacting with protein synthesis in postsynaptic microdomains. In transgenic mice overexpressing a combination of APPswe and human APP with the V717F Indiana mutation (APPind; Tg19959 mice) miR-103 and miR-107 were found to be decreased, which was linked to increased cofilin expression [602]. Cofilin is a pivotal player in cytoskeletal integrity and is thought to influence microtubule stability, neuronal transport and synaptic functioning [603].

Compared to miRNAs, evidence for the involvement of other ncRNAs in AD pathology is sparse. RNAPIII-dependent ncRNA neuroblastoma differentiation marker (NDM) 29 was found to facilitate the production and secretion of $A \beta$ by influencing APP processing [604], whereas the IncRNA BACE1-antisense (BACE1-AS) positively affects BACE1 expression [313]. BACE1-AS has a length of about $2 \mathrm{~kb}$ and is transcribed from the DNA strand complementary to the BACE1 gene [605]. It is thought to enhance the stability of BACE1 mRNA, facilitating BACE1 protein production. Interestingly, BACE1-AS transcription is enhanced in response to $A \beta$ exposure, initiating a vicious cycle, as its positive effects on BACE1 expression in turn enhances $A \beta$ production. In both $A D$ patients and Tg19959 mice, BACE1-AS was indeed found to be overexpressed. Although only confirmed for the nonconventional miR-485-5p, evidence suggests that the binding of BACE1-AS to BACE1 mRNA enhances mRNA stability by competing with miRNA binding [573]. The ncRNA 17a has been observed to promote $A \beta$ secretion and accumulation and is elevated in the cerebral cortex of AD cases, which is thought to be the result of inflammatory factors [606]. 


\subsubsection{Non-coding RNAs and Parkinson's disease}

Apart from epigenetic transcriptional regulation of SNCA, some miRNAs have been identified that regulate its function on a translational level. One of these is miR-7, which negatively regulates a-synuclein expression through binding to the 3' UTR of a-synuclein mRNA and is mainly expressed in neurons [607]. Through its suppression of a-synuclein, including cytotoxic mutant forms, it is thought to have a neuroprotective role in PD. Interestingly, miR-7 levels were shown to be decreased in vitro and in animal models after exposure to the toxic metabolite of MPTP, 1-methyl-4-phenyl-pyridinium ion (MPP+), increasing a-synuclein expression. Downregulation of miR-7 might thus, at least in part, explain how MPTP induces PD-like pathology.

Another miRNA, miR-153, represses a-synuclein production both at a mRNA and protein level [608]. Indirectly, miR-433 has also been implicated in SNCA expression, via its regulation of the fibroblast growth factor 20 (FGF20). FGF20 expression has been positively correlated with a-synuclein expression, and a 3' UTR SNP (rs1270208) has been linked to an increased risk to develop PD. This SNP interferes with miR-433 binding, increasing FGF20 expression [609]. Conversely, a-synuclein has been shown to affect the expression levels of certain miRNAs in in vivo models were a-synuclein was overexpressed. Levels of various miRNAs were affected in a transgenic mouse model overexpressing human A30P a-synuclein [610] (Table 11). In a transgenic $C$. elegans model expressing human a-synuclein, alterations in levels of 12 miRNAs were found [611]. The significance for the human situation, however, remains to be elucidated as the human orthologs of these miRNAs remain to be identified.

In addition to SNCA, the expression of $L R R K 2$, a gene implicated in both fPD and SPD, is also regulated by miRNAs. MiR-205 targets the 3' UTR of $L R R K 2 \mathrm{mRNA}$ and was found to be downregulated in SPD cases in which leucine-rich repeat kinase 2 (LRRK2) protein levels were increased, whereas miR-205 was able to mitigate the aberrant neurite growth induced by LRRK2 mutation R1411G in vitro [612]. Conversely, mutant LRRK2 (I1915T or G2019S) was observed to inhibit the actions of let-7 and miR-184*. These miRNAs regulate E2F transcription factor 1 (E2F1) and differentiation regulated transcription factor protein (DP) levels, transcription factors associated with cell cycle regulation and cell survival. LRRK2 thus induces E2F1 and DP expression, which is associated with reduced dopaminergic neuron numbers and locomotor activity in Drosophila, effects that have also been linked to mutant LRRK2 [613]. 


\begin{tabular}{|c|c|c|c|c|c|c|}
\hline Regulation & ncRNA & Observed in & $\begin{array}{l}\text { Sample } \\
\text { size }\end{array}$ & Approach & Methods & Reference \\
\hline$\downarrow$ & miR-124 & AD patients & 11 & Targeted & $\begin{array}{l}\text { WB, RT- } \\
\text { PCR }\end{array}$ & [564] \\
\hline$\downarrow$ & miR-195 & $\begin{array}{c}\text { AD mouse } \\
\text { model (SAMP8 } \\
\text { mice) }\end{array}$ & N.S. & $\begin{array}{c}\text { Targeted } \\
\text { (5 miRNAs) }\end{array}$ & RT-PCR & [567] \\
\hline$\downarrow$ & miR-29a/b-1 & $\begin{array}{l}\text { sAD patients } \\
\text { (ATC) }\end{array}$ & 34 & Genome-wide & $\begin{array}{l}\text { miRNA } \\
\text { microarray }\end{array}$ & [566] \\
\hline$\uparrow$ & miR-29c & $\begin{array}{l}\text { AD mouse } \\
\text { model (APP/ } \\
\text { PSdE9 mice) }\end{array}$ & 6 & Targeted & RT-PCR & [568] \\
\hline \multirow{2}{*}{$\downarrow$} & \multirow{2}{*}{ miR-107 } & AD patients & 7,10 & Genome-wide & $\begin{array}{c}\text { miRNA } \\
\text { microarray, } \\
\text { rna22 } \\
\text { algorithm }\end{array}$ & {$[569,570]$} \\
\hline & & $\begin{array}{c}\text { AD mouse } \\
\text { model } \\
(\mathrm{Tg} 19959 \\
\text { mice })\end{array}$ & 7 & Targeted & RT-PCR & [602] \\
\hline$\downarrow$ & $m i R-106 b$ & $\begin{array}{l}\text { AD patients } \\
\text { (ATC) }\end{array}$ & 19 & $\begin{array}{c}\text { Targeted } \\
\text { (200 miRNAs) }\end{array}$ & $\begin{array}{c}\text { miRNA } \\
\text { microarray, } \\
\text { NB }\end{array}$ & [579] \\
\hline$\downarrow$ & $\begin{array}{l}\text { miR-9, miR- } \\
181 \mathrm{c}\end{array}$ & AD patients & $\begin{array}{c}15 \\
\text { (brain) } \\
10 \\
\text { (CSF) }\end{array}$ & $\begin{array}{c}\text { Targeted } \\
\text { (48 miRNAs) }\end{array}$ & PCR & [588] \\
\hline$\downarrow$ & miR-132 & $\begin{array}{l}\text { AD patients } \\
\text { (hippocampus, } \\
\text { cerebellum, } \\
\text { MedFG) }\end{array}$ & 6 & $\begin{array}{c}\text { Targeted } \\
(15 \text { miRNAs) }\end{array}$ & NB & [599] \\
\hline$\downarrow$ & miR-15 & $\begin{array}{l}\text { Dicer cKO } \\
\text { mice }\end{array}$ & $8-10$ & $\begin{array}{c}\text { Targeted } \\
\text { (200 miRNAs) }\end{array}$ & $\begin{array}{c}\text { miRNA } \\
\text { microarray }\end{array}$ & [595] \\
\hline$\neq$ & miR-26a & $A D$ patients & $\begin{array}{c}15 \\
\text { (brain) } \\
10 \\
\text { (CSF) }\end{array}$ & $\begin{array}{c}\text { Targeted } \\
(48 \text { miRNAs) }\end{array}$ & PCR & [588] \\
\hline$\downarrow$ & miR-101 & $\begin{array}{c}\text { HeLa, } \\
\text { HEK293T, } \\
\text { U373, SK- } \\
\text { N-SH, PC12 } \\
\text { cells }\end{array}$ & - & $\begin{array}{l}\text { Targeted } \\
(1 \text { miRNA })\end{array}$ & PCR & [559] \\
\hline$\uparrow$ & $m i R-125 b$ & $\begin{array}{c}\text { AD patients } \\
\text { (hippocampus, } \\
\text { cerebellum, } \\
\text { medial frontal } \\
\text { gyrus) }\end{array}$ & 6 & $\begin{array}{c}\text { Targeted } \\
(15 \text { miRNAs) }\end{array}$ & NB & [599] \\
\hline$\uparrow$ & \multirow{2}{*}{ BC200 } & $\begin{array}{c}\text { AD patients } \\
\text { (hippocampus, } \\
\text { SFG) }\end{array}$ & \multirow{2}{*}{12} & \multirow{2}{*}{$\begin{array}{c}\text { Targeted } \\
\text { (2 miRNAs) }\end{array}$} & \multirow{2}{*}{ NB, ISH } & \multirow{2}{*}{ [601] } \\
\hline$\downarrow$ & & $\begin{array}{c}\text { AD patients } \\
\text { (temporal } \\
\text { neocortex) }\end{array}$ & & & & \\
\hline$\downarrow$ & miR-103 & $\begin{array}{l}\text { AD mouse } \\
\text { model } \\
(\mathrm{Tg} 19959 \\
\text { mice })\end{array}$ & 7 & $\begin{array}{l}\text { Targeted } \\
\text { (2 miRNAs) }\end{array}$ & RT-PCR & [602] \\
\hline$\uparrow$ & BACE1-AS & AD patients & 2 & $\begin{array}{c}\text { Targeted } \\
\text { (48 probes) }\end{array}$ & $\begin{array}{l}\text { HTS, ECA } \\
\text { ECA }\end{array}$ & [573] \\
\hline$\uparrow$ & ncRNA 17a & AD patients & 11 & $\begin{array}{l}\text { Targeted } \\
\text { (5 probes) }\end{array}$ & RT-PCR & [606] \\
\hline
\end{tabular}

Overexpression of let-7 or miR-184* reversed the deleterious effects of mutant LRRK2 expression. Note, however, that let-7b was also found to progressively inhibit neural stem cell proliferation in the subventricular zone with age [614]. Interestingly, the disruption of let-7 and miR-184*
웅

TABLE 10. Epigenetic dysregulation in Alzheimer's disease: non-coding RNAs.

$\uparrow$ indicates increased levels, $\Downarrow$ indicates decreased levels, and $\neq$ indicates altered, not further specified.

ABBREVIATIONS: (s)AD, (sporadic) Alzheimer's disease; APP/PSldE9 mice, transgenic mice overexpressing human APP isoform 695 with the double KM670/671NL Swedish mutation and human PSl with the deleted exon 9 mutation; ATC, anterior temporal cortex; BACE1-AS, b-secretase 1-antisense; BC200, brain cytoplasmic RNA 200; CSF, cerebrospinal fluid; Dicer cKO mice, conditional Dicerl knockout mouse model; ECA, enzyme complementation assay; HEK293 cells, human embryonic kidney 293 cell line; HeLa cells, cell line established from cervical cancer cells; HTS, high-throughput sequencing; ISH, in situ hybridization; MedFG, medial frontal gyrus; miR (NA), micro RNA; NB, northern blot; ncRNA, non-coding RNA; NS, not specified; PC12 cells, rat pheochromocytoma-derived cell line; $\mathrm{PCR}$, polymerase chain reaction; RT-PCR, real-time PGR; SAMP8 mouse, senescence-accelerated prone mouse 8; SFG, superior frontal gyrus; SK-N-SH cells, human neuroblastoma cell line; Tg19959 mice, transgenic mouse model overexpressing mutant human APP with the double KM670/671NL Swedish and V717F Indiana mutations; U373 cells, human glioblastoma astrocytoma-derived cell line; WB, western blot. 
activity by mutant LRRK2 is thought to be an indirect effect, as the increased activity of mutant LRRK2 increases the phosphorylation of eukaryotic translation initiation factor 4E binding protein (4E-BP). 4E-BP interacts with Argonaute 2, a pivotal constituent of the RISC, which in turn is required for proper let-7 and miR-184* functioning [615]. The negative regulation of these miRNAs by LRRK2 thus depends on gain of function mutations, such as I1915T and G2019S [615, 616]. Indeed, mutant LRRK2 without enzymatic activity does not affect miRNA repression [613]. Additionally, LRRK2 might also affect Dicer, another protein integral to the RNA interference (RNAi) pathway, as knocking down LRRK2 was able to attenuate some of the pathology in the Drosophila model related to decreased Dicer activity.

MiRNA profiling of PD brains at different stages of the disease pointed towards a miR-34b/c downregulation, mainly at the early premotor stages (1-3) [617]. MiR-34b/c is thought to modulate mitochondrial functioning via its modulation of deglycase DJ-1 and E3 ubiquitin-protein ligase parkin, proteins that have both been associated with fPD. In the blood, comparing healthy individuals with untreated PD patients, miR-1, -22*, and -29 were found to be differentially expressed, while miR-16-2*, $-26 \mathrm{a} 2^{*}$, and $-30 \mathrm{a}$ were differentially expressed comparing treated and untreated PD patients [618]. Table 11 contains the most important findings regarding ncRNAs associated with PD.

TABLE 11. Epigenetic dysregulation in Parkinson's disease: non-coding RNAs.

$\Downarrow$ indicates decreased levels and $\neq$ indicates altered, not further specified.

ABBREVIATIONS: HEK293T cells, human embryonic kidney cell line; miR, micro RNA; NS20Y, mouse cholinergic neuroblastoma cell line; PCR, polymerase chain reaction; SH-SY5Y cells, human neuroblastoma cell line; (s)PD, (sporadic) Parkinson's disease.

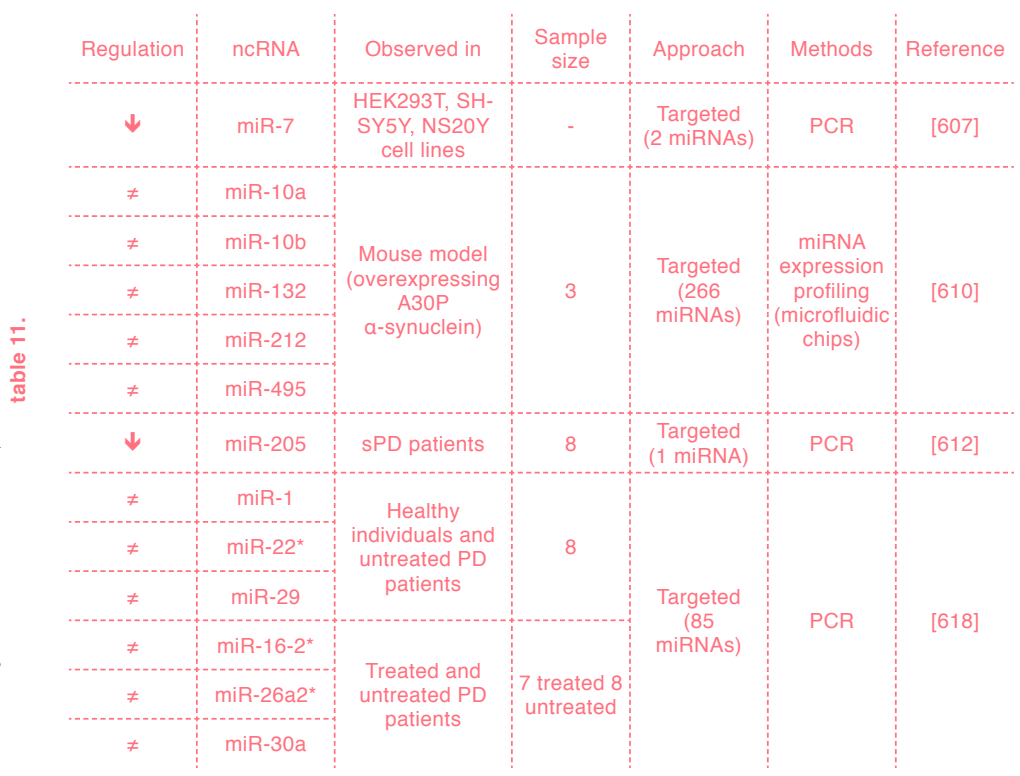




\subsubsection{Non-coding RNAs and Huntington's disease}

In accordance with the widespread dysregulation of gene expression, the expression of miRNAs is also affected in HD (Table 12). In HD models and patients neuronal miRNA expression was found to be decreased in general, resulting in an upregulation of their target mRNAs [144, 619, 620] (Table 12). In addition, it was observed that mutant HTT expression decreased miR-125b and miR-150 expression [621]. These miRNAs have P53 among their targets, which is known to repress NF-KB and miR-146a expression. Further interactions between P53 and mutant HTT mediate nuclear and mitochondrial damage in HD models and patients [622].

\subsection{Epigenetic-based diagnos- tics and therapies}

The available treatment strategies for most progressive neurodegenerative diseases only provide symptomatic relief, stressing the need to develop innovative, realistic therapeutic approaches that can effectively modulate the disease process. The factor common to all of the conditions discussed in this review is their neurodegenerative nature. Treatments providing a general neuroprotective effect could thus potentially be beneficial for any of them. Among such treatments, HDAC and DNMT inhibitors represent interesting options to act upon the epigenetic machinery. These are already used in the treatment of other disorders such as epilepsy and cancer [623]. The versatile and reversible nature of epigenetic changes makes epigenetic mechanisms ideal targets for the development of efficient, novel treatment strategies [624]. The adverse role of HDAC2 in memory facilitation has, for instance, led to the investigation of HDACls as a potential treatment for memory impairment, for example in AD [95].

\subsubsection{Strategies targeting DNA methylation}

Neurodegenerative disorders may involve a dysregulated SAM metabolism, resulting in global DNA hypomethylation, as well as the hypermethylation of some crucial genes. It is thus not surprising that strategies aiming to increase or decrease DNA methylation have been investigated. Enhancing DNA methylation can be achieved by boosting 


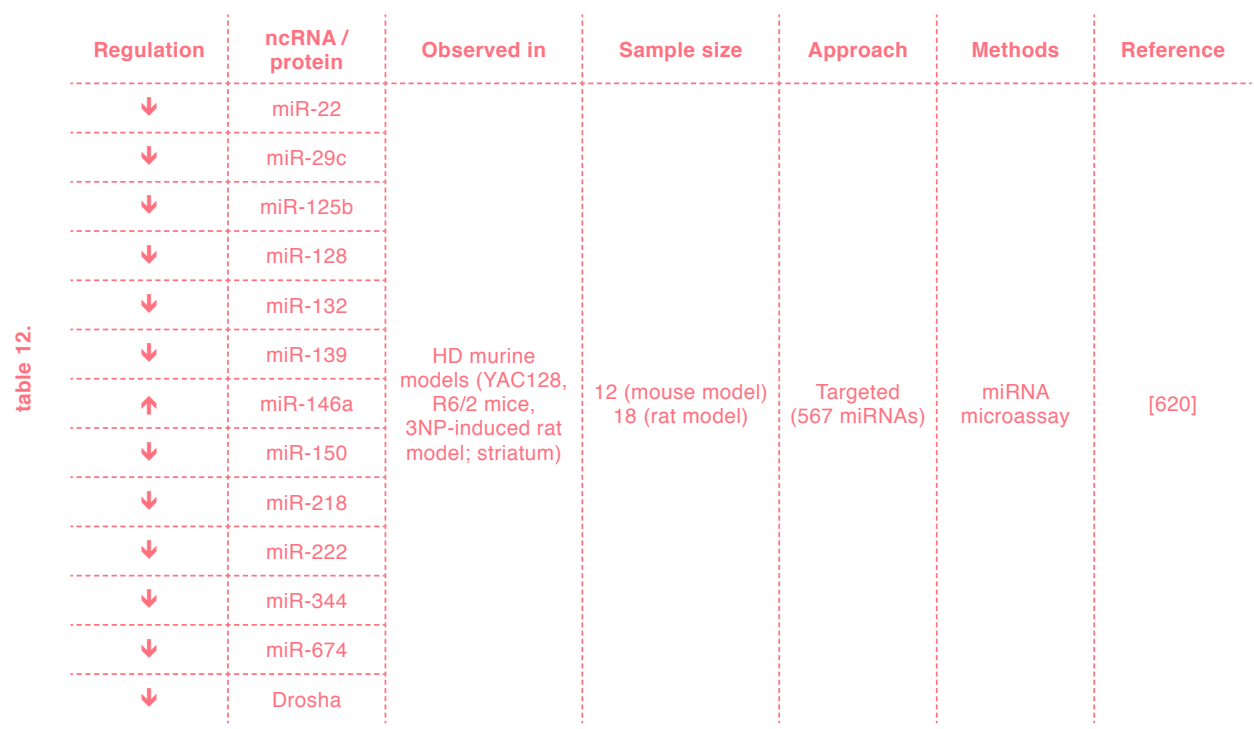

SAM metabolism, for example through the administration of SAM itself, and by vitamin B12 and folate supplementation was shown to be effective [625-627]. Reducing the levels of methylated DNA can be accomplished with DNA demethylating agents, such as DAC [32]. However, these treatment options are highly unspecific, which may, especially in the case of DNA demethylating compounds, result in considerable adverse effects. Apart from therapies targeting DNA methylation, it has also been suggested that differential genomic and mtDNA methylation patterns may serve as diagnostic biomarkers [628, 629].

\subsubsection{Alzheimer's disease}

TABLE 12. Epigenetic dysregulation in Huntington's disease: non-coding RNAs.

$\uparrow$ indicates increased levels and $\Downarrow$ indicates decreased levels.

ABBREVIATIONS: 3NP, 3-nitropropionic acid; HD, Huntington's disease; miR(NA), micro RNA ; R6/2 mice, transgenic mice overexpressing exon 1 of human huntingtin with an expanded cytosine-adenine-guanine repeat length; YAC128 mice, transgenic mouse model expressing the human huntingtin gene with 128 cytosine-adenine-guanine repeats.
Scarpa et al. [627] argued that the Hcy accumulation often seen in AD might be an indication for an abnormal SAM metabolism. The resulting decrement in SAM levels could explain a global decrease in DNA methylation, which in turn could lead to an overexpression of multiple genes, including ones involved in AD pathology. Interestingly, in vitro SAM administration led to a repression of PS1 gene expression and $A \beta$ production. Accordingly, folate and vitamin B12 supplementation have been found to enhance cognitive functioning and slow the development of dementia [625, 626]. There are, however, also other studies that could not detect a positive effect of folate and vitamin B12 supplementation [630, 631], and it has been reported that folic acid supplementation, in addition to other side-effects, might exacerbate neuropathology in patients with low vitamin B12 levels [632]. The observation that some crucial genes are hypermethylated in AD has led to the suggestion that the DNA demethylating agent DAC could be used to restore normal expression 
levels of these genes. AD, however, is also associated with general hypomethylation and due to the non-specific nature of DAC it might in fact cause more harm than good [32].

\subsubsection{Parkinson's disease}

Similarly to AD, a disturbed SAM metabolism has also been associated with PD, and decreased methylation was linked to cognitive decline [249]. A viable option to counteract this decline would be to increase the levels of SAM, through administration of methionine, choline, folates or vitamin B12, among other possibilities [623].

\subsubsection{Strategies targeting chromatin modifications}

One of the most promising epigenetics-based treatment options in relation to neurodegeneration are HDACls. There are many HDACls, which can be subdivided into four classes, including short-chain fatty acids, hydroxamic acids, epoxyketones and benzamides. Of these, sodium butyrate (SB) has received most of the attention for clinical use. The bioavailability of SB in the central nervous system has been characterized and is well tolerated in animals and in humans due to its low toxicity [633-635]. Chen et al. [636] investigated the short-chain fatty acid valproate (valproic acid, VPA), a drug used as a mood stabilizer and anti-epileptic that was found to be an HDACI. VPA is thought to enhance $\mathrm{H} 3$ acetylation indirectly, possibly through the recruitment of the KAT EP300 [637]. This study found that VPA exerts a neurotrophic effect, involving the repression of pro-inflammatory factors released by microglia and a stimulation of neurotropic factor expression, including glial cell linederived neurotrophic factor (GDNF) and BDNF, by astrocytes. VPA may thus represent a viable treatment option to counteract neurodegeneration. Comparable effects have been attributed to other HDACls, including TSA, suberoylanilide hydroxamic acid (SAHA) and SB, as well as MS-275 and apicidin, which specifically inhibit class I HDACs [637-642]. Some HDACls, such as 4-phenylbutyrate (4PBA), VPA, and urocortin, might also exert some of their neuroprotective effects independent of their effects on HDACs [643-645].

The use of HDACls in the treatment of neurodegenerative diseases is thus promising and deserves attention. However, several issues, especially concerning the non-specific action of most tested HDACIs, must be overcome for HDACls to be ready for clinical use. For example, 
as some HDACls are already being used in cancer therapy, it was

observed that they induce cell death and cell-cycle arrest, which has also been reported to affect neurons [646-649]. HDACls have additionally been observed to disturb the immune system [650,651]. It has thus been found that targeting specific HDACs would be more preferable over the more general HDACls. Some examples of specific HDACls are tubacin, a selective HDAC6 inhibitor, and suramin, a selective SIRT1 and SIRT2 inhibitor [652, 653].

\subsubsection{Alzheimer's disease}

A decrease in BDNF expression, a pivotal player in memory processes [654], has been implicated as an early marker in the development of AD [490] and TSA treatment has been shown to enhance BDNF expression in vitro, possibly through restoring BDNF promoter histone acetylation levels $[655,656]$. Another HDACI, VPA, can counter A $\beta$ production in human embryonic kidney 293 (HEK293) cells expressing APPswe isoform 751 and in a transgenic mouse model overexpressing APPind (PDAPP mice) [657]. Using a transgenic mouse model with a 7-fold overexpression of APPswe (APP23 mice), this decrease in A $\beta$ was shown to be due to an inhibition of GSK-3 $\beta$-mediated $\gamma$-secretase cleavage of APP by VPA, which was also found to improve behavioral impairments [658]. Another $\mathrm{HDACl}, 4 \mathrm{PBA}$, was shown to reverse learning and memory problems in Tg2576 mice, without affecting $A \beta$ levels, but decreasing tau phosphorylation [659]. This was accompanied by increases in GSK-3 $\beta$, histone acetylation, as well as GluR1, postsynaptic density protein (PSD) 95 and microtubule-associated protein (MAP) 2, the later three being involved in synaptic plasticity [659]. A subsequent study using the same mouse model showed that 4PBA elevated intraneuronal $A \beta$ clearance, paired with an increase in plasticity-related proteins and subsequent restoring of dendritic spine densities in the hippocampus [660]. Treatment in mice with the HDACI SAHA achieved an increase in H4K12 acetylation levels and accordingly restored expression levels of genes associated with learning [281]. VPA and SAHA were also reported to restore CLU expression in vitro [661]. Effective VPA, SB, and SAHA treatment in AD models has additionally been linked to elevating $\mathrm{H} 4$ acetylation levels and alleviation of memory deficits [482]. Interestingly, although VPA, SB, and SAHA by elevating $\mathrm{H} 4$ acetylation are likely to generally affect gene expression, the HDACI TSA was found to specifically enhance expression of those genes involved in memory consolidation [662]. Curiously, inhibition of SIRTs, the class III HDACs, with nicotinamide was observed to restore cognitive impairments in $3 x T g-A D$ mice, by indirectly promoting microtubule stability, which is affected by hyperphosphorylated tau in $A D$ [663]. Recently, Forum Pharmaceuticals compound 0334 (FRM-0334), 
a class I HDACI specifically designed to cross the blood-brain barrier (BBB) was developed, addressing the problem of BBB permeability [664]. FRM-0334 is one the first HDACl that is specifically being tested for the treatment of $A D$, with most others having an approved indication in cancer treatment. In relation to specific HDAC inhibition, the selective HDAC6 tubacin has been reported to affect tau phosphorylation in vitro [496]. In addition to HDACls, KAT agonists are being developed [665] and it has also been suggested that targeting HKMTs and HKDMs may prove to be a viable treatment strategy for $A D$ [503].

\subsubsection{Parkinson's disease}

As stated above, preventing histone deacetylation may alleviate memory problems, such as those associated with AD [95]. Similar approaches in PD models suggest that HDAC inhibition could be neuroprotective. In in vitro and Drosophila models the HDACIs SB and SAHA attenuated a-synuclein-induced toxic effects [529], illustrating the prominent role of disrupted histone acetylation in the neurotoxic effects of a-synuclein, caused by its direct binding to histones. TSA was able to rescue mitochondrial fragmentation and cell death induced by MPP+ in human neuroblastoma cells [666]. Similar results were obtained when inhibiting the HDAC SIRT2 with 2-cyano-3-(5-(2,5-dichlorophenyl)-2-furanyl)-N-5quinolinyl-2-propenamide (AGK2) [530]. Additionally, pretreatment with VPA has been shown to protect midbrain dopaminergic neurons from inflammation and a-synuclein-induced neurotoxicity [636, 667-669].

Currently, one of the main treatments for PD is the dopamine precursor L-DOPA, which provides some symptom alleviation. Although not intended as such, chronic L-DOPA treatment was observed to induce epigenetic alterations. Specifically, the development of L-DOPA-induced dyskinesia presented with decreased $\mathrm{H} 3 \mathrm{~K} 4 \mathrm{me} 3$ levels, whereas L-DOPA induced hyperkinesia was associated with decreased acetylation levels of H4K5, H4K8, H4K12 and H4K16, in the striatum of animal models [537]. Additionally, it was shown that L-DOPA-induced dyskinesia paralleled $\mathrm{H} 3$ phosphoacetylation, suggesting that the inhibition of striatal $\mathrm{H} 3$ phosphoacetylation when using L-DOPA might prevent the development of dyskinesia [670].

\subsubsection{Huntington's disease}

In $\mathrm{HD}$, reversing the reduced expression of crucial genes due to histone hypoacetylation has been attempted through the application of HDACls, showing promising results, both in terms of neuropathology 
and motor symptoms [419, 540, 542, 543, 545, 671-673]. HDACls improved memory and behavior in CREBBP deficiency or KAT deletion animal models [285, 286, 674]. Additionally, in an in vitro model based on the administration of toxic polyglutamine, a model that also exhibits histone hypoacetylation, HDACls were able to mitigate the toxic effects of polyglutamine [675]. As in PD, SAHA, and SB also were effective in transgenic HD mice [676]. HDAC inhibition, either through SAHA or SB administration, or HDAC2 knock-out, improved memory in mice [677]. SB-treated transgenic mice overexpressing exon 1 of human HTT with an expanded CAG repeat length (R6/2 mice), however showed improved motor performance and decreased neuropathology, and survived significantly longer than non-treated mice [678]. Alternatively, 4PBA may represent a promising candidate treatment for $\mathrm{HD}$, as it is already FDA-approved and data about pharmacokinetics, toxicity, and dosing are available. Although 4PBA itself has no inhibitory effect on HDACs, its metabolite phenylacetate does, in addition to having a high bioavailability in the brain [679]. As with SB, treatment with 4PBA improved motor symptoms and neuropathology in a transgenic HD mouse model [542]. Unfortunately, a multicenter, double-blind, placebo-controlled clinical trial of 4PBA to determine safety and tolerability in HD, patients showed that its efficacy was very low, necessitating the use of high doses [680,681]. Therefore, although promising in animal models, the use of 4PBA in the treatment of $\mathrm{HD}$ is not optimal. A novel $\mathrm{HDACl}$, the pimelic diphenylamide $\mathrm{HDACl} 4 \mathrm{~b}$, has also shown to be effective in R6/2 mice, improving the $\mathrm{HD}$-related transcriptional abnormalities, including $\mathrm{H} 3$ acetylation and mRNA levels, and behavioral phenotype [419]. Additionally, in a different HD model, that expresses the first 171 amino acids of HTT with 82 CAG repeats at a relatively low steady-state level (N171-82Q mice), HDACl 4b enhanced body weight, motor function and cognitive performance, which may be mediated by modulatory effects of $\mathrm{HDACl} 4 \mathrm{~b}$ on post-translational mechanisms, such as protein phosphorylation and ubiquitination [682]. Accordingly, activation of inhibitor of kappaB kinase (IKK) by HDACI $4 \mathrm{~b}$ enhanced phosphorylation and acetylation of HTT, and subsequent clearance effected by the ubiquitin-proteasomal and autophagy systems. The selectivity of HDACI $4 \mathrm{~b}$ to inhibit class I and class II HDACs, and restore proper gene expression, was also explored in various HD models, including mice, flies, and cells [683]. Targeted inhibition of HDAC1 and HDAC3 was observed to mitigate mutant HTT-induced degeneration of the eyes and brain in Drosophila, and subdued some of the metabolic defects seen in STHdhQ111 mutant HTT knock-in striatal cells. In addition to $\mathrm{HDACl} 4 \mathrm{~b}$, some additional compounds were tested, revealing that one of them, compound 136, could effectively inhibit HDAC3 and restore proper gene expression in HD models. Although the exact targeting mechanisms remain elusive, HDACls upregulate pro-survival genes selectively, while downregulating pro-death genes [684]. 
Apart from drugs targeting HDACs, DNA-binding drugs have also received some attention in the context of HD. These efforts are mainly focused on the DNA intercalating anthracyclines, such as mithramycin $A$ and chromomycin A3, which were isolated from Streptomyces argillaceus and Streptomyces griseus, respectively [685-687]. Mithramycin A and chromomycin A3 inhibit the replication and translation processes in cells, processes that are especially indispensable to tumors. Mithramycin A has already been used to treat Paget's disease, hypercalcemia in malignancy, and various types of cancer [688-690]. These DNA intercalating agents specifically block the binding of transcription activators and repressors that bind to GC-rich regions of gene promoters, thereby affecting gene expression [688, 691, 692]. Their interference with transcription factors SP1 and SP3 are thought to be neuroprotective, as these induce detrimental responses after oxidative stress and DNA damage [693]. In R6/2 mice, mithramycin A was found to reduce clinical and neuropathological symptoms, as well as significantly increase survival rate, probably via the reduction of pericentromeric heterochromatin condensation through an epigenetic mechanism [540, 544]. Mithramycin A can repress the HKMT SETDB1 and thereby reverse the H3K9 hypermethylation seen in R6/2 mice [540]. The effects of chromomycin A3 have been investigated in both $N 171-82 \mathrm{Q}$ and $\mathrm{R} 6 / 2$ mice, showing that it can beneficially tip the methylation-acetylation balance at $\mathrm{H} 3 \mathrm{~K} 9$ in favor of acetylation, reactivating the chromatin and improving the HD phenotype [547]. Despite already being used as chemotherapy in cancer, mithramycin $A$ and chromomycin A3 are not well-suited for chronic use, which would be required for $\mathrm{HD}$ treatment, due to their relatively high, dose-dependent, toxicity in humans. Nevertheless, they may serve as a template in the development of less toxic DNA-binding compounds to treat HD.

\subsubsection{Strategies targeting non-coding RNAs}

Due to their relatively high specificity, miRNAs have been investigated as potential therapeutic targets for the treatment of neurodegenerative disorders. Alternatively, miRNA mimics, miRNA precursor analogs, and anti-miRNAs could also be employed to restore miRNA homeostasis in such conditions [694]. Although these RNA-based strategies are specific, a major obstacle, as with HDACls, remains access and distribution to the brain. For instance, simple intravenous administration of antimiRNAs conjugated to cholesterol molecules ("antagomirs"), while showing promise, failed to cross the BBB [695]. Additionally, although cholesterol facilitates cell entry, it might induce undesirable side effects [694]. More invasive, direct injections into the ventricles may represent 
an effective way of circumventing the BBB to enhance the performance of such treatments [696, 697]. Packaging siRNAs into exosomes has been suggested as a less invasive strategy to pass the BBB [698, 699].

\subsubsection{Alzheimer's disease}

Suggested miRNA targets for the treatment of AD include miR-124 and miR-195, which, when increased, could lower the levels of BACE1 and subsequently $A \beta[565,567]$. Alternatively, miR-323-3p, which is associated with inflammatory responses, has been proposed as a target for therapy in $A D$ [700]. Apart from being promising treatment targets, miRNA levels have also been investigated as potential diagnostic and prognostic markers for AD. For instance, Schipper et al. [553] investigated miRNA expression in blood mononuclear cells of mild SAD patients, finding miR-34a and miR-181b to be upregulated in these patients. Although it remains to be elucidated whether these miRNAs play a significant role in $A D$ pathology, they might serve as valuable prognostic biomarkers, especially as they can be relatively easily measured in the blood. Identifying changes in miRNA expression in very early, nonsymptomatic stages of $A D$ will substantially enhance $A D$ diagnostic and treatment efficacy.

\subsubsection{Huntington's disease}

Because HD is caused by the expression of mutant HTT, directly targeting its mRNA through RNAi is an attractive treatment strategy [701-703]. Due to the cardinal role of normal HTT in neuronal survival and functioning, it is crucial that such a treatment specifically target only mutant HTT. Choosing for adeno-associated virus short hairpin RNA (shRNA)-mediated RNAi, Harper et al. [704] were able to improve motor function and neuropathology in transgenic $\mathrm{N} 171-82 \mathrm{Q}$ mice. Subsequently, studies using adenovirus-shRNA, lentivirus-shRNA, adeno-associated virus-miRNA, or cholesterol-conjugated siRNA were successful in downregulating mutant $\mathrm{HTT}$, reducing aggregates and improving motor functions and neuropathology [705-712]. Interestingly, chemically modified single-stranded siRNAs (ss-siRNAs) with mismatched bases have a 100-times higher mutant HTT targeting efficacy when compared to unmodified RNA, as tested in an HD mouse model expressing one mutant HTT copy with 150 CAG repeats and a normal HTT copy with 7 CAG repeats (HdhQ150/Q7 mice) after intraventricular infusion [697]. This increased potency likely stems from the ability of these ss-siRNAs to distinguish mutant from normal HTT optimally, in collaboration with RISC, in a similar fashion as miRNAs. 


\subsection{Discussion and future per- spectives}

Epigenetic dysregulation currently garners much attention as a potentially pivotal player in aging and age-related neurodegenerative disorders, mediating interactions between genetic and environmental risk factors, or directly interacting with disease-specific pathological factors. Despite the profound differences in the epigenetic aberrancies, some similar patters begin to emerge and key-player molecules arise and build bridges between the seemingly diverse psychopathophysiology of neurodegenerative diseases, such as AD, PD, and HD. For instance, careful consideration of the (de)methylation dysregulations reveals a differential methylation pattern in genes that contribute genetic predisposition to $A D$ and $P D$; namely $A P P, P S 1, B A C E$ and $A P O E$ for $A D$ and SNCA and PARKIN16 for PD. Moreover, there is derailed histone acetylation in all three diseases discussed and, more specifically, in $A D$ and $P D$, a genome wide deacetylation of histones is observed. The various modifications on $\mathrm{H} 3$ are another common factor of these diseases that cannot be overlooked and especially the upregulated tri-methylation of $\mathrm{H} 3 \mathrm{~K} 9$ in both $A D$ and $H D$. Finally, the deviant expression of specific ncRNAs in all the three discussed diseases posits key-player roles in their pathophysiology. Briefly, the differential expression of miR-132 and miR-29 is a common observation not only among all three age-related neurodegenerative disorders but also normal aging. MiR-22, miR-26a and miR-125 also present a differential expression pattern that is common in these diseases.

Even though epigenetic research is expanding, covering more neurodegenerative disorders, the common points remain rather faint and sporadic, impeding the advancement towards innovative therapeutic strategies targeting neurodegeneration in general, instead of diseasespecific processes. This notion stems from the fact that large, empirical and broad studies are rare, with most investigations using only small samples with low statistical power, focusing on very specific tissues, cell types, or genes, and looking only at one or a few epigenetic modifications [33]. This substantial heterogeneity in research makes it hard to draw concrete conclusions about the exact involvement of epigenetics in neurodegeneration, stressing the need for studies with larger sample sizes, longitudinal designs with repeated sampling schemes, study designs with tissue and cell-specific analyses - but not just one type at a time - the inclusion of multiple epigenetic markers and levels, and genome-wide approaches. Although epigenome-wide association studies are performed, it should be noted that the Illumina 450k Methylation Beadchip array, which is the most commonly used platform for such 
studies, does not cover the complete methylome. Although this array covers most CpG-rich promoters, it may miss important phenotypically relevant variations in the methylome. Recent investigations have stressed the importance of DNA methylation at non-promoter and CpG-poor sites [713-715]. On a similar note, microarray-based transcriptome analyses are limited to known exons and transcripts [716]. For a whole transcriptome approach, including known and potentially novel ncRNAs, strategies based on next-generation sequencing should be employed, complemented with proper bioinformatic analyses. When compared to proteins, a much larger proportion of the human genome is transcribed into ncRNAs [117]. Nevertheless, due to their codon-bias, open reading frames and strong sequence conservation, protein genes can be detected more reliably than ncRNAs [717]. Raasch et al. [717] have therefore proposed a procedure combining multiple ncRNA identification strategies for increased sensitivity, but which is limited in its use for large genomes due to its high computational requirements.

An additional caveat of many published studies on epigenetics is the specificity of the detection techniques used. In the case of DNA modifications this is partly the result of the discovery of novel modifications. DNA methylation can be detected with techniques such as those based on sodium bisulfite sequencing or methylationsensitive restriction enzyme cleavage. With the discovery of DNA hydroxymethylation, however, it was found that these methods cannot distinguish between methylated and hydroxymethylated DNA [49]. By a method of quantitative subtraction, oxidative bisulfate sequencing can be used to identify DNA methylation and hydroxymethylation in parallel. This procedure involves the oxidation of $5-\mathrm{hmC}$ to $5-\mathrm{fC}$ and subsequently to uracil. 5-fC, however, has recently been observed to play a role in epigenetic priming, and thus has an independent function from $5-\mathrm{mC}$ and 5-hmC [57]. Epigenetic priming of 5-fC occurs mainly at poised enhancer sequences and is thought to activate these sites, possibly through the recruitment of transcriptional coactivator EP300. To specifically detect 5-fC, Song et al. [57] have described two methods, one of which has a single-base resolution and is also based on bisulfite sequencing. To detect $5-\mathrm{fC}$, this chemically assisted bisulfite sequencing method uses hydroxylamine protection of $5-\mathrm{fC}$ to prevent it from bisulfite-mediated deamination and reduction to $5-\mathrm{hmC}$. The genomic location of $5-\mathrm{fC}$ can then be determined by comparing hydroxylamine-treated bisulfite sequencing with traditional bisulfite sequencing. Sequencing of one of the various epigenetic DNA modifications should thus not be done without taking into account the other, functionally different, DNA modifications. Although epigenomic profiling provides valuable gene-specific information, the input material for profiling studies often consists of tissue homogenates. Investigations into the regional and cellular specific 
effects of diseases illustrate that certain regions and cell-types are often differentially affected and using homogenates may thus prevent the proper detection of potentially crucial epigenetic changes that only occur in a limited number of cells [718]. Indeed, in the healthy brain regionspecific differentially methylated regions can be distinguished [261, 713]. Even when using a homogenate of a specific brain region of interest, different cell types could still give interfering read-outs, for instance when considering the widely different levels of $5-\mathrm{mC}$ and $5-\mathrm{hmC}$ between cerebellar Purkinje and granule cells [54]. Although attempts are being made to investigate cell-specific epigenetic profiles these studies are few and are mostly limited to DNA methylation [12]. Interestingly, Guintivano et al. [719] have developed a model to correct DNA methylation patterns for cellular heterogeneity in the brain. Additionally, various methods to isolate cells of interest are nowadays available, including density gradients [720], laser capture microdissection [721], fluorescenceactivated cell sorting [722] and magnetic affinity cell sorting [723]. These methods have only been sparingly used for epigenetic studies and need to be validated for this purpose. It has been suggested that the isolation processes themselves could already influence gene expression [33]. When looking at the potentially high variability of epigenetic markers across different tissue and cell types it may thus be worth investing in novel techniques such as CLARITY [724] and fluorescent in situ RNA sequencing [725] to determine the regional distribution of epigenetic markers and how this may result in regional differences in RNA and protein expression. To complicate matters further, there is increasing evidence that mitochondrial gene expression is also epigenetically regulated, the investigation of which presents a whole new set of challenges [628].

For molecular studies of the human brain, most investigations depend on postmortem tissue donated by patients. Apart from possible influences of cell isolation techniques on epigenetics markers, various peri- and postmortem factors, such as postmortem interval, are known to affect tissue components, including RNA, and which could thus potentially affect epigenetic analyses [726, 727]. Such factors are thus most likely to influence ncRNA quality, but are in addition likely to compromise chromatin structure and possibly some DNA modifications. DNA methylation, however, is thought to be relatively stable and thereby represents one of the more reliable epigenetic markers when analyzing postmortem tissue [728].

Although many epigenetic changes are associated with aging and neurodegeneration, it remains unclear whether they are integral to the aging and neurodegenerative processes, or are an epiphenomenon; the result of other factors such as increased oxidative stress. Investigating 
causality with respect to epigenetic alterations is challenging in epidemiological studies and especially in studies relying on postmortem human tissue [728, 729]. Epigenetic alterations identified through the comparison of epigenetic profiles of post-mortem tissue between disease states and controls could be a combination of disease instigating alterations, but also epigenetic changes that are secondary to disease pathology [730], as well as changes that are an effect of medication [731]. Thus, when disease related epigenetic alterations are identified in epigenome-wide association studies, a major issue is to determine whether such changes actually played a role in the etiopathogenesis of the disease. An approach to overcome this hurdle would be to compare postmortem brain samples of subjects with varying stages of disease severity and including samples from preclinical, possibly prodromal stages of the disease [33]. Control samples should be very carefully selected, as for example amyloid plaques, a pathological hallmark of $A D$, also occur in subjects without any overt symptoms of the disease. Additionally, comparisons between familial and sporadic cases could help in the identification of causal epigenetic alternations and those that might be the secondary result of genetic mutations. Disease-specific epigenetic changes could in addition be identified by comparing patients with the target disease, with patients with similar diseases, such as frontotemporal lobar degeneration, and dementia with Lewy bodies when looking at $A D$, $\mathrm{PD}$, and HD.

Alternatively, determining the exact role of epigenetic alterations in progressive, age-related neurodegenerative diseases could be achieved through the longitudinal assessment of the epigenome, starting with individuals in a preclinical stage of the disease. However, assessing the epigenome of living individuals is only achievable in easily accessible tissues, such as peripheral blood. Although robust disease associated epigenetic markers in the blood have great potential as diagnostic and prognostic markers, thorough comparisons between such markers in the blood and brain should be made before their relevance to the disease process can be established. Although many tissue-specific differentially methylated regions related to tissue-specific gene expression can be identified, an important study by Davies et al. [713] indicates that at least some inter-individual methylomic variation is represented in both brain and blood. Blood sampling could be used to investigate epigenetic markers in the brain in such cases. In addition to DNA methylation, chromatin status and ncRNAs in peripheral mononuclear cells have been identified as potential diagnostic markers for brain-related conditions [732, 733]. Currently, for AD, PD, and HD, it is largely unknown whether epigenetic alterations relevant to the disease process are present in blood. Nevertheless, in the case of AD, some changes in the blood transcriptome reflect disease-related changes in the brain [734]. 
Animal models could potentially be used to determine the relationships between disease-associated epigenetic markers in the brain and those in blood. Additionally, the epigenetic effects of specific environmental factors, such as medication, can be investigated in isolation from other possible confounding factors [33]. Presently, the most used models are transgenic mouse models that express mutated human genes associated with familial disease forms [735]. Overexpressing human mutant APP in mice may, however, result in unwanted side-effects as these models will likely also have elevated levels of APP-related products such as $\mathrm{C}$-terminal fragment- $\beta / \alpha$ and amyloid precursor protein intracellular domain [736]. As $A \beta$ plays an important role in AD, the increased presence of these additional APP-related products and APP itself may limited the usefulness of such models. Saito et al. [736] have recently circumvented these problems of APP overexpression by directly manipulating the mouse $A p p$ gene, inducing fAD-related mutations that selectively enhance $A \beta$ production and the $A \beta 42$ to $A \beta 40$ ratio, without affecting APP expression. Nevertheless, there are so far only few animal models of the more common late-onset sporadic forms of $A D$ and $P D$. Some examples of SAD models are those based on APOE [737], or specific environmental/pharmacological interventions such as colchicine [738], cholesterol [739], or inhibition of the neuronal insulin receptor [740]. In case of SPD, models based on toxins are mainly used, such as those using the MPTP neurotoxin, which induces a permanent PD-like syndrome [741].

Using rodents to model diseases that occur mainly at the end of the life-span is attractive as they age relatively quickly. Rodent physiology, however, might prove to be too different to allow for the generation of a true model of sporadic late-onset neurodegenerative diseases. Moreover, as there are no natural counterparts of most of these diseases in rodents the successful generation of a true model depends on the available knowledge about the disease, which is in the case of sporadic lateonset neurodegenerative diseases very limited. Therefore, other model organisms may be more suitable, including non-human primates, which can naturally develop limited AD-like pathology [742], and in vitro models. Especially human primary cultures and induced pluripotent stem cells (iPSCs) represent highly promising alternatives to animal models [743, 744]. A number of methods have been described to generate iPSCs from easily accessible fibroblasts that can be differentiated into neurons, or induce neural progenitor-like cells (iNPCs) directly [745-748]. However, these methods have not been fully optimized yet and involve procedures that induce widespread epigenetic alterations [749].

To fully map the sequence of events leading to the development of complex diseases, epigenomic data should not be investigated in 


\section{REFERENCES}

[1] Choudhuri S. From

Waddington's epigenetic landscape to small noncoding RNA: some important milestones in the history of epigenetics research. Toxicol Mech Methods 2011; 21: 252-274.

[2] Müller GB, Olsson L. Epigenesis and epigenetics. In: Keywords and concepts in evolutionary developmental biology. Amsterdam: Elsevier Science B.V., 2003, pp. 114-123.

[3] Handel AE, Ebers GC,

Ramagopalan S V. Epigenetics: molecular mechanisms and implications for disease. Trends Mol Med 2010; 16: 7-16. [4] Hsieh J, Eisch AJ. Epigenetics, hippocampal neurogenesis, and neuropsychiatric disorders: Unraveling the genome to understand the mind. Neurobiol Dis 2010; 39:

$73-84$.

[5] Hu X-L, Wang Y, Shen Q.

Epigenetic control on cell fate choice in neural stem cells. Protein Cell 2012; 3: 278-290.

[6] Ma DK, Marchetto MC, Guo JU, et al. Epigenetic choreographers of neurogenesis in the adult mammalian brain. Nat Neurosci 2010; 13: 1338-1344.

[7] Henikoff S, Matzke MA, Mauro E Di. Exploring and explaining epigenetic effects. Trends Genet 1997; 13: $293-5$.

[8] Liu L, Li Y, Tollefsbol TO. Gene-environment interactions and epigenetic basis of human diseases. Curr Issues Mol Biol 2008; 10: 25-36. [9] Feinberg AP. Epigenetics at the epicenter of modern medicine. JAMA 2008; 299: 134.5.

[10] Adwan L, Zawia NH. Epigenetics: A novel therapeutic approach for the treatment of Alzheimer's disease. Pharmacol Ther 2013; 139: 41-50. isolation, but should be complemented with other modalities, including genomic, transcriptomic and proteomic data [750]. As such, through the integration of genetic and epigenetic approaches [334], noncoding genetic variation might be found to influence gene expression through epigenetic mechanisms. Such integrated data may also help in determining where in the etiopathogenesis of complex neurodegenerative conditions epigenetic players start to play a role. Integrated knowledge may additionally help to reveal whether therapeutic strategies targeting epigenetic mechanisms should have a general mode of action, aiming at, for example, DNA methylation at large [751], or a more targeted approach, for example changing the DNA methylation status of a specific DNA sequence [752].

Concluding, although it is clear that various levels of epigenetic regulation, including DNA and chromatin modifications, and ncRNAs, are affected during aging, $A D, P D$, and $H D$, it remains to be elucidated exactly how these epigenetic processes fit into the etiopathogenesis of these disorders and whether they play a causal role. Such knowledge is crucial for the exploration of novel therapeutic avenues, which are sorely needed to combat these devastating neurodegenerative diseases.

\section{Acknowledgements}

We thank T. Vaessen for helping with the figures. Funds have been provided by the Internationale Stichting Alzheimer Onderzoek (ISAO) grants 07551 and 11532 (D.L.A.vdH.), by the ISAO grants 09552 and 13515, and the Netherlands Organization for Scientific Research (NWO), grant 916.11.086 (Veni Award) (B.P.F.R.), the Maastricht University Medical Centre+ (Koostra Talent Fellowship) (R.L.), and the National Institutes of Health grant P50 AG005138 (P.R.H.). The authors declare that there are no conflicts of interest. 
methylation and gene function. Science 1980; 210: 604-10.

[12] Guo JU, Su Y, Shin JH, et al. Distribution, recognition and regulation of non-CpG methylation in the adult mammalian brain. Nat Neurosci 2013; 17: 215-222.

[13] Thomas B, Matson S, Chopra V, et al. A novel method for detecting 7-methyl guanine reveals aberrant methylation levels in Huntington disease. Anal Biochem 2013; 436: 112-120.

[14] Miller CA, Sweatt JD, Park A, et al. Covalent modification of DNA regulates memory formation. Neuron 2007; 53: 857-69.

[15] Silva PNO, Gigek CO, Leal

$\mathrm{MF}$, et al. Promoter methylation analysis of SIRT3, SMARCA5, HTERT and $C D H 1$ genes in aging and Alzheimer's disease. J Alzheimers Dis 2008; 13 : 173-6.

[16] Flores K, Wolschin F, Corneveaux JJ, et al. Genome-wide association between DNA methylation and alternative splicing in an invertebrate. BMC Genomics 2012; 13: 480.

[17] Lyko F, Foret S, Kucharski

R, et al. The Honey Bee Epigenomes:

Differential Methylation of Brain DNA in Queens and Workers. PLoS Biol 2010; 8: e1000506.

[18] Ziller MJ, Gu H, Müller F, et al. Charting a dynamic DNA methylation landscape of the human genome. Nature 2013; 500: 477-81.

[19] Klose RJ, Bird AP. Genomic DNA methylation: the mark and its mediators. Trends Biochem Sci 2006; 31: 89-97.

[20] Portela A, Esteller M. Epigenetic modifications and human disease. Nat Biotechnol 2010; 28:

1057-1068.
[21] Bhattacharya SK, Ramchandani S, Cervoni N, et al. A mammalian protein with specific demethylase activity for $\mathrm{mCpG}$ DNA. Nature 1999; 397: 579-583.

[22] Levenson JM, Roth TL,

Lubin FD, et al. Evidence that DNA (cytosine-5) methyltransferase regulates synaptic plasticity in the hippocampus. J Biol Chem 2006; 281: 15763-73.

[23] Weaver ICG, Cervoni N, Champagne FA, et al. Epigenetic programming by maternal behavior. Nat Neurosci 2004; 7: 847-854.

[24] Bergman Y, Cedar H. DNA methylation dynamics in health and disease. Nat Struct Mol Biol 2013; 20 : 274-281.

[25] Guerrero-Bosagna C. Environmentally induced epigenetic transgenerational inheritance of phenotype and disease. Mol Cell Endocrinol 2012; 354: 3-8.

[26] Mastroeni D, Grover A,

Delvaux E, et al. Epigenetic changes in Alzheimer's disease: Decrements in DNA methylation. Neurobiol Aging 2010; 31: 2025-2037.

[27] Jurkowski TP, Meusburger

M, Phalke S, et al. Human DNMT2 methylates tRNA(Asp) molecules using a DNA methyltransferase-like catalytic mechanism. RNA 2008; 14: 1663-70.

[28] Ooi SKT, Qiu C, Bernstein E, et al. DNMT3L connects unmethylated lysine 4 of histone $\mathrm{H} 3$ to de novo methylation of DNA. Nature 2007; 448: 714-717.

[29] Gordon CA, Hartono SR, Chédin F, et al. Inactive DNMT3B splice variants modulate de novo DNA methylation. PLoS One 2013; 8: e69486.

[30] Okano M, Bell DW, Haber
DA, et al. DNA methyltransferases

Dnmt3a and Dnmt3b are essential for de novo methylation and mammalian development. Cell 1999; 99: 247-257. [31] Mastroeni D, Grover A, Delvaux E, et al. Epigenetic mechanisms in Alzheimer's disease. Neurobiol Aging 2011; 32: 1161-1180.

[32] Wang J, Yu J-T, Tan M-S, et al. Epigenetic mechanisms in Alzheimer's disease: Implications for pathogenesis and therapy. Ageing Res Rev 2013; 12: 1024-1041.

[33] Lunnon K, Mill J. Epigenetic studies in Alzheimer's disease: current findings, caveats, and considerations for future studies. $A m J$ Med Genet B Neuropsychiatr Genet 2013; 162B: 789-99.

[34] Mattson MP. Methylation and acetylation in nervous system development and neurodegenerative disorders. Ageing Res Rev 2003; 2: 329-342.

[35] Fan G, Beard C, Chen RZ, et al. DNA hypomethylation perturbs the function and survival of CNS neurons in postnatal animals.

J Neurosci; http://www.jneurosci.org/ content/21/3/788.short (2001, accessed 3 April 2017).

[36] Feng J, Zhou Y, Campbell

SL, et al. Dnmtl and Dnmt3a maintain DNA methylation and regulate synaptic function in adult forebrain neurons. Nat Neurosci 2010; 13: 423-430.

[37] Iskandar BJ, Rizk E, Meier $\mathrm{B}$, et al. Folate regulation of axonal regeneration in the rodent central nervous system through DNA methylation. J Clin Invest 2010; 120: 1603-16. [38] Chan MF, van Amerongen $\mathrm{R}$, Nijjar T, et al. Reduced rates of gene loss, gene silencing, and gene mutation in Dnmtl-deficient embryonic stem cells. Mol Cell Biol 2001; 21: 
7587-600.

[39]

Espada J, Ballestar E,

Santoro R, et al. Epigenetic disruption of ribosomal RNA genes and nucleolar architecture in DNA methyltransferase 1 (Dnmtl) deficient cells. Nucleic Acids Res 2007; 35: 2191-2198.

[40] Jackson M, Krassowska A, Gilbert N, et al. Severe global DNA hypomethylation blocks differentiation and induces histone hyperacetylation in embryonic stem cells. Mol Cell Biol $2004 ; 24: 8862-71$.

[41] Milutinovic S, Brown SE, Zhuang Q, et al. DNA methyltransferase 1 knock down induces gene expression by a mechanism independent of DNA methylation and histone deacetylation. J Biol Chem 2004; 279: 27915-27.

[42] Ooi SKT, Bestor TH. The colorful history of active DNA demethylation. Cell 2008; 133: 1145-1148.

[43] Sharma RP, Tun N, Grayson DR. Depolarization induces downregulation of DNMT1 and DNMT3 in primary cortical cultures. Epigenetics 2008; 3: 74-80.

[44] Barreto G, Schäfer A, Marhold J, et al. Gadd45a promotes epigenetic gene activation by repair-mediated DNA demethylation. Nature 2007; 445: 671-675.

[45] Gavin DP, Chase KA, Sharma RP. Active DNA demethylation in post-mitotic neurons: A reason for optimism. Neuropharmacology 2013; 75 : 233-245.

[46] Zhu B, Zheng Y, Ang-

liker H, et al. 5-Methylcytosine DNA glycosylase activity is also present in the human MBD4 (G/T mismatch glycosylase) and in a related avian sequence. Nucleic Acids Res 2000; 28 : 4157-4165. al. 5-methylcytosine-DNA glycosylase activity is present in a cloned G/T mismatch DNA glycosylase associated with the chicken embryo DNA demethylation complex. Proc Natl Acad Sci U S A 2000; 97: 5135-9.

[48] Guo JU, Su Y, Zhong C, et al. Hydroxylation of 5-methylcytosine by TETl promotes active DNA demethylation in the adult brain. Cell 2011; 145: 423-434.

[49] Ito S, Shen L, Dai Q, et al. Tet proteins can convert 5-methylcytosine to 5 -formylcytosine and 5-carboxylcytosine. Science; http://science.sciencemag.org/content/333/6047/1300 (2011, accessed 3 April 2017).

[50] Delatte B, Fuks F. TET proteins: on the frenetic hunt for new cytosine modifications. Brief Funct Genomics 2013; 12: 191-204.

[51] van den Hove DLA, Chouliaras L, Rutten BPF. The role of 5-hydroxymethylcytosine in aging and Alzheimer's disease: current status and prospects for future studies. Curr Alzheimer Res 2012; 9: 545-9.

[52] Jin S-G, Wu X, Li AX, et al. Genomic mapping of 5-hydroxymethylcytosine in the human brain. Nucleic Acids Res 2011; 39: 5015-5024.

[53] Globisch D, Münzel M,

Müller M, et al. Tissue distribution of 5 -hydroxymethylcytosine and search for active demethylation intermediates. PLoS One; 5. Epub ahead of print 2010. DOI: 10.1371/journal. pone.0015367.

[54] Kriaucionis S, Heintz

N. The nuclear DNA base 5-hydroxymethylcytosine is present in Purkinje neurons and the brain. Science (80- ) 2009; 324: 929-30. [55] Orr BA, Haffner MC, Nelson WG, et al. Decreased 5-hy- droxymethylcytosine is associated with neural progenitor phenotype in normal brain and shorter survival in malignant glioma. PLoS One 2012; 7: e41036.

[56] Raiber E-A, Beraldi D, Ficz $\mathrm{G}$, et al. Genome-wide distribution of 5 -formylcytosine in embryonic stem cells is associated with transcription and depends on thymine DNA glycosylase. Genome Biol 2012; 13: R69.

[57] Song C-X, He C. Potential functional roles of DNA demethylation intermediates. Trends Biochem Sci 2013; 38: 480-484.

[58] Popp C, Dean W, Feng S, et al. Genome-wide erasure of DNA methylation in mouse primordial germ cells is affected by AID deficiency. Nature 2010; 463: 1101-1105.

[59] Cortellino S, Xu J, Sannai M, et al. Thymine DNA glycosylase is essential for active DNA demethylation by linked deamination-base excision repair. Cell 2011; 146: 67-79. [60] He Y-F, Li B-Z, Li Z, et al. Tet-mediated formation of 5-carboxylcytosine and its excision by TDG in mammalian DNA. Science; http://science.sciencemag.org/content/333/6047/1303 (2011, accessed 3 April 2017).

[61] Matsubara M, Tanaka T, Terato H, et al. Mutational analysis of the damage-recognition and catalytic mechanism of human SMUG1 DNA glycosylase. Nucleic Acids Res 2004; 32 : 5291-5302.

[62] Ma DK, Jang M-H, Guo

JU, et al. Neuronal activity-induced Gadd45b promotes epigenetic DNA demethylation and adult neurogenesis. Science; http://science.sciencemag. org/content/323/5917/1074 (2009, accessed 3 April 2017).

[63]

Rai K, Huggins IJ, James 
SR, et al. DNA demethylation in zebrafish involves the coupling of a deaminase, a glycosylase, and Gadd45. Cell 2008; 135: 1201-1212.

[64] Schmitz K-M, Schmitt N, Hoffmann-Rohrer U, et al. TAF12 recruits Gadd45a and the nucleotide excision repair complex to the promoter of rRNA genes leading to active DNA demethylation. Mol Cell 2009; 33: $344-353$.

[65] Richmond TJ, Luger K, Mäder AW, et al. Crystal structure of the nucleosome core particle at $2.8 \mathrm{~A}$ resolution. Nature 1997; 389: 251-260. [66] Murray K. The occurrence

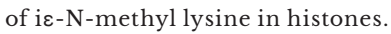

Biochemistry 1964; 3: 10-15.

[67] Gershey EL, Vidali G, Allfrey VG. Chemical studies of histone acetylation. The occurrence of epsilon-N-acetyllysine in the $\mathrm{f} 2 \mathrm{al}$ histone. J Biol Chem 1968; 243: 5018-22.

[68] Kleinsmith LJ, Allfrey VG, Mirsky AE. Phosphorylation of nuclear protein early in the course of gene activation in lymphocytes. Science 1966; 154: 780-1.

[69] Hunt LT, Dayhoff MO.

Amino-terminal sequence identity of ubiquitin and the nonhistone component of nuclear protein A24. Biochem

Biophys Res Commun 1977; 74: 650-655.

[70] Ueda K, Omachi A,

Kawaichi M, et al. Natural occurrence of poly(ADP-ribosyl) histones in rat liver. Proc Natl Acad Sci U S A 1975; 72: 205-9.

[71] Tan M, Luo H, Lee S, et al. Identification of 67 histone marks and histone lysine crotonylation as a new type of histone modification. Cell 2011; 146: 1016-1028.

[72] Houston I, Peter CJ, Mitchell A, et al. Epigenetics in the human brain. Neuropsychopharmacology 2013;

38: 183-197.

[73] Kouzarides T. Chromatin modifications and their function. Cell 2007; 128: 693-705.

[74] Shiio Y, Eisenman RN. Histone sumoylation is associated with transcriptional repression. Proc Natl Acad Sci U S A 2003; 100: 13225-30.

[75] Jenuwein T, Allis CD.

Translating the histone code. Science; http://science.sciencemag.org/content/293/5532/1074 (2001, accessed 3 April 2017).

[76] Day JJ, Sweatt JD. Epigenetic mechanisms in cognition. Neuron 2011; 70: 813-829.

[77] Allis CD, Berger SL, Cote

J, et al. New nomenclature for chro-

matin-modifying enzymes. Cell 2007; 131: $633-636$.

[78] Marmorstein R, Roth SY.

Histone acetyltransferases: function, structure, and catalysis. Curr Opin Genet Dev 2001; 11: 155-161.

[79] Huynh JL, Casaccia P.

Epigenetic mechanisms in multiple sclerosis: implications for pathogenesis and treatment. Lancet Neurol 2013; 12: 195-206.

[80] Dokmanovic M, Clarke C, Marks PA. Histone deacetylase inhibitors: overview and perspectives. Mol Cancer Res; http://mcr.aacrjournals. org/content/5/10/981 (2007, accessed 3 April 2017).

[81] Gregoretti I, Lee Y-M,

Goodson H V. Molecular evolution of the histone deacetylase family: functional implications of phylogenetic analysis. J Mol Biol 2004; 338: 17-31. [82] Marks PA, Breslow R.

Dimethyl sulfoxide to vorinostat: development of this histone deacetylase inhibitor as an anticancer drug. Nat

Biotechnol 2007; 25: 84-90.

[83] Minucci S, Pelicci PG.
Histone deacetylase inhibitors and the promise of epigenetic (and more) treatments for cancer. Nat Rev Cancer 2006; 6: 38-51.

[84] Rosato RR, Grant S. Histone deacetylase inhibitors: insights into mechanisms of lethality. Expert Opin Ther Targets 2005; 9: 809-824.

[85] Xu WS, Parmigiani RB, Marks PA. Histone deacetylase inhibitors: molecular mechanisms of action. Oncogene 2007; 26: 5541-5552.

[86] Chang S, Young BD, Li S, et al. Histone deacetylase 7 maintains vascular integrity by repressing matrix metalloproteinase 10. Cell 2006; 126: 321-334.

[87] Harms KL, Chen X.

Histone deacetylase 2 modulates p53 transcriptional activities through regulation of p53-DNA binding activity. Cancer Res; 67http://cancerres. aacrjournals.org/content/67/7/3145 (2007, accessed 3 April 2017).

[88] Kim S-H, Jeong J-W, Park JA, et al. Regulation of the HIF-lalpha stability by histone deacetylases. Oncol Rep 2007; 17: 647-51.

[89] Lagger G. Essential

function of histone deacetylase 1 in proliferation control and CDK inhibitor repression. EMBO J 2002; 21: 2672-2681.

[90] Parra M, Mahmoudi

T, Verdin E. Myosin phosphatase dephosphorylates HDAC7, controls its nucleocytoplasmic shuttling, and inhibits apoptosis in thymocytes. Genes Dev 2007; 21: 638-43.

[91] Vega RB, Matsuda K, Oh J, et al. Histone deacetylase 4 controls chondrocyte hypertrophy during skeletogenesis. Cell 2004; 119: 555-566.

[92] Zhang CL, McKinsey

TA, Chang S, et al. Class II histone 
deacetylases act as signal-responsive repressors of cardiac hypertrophy. Cell 2002; 110: 479-488.

[93] Hirschey MD. Old

enzymes, new tricks: sirtuins are NAD+-dependent de-acylases. Cell Metab 2011; 14: 718-719.

[94] Michan S, Sinclair D. Sirtuins in mammals: insights into their biological function. Biochem $J$; http:// www.biochemj.org/content/404/1/1 (2007, accessed 3 April 2017).

[95] Guan J-S, Haggarty SJ,

Giacometti E, et al. HDAC2 negatively regulates memory formation and synaptic plasticity. Nature 2009; 459: $55-60$.

[96] MacDonald JL, Roskams AJ. Histone deacetylases 1 and 2 are expressed at distinct stages of neuro-glial development. Dev Dyn 2008; 237: 2256-2267.

[97] Chang B, Chen Y, Zhao Y, et al. JMJD6 is a histone arginine demethylase. Science; http://science. sciencemag.org/content/318/5849/444 (2007, accessed 3 April 2017).

[98] Habibi E, Masoudi-Nejad A, Abdolmaleky HM, et al. Emerging roles of epigenetic mechanisms in Parkinson's disease. Funct Integr Genomics 2011; 11: 523-537.

\section{[99] Brami-Cherrier K, Roze}

E, Girault J-A, et al. Role of the ERK/ MSKl signalling pathway in chromatin remodelling and brain responses to drugs of abuse. J Neurochem 2009; 108: 1323-1335.

[100] Koshibu K, Gräff J, Beullens $\mathrm{M}$, et al. Protein phosphatase $1 \mathrm{regu}-$ lates the histone code for long-term memory. J Neurosci; 29http://www. jneurosci.org/content/29/41/13079 (2009, accessed 3 April 2017).

[101] Sawicka A, Seiser C.

Histone H3 phosphorylation - A versatile chromatin modification for different occasions. Biochimie 2012; 94: 2193-2201.

\section{[102] Bernstein BE, Mikkelsen}

TS, Xie X, et al. A bivalent chromatin structure marks key developmental genes in embryonic stem cells. Cell 2006; 125: 315-326.

[103] Greer EL, Shi Y. Histone methylation: a dynamic mark in health, disease and inheritance. Nat Rev Genet 2012; 13: 343-357.

[104] Hwang J-Y, Aromolaran

KA, Zukin RS. Epigenetic mechanisms in stroke and epilepsy. Neuropsychopharmacology 2013; 38: 167-182.

[105] Barski A, Cuddapah S, Cui

$\mathrm{K}$, et al. High-resolution profiling of histone methylations in the human genome. Cell 2007; 129: 823-837.

[106] Kondo Y, Issa J-PJ. Enrichment for histone H3 lysine 9 methylation at Alu repeats in human cells. $J$ Biol Chem 2003; 278: 27658-62.

[107] Martens JHA, O'Sullivan RJ, Braunschweig U, et al. The profile of repeat-associated histone lysine methylation states in the mouse epigenome. EMBO J 2005; 24: 800-812.

[108] Pauler FM, Sloane MA,

Huang R, et al. H3K27me3 forms BLOCs over silent genes and intergenic regions and specifies a histone banding pattern on a mouse autosomal chromosome. Genome Res 2008; 19: 221-233.

[109] Huda A, Jordan IK. Epigenetic regulation of mammalian genomes by transposable elements. Ann N Y Acad Sci 2009; 1178: 276-284. [110] Clapier CR, Cairns BR.

The biology of chromatin remodeling complexes. Annu Rev Biochem 2009; 78: 273-304.

[111] Hargreaves DC, Crab-

tree GR. ATP-dependent chromatin remodeling: genetics, genomics and mechanisms. Cell Res 2011; 21: $396-420$.

[112] Vogel-Ciernia A, Wood MA. Neuron-specific chromatin remodeling: A missing link in epigenetic mechanisms underlying synaptic plasticity, memory, and intellectual disability disorders. Neuropharmacology 2014; 80: 18-27.

[113] Ho L, Crabtree GR. Chromatin remodelling during development. Nature 2010; 463: 474-484.

[114] Ronan JL, Wu W, Crabtree GR. From neural development to cognition: unexpected roles for chromatin. Nat Rev Genet 2013; 14: 347-59.

[115] Wang X, Nagl NG, Wilsker

$\mathrm{D}$, et al. Two related ARID family proteins are alternative subunits of human SWI/SNF complexes. Biochem $J$; http://www.biochemj.org/content/383/2/319 (2004, accessed 3 April 2017).

[116] Vogel-Ciernia A, Matheos DP, Barrett RM, et al. The neuron-specific chromatin regulatory subunit BAF53b is necessary for synaptic plasticity and memory. Nat Neurosci 2013; 16: 552-561.

[117] Amaral PP, Dinger ME, Mercer TR, et al. The eukaryotic genome as an RNA machine. Science; http://science.sciencemag.org/content/319/5871/1787 (2008, accessed 3 April 2017).

[118] Jády BE, Bertrand E, Kiss T. Human telomerase RNA and box H/ ACA scaRNAs share a common Cajal body-specific localization signal. $J$ Cell Biol; http://jcb.rupress.org/content/164/5/647 (2004, accessed 3 April 2017).

[119] Mattick JS. The central role of RNA in human development and cognition. FEBS Lett 2011; 585: 
1600-1616.

[120] Preker P, Nielsen J,

Kammler S, et al. RNA exosome depletion reveals transcription upstream of active human promoters. Science; http://science.sciencemag.org/content/322/5909/1851 (2008, accessed 3 April 2017).

[121] Schouten M, Buijink MR, Lucassen PJ, et al. New neurons in aging brains: molecular control by small non-coding RNAs. Front Neurosci 2012; 6: 25 .

[122] Seila AC, Calabrese JM, Levine SS, et al. Divergent transcription from active promoters. Science; http://science.sciencemag.org/content/322/5909/1849 (2008, accessed 3 April 2017).

\section{[123] Johnson R, Guigó R.}

The RIDL hypothesis: transposable elements as functional domains of long noncoding RNAs. RNA 2014; 20 : 959-76.

\section{[124] Kapranov P, Cheng J,}

Dike S, et al. RNA maps reveal new RNA classes and a possible function for pervasive transcription. Science; http://science.sciencemag.org/content/316/5830/1484 (2007, accessed 3 April 2017).

[125] Khalil AM, Guttman M, Huarte M, et al. Many human large intergenic noncoding RNAs associate with chromatin-modifying complexes and affect gene expression. Proc Natl Acad Sci U S A 2009; 106: 11667-72. [126] Kim T-K, Hemberg M, Gray JM, et al. Widespread transcription at neuronal activity-regulated enhancers. Nature 2010; 465: 182-187. [127] Nakamori M, Thornton C. Epigenetic changes and non-coding expanded repeats. Neurobiol Dis 2010; 39: 21-27. sense transcripts. RNA Biol 2005; 2: 53-62.

[129] Cabili MN, Trapnell C, Goff

$\mathrm{L}$, et al. Integrative annotation of human large intergenic noncoding RNAs reveals global properties and specific subclasses. Genes Dev 2011; 25: 1915-27. [130] Chooniedass-Kothari S, Emberley E, Hamedani MK, et al. The steroid receptor RNA activator is the first functional RNA encoding a protein. FEBS Lett 2004; 566: 43-47. [131] Djebali S, Davis CA, Merkel A, et al. Landscape of transcription in human cells. Nature 2012; 489: 101-108.

[132] Johnsson P, Ackley A, Vidarsdottir L, et al. A pseudogene long-noncoding-RNA network regulates PTEN transcription and translation in human cells. Nat Struct Mol Biol 2013; $20: 440-446$.

[133] Ashe HL, Monks J, Wijgerde $\mathrm{M}$, et al. Intergenic transcription and transinduction of the human beta-globin locus. Genes Dev 1997; 11: 2494-509.

[134] Dinger ME, Gascoigne DK, Mattick JS. The evolution of RNAs with multiple functions. Epub ahead of print 2011. DOI: 10.1016/j. biochi.2011.07.018.

[135] Mercer TR, Wilhelm D, Dinger ME, et al. Expression of distinct RNAs from 3' untranslated regions. Nucleic Acids Res 2011; 39: 2393-2403.

[136] Jeon Y, Sarma K, Lee JT.

New and Xisting regulatory mechanisms of $\mathrm{X}$ chromosome inactivation. Curr Opin Genet Dev 2012; 22: 62-71.

[137] Lisch D. Regulation of transposable elements in maize. Curr Opin Plant Biol 2012; 15: 511-516. [138] Morrissy AS, Griffith M, Marra MA. Extensive relationship between antisense transcription and alternative splicing in the human genome. Genome Res 2011; 21: 1203-12. [139] Koziol MJ, Rinn JL. RNA traffic control of chromatin complexes. Curr Opin Genet Dev 2010; 20: $142-148$

[140] Mercer TR, Mattick JS.

Structure and function of long noncoding RNAs in epigenetic regulation. Nat Struct Mol Biol 2013; 20: 300-307. [141] Di Ruscio A, Ebralidze AK, Benoukraf T, et al. DNMT1-interacting RNAs block gene-specific DNA methylation. Nature 2013; 503: $371-376$.

[142] Han J, Pedersen JS,

Kwon SC, et al. Posttranscriptional Crossregulation between Drosha and DGCR8. Cell 2009; 136: 75-84.

[143] Ambros V. The functions of animal microRNAs. Nature 2004; 431: $350-355$.

[144] Han J, Lee Y, Yeom K-H, et al. The Drosha-DGCR8 complex in primary microRNA processing. Genes Dev 2004; 18: 3016-27.

[145] He L, Hannon GJ. MicroRNAs: small RNAs with a big role in gene regulation. Nat Rev Genet 2004; 5: 522-531.

[146] Lim LP, Lau NC, Garrett-Engele $\mathrm{P}$, et al. Microarray analysis shows that some microRNAs downregulate large numbers of target mRNAs. Nature 2005; 433: 769-773.

[147] Zeng Y, Yi R, Cullen BR.

MicroRNAs and small interfering RNAs can inhibit mRNA expression by similar mechanisms. Proc Natl Acad Sci US A 2003; 100: 9779-84.

[148] Eulalio A, Huntzinger E, Izaurralde E. Getting to the root of miRNA-mediated gene silencing. Cell 2008; 132: 9-14.

[149] Fabian MR, Sonenberg N, 
Filipowicz W. Regulation of mRNA translation and stability by microRNAs. Annu Rev Biochem 2010; 79 :

351-379.

[150] The University of Manchester. miRBase; http://www.mirbase.org/ (2014).

[151] Gokey NG, Srinivasan R, Lopez-Anido C, et al. Developmental regulation of microRNA expression in Schwann cells. Mol Cell Biol 2012; 32: 558-68.

[152] Hu HY, Guo S, Xi J, et al. MicroRNA expression and regulation in human, chimpanzee, and macaque brains. PLoS Genet 2011; 7: el002327. [153] Qu Z, Adelson DL. Evolutionary conservation and functional roles of ncRNA. Front Genet 2012; 3: 205.

[154] Santosh B, Varshney A, Yadava PK. Non-coding RNAs: biological functions and applications. Cell Biochem Funct 2015; 33: 14-22.

[155] Bass BL. RNA editing by adenosine deaminases that act on RNA. Annu Rev Biochem 2002; 71: 817-846.

[156] Mattick JS. RNA as the substrate for epigenome-environment interactions. BioEssays 2010; 32: 548-552.

[157] Mattick JS, Mehler MF. RNA editing, DNA recoding and the evolution of human cognition. Trends Neurosci 2008; 31: 227-233.

[158] Athanasiadis A, Rich A, Maas S, et al. Widespread A-to-I RNA editing of Alu-containing mRNAs in the human transcriptome. PLoS Biol 2004; 2: e391.

[159] Kim DDY, Kim TTY, Walsh

$\mathrm{T}$, et al. Widespread RNA editing of embedded Alu elements in the human transcriptome. Genome Res 2004; 14: 1719-25.
[160] Lander ES, Linton LM,

Birren B, et al. Initial sequencing and analysis of the human genome. Nature 2001; 409: 860-921.

[161] Levanon EY, Eisenberg E,

Yelin R, et al. Systematic identification of abundant A-to-I editing sites in the human transcriptome. Nat Biotechnol 2004; 22: 1001-1005.

[162] Umylny B, Presting G, Efird

JT, et al. Most human Alu and Murine $\mathrm{B} 1$ repeats are unique. $J$ Cell Biochem 2007; 102: 110-121.

[163] Valente L, Nishikura K. ADAR gene family and A-to-I RNA editing: diverse roles in posttranscriptional gene regulation. Prog Nucleic Acid Res Mol Biol 2005; 79: 299-338.

[164] Conticello SG, Liang Y, Li L, et al. The AID/APOBEC family of nucleic acid mutators. Genome Biol 2008; 9: 229.

[165] Navaratnam N, Sarwar R. An overview of cytidine deaminases. Int $J$ Hematol 2006; 83: 195-200.

[166] Muramatsu M, Sankaranand VS, Anant S, et al. Specific expression of activation-induced cytidine deaminase (AID), a novel member of the RNA-editing deaminase family in germinal center B cells. J Biol Chem 1999; 274: 18470-6.

[167] Blow M, Grocock R, van Dongen S, et al. RNA editing of human microRNAs. Genome Biol 2006; 7: R27.

[168] Kawahara Y, Megraw M,

Kreider E, et al. Frequency and fate of microRNA editing in human brain. Nucleic Acids Res 2008; 36: 5270-5280. [169] Nishikura K. Editor meets silencer: crosstalk between RNA editing and RNA interference. Nat Rev Mol Cell Biol 2006; 7: 919-931.

[170] Morse DP, Aruscavage PJ, Bass BL. RNA hairpins in noncoding regions of human brain and Caenorhabditis elegans mRNA are edited by adenosine deaminases that act on RNA. Proc Natl Acad Sci U S A 2002; 99: 7906-11.

[171] Conticello SG, Thomas CJF, Petersen-Mahrt SK, et al. Evolution of the AID/APOBEC family of polynucleotide (deoxy)cytidine deaminases. Mol Biol Evol 2004; 22: 367-377.

[172] Sawyer SL, Emerman M, Malik HS. Ancient adaptive evolution of the primate antiviral DNA-editing enzyme APOBEC3G. PLoS Biol 2004; 2: E275.

[173] Aguiar RS, Peterlin BM. APOBEC3 proteins and reverse transcription. Virus Res 2008; 134: 74-85. [174] Schumann GG. APOBEC3 proteins: major players in intracellular defence against LINE-1-mediated retrotransposition. Biochem Soc Trans; http://www.biochemsoctrans.org/content/35/3/637 (2007, accessed 3 April 2017).

[175] Coufal NG, Garcia-Perez JL, Peng GE, et al. Ll retrotransposition in human neural progenitor cells. Nature 2009; 460: 1127-1131.

[176] Dunlap KA, Palmarini M, Varela M, et al. Endogenous retroviruses regulate periimplantation placental growth and differentiation. Proc Natl Acad Sci U S A 2006; 103: 14390-5. [177] Faulkner GJ, Kimura Y, Daub CO, et al. The regulated retrotransposon transcriptome of mammalian cells. Nat Genet 2009; 41: 563-571.

[178] Sebastiani P, Montano M, Puca A, et al. RNA editing genes associated with extreme old age in humans and with lifespan in C. elegans. PLoS One 2009; 4: e8210.

[179] Holmes AP, Wood SH, Merry BJ, et al. A-to-I RNA editing does not change with age in the healthy 
male rat brain. Biogerontology 2013; 14: 395-400.

[180] Nicholas A, de Magalhaes

JP, Kraytsberg Y, et al. Age-related gene-specific changes of A-to-I mRNA editing in the human brain. Epub ahead of print 2010. DOI: $10.1016 / \mathrm{j}$. mad.2010.06.001.

[181] Stilling RM, Benito E, Barth J, et al. De-regulation of gene expression and alternative splicing affects distinct cellular pathways in the aging hippocampus. Front Cell Neurosci 2014; 8: 373.

[182] Akbarian S, Smith MA, Jones EG. Editing for an AMPA receptor subunit RNA in prefrontal cortex and striatum in Alzheimer's disease, Huntington's disease and schizophrenia. Brain Res 1995; 699: 297-304.

[183] Gaisler-Salomon I, Kravitz E, Feiler Y, et al. Hippocampus-specific deficiency in RNA editing of GluA2 in Alzheimer's disease. Neurobiol Aging 2014; 35: 1785-1791.

[184] Desrosiers R, Friderici K, Rottman F. Identification of methylated nucleosides in messenger RNA from Novikoff hepatoma cells. Proc Natl Acad Sci U S A 1974; 71: 3971-5. [185] Rottman F, Shatkin AJ, Perry RP. Sequences containing methylated nucleotides at the $5^{\prime}$ termini of messenger RNAs: Possible implications for processing. Cell 1974; 3: 197-199.

[186] Behm-Ansmant I, Helm M, Motorin Y. Use of specific chemical reagents for detection of modified nucleotides in RNA. J Nucleic Acids 2011; 2011: 408053.

[187] Kellner S, Burhenne J, Helm M. Detection of RNA modifications. RNA Biol 2010; 7: 237-247.

[188] Meyer KD, Saletore Y, Zumbo P, et al. Comprehensive analysis of mRNA methylation reveals enrichment in $3^{\prime}$ UTRs and near stop codons. Cell 2012; 149: 1635-1646.

\section{[189] Fu L, Guerrero CR, Zhong}

$\mathrm{N}$, et al. Tet-mediated formation of 5 -hydroxymethylcytosine in RNA. $J$ Am Chem Soc 2014; 136: 11582-11585.

[190] Bringmann P, Lührmann

R. Antibodies specific for N 6 -methyladenosine react with intact snRNPs U2 and U4/U6. FEBS Lett 1987; 213: 309-315.

[191] Choi YC, Busch H. Modi-

fied nucleotides in T1 RNase oligonucleotides of $18 \mathrm{~S}$ ribosomal RNA of the Novikoff hepatoma. Biochemistry 1978; 17: 2551-2560.

[192] Epstein P, Reddy R, Henning D, et al. The nucleotide sequence of nuclear U6 (4.7 S) RNA. J Biol Chem 1980; 255: 8901-6.

[193] Harada F, Kato N, Nishimura $S$. The nucleotide sequence of nuclear 4.8S RNA of mouse cells. Biochem Biophys Res Commun 1980; 95: 1332-40. [194] Munns TW, Sims HF, Liszewski MK. Immunospecific retention of oligonucleotides possessing N6-methyladenosine and 7-methylguanosine. $J$ Biol Chem 1977; 252 : 3102-4.

[195] Perry RP, Kelley DE,

Friderici K, et al. The methylated constituents of L cell messenger RNA: Evidence for an unusual cluster at the 5 ' terminus. Cell 1975; 4: 387-394.

[196] Shimba S, Bokar JA, Rott-

man F, et al. Accurate and efficient $\mathrm{N}$-6-adenosine methylation in spliceosomal U6 small nuclear RNA by HeLa cell extract in vitro. Nucleic Acids Res 1995; 23: 2421-6.

[197] Tanaka T, Weisblum B. Systematic difference in the methylation of ribosomal ribonucleic acid from gram-positive and gram-negative bacteria. J Bacteriol 1975; 123: 771-4.

[198] Dominissini D,

Moshitch-Moshkovitz S, Schwartz

$\mathrm{S}$, et al. Topology of the human and mouse m6A RNA methylomes revealed by m6A-seq. Nature 2012; 485: 201-206.

[199] Bujnicki JM, Feder M, Radlinska M, et al. Structure prediction and phylogenetic analysis of a functionally diverse family of proteins homologous to the MT-A70 subunit of the human mRNA:m6A methyltransferase. J Mol Evol 2002; 55: 431-444. [200] Ping X-L, Sun B-F, Wang L, et al. Mammalian WTAP is a regulatory subunit of the RNA N6-methyladenosine methyltransferase. Cell Res 2014; 24: 177-189.

[201] Wang Y, Li Y, Toth JI, et al. N6-methyladenosine modification destabilizes developmental regulators in embryonic stem cells. Nat Cell Biol 2014; 16: 191-198.

[202] Jia G, Fu Y, Zhao X, et al. N6-Methyladenosine in nuclear RNA is a major substrate of the obesity-associated FTO. Nat Chem Biol 2011; 7 : 885-887.

[203] Zheng G, Dahl JA, Niu Y, et al. ALKBH5 is a mammalian RNA demethylase that Impacts RNA metabolism and mouse fertility. Mol Cell 2013; 49: 18-29.

[204] Fu Y, Jia G, Pang X, et

al. FTO-mediated formation of N6-hydroxymethyladenosine and N6-formyladenosine in mammalian RNA. Nat Commun 2013; 4: 1798.

[205] Chandola U, Das R, Panda

B. Role of the N6-methyladenosine RNA mark in gene regulation and its implications on development and disease. Brief Funct Genomics 2015; 14: 169-179.

[206] Wang X, Lu Z, Gomez A, et 
al. N6-methyladenosine-dependent regulation of messenger RNA stability. Nature 2013; 505: 117-120.

[207] Bachellerie J-P, Cavaillé J, Hüttenhofer A. The expanding snoRNA world. Biochimie 2002; 84: 775-790. [208] Maxwell E, Fournier M. The small nucleolar RNAs. Annu Rev Biochem 1995; 64: 897-934.

[209] Chen PY, Weinmann L, Gaidatzis D, et al. Strand-specific 5'-O-methylation of siRNA duplexes controls guide strand selection and targeting specificity. RNA 2008; 14: $263-74$.

[210] Ender C, Krek A, Friedländer MR, et al. A Human snoRNA with MicroRNA-Like Functions. Mol Cell 2008; 32: 519-528. [211] Ritland Politz JC, Hogan EM, Pederson T. MicroRNAs with a nucleolar location. $R N A 2009 ; 15$ : 1705-1715.

[212] Saraiya AA, Wang CC, Nohynkova E, et al. snoRNA, a novel precursor of microRNA in Giardia lamblia. PLoS Pathog 2008; 4: el000224.

[213] Taft RJ, Glazov EA, Lassmann T, et al. Small RNAs derived from snoRNAs. RNA 2009; 15: $1233-40$.

[214] Schaefer M, Hagemann S, Hanna K, et al. Azacytidine inhibits RNA methylation at DNMT2 target sites in human cancer cell lines. Cancer Res; http://cancerres.aacrjournals.org/content/69/20/8127 (2009, accessed 3 April 2017).

[215] Phalke S, Nickel O, Walluscheck D, et al. Retrotransposon silencing and telomere integrity in somatic cells of Drosophila depends on the cytosine- 5 methyltransferase DNMT2. Nat Genet 2009; 41: 696-702. Rai K, Chidester S, Zavala
C V, et al. Dnmt2 functions in the cytoplasm to promote liver, brain, and retina development in zebrafish. Genes Dev 2007; 21: 261-6.

[217] Anderson J, Phan L, Hinnebusch AG. The Gcd10p/Gcd14p complex is the essential two-subunit tRNA(1-methyladenosine) methyltransferase of Saccharomyces cerevisiae. Proc Natl Acad Sci U S A 2000; 97: 5173-8.

[218] Björk GR, Wikström PM, Byström AS. Prevention of translational frameshifting by the modified nucleoside 1-methylguanosine. Science 1989; 244: 986-9.

[219] Jackman JE, Montange RK, Malik HS, et al. Identification of the yeast gene encoding the tRNA mlG methyltransferase responsible for modification at position 9. RNA 2003; 9: $574-85$.

[220] Saikia M, Fu Y, Pavon-Eter$\operatorname{nod} \mathrm{M}$, et al. Genome-wide analysis of N1-methyl-adenosine modification in human tRNAs. RNA 2010; 16: 1317-27. [221] Giordano M, Cristiani C, Crocco P, et al. Methylation of the human mitochondrial 12S rRNA gene is correlated with aging. 12th Int FISV 2012.

[222] Byun H-M, Baccarelli AA. Environmental exposure and mitochondrial epigenetics: study design and analytical challenges. Hum Genet 2014; 133: 247-257.

[223] Kudriashova IB, Kirnos MD, Vaniushin BF. [DNA-methylase activities from animal mitochondria and nuclei: different specificity of DNA methylation]. Biokhimiia 1976; 41: 1968-77.

\section{[224] Nass MMK. Differential}

methylation of mitochondrial and nuclear DNA in cultured mouse, hamster and virus-transformed hamster cells
In vivo and in vitro methylation. $J \mathrm{Mol}$ Biol 1973; 80: 155-175.

[225] Shmookler Reis RJ, Goldstein S. Mitochondrial DNA in mortal and immortal human cells. Genome number, integrity, and methylation. $J$ Biol Chem 1983; 258: 9078-85.

[226] Shock LS, Thakkar P V, Peterson EJ, et al. DNA methyltransferase 1 , cytosine methylation, and cytosine hydroxymethylation in mammalian mitochondria. Proc Natl Acad Sci U S A 2011; 108: 3630-5.

[227] Hong EE, Okitsu CY, Smith $\mathrm{AD}$, et al. Regionally specific and genome-wide analyses conclusively demonstrate the absence of $\mathrm{CpG}$ methylation in human mitochondrial DNA. Mol Cell Biol 2013; 33: 2683-90. [228] Choi Y-S, Hoon Jeong J, Min H-K, et al. Shot-gun proteomic analysis of mitochondrial D-loop DNA binding proteins: identification of mitochondrial histones. Mol Biosyst 2011; 7: 1523.

[229] Barrey E, Saint-Auret G, Bonnamy B, et al. Pre-microRNA and mature microRNA in human mitochondria. PLoS One 2011; 6: e20220. [230] Chestnut BA, Chang Q, Price A, et al. Epigenetic regulation of motor neuron cell death through DNA methylation. J Neurosci; http://www. jneurosci.org/content/31/46/16619 (2011, accessed 3 April 2017).

[231] Dzitoyeva S, Chen H, Manev H, et al. Effect of aging on 5 -hydroxymethylcytosine in brain mitochondria. Neurobiol Aging 2012; 33: 2881-2891.

[232] Iacobazzi V, Castegna A, Infantino V, et al. Mitochondrial DNA methylation as a next-generation biomarker and diagnostic tool. Mol Genet Metab 2013; 110: 25-34.

[233] Manev H, Dzitoyeva S, 
Chen H. Mitochondrial DNA: a blind spot in neuroepigenetics. Biomol Concepts 2012; 3: 107-115.

[234] Bellizzi D, D'Aquila P,

Scafone T, et al. The control region of mitochondrial DNA shows an unusual $\mathrm{CpG}$ and non-CpG methylation pattern. DNA Res 2013; 20: 537-547.

[235] Sun Z, Terragni J, Borgaro JG, et al. High-resolution enzymatic mapping of genomic 5-hydroxymethylcytosine in mouse embryonic stem cells. Cell Rep 2013; 3: 567-576.

[236] Feng S, Xiong L, Ji Z, et al. Correlation between increased ND2 expression and demethylated displacement loop of mtDNA in colorectal cancer. Mol Med Rep 2012; 6: 125-30.

\section{[237] Pirola CJ, Gianotti TF,}

Burgueño AL, et al. Epigenetic modification of liver mitochondrial DNA is associated with histological severity of nonalcoholic fatty liver disease. Gut 2013; 62: 1356-1363.

[238] Grewal SIS, Jia S. Heterochromatin revisited. Nat Rev Genet 2007; 8: $35-46$.

\section{[239] Zhang Y, Reinberg D.}

Transcription regulation by histone methylation: interplay between different covalent modifications of the core histone tails. Genes Dev 2001; 15: 2343-60.

[240] Wu H, Coskun V, Tao J, et al. Dnmt3a-dependent nonpromoter DNA methylation facilitates transcription of neurogenic genes. Science; http://science.sciencemag.org/ content/329/5990/444 (2010, accessed 4 April 2017).

[241] Liu C, Teng Z-Q, Santistevan NJ, et al. Epigenetic regulation of miR-184 by MBD1 governs neural stem cell proliferation and differentiation. Cell Stem Cell 2010; 6:
$433-44$.

[242] Detich N, Bovenzi V, Szyf

M. Valproate induces replication-independent active DNA demethylation. J Biol Chem 2003; 278: 27586-92.

[243] Sun G, Yu RT, Evans RM, et al. Orphan nuclear receptor TLX recruits histone deacetylases to repress transcription and regulate neural stem cell proliferation. Proc Natl Acad Sci U S A 2007; 104: 15282-7.

[244] Saito Y, Jones PM. Epigenetic activation of tumor suppressor microRNAs in human cancer cells. Cell Cycle 2006; 5: 2220-2222.

[245] Bocklandt S, Lin W, Sehl

$\mathrm{ME}$, et al. Epigenetic predictor of age. PLoS One 2011; 6: el4821.

[246] Horvath S. DNA methylation age of human tissues and cell types. Genome Biol 2013; 14: R115. [247] Beckman KB, Ames BN. The free radical theory of aging matures. Physiol Rev 1998; 78: 547-81. [248] Cencioni C, Spallotta F, Martelli F, et al. Oxidative stress and epigenetic regulation in ageing and age-related diseases. Int J Mol Sci 2013; 14: 17643-17663.

[249] Obeid R, Schadt A, Dillmann U, et al. Methylation status and neurodegenerative markers in parkinson disease. Clin Chem; http://clinchem.aaccjnls.org/content/55/10/1852 (2009, accessed 4 April 2017).

[250] Siegmund KD, Connor CM, Campan M, et al. DNA methylation in the human cerebral cortex is dynamically regulated throughout the life span and involves differentiated neurons. PLoS One 2007; 2: e895.

[251] Tohgi H, Utsugisawa K, Nagane Y, et al. The methylation status of cytosines in a $\mathrm{t}$ gene promoter region alters with age to downregulate tran- scriptional activity in human cerebral cortex. Epub ahead of print 1999. DOI: 10.1016/S0304-3940(99)00731-4.

[252] Chouliaras L, van den Hove DLA, Kenis G, et al. Caloric restriction attenuates age-related changes of DNA methyltransferase $3 \mathrm{a}$ in mouse hippocampus. Brain Behav Immun 2011; 25 : 616-623.

[253] Chouliaras L, van den Hove DLA, Kenis G, et al. Prevention of age-related changes in hippocampal levels of 5-methylcytidine by caloric restriction. Neurobiol Aging 2012; 33 : $1672-81$.

[254] Lopatina N, Haskell JF, Andrews LG, et al. Differential maintenance and de novo methylating activity by three DNA methyltransferases in aging and immortalized fibroblasts. $J$ Cell Biochem 2002; 84: 324-334.

\section{[255] Oliveira AMM, Hemstedt}

TJ, Bading H. Rescue of aging-associated decline in Dnmt3a2 expression restores cognitive abilities. Nat Neurosci 2012; 15: 1111-1113.

[256] Hernandez DG, Nalls MA, Gibbs JR, et al. Distinct DNA methylation changes highly correlated with chronological age in the human brain. Hum Mol Genet 2011; 20: 1164-1172.

[257] Bollati V, Schwartz J, Wright $\mathrm{R}$, et al. Decline in genomic DNA methylation through aging in a cohort of elderly subjects. Mech Ageing Dev 2009; 130: 234-239.

[258] Pan K, Chen Y, Roth M, et al. HBPl-mediated transcriptional regulation of DNA methyltransferase 1 and its impact on cell senescence. $\mathrm{Mol}$ Cell Biol 2013; 33: 887-903.

[259] Mazin AL. Suicidal function of DNA methylation in age-related genome disintegration. Ageing Res Rev 2009; 8: 314-327.

[260] Brown SE, Weaver ICG, 
Meaney MJ, et al. Regional-specific global cytosine methylation and DNA methyltransferase expression in the adult rat hippocampus. Epub ahead of print 2008. DOI: 10.1016/j. neulet.2008.05.028.

[261] Ladd-Acosta C, Pevsner J, Sabunciyan S, et al. DNA methylation signatures within the human brain. Am J Hum Genet 2007; 81: 1304-15. [262] Thompson RF, Atzmon G, Gheorghe C, et al. Tissue-specific dysregulation of DNA methylation in aging. Aging Cell 2010; 9: 506-518.

[263] Fraga MF, Ballestar E, Paz

$\mathrm{MF}$, et al. Epigenetic differences arise during the lifetime of monozygotic twins. Proc Natl Acad Sci U S A 2005; 102: 10604-9.

[264] Chouliaras L, van den Hove DLA, Kenis G, et al. Age-related increase in levels of 5-hydroxymethylcytosine in mouse hippocampus is prevented by caloric restriction. Curr Alzheimer Res 2012; 9: 536-44.

[265] Münzel M, Globisch D, Brückl T, et al. Quantification of the sixth DNA base hydroxymethylcytosine in the brain. Angew Chemie Int Ed 2010; 49: 5375-5377.

[266] Song C-X, Szulwach KE,

$\mathrm{Fu}$ Y, et al. Selective chemical labeling reveals the genome-wide distribution of 5-hydroxymethylcytosine. Nat

Biotechnol 2011; 29: 68-72.

[267] Madrigano J, Baccarelli

AA, Mittleman MA, et al. Aging and epigenetics: Longitudinal changes in gene-specific DNA methylation. Epigenetics 2012; 7: 63-70.

[268] So K, Tamura G, Honda

$\mathrm{T}$, et al. Quantitative assessment of $R U N X 3$ methylation in neoplastic and non-neoplastic gastric epithelia using a DNA microarray. Pathol Int 2006; 56: 571-575.
[269] Westberry JM, Trout AL, Wilson ME. Epigenetic regulation of estrogen receptor beta expression in the rat cortex during aging. Neuroreport 2011; 22: 428-432.

\section{[270] Ryan JM, Cristofalo VJ.}

Histone acetylation during aging of human cells in culture. Biochem Bio-

phys Res Commun 1972; 48: 735-742.

[271] Thakur MK, Kanungo MS.

Methylation of chromosomal proteins and DNA of rat brain and its modulation by estradiol and calcium during aging. Exp Gerontol 1981; 16: 331-336. [272] Pina B, Martinez P, Suau P. Differential acetylation of core histones in rat cerebral cortex neurons during development and aging. Eur $J$ Biochem 1988; 174: 311-315.

\section{[273] Kawakami K, Nakamura}

A, Ishigami A, et al. Age-related difference of site-specific histone modifications in rat liver. Biogerontology 2009; 10: $415-421$.

[274] Nakamura A, Kawakami

K, Kametani F, et al. Biological significance of protein modifications in aging and calorie restriction. Ann NY Acad Sci 2010; 1197: 33-39.

[275] Wang CM, Tsai SN, Yew

TW, et al. Identification of histone methylation multiplicities patterns in the brain of senescence-accelerated prone mouse 8. Biogerontology 2010; 11: 87-102.

\section{[276] Sarg B, Koutzamani}

E, Helliger W, et al. Postsynthetic trimethylation of histone $\mathrm{H} 4$ at lysine 20 in mammalian tissues is associated with aging. J Biol Chem 2002; 277: 39195-201.

[277] Agger K, Cloos PAC,

Rudkjaer L, et al. The H3K27me3

demethylase JMJD3 contributes to the activation of the INK4A-ARF locus in response to oncogene- and stress-in- duced senescence. Genes Dev 2009; 23: 1171-6.

[278] Jung J-W, Lee S, Seo M-S, et al. Histone deacetylase controls adult stem cell aging by balancing the expression of polycomb genes and jumonji domain containing 3. Cell $\mathrm{Mol}$ Life Sci 2010; 67: 1165-1176.

[279] Chouliaras L, van den Hove DLA, Kenis G, et al. Histone deacetylase 2 in the mouse hippocampus: attenuation of age-related increase by caloric restriction. Curr Alzheimer Res 2013; 10: 868-76.

[280] Perry M, Chalkley R.

Histone acetylation increases the solubility of chromatin and occurs sequentially over most of the chromatin. A novel model for the biological role of histone acetylation. $\mathrm{J}$ Biol Chem 1982; 257: 7336-47.

[281] Peleg S, Sananbenesi F,

Zovoilis A, et al. Altered histone acetylation is associated with age-dependent memory impairment in mice. Science 2010; 328: 753-6.

[282] Fischer A, Sananbenesi F, Wang X, et al. Recovery of learning and memory is associated with chromatin remodelling. Nature 2007; 447: 178-182.

[283] Wellen KE, Hatzivassiliou G, Sachdeva UM, et al. ATP-citrate lyase links cellular metabolism to histone acetylation. Science; http:// science.sciencemag.org/content/324/5930/1076 (2009, accessed 4 April 2017).

[284] Jiang N, Yan X, Zhou W, et al. NMR-based metabonomic investigations into the metabolic profile of the senescence-accelerated mouse. $J$ Proteome Res 2008; 7: 3678-3686.

[285] Alarcón JM, Malleret G, Touzani K, et al. Chromatin acetylation, memory, and LTP are impaired 
in $\mathrm{CBP}+/-$ mice: a model for the cognitive deficit in Rubinstein-Taybi syndrome and its amelioration. $\mathrm{Neu}$ ron 2004; 42: 947-59.

[286] Korzus E, Rosenfeld MG, Mayford M, et al. CBP histone acetyltransferase activity is a critical component of memory consolidation. Neuron 2004; 42: 961-72.

[287] Gupta S, Kim SY, Artis S, et al. Histone methylation regulates memory formation. $\mathrm{J} \mathrm{Neu}$ rosci; http://www.jneurosci.org/ content/30/10/3589 (2010, accessed 4 April 2017).

[288] Chwang WB, O'Riordan KJ, Levenson JM, et al. ERK/MAPK regulates hippocampal histone phosphorylation following contextual fear conditioning. Learn Mem 2006; 13 : 322-8.

[289] Quintas A, de Solís AJ, Díez-Guerra FJ, et al. Age-associated decrease of SIRT1 expression in rat hippocampus: Prevention by late onset caloric restriction. Epub ahead of print 2012. DOI: 10.1016/j. exger.2011.11.010.

[290] Sommer M, Poliak N, Upadhyay $S$, et al. $\Delta$ Np63alpha overexpression induces downregulation of Sirtl and an accelerated aging phenotype in the mouse. Cell Cycle 2006; 5: 2005-2011.

[291] Sasaki T, Maier B, Bartke

A, et al. Progressive loss of SIRT1 with cell cycle withdrawal. Aging Cell 2006; 5: 413-422.

[292] Pruitt K, Zinn RL, Ohm JE, et al. Inhibition of SIRT1 reactivates silenced cancer genes without loss of promoter DNA hypermethylation. PLoS Genet 2006; 2: e40.

[293] Vaquero A, Scher M, Erdjument-Bromage H, et al. SIRT1 regulates the histone methyl-transfer- ase SUV39H1 during heterochromatin formation. Nature 2007; 450: 440-444. [294] Narita M, Nũnez S, Heard $\mathrm{E}$, et al. Rb-mediated heterochromatin formation and silencing of E2F target genes during cellular senescence. Cell 2003; 113: 703-16.

[295] Ye X, Zerlanko B, Zhang R, et al. Definition of pRB- and p53-dependent and -independent steps in HIRA/ASFla-mediated formation of senescence-associated heterochromatin foci. Mol Cell Biol 2007; 27: 2452-65.

[296] Schmeisser K, Mansfeld

J, Kuhlow D, et al. Role of sirtuins in lifespan regulation is linked to methylation of nicotinamide. Nat Chem Biol 2013; 9: 693-700.

[297] Ristow M, Zarse K. How increased oxidative stress promotes longevity and metabolic health: The concept of mitochondrial hormesis (mitohormesis). Exp Gerontol 2010; 45: $410-418$

[298] Guarente L, Picard F, Ramsey CS, et al. Calorie restrictionthe SIR2 connection. Cell 2005; 120 : $473-482$.

[299] Rutten BPF, Brasnjevic I, Steinbusch HWM, et al. Caloric restriction and aging but not overexpression of SODl affect hippocampal volumes in mice. Mech Ageing Dev 2010; 131: 574-579.

[300] Baur JA, Pearson KJ, Price NL, et al. Resveratrol improves health and survival of mice on a high-calorie diet. Nature 2006; 444: 337-342.

[301] Haigis MC, Sinclair DA. Mammalian sirtuins: biological insights and disease relevance. Annu Rev Pathol Mech Dis 2010; 5: 253-295.

[302] Lee RC, Feinbaum RL, Ambros V, et al. The C. elegans heterochronic gene lin-4 encodes small
RNAs with antisense complementarity to lin-14. Cell 1993; 75: 843-54.

[303] Boulias K, Horvitz HR, Suchanek-Kavipurapu M, et al. The C. elegans microRNA mir-71 acts in neurons to promote germline-mediated longevity through regulation of DAF-16/FOXO. Cell Metab 2012; 15: $439-450$.

[304] Maes OC, An J, Sarojini H, et al. Murine microRNAs implicated in liver functions and aging process. Mech Ageing Dev 2008; 129: 534-541. [305] Noren Hooten N, Abdelmohsen K, Gorospe M, et al. microRNA expression patterns reveal differential expression of target genes with age. PLoS One 2010; 5: e10724.

[306] Boon RA, Iekushi K, Lechner S, et al. MicroRNA-34a regulates cardiac ageing and function. Nature 2013; 495: 107-110.

[307] Boon RA, Seeger T, Heydt $\mathrm{S}$, et al. MicroRNA-29 in aortic dilation: implications for aneurysm formation novelty and significance. Circ Res; http://circres.ahajournals.org/ content/109/10/1115 (2011, accessed 4 April 2017).

[308] Menghini R, Casagrande V, Cardellini M, et al. MicroRNA 217 modulates endothelial cell senescence via silent information regulator 1. Circulation; http://circ.ahajournals.org/ content/120/15/1524 (2009, accessed 4 April 2017).

[309] Olivieri F, Lazzarini R, Recchioni R, et al. MiR-146a as marker of senescence-associated pro-inflammatory status in cells involved in vascular remodelling. Age (Omaha) 2013; 35: 1157-1172.

[310] Vasa-Nicotera M, Chen H, Tucci P, et al. miR-146a is modulated in human endothelial cell with aging. Atherosclerosis 2011; 217: 326-30. 
[311]

Hackl M, Brunner S,

Fortschegger K, et al. miR-17, miR-19b, miR-20a, and miR-106a are down-regulated in human aging. Aging Cell 2010; 9: 291-296.

[312] Magenta A, Cencioni C, Fasanaro P, et al. miR-200c is upregulated by oxidative stress and induces endothelial cell apoptosis and senescence via ZEB1 inhibition. Cell Death

Differ 2011; 18: 1628-1639.

[313] Hébert SS, De Strooper B.

Alterations of the microRNA network cause neurodegenerative disease.

Trends Neurosci 2009; 32: 199-206.

[314] Somel M, Guo S, Fu N, et al. MicroRNA, mRNA, and protein expression link development and aging in human and macaque brain. Genome Res 2010; 20: 1207-18.

[315] Persengiev S, Kondova I, Otting N, et al. Genome-wide analysis of miRNA expression reveals a potential role for miR-144 in brain aging and spinocerebellar ataxia pathogenesis. Neurobiol Aging 2011; 32: 2316. el7-2316.e27.

[316] Li N, Bates DJ, An J, et al. Up-regulation of key microRNAs, and inverse down-regulation of their predicted oxidative phosphorylation target genes, during aging in mouse brain. Neurobiol Aging 2011; 32: 944-55.

[317] Liu W, Liu C, Zhu J, et al.

MicroRNA-16 targets amyloid precursor protein to potentially modulate Alzheimer's-associated pathogenesis in SAMP8 mice. Neurobiol Aging 2012; 33: $522-34$.

[318] Coppedè F, Mancuso M, Siciliano G, et al. Genes and the environment in neurodegeneration. Biosci Rep; http://www.bioscirep.org/content/26/5/341 (2006, accessed 4 April 2017).
[319] Trapp BD, Nave K-A. Multiple sclerosis: an immune or neurodegenerative disorder? Annu Rev Neurosci 2008; 31: 247-269.

[320] Bates EA, Victor M, Jones

AK, et al. Differential contributions of Caenorhabditis elegans histone deacetylases to huntingtin polyglutamine toxicity. J Neurosci; http://www. jneurosci.org/content/26/10/2830 (2006, accessed 4 April 2017).

[321] Chouliaras L, Rutten BPF,

Kenis G, et al. Epigenetic regulation in the pathophysiology of Alzheimer's disease. Prog Neurobiol 2010; 90: 498-510.

[322] Chouliaras L, Sierksma

ASR, Kenis G, et al. Gene-environment interaction research and transgenic mouse models of Alzheimer's disease. Int J Alzheimers Dis; 2010. Epub ahead of print January 2010. DOI: $10.4061 / 2010 / 859101$.

\section{[323] Migliore L, Coppedè F.}

Genetics, environmental factors and the emerging role of epigenetics in neurodegenerative diseases. Mutat Res Mol Mech Mutagen 2009; 667: 82-97. [324] Babenko O, Kovalchuk I, Metz GA. Epigenetic programming of neurodegenerative diseases by an adverse environment. Brain Res 2012; 1444: 96-111.

[325] Jaenisch R, Bird A. Epigenetic regulation of gene expression: how the genome integrates intrinsic and environmental signals. Nat Genet 2003; 33: 245-254.

[326] Selkoe DJ. Preventing Alzheimer's Disease. Science (80- ); 337http://science.sciencemag.org/ content/337/6101/1488 (2012, accessed 4 April 2017).

[327] Bediou B, Ryff I, Mercier

B, et al. Impaired social cognition in mild alzheimer disease. $J$ Geriatr
Psychiatry Neurol 2009; 22: 130-140.

[328] Budson AE, Solomon PR.

New criteria for Alzheimer disease and mild cognitive impairment: implications for the practicing clinician. Neurologist 2012; 18: 356-363.

[329] Hardy J, Saura CA, Choi SY, et al. A hundred years of Alzheimer's disease research. Neuron 2006; 52: $3-13$.

[330] Wenk GL. Neuropathologic changes in Alzheimer's disease. J Clin Psychiatry 2003; 64 Suppl 9: 7-10.

[331] Fukutani Y, Cairns NJ,

Rossor MN, et al. Purkinje cell loss and astrocytosis in the cerebellum in familial and sporadic Alzheimer's disease. Neurosci Lett 1996; 214: 33-36. [332] Alzheimer's Association. 2013 Alzheimer's disease facts and figures. Alzheimer's Dement 2013; 9 : 208-245.

[333] Tiraboschi P, Hansen LA, Thal LJ, et al. The importance of neuritic plaques and tangles to the development and evolution of AD. Neurology 2004; 62: 1984-9.

[334] Mill J. Toward an integrated genetic and epigenetic approach to Alzheimer's disease. Neurobiol Aging 2011; 32: 1188-1191.

[335] Citron M, Teplow DB, Selkoe DJ, et al. Generation of amyloid beta protein from its precursor is sequence specific. Neuron 1995; 14: 661-70.

[336] Shoji M, Golde TE, Ghiso

J, et al. Production of the Alzheimer amyloid beta protein by normal proteolytic processing. Science 1992; 258: 126-9.

[337] van Groen T. DNA methylation and Alzheimer's disease. In: Epigenetics of Aging. New York, NY: Springer New York, pp. 315-326.

[338] Hardy JA, Higgins GA. Alz- 
heimer's disease: the amyloid cascade hypothesis. Science 1992; 256: 184-5. [339] Mattson MP, Cheng B, Davis D, et al. beta-Amyloid peptides destabilize calcium homeostasis and render human cortical neurons vulnerable to excitotoxicity. $J$ Neurosci 1992; 12: 376-89.

[340] Selkoe DJ. Physiological production of the $\beta$-amyloid protein and the mechanism of Alzheimer's disease. Trends Neurosci 1993; 16: 403-409.

[341] Tanzi RE. The genetics of Alzheimer disease. Cold Spring Harb Perspect Med; 2. Epub ahead of print 2012. DOI: $10.1101 /$ cshperspect. a006296.

[342] Czech C, Tremp G, Pradier

L. Presenilins and Alzheimer's disease: biological functions and pathogenic mechanisms. Prog Neurobiol 2000; 60: 363-384.

[343] De Strooper B, Saftig P, Craessaerts K, et al. Deficiency of presenilin-1 inhibits the normal cleavage of amyloid precursor protein. Nature 1998; 391: 387-390.

\section{[344] Goate A, Chartier-Harlin}

M-C, Mullan M, et al. Segregation of a missense mutation in the amyloid precursor protein gene with familial Alzheimer's disease. Nature 1991; 349: $704-706$.

[345] Sherrington R, Rogaev EI, Liang Y, et al. Cloning of a gene bearing missense mutations in early-onset familial Alzheimer's disease. Nature 1995; 375: 754-760.

[346] Tanzi RE, Kovacs DM, Kim $\mathrm{T}-\mathrm{W}$, et al. The gene defects responsible for familial Alzheimer's disease. Neurobiol Dis 1996; 3: 159-168.

[347] Lemere CA, Lopera F, Kosik KS, et al. The E280A presenilin 1 Alzheimer mutation produces increased A beta 42 deposition and severe cerebellar pathology. Nat Med 1996; 2: 1146-1150.

[348] van Goethem NP, Lardenoije R, Kompotis K, et al. Cognitive disorders: impairment, aging, and dementia. In: In Vivo Models for Drug Discovery. Wiley-VCH Verlag $\mathrm{GmbH} \&$ Co. KGaA, pp. 349-366.

[349] Cacabelos R. Pharmacogenomics and therapeutic prospects in Alzheimer's disease. Expert Opin Pharmacother 2005; 6: 1967-1987.

[350] Cacabelos R. Pharmacogenetic basis for therapeutic optimization in Alzheimer's disease. Mol Diagn Ther 2007; 11: 385-405.

[351] Slooter AJC, Cruts M,

Kalmijn S, et al. Risk estimates of dementia by apolipoprotein E genotypes from a population-based incidence study: The Rotterdam Study. Arch Neurol 1998; 55: 964.

[352] Harold D, Abraham R, Hollingworth P, et al. Genome-wide association study identifies variants at $C L U$ and PICALM associated with Alzheimer's disease. Nat Genet 2009; 41: 1088-1093.

[353] Hollingworth P, Harold D, Sims R, et al. Common variants at $A B C A 7, M S 4 A 6 A / M S 4 A 4 E, E P H A 1$, $C D 33$ and $C D 2 A P$ are associated with Alzheimer's disease. Nat Genet 2011; 43: 429-435.

\section{[354] Naj AC, Jun G, Beecham}

$\mathrm{GW}$, et al. Common variants at

$M S 4 A 4 / M S 4 A 6 E, C D 2 A P, C D 33$ and

EPHA1 are associated with late-onset

Alzheimer's disease. Nat Genet 2011; 43: 436-441.

[355] Sleegers K, Lambert J-C, Bertram L, et al. The pursuit of susceptibility genes for Alzheimer's disease: progress and prospects. Trends Genet 2010; 26: 84-93.
[356] Guerreiro R, Wojtas A, Bras $\mathrm{J}$, et al. TREM2 variants in Alzheimer's disease. N Engl J Med 2013; 368: 117-127.

[357] Jonsson T, Stefansson H, Steinberg S, et al. Variant of TREM2 associated with the risk of Alzheimer's disease. N Engl J Med 2013; 368: 107-116.

[358] Neumann H, Daly MJ. Variant TREM2 as risk factor for $\mathrm{Alz}$ heimer's disease. N Engl J Med 2013; 368: 182-184.

[359] Yamagata K, Urakami K, Ikeda K, et al. High expression of apolipoprotein E mRNA in the brains with sporadic Alzheimer's disease. Dement Geriatr Cogn Disord; 12: 57-62.

[360] Caesar I, Gandy S. Evidence that an APOE $\varepsilon 4$ 'double whammy' increases risk for Alzheimer's disease. BMC Med 2012; 10: 36.

[361] Castellano JM, Kim J, Stewart FR, et al. Human apoE isoforms differentially regulate brain amyloid- $\beta$ peptide clearance. Sci Transl Med; http://stm.sciencemag.org/ content/3/89/89ra57 (2011, accessed 4 April 2017).

[362] Mawuenyega KG, Sigurdson W, Ovod V, et al. Decreased clearance of CNS $\beta$-amyloid in Alzheimer's disease. Science; http://science.sciencemag.org/content/330/6012/1774 (2010, accessed 4 April 2017).

[363] Yu J-T, Tan L. The role of clusterin in Alzheimer's disease: pathways, pathogenesis, and therapy. Mol Neurobiol 2012; 45: 314-326.

[364] Schrijvers EMC, Koudstaal PJ, Hofman A, et al. Plasma clusterin and the risk of Alzheimer disease. JAMA 2011; 305: 1322.

[365] Thambisetty M, Simmons A, Velayudhan L, et al. Association of plasma clusterin concentration with 
severity, pathology, and progression

in Alzheimer disease. Arch Gen Psychiatry 2010; 67: 739.

[366] Weingarten MD, Lockwood AH, Hwo SY, et al. A protein factor essential for microtubule assembly. Proc Natl Acad Sci U S A 1975; 72: 1858-62.

[367] Lee VM-Y, Goedert M,

Trojanowski JQ. Neurodegenerative tauopathies. Annu Rev Neurosci 2001; 24: 1121-1159.

[368] Lovestone S, Reynolds CH. The phosphorylation of tau: a critical stage in neurodevelopment and neurodegenerative processes. Neuroscience 1997; 78: 309-24.

[369] Alonso AD, Grundke-Iqbal

I, Barra HS, et al. Abnormal phosphorylation of tau and the mechanism of Alzheimer neurofibrillary degeneration: sequestration of microtubule-associated proteins 1 and 2 and the disassembly of microtubules by the abnormal tau. Proc Natl Acad Sci U S A 1997; 94: 298-303.

[370] Iqbal K, Liu F, Gong C-X, et al. Mechanisms of tau-induced neurodegeneration. Acta Neuropathol 2009; 118: 53-69.

[371] Klafki H-W, Staufenbiel M, Kornhuber J, et al. Therapeutic approaches to Alzheimer's disease. Brain 2006; 129: 2840-2855.

[372] Khairallah MI, Kassem

LAA. Alzheimer's disease: current status of etiopathogenesis and therapeutic strategies. Pakistan J Biol Sci PJBS 2011; 14: 257-72.

[373] Hardy J, Selkoe DJ. The amyloid hypothesis of Alzheimer's disease: progress and problems on the road to therapeutics. Science; http://science.sciencemag.org/content/297/5580/353 (2002, accessed 4 April 2017).

[374] Lahiri DK, Maloney B,
Zawia NH. The LEARn model: an epigenetic explanation for idiopathic neurobiological diseases. Mol Psychiatry 2009; 14: 992-1003.

[375] Jankovic J. Parkinson's disease: clinical features and diagnosis.

J Neurol Neurosurg Psychiatry 2008; 79: 368-376.

[376] Aarsland D, Larsen JP,

Cummins JL, et al. Prevalence and clinical correlates of psychotic symptoms in Parkinson disease: a community-based study. Arch Neurol 1999; 56: 595-601.

[377] Naimark D, Jackson E,

Rockwell E, et al. Psychotic symptoms in Parkinson's disease patients with dementia. J Am Geriatr Soc 1996; 44: 296-299.

[378] Riedel O, Klotsche J,

Spottke A, et al. Cognitive impairment in 873 patients with idiopathic Parkinson's disease. $J$ Neurol 2008; 255 : 255-264.

[379] Francis PT, Perry EK. Cholinergic and other neurotransmitter mechanisms in Parkinson's disease, Parkinson's disease dementia, and dementia with Lewy bodies. Mov Disord 2007; 22: S351-S357.

[380] van de Berg WDJ, Hepp

DH, Dijkstra AA, et al. Patterns of a-synuclein pathology in incidental cases and clinical subtypes of Parkinson's disease. Parkinsonism Relat Disord 2012; 18 Suppl 1: S28-30.

[381] Jellinger KA. Formation and development of Lewy pathology: a critical update. $J$ Neurol 2009; 256 : 270-279.

[382] Singleton AB, Farrer M, Johnson J, et al. a-Synuclein locus triplication causes Parkinson's disease. Science; http://science.sciencemag.org/ content/302/5646/841 (2003, accessed 4. April 2017).
[383] Thomas B, Beal MF. Molecular insights into Parkinson's disease. F1000 Med Rep 2011; 3: 7.

[384] Edwards TL, Scott WK, Almonte C, et al. Genome-wide association study confirms SNPs in SNCA and the $M A P T$ region as common risk factors for Parkinson disease. Ann Hum Genet 2010; 74: 97-109.

[385] Simón-Sánchez J, Schulte

C, Bras JM, et al. Genome-wide association study reveals genetic risk underlying Parkinson's disease. Nat Genet 2009; 41: 1308-1312.

[386] Ammal Kaidery N,

Tarannum S, Thomas B. Epigenetic landscape of Parkinson's disease: emerging role in disease mechanisms and therapeutic modalities. Neurotherapeutics 2013; 10: 698-708.

[387] Houlden H, Singleton AB The genetics and neuropathology of Parkinson's disease. Acta Neuropathol 2012; 124: 325-338.

[388] Veldman BA, Wijn AM, Knoers N, et al. Genetic and environmental risk factors in Parkinson's disease. Clin Neurol Neurosurg 1998; 100: 15-26.

[389] de Lau LML, Breteler MMB, Louis E, et al. Epidemiology of Parkinson's disease. Lancet Neurol 2006; 5: 525-35.

[390] Fukuda T. Neurotoxicity of MPTP. Neuropathology 2001; 21: $323-332$.

[391] Kopin IJ. Toxins and Parkinson's disease: MPTP parkinsonism in humans and animals. Adv Neurol 1987; 4.5: 137-44.

[392] Franco R, Li S, Rodriguez-Rocha H, et al. Molecular mechanisms of pesticide-induced neurotoxicity: Relevance to Parkinson's disease. Chem Biol Interact 2010; 188: 289-300. 
[393] Kanthasamy A, Jin H, Anantharam V, et al. Emerging neurotoxic mechanisms in environmental factors-induced neurodegeneration.

Neurotoxicology 2012; 33: 833-837.

[394] Ryu H, Rosas HD, Hersch

$\mathrm{SM}$, et al. The therapeutic role of creatine in Huntington's disease. Pharmacol Ther 2005; 108: 193-207.

[395] The Huntington's Disease

Collaborative Research Group. A novel gene containing a trinucleotide repeat that is expanded and unstable on Huntington's disease chromosomes. Cell 1993; 72: 971-83.

[396] Kremer B, Goldberg P, Andrew SE, et al. A worldwide study of the Huntington's disease mutation: the sensitivity and specificity of measuring CAG repeats. $N$ Engl J Med 1994; 330: 1401-1406.

[397] Myers RH, MacDonald ME, Koroshetz WJ, et al. De novo expansion of a (CAG)n repeat in sporadic Huntington's disease. Nat Genet 1993; 5: 168-173.

[398] Okazawa H. Polyglutamine diseases: a transcription disorder? Cell Mol Life Sci 2003; 60: 1427-1439.

[399] Sugars KL, Rubinsztein DC. Transcriptional abnormalities in Huntington disease. Trends Genet 2003; 19: 233-8.

[400] Thomas EA. Striatal specificity of gene expression dysregulation in Huntington's disease. $J$ Neurosci Res 2006; 84: 1151-1164.

[401] Ferrante RJ, Kowall NW, Beal MF, et al. Morphologic and histochemical characteristics of a spared subset of striatal neurons in Huntington's disease. J Neuropathol Exp Neurol 1987; 46: 12-27.

[402] Ferrante RJ, Kowall NW, Beal MF, et al. Selective sparing of a class of striatal neurons in Hunting- ton's disease. Science 1985; 230: 561-3. [403] Ferrante RJ, Kowall NW, Richardson EP, et al. Topography of enkephalin, substance $\mathrm{P}$ and acetylcholinesterase staining in Huntington's disease striatum. Neurosci Lett 1986; 71: 283-288.

[404] Ferrante RJ, Kowall NW, Richardson EP. Proliferative and degenerative changes in striatal spiny neurons in Huntington's disease: a combined study using the section-Golgi method and calbindin D28k immunocytochemistry. $J$ Neurosci 1991; 11: 3877-87.

[405] Graveland GA, Williams

RS, DiFiglia M. Evidence for degenerative and regenerative changes in neostriatal spiny neurons in Huntington's disease. Science 1985; 227: 770-3.

[406] Kowall NW, Ferrante RJ,

Martin JB. Patterns of cell loss in Huntington's disease. Trends Neurosci 1987; 10: $24-29$.

[407] Lee J, Hwang YJ, Kim KY, et al. Epigenetic Mechanisms of Neurodegeneration in Huntington's Disease. Neurotherapeutics 2013; 10: 664-676. [408] Vonsattel J-P, Myers RH, Stevens TJ, et al. Neuropathological Classification of Huntington's Disease. $J$ Neuropathol Exp Neurol 1985; 44: 559-577.

[409] Ross CA, Koshy BT, Cummings CJ, et al. Intranuclear neuronal inclusions: a common pathogenic mechanism for glutamine-repeat neurodegenerative diseases? Neuron 1997; 19: 1147-50.

[410] Cattaneo E. Dysfunction of wild-type huntingtin in Huntington disease. Physiology; http://physiologyonline.physiology.org/content/18/1/34 (2003, accessed 4 April 2017).

[411] Mangiarini L, Sathasivam
K, Seller M, et al. Exon 1 of the HD gene with an expanded CAG repeat is sufficient to cause a progressive neurological phenotype in transgenic mice. Cell 1996; 87: 493-506.

\section{[412] Nucifora Jr. FC, Sasaki}

M, Peters MF, et al. Interference by huntingtin and atrophin-1 with CBP-mediated transcription leading to cellular toxicity. Science 2001; 291: 2423-2428.

[413] Ross CA, Poirier MA.

Protein aggregation and neurodegenerative disease. Nat Med 2004; 10 : S10-S17.

[414] Zhang Y, Li M, Drozda M, et al. Depletion of wild-type huntingtin in mouse models of neurologic diseases. J Neurochem 2003; 87: 101-6. [415] Beal MF, Ferrante RJ.

Experimental therapeutics in transgenic mouse models of Huntington's disease. Nat Rev Neurosci 2004; 5 : 373-384.

[416] Szebenyi G, Morfini GA, Babcock A, et al. Neuropathogenic forms of huntingtin and androgen receptor inhibit fast axonal transport. Neuron 2003; 40: 41-52.

[417] Trushina E, Dyer RB, Badger JD, et al. Mutant huntingtin impairs axonal trafficking in mammalian neurons in vivo and in vitro. Mol Cell Biol 2004; 24: 8195-209.

[418] Li S-H, Cheng AL, Zhou

$\mathrm{H}$, et al. Interaction of Huntington disease protein with transcriptional activator Sp1. Mol Cell Biol 2002; 22: 1277-87.

[419] Thomas EA, Coppola G, Desplats PA, et al. The HDAC inhibitor $4 \mathrm{~b}$ ameliorates the disease phenotype and transcriptional abnormalities in Huntington's disease transgenic mice. Proc Natl Acad Sci U S A 2008; 105:

15564-9. 
LE. Hypomethylation of the amyloid precursor protein gene in the brain of an Alzheimer's disease patient. $J \mathrm{Mol}$ Neurosci 1995; 6: 141-146.

[421] Barrachina M, Ferrer I.

DNA methylation of Alzheimer disease and tauopathy-related genes in postmortem brain. $J$ Neuropathol Exp Neurol 2009; 68: 880-891.

[422] Fuso A, Seminara L, Cavallaro RA, et al. S-adenosylmethionine/homocysteine cycle alterations modify DNA methylation status with consequent deregulation of PSI and BACE and beta-amyloid production. Mol Cell Neurosci 2005; 28: 195-204. [423] Brohede J, Rinde M, Winblad B, et al. A DNA methylation study of the amyloid precursor protein gene in several brain regions from patients with familial Alzheimer disease. $J$ Neurogenet 2010; 24: 179-181.

[424] Wang S-C, Oelze B,

Schumacher A, et al. Age-specific epigenetic drift in late-onset Alzheimer's disease. PLoS One 2008; 3: e2698.

[425] Morrison LD, Smith DD,

Kish SJ. Brain S-adenosylmethionine levels are severely decreased in Alzheimer's disease. J Neurochem 1996; 67: $1328-31$.

[426] Bottiglieri T, Godfrey P, Flynn T, et al. Cerebrospinal fluid $\mathrm{S}$-adenosylmethionine in depression and dementia: effects of treatment with parenteral and oral S-adenosylmethionine. J Neurol Neurosurg Psychiatry 1990; 53: 1096-1098.

[427] Serot JM, Christmann D, Dubost T, et al. CSF-folate levels are decreased in late-onset AD patients. $J$ Neural Transm 2001; 108: 93-99.

[428] Kennedy BP, Bottiglieri T, Arning E, et al. Elevated S-adenosylhomocysteine in Alzheimer brain: influence on methyltransferases and cognitive function. J Neural Transm 2004; 111: 547-567.

[429] Coppedè F, Tannorella $P$, Pezzini I, et al. Folate, homocysteine, vitamin B12, and polymorphisms of genes participating in one-carbon metabolism in late-onset Alzheimer's disease patients and healthy controls. Antioxid Redox Signal 2012; 17: 195-204.

[430] Sontag E, Nunbhakdi-Craig V, Sontag J-M, et al. Protein phosphatase 2A methyltransferase links homocysteine metabolism with tau and amyloid precursor protein regulation. J Neurosci; http://www.jneurosci. org/content/27/11/2751 (2007, accessed 4. April 2017).

[431] Zhou X-W, Gustafsson J-Å, Tanila H, et al. Tau hyperphosphorylation correlates with reduced methylation of protein phosphatase 2A. Neurobiol Dis 2008; 31: 386-394. [432] Yoon SY, Choi HI, Choi JE, et al. Methotrexate decreases PP2A methylation and increases tau phosphorylation in neuron. Biochem Biophys Res Commun 2007; 363: 811-816. [433] Zhang C-E, Tian Q, Wei W, et al. Homocysteine induces tau phosphorylation by inactivating protein phosphatase $2 \mathrm{~A}$ in rat hippocampus. Neurobiol Aging 2008; 29: 1654-65. [434] Cacciapuoti F. Lowering homocysteine levels with folic acid and B-vitamins do not reduce early atherosclerosis, but could interfere with cognitive decline and Alzheimer's disease. $J$ Thromb Thrombolysis 2013; 36: 258-262.

[435] Fuso A, Nicolia V, Cavallaro

RA, et al. B-vitamin deprivation induces hyperhomocysteinemia and brain S-adenosylhomocysteine, depletes brain $\mathrm{S}$-adenosylmethionine, and enhances PSl and BACE expression and amyloid-beta deposition in mice. Mol Cell Neurosci 2008; 37: 731-46.

[436] Fuso A, Nicolia V, Ricceri L, et al. S-adenosylmethionine reduces the progress of the Alzheimer-like features induced by B-vitamin deficiency in mice. Neurobiol Aging 2012; 33: 1482.el-16.

[437] Fuso A, Nicolia V, Pasqualato A, et al. Changes in Presenilin 1 gene methylation pattern in diet-induced B vitamin deficiency. Neurobiol Aging 2011; 32: 187-199.

[438] Lee S, Lemere CA, Frost JL, et al. Dietary supplementation with S-adenosyl methionine delayed amyloid- $\beta$ and tau pathology in $3 \times \mathrm{Tg}$ AD mice. J Alzheimers Dis 2012; 28: 423-31.

[439] Chan A, Paskavitz J,

Remington R, et al. Efficacy of a vitamin/nutriceutical formulation for early-stage Alzheimer's disease: a 1-year, open-label pilot study with an 16-month caregiver extension. Am J Alzheimers Dis Other Demen 2008; 23 : 571-85.

[440] Remington R, Chan A, Paskavitz J, et al. Efficacy of a vitamin/ nutriceutical formulation for moderate-stage to later-stage Alzheimer's disease: a placebo-controlled pilot study. Am J Alzheimers Dis Other Demen 2009; 24: 27-33.

[441] Chouliaras L, Mastroeni D, Delvaux E, et al. Consistent decrease in global DNA methylation and hydroxymethylation in the hippocampus of Alzheimer's disease patients. Neurobiol Aging 2013; 34: 2091-2099. [442] Hoyaux D, Decaestecker C, Heizmann CW, et al. S100 proteins in Corpora Amylacea from normal human brain. Brain Res 2000; 867: 
$280-288$.

[443] Urdinguio RG, San-

chez-Mut J V, Esteller M, et al. Epigenetic mechanisms in neurological diseases: genes, syndromes, and therapies. Lancet Neurol 2009; 8: 1056-72.

[444] De Jager PL, Srivastava G, Lunnon K, et al. Alzheimer's disease: early alterations in brain DNA methylation at $A N K 1, B I N 1$, RHBDF2 and other loci. Nat Neurosci 2014; 17: 1156-1163.

[445] Lunnon K, Smith R, Hannon E, et al. Methylomic profiling implicates cortical deregulation of $A N K 1$ in Alzheimer's disease. Nat Neurosci 2014; 17: 1164-1170.

[446] Chen K-L, Wang SS-S, Yang Y-Y, et al. The epigenetic effects of amyloid-beta(1-40) on global DNA and neprilysin genes in murine cerebral endothelial cells. Biochem Biophys Res Commun 2009; 378: 57-61.

[447] Müerköster SS, Werbing V, Koch D, et al. Role of myofibroblasts in innate chemoresistance of pancreatic carcinoma-Epigenetic downregulation of caspases. Int J Cancer 2008; 123: 1751-1760.

[448] Sommer G, Kralisch S, Lipfert J, et al. Amyloid precursor protein expression is induced by tumor necrosis factor $\hat{\mathrm{I}} \pm$ in $3 \mathrm{~T} 3-\mathrm{L} 1$ adipocytes. $J$ Cell Biochem 2009; 108: 1418-1422.

[449] Wilson AG. Epigenetic regulation of gene expression in the inflammatory response and relevance to common diseases. J Periodontol 2008; 79: 1514-1519.

[450] Xiong M, Zhang T, Zhang L-M, et al. Caspase inhibition attenuates accumulation of $\beta$-amyloid by reducing $\beta$-secretase production and activity in rat brains after stroke.

Neurobiol Dis 2008; 32: 433-441.

[451] Mukaetova-Ladinska
EB, Harrington CR, Roth M, et al.

Alterations in tau protein metabolism

during normal aging. Dement Geriatr

Cogn Disord 1996; 7: 95-103.

[452] Rauhala HE, Porkka KP,

Saramäki OR, et al. Clusterin is epigenetically regulated in prostate cancer. Int J Cancer 2008; 123: 1601-1609.

[453] Nuutinen T, Suuronen

$\mathrm{T}$, Kyrylenko S, et al. Induction of clusterin/apoJ expression by histone deacetylase inhibitors in neural cells. Neurochem Int 2005; 47: 528-538.

[454] Suuronen T, Nuutinen

T, Ryhänen T, et al. Epigenetic regulation of clusterin/apolipoprotein $\mathrm{J}$ expression in retinal pigment epithelial cells. Biochem Biophys Res Commun 2007; 357: 397-401.

[455] Jee C Do, Lee HS, Bae SI, et al. International journal of oncology; https://www.spandidos-publications. com/10.3892/ijo.26.5.1265 (2005, accessed 4 April 2017).

[456] Moreira PR, Guimarães MM, Guimarães ALS, et al. Methylation of P16, P21, P27, RB1 and $P 53$ genes in odontogenic keratocysts. J Oral Pathol Med 2008; 38: 99-103. [457] Robertson KD, Jones PA. The human ARF cell cycle regulatory gene promoter is a $\mathrm{CpG}$ island which can be silenced by DNA methylation and down-regulated by wild-type p53. Mol Cell Biol 1998; 18: 6457-73.

[458] Tschöp K, Engeland K. Cell cycle-dependent transcription of cyclin B2 is influenced by DNA methylation but is independent of methylation in the CDE and CHR elements. FEBS $J$ 2007; 274: 5235-5249.

[459] Rao JS, Keleshian VL, Klein

$S$, et al. Epigenetic modifications

in frontal cortex from Alzheimer's disease and bipolar disorder patients. Transl Psychiatry 2012; 2: el32.
[460] Bollati V, Galimberti D,

Pergoli L, et al. DNA methylation in repetitive elements and Alzheimer disease. Brain Behav Immun 2011; 25: 1078-1083.

[461] Condliffe D, Wong A,

Troakes C, et al. Cross-region reduction in 5-hydroxymethylcytosine in Alzheimer's disease brain. Neurobiol Aging 2014; 35: 1850-1854.

[462] Coppieters N, Dieriks B V, Lill C, et al. Global changes in DNA methylation and hydroxymethylation in Alzheimer's disease human brain. Neurobiol Aging 2014; 35: 1334-1344. [463] Bradley-Whitman MA, Lovell MA. Epigenetic changes in the progression of Alzheimer's disease. Mech Ageing Dev 2013; 134: 486-95. [464] Hutnick LK, Golshani P, Namihira M, et al. DNA hypomethylation restricted to the murine forebrain induces cortical degeneration and impairs postnatal neuronal maturation. Hum Mol Genet 2009; 18: 2875-2888. [465] Morgan AR, Hamilton G, Turic D, et al. Association analysis of 528 intra-genic SNPs in a region of chromosome 10 linked to late onset Alzheimer's disease. Am J Med Genet Part B Neuropsychiatr Genet 2008; 147B: 727-731.

[466] Desplats P, Spencer B, Coffee E, et al. Alpha-synuclein sequesters Dnmtl from the nucleus: a novel mechanism for epigenetic alterations in Lewy body diseases. J Biol Chem 2011; 286: 9031-7.

[467] Trojanowski JQ, Goedert M, Iwatsubo T, et al. Fatal attractions: abnormal protein aggregation and neuron death in Parkinson's disease and Lewy body dementia. Cell Death Differ 1998; 5: 832-837.

[468] Jowaed A, Schmitt I, Kaut O, et al. Methylation regulates 
alpha-synuclein expression and is decreased in Parkinson's disease patients' brains. J Neurosci; http://www. jneurosci.org/content/30/18/6355 (2010, accessed 4 April 2017).

[469] Matsumoto L, Takuma H, Tamaoka A, et al. CpG demethylation enhances alpha-synuclein expression and affects the pathogenesis of Parkinson's disease. PLoS One 2010; 5: el5522.

[470] de Boni L, Tierling S,

Roeber S, et al. Next-generation sequencing reveals regional differences of the a-synuclein methylation state independent of Lewy body disease. NeuroMolecular Med 2011; 13: 310-320. [471] International Parkinson's

Disease Genomics Consortium (IPDGC), Wellcome Trust Case Control Consortium 2 (WTCCC2). A two-stage meta-analysis identifies several new loci for Parkinson's disease. PLoS Genet 2011; 7: el002142.

[472] Moore K, McKnight AJ, Craig D, et al. Epigenome-wide association study for Parkinson's disease. NeuroMolecular Med 2014; 16: 845-855. [473] Agirre X, Román-Gómez J, Vázquez I, et al. Abnormal methylation of the common PARK2 and $P A C R G$ promoter is associated with downregulation of gene expression in acute lymphoblastic leukemia and chronic myeloid leukemia. Int J Cancer 2006; 118: 1945-1953.

[474] Cai M, Tian J, Zhao G, et al. Study of methylation levels of parkin gene promoter in Parkinson's disease patients. Int $J$ Neurosci 2011; 121:

497-502.

[475] Coppedè F. Genetics and epigenetics of Parkinson's disease. Sci World J 2012; 2012: 1-12. [476] Ng CW, Yildirim F, Yap YS, et al. Extensive changes in DNA meth- ylation are associated with expression of mutant huntingtin. Proc Natl Acad Sci U S A 2013; 110: 2354-9.

[477] Villar-Menéndez I,

Blanch M, Tyebji S, et al. Increased

5 -methylcytosine and decreased

5 -hydroxymethylcytosine levels are associated with reduced striatal A2AR levels in Huntington's disease. NeuroMolecular Med 2013; 15: 295-309.

[478] Wood H. Neurodegenera-

tive disease: Altered DNA methylation and RNA splicing could be key mechanisms in Huntington disease. Nat Rev Neurol 2013; 9: 119-119.

[479] Zhang K, Schrag M, Crofton A, et al. Targeted proteomics for quantification of histone acetylation in Alzheimer's disease. Proteomics 2012; 12: 1261-1268.

[480] Gräff J, Kim D, Dobbin MM, et al. Epigenetic regulation of gene expression in physiological and pathological brain processes. Physiol Rev; http://physrev.physiology.org/ content/91/2/603 (2011, accessed 4 April 2017).

[481] Marques SCF, Lemos R, Ferreiro E, et al. Epigenetic regulation of BACEl in Alzheimer's disease patients and in transgenic mice.

Neuroscience 2012; 220: 256-66.

[482] Kilgore M, Miller CA, Fass

DM, et al. Inhibitors of class 1 histone deacetylases reverse contextual memory deficits in a mouse model of Alzheimer's disease. Neuropsychopharmacology 2010; 35: 870-80.

[483] Francis YI, Fà M, Ashraf H, et al. Dysregulation of histone acetylation in the APP/PS1 mouse model of Alzheimer's disease. J Alzheimers Dis 2009; 18: 131-9.

[484] Liu R, Lei JX, Luo C, et al. Increased EIDl nuclear translocation impairs synaptic plasticity and memo- ry function associated with pathogenesis of Alzheimer's disease. Neurobiol Dis 2012; 45: 902-912.

[485] Caccamo A, Maldonado MA, Bokov AF, et al. CBP gene transfer increases BDNF levels and ameliorates learning and memory deficits in a mouse model of Alzheimer's disease. Proc Natl Acad Sci U S A 2010; 107: 22687-92.

[486] Oliveira AMM, Estévez

MA, Hawk JD, et al. Subregion-specific p300 conditional knock-out mice exhibit long-term memory impairments. Learn Mem 2011; 18: 161-9.

[487] Oliveira AMM, Wood MA, McDonough CB, et al. Transgenic mice expressing an inhibitory truncated form of p300 exhibit long-term memory deficits. Learn Mem 2007; 14: 564-72.

[488] Maurice T, Duclot F, Meunier J, et al. Altered memory capacities and response to stress in p300/ CBP-associated factor (PCAF) histone acetylase knockout mice. Neuropsychopharmacology 2008; 33: 1584-1602.

[489] Duclot F, Meffre J, Jacquet C, et al. Mice knock out for the histone acetyltransferase p300/CREB binding protein-associated factor develop a resistance to amyloid toxicity. Neuroscience 2010; 167: 850-863.

[490] Walker MP, LaFerla FM, Oddo SS, et al. Reversible epigenetic histone modifications and Bdnf expression in neurons with aging and from a mouse model of Alzheimer's disease. Age (Dordr) 2013; 35: 519-31. [491] Ryu H, Barrup M, Kowall NW, et al. P3-260: Epigenetic modification in a monozygotic twin with Alzheimer's disease. Alzheimer's Dement 2008; 4: T598.

[492] Lithner CU, Hernandez CM, Nordberg A, et al. Epigenetic 
changes related to beta-amyloid-implications for Alzheimer's disease.

Alzheimer's Dement J Alzheimer's Assoc 2009; 5: P304.

[493] Gräff J, Rei D, Guan J-S, et al. An epigenetic blockade of cognitive functions in the neurodegenerating brain. Nature 2012; 483: 222-226.

[494] Zhang Z-Y, Schluesener HJ. Oral administration of histone deacetylase inhibitor MS-275 ameliorates neuroinflammation and cerebral amyloidosis and improves behavior in a mouse model. $J$ Neuropathol Exp Neurol 2013; 72: 178-185.

[495] McQuown SC, Barrett RM, Matheos DP, et al. HDAC3 is a critical negative regulator of long-term memory formation. J Neurosci; http://www. jneurosci.org/content/31/2/764 (2011, accessed 4 April 2017).

[496] Ding H, Dolan PJ, Johnson

GVW. Histone deacetylase 6 interacts with the microtubule-associated protein tau. J Neurochem 2008; 106 : 2119-2130.

[497] Simões-Pires C, Zwick V, Nurisso A, et al. HDAC6 as a target for neurodegenerative diseases: what makes it different from the other HDACs? Mol Neurodegener 2013; 8: 7. [498] Cook C, Carlomagno Y, Gendron TF, et al. Acetylation of the KXGS motifs in tau is a critical determinant in modulation of tau aggregation and clearance. $\mathrm{Hum} \mathrm{Mol}$ Genet 2014; 23: 104-116.

[499] Cook C, Gendron TF, Scheffel K, et al. Loss of HDAC6, a novel CHIP substrate, alleviates abnormal tau accumulation. Hum $\mathrm{Mol}$ Genet 2012; 21: 2936-2945.

[500] Perez M, Santa-Maria I, de Barreda EG, et al. Tau - an inhibitor of deacetylase HDAC6 function. $J \mathrm{Neu}$ rochem 2009; 109: 1756-1766.
[501] Govindarajan N, Rao P,

Burkhardt S, et al. Reducing HDAC6

ameliorates cognitive deficits in a mouse model for Alzheimer's disease.

EMBO Mol Med 2013; 5: 52-63.

[502] Xiong Y, Zhao K, Wu J, et

al. HDAC6 mutations rescue human

tau-induced microtubule defects in

Drosophila. Proc Natl Acad Sci U S A

2013; 110: 4604-9.

[503] Fischer A. Targeting

histone-modifications in Alzheimer's disease. What is the evidence that this is a promising therapeutic avenue? Neuropharmacology 2014; 80: 95-102. [504] Sarthi J, Elefant F. dTip60

HAT activity controls synaptic bouton expansion at the Drosophila neuromuscular junction. PLoS One 2011; 6 : e26202.

[505] Pirooznia SK, Sarthi J, Johnson AA, et al. Tip60 HAT activity mediates APP induced lethality and apoptotic cell death in the CNS of a Drosophila Alzheimer's disease model. PLoS One 2012; 7: e41776.

[506] Johnson AA, Sarthi J, Pirooznia SK, et al. Increasing Tip60 HAT levels rescues axonal transport defects and associated behavioral phenotypes in a Drosophila Alzheimer's disease model. J Neurosci; http:// www.jneurosci.org/content/33/17/7535 (2013, accessed 4 April 2017).

[507] Kaidi A, Jackson SP. KAT5 tyrosine phosphorylation couples chromatin sensing to ATM signalling. Nature 2013; 498: 70-74.

[508] Müller T, Schrötter A, Loosse C, et al. A ternary complex consisting of AICD, FE65, and TIP60 down-regulates Stathminl. Biochim Biophys Acta - Proteins Proteomics 2013; 1834: 387-394.

[509] Agis-Balboa RC, Pavelka Z, Kerimoglu C, et al. Loss of HDAC5 im- pairs memory function: implications for Alzheimer's disease. J Alzheimers Dis 2013; 33: 35-44.

[510] Kim M-S, Akhtar MW, Adachi M, et al. An essential role for histone deacetylase 4 in synaptic plasticity and memory formation.

J Neurosci; 32http://www.jneurosci. org/content/32/32/10879 (2012, accessed 4 April 2017).

[511] Julien C, Tremblay C, Émond V, et al. Sirtuin 1 reduction parallels the accumulation of tau in Alzheimer disease. $J$ Neuropathol Exp Neurol 2009; 68: 48-58.

[512] Morris BJ. Seven sirtuins for seven deadly diseases of aging. Free Radic Biol Med 2013; 56: 133-171. [513] Kim D, Nguyen MD, Dobbin MM, et al. SIRT1 deacetylase protects against neurodegeneration in models for Alzheimer's disease and amyotrophic lateral sclerosis. EMBO J 2007; 26: 3169-3179.

[514] Donmez G, Wang D, Cohen DE, et al. SIRT1 suppresses beta-amyloid production by activating the alpha-secretase gene ADAM10. Cell 2010; 142: 320-32.

[515] Min S-W, Cho S-H, Zhou $\mathrm{Y}$, et al. Acetylation of tau inhibits its degradation and contributes to tauopathy. Neuron 2010; 67: 953-66.

[516] Araki T, Sasaki Y, Mil-

brandt J. Increased nuclear NAD biosynthesis and SIRT1 activation prevent axonal degeneration. Science; http://science.sciencemag.org/content/305/5686/1010 (2004, accessed 4 April 2017).

[517] Chen J, Zhou Y, Mueller-Steiner S, et al. SIRT1 protects against microglia-dependent amyloidtoxicity through inhibiting NF- B signaling. J Biol Chem 2005; 280:

40364-40374. 
man II, et al. Evidence of DNA damage in Alzheimer disease: phosphorylation of histone H2AX in astrocytes. Age (Omaha) 2008; 30: 209-215.

[519] Ogawa O, Zhu X, Lee H-G, et al. Ectopic localization of phosphorylated histone H3 in Alzheimer's disease: a mitotic catastrophe? Acta Neuropathol 2003; 105: 524-8.

[520] Hyman BT, Elvhage TE, Reiter J. Extracellular signal regulated kinases. Localization of protein and mRNA in the human hippocampal formation in Alzheimer's disease. Am J Pathol 1994; 144: 565-72.

[521] Perry G, Roder H,

Nunomura A, et al. Activation of neuronal extracellular receptor kinase (ERK) in Alzheimer disease links oxidative stress to abnormal phosphorylation. Neuroreport 1999; 10: 2411-5.

[522] Zhu X, Castellani RJ,

Takeda A, et al. Differential activation of neuronal ERK, JNK/SAPK and p38 in Alzheimer disease: the 'two hit' hypothesis. Mech Ageing Dev 2001; 123 : $39-46$.

\section{[523] Iqbal K, Tellez-Nagel I,}

Grunke-Iqbal I. Protein abnormalities in Huntington's chorea. Epub ahead of print 1974. DOI: 10.1016/00068993(74)90527-7.

[524] Mastroeni D, Chouliaras L, Grover A, et al. Reduced RAN expression and disrupted transport between cytoplasm and nucleus; a key event in Alzheimer's disease pathophysiology. PLoS One; 8. Epub ahead of print 2013. DOI: 10.1371/journal.pone.0053349.

[525] Fontán-Lozano Á, Suárez-Pereira I, Horrillo A, et al. Histone $\mathrm{Hl}$ poly[ADP]-ribosylation regulates the chromatin alterations required for learning consolidation. J Neurosci; http://www.jneurosci.org/ content/30/40/13305 (2010, accessed 4 April 2017).

[526] Abeti R, Abramov AY,

Duchen MR. Beta-amyloid activates

PARP causing astrocytic metabolic

failure and neuronal death. Brain

2011; 134: 1658-1672.

[527] Liu H-P, Lin W-Y, Wu B-T,

et al. Evaluation of the poly(ADP-ribose) polymerase-1 gene variants in Alzheimer's disease. J Clin Lab Anal 2010; 24: 182-186.

[528] Strosznajder JB, Czapski

GA, Adamczyk A, et al. Poly(ADP-ribose) polymerase-1 in amyloid beta toxicity and Alzheimer's disease. $\mathrm{Mol}$ Neurobiol 2012; 46: 78-84.

[529] Kontopoulos E, Parvin JD, Feany MB. Alpha-synuclein acts in the nucleus to inhibit histone acetylation and promote neurotoxicity. $\mathrm{Hum} \mathrm{Mol}$ Genet 2006; 15: 3012-3023.

[530] Outeiro TF, Kontopoulos E, Altmann SM, et al. Sirtuin 2 inhibitors rescue a-synuclein-mediated toxicity in models of Parkinson's disease.

Science; http://science.sciencemag.org/ content/317/5837/516 (2007, accessed 4 April 2017).

[531] St. Laurent R, O'Brien LM, Ahmad ST. Sodium butyrate improves locomotor impairment and early mortality in a rotenone-induced Drosophila model of Parkinson's disease. Neuroscience 2013; 246: 382-390. [532] Siddiqui A, Chinta SJ, Mallajosyula JK, et al. Selective binding of nuclear alpha-synuclein to the PGC1alpha promoter under conditions of oxidative stress may contribute to losses in mitochondrial function: Implications for Parkinson's disease. Free Radic Biol Med 2012; 53: 993-1003.

[533] Zheng B, Liao Z, Locascio

JJ, et al. PGC-1, A potential therapeutic target for early intervention in Parkinson's disease. Sci Transl Med 2010; 2: 52ra73-52ra73.

[534] Kirilyuk A, Shimoji M, Catania J, et al. An intrinsically disordered region of the acetyltransferase p300 with similarity to prion-like domains plays a role in aggregation. PLoS One 2012; 7: e48243.

[535] Jin H, Kanthasamy A, Ghosh A, et al. a-Synuclein negatively regulates protein kinase C $\delta$ expression to suppress apoptosis in dopaminergic neurons by reducing p300 histone acetyltransferase activity. J Neuro$s c i$; http://www.jneurosci.org/content/31/6/2035 (2011, accessed 4 April 2017).

[536] Voutsinas GE, Stavrou EF, Karousos G, et al. Allelic imbalance of expression and epigenetic regulation within the alpha-synuclein wild-type and p.Ala53Thr alleles in Parkinson disease. Hum Mutat 2010; 31: 685-691. [537] Nicholas AP, Lubin FD, Hallett PJ, et al. Striatal histone modifications in models of levodopa-induced dyskinesia. J Neurochem 2008; 106: 486-494.

[538] Song C, Kanthasamy A, Anantharam V, et al. Environmental neurotoxic pesticide increases histone acetylation to promote apoptosis in dopaminergic neuronal cells: relevance to epigenetic mechanisms of neurodegeneration. Mol Pharmacol; http://molpharm.aspetjournals.org/ content/77/4/621 (2010, accessed 4 April 2017).

[539] Song C, Kanthasamy A, Jin $\mathrm{H}$, et al. Paraquat induces epigenetic changes by promoting histone acetylation in cell culture models of dopaminergic degeneration. Neurotoxicology 2011; 32: 586-595.

[540] Ferrante RJ, Ryu H, Kubilus JK, et al. Chemotherapy for 
the brain: the Antitumor antibiotic mithramycin prolongs survival in a mouse model of Huntington's disease. $J$ Neurosci; http://www.jneurosci.org/ content/24/46/10335 (2004, accessed 4 April 2017).

[541] Suzuki MM, Bird A. DNA methylation landscapes: provocative insights from epigenomics. Nat Rev Genet 2008; 9: 465-476.

[542] Gardian G, Browne SE, Choi D-K, et al. Neuroprotective effects of phenylbutyrate in the N171-82Q transgenic mouse model of Huntington's disease. $J$ Biol Chem 2005; 280: 556-63.

[543] McFarland KN, Das S, Sun TT, et al. Genome-wide histone acetylation is altered in a transgenic mouse model of Huntington's disease. PLoS One 2012; 7: e41423.

[544] Ryu H, Lee J, Hagerty SW, et al. ESET/SETDBl gene expression and histone H3 (K9) trimethylation in Huntington's disease. Proc Natl Acad Sci U S A 2006; 103: 19176-81.

\section{[545] Sadri-Vakili G, Bouzou}

B, Benn CL, et al. Histones associated with downregulated genes are hypo-acetylated in Huntington's disease models. Hum Mol Genet 2007; 16: 1293-1306.

[546] Lee J, Hagerty S, Cormier KA, et al. Monoallele deletion of CBP leads to pericentromeric heterochromatin condensation through ESET expression and histone H3 (K9) methylation. Hum Mol Genet 2008; 17: 1774-1782.

[547] Stack EC, Del Signore SJ, Luthi-Carter R, et al. Modulation of nucleosome dynamics in Huntington's disease. Hum Mol Genet 2007; 16: $1164-1175$.

[548] Lee J, Hwang YJ, Shin J-Y, et al. Epigenetic regulation of cholin- ergic receptor M1 (CHRM1) by histone H3K9me3 impairs Ca2+ signaling in Huntington's disease. Acta Neuropathol 2013; 125: 727-739.

[549] Calabresi P, Centonze D, Gubellini P, et al. Acetylcholine-mediated modulation of striatal function. Trends Neurosci 2000; 23: 120-6. [550] Cha JH, Kosinski CM, Kerner JA, et al. Altered brain neurotransmitter receptors in transgenic mice expressing a portion of an abnormal human huntington disease gene. Proc Natl Acad Sci U S A 1998; 95: 6480-5.

[551] Wang Z, Kai L, Day M, et al. Dopaminergic control of corticostriatal long-term synaptic depression in medium spiny neurons is mediated by cholinergic interneurons. Neuron 2006; 50: 443-52.

[552] Sonntag K-C. MicroRNAs and deregulated gene expression networks in neurodegeneration. Brain Res 2010; 1338: 48-57.

[553] Schipper HM, Maes OC, Chertkow HM, et al. MicroRNA expression in Alzheimer blood mononuclear cells. Gene Regul Syst Bio 2007; 1: 263-74.

[554] Van den Hove DL, Kompotis K, Lardenoije R, et al. Epigenetically regulated microRNAs in Alzheimer's disease. Neurobiol Aging 2014; 35: 731-745.

[555] Bicchi I, Morena F,

Montesano S, et al. MicroRNAs and molecular mechanisms of neurodegeneration. Genes 2013, Vol 4, Pages 244-263 2013; 4: 244-263.

[556] Delay C, Calon F, Mathews $\mathrm{P}$, et al. Alzheimer-specific variants in the 3'UTR of Amyloid precursor protein affect microRNA function.

Mol Neurodegener 201161 2011; 286 : 241-251.
[557]

Fan X, Liu Y, Jiang J, et al.

Regulated intramembrane proteolysis of amyloid precursor protein and regulation of expression of putative target genes. EMBO Rep 2010; 7: $739-745$.

[558] Liang C, Zhu H, Xu Y, et al. MicroRNA-153 negatively regulates the expression of amyloid precursor protein and amyloid precursor-like protein 2. Brain Res 2012; 1455: 103-13. [559] Long JM, Lahiri DK.

MicroRNA-101 downregulates Alzheimer's amyloid- $\beta$ precursor protein levels in human cell cultures and is differentially expressed. Epub ahead of print 2011. DOI: 10.1016/j. bbrc.2010.12.053.

[560] Niwa R, Zhou F, Li C, et al. The expression of the Alzheimer's amyloid precursor protein-like gene is regulated by developmental timing microRNAs and their targets in Caenorhabditis elegans. Dev Biol 2008; 315: 418-425.

[561] Patel N, Hoang D, Miller N, et al. MicroRNAs can regulate human APP levels. Mol Neurodegener 200831 2008; 93: 1600-1608.

[562] Long JM, Ray B, Lahiri DK. MicroRNA-153 physiologically inhibits expression of amyloid- $\beta$ precursor protein in cultured human fetal brain cells and is dysregulated in a subset of Alzheimer disease patients. J Biol Chem 2012; 287: 31298-310.

[563] Cheng L-C, Pastrana E, Tavazoie M, et al. miR-124 regulates adult neurogenesis in the subventricular zone stem cell niche. Nat Neurosci 2009; 12: 399-408.

[564] Smith P, Al Hashimi A, Girard J, et al. In vivo regulation of amyloid precursor protein neuronal splicing by microRNAs. J Neurochem 2011; 116: 240-247. 
[565] Fang M, Wang J, Zhang

$\mathrm{X}$, et al. The miR-124 regulates the expression of BACE1/ $\beta$-secretase correlated with cell death in Alzheimer's disease. Toxicol Lett 2012; 209: 94-105.

[566] Hébert SS, Horré K, Nicolaï

L, et al. Loss of microRNA cluster miR-29a/b-1 in sporadic Alzheimer's disease correlates with increased BACE1/beta-secretase expression. Proc Natl Acad Sci U S A 2008; 105: 6415-20. [567] Zhu H-C, Wang L-M, Wang M, et al. MicroRNA-195 downregulates Alzheimer's disease amyloid- $\beta$ production by targeting BACE1. Brain Res Bull 2012; 88: 596-601.

[568] Zong Y, Wang H, Dong W, et al. miR-29c regulates BACE1 protein expression. Brain Res 2011; 1395 : 108-115.

[569] Wang W-X, Rajeev BW, Stromberg AJ, et al. The expression of microRNA miR-107 decreases early in Alzheimer's disease and may accelerate disease progression through regulation of $\beta$-site amyloid precursor protein-cleaving enzyme 1. J Neurosci; http://www.jneurosci.org/ content/28/5/1213 (2008, accessed 4 April 2017).

[570] Wang W-X, Huang Q, Hu Y, et al. Patterns of microRNA expression in normal and early Alzheimer's disease human temporal cortex: white matter versus gray matter. Acta Neuropathol 2011; 121: 193-205.

[571] Wang W-X, Wilfred BR, Madathil SK, et al. miR-107 regulates granulin/progranulin with implications for traumatic brain injury and neurodegenerative disease. $\mathrm{Am} \mathrm{J}$ Pathol 2010; 177: 334-345.

[572] Boissonneault V, Plante I, Rivest S, et al. MicroRNA-298 and microRNA-328 regulate expression of mouse beta-amyloid precursor pro- tein-converting enzyme 1. J Biol Chem 2009; 284: 1971-81.

[573] Faghihi MA, Zhang M, Huang J, et al. Evidence for natural antisense transcript-mediated inhibition of microRNA function. Genome Biol 2010; 11: R56.

[574] Geekiyanage H, Chan

C. MicroRNA-137/181c regulates serine palmitoyltransferase and in turn amyloid $\beta$, novel targets in sporadic Alzheimer's disease. $J$ Neurosci; http://www.jneurosci.org/ content/31/41/14820 (2011, accessed 4 April 2017).

[575] Smrt RD, Szulwach KE, Pfeiffer RL, et al. MicroRNA miR-137 regulates neuronal maturation by targeting ubiquitin ligase mind bomb-1. Stem Cells 2010; 28: 1060-1070.

\section{[576] Szulwach KE, Li X, Smrt}

RD, et al. Cross talk between microRNA and epigenetic regulation in adult neurogenesis. J Cell Biol 2010; 189 : 127-141.

[577] Wang H, Liu J, Zong Y, et al. miR-106b aberrantly expressed in a double transgenic mouse model for Alzheimer's disease targets TGF- $\beta$ type II receptor. Brain Res 2010; 1357: 166-174.

[578] Schonrock N, Humphreys

DT, Preiss T, et al. Target gene repression mediated by miRNAs miR-181c and miR-9 both of which are down-regulated by amyloid- $\beta$. $J \mathrm{Mol}$ Neurosci 2012; 46: 324-335.

[579] Hébert SS, Horré K, Nicolaï L, et al. MicroRNA regulation of Alzheimer's Amyloid precursor protein expression. Neurobiol Dis 2009; 33: $422-428$.

[580] Akram A, Schmeidler J, Katsel $\mathrm{P}$, et al. Increased expression of cholesterol transporter ABCAl is highly correlated with severity of dementia in AD hippocampus. Brain

Res 2010; 1318: 167-177.

[581] Brett JO, Renault VM, Rafalski VA, et al. The microRNA cluster miR-106b 25 regulates adult neural stem/progenitor cell proliferation and neuronal differentiation. Aging (Albany NY) 2011; 3: 108-124.

[582] Delaloy C, Liu L, Lee J-A, et al. MicroRNA-9 coordinates proliferation and migration of human embryonic stem cell-derived neural progenitors. Cell Stem Cell 2010; 6: 323-35.

[583] Zhao C, Sun G, Li S, et al. A feedback regulatory loop involving microRNA-9 and nuclear receptor TLX in neural stem cell fate determination. Nat Struct Mol Biol 2009; 16: $365-371$.

[584] Hébert SS, Sergeant N, Buée L. MicroRNAs and the regulation of tau metabolism. Int J Alzheimers Dis 2012; 2012: 1-6.

[585] Smith PY, Delay C, Girard J, et al. MicroRNA-132 loss is associated with tau exon 10 inclusion in progressive supranuclear palsy. Hum Mol Genet 2011; 20: 4016-4024.

[586] Liu F, Gong C-X. Tau exon 10 alternative splicing and tauopathies. Mol Neurodegener 2008; 3: 8. [587] Caffrey TM, Joachim C, Paracchini S, et al. Haplotype-specific expression of exon 10 at the human MAPT locus. Hum Mol Genet 2006; 15: 3529-3537.

[588] Cogswell JP, Ward J, Taylor IA, et al. Identification of miRNA changes in Alzheimer's disease brain and CSF yields putative biomarkers and insights into disease pathways. $J$ Alzheimers Dis 2008; 14: 27-41.

[589] Hébert SS, Wang W-X, Zhu $\mathrm{Q}$, et al. A study of small RNAs from cerebral neocortex of pathology-ver- 
ified Alzheimer's disease, dementia with lewy bodies, hippocampal sclerosis, frontotemporal lobar dementia, and non-demented human controls. J Alzheimers Dis 2013; 35: 335-48. [590] Mohamed JS, Lopez MA, Boriek AM. Mechanical stretch up-regulates microRNA-26a and induces human airway smooth muscle hypertrophy by suppressing glycogen synthase kinase-3ß. J Biol Chem 2010; 285: 29336-47.

[591] Zovoilis A, Agbemenyah HY, Agis-Balboa RC, et al. microRNA-34c is a novel target to treat dementias. EMBO J 2011; 30: 4299-4308. [592] Carrettiero DC, Hernandez I, Neveu P, et al. The cochaperone BAG2 sweeps paired helical filamentinsoluble tau from the microtubule. J Neurosci; http://www.jneurosci.org/ content/29/7/2151 (2009, accessed 4 April 2017).

[593] Lukiw WJ. Micro-RNA speciation in fetal, adult and Alzheimer's disease hippocampus. Neuroreport 2007; 18: 297-300.

[594] Bilen J, Liu N, Burnett BG, et al. MicroRNA pathways modulate polyglutamine-induced neurodegeneration. Mol Cell 2006; 24: 157-63.

[595] Hébert SS, Papadopoulou AS, Smith P, et al. Alterations in glucose metabolism induce hypothermia leading to tau hyperphosphorylation through differential inhibition of kinase and phosphatase activities: implications for Alzheimer's disease. J Neurosci 2010; 24: 2401-2411.

[596] Cui JG, Li YY, Zhao Y, et al. Differential regulation of interleukin-1 receptor-associated kinase-1 (IRAK-1) and IRAK-2 by microRNA-146a and NF-kappaB in stressed human astroglial cells and in Alzheimer disease. J Biol Chem 2010; 285 :
38951-60.

[597] Taganov KD, Boldin MP, Chang K-J, et al. NF-kappaB-dependent induction of microRNA miR-146, an inhibitor targeted to signaling proteins of innate immune responses. Proc Natl Acad Sci U S A 2006; 103: 12481-6.

[598] Lukiw WJ, Zhao Y, Gui

JG. An NF-kappaB-sensitive micro RNA-146a-mediated inflammatory circuit in Alzheimer disease and in stressed human brain cells. J Biol Chem 2008; 283: 31315-22.

[599] Sethi P, Lukiw WJ. Micro-RNA abundance and stability in human brain: Specific alterations in Alzheimer's disease temporal lobe neocortex. Epub ahead of print 2009. DOI: 10.1016/j.neulet.2009.04.052.

[600] Lukiw WJ, Handley P,

Wong L, et al. BC200 RNA in normal human neocortex, non-Alzheimer dementia (NAD), and senile dementia of the Alzheimer type (AD). Neurochem Res 1992; 17: 591-597.

[601] Mus E, Hof PR, Tiedge H.

Dendritic BC200 RNA in aging and in Alzheimer's disease. Proc Natl Acad Sci US A 2007; 104: 10679-84.

[602] Yao J, Hennessey T, Flynt

A, et al. MicroRNA-related cofilin abnormality in Alzheimer's disease. PLoS One 2010; 5: el5546.

[603] Minamide LS, Striegl AM, Boyle JA, et al. Neurodegenerative stimuli induce persistent ADF/ cofilin-actin rods that disrupt distal neurite function. Nat Cell Biol 2000; 2: 628-636.

[604] Massone S, Ciarlo E, Vella

$\mathrm{S}$, et al. NDM29, a RNA polymerase III-dependent non coding RNA, promotes amyloidogenic processing of APP and amyloid $\beta$ secretion. Biochim Biophys Acta - Mol Cell Res 2012; 1823:
$1170-1177$

[605] Faghihi MA, Modarresi F, Khalil AM, et al. Expression of a noncoding RNA is elevated in Alzheimer's disease and drives rapid feed-forward regulation of $\beta$-secretase. Nat Med 2008; 14: 723-730.

[606] Massone S, Vassallo I,

Fiorino G, et al. 17A, a novel non-coding RNA, regulates GABA B alternative splicing and signaling in response to inflammatory stimuli and in Alzheimer disease. Neurobiol Dis 2011; 41: $308-317$.

[607] Junn E, Lee K-W, Jeong BS, et al. Repression of alpha-synuclein expression and toxicity by microRNA-7. Proc Natl Acad Sci U S A 2009; 106: 13052-7.

[608] Doxakis E. Post-transcriptional regulation of alpha-synuclein expression by mir-7 and mir-153. J Biol Chem 2010; 285: 12726-34. [609] Wang G, van der Walt JM, Mayhew G, et al. Variation in the miRNA-433 binding site of FGF20 confers risk for Parkinson disease by overexpression of alpha-synuclein. $\mathrm{Am}$ J Hum Genet 2008; 82: 283-9.

[610] Gillardon F, Mack M, Rist

W, et al. MicroRNA and proteome expression profiling in early-symptomatic a-synuclein(A30P)-transgenic mice. PROTEOMICS - Clin Appl 2008; 2: 697-705.

[611] Asikainen S, Rudgalvyte M, Heikkinen L, et al. Global microRNA expression profiling of Caenorhabditis elegans Parkinson's disease models. $J$ Mol Neurosci 2010; 41: 210-218.

[612] Cho HJ, Liu G, Jin SM, et al. MicroRNA-205 regulates the expression of Parkinson's disease-related leucine-rich repeat kinase 2 protein. Hum Mol Genet 2013; 22: 608-620.

[613] Gehrke S, Imai Y, Sokol N, 
et al. Pathogenic LRRK2 negatively

regulates microRNA-mediated translational repression. Nature 2010; 466: 637-41.

[614] Nishino J, Kim I, Chada K, et al. Hmga2 promotes neural stem cell self-renewal in young but not old mice by reducing pl6Ink4a and pl9Arf Expression. Cell 2008; 135: 227-39.

[615] Imai Y, Gehrke S, Wang $\mathrm{H}-\mathrm{Q}$, et al. Phosphorylation of 4E-BP by LRRK2 affects the maintenance of dopaminergic neurons in Drosophila. EMBO J 2008; 27: 2432-2443.

[616] Smith WW, Pei Z, Jiang H, et al. Kinase activity of mutant LRRK2 mediates neuronal toxicity. Nat Neurosci 2006; 9: 1231-1233.

[617] Minones-Moyano E, Porta S, Escaramis G, et al. MicroRNA profiling of Parkinson's disease brains identifies early downregulation of miR-34b/c which modulate mitochondrial function. Hum Mol Genet 2011; 20: 3067-3078.

[618] Margis R, Margis R, Rieder CRM. Identification of blood microRNAs associated to Parkinsońs disease. J Biotechnol 2011; 152: 96-101.

[619] Johnson R, Zuccato C,

Belyaev ND, et al. A microRNA-based gene dysregulation pathway in Huntington's disease. Neurobiol Dis 2008; 29: 438-445.

[620] Lee S-T, Chu K, Im W-S, et al. Altered microRNA regulation in Huntington's disease models. Exp Neurol 2011; 227: 172-179.

[621] Ghose J, Sinha M, Das E, et al. Regulation of miR-146a by RelA/ NFkB and p53 in STHdhQ111/HdhQ111 cells, a cell model of Huntington's disease. PLoS One 2011; 6: e23837.

[622] Bae B-I, Xu H, Igarashi S, et al. p53 mediates cellular dysfunction and behavioral abnormalities in
Huntington's disease. Neuron 2005; 47: $29-41$

[623] Xu Z, Li H, Jin P. Epi-

genetics-based therapeutics for

neurodegenerative disorders. Curr

Transl Geriatr Exp Gerontol Rep 2012; 1: 229-236.

[624] Coppedè F. The potential

of epigenetic therapies in neurodegenerative diseases. Front Genet 2014; 5: 220 .

[625] Durga J, van Boxtel MPJ, Schouten EG, et al. Effect of 3-year folic acid supplementation on cognitive function in older adults in the FACIT trial: a randomised, double blind, controlled trial. Lancet (London, England) 2007; 369: 208-16.

[626] Haan MN, Miller JW, Aiello AE, et al. Homocysteine, B vitamins, and the incidence of dementia and cognitive impairment: results from the Sacramento Area Latino Study on Aging. Am J Clin Nutr 2007; 85: 511-7. [627] Scarpa S, Fuso A, D’Ansel$\mathrm{mi}$, et al. Presenilin 1 gene silencing by $\mathrm{S}$-adenosylmethionine: a treatment for Alzheimer disease? FEBS Lett 2003; 541: 145-148.

[628] Devall M, Mill J, Lunnon K. The mitochondrial epigenome: a role in Alzheimer's disease? Epigenomics 2014; 6: 665-675.

[629] How Kit A, Nielsen HM,

Tost J. DNA methylation based biomarkers: Practical considerations and applications. Biochimie 2012; 94: 2314-2337.

[630] Malouf R, Grimley Evans J, Areosa Sastre A. Folic acid with or without vitamin B12 for cognition and dementia. In: Malouf R (ed) The Cochrane Database of Systematic Reviews. Chichester, UK: John Wiley \& Sons, Ltd, p. CD004514.

[631] McMahon JA, Green TJ,
Skeaff CM, et al. A controlled trial of homocysteine lowering and cognitive performance. $N$ Engl J Med 2006; 354: 2764-2772.

[632] Campbell NRC. How safe are folic acid supplements? Arch Intern Med 1996; 156: 1638.

[633] Daniel P, Brazier M, Cerutti

I, et al. Pharmacokinetic study of butyric acid administered in vivo as sodium and arginine butyrate salts. Clin Chim Acta 1989; 181: 255-263.

[634] Egorin MJ, Yuan Z-M, Sentz DL, et al. Plasma pharmacokinetics of butyrate after intravenous administration of sodium butyrate or oral administration of tributyrin or sodium butyrate to mice and rats. Cancer Chemother Pharmacol 1999; 43: $445-453$.

[635] Miller AA, Kurschel E, Osieka R, et al. Clinical pharmacology of sodium butyrate in patients with acute leukemia. Eur J Cancer Clin Oncol 1987; 23: 1283-1287.

[636] Chen P-S, Peng G-S, Li G, et al. Valproate protects dopaminergic neurons in midbrain neuron/glia cultures by stimulating the release of neurotrophic factors from astrocytes. Mol Psychiatry 2006; 11: 1116-1125.

[637] Marinova Z, Ren M, Wendland JR, et al. Valproic acid induces functional heat-shock protein 70 via Class I histone deacetylase inhibition in cortical neurons: a potential role of Spl acetylation. J Neurochem 2009; 111: 976-987.

[638] Marinova Z, Leng Y, Leeds $\mathrm{P}$, et al. Histone deacetylase inhibition alters histone methylation associated with heat shock protein 70 promoter modifications in astrocytes and neurons. Neuropharmacology 2011; 60: 1109-1115.

[639] Chen H, Dzitoyeva S, 
Manev H. Effect of valproic acid on mitochondrial epigenetics. Eur J Pharmacol 2012; 690: 51-59.

[640] Kidd SK, Schneider JS.

Protection of dopaminergic cells from MPP+-mediated toxicity by histone deacetylase inhibition. Brain Res 2010; 1354: 172-178.

[641] Leng $Y$, Marinova Z,

Reis-Fernandes MA, et al. Potent neuroprotective effects of novel structural derivatives of valproic acid: Potential roles of HDAC inhibition and HSP70 induction. Epub ahead of print 2010. DOI: 10.1016/j.neulet.2010.04.013. [642] Wu X, Chen PS, Dallas S, et al. Histone deacetylase inhibitors up-regulate astrocyte GDNF and BDNF gene transcription and protect dopaminergic neurons. Int $J$ Neuropsychopharmacol 2008; 11: 1123.

[643] Zhou W, Bercury K, Cummiskey J, et al. Phenylbutyrate up-regulates the DJ-1 protein and protects neurons in cell culture and in animal models of Parkinson disease.

J Biol Chem 2011; 286: 14941-51.

[644] Huang H-Y, Lin S-Z, Chen

W-F, et al. Urocortin modulates dopaminergic neuronal survival via inhibition of glycogen synthase kinase- $3 \beta$ and histone deacetylase. Neurobiol Aging 2011; 32: 1662-77.

[645] Roy A, Ghosh A, Jana A, et al. Sodium phenylbutyrate controls neuroinflammatory and antioxidant activities and protects dopaminergic neurons in mouse models of Parkinson's disease. PLoS One 2012; 7: e38113. [646] Brahe C, Vitali T, Tiziano FD, et al. Phenylbutyrate increases SMN gene expression in spinal muscular atrophy patients. Eur J Hum Genet 2005; 13: 256-259.

[647] Marks PA. The clinical development of histone deacetylase inhibitors as targeted anticancer drugs. Expert Opin Investig Drugs 2010; 19: 1049-1066.

[648] Marks PA, Xu W-S. Histone deacetylase inhibitors: Potential in cancer therapy. J Cell Biochem 2009; 107: 600-608.

[649] Salminen A, Tapiola T,

Korhonen P, et al. Neuronal apoptosis induced by histone deacetylase inhibitors. Mol Brain Res 1998; 61: 203-206. [650] Kelly-Sell MJ, Kim YH, Straus S, et al. The histone deacetylase inhibitor, romidepsin, suppresses cellular immune functions of cutaneous T-cell lymphoma patients. $\mathrm{Am} J$ Hematol 2012; 87: 354-360.

[651] Rossi LE, Avila DE, Spallanzani RG, et al. Histone deacetylase inhibitors impair NK cell viability and effector functions through inhibition of activation and receptor expression. J Leukoc Biol 2012; 91: 321-31.

[652] Haggarty SJ, Koeller KM,

Wong JC, et al. Domain-selective small-molecule inhibitor of histone deacetylase 6 (HDAC6)-mediated tubulin deacetylation. Proc Natl Acad Sci U S A 2003; 100: 4389-94.

[653] Trapp J, Meier R, Hongwiset D, et al. Structure-activity studies on suramin analogues as inhibitors of NAD+-dependent histone deacetylases (sirtuins). ChemMedChem 2007; 2: 1419-1431.

[654] Yamada K, Mizuno M,

Nabeshima T. Role for brain-derived neurotrophic factor in learning and memory. Life Sci 2002; 70: 735-744. [655] Ishimaru N, Fukuchi M, Hirai A, et al. Differential epigenetic regulation of BDNF and NT-3 genes by trichostatin A and 5-aza- ${ }^{\prime}$-deoxycytidine in Neuro-2a cells. Epub ahead of print 2010. DOI: 10.1016/j. bbrc.2010.02.139.
[656] Tian F, Marini AM, Lipsky

RH. Effects of histone deacetylase inhibitor Trichostatin A on epigenetic changes and transcriptional activation of Bdnf promoter 1 by rat hippocampal neurons. Ann N Y Acad Sci 2010; 1199: 186-193.

[657] Yuan Su *,‡, John Ryder $\ddagger$,\#, Baolin Li drug for bipolar disorder treatment, regulates amyloid- $\beta$ precursor protein processing. Epub ahead of print 2004. DOI: 10.1021/BI035627J.

[658] Qing H, He G, Ly PTT, et al. Valproic acid inhibits $A \beta$ production, neuritic plaque formation, and behavioral deficits in Alzheimer's disease mouse models. J Exp Med; http:// jem.rupress.org/content/205/12/2781 (2008, accessed 4 April 2017).

[659] Ricobaraza A, Cuadrado-Tejedor M, Pérez-Mediavilla A, et al. Phenylbutyrate ameliorates cognitive deficit and reduces tau pathology in an Alzheimer's disease mouse model. Neuropsychopharmacology 2009; 34: 1721-1732.

[660] Ricobaraza A, Cuadrado-Tejedor M, Marco S, et al. Phenylbutyrate rescues dendritic spine loss associated with memory deficits in a mouse model of Alzheimer disease.

Hippocampus 2012; 22: 1040-1050.

[661] Nuutinen T, Suuronen

T, Kauppinen A, et al. Valproic acid stimulates clusterin expression in human astrocytes: Implications for Alzheimer's disease. Epub ahead of print 2010. DOI: 10.1016/j. neulet.2010.03.041.

[662] Vecsey CG, Hawk JD, Lattal $\mathrm{KM}$, et al. Histone deacetylase inhibitors enhance memory and synaptic plasticity via CREB: CBP-dependent transcriptional activation. $J$ Neuro$s c i$; http://www.jneurosci.org/con- 
tent/27/23/6128 (2007, accessed 4 April 2017).

[663] Green KN, Steffan JS, Martinez-Coria H, et al. Nicotinamide restores cognition in Alzheimer's disease transgenic mice via a mechanism involving sirtuin inhibition and selective reduction of Thr231-phosphotau.

J Neurosci 2008; 28: 11500-10.

[664] Arrowsmith CH, Bountra

C, Fish P V., et al. Epigenetic protein families: a new frontier for drug discovery. Nat Rev Drug Discov 2012; 11: 384-400.

[665] Chatterjee S, Mizar P, Cassel R, et al. A novel activator of CBP/p300 acetyltransferases promotes neurogenesis and extends memory duration in adult mice. $J$ Neurosci; http://www.jneurosci.org/ content/33/26/10698 (2013, accessed 4 April 2017).

[666] Zhu M, Li W-W, Lu C-Z. Histone decacetylase inhibitors prevent mitochondrial fragmentation and elicit early neuroprotection against MPP+. CNS Neurosci Ther 2014; 20: 308-316.

[667] Kidd SK, Schneider JS. Protective effects of valproic acid on the nigrostriatal dopamine system in a 1-methyl-4-phenyl-1,2,3,6-tetrahydropyridine mouse model of Parkinson's disease. Neuroscience 2011; 194: 189-194.

[668] Chen PS, Wang C-C, Bortner CD, et al. Valproic acid and other histone deacetylase inhibitors induce microglial apoptosis and attenuate lipopolysaccharide-induced dopaminergic neurotoxicity. Neuroscience 2007; 149: 203-212.

[669] Peng G-S, Li G, Tzeng N-S, et al. Valproate pretreatment protects dopaminergic neurons from LPS-induced neurotoxicity in rat primary midbrain cultures: role of microglia.

Mol Brain Res 2005; 134: 162-169.

[670] Darmopil S, Martín AB, De

Diego IR, et al. Genetic inactivation

of dopamine D1 but not D2 receptors

inhibits L-DOPA-induced dyskinesia and histone activation. Biol Psychiatry 2009; 66: 603-613.

[671] Igarashi S, Morita H,

Bennett KM, et al. Inducible PC12 cell model of Huntington's disease shows toxicity and decreased histone acetylation. Neuroreport 2003; 14: 565-8.

[672] Steffan JS, Bodai L, Pallos

$\mathrm{J}$, et al. Histone deacetylase inhibitors arrest polyglutamine-dependent neurodegeneration in Drosophila. Nature 2001; 413: 739-743.

[673] Sugai F, Yamamoto Y,

Miyaguchi K, et al. Benefit of valproic acid in suppressing disease progression of ALS model mice. Eur J Neurosci 2004; 20: 3179-3183.

\section{[674] Wood MA, Attner MA,}

Oliveira AMM, et al. A transcription factor-binding domain of the coactivator CBP is essential for long-term memory and the expression of specific target genes. Learn Mem 2006; 13: 609-17.

\section{[675] McCampbell A, Taye AA,}

Whitty L, et al. Histone deacetylase inhibitors reduce polyglutamine toxicity. Proc Natl Acad Sci U S A 2001; 98: $15179-84$.

\section{[676] Hockly E, Richon VM,}

Woodman B, et al. Suberoylanilide hydroxamic acid, a histone deacetylase inhibitor, ameliorates motor deficits in a mouse model of Huntington's disease. Proc Natl Acad Sci U S A 2003; 100: 2041-6.

\section{[677] Mielcarek M, Benn CL,}

Franklin SA, et al. SAHA decreases HDAC 2 and 4 levels in vivo and improves molecular phenotypes in the
R6/2 mouse model of Huntington's disease. PLoS One 2011; 6: e27746.

[678] Ferrante RJ, Kubilus JK, Lee

J, et al. Histone deacetylase inhibition by sodium butyrate chemotherapy ameliorates the neurodegenerative phenotype in Huntington's disease mice. J Neurosci 2003; 23: 9418-27. [679] Dasgupta S, Zhou Y, Jana M, et al. Sodium phenylacetate inhibits adoptive transfer of experimental allergic encephalomyelitis in SJL/J mice at multiple steps. J Immunol; http://www.jimmunol.org/content/170/7/3874 (2003, accessed 4 April 2017).

[680] Ebbel EN, Leymarie N, Schiavo S, et al. Identification of phenylbutyrate-generated metabolites in Huntington disease patients using parallel liquid chromatography/electrochemical array/mass spectrometry and off-line tandem mass spectrometry. Anal Biochem 2010; 399: 152-161. [681] Hogarth P, Lovrecic L, Krainc D. Sodium phenylbutyrate in Huntington's disease: A dose-finding study. Mov Disord 2007; 22: 1962-1964. [682] Jia H, Kast RJ, Steffan JS, et al. Selective histone deacetylase (HDAC) inhibition imparts beneficial effects in Huntington's disease mice: implications for the ubiquitin-proteasomal and autophagy systems. Hum Mol Genet 2012; 21: 5280-5293.

[683] Jia H, Pallos J, Jacques V, et al. Histone deacetylase (HDAC) inhibitors targeting HDAC3 and HDACl ameliorate polyglutamine-elicited phenotypes in model systems of Huntington's disease. Neurobiol Dis 2012; 46: 351-361.

[684] Hu Y, Chopra V, Chopra R, et al. Transcriptional modulator $\mathrm{H} 2 \mathrm{~A}$ histone family, member Y (H2AFY) marks Huntington disease activity in 
man and mouse. Proc Natl Acad Sci

US A 2011; 108: 17141-6.

[685] Blanco G, Fu H, Mendez

C, et al. Deciphering the biosynthetic origin of the aglycone of the aureolic acid group of anti-tumor agents.

Chem Biol 1996; 3: 193-6.

[686] Chakrabarti S, Bhattacharyya D, Dasgupta D. Structural basis of DNA recognition by anticancer antibiotics, chromomycin A3, and mithramycin: Roles of minor groove width and ligand flexibility. Biopolymers 2000; 56: 85-95.

[687] Prado L, Lombó F, Braña AF, et al. Analysis of two chromosomal regions adjacent to genes for a type II polyketide synthase involved in the biosynthesis of the antitumor polyketide mithramycin in Streptomyces argillaceus. Mol Gen Genet $M G G$ 1999; 261: 216-225.

[688] Ralston SH. Pathogenesis and management of cancer associated hypercalcaemia. Cancer Surv 1994; 21 : $179-96$.

[689] Ryan WG. Treatment of Paget's disease of bone with mithramycin. Clin Orthop Relat Res 1977; 106-10.

[690] Kennedy BJ. Mithramycin therapy in testicular cancer. $J$ Urol 1972; 107: 429-32.

[691] Hagen G, Dennig J, Preiss

A, et al. Functional analyses of the transcription factor $\mathrm{Sp} 4$ reveal properties distinct from Spl and Sp3. J Biol Chem 1995; 270: 24989-94.

[692] Majello B, De Luca P, Suske G, et al. Differential transcriptional regulation of c-myc promoter through the same DNA binding sites targeted by Spl-like proteins. Oncogene 1995; 10: 1841-8.

[693] Chatterjee S, Zaman K, Ryu H, et al. Sequence-selective DNA binding drugs mithramycin A and chromomycin A3 are potent inhibitors of neuronal apoptosis induced by oxidative stress and DNA damage in cortical neurons. Ann Neurol 2001; 49: 345-54.

[694] Junn E, Mouradian MM.

MicroRNAs in neurodegenerative diseases and their therapeutic potential.

Pharmacol Ther 2012; 133: 142-150.

[695] Krützfeldt J, Rajewsky N,

Braich R, et al. Silencing of microR-

NAs in vivo with 'antagomirs'. Nature 2005; 438: 685-689.

[696] Kuhn DE, Nuovo GJ, Terry

A V, et al. Chromosome 21-derived microRNAs provide an etiological basis for aberrant protein expression in human Down syndrome brains. $J$ Biol Chem 2010; 285: 1529-43.

[697] Yu D, Pendergraff H, Liu J, et al. Single-stranded RNAs use RNAi to potently and allele-selectively inhibit mutant huntingtin expression. Cell 2012; 150: 895-908.

[698] Alvarez-Erviti L, Seow Y, Yin H, et al. Delivery of siRNA to the mouse brain by systemic injection of targeted exosomes. Nat Biotechnol 2011; 29: 341-345.

[699] Lakhal S, Andaloussi S El, O'Loughlin AJ, et al. RNAi therapeutic delivery by exosomes. Springer US, pp. 185-205.

[700] Xu T, Li L, Huang C, et al. MicroRNA-323-3p with clinical potential in rheumatoid arthritis, Alzheimer's disease and ectopic pregnancy. Expert Opin Ther Targets 2014; 18: 153-158.

[701] $\mathrm{Hu}$ J, Matsui M, Gagnon $\mathrm{KT}$, et al. Allele-specific silencing of mutant huntingtin and ataxin-3 genes by targeting expanded CAG repeats in mRNAs. Nat Biotechnol 2009; 27: 478-484
[702] Lombardi MS, Jaspers L, Spronkmans C, et al. A majority of Huntington's disease patients may be treatable by individualized allele-specific RNA interference. Exp Neurol 2009; 217: 312-319.

[703] Zhang Y, Friedlander RM. Using non-coding small RNAs to develop therapies for Huntington's disease. Gene Ther 2011; 18: 1139-1149. [704] Harper SQ, Staber PD, He $\mathrm{X}$, et al. RNA interference improves motor and neuropathological abnormalities in a Huntington's disease mouse model. Proc Natl Acad Sci USA 2005; 102: 5820-5.

[705] Boudreau RL, McBride JL, Martins I, et al. Nonallele-specific silencing of mutant and wild-type huntingtin demonstrates therapeutic efficacy in Huntington's disease mice. Mol Ther 2009; 17: 1053-1063.

[706] DiFiglia M, Sena-Esteves M, Chase K, et al. Therapeutic silencing of mutant huntingtin with siRNA attenuates striatal and cortical neuropathology and behavioral deficits. Proc Natl Acad Sci U S A 2007; 104: 17204-9. [707] Drouet V, Perrin V, Hassig $\mathrm{R}$, et al. Sustained effects of nonallele-specific Huntingtin silencing. Ann Neurol 2009; 65: 276-285.

[708] Franich NR, Fitzsimons HL, Fong DM, et al. AAV vector-mediated RNAi of mutant huntingtin expression is neuroprotective in a novel genetic rat model of Huntington's disease. Mol Ther 2008; 16: 947-956.

[709] Huang B, Schiefer J, Sass C, et al. High-capacity adenoviral vector-mediated reduction of huntingtin aggregate load in vitro and in vivo. Hum Gene Ther 2007; 18: 303-311.

[710] Machida Y, Okada T, Kurosawa M, et al. rAAV-mediated shRNA ameliorated neuropathology 
in Huntington disease model mouse.

Biochem Biophys Res Commun 2006;

343: 190-197.

[711] McBride JL, Boudreau RL, Harper SQ, et al. Artificial miRNAs mitigate shRNA-mediated toxicity in the brain: implications for the therapeutic development of RNAi. Proc Natl Acad Sci U S A 2008; 105: 5868-73.

[712] Rodriguez-Lebron E, Denovan-Wright EM, Nash K, et al. Intrastriatal rAAV-mediated delivery of anti-huntingtin shRNAs induces partial reversal of disease progression in R6/1 Huntington's disease transgenic mice. Mol Ther 2005; 12: 618-633.

[713] Davies MN, Volta M, Pidsley R, et al. Functional annotation of the human brain methylome identifies tissue-specific epigenetic variation across brain and blood. Genome Biol 2012; 13: R43.

[714] Hansen KD, Timp W, Bravo HC, et al. Increased methylation variation in epigenetic domains across cancer types. Nat Genet 2011; 43: $768-775$.

[715] Lister R, Pelizzola M, Dowen RH, et al. Human DNA methylomes at base resolution show widespread epigenomic differences. Nature 2009; 462: 315-322.

[716] Guffanti A, Simchovitz A, Soreq H. Emerging bioinformatics approaches for analysis of NGS-derived coding and non-coding RNAs in neurodegenerative diseases. Front Cell Neurosci 2014; 8: 89.

[717] Raasch P, Schmitz U, Patenge N, et al. Non-coding RNA detection methods combined to improve usability, reproducibility and precision. BMC Bioinformatics 2010; 11: 491.

[718] Blalock EM, Buechel HM, Popovic J, et al. Microarray analy- ses of laser-captured hippocampus reveal distinct gray and white matter signatures associated with incipient Alzheimer's disease. J Chem Neuroanat 2011; 42: 118-126.

[719] Guintivano J, Aryee MJ,

Kaminsky ZA. A cell epigenotype specific model for the correction of brain cellular heterogeneity bias and its application to age, brain region and major depression. Epigenetics 2013; 8: 290-302.

[720] Whittemore SR, Sanon HR, Wood PM. Concurrent isolation and characterization of oligodendrocytes, microglia and astrocytes from adult human spinal cord. Int J Dev Neurosci 1993; 11: 755-764.

[721] Suarez-Quian CA, Goldstein SR, Pohida T, et al. Laser capture microdissection of single cells from complex tissues. Biotechniques 1999; 26: 328-35.

[722] Uchida N, Buck DW, He D, et al. Direct isolation of human central nervous system stem cells. Proc Natl Acad Sci U S A 2000; 97: 14720-5.

[723] Yu S, Zhang JZ, Zhao CL, et al. Isolation and characterization of the CD133 + precursors from the ventricular zone of human fetal brain by magnetic affinity cell sorting. Biotechnol Lett 2004; 26: 1131-1136.

[724] Chung K, Wallace J, Kim S-Y, et al. Structural and molecular interrogation of intact biological systems. Nature 2013; 497: 332-337. [725] Lee JH, Daugharthy ER, Scheiman J, et al. Highly multiplexed subcellular RNA sequencing in situ. Science; http://science.sciencemag.org/ content/343/6177/1360 (2014, accessed 5 April 2017).

[726] Barton AJL, Pearson RCA,

Najlerahim A, et al. Pre-and Postmortem Influences on Brain RNA.
J Neurochem 1993; 61: 1-11.

[727] Stan AD, Ghose S, Gao

$\mathrm{X}-\mathrm{M}$, et al. Human postmortem tissue: What quality markers matter? Brain Res 2006; 1123: 1-11.

[728] Pidsley R, Mill J. Epigenetic studies of psychosis: current findings, methodological approaches, and implications for postmortem research. Biol Psychiatry 2011; 69: 146-156.

[729] Mill J, Heijmans BT. From promises to practical strategies in epigenetic epidemiology. Nat Rev Genet 2013; 14: 585-594.

[730] Relton CL, Davey Smith G. Two-step epigenetic Mendelian randomization: a strategy for establishing the causal role of epigenetic processes in pathways to disease. Int J Epidemiol 2012; 41: 161-176.

[731] Boks MP, de Jong NM, Kas $\mathrm{MJH}$, et al. Current status and future prospects for epigenetic psychopharmacology. Epigenetics 2012; 7: 20-28. [732] Pasinetti GM, Ho L, Dooley C, et al. Select non-coding RNA in blood components provide novel clinically accessible biological surrogates for improved identification of traumatic brain injury in OEF/OIF Veterans. Am J Neurodegener Dis 2012; 1: $88-98$.

[733] Sharma RP. Blood chromatin as a biosensor of the epigenetic milieu: a tool for studies in living psychiatric patients. Epigenomics 2012; 4: 551-559.

[734] Lunnon K, Ibrahim

Z, Proitsi P, et al. Mitochondrial dysfunction and immune activation are detectable in early Alzheimer's disease blood. J Alzheimers Dis 2012; 30: 685-710.

[735] German DC, Eisch AJ. Mouse models of Alzheimer's disease: insight into treatment. Rev Neurosci 
2004; 15: 353-69.

[736] Saito T, Matsuba Y, Mihira

N, et al. Single App knock-in mouse

models of Alzheimer's disease. Nat

Neurosci 2014; 17: 661-663.

[737] Raber J, Wong D, Buttini

M, et al. Isoform-specific effects of

human apolipoprotein $\mathrm{E}$ on brain

function revealed in ApoE knockout

mice: increased susceptibility of

females. Proc Natl Acad Sci U S A 1998;

95: 10914-9.

[738] Kumar A, Seghal N,

Naidu PS, et al. Colchicines-induced neurotoxicity as an animal model

of sporadic dementia of Alzheimer's

type. Pharmacol Rep; 59: 274-83.

[739] Sparks DL, Scheff SW,

Hunsaker JC, et al. Induction of

Alzheimer-like $\beta$-amyloid immuno-

reactivity in the brains of rabbits with

dietary cholesterol. Exp Neurol 1994;

126: 88-94.

[740] Hoyer S, Lee SK, Löffler

$\mathrm{T}$, et al. Inhibition of the neuronal

insulin receptor. An in vivo model for sporadic Alzheimer disease? Ann N Y Acad Sci 2000; 920: 256-8.

[741] Przedborski S, Vila M. The 1-methyl-4-phenyl-1,2,3,6-tetrahy-

dropyridine mouse model: a tool to explore the pathogenesis of Parkinson's disease. Ann N Y Acad Sci 2003; 991: 189-98.

[742] Podlisny MB, Tolan DR,

Selkoe DJ. Homology of the amyloid beta protein precursor in monkey and human supports a primate model for beta amyloidosis in Alzheimer's disease. Am J Pathol 1991; 138: 1423-35. [743] Israel MA, Yuan SH, Bardy C, et al. Probing sporadic and familial Alzheimer's disease using induced pluripotent stem cells. Nature 2012; 482: 216-20.

Alzheimer's disease modeling: ups, downs, and perspectives for human induced pluripotent stem cells. J Alzheimers Dis 2013; 34: 563-88.

[745] Qiang L, Fujita R, Yamashita T, et al. Directed conversion of Alzheimer's disease patient skin fibroblasts into functional neurons. Cell 2011; 146: 359-71.

[746] Takahashi K, Tanabe K, Ohnuki M, et al. Induction of pluripotent stem cells from adult human fibroblasts by defined factors. Cell 2007; 131: 861-872.

[747] Tian C, Liu Q, Ma K, et al. Characterization of induced neural progenitors from skin fibroblasts by a novel combination of defined factors. Sci Rep 2013; 3: 1345.

[748] Verma A, Verma N.

Induced pluripotent stem cells and promises of neuroregenerative medicine. Neurol India 2011; 59: 555-7.

[749] Kim K, Doi A, Wen B, et al. Epigenetic memory in induced pluripotent stem cells. Nature 2010; 467: 285-90.

[750] Meaburn EL, Schalkwyk

LC, Mill J. Allele-specific methylation in the human genome. Epigenetics 2010; 5: 578-582.

[751] Szyf M. Epigenetics, a key for unlocking complex CNS disorders? Therapeutic implications. Eur Neuropsychopharmacol 2015; 25: 682-702.

[752] de Groote ML, Verschure PJ, Rots MG. Epigenetic Editing: targeted rewriting of epigenetic marks to modulate expression of selected target genes. Nucleic Acids Res 2012; 40: 10596-10613. 




\section{RODENT MODELS OF} COGNITIVE DISORDERS:

\section{IMPAIRMENT, AGING \& DEMENTIA}

ROY LARDENOIJE ${ }^{A, *}$, NICK P. VAN GOETHEM ${ }^{A, *}$, KONSTANTINOS KOMPOTIS $^{A}$, BART P.F. RUTTEN ${ }^{\text {, }}$, JOS PRICKAERTS ${ }^{A}$, HARRY W.M. STEINBUSCH ${ }^{A}$

ASCHOOL FOR MENTAL HEALTH AND NEUROSCIENCE (MHENS), DEPARTMENT OF PSYCHIATRY AND NEUROPSYCHOLOGY, MAASTRICHT UNIVERSITY, UNIVERSITEITSSINGEL 50, 6200 MD MAASTRICHT, THE NETHERLANDS

*THESE AUTHORS CONTRIBUTED EQUALLY TO THIS WORK. 


\subsection{Introduction}

Cognitive dysfunction is a feature often encountered in a broad spectrum of neurological and psychiatric conditions. The property of animal models to study the development of a disease, and not just late-stage pathology, is crucial for disease models involving cognitive deficits, as such deficits are often the result of neurodegeneration. Considering the limited regenerative capacity of the brain, it is thus pivotal to treat neurodegenerative diseases as early as possible [1]. Since ameliorating these dysfunctions can dramatically improve the quality of life of patients, developing treatments, or "cognition enhancers," is a major area of interest for the pharmaceutical industry. Accordingly, over the past few decades certain drugs have been approved for the treatment of cognitive impairments related to specific neurological and psychiatric conditions (for a recent review, see [2]). A diverse range of animal models are being used to identify potential cognition-enhancing drugs and such models can be based on pharmacological deficits, the naturally occurring aging process, and/or introduction of transgenic constructs in rodents. The first part of this chapter describes the most commonly used rodent pharmacological deficit models. Hereafter, animal models of aging and transgenic animal models will be discussed.

\subsection{Pharmacological models}

In pharmacological deficit models, specific drugs are administered to animals in order to induce cognitive deficits. The targets of these cognition-impairing drugs are hypothesis based and are often directed to alter distinct neurotransmitter systems, with different disorders showing specific dysregulation or impairments.

\subsubsection{Inhibition of energy/ glucose metabolism}

A variety of studies in both rodents and humans have shown that slight increases in circulating glucose concentrations exhibit beneficial effects in brain functions relating to learning and memory [3]. Administrating glucose has been shown to facilitate rodent performance and furthermore reverses both drug- and age-related cognitive deficits. The putative mechanism of action underlying these precognitive effects probably relates to altered neuronal metabolism, neuronal activity, or neurotransmitter synthesis [4]. 
The most straightforward way of inhibiting energy/glucose metabolism is glucose deprivation. In vitro studies often use oxygen-glucose deprivation to mimic ischemic injury and subsequently study acute stroke pathology [5]. In vivo studies, which use oxygen-glucose deprivation, mostly do this via middle cerebral artery occlusion [6]. $\mathrm{N}$-methyl-D-aspartate (NMDA) receptor antagonists have been shown to be neuroprotective against excitotoxicity in both in vitro and in vivo models of ischemia or neurodegeneration $[7,8]$. Another way of inhibiting energy/glucose metabolism is by treatment with the glycolytic inhibitor, 2-deoxyglucose. Although mostly used for glucose uptake measurement, 2-deoxyglucose has been shown to dose-dependently affect cognitive performance of rodents [9].

Another possible animal model for metabolic dysfunction (and/ or generation of oxidative stress) is intracerebral ventricular (i.c.v.) administration of streptozotocin $[10,11]$. Streptozotocin is a naturally occurring chemical that was discovered in the late 1950s and a little later identified as an antibacterial antibiotic [12]. Subsequently, it was discovered that i.c.v. administration of streptozotocin decreases the central metabolism of glucose and hence offers a useful animal/ rodent model of neurodegeneration [13]. Furthermore, i.c.v. streptozotocin administration also reduces the concentrations of different neurotransmitters, including acetylcholine (ACh) [14, 15]. As will be described in the next section, this cholinergic reduction further contributes to the use of this animal model of neurodegeneration. Accordingly, middle-aged and old rats that have been treated with streptozotocin (i.c.v.) show cognitive deficits in tasks assessing learning and memory. These deficits can be reversed with specific cognition-enhancing drugs $[13,16]$.

\subsubsection{Cholinergic interven- tions}

\subsubsection{Cholinergic toxins}

The use of pharmacological deficit models targeting the cholinergic system became popular after the cholinergic hypothesis of geriatric memory dysfunction was postulated. This hypothesis states that the age-related decline in cognition is predominately caused by a decrement of cholinergic neurotransmission [17]. Nowadays, with the exception of one NMDA receptor antagonist (see also above), all approved drugs for the treatment of cognitive dysfunction in Alzheimer's disease (AD) aim at increasing cholinergic neurotransmission. Different approaches have 
been used to induce cholinergic hypofunction in order to mimic AD-, and age-related cognitive decline. To achieve chronic dysregulation of the cholinergic system, cholinergic toxins have been used. The exact role of ACh in cognition is not fully understood, but ACh regulation has been associated with attention, learning, and memory processes [18].

Many of the early rat studies made use of excitotoxic lesions by means of central administrations of ibotenic acid or quisqualic acid. The excitotoxic lesions (especially with ibotenic acid) of cholinergic neurons revealed a vast range of cognitive impairments $[19,20]$. However, a fundamental problem with this approach was the lack of a specific cholinergic toxin, introducing the possibility that such impairments may be due to damage to noncholinergic neurons. A more selective way to destruct cholinergic cells can be accomplished by locally injecting 192 lgG-saporin. 192 lgGsaporin is an antineuronal immunotoxin that consists of a monoclonal antibody (192 lgG) to the nerve growth factor (NGF) receptor that has been armed with saporin, a ribosome-inactivating protein [21, 22]. Injection of this 192 lgG-saporin complex produces long-lasting depletions in cholinergic markers throughout the forebrain of rats [23]. 192 IgG-saporin administration has been used to induce cognitive impairments in rodents to investigate the role of the cholinergic system in particular brain structures $[24,25]$.

\subsubsection{Cholinergic antagonists}

Induction of more transient or acute disruption of the cholinergic system can be induced with cholinergic antagonists. ACh has two types of receptors: the metabotropic muscarinic receptors (five subtypes in the central nervous system [CNS]) and the ionotropic nicotinic receptors (two major subtypes in the CNS). There are specific antagonists for each ACh receptor type. A further division can be made between selective and nonselective cholinergic antagonists. This applies to the selectivity/affinity of an antagonist to the isoforms of ACh receptor (sub)types.

The most widely used nonselective competitive cholinergic antagonists are the tropane alkaloids scopolamine hydrobromide and atropine. The nonselective muscarinic antagonist scopolamine is probably the most often used cognition deficit-inducing drug in (preclinical) rodent research. Since scopolamine induces amnesia that is caused by a blockade of cholinergic signaling, this drug is used to model cognitive deficits associated with aging and dementia [26]. In preclinical testing, scopolamine is often coadministered with putative cognition-enhancing drugs in order to test whether a new drug is effective in reversing a scopolamine induced cognitive deficit [27]. The rationale is that if a 
new experimental drug can reverse such a deficit, it might also improve cognitive function in healthy participants or people diagnosed with a neuropsychiatric disorder [26].

Since scopolamine is a nonselective muscarinic antagonist, efforts have been made to promote the use of more selective muscarinic antagonists. Since muscarinic receptors are both centrally and peripherally present, it would be more "clean" to use a more centrally selective muscarinic antagonist. Of the five known muscarinic receptors (M1-M5), M1 might be a promising target since this receptor is predominantly located in the cortex and the hippocampus, brain regions known to be important for attention, learning, and memory. Peripheral presence of the $\mathrm{M} 1$ receptor is relatively limited [28]. The selective muscarinic M1 receptor antagonist biperiden is, therefore, an interesting drug candidate to more selectively induce cognitive, in particular memory, deficits in rodent models [29].

The other class of cholinergic receptors is the class of ionotropic nicotinic receptors ( $\mathrm{nAChRs}$ ). These receptors belong to a family of ligand-gated ion channel receptors that include type 3 serotonin (5HT3), gamma-aminobutyric acid A (GABAA) and strychnine-sensitive glycine receptors. $n A C h R s$ in the brain are composed of five subunits, which can be either $a$-subunits (nine identified subunits: $\alpha 2-a 10)$ or $\beta$-subunits (three identified subunits: $\beta 2-\beta 4$ ). These subunits can combine to result in different isoforms. In the CNS, the heteropentameric $\alpha 4 \beta 2$ and the homopentameric a7 nAChRs comprise $>90 \%$ of the nAChR subtypes [30]. Since $n A C h R s$ have been shown to be involved in learning and memory [27] and postmortem research shows that $\mathrm{nAChR}$ densities are markedly decreased in the brains of both patients with $A D$ and schizophrenia, the pharmaceutical industry has been developing different nAChR agonists in order to try to ameliorate the cognitive deficits that accompany these disorders [30]. Accordingly, antagonists of these nAChRs cause cognitive impairments in rodents, and hence certain drugs are used to mimic cognitive deficits seen in both AD and schizophrenia.

Mecamylamine is such a nonselective $\mathrm{nAChR}$ antagonist shown to induce learning and memory deficits (at high enough doses) in rodents [31]. In order to more specifically investigate the role of the different nAChR subtypes, selective nAChR antagonists are used. Methyllycaconitine (MLA) is a selective a7 $n A C h R$ competitive antagonist, and dihydro-betaerythroidine $(\mathrm{DHbE})$ is a selective a $4 \beta 2 \mathrm{nAChR}$ competitive antagonist. Both of these drugs have been shown to induce memory deficits in rodents [32], when administered at a high enough dose [33]. Besides inducing cognitive deficits on their own, these drugs are also used to counteract the procognitive effect of agonists at their corresponding nAChR subtype. This approach is used in order to confirm that the 
procognitive effects of a selective $\mathrm{nAChR}$ agonist are indeed mediated via a specific $n A C h R[27,33]$.

\subsubsection{Glutamatergic antag- onists}

Another important neurotransmitter directly involved in cognitive processes is glutamate. Glutamate is an abundantly present excitatory neurotransmitter, which acts through the ionotropic NMDA receptor (besides the alpha-amino-3-hydroxy-5-methyl-4-isoxazolepropionic acid [AMPA] receptor). NMDA receptors have been implicated in cognitive processes, in particular memory formation [34].

Following this rationale, NMDA antagonists have been used to function as cognition-deficit models in rodents and of these the most widely used cognition impairers are noncompetitive NMDA receptor-channel blockers. The most frequently used NMDA receptor-channel blockers in rodent models are MK-801 (dizocilpine), phencyclidine (PCP), and ketamine. These receptor-channel blockers bind to specific sites within the NMDA receptor channel pore and subsequently block the channel, thereby inducing cognitive impairments.

MK-801 has been assessed in a broad range of rodent test paradigms and is considered a valid model to induce acute cognitive dysfunction provided the right dose is used (without inducing noncognitive side effects) [34]. PCP in rodents is mainly used in a (sub)chronic manner to mimic the impairments seen in schizophrenia patients. In contrast to MK801, PCP was also tested in humans; hence, more direct comparisons between rodent and human behavior can be made [35]. PCP is believed to bind to a site within the NMDA receptor-channel pore (the PCP binding site) that is only accessible when the channel is open. Therefore, the antagonism is "use dependent." PCP thus acts at the same site as other "open-channel" blockers such as MK-801 or ketamine [36]. Besides acting on the NMDA receptor channel, PCP also binds to the dopamine uptake site. MK-801 is considerably more potent than PCP in producing a noncompetitive blockade at the NMDA receptor. However, MK-801 lacks the direct action on dopamine uptake, which accounts for the argument that PCP might be a more suitable deficit model for schizophrenia specifically, since PCP intoxication is associated with more psychotic features. Ketamine also acts as a type 2 dopamine partial agonist, but is a weaker blocker of the NMDA ion channel. Therefore, for mimicking psychosis, PCP might represent a more (and ketamine a less) complete model. Although MK-801 is much less complex in its pharmacological 
profile, it has proved to be valuable in animal studies because of its high selectivity for the NMDA receptor [35]. After scopolamine, MK-801 is probably the most widely used drug for the induction of cognitive impairments in rodents [34].

\subsubsection{Serotonergic inter- vention}

The serotonergic system has been implicated in cognitive processes as well. This system may have only minor effects on cognitive function on its own, but is assumed to interact with the cholinergic system. This serotonergic-cholinergic interaction probably plays an important role in the mediation of behavioral, including cognitive, performance [37].

A model used to decrease serotonin (5HT) entails the lowering of $5 \mathrm{HT}$ levels. Decreasing $5 \mathrm{HT}$ levels can be accomplished by manipulating the availability of the essential amino acid tryptophan via the food. Tryptophan has multiple functions, one of which is that it functions as a biochemical precursor for $5 \mathrm{HT}$. Acute tryptophan depletion is used as a pharmacological deficit model to lower central $5 \mathrm{HT}$ levels. The acute tryptophan depletion method is widely used both preclinically and clinically as a model to investigate the implication of the $5 \mathrm{HT}$ system in affective disorders [38, 39]. This serotonergic-deficit model has been frequently used to study putative cognition enhancers in rats $[39,40]$.

\subsection{Aging and transgenic models}

Over the past few decades, ample transgenic rodent models modeling specific diseases and exhibiting cognitive deficits have been generated. It should, however, be noted that most of the diseases discussed below are not of simple genetic origin. Indeed, the exact etiology of most remains to be elucidated. This means that the specific mutations used to create a model may only have a small hand in the actual pathology. Single mutations might not even result in any detectable pathology and multiple mutations, or specific environmental interactions may be required to instigate disease pathology [41]. Described in this section is a selection of transgenic rodent models of some of the most common neurodegenerative diseases involving cognitive impairments, which are most widely used or have provided critical insights. 


\subsubsection{Normal aging}

Of most cognitive disorders, aging is the top risk factor, while at the same time aging itself is also associated with cognitive decline [42]. Although aging is a natural process, it can result in quite severe functional limitations at the end of the life span, resulting inevitably in death. Rats and mice are useful laboratory species for studying the aging process, as they have relatively short life spans (up to 4 years for mice and up to 5 years for rats), are small and thus easy to keep, and reproduce fast [43]. For instance, nontransgenic mice can be used to study epigenetic, physiological, morphological, and behavioral changes as they occur during the aging process [44-46]. Importantly, interventions that may have a positive effect on age-related decline, such as caloric restriction, can be tested in these animals in a relatively short amount of time [46]. An even faster rodent model of aging is the senescence-accelerated mouse. This model consists of a collection of series created through the selective breeding of AKR/J mice, which already showed signs of accelerated aging, including multiple senescence-prone ( $P$ series) and senescence-resistant ( $R$ series) series [47, 48]. Of particular interest are the senescence-accelerated prone SAMP8 mice, which show ample age-related changes early in life, leading to a median survival time of only around 10 months. SAMP 8 mice naturally present with neuropathological and neurochemical changes, including $A \beta$ deposition, hyperphosphorylation of tau, and hampered dendritic spine development, as well as NMDA-, acetylcholine-, and noradrenaline-associated abnormalities [49]. This makes the SAMP8 model attractive for the study of, for example, age-related Parkinson's disease (PD) and AD. SAMP8 mice develop learning and memory impairments at a young age. Such deficits start at 2 months of age, as assessed with such tests as the water maze, T-maze, passive avoidance, and one-way active avoidance paradigms $[49,50]$.

The greatest advantage of mice and rats may, however, also be one of their greatest limitations as models of human aging. The large gap between the life spans of humans, and that of mice and rats, may be indicative of the latter being unable to fully elucidate the mechanisms influencing human aging [43]. For this reason, some investigators have chosen to use animal models that live longer, among which are also other rodent models. Some of these model organisms, including the naked mole rat, porcupines, and beavers, reach life spans of over 20 years. Comparing species of the same order of Rodentia with such diverging life spans may offer insights into the general mechanisms that increase a species age. 


\subsubsection{Alzheimer's disease 3.3.2.1. APP}

Despite its relative rarity, familial $A D$ ( $f A D$ ) has garnered the most attention due to its large genetic component. It is thus not surprising that the first transgenic mouse model for AD, the PDAPP model made in 1995 , is based on a mutation in the fAD-associated amyloid precursor protein (APP) gene [51, 52]. PDAPP mice express human APP cDNA with the Indiana mutation (V717F). In this model, plaque pathology arises between 6 and 9 months, paired with synapse, but no severe cell loss, or neurofibrillary tangle (NFT) deposition. Aged mice of this model display an impaired learning ability in the Morris water maze, the radial arm water maze, the cue task, and serial spatial reversal task [53].

Although some neuropathology occurs in this first model, it is the second transgenic model, Tg2576, implementing a double APP mutation (K670N and M671L), that successfully models an age-dependent buildup of amyloid plaques and related cognitive decline, as associated with $A D$ [54]. The mutant APP expressed by Tg2576 mice is also referred to as APPswe, and is under control of the hamster prion promoter. Cognitive decline in these widely-used mice occurs progressively from 6 to 9 months of age. By the age of 12 months, this model shows an impaired performance on spatial and working memory tasks, including the Y-maze spontaneous alternation and visible platform recognition tasks, as well as amygdala-dependent fear conditioning tasks.

A more aggressive AD model, the TgCRND8 transgenic mouse model, combines the Swedish and Indiana mutations, expressing the human bAPP695 transgene under control of the Syrian hamster prion promoter, on a $\mathrm{C} 3 \mathrm{H} / \mathrm{B} 6$ background [55]. This combination results in rapid extracellular plaque formation in the hippocampus and frontal cortex, similar to human $A D$, paired with defunct spatial learning in the Morris water maze task at 3 months of age, and impaired nonspatial episodic memory, as determined with the object recognition task at 8 weeks of age.

\subsubsection{2. $\mathrm{PS} 1, \mathrm{PS} 2$, and $\mathrm{PS} 1 \times \mathrm{APP}$}

Apart from mutations in the APP gene, mutations in presenilin (PS) genes have also been used to generate transgenic mouse models. For instance, the PS1M146L, PS1M146V, and PS2N141I models were used to demonstrate in vivo that mutant PS1 and PS2 are able to selectively enhance $A \beta 42$ levels [56]. This increased $A \beta 42$ presence is, however, 
without significant plaque pathology and cognitive deficits. It seems that the interaction between the PS and APP genes is of vital importance in the pathophysiology of $A D$ and, therefore, PS mutations are usually combined with a mutated APP transgene. The biogenic PSAPP model, a crossing between APP and PS1 transgenic models (e.g., Tg2576 × PS1M146L, PS1-A246 + APPswe, and APPswe/PS1dE9), shows a grave acceleration in pathology, compared with mutant APP-only models [57]. This includes an earlier onset of cognitive impairments, as measured with the Morris water maze and radial arm water maze tests for working memory.

One of the most early-onset and aggressive amyloid models is the 5xFAD transgenic mouse model, sporting five fAD-associated mutations [58]. 5xFAD mice carry two transgenes under the mouse Thy-1 promoter: APPswe/Ind/fl and PS1M146L/L286V (on a B6/SJL background), resulting in a grossly exaggerated $A \beta 42$ production. Consequently, amyloid deposits in the hippocampus start to form at the young age of 2 months. By the age of 6 months, massive amyloid pathology can be observed throughout the hippocampus and cortex of these mice, paired with impaired spatial working memory, as tested with the spontaneous alternation Y-maze. At this age 5xFAD mice also show impaired hippocampal-dependent contextual fear memory [59].

\subsubsection{MAPT}

APP, PS1, and PS2 transgenic models are able to capture some of the $A \beta$-associated pathology seen in AD. Most of these models, however, fail to recapitulate the widespread neurodegeneration and tangle pathology, which is critical for a suitable phenocopy of AD. A model that achieves just that is the TauP301S transgenic mouse model, based on the shortest isoform of $4 \mathrm{R}$ microtubule-associated protein tau (MAPT) with the P301S mutation, controlled by the mouse Thy-1 promoter on a C57BL/J background [60]. Around 5-6 months of age, widespread NFT pathology can be observed in the brain and spinal cord, as well as neuronal loss in the latter area, paired with severe paraparesis in mice of this model. Cognitive deficits at 5-6 months of age include decreased spontaneous alternation in the Y-maze test, impaired sociability and object recognition memory in Crawley's social interaction test, hampered spatial memory in the Morris water maze test, and slightly impaired contextual memory in the contextual and cued fear conditioning tests.

The peculiar TauV337M model, which expresses 4R MAPT with the V337M mutation controlled by the platelet-derived growth factor promoter (also exists with the mouse Thy-1 promoter) on a B6SJL background, 
is characterized by a low-level synthesis of $4 \mathrm{R}$ MAPT, which is only $1 / 10$ of endogenous mouse MAPT production [61]. The observation of neurofibrillary pathology in this model indicates that it may not be the absolute MAPT levels, but the nature of MAPT that instigates tangle pathology. At the age of 12 months, TauV337M mice seem to have defunct olfactory memory, as tested with the social transmission of food preferences task, and deficits in impulse control, as determined with the five-choice serial reaction time task, at 24 months of age and at 12 months of age when the intertrial intervals were increased. Note that in contrast to most other tau-based models, this model does not exhibit motor abnormalities until at least 24 months of age.

To investigate the reversibility of tangle pathology, the rTg4510 model was created [62]. These transgenic mice express MAPT with the P301L mutation under control of the TET-off system, making the transgene inducible. When the mutant MAPT is expressed, these mice show progressive NFT development and cell loss from 1 month of age, including severe hippocampal CA1 neuron death at the age of 5 months. From the age of 2.5 months, this model starts to display impaired spatial reference memory, as examined with the Morris water maze. Interestingly, turning off production of the mutant MAPT after 4 months of age leads to a recovery of cognitive performance, but a worsening of the tangle pathology, indicating that at this age tau pathology becomes independent of transgenic MAPT expression.

When considering the MAPT-based models discussed earlier as models for $A D$, it is important to realize that NFT pathology in AD arises in the absence of mutations in the MAPT gene; indeed, most of the mutations these models are based on are from other tauopathies such as frontotemporal dementia (FTD) [56]. Furthermore, most of the other transgenic models do not take into account endogenous gene expression of the model organism. For instance, all of the abovementioned transgenic mouse models that express a mutant form of MAPT also express mouse MAPT. The htau transgenic mouse model was created keeping the following point in mind; expressing nonmutant human genomic MAPT in a mouse MAPT knockout background (maintained on a Swiss Webster/129/SvJae/C57BL/6 background) [63]. This model presents with AD-like tau pathology, starting with pretangle-like hyperphosphorylated MAPT accumulation after 3 months, spreading at an age of 9 months through hippocampal and neocortical regions. At the age of 12 months, these htau mice start displaying cognitive impairments in the object recognition task and the Morris water maze, paired with disrupted long-term potentiation in the hippocampal CA1 region [64]. 


\subsubsection{4. $\mathrm{PS} 1 \times \mathrm{APP} \times \mathrm{MAPT}$}

One of the most used transgenic models for $A D$ is the triple transgenic mouse model, which combines mutated PS1, APP, and MAPT genes into one model. This 3xTgAD model expresses mutant APPswe and MAPTP301L, under control of the mouse Thy-1 promoter, on a PSEN1M146V knock-in background (PSEN1-KI) [65]. Plaques develop from an age of 6 months in 3xTgAD mice, and tangle pathology arises by the age of 12 months. Although not completely mimicking $A D$, this is one of the best models available - developing progressive synaptic dysfunction, amyloid plaques, and neurofibrillary tangles in a temporal and spatial pattern that is similar to human AD. Around 4 months of age, 3xTgAD mice start to present with impaired spatial memory and long-term retention, as tested with the Morris water maze task, and at 6 months their short- and long-term retention for contextual fear also becomes significantly reduced [66]. Aged 3xTgAD mice show deficits in object discrimination memory in the object discrimination task, together with derailed long-term potentiation and paired-pulse facilitation.

\subsubsection{APOE4}

When looking at the genes used in the triple transgenic AD model, it can be argued that it is primarily a model of $f A D$ and not of the far more common sporadic $A D$ ( $S A D$ ). Models employing the highest genetic risk factor for $S A D$, allele APOE4, have been constructed - expressing human APOE4 under control of the neuron-specific enolase (NSE) promoter in transgenic mice devoid of endogenous mouse APOE [67]. This NSE-APOE4 model exhibits a less severe phenotype than most other transgenic models of $A D$, failing to recapitulate most of the pathological hallmarks associated with the disease. Nevertheless, the NSE-APOE4 model displays impaired excitatory synaptic transmission, a decline in dendritic density and complexity, and cognitive impairments in a water maze task at the age of 6 months.

\subsubsection{Parkinson's disease 3.3.3.1. a-Syn}

a-Synuclein (a-Syn) transgenic mice overexpress human wild-type or mutant a-Syn usually under the regulatory control of the human $P D G F-\beta$ promoter. $\alpha$-Syn is expressed in high levels, resulting in an age-dependent increase of brain inclusions consisting of a-Syn, ubiquitin, and other proteins. Severity of the brain pathology correlates with increasing age. By 6 months of age, these transgenic mice exhibit deficits in cognition shown 
by an increased time to find the platform in the water maze task [68]. Mice overexpressing wild-type a-Syn under regulation of the human PDGF- $\beta$ promoter also display a progressive increase in a-Syn aggregation in multiple brain regions, a loss of dopaminergic terminals in the striatum, and mild changes in motor activity as shown by a decreased latency to fall on a rotarod. Another variation of these mice uses the human Thy1 promoter for overexpressing a-Syn. Cognitive changes (Y-maze, novel object recognition, and operant reversal learning) are also evident in the Thy1a-Syn mice beginning $4-6$ months of age [69].

Nuber et al. in 2008 [70] created a conditional mouse model for the overexpression of WT a-Syn under the CAMK2A promoter, using a tetracycline-regulated TET-off system. These mice displayed a progressive motor decline after 7 months (rotarod) of age, modest impairment in reference memory after 12 months (water maze), and a-Syn accumulation in the substantia nigra, hippocampus, and olfactory bulb.

\subsubsection{DJ1(PARK7)KO}

DJ1KO mice have a deficiency in expressing the Park7 protein, due to a knockout of the respective gene, namely, DJ1. DJ1-/- mice between 13 and 14 months of age show cognitive deficits, as characterized by reduced performance in an object recognition task [71].

\subsubsection{Parkin(PARK2)KO}

This PD mouse model is produced by a knockout in the PARK2 gene, responsible for the expression of a protein called parkin. Parkin-/- mice display increased anxiety, as shown in the open-field and light/dark preference tests, and cognitive impairment exhibited as spatial memory deficits in the Morris water maze [72]. Mice that lack exon 3 in the parkin gene do not demonstrate loss of dopaminergic neurons; nevertheless, they show signs of altered synaptic transmission in the nigrostriatal circuit [73].

\subsubsection{Huntington's disease}

Various transgenic rodent models of Huntington's disease (HD) have been found to exhibit affective and cognitive abnormalities reflecting clinical data in HD patients. For example, R6/1 and R6/2 transgenic lines of HD mice have behavioral deficits that include impaired hippocampal-dependent spatial cognition [74, 75]. However, depression-like behavior also manifests in R6/1 HD mice prior to cognitive and motor symptoms [76]. 


\subsubsection{R6/2}

Of the transgenic chimeric models that express truncated forms of the human mutant $\mathrm{HD}$ allele, the R6/2 line is the most widely used. This line expresses an exon 1 fragment of $h H t t$ with a range of 148-153 repeats, expressed from an unknown location in the mouse genome. R6/2 mice exhibit learning and memory tasks abnormalities as early as 3.5 weeks of age (water Morris maze), which follow them throughout their life span, as evaluated by various cognitive tests (T-maze, two-choice swim tank, and visual discriminate learning) [77-79]. Moreover, they show behavioral deficits by 5 weeks, neuroanatomic abnormalities including progressive reduction in brain and striatal volume, substantially reduced striatal neuron number by 12 weeks, and death by 12-15 weeks [79-81]. As the $\mathrm{R} 6 / 2$ model exhibits severe, early-onset and diffuse pathology, it is potentially a good model of juvenile-onset HD, displaying an aggressive phenotype and provides clear experimental endpoints.

\subsubsection{YAC128}

The YAC128 is a widely-used yeast-artificial-chromosome full-length human mutant HD transgenic model generated and characterized by the Hayden laboratory [82, 83]. Van Raamsdonk et al. in 2005 evaluated YAC128 mice with a variety of more cognitively oriented tests, demonstrating progressive cognitive deficits at 8 weeks (accelerated rotarod) and 32 weeks (water Morris maze, open-field habituation, and T-maze). Unlike the R6/2 mice, where there is probably a diffuse loss of brain volume, some regions of the YAC128 brain, such as the cerebellum and hippocampus, exhibit normal volume [84]. YAC128 mice also exhibit motor abnormalities as early as 3 months with increased open-field activity, followed by rotarod performance abnormalities at 6 months.

\subsubsection{3. tgHD Rats}

This transgenic rat model of $\mathrm{HD}$, with a mutated huntingtin gene containing 51 CAG repeats, expresses adult-onset neurological phenotypes, cognitive impairments, progressive motor dysfunction, and neuronal nuclear inclusions in the brain [85]. The transgenic rat model exhibits a late-onset neurological phenotype, cognitive decline in spatial learning at 10 months (radial arm maze), and significantly impaired object recognition performance at 16 months [86], develops gradually progressive motor abnormalities, and dies between 15 and 24 months. However, according to a recent report by Fielding et al. in 2012 [87], the tgHD rat model does not show consistent, reliable, and progressive 
impairment in a range of cognitive tests. The consistent failure to reveal impairments at any age on a range of tests of cognition and learning suggest that the tgHD rat is not a reliable model of the cognitive and behavioral impairments of human HD.

\subsubsection{Frontotemporal dementia \\ 3.3.5.1. TDP43}

Transgenic murine models used in frontotemporal lobar degeneration (FTLD) research overexpress either wild-type or mutant human TAR DNA-binding protein (TDP) 43 knock-in (KI) via a human promoter. TDP43 is a multifunctional, nuclear protein that binds both DNA and RNA, as well as a member of the heterogeneous nuclear ribonuclear protein (hnRNP) family, and regulates several aspects of RNA processing, including alternative splicing, miRNA production, and mRNA trafficking and stabilization. Missense changes in the glycine-rich domain of TDP43 lead to a shift in its localization from the nucleus to the cytoplasm, resulting in FTLD-TDP pathology [88]. This mouse model exhibits cognitive deficits at the age of 7 or 9 months, depending on the use of a mutated or a WT TDP43KI, respectively, as shown by passive avoidance test and Barns maze. Cognitive impairments for this murine model reach a peak at the age of $11-13$ months [88, 89].

\subsubsection{Down syndrome 3.3.6.1. TgDyrk1A}

Apart from the trisomic mice used in Down syndrome research, transgenic models have also been constructed carrying human genes mapped in the repeated fragment of chromosome 21. One such model, namely, TgDyrk1a, overexpresses $D Y R K 1 A$, a gene encoding a serinethreonine kinase, which is probably involved in neuroblast proliferation [90]. In the Morris water maze, TgDyrk1A mice show significant deficits in spatial learning and cognitive flexibility, due to hippocampal and prefrontal cortical dysfunction, a defect that was related specifically to reference memory. TgDyrk1A mice also exhibit delayed craniocaudal maturation, altered motor skill acquisition, and hyperactivity [91]. 


\subsection{Translation to clinics: limitations and difficulties}

To date, pharmacological, transgenic, and naturally aging rodent models have provided new insights into behavioral function. Although these models have given invaluable information, it is important to remember that they only provide approximations of the molecular and cellular mechanisms and cognitive impairments as seen in humans. In addition, whereas motor phenotypes can be readily assessed in rodent models, it is more challenging to characterize cognitive phenotypes. The frontal cortex of rodents is anatomically different from that of humans [92], and it is therefore difficult to model executive dysfunction, not to mention the existence of significant limitations in modeling complex behaviors in rats and mice, since they already differ in their own cognitive and social functions. Due to these obstacles, face validity gets compromised (Table 1). It has thus been suggested that the research community should take an "agnostic" approach as new models emerge and characterize their behavior as fully as possible. At present, face validity of the behavioral tests used is, in general, the same for pharmacological, aging, and transgenic rodent models (Table 2 and Table 3 ). This results from the fact that, independent of the manipulation used (pharmacological, age, or genetic), the same symptoms are being screened for. In addition, it is important to recognize that not all animal models currently in use or under development will be appropriate for mechanistic research, whereas other certain models exhibit phenotypes that are practical for therapy development. Compared with pharmacological models, transgenic models comprise more construct validity, and hence are more suited for mechanistic and fundamental research. On the other hand, pharmacological models comprise more predictive validity, and therefore contribute to more reliable testing of new (pharmacological) therapies (Table 2 and Table 3). Subjecting rodents to a comprehensive battery of tests provides a better framework not only for understanding the overall behavioral phenotype of the animal but also for more fully recognizing the limitations of the specific model. Nevertheless, it should be emphasized that the goal of animal research is to mimic as much as possible the human disease pathophysiology, and thus improving construct and face validity will provide greater insights into basic genetic and molecular mechanisms involved in expression of the behavior [93]. At the same time, once these mechanisms are better understood, predictive validity will improve and better efficacious therapeutic strategies can be explored and developed for treating cognitive deficits in human patients. 


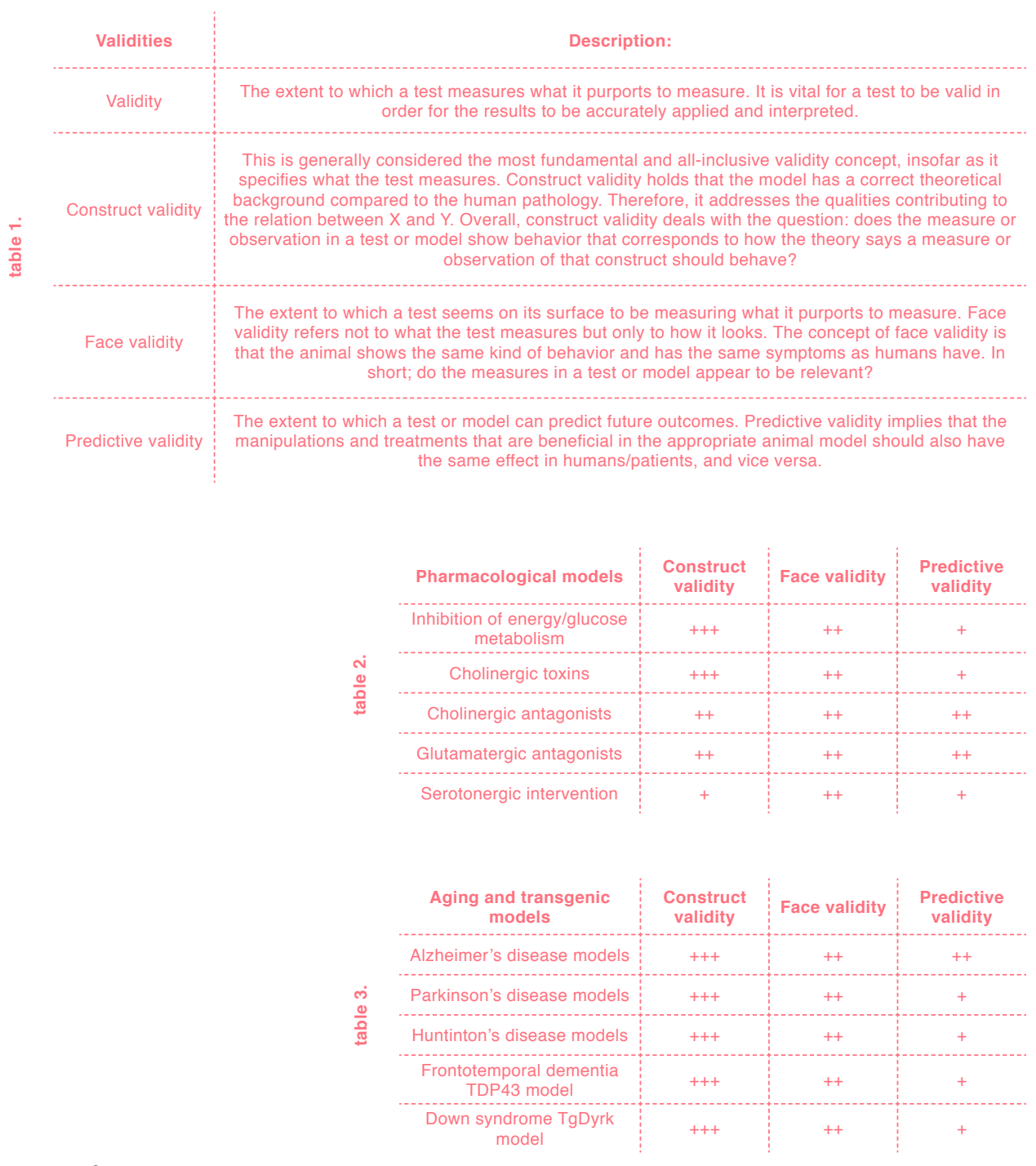

TABLE 1. Descriptions of construct, face, and, predictive validity.

TABLE 2. Validity of pharmacological models.

++++ : meets validity perfectly, +++ :

meets validity good, ++ : meets validity

somewhat, + : meets validity poorly

TABLE 3. Validity of aging and transgenic models in general.

++++ : meets validity perfectly, +++ :

meets validity good, ++ : meets validity

somewhat, +: meets validity poorly 


\section{REFERENCES}

[1]

Faigle R, Song H. Signaling

mechanisms regulating adult neural stem cells and neurogenesis. Biochim Biophys Acta - Gen Subj 2013; 1830: 2435-2448.

[2] Froestl W, Muhs A, Pfeifer A. Cognitive enhancers (nootropics). Part 1: drugs interacting with receptors. J Alzheimers Dis 2012; 32 : $793-887$.

[3] Gold PE. Role of glucose in regulating the brain and cognition. Am J Clin Nutr 1995; 61: 987S-995S. [4] Korol DL, Gold PE. Glucose, memory, and aging. Am J Clin Nutr 1998; 67: 764S-771S.

[5] Cho S, Wood A, Bowlby MR. Brain slices as models for neurodegenerative disease and screening platforms to identify novel therapeutics. Curr Neuropharmacol 2007; 5: $19-33$.

[6] Bederson JB, Pitts LH, Tsuji M, et al. Rat middle cerebral artery occlusion: evaluation of the model and development of a neurologic examination. Stroke; 17: 472-6.

[7] Arias RL, Tasse JR, Bowlby MR. Neuroprotective interaction effects of NMDA and AMPA receptor antagonists in an in vitro model of cerebral ischemia. Brain Res 1999; 816: 299-308.

[8] Danysz W, Parsons CG. The NMDA receptor antagonist memantine as a symptomatological and neuroprotective treatment for Alzheimer's disease: preclinical evidence. Int $J$ Geriatr Psychiatry 2003; 18: S23-S32.

[9] Ockuly JC, Gielissen

JM, Levenick C V., et al. Behavioral, cognitive, and safety profile of 2-deoxy-2-glucose (2DG) in adult rats. Epilepsy Res 2012; 101: 246-252.

[10] Mayer G, Nitsch R, Hoyer
S. Effects of changes in peripheral and cerebral glucose metabolism on locomotor activity, learning and memory in adult male rats. Brain Res 1990; 532: 95-100.

[11] Nitsch R, Mayer G, Hoyer

$\mathrm{S}$. The intracerebroventriculary streptozotocin-treated rat: Impairment of cerebral glucose metabolism resembles the alterations of carbohydrate metabolism of the brain in Alzheimer's disease. J Neural Transm - Park Dis Dement Sect 1989; 1: 109-110. [12] Vavra JJ, Deboer C, Dietz A, et al. Streptozotocin, a new antibacterial antibiotic. Antibiot Annu; 7: 230-5. [13] Prickaerts J, Blokland A, Honig W, et al. Spatial discrimination learning and choline acetyltransferase activity in streptozotocin-treated rats: effects of chronic treatment with acetyl-L-carnitine. Brain Res 1995; 674: $142-6$.

\section{[14] Ding A, Nitsch R, Hoyer}

$\mathrm{S}$. Changes in brain monoaminergic neurotransmitter concentrations in rat after intracerebroventricular injection of streptozotocin. J Cereb Blood Flow Metab 1992; 12: 103-109.

[15] Hellweg R, Nitsch R, Hock C, et al. Nerve growth factor and choline acetyltransferase activity levels in the rat brain following experimental impairment of cerebral glucose and energy metabolism. J Neurosci Res 1992; 31: 479-486.

[16] Blokland A, Jolles J. Spatial learning deficit and reduced hippocampal ChAT activity in rats after an ICV injection of streptozotocin. Pharmacol Biochem Behav 1993; 44: 491-4.

[17] Bartus RT, Dean RL, Beer

$\mathrm{B}$, et al. The cholinergic hypothesis of geriatric memory dysfunction. Science 1982; 217: 408-14.

[18] Blokland A. Acetylcholine: a neurotransmitter for learning and memory? Brain Res Brain Res Rev 1995; 21: $285-300$.

[19] Steckler T, Andrews JS, Marten P, et al. Effects of NBM lesions with two neurotoxins on spatial memory and autoshaping. Pharmacol Biochem Behav 1993; 44: 877-89.

[20] Peternel A, Hughey D, Wenk G, et al. Basal forebrain and memory: Neurotoxic lesions impair serial reversals of a spatial discrimination. Psychobiology; 16: 54-58.

[21] Wiley RG, Oeltmann TN, Lappi DA. Immunolesioning: selective destruction of neurons using immunotoxin to rat NGF receptor. Brain Res 1991; 562: 149-53.

[22] Wenk GL, Stoehr JD, Quintana $\mathrm{G}$, et al. Behavioral, biochemical, histological, and electrophysiological effects of $192 \mathrm{IgG}$-saporin injections into the basal forebrain of rats. $J \mathrm{Neu}$ rosci 1994; 14: 5986-95.

[23] Book AA, Wiley RG, Schweitzer JB. Specificity of 192 IgGsaporin for NGF receptor-positive cholinergic basal forebrain neurons in the rat. Brain Res 1992; 590: 350-5.

[24] Walsh TJ, Herzog CD, Gandhi C, et al. Injection of $\operatorname{IgG}$ 192 -saporin into the medial septum produces cholinergic hypofunction and dose-dependent working memory deficits. Brain Res 1996; 726: 69-79. [25] Lehmann O, Grottick AJ, Cassel J-C, et al. A double dissociation between serial reaction time and radial maze performance in rats subjected to $192 \mathrm{IgG}$-saporin lesions of the nucleus basalis and/or the septal region. Eur J Neurosci 2003; 18: 651-66.

[26] Klinkenberg I, Blokland A. The validity of scopolamine as a pharmacological model for cognitive impairment: A review of animal be- 
havioral studies. Neurosci Biobehav Rev 2010; 34: 1307-1350.

[27] Prickaerts J, van Goethem NP, Chesworth R, et al. EVP-6124, a novel and selective a7 nicotinic acetylcholine receptor partial agonist, improves memory performance by potentiating the acetylcholine response of a7 nicotinic acetylcholine receptors. Neuropharmacology 2012; 62: 1099-1110.

[28] Caulfield MP. Muscarinic

receptors--characterization, coupling and function. Pharmacol Ther 1993; 58 : 319-79.

[29] Klinkenberg I, Blokland

A. A comparison of scopolamine and biperiden as a rodent model for cholinergic cognitive impairment. Psychopharmacology (Berl) 2011; 215: 549-66.

[30] Toyohara J, Hashimoto K. a7 Nicotinic receptor agonists: potential therapeutic drugs for treatment of cognitive impairments in schizophrenia and Alzheimer's disease. Open Med Chem J 2010; 4: 37-56.

[31] Levin ED. Nicotinic systems and cognitive function. Psychopharmacology (Berl) 1992; 108: 417-31. [32] Addy NA, Nakijama A, Levin ED. Nicotinic mechanisms of memory: effects of acute local DHbetaE and MLA infusions in the basolateral amygdala. Brain Res Cogn Brain Res 2003; 16: 51-7.

[33] Hahn B, Shoaib M, Stolerman IP. Selective nicotinic receptor antagonists: effects on attention and nicotine-induced attentional enhancement. Psychopharmacology (Berl) 2011; 217: 75-82.

[34] van der Staay FJ, Rutten K, Erb C, et al. Effects of the cognition impairer MK-801 on learning and memory in mice and rats. Behav Brain

Res 2011; 220: 215-229.

[35] Ellison G. The $N$-meth-

yl-D-aspartate antagonists phencyclidine, ketamine and dizocilpine as both behavioral and anatomical models of the dementias. Brain Res Brain Res Rev 1995; 20: 250-67.

[36] Morris BJ, Cochran SM, Pratt JA. PCP: from pharmacology to modelling schizophrenia. Curr Opin Pharmacol 2005; 5: 101-106.

[37] Steckler T, Sahgal A. The role of serotonergic-cholinergic interactions in the mediation of cognitive behaviour. Behav Brain Res 1995; 67: 165-99.

[38] Booij L, Van der Does AJW, Riedel WJ. Monoamine depletion in psychiatric and healthy populations: review. Mol Psychiatry 2003; 8: 951-973.

[39] van Donkelaar EL, Rutten

K, Blokland A, et al. Phosphodiesterase 2 and 5 inhibition attenuates the object memory deficit induced by acute tryptophan depletion. Eur $J$ Pharmacol 2008; 600: 98-104.

[40] Rutten K, Lieben C, Smits L, et al. The PDE4 inhibitor rolipram reverses object memory impairment induced by acute tryptophan depletion in the rat. Psychopharmacology (Berl) 2007; 192: 275-82.

\section{[41] Chouliaras L, Sierksma}

ASR, Kenis G, et al. Gene-environment interaction research and transgenic mouse models of Alzheimer's disease. Int J Alzheimers Dis; 2010. Epub ahead of print January 2010. DOI: 10.4061/2010/859101.

[42] Gu Y, Huang C-S, Inoue $\mathrm{T}$, et al. Drinking hydrogen water ameliorated cognitive impairment in senescence-accelerated mice. J Clin Biochem Nutr 2010; 46: 269-76.

[43] Gorbunova V, Bozzella MJ,
Seluanov A. Rodents for comparative aging studies: from mice to beavers. Age (Dordr) 2008; 30: 111-9.

[44] Chouliaras L, van den Hove DLA, Kenis G, et al. Caloric restriction attenuates age-related changes of DNA methyltransferase $3 \mathrm{a}$ in mouse hippocampus. Brain Behav Immun 2011; 25 : 616-623.

[45] Chouliaras L, van den Hove DLA, Kenis G, et al. Prevention of age-related changes in hippocampal levels of 5-methylcytidine by caloric restriction. Neurobiol Aging 2012; 33 : $1672-81$.

[46] Rutten BPF, Brasnjevic I, Steinbusch HWM, et al. Caloric restriction and aging but not overexpression of SODl affect hippocampal volumes in mice. Mech Ageing Dev 2010; 131: 574-579.

[47] Takeda T, Hosokawa M,

Higuchi K. Senescence-accelerated mouse (SAM): a novel murine model of accelerated senescence. J Am Geriatr Soc 1991; 39: 911-9.

[48] Takeda T, Hosokawa M,

Takeshita S, et al. A new murine model of accelerated senescence. Mech Ageing Dev 1981; 17: 183-94.

[49] Tomobe K, Nomura Y.

Neurochemistry, neuropathology, and heredity in SAMP8: a mouse model of senescence. Neurochem Res 2009; 34: 660-669.

[50] Miyamoto M, Kiyota $\mathrm{Y}$, Yamazaki N, et al. Age-related changes in learning and memory in the senescence-accelerated mouse (SAM). Physiol Behav 1986; 38: 399-406.

[51] Giuliani F, Vernay A, Leuba G, et al. Decreased behavioral impairments in an Alzheimer mice model by interfering with TNF-alpha metabolism. Brain Res Bull 2009; 80: 302-308. 
GW, Leighty RE, et al. Cognitive impairment in PDAPP mice depends on ApoE and ACT-catalyzed amyloid formation. Neurobiol Aging 2004; 25 : 1153-1167.

[53] Chen G, Chen KS, Kobayashi D, et al. Active $\beta$-amyloid immunization restores spatial learning in PDAPP mice displaying very low levels of $\beta$-amyloid. $J$ Neurosci; http://www.jneurosci.org/ content/27/10/2654 (2007, accessed 6 April 2017).

[54] King DL, Arendash GW. Behavioral characterization of the Tg2576 transgenic model of Alzheimer's disease through 19 months. Physiol Behav 2002; 75: 627-42.

[55] Francis BM, Kim J, Barakat $\mathrm{ME}$, et al. Object recognition memory and BDNF expression are reduced in young TgCRND8 mice. Neurobiol Aging 2012; 33: 555-563.

[56] McGowan E, Eriksen J, Hutton M. A decade of modeling Alzheimer's disease in transgenic mice. Trends Genet 2006; 22: 281-289. [57] Arendash GW, King DL, Gordon MN, et al. Progressive, age-related behavioral impairments in transgenic mice carrying both mutant amyloid precursor protein and presenilin-1 transgenes. Brain Res 2001; 891: 42-53.

[58] Ohno M, Cole SL, Yasvoina M, et al. BACEl gene deletion prevents neuron loss and memory deficits in 5XFAD APP/PS1 transgenic mice. Neurobiol Dis 2007; 26: 134-145. [59] Kimura R, Ohno M. Impairments in remote memory stabilization precede hippocampal synaptic and cognitive failures in 5XFAD Alzheimer mouse model. Neurobiol Dis 2009; 33: 229-35.
[60] Takeuchi H, Iba M, Inoue

$\mathrm{H}$, et al. P301S mutant human tau transgenic mice manifest early symptoms of human tauopathies with dementia and altered sensorimotor gating. PLoS One 2011; 6: e21050.

[61] Lambourne SL, Sellers

LA, Bush TG, et al. Increased tau phosphorylation on mitogen-activated protein kinase consensus sites and cognitive decline in transgenic models for Alzheimer's disease and FTDP-17: evidence for distinct molecular processes underlying tau abnormalities.

Mol Cell Biol 2005; 25: 278-293.

[62] SantaCruz K, Lewis J,

Spires T, et al. Tau suppression in a neurodegenerative mouse model improves memory function. Science 2005; 309: 476-481.

[63] Andorfer C, Kress Y, Espinoza M, et al. Hyperphosphorylation and aggregation of tau in mice expressing normal human tau isoforms. J Neurochem 2003; 86: 582-90.

[64] Polydoro M, Acker CM, Duff K, et al. Age-dependent impairment of cognitive and synaptic function in the htau mouse model of tau pathology. J Neurosci 2009; 29: 10741-10749.

[65] Oddo S, Caccamo A, Kitazawa M, et al. Amyloid deposition precedes tangle formation in a triple transgenic model of Alzheimer's disease. Neurobiol Aging 2003; 24: 1063-70.

\section{[66] Billings LM, Oddo S,}

Green KN, et al. Intraneuronal Abeta causes the onset of early Alzheimer's disease-related cognitive deficits in transgenic mice. Neuron 2005; 45: $675-88$.

[67] Raber J, Wong D, Buttini $\mathrm{M}$, et al. Isoform-specific effects of human apolipoprotein $\mathrm{E}$ on brain function revealed in ApoE knockout mice: increased susceptibility of females. Proc Natl Acad Sci U S A 1998; 95: 10914-9.

[68] Masliah E, Rockenstein E, Mante M, et al. Passive immunization reduces behavioral and neuropathological deficits in an alpha-synuclein transgenic model of Lewy body disease. PLoS One 2011; 6: el9338.

[69] Magen I, Chesselet M-F. Genetic mouse models of Parkinson's disease. In: Progress in brain research, pp. $53-87$.

[70] Nuber S, Petrasch-Parwez

E, Winner B, et al. Neurodegeneration and motor dysfunction in a conditional model of Parkinson's disease.

J Neurosci 2008; 28: 2471-2484.

[71] Pham TT, Giesert F, Röthig A, et al. DJ-1-deficient mice show less $\mathrm{TH}$-positive neurons in the ventral tegmental area and exhibit non-motoric behavioural impairments. Genes, Brain Behav 2010; 9: 305-317.

[72] Zhu X-R, Maskri L, Herold C, et al. Non-motor behavioural impairments in parkin-deficient mice. Eur J Neurosci 2007; 26: 1902-1911.

[73] Goldberg MS, Fleming SM, Palacino JJ, et al. Parkin-deficient mice exhibit nigrostriatal deficits but not loss of dopaminergic neurons.

J Biol Chem 2003; 278: 43628-43635.

[74] Nithianantharajah J,

Barkus C, Murphy M, et al. Gene-environment interactions modulating cognitive function and molecular correlates of synaptic plasticity in Huntington's disease transgenic mice. Neurobiol Dis 2008; 29: 490-504.

[75] Pang TYC, Stam NC, Nithianantharajah J, et al. Differential effects of voluntary physical exercise on behavioral and brain-derived neurotrophic factor expression deficits in 
huntington's disease transgenic mice.

Neuroscience 2006; 141: 569-584.

[76] Pang TYC, Du X, Zajac

MS, et al. Altered serotonin receptor

expression is associated with depres-

sion-related behavior in the R6/1

transgenic mouse model of Hunting-

ton's disease. Hum Mol Genet 2009; 18:

$753-766$.

[77] Carter RJ, Lione LA,

Humby T, et al. Characterization of progressive motor deficits in mice transgenic for the human Huntington's disease mutation. J Neurosci 1999; 19: 3248-57.

[78] Lione LA, Carter RJ,

Hunt MJ, et al. Selective discrimination learning impairments in mice expressing the human Huntington's disease mutation. J Neurosci 1999; 19: 10428-37.

[79] Stack EC, Kubilus JK, Smith K, et al. Chronology of behavioral symptoms and neuropathological sequela in R6/2 Huntington's disease transgenic mice. J Comp Neurol 2005; 490: 354-370.

[80] Hickey M, Gallant K, Gross G, et al. Early behavioral deficits in $\mathrm{R} 6 / 2$ mice suitable for use in preclinical drug testing. Neurobiol Dis 2005; 20: 1-11.

[81] Morton AJ, Hunt MJ,

Hodges AK, et al. A combination drug therapy improves cognition and reverses gene expression changes in a mouse model of Huntington's disease. Eur J Neurosci 2005; 21: 855-870.

[82] Slow EJ, van Raamsdonk J, Rogers D, et al. Selective striatal neuronal loss in a YAC128 mouse model of Huntington disease. Hum Mol Genet 2003; 12: 1555-67.

[83] Van Raamsdonk JM, Pearson J, Bailey CDC, et al. Cystamine treatment is neuroprotective in the

YAC128 mouse model of Huntington disease. J Neurochem 2005; 95 : 210-220.

[84] Van Raamsdonk JM,

Pearson J, Slow EJ, et al. Cognitive dysfunction precedes neuropathology and motor abnormalities in the YAC128 mouse model of Huntington's disease. J Neurosci 2005; 25: 41694180 .

[85] von Hörsten S, Schmitt

I, Nguyen HP, et al. Transgenic rat model of Huntington's disease. Hum

Mol Genet 2003; 12: 617-24.

[86] Zeef DH, van Goethem NP,

Vlamings R, et al. Memory deficits in the transgenic rat model of Huntington's disease. Behav Brain Res 2012; 227: 194-198.

[87] Fielding SA, Brooks SP, Klein A, et al. Profiles of motor and cognitive impairment in the transgenic rat model of Huntington's disease. Brain Res Bull 2012; 88: 223-236.

[88] Swarup V, Phaneuf D,

Bareil C, et al. Pathological hallmarks of amyotrophic lateral sclerosis/ frontotemporal lobar degeneration in transgenic mice produced with TDP43 genomic fragments. Brain 2011; 134: 2610-2626.

[89] Tsai K-J, Yang C-H, Fang

Y-H, et al. Elevated expression of TDP-43 in the forebrain of mice is sufficient to cause neurological and pathological phenotypes mimicking FTLD-U. J Exp Med 2010; 207: 1661-1673.

[90] Altafaj X, Dierssen M, Baamonde C, et al. Neurodevelopmental delay, motor abnormalities and cognitive deficits in transgenic mice overexpressing DyrklA (minibrain), a murine model of Down's syndrome. Hum Mol Genet 2001; 10: 1915-23.

[91] Ahn K-J, Jeong HK, Choi
H-S, et al. DYRK1A BAC transgenic mice show altered synaptic plasticity with learning and memory defects. Neurobiol Dis 2006; 22: 463-472.

[92] Uylings HBM, Groenewegen HJ, Kolb B. Do rats have a prefrontal cortex? Behav Brain Res 2003; 146: $3-17$.

[93] D'Mello GD, Steckler T. Animal models in cognitive behavioural pharmacology: an overview. Brain Res Cogn Brain Res 1996; 3: 345-52. 



\section{EPIGENETIC}

MODIFICATIONS IN

MOUSE CEREBELLAR

PURKINJE CELLS:

EFFECTS OF AGING,

CALORIC RESTRICTION,

AND OVEREXPRESSION

OF SUPEROXIDE

DISMUTASE 1 ON

5-METHYLCYTOSINE

\section{AND 5-HYDROXYMETH- YLCYTOSINE}

ROY LARDENOIJE ${ }^{A}$, DANIËL L.A. VAN DEN HOVE ${ }^{A, B}$, THOMAS S.J. VAESSEN $^{A}$, ARTEMIS IATROU ${ }^{A}$, KOEN P.V. MEUWISSEN ${ }^{A}$, BRITT T.J. VAN HAGEN ${ }^{A}$, GUNTER KENIS ${ }^{A}$, HARRY W.M. STEINBUSCH ${ }^{A}$, CHRISTOPH SCHMITZ ${ }^{C}$, BART P.F. RUTTEN ${ }^{A}$

ASCHOOL FOR MENTAL HEALTH AND NEUROSCIENCE (MHENS), DEPARTMENT OF PSYCHIATRY AND NEUROPSYCHOLOGY, MAASTRICHT UNIVERSITY, UNIVERSITEITSSINGEL 50, 6200 MD MAASTRICHT, THE NETHERLANDS BLABORATORY OF TRANSLATIONAL NEUROSCIENCE, DEPARTMENT OF PSYCHIATRY, PSYCHOSOMATICS AND PSYCHOTHERAPY, UNIVERSITY OF WUERZBURG, FUECHSLEINSTRASSE 15, 97080 WUERZBURG, GERMANY CDEPARTMENT OF NEUROANATOMY, LUDWIG-MAXIMILIANSUNIVERSITY OF MUNICH, MUNICH, GERMANY 


\section{Abstract}

The aim of the present study was to assess alterations in DNA methylation and hydroxymethylation during aging in cerebellar Purkinje cells and to determine the effects of putatively preventative measures to such age-related changes. Employing immunohistochemical techniques, 5-methylcytosine (5-mC) and 5-hydroxymethylcytosine (5-hmC) immunoreactivity (IR) in cerebellar Purkinje cells of 12-month-, and 24-month-old mice was interrogated. Additionally, the modulatory effects of caloric restriction (CR) and normal human Cu/Zn super oxide dismutase (SOD) 1 overexpression on these changes were assessed. We show that aging is associated with an increase of $5-\mathrm{mC}$ and $5-\mathrm{hmC}$ IR in mouse cerebellar Purkinje cells. These age-related increases were mitigated by $\mathrm{CR}$, but not SOD1 overexpression. Additionally, the ratio between $5-\mathrm{mC}$ and $5-\mathrm{hmC}$ decreased with age and $\mathrm{CR}$ treatment, suggesting that CR has a stronger effect on DNA methylation than DNA hydroxymethylation. These findings enforce the notion that aging is closely connected to marked epigenetic changes, affecting multiple brain regions, and that $\mathrm{CR}$ is an effective means to prevent or counteract deleterious age-related epigenetic alterations.

KEYWORDS: DNA methylation; DNA hydroxymethylation; Purkinje cells; aging; caloric restriction; antioxidant overexpression 


\subsection{Introduction}

Preventing the global deterioration of the aging human body to extend the life span has been heavily researched and remains a lively field. The aging brain has been shown to undergo region-specific gene expression, morphological, and functional changes [1-8]. A downregulation of genes involved in synaptic plasticity, neurotropic support and an upregulation of immune-related genes, concomitant with progressive synaptic dysfunction, eventually leads to cognitive decline. Indeed, areas important for proper cognitive functioning, such as the neocortical and hippocampal networks, as well as the more recently identified cerebello-hippocampal network, seem to be particularly prone to deteriorate with age [9-13].

Dietary restriction of caloric intake and enhanced levels of endogenous and exogenous antioxidants are approaches that are potentially able to mitigate age-related deterioration of the brain [14-19]. While increasing exogenous antioxidants can simply be achieved through dietary supplementation, endogenous antioxidants can be experimentally upregulated in transgenic animal models [20]. This latter approach, applied to boost normal human Cu/Zn super oxide dismutase (SOD) 1 in mice, has been shown to attenuate age-related accumulation of oxidative damage to DNA and proteins in the brainstem [21] and neurodegeneration after various forms of trauma in the mammalian brain [20-26]. In addition, caloric restriction (CR), without undernutrition, appears to be a promising strategy to extend the lifespan and counteract detrimental age-related alterations in various species [14, 15, 27-34], although studies in humans are very limited and with mixed results [35].

Over the past few years, evidence has accumulated that epigenetic mechanisms may be critically involved in mediating age-related changes of the brain [36-40]. The epigenetic machinery comprises multiple pathways involved in the regulation of gene expression, including histone modifications, DNA methylation and DNA hydroxymethylation $[41,42]$. These various epigenetic mechanisms work in concert to fine-tune gene expression at multiple levels, for instance to ensure that the correct genes are activated or deactivated during cell division and differentiation. Furthermore, these mechanisms are also thought to be critically involved in regulating synaptic plasticity and related memory functions [38, 43-45]. DNA methylation has been investigated intensively in relation to aging and age-related neurodegeneration [36, 37, 46-53], whereas DNA hydroxymethylation (i.e. the closely related, but functionally distinct DNA modification) has only recently received attention in this respect [42, 54-57]. DNA methylation is performed by DNA methyltransferases (DNMTs), which are able to covalently bind a methyl group to cytosine bases of the DNA, creating 5-methylcytosine 
(5-mC) [58-61]. The ten-eleven translocation (TET) enzyme family is responsible for DNA hydroxymethylation [62]. TET enzymes convert 5-mC into 5-hydroxymethylcytosine $(5-\mathrm{hmC})$ through the hydroxymethylation of methylated cytosines [63]. TET can further oxidize 5-hmC to 5-formylcytosine (5-fC) and 5-carboxylcytosine (5-caC), the exact functional roles of which, apart from being demethylation intermediates, remain to be determined [64-66]. As 5-hmC is created from pre-existing 5-mC, DNA methylation and DNA hydroxymethylation are closely related processes [67]. Nevertheless, as DNA methylation is generally associated with repression of gene expression through attraction of methyl-binding proteins (MBPs), thereby decreasing the accessibility of the DNA for the transcription apparatus, DNA hydroxymethylation has been reported to have a lower affinity for MBPs [68], and generally leads to an opening of the chromatin and promotion of transcription [69, 70]. Note, however, that recent findings suggest that the precise roles of $5-\mathrm{mC}$ and $5-\mathrm{hmC}$ in the regulation of gene transcription are likely much more complex than initially assumed [71-74].

DNA methylation and especially hydroxymethylation have been shown to be dynamic and are known to undergo widespread changes during development $[71,72,75,76]$. The behavior of these epigenetic modifications in the aging brain, especially outside the hippocampus and frontal cortex, is largely unknown. Recently, our group has investigated how CR and SOD1 overexpression might affect age-related epigenetic changes in the hippocampus and reported striking age-related increases in hippocampal DNMT3A, 5-mC, 5-hmC and histone deacetylase (HDAC) 2 immunoreactivity (IR), and that these changes were attenuated by $C R$, but not by overexpression of SOD1 [47, 48, 54, 77]. Although the previous studies provided new insights into age-related epigenetic changes, they were limited to the hippocampus. The present study expands upon this previous work by focusing on cerebellar Purkinje cells. Purkinje cells, the output cells of the cerebellar cortex, represent an interesting target, as $5-\mathrm{hmC}$ was discovered in these cells and they appear to exhibit some of the highest levels of 5-hmC [78]. Note that the first hint at the existence of 5-hmC was published much earlier by Penn et al. [79]. Purkinje cells are among the largest cells in the brain, whereas the other prominent class of neurons present in the cerebellum, the granule cells, which are several hundred times more abundant, are the smallest [80, 81]. Moreover, Purkinje cells have an unusually large nucleus, with a centrally located nucleolus, and are known to be highly heterogeneous in their production of neurotransmitters and other cell markers [81, 82]. Purkinje cells receive excitatory input from thousands of connections, resulting in huge surges of calcium, which results in a high metabolic rate to keep this in check and is thought to contribute to a selective vulnerability to various factors, including oxidative stress, hypoxia, metabolic insufficiencies and 
many environmental toxins [83]. Additionally, their numbers are known to decrease with age [12]. Given previous observations of (i) age-related changes in 5-mC and 5-hmC in the mouse hippocampus [48,54], (ii) the presence of high levels of 5-hmC in Purkinje cells [78], and (iii) major changes during development and aging in Purkinje cells [84-86], it is postulated that Purkinje cell 5-mC and 5-hmC levels increase with age and that this increase is attenuated by $\mathrm{CR}$ and SOD1 overexpression. To assess this hypothesis, $5-\mathrm{mC}$ and 5 -hmC IR was measured by employing design-based quantitative immunohistochemical techniques in 12- and 24-month-old wild-type (WT) and SOD1 transgenic mice kept on either CR or a control diet (CD).

\subsection{Materials and methods 4.2.1. Animals}

This study included brain sections of 12 12-month-old WT C57B16J mice, 12 24-month-old WT C57B16J mice, 12 12-month-old transgenic mice overexpressing SOD1 (SOD1 mice) and 12 24-month-old SOD1 mice. These mice stem from a large aging cohort of 240 male mice and are the same as those previously used to investigate epigenetic alterations in the hippocampus [47, 48, 54, 77]. The generation, diet, weight and survival curves of this cohort have been described previously [32, 34, 47]. In short, the transgenic mice were created by introducing 7 copies of the entire human SOD1 sequence into chromosome 3 of C57B16 mice, leading to enhanced SOD1 activity in the brain and other tissues [87]. Confirmation of human SOD1 expression specifically for the transgenic mice in the used cohort has been reported previously [32]. The cohort was initiated at the Central Animal Facilities (Maastricht University, Maastricht, the Netherlands), with 4 breeder pairs of female WT C57BI6J mice and male transgenic mice hemizygous for the SOD1 transgene. After weaning at 21 days after birth, the mice were immediately assigned to different diet groups, which were maintained throughout the entire lifespan of the animals. Half of the mice of each genotype were provided with a CR diet of approximately $50 \%$ less calories than ad libitum consumption, while the other half received a CD of approximately $15 \%$ less calories [34]. This 15\% reduction in calorie intake for the control group was chosen to have full control over calorie consumption, as ad libitum food consumption might introduce unwanted variation in the control diet groups. As reported previously, this CR paradigm resulted in a $46 \%$ reduction in body weights, as compared with the CD, and increased mean and maximum life span [32]. 6 mice per group were sacrificed at either 12, or 24-months of age for the current analyses. All animals were housed individually on a 12/12 hours light/dark cycle, under standard 
temperature, humidity and specified pathogen free (SPF) conditions, and with ad libitum access to water. All the experimental procedures were approved by the Animal Ethics Board of Maastricht University.

This study had a $2 \times 2 \times 2$ experimental design, based on genotype, age and diet. The 8 groups in total are thus (i) 12-months-old WT mice on the CD (12WT-CD), (ii) 24-months-old WT mice on the CD (24WT-CD), (iii) 12-months-old SOD1 mice on the CD (12SOD-CD), (iv) 24-months-old SOD1 mice on the CD (24SOD-CD), (v) 12-months-old WT mice on the CR diet (12WT-CR), (vi) 24-months-old WT mice on the CR diet (24WTCR), (vii) 12-months-old SOD1 mice on the CR diet (12SOD-CR) and (viii) 24-months-old SOD1 mice on the CR diet (24SOD-CR).

\subsubsection{Tissue processing}

The brains were processed as described previously [32, 47]. Briefly, after deep anesthesia the animals underwent transcardial perfusion with $20 \mathrm{ml}$ tyrode solution and 2 fixative solutions $(20 \mathrm{ml}$ of $4 \%$ paraformaldehyde, $0.9 \% \mathrm{NaCl}, 1 \%$ acetic acid and $30 \mathrm{ml}$ of $8 \%$ paraformaldehyde, $0.9 \%$ $\mathrm{NaCl}, 1 \%$ acetic acid). The heads were then removed and the opened skulls including the brain were postfixed for 24 hours in the $8 \%$ paraformaldehyde solution, but without acetic acid, at $4^{\circ} \mathrm{C}$. Following fixation, the brains were removed and hemisected along the midsaggital line, cryoprotected in sucrose solutions (consecutively, 10\%, 20\% and $30 \%$ sucrose, in Tris- $\mathrm{HCl}$ buffer, twice for 12 hours per solution, at $4^{\circ} \mathrm{C}$ ) and embedded in Tissue Tek® (Sakura Finetec Europe, Zoeterwoude, The Netherlands). For this study, only the left brain halves were used. They were frozen quickly and kept at $-80^{\circ} \mathrm{C}$ until being cut serially with a cryostat (type HM 500 OMV, Microm, Walldorf, Germany), in $30 \mu \mathrm{m}$-thick free-floating coronal sections. The brains were split into anterior $(A)$, medial $(B)$ and posterior $(C)$ parts, each of which was again divided in 10 subseries of every 10th section. After cutting, the sections were stored at $-80^{\circ} \mathrm{C}$ until further use. The current study only used the $\mathrm{C}$ series, which included the cerebellum.

\subsubsection{Immunohistochem- ical detection of $5-\mathrm{mC}$ and 5 -hmG}

Two full series of free-floating sections containing the cerebellum were immunohistochemically stained, one for $5-\mathrm{mC}$ and one for $5-\mathrm{hmC}$. 
Sections first underwent antigen retrieval with $14 \mathrm{mM}$ citric acid buffer $\mathrm{pH}$ 6.0) in a water bath at $95^{\circ} \mathrm{C}$ for 20 minutes, quenching of endogenous peroxidase activity with $0.1 \% \mathrm{H}_{2} \mathrm{O}_{2}$ for 1 hour at room temperature (RT), and blocking with $3 \%$ normal donkey serum for 30 minutes at RT. Sections were then incubated overnight at $4{ }^{\circ} \mathrm{C}$ with either mouse monoclonal anti-5-mC antibody (dilution 1:500; GenWay Biotech Inc., San Diego, CA, USA), or rabbit polyclonal anti-5-hmC antiserum (dilution 1:25,000; Active Motif, Rixensart, Belgium) as a primary antibody. Subsequently, the sections were incubated with biotinylated donkey anti-mouse secondary antibody (dilution 1:200; Jackson, Westgrove, $\mathrm{PA}$, USA) in case of 5-mC, or biotinylated donkey anti-rabbit (dilution 1:1,000; Jackson Westgrove, PA, USA) secondary antibody in case of 5-hmC, for 2 hours at RT, followed by an incubation with avidin-biotinperoxidase complex (diluted 1:500; Vector laboratories, Burlingame, CA, USA) for 2 hours at RT. In order to visualize the horseradish peroxide reaction product, the sections were reacted with a 3,3'-diaminobenzidine tetrahydrochloride (DAB) solution (1:1 DAB:Tris- $\mathrm{HCl}, 0.01 \% \mathrm{H}_{2} \mathrm{O}_{2}$; Sigma, Uithoorn, The Netherlands) at RT for 6 minutes. The sections were then mounted on gelatin-coated microscope slides, dehydrated, and coverslipped using Pertex (Histolab Products ab, Göteborg, Sweden). Sections from all 48 animals were stained at the same time, and handled in a randomized order.

For qualitative purposes, a 5-mC, 5-hmC and 4',6-diamidino-2phenylindole dihydrochloride (DAPI; a fluorescent dye binding AT-rich regions of the DNA) triple immunofluorescent labeling was performed in a similar fashion. After antigen retrieval and blocking, the sections were first incubated overnight at $4^{\circ} \mathrm{C}$ with rabbit polyclonal anti-5-hmC antiserum (dilution 1:10,000), and then with donkey anti-rabbit Alexa 594 conjugated secondary antibody (dilution 1:1,000; Invitrogen, Eugene, OR, USA) for 2 hours at RT. After an additional blocking step, the sections were stained for $5-\mathrm{mC}$ by first incubating them overnight at $4^{\circ} \mathrm{C}$ with mouse monoclonal anti-5-mC antibody (dilution 1:500), then with biotinylated donkey antimouse secondary antibody (dilution 1:1,000) for 2 hours at RT, followed by a 2 hour incubation at RT with streptavidin Alexa 488 conjugate (dilution 1:400; Invitrogen, Eugene, OR, USA). Finally, the sections were counterstained with DAPI (dilution 1:100,000; Sigma Aldrich, Zwijndrecht, The Netherlands). The sections were mounted on gelatin-coated microscope slides, shortly air-dried and coverslipped with $80 \%$ glycerol.

Of note, reversing the order of $5-\mathrm{mC}$ and $5-\mathrm{hmC}$ antibody incubation did not influence the fluorescent staining pattern (data not shown). For all staining procedures, negative controls, without primary antibody, were included and evaluated (data not shown). The specificity of the anti-5-mC and anti5-hmC primary antibodies has been shown previously [48, 54, 88]. 


\subsubsection{Analysis of 5-mC and 5-hmC immunoreactivity}

To quantify the level of 5-mC and $5-\mathrm{hmC}$ immunoreactivity in cerebellar Purkinje cells, all sections of the cerebellum were selected (between Bregma levels $-4.83 \mathrm{~mm}$ and $-7.55 \mathrm{~mm}$ [89]) and, per animal, approximately 100 images of individual Purkinje cells within the Purkinje cell layer, spread across the different lobules of the cerebellum, were taken. Brightfield images were taken with a digital camera (F-view; Olympus, Tokyo, Japan) connected to an Olympus AX70 brightfield microscope (analysis; Imaging System, Münster, Germany), using the $100 \times$ objective and an IF550 filter. All slides were photographed in a randomized order.

Of the fluorescent 5-mC, 5-hmC and DAPI triple staining, 2-5 $\mu \mathrm{m}$ thick image stacks were taken, with an image spacing of $0.1 \mu \mathrm{m}$, using a Stereo Investigator Confocal Spinning Disk (SI-SD) system (MBF Bioscience, Williston, VT, USA), using the $100 \times$ objective. The SI-SD system consisted of a modified Olympus BX51 fluorescence microscope with a customized spinning disk unit (Olympus), computer-controlled excitation and emission filter wheels (Olympus), three axis high-accuracy computer controlled stepping motor $4 \times 4$ Grid Encoded Stage (Ludl Electronic Products, Hawthorne, NY, USA), linear z-axis position encoder (Ludl Electronic Products), ultra-high sensitivity electron multiplier charge-coupled device camera (1000 × 1000 pixels, C9100-02, Hamamatsu Photonics, Hamamatsu City, Japan) and Stereo Investigator controlling software (MBF Bioscience). Using the image stacks, three-dimensional section reconstructions were generated using the Imaris software (Bitplane AG, Zurich, Switzerland). The image stacks were also used for colocalization analyses of 5-mC, 5-hmC and DAPI in Purkinje cell nuclei, using the Coloc 2 plugin that comes with the Fiji software package [90].

A total of 4 mice were excluded from the 5-mC (1 of the 12WT-CD group, 1 of the 24SOD-CD group, and 2 of the 12SOD-CD group) image analysis, and 2 mice for the $5-\mathrm{hmC}$ ( 1 of the 24SOD-CD group and 1 of the 12SODCD group) image analysis, because of insufficient numbers of sections per animal (due to histological processing). The mean IR of the photographed Purkinje cells was determined with the ImageJ software program (version $1.48 \mathrm{v}$, Wayne Rasband, National Institutes of Health, Bethesda, Maryland, USA), by measuring the area of immunoreactivity in the nucleus and the mean grey value of this area. The grey value measurements were noisecorrected by subtracting the signal that was measured when the camera shutter was closed, and corrected for background variation by dividing the mean grey value of the Purkinje cell nucleus of each individual image 
by the mean background grey value of areas of tissue without cells (in the molecular layer), of that same image. After analyzing all individual Purkinje cell images of a staining, the measured values were averaged for each animal. These animal means were used for further analyses. To get a complete picture of $5-\mathrm{mC}$ and $5-\mathrm{hmC} \mathrm{IR}$, the integrated density was calculated by multiplying the area and grey value measurements, and these values were then normalized to the data of the 12WT-CD group. Additionally, in order to compare the relative degree of DNA methylation and hydroxymethylation between groups, the ratio between the $5-\mathrm{mC}$ and 5-hmC integrated densities was determined.

\subsubsection{Statistical analysis}

All data displayed below is presented as the mean and standard error of means. Comparisons between groups were made with the general linear model univariate analysis of variance (ANOVA), taking into account main and interactive effects of genotype, diet and age. In addition, pair-wise comparisons with a Bonferroni post-hoc test were done. Normality was inspected via a quantile-quantile plot of the ANOVA residuals and the Lilliefors adaptation of the Kolmogorov-Smirnov test. Heteroscedasticity was checked by plotting the ANOVA residuals versus the predicted values in addition to a Breusch-Pagan test. The correlation between 5-mC and 5-hmC IR was determined by calculating the Pearson's correlation coefficient $(r)$. Statistical significance for all tests was set at $a=0.05$. All statistical calculations were performed using $R$ (www.r-project.org) and RStudio (www.rstudio.com). Graphs were constructed in GraphPad Prism (Version 4, GraphPad Software, San Diego, USA).

\subsection{Results}

\subsubsection{Qualitative analysis of 5-mC and 5-hmC immuno- reactivity}

As can be seen in Figure 1 and Figure 2, most cells of the mouse cerebellum exhibited nuclear 5-mC and 5-hmC IR. In Purkinje cells, the expression patterns of $5-\mathrm{mC}$ and $5-\mathrm{hmC}$ were very different from the other cerebellar cell types. While 5-hmC appeared more diffusely throughout the nucleus, with darker spots, 5-mC was much more localized, mostly limited to one large site, with sporadically additional smaller sites of high IR. The nucleolus itself generally showed little to no $5-\mathrm{mC}$ or 5-hmC IR. 

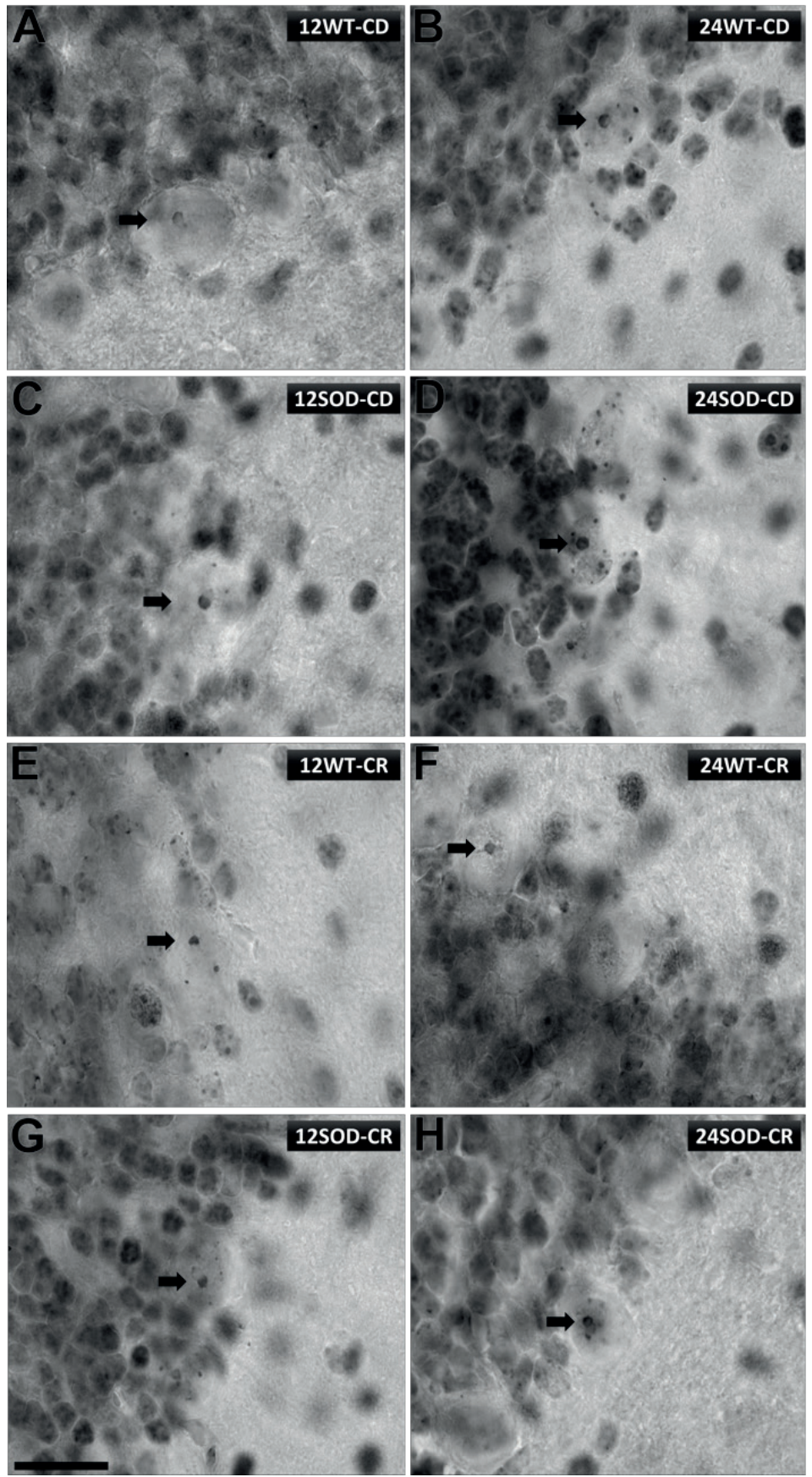

Figure 3 and the colocalization analyses showed that there is only limited overlap in Purkinje cell nuclear $5-\mathrm{mC}$ and $5-\mathrm{hmC} \mathrm{IR}(r=0.19)$, as well as
FIGURE 1. High magnification images of cerebellar 5-methylcytosine (5-mC) immunoreactivity (IR). Representative images of a wild-type (WT) mouse on control diet (CD) of 12 months (12WT-CD; A) and of 24 months (24WT-CD; B), of a transgenic $\mathrm{Cu} / \mathrm{Zn}$ super oxide dismutase (SOD) 1 overexpressing mouse on CD of 12 months (12SOD-CD; C) and of 24 months (24SOD-CD; D), of a WT mouse on caloric restriction (CR) of 12 months (12WT-CR; E) and of 24 months (24WT-CR; F), and of a SOD1 mouse on CR of 12 months (12SODCR; G) and of 24 months (24SOD-CR; $\mathrm{H})$. Arrows indicate Purkinje cell nuclei. Note the higher 5-mC IR, especially the increased amount of reactive boutons, in the $24 \mathrm{WT}-\mathrm{CD}$ (B) and 24SOD-CD (D) groups, as compared to the other groups, indicating an age-related increase in 5-mC IR, which is not visible in the CR groups, as 24WT-CR (F) and 24.SOD-CR (H) do not display a higher 5-mC IR as compared to their 12 months old counterparts ( $\mathrm{E}$ and $\mathrm{G}$, respectively). Scale bar represents $20 \mu \mathrm{m}$. 
FIGURE 2. High magnification images of cerebellar 5-hydroxymethylcytosine $(5-\mathrm{hmC})$ immunoreactivity (IR). Representative images of a wildtype (WT) mouse on control diet (CD) of 12 months (12WT-CD; A) and of 24 months (24WT-CD; B), of a transgenic $\mathrm{Cu} / \mathrm{Zn}$ super oxide dismutase (SOD) 1 overexpressing mouse on $\mathrm{CD}$ of 12 months (12SOD-CD; C) and of 24 months (24SOD-CD; D), of a WT mouse on caloric restriction (CR) of 12 months (12WT-CR; E) and of 24 months (24WT-CR; F), and of a SODl mouse on CR of 12 months (12SODCR; G) and of 24 months (24SOD-GR; H). Arrows indicate Purkinje cell nuclei. Note the higher 5 -hmC IR in the $24 \mathrm{WT}-\mathrm{CD}$ (B) and $24 \mathrm{SOD}-\mathrm{CD}$ (D) groups, as compared to the other groups, indicating an age-related increase in 5-hmC IR, which is not visible in the GR groups, as 24WT-CR (F) and 24SOD-CR $(\mathrm{H})$ do not display a higher 5 -mC IR as compared to their 12 months old counterparts (E and G, respectively). Scale bar represents $20 \mu \mathrm{m}$.
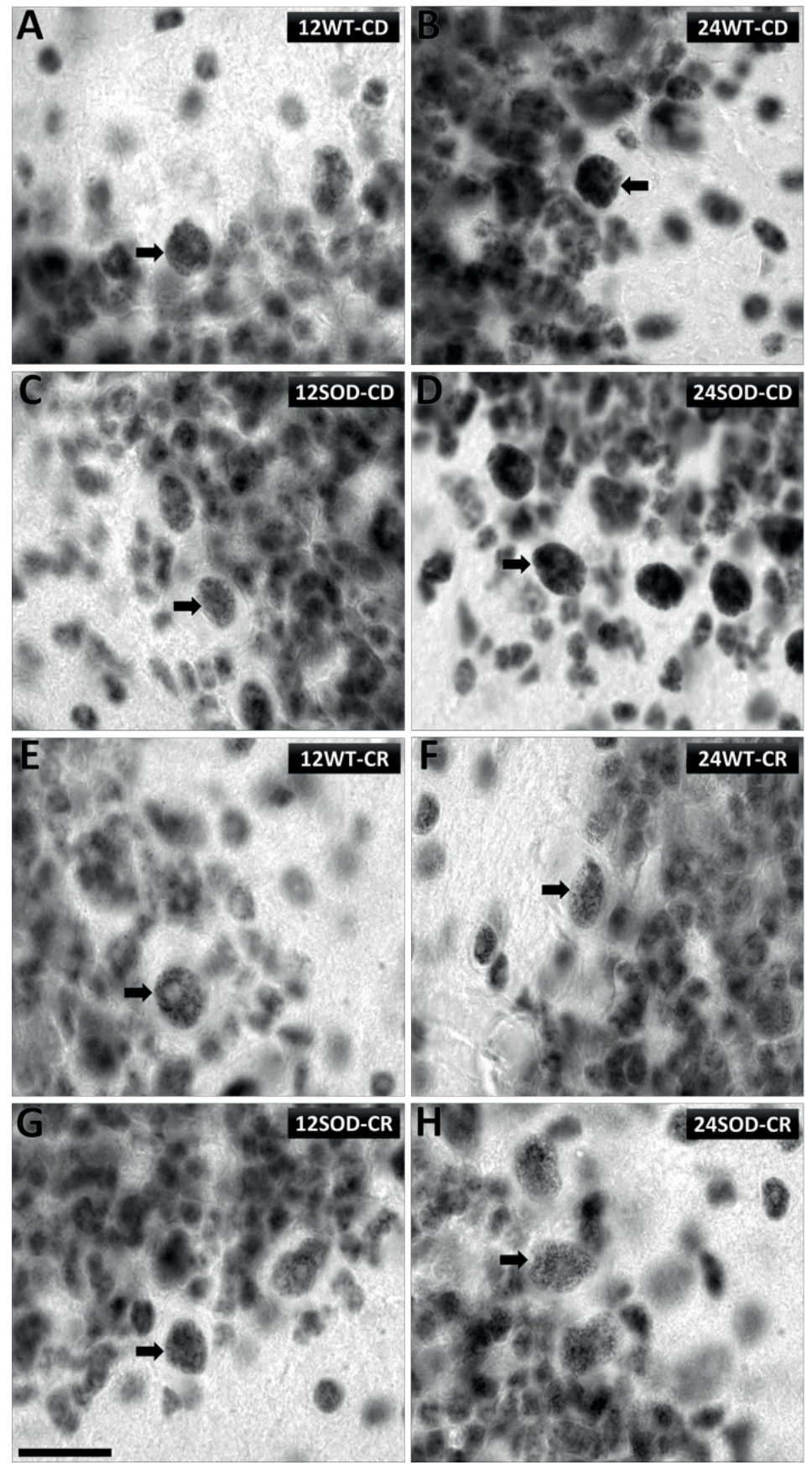

5-hmC and DAPI IR $(r=0.15)$, whereas 5-mC and DAPI IR had a high colocalization $(r=0.71)$. 

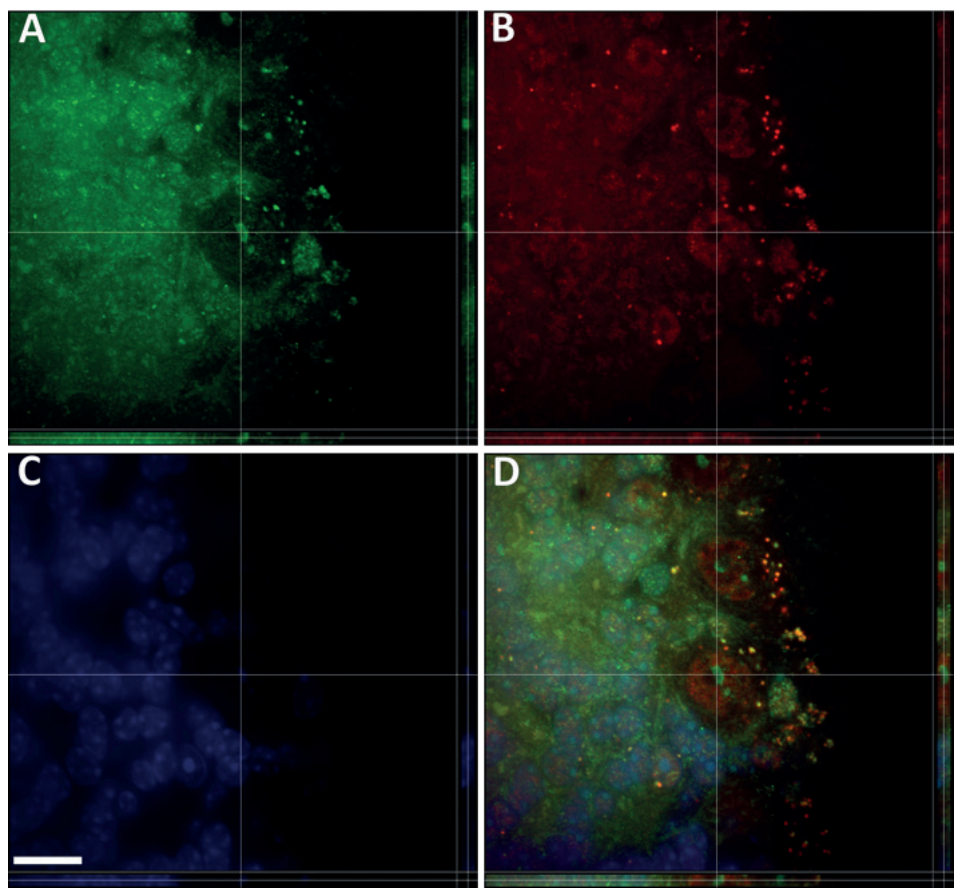

Further visual inspection of 5-mC and 5-hmC IR showed an age-related increase of 5-hmC IR intensity in the CD groups, as the Purkinje cells of the 24WT-CD and 24SOD-CD groups appeared to be strikingly darker than those of the other groups (Figure 1). While this increase in $\mathrm{IR}$ intensity was not as obvious in case of 5-mC, the number of 5-mC immunoreactive sites appeared to increase with age in the CD groups and not the CR groups (Figure 2). The intensity of the 5-mC and 5-hmC IR of the Purkinje cells was further quantitatively analyzed to corroborate these observations.

\subsubsection{Semi-quantita- tive analysis of 5-mC and 5-hmC immunoreactivity}

Results are summarized in Table 1 and Figure 4. Inspection of the quantile-quantile plots of the ANOVA residuals and the Lilliefors adaptation of the Kolmogorov-Smirnov tests $(p>0.05)$ indicated that the assumption of normality was met. No significant heteroscedasticity was detected in the residuals versus predicted values plots and the Breusch-Pagan tests $(p>0.05)$. The ANOVA showed statistically significant main effects of diet

FIGURE 3. Triple fluorescent staining of 5-methylcytosine (5-mC), 5 -hydroxymethylcytosine $(5-\mathrm{hmC})$ and 4',6-diamidino-2-phenylindole dihydrochloride (DAPI). Representative high magnification $3 \mathrm{D}$ reconstructions of image stacks of a 12 month wildtype mouse receiving the control diet. 5-mC visualization with Alexa 488 (A; green), 5 -mC visualization with Alexa 594 (B; red), and DAPI counterstain (C; blue). The images are merged to show overlap between the 5 -mC, 5 -mC, and DAPI signals (D). See text for the results of the colocalization analysis. The most right and bottom parts of the images show a front and side view of the $3 \mathrm{D}$ reconstructions. Scale bar represents $20 \mu \mathrm{m}$. 
on both 5-mC and 5-hmC IR (5-mC: $p<0.001 ; 5-\mathrm{hmC}: p<0.01)$, and of age on 5-hmC IR $(p<0.001)$. Significant interactions between diet and age were found for both $5-\mathrm{mC}$ and $5-\mathrm{hmC}$ IR $(5-\mathrm{mC}: p<0.001 ; 5-\mathrm{hmC}$ : $p<0.01)$. Stratified analyses showed statistically significant increases in $5-\mathrm{mC}$ IR with age in the CD groups $(p<0.05)$ and a statistical significant decrease in the $\mathrm{CR}$ groups $(p<0.05)$ (Figure $4 \mathrm{~A})$. Pairwise comparisons furthermore indicated that 5 -hmC IR increased statistically significantly with age in the CD groups $(p<0.001)$, but not in the CR groups $(p>0.05)$, and that these effects were independent of genotype (Figure 4B).

When looking at the ratio between $5-\mathrm{mC}$ and $5-\mathrm{hmC}$, main effects of diet $(p<0.01)$ and age $(p<0.001)$ were observed, as well as interaction effects between genotype and diet $(p<0.05)$, and diet and age $(p<0.001)$. The Bonferroni corrected pairwise comparisons showed a statistically significant age-related decrease in $5-\mathrm{mC}$ and $5-\mathrm{hmC}$ ratio in the CR groups only ( $p<0.001$ ) (Figure $4 \mathrm{C})$. At the same time, correlation analysis between $5-\mathrm{mC}$ and $5-\mathrm{hmC}$ IR indicates there is a positive correlation between 5-mC and 5-hmC IR $(r=0.484, p<0.001)$.

\begin{tabular}{|c|c|c|c|}
\hline$P$-values & $5-\mathrm{mC}$ & 5-hmC & Ratio \\
\hline Age & 0.806 & $<0.001^{* \star *}$ & $<0.001^{* * *}$ \\
\hline Genotype & 0.791 & 0.572 & 0.643 \\
\hline Diet & $<0.001^{\star \star \star}$ & $0.001^{\star \star}$ & $0.002^{\star *}$ \\
\hline Age x Genotype & 0.604 & 0.687 & 0.819 \\
\hline Age $\times$ Diet & $<0.001^{\star \star \star}$ & $0.001^{\star *}$ & $<0.001^{* * *}$ \\
\hline Genotype x Diet & 0.081 & 0.508 & $0.049^{*}$ \\
\hline Age x Genotype x Diet & 0.877 & 0.849 & 0.867 \\
\hline
\end{tabular}

TABLE 1. $P$-values of the general linear model univariate analysis of variance tests of the integrated density measurements.

ABBREVIATIONS: 5 -mC, 5 -methylcytosine; 5 -hmC, 5 -hydroxymethylcytosine; ${ }^{*} p<0.05 ;{ }^{* *} p<0.01$; *** $p<0.001$.

\subsection{Discussion}

5-mC and 5-hmC IR in cerebellar Purkinje cells was immunohistochemically investigated in WT and transgenic SOD1 mice, fed with either a CR or CD, and divided in 12- and 24-month-old groups. Qualitative analysis revealed an age-related increase in 5-hmC IR in the CD groups only. A semi-quantitative analysis of $5-\mathrm{mC}$ and $5-\mathrm{hmC}$ IR corroborated these observations, indicating that IR significantly increased with age, CR seems to mitigate this epigenetic change, SOD1 overexpression does not affect this age-related increase, and that lifelong CR appears to favor DNA hydroxymethylation over methylation with age. 
A

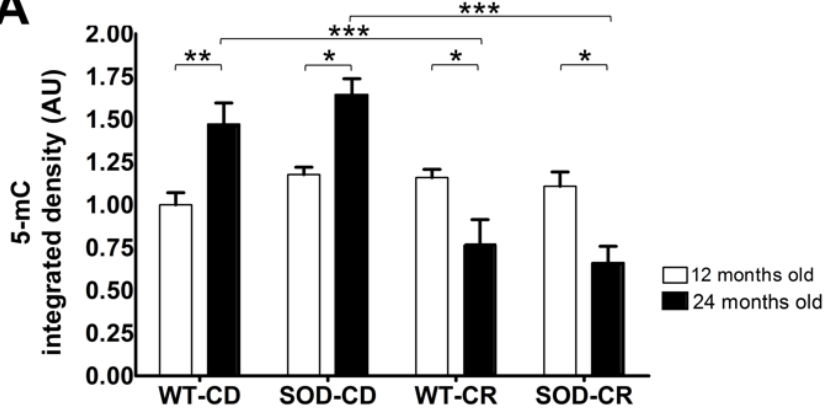

B

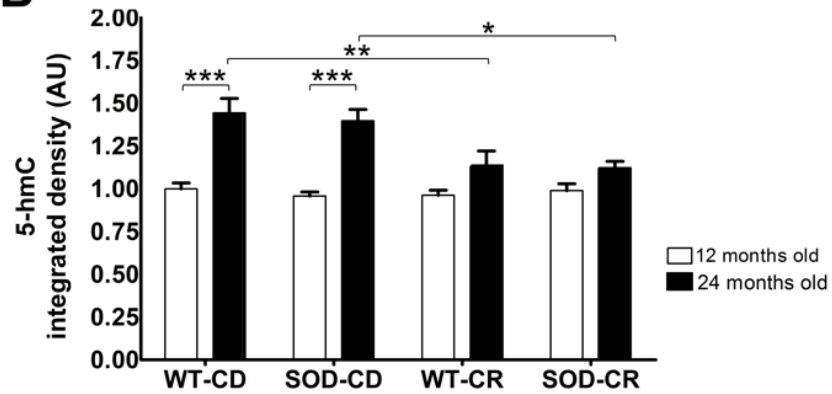

C

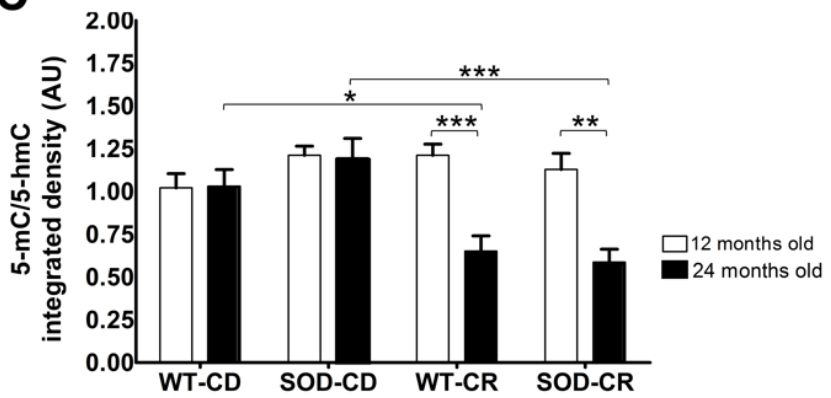

\subsubsection{Nuclear staining pattern of 5-mC and 5-hmG immunoreactivity}

The immunohistochemical staining of 5-mC in mouse cerebellar Purkinje cells was limited to sites of high IR, while $5-\mathrm{hmC}$ was located throughout the nucleus. 5-mC and 5-hmC staining showed only minor colocalization, while 5-mC and DAPI staining showed profound colocalization. This is in line with previous research on the localization of $5-\mathrm{mC}$ and $5-\mathrm{hmC}$ in the nuclei of hippocampal $[48,54]$ and embryonic stem cells [69] in
FIGURE 4. Integrated density of 5 -methylcytosine $(5-\mathrm{mC})(\mathrm{A})$, 5 -hydroxymethylcytosine $(5-\mathrm{hmC})$ (B), and the ratio between $5-\mathrm{mC}$ and 5-hmC (C). Presented are the mean and standard error of means. Pooled data of the wild-type control diet (WT-CD), transgenic Cu/Zn super oxide dismutase 1 overexpressing control diet (SOD-CD), wild-type caloric restriction (WT-CR) and $\mathrm{Cu} /$ Zn super oxide dismutase 1 overexpressing caloric restriction (SOD-CR) groups are displayed separately at 12 months of age (white bars; WT-CD $5-\mathrm{mCn}=5,5-\mathrm{hmC} \mathrm{n}=6$, ratio $\mathrm{n}=5$; SOD-CD $5-\mathrm{mC} \mathrm{n}=4,5-\mathrm{hmC} \mathrm{n}=5$, ratio $\mathrm{n}=4 ; \mathrm{WT}-\mathrm{CR} 5-\mathrm{mC} \mathrm{n}=6,5-\mathrm{hmC}$ $\mathrm{n}=6$, ratio $\mathrm{n}=6$; SOD - CR $5-\mathrm{mC} \mathrm{n}=$ $6,5-\mathrm{hmC} \mathrm{n}=6$, ratio $\mathrm{n}=6$ ) and at 24 months of age (black bars; WT-CD 5 - $\mathrm{mC} \mathrm{n}=6,5-\mathrm{hmC} \mathrm{n}=6$, ratio $\mathrm{n}=6$; SOD-CD $5-\mathrm{mC} \mathrm{n}=5,5-\mathrm{hmC} \mathrm{n}=5$, ratio $\mathrm{n}=5$; WT-CR $5-\mathrm{mC} \mathrm{n}=6,5-\mathrm{hmC}$ $\mathrm{n}=6$, ratio $\mathrm{n}=6$; $\mathrm{SOD}-\mathrm{CR} 5-\mathrm{mC} \mathrm{n}=6$, 5 - $\mathrm{hmC} \mathrm{n}=6$, ratio $\mathrm{n}=6$ ). Indicated in the graph are the pairwise comparisons. AU: arbitrary units. ${ }^{*} \mathrm{p}<0.05$; ${ }^{* *}$ $\mathrm{p}<0.01 ;{ }^{* * *} \mathrm{p}<0.001$ (after Bonferroni correction). 
the mouse. These studies indicated that 5-mC co-localizes with DAPI, which is thought to primarily mark densely packed heterochromatin, whereas 5 -hmC barely co-localizes with either, suggesting that 5 -hmC favors the loosely packed euchromatin. This supports the suggested differential roles of $5-\mathrm{hmC}$ and $5-\mathrm{mC}$ in the regulation of gene expression $[69,70,91]$. The localization of $5-\mathrm{hmC}$ to euchromatin, regions that are transcriptionally active, is in line with its suggested generally transcription-promoting function [62, 63], whereas the localization of $5-\mathrm{mC}$ to heterochromatin, regions that are transcriptionally inactive, is in line with its suggested overall transcription-inhibiting function [91]. Note, however, that these suggested roles of 5-hmC and 5-mC are rather general and that how they exactly affect gene transcription is far from clear and appears to be much more complex than initially assumed [42, $67,92-95]$. For example, the effect on gene transcription seems to be gene-specific and highly dependent on the exact location of the DNA modification(s) in that particular gene (e.g. promoter region, intragenic) [73].

\subsubsection{5-mC and 5-hmC levels increase with age}

Visual inspection and subsequent semi-quantitative analysis showed an age-related increase of Purkinje cell 5-mC and 5-hmC IR in the CD groups, similar to previous observations in the hippocampus by our group and by others $[48,54,96]$. It, however, remains to be elucidated what the functional impact of these age-related changes in $5-\mathrm{mC}$ and $5-\mathrm{hmC}$ are. For this reason, it is important to investigate which genes are affected by these DNA modifications and how these epigenetic changes impact upon gene expression. A good start would be to look the genes whose expression is known to alter with age, in general, and more specifically in the cerebellum $[1,97,98]$. Additionally, it might be fruitful to explore the relation between $5-\mathrm{mC}$ and $5-\mathrm{hmC}$ levels and age-related changes in splicing in the cerebellum. Recently, it has been shown that more than a third of brain-specific genes undergo age-related changes in splicing in the cerebellum and other brain areas and that a portion of these changes are region-specific [99].

In order to successfully determine the exact role of 5-mC and 5-hmC in the aging cerebellum it is, however, crucial to further delve into the fundamental mechanisms underlying $5-\mathrm{mC}$ and especially 5 -hmCmediated regulation of gene expression, as these are still far from clear. Studies have shown that in the body, 5 -hmC is most abundant in the brain and that, in contrast to $5-\mathrm{mC}$, it specifically targets portions of the DNA 
encoding genes [73]. As mentioned above, it has been suggested that the formation of $5-\mathrm{hmC}$ from $5-\mathrm{mC}$ is an intermediate stage in the process of active DNA demethylation and while 5-mC is thought to repress gene expression, it is argued that $5-\mathrm{hmC}$ enhances gene expression [100, 101]. Observations from DNMT knock-out experiments in cell cultures indicate that $5-\mathrm{hmC}$ can only be generated in the presence of $5-\mathrm{mC}$, supporting the notion that formation of $5-\mathrm{hmC}$ is a step in DNA demethylation [67]. Evidence for a transcription-promoting role shows that $5-\mathrm{hmC}$ has a relatively low affinity for MBPs 1,2 and 4 , as compared to $5-\mathrm{mC}[68,102]$. These proteins can bind to $5-\mathrm{mC}$ and play a pivotal role in its function as a transcriptional repressor. Although these findings seem to point towards an antagonistic role of $5-\mathrm{hmC}$ in relation to $5-\mathrm{mC}$, recent studies have shown its function to be more complex. For instance, methyl-CpG binding protein (MeCP) 2 appears to have a higher affinity for 5-hmC in the brain [74], and only 5-hmC located in gene bodies, but not in promoter regions, seems to correlate positively with gene expression $[73,78,96]$. Furthermore, high levels of 5-hmC do not appear to be indicative of high levels of DNA demethylation [72]. These findings illustrate that the exact function of 5 -hmC might be dependent on cell-type and location in the genome.

How changes in 5-mC and 5-hmC exactly relate to the aging process thus remains elusive. Nevertheless, levels of DNA damage, a factor that is thought to increase with age, do not appear to be related to $5-\mathrm{hmC}$ levels in cerebellar and hippocampal cells, as observed in adult mice [78, 96]. Additionally, some of the genes found to exhibit increasing 5-hmC content with age in the cerebellum, such as those encoding secretases and presenilins, are associated with neurodegeneration [57], while the $A P P$ promoter region becomes hypomethylated with age [103] and several tumor-suppressor gene promoter regions become hypermethylated [104].

Previous research utilizing the same aging mice cohort as in the present study, focused on the hippocampus and found an age-related increase of 5-hmC, 5-mC and DNMT3A IR [47, 48, 54]. This brain structure is known for its importance for learning and memory, and DNA methylation and demethylation are thought to be critically involved in these processes, as well as synaptic plasticity and neurogenesis [105-107]. Additionally, age-related changes in the hippocampal methylation status of genes linked to memory have been observed $[40,108,109]$. The cerebellum is mostly associated with motor functions and its exact role in regulating cognition remains unclear. Purkinje cells are, however, cardinal for motor learning and recently have been implicated in the spatial navigation system, a cerebello-hippocampal network responsible for goal-directed navigation $[10,11]$. Interestingly, spatial navigation is a cognitive process that has recently been shown to deteriorate with aging [110]. 
Since the age-related increases in cerebellar 5-mC and 5-hmC IR positively correlate, which was previously also found in the hippocampus, it could be that the observed increases in DNA methylation might reflect an increased methylation potential, which in turn could indicate an increased hydroxymethylation potential [54]. There are, however, reports that the methylation potential, which is critically dependent on the levels of the methyl-group donor S-adenosylmethionine (SAM) [111], actually decreases with age [112]. An alternative explanation of how changes in 5-mC and 5-hmC patterns might result in gene expression changes with age would be that an aberrant methylation-hydroxymethylation balance is responsible [54]. Clearly, additional efforts to unveil the precise role of 5-mC and 5-hmC in aging are warranted.

\subsubsection{Diet affects age-re- lated changes in 5-mC and 5-hmC}

The detection of a significant interaction between diet and age signifies that the age-related increase of 5-mC and 5-hmC IR depended on the diet. This finding is in concert with observations in the hippocampus, where it was found that $\mathrm{CR}$ was able to attenuate age-related alterations in $5-\mathrm{mC}$ and 5 -hmC IR [48, 54]. Interestingly, the present results seem to suggest that 5-mC levels and the 5-mC:5-hmC ratio may actually decrease with age in the CR groups, whereas 5 -hmC levels remain unchanged. This indicates that CR may differentially affect DNA methylation and hydroxymethylation in cerebellar Purkinje cells. The high decrease in 5-mC levels may be a result of either a decrease in DNA methylation, or an increase in active DNA demethylation. CR has been reported to affect DNA methyltransferases DNMT1 and DNMT3A [47, 113], but it remains to be established how CR may promote demethylation in cerebellar Purkinje cells. Although some demethylating agents, such as green tea polyphenols and soybean genistein, are thought to be beneficial in the treatment and prevention of cancer [114], more evidence in the case of aging is still needed.

Despite rigorous investigations into the life-prolonging effects of $\mathrm{CR}$, it remains unknown exactly how CR modulates DNA methylation and hydroxymethylation. Current hypotheses mainly revolve around either a dietary impact of $\mathrm{CR}$ on the one-carbon metabolism, which determines the availability of methyl-groups necessary for DNA methylation and hydroxymethylation [115-117], or a chromatin remodeling process involving sirtuins, a class of HDACs that are known to influence life span [28, 118-123]. 


\subsubsection{SOD1 does not affect age-related changes in 5-mC and 5-hmC levels}

Despite previous reports on the neuroprotective effects of increased antioxidant levels [21-26], no effect of SOD1 overexpression on the age-related increase of 5-mC and 5-hmC IR was observed, nor was an additional or synergistic effect of combining CR and SOD1 overexpression detected. This finding complies with previous studies employing the same transgenic SOD1 overexpression model, which found that SOD1 overexpression did not affect lifespan and age-related changes in hippocampal volumes, and DNMT3A, 5-mC, 5-hmC and HDAC2 IR [32, 47, 48, 54, 77].

Despite these observations, it could be possible that SOD1 overexpression succeeds in reducing oxidative damage, but that this reduction is independent of epigenetic markers such as those previously investigated (i.e. DNMT3A, 5-mC, 5-hmC and HDAC2). This would, however, be in contrast of previous reports stating that oxidative stress is able to induce epigenetic changes $[124,125]$. Alternatively, as suggested previously $[48,54]$, it might be that the effects of SOD1 overexpression are only observable in other brain regions than hippocampus or cerebellum. The observation that SOD1 overexpression does not appear to have an effect in both the hippocampus and the cerebellum points towards another option, i.e. that the level of SOD1 overexpression was insufficient to incite any detectable alterations, despite the observation that apart from SOD1, these mice also present with increased levels of other antioxidants, including catalase and Mn-SOD [32, 87, 126]. In light of previously reported beneficial results of increasing antioxidant levels, it would be interesting to investigate the effects of other antioxidants, such as glutathione, which have been shown to be pivotally involved in the aging process [126-128].

Curiously, although the genotype may not affect age-related changes in

5-mC and 5-hmC, the significant genotype and diet interaction observed for the ratio between $5-\mathrm{mC}$ and $5-\mathrm{hmC}$ suggests that $5-\mathrm{mC}$ levels are increased in the SOD mice, but only in those that received the CD. This effect should, however, be confirmed in additional studies. 


\subsubsection{Strengths, limitations, and future perspectives}

Strengths of the current study include the strictly controlled conditions the mouse cohort was maintained under, the implementation of established histological techniques that allowed for the qualitative and quantitative analyse of 5-mC and 5-hmC IR at a cellular level and the availability of 12 and 24 months old mice to allow for the detection of age-related alterations. The discovery of $5-\mathrm{hmC}$ as an epigenetic marker very similar to, but functionally distinct from 5-mC spawned a controversy concerning 5-mC detection methods. Apparently, many techniques commonly used to measure $5-\mathrm{mC}$ are unable to distinguish $5-\mathrm{mC}$ from $5-\mathrm{hmC}$, meaning that reports on 5-mC relying on these techniques should be interpreted with caution [42]. The present study, however, uses highly specific antibodies for the selective detection of 5-hmC, without non-specific interference of $5-\mathrm{mC}[54,69]$. Indeed, detection methods involving antibodies have been shown to be specific, while alternative methods, such as classical bisulfite conversion, are unable to distinguish 5-hmC from 5-mC [102, 129-131].

A limitation of the study is that the strictly monitored dietary intake necessitated individual housing [34], and as a consequence confounding effects of social isolation cannot be excluded [132]. The choice to refrain from behavioral and cognitive testing might be pointed out as another limitation. However, since it is known that stress, learning, or other factors connected to testing are able to induce epigenetic changes by themselves, testing was not performed to prevent the introduction of unwanted bias into the dependent variables. Furthermore, a tradeoff was made by choosing for an immunohistochemical approach that allows for a cell-type specific semi-quantitative analysis, but is unable to determine absolute 5-mC and 5-hmC levels. Although a cell-type specific analysis was performed, the different cerebellar subregions were grouped together, as not all animals had sections of all the subregions. As it has been reported that the total numbers of Purkinje cells show age related decreases [12], it is possible that the loss of a specific subset of Purkinje cells in the older animals may have influenced the average 5-mC and 5 -hmC IR levels as determined in this study. Finally, interpreting agerelated alterations in 5-mC and 5-hmC, or other epigenetic markers, should be done with caution, as causal relationships remain elusive. Changes in epigenetic DNA modifications could either contribute to the aging process or be a consequence of it, or both. Future studies, possibly investigating whether the induction of age-related epigenetic changes results in an age-related phenotype, are required to establish such a causal relationship. Additionally, future studies should not only elucidate which genes are regulated by $5-\mathrm{mC}$ and $5-\mathrm{hmC}$, and how this regulation 
is influenced by age, but should do so in a region- and cell-type-specific manner. Furthermore, 5-mC and 5-hmC should preferably not be viewed and investigated as isolated epigenetic mechanisms, but rather as two out of numerous connected components in the complex epigenetic machinery that regulates gene expression. Apart from studies on a molecular, cellular and genetic level, it is also crucial to relate changes on these various levels with behavioral outcomes, such as alterations in cognition and motor skills.

\subsection{Conclusion}

The findings presented here indicate that mouse cerebellar Purkinje cells display an age-related increase in levels of DNA methylation and hydroxymethylation. It was furthermore shown that these age-related alterations could be mitigated through $\mathrm{CR}$, but that SOD1 overexpression failed to exert any effect on the observed changes during aging. These results are mostly in agreement with previous investigations in the hippocampus, but in addition indicate that $C R$ has a stronger effect on DNA methylation than DNA hydroxymethylation with age, leading to a decreased methylated DNA to hydroxymethylated DNA ratio. This corroborates the notion that widespread epigenetic changes occur during the aging process and that $\mathrm{CR}$ is able to strongly impact on these epigenetic mechanisms.

\section{Acknowledgments}

Funds have been provided by the Internationale Stichting Alzheimer Onderzoek (ISAO) grants 07551 and 11532 (D.L.A.vdH.), by the ISAO grants 09552 and 13515, and the Netherlands Organization for Scientific Research (NWO), grant 916.11.086 (Veni Award) (B.P.F.R.), by the NWO grant 911.06 .086 (C.S.), by a fellowship as part of the NWO grant 022.005.019 and the Maastricht University Medical Centre+ (Koostra Talent Fellowship) (R.L.). We thank H. P. J. Steinbusch and D. Mastroeni for expert technical advice, and K. Kompotis and J. Dela Cruz for assistance during the laboratory experiments. The authors declare that there are no conflicts of interest.

\section{REFERENCES}

[1] Berchtold NC, Cribbs DH, Coleman PD, et al. Gene expression changes in the course of normal brain aging are sexually dimorphic. Proc Natl Acad Sci 2008; 105: 15605-15610.

[2] Dickstein DL, Kabaso D, Rocher AB, et al. Changes in the structural complexity of the aged brain. Aging Cell 2007; 6: 275-84.

[3] Hegde ML, Mantha AK, Hazra TK, et al. Oxidative genome damage and its repair: Implications in aging and neurodegenerative diseases. Mech Ageing Dev 2012; 133: 157-168.

[4] Hof PR, Morrison JH.

The aging brain: morphomolecular senescence of cortical circuits. Trends Neurosci 2004; 27: 607-613.

[5] Lee C-K, Weindruch R, Prolla TA. Gene-expression profile of the ageing brain in mice. Nat Genet 2000; 25: 294-297.

[6] Lu T, Pan Y, Kao S-Y, et al. Gene regulation and DNA damage in the ageing human brain. Nature 2004; 429: 883-891.

[7] Morrison JH, Hof PR. Life and death of neurons in the aging brain. Science 1997; 278: 412-9.

[8] Rutten BPF, Korr H, Steinbusch HWM, et al. The aging brain: less neurons could be better. Mech Ageing Dev 2003; 124: 349-55.

[9] Luebke JI, Weaver CM, Rocher AB, et al. Dendritic vulnerability in neurodegenerative disease: insights from analyses of cortical pyramidal neurons in transgenic mouse models. Brain Struct Funct 2010; 214: 181-199.

[10] Rochefort C, Arabo A, André M, et al. Cerebellum shapes hippocampal spatial code. Science; http://science.sciencemag.org/content/334/6054/385 (2011, accessed 7 
April 2017).

[11] Rochefort C, Lefort J, Rondi-Reig L. The cerebellum: a new key structure in the navigation system.

Front Neural Circuits 2013; 7: 35.

[12] Rutten BPF, Schmitz C, Gerlach $\mathrm{OHH}$, et al. The aging brain: Accumulation of DNA damage or neuron loss? Neurobiol Aging 2007; 28: 91-98.

[13] Wu W, Brickman AM, Luchsinger J, et al. The brain in the age of old: The hippocampal formation is targeted differentially by diseases of late life. Ann Neurol 2008; 64: 698-706.

[14] Anderson RM, Shanmuganayagam D, Weindruch $\mathrm{R}$. Caloric restriction and aging: studies in mice and monkeys. Toxicol Pathol 2009; 37: 47-51.

[15] Colman RJ, Anderson RM, Johnson SC, et al. Caloric restriction delays disease onset and mortality in rhesus monkeys. Science 2009; 325 : 201-4.

[16] Devore EE, Grodstein F, van Rooij FJA, et al. Dietary antioxidants and long-term risk of dementia. Arch Neurol 2010; 67: 819-25.

[17] Fontana L, Partridge L, Longo VD. Extending healthy life span--from yeast to humans. Science 2010; 328: 321-326.

[18] Joseph J, Cole G, Head E, et al. Nutrition, Brain aging, and neurodegeneration. J Neurosci; http://www. jneurosci.org/content/29/41/12795 (2009, accessed 7 April 2017).

[19] Llewellyn DJ, Lang IA,

Langa KM, et al. Vitamin D and risk of cognitive decline in elderly persons.

Arch Intern Med 2010; 170: 1135.

[20] Rutten BPF, Steinbusch

HWM, Korr H, et al. Antioxidants and Alzheimer's disease: from bench to bedside (and back again). Curr Opin

Clin Nutr Metab Care 2002; 5: 645-51.

[21] Cardozo-Pelaez F, Song S,

Parthasarathy A, et al. Attenuation

of age-dependent oxidative damage to DNA and protein in brainstem of $\mathrm{Tg} \mathrm{Cu} / \mathrm{Zn}$ SOD mice. Neurobiol Aging 1998; 19: 311-6.

[22] Borg J, Chereul E. Differential MRI patterns of brain atrophy in double or single transgenic mice for APP and/or SOD. J Neurosci Res 2008; 86: 3275-3284.

[23] Borg J, London J. Copper/ zinc superoxide dismutase overexpression promotes survival of cortical neurons exposed to neurotoxins in vitro. J Neurosci Res 2002; 70: 180-189. [24] Cadet JL, Sheng P, Ali S, et al. Attenuation of methamphetamine-induced neurotoxicity in copper/zinc superoxide dismutase transgenic mice. J Neurochem 1994; 62 : $380-3$.

[25] Chan PH, Epstein CJ,

Kinouchi H, et al. SOD-1 transgenic mice as a model for studies of neuroprotection in stroke and brain trauma. Ann N Y Acad Sci 1994; 738: 93-103.

[26] Sha SH, Zajic G, Epstein

CJ, et al. Overexpression of copper/ zinc-superoxide dismutase protects from kanamycin-induced hearing loss. Audiol Neurootol 2001; 6: 117-23. [27] Adams MM, Shi L, Linville $\mathrm{MC}$, et al. Caloric restriction and age affect synaptic proteins in hippocampal CA3 and spatial learning ability.

Exp Neurol 2008; 211: 141-149.

[28] Bordone L, Guarente L.

Calorie restriction, SIRT1 and metabolism: understanding longevity. Nat Rev Mol Cell Biol 2005; 6: 298-305. [29] Levenson CW, Rich NJ. Eat less, live longer? New insights into the role of caloric restriction in the brain.
Nutr Rev 2007; 65: 412-5.

[30] Mattson MP, Chan SL,

Duan W. Modification of brain aging and neurodegenerative disorders by genes, diet, and behavior. Physiol Rev 2002; 82: 637-672.

[31] Mattson MP, Duan W,

Chan SL, et al. Neuroprotective and neurorestorative signal transduction mechanisms in brain aging: modification by genes, diet and behavior. Neurobiol Aging 2002; 23: 695-705. [32] Rutten BPF, Brasnjevic I, Steinbusch HWM, et al. Caloric restriction and aging but not overexpression of SODl affect hippocampal volumes in mice. Mech Ageing Dev 2010; 131: 574-579.

[33] Sohal RS, Weindruch R. Oxidative stress, caloric restriction, and aging. Science 1996; 273: 59-63.

[34] Weindruch R, Walford

RL, Fligiel S, et al. The retardation of aging in mice by dietary restriction: longevity, cancer, immunity and lifetime energy intake. $J$ Nutr 1986; 116: 641-54.

[35] Lee S-H, Min K-J. Caloric restriction and its mimetics. $B M B$ Rep 2013; 46: 181-7.

[36] Calvanese V, Lara E, Kahn $\mathrm{A}$, et al. The role of epigenetics in aging and age-related diseases. Ageing Res Rev 2009; 8: 268-276.

[37] Chouliaras L, Rutten BPF, Kenis G, et al. Epigenetic regulation in the pathophysiology of Alzheimer's disease. Prog Neurobiol 2010; 90: 498-510.

[38] Day JJ, Sweatt JD. DNA methylation and memory formation. Nat Neurosci 2010; 13: 1319-1323.

[39] Peleg S, Sananbenesi F,

Zovoilis A, et al. Altered histone acetylation is associated with age-dependent memory impairment in mice. 
Science 2010; 328: 753-6.

[40] Penner MR, Roth TL,

Barnes CA, et al. An epigenetic hypothesis of aging-related cognitive dysfunction. Front Aging Neurosci 2010; 2: 9.

[41] Goldberg AD, Allis CD, Bernstein E. Epigenetics: a landscape takes shape. Cell 2007; 128: 635-638. [42] van den Hove DLA, Chouliaras L, Rutten BPF. The role of 5-hydroxymethylcytosine in aging and Alzheimer's disease: current status and prospects for future studies. Curr Alzheimer Res 2012; 9: 545-9.

[43] Day JJ, Sweatt JD. Cognitive neuroepigenetics: A role for epigenetic mechanisms in learning and memory. Neurobiol Learn Mem 2011; 96: 2-12.

[44] Fitzsimons CP, van Bode-

graven E, Schouten M, et al. Epigenetic regulation of adult neural stem cells: implications for Alzheimer's disease. Mol Neurodegener 2014; 9: 25. [45] Serrano L, Vazquez BN, Tischfield J. Chromatin structure, pluripotency and differentiation. Exp Biol Med 2013; 238: 259-270.

[46] Ben-Avraham D, Muzumdar RH, Atzmon G. Epigenetic genome-wide association methylation in aging and longevity. Epigenomics 2012; 4: 503-509.

[47] Chouliaras L, van den Hove DLA, Kenis G, et al. Caloric restriction attenuates age-related changes of DNA methyltransferase $3 \mathrm{a}$ in mouse hippocampus. Brain Behav Immun 2011; 25 : 616-623.

[48] Chouliaras L, van den Hove DLA, Kenis G, et al. Prevention of age-related changes in hippocampal levels of 5-methylcytidine by caloric restriction. Neurobiol Aging 2012; 33 : $1672-81$. genetics and aging: the targets and the marks. Trends Genet 2007; 23: 413-418. [50] Hernandez DG, Nalls MA, Gibbs JR, et al. Distinct DNA methylation changes highly correlated with chronological age in the human brain. Hum Mol Genet 2011; 20: 1164-1172.

[51] Lunnon K, Smith R, Hannon E, et al. Methylomic profiling implicates cortical deregulation of $A N K 1$ in Alzheimer's disease. Nat Neurosci 2014; 17: 1164-1170.

\section{[52] Mastroeni D, Grover A,}

Delvaux E, et al. Epigenetic changes in Alzheimer's disease: Decrements in DNA methylation. Neurobiol Aging 2010; 31: 2025-2037.

\section{[53] Thompson RF, Atzmon}

G, Gheorghe C, et al. Tissue-specific dysregulation of DNA methylation in aging. Aging Cell 2010; 9: 506-518.

\section{[54] Chouliaras L, van den}

Hove DLA, Kenis G, et al. Age-related increase in levels of 5-hydroxymethylcytosine in mouse hippocampus is prevented by caloric restriction. Curr Alzheimer Res 2012; 9: 536-44.

[55] Condliffe D, Wong A,

Troakes C, et al. Cross-region reduction in 5-hydroxymethylcytosine in Alzheimer's disease brain. Neurobiol Aging 2014; 35: 1850-1854.

[56] Coppieters N, Dieriks B V, Lill C, et al. Global changes in DNA methylation and hydroxymethylation in Alzheimer's disease human brain. Neurobiol Aging 2014; 35: 1334-1344. [57] Song C-X, Szulwach KE, $\mathrm{Fu} \mathrm{Y}$, et al. Selective chemical labeling reveals the genome-wide distribution of 5-hydroxymethylcytosine. Nat

Biotechnol 2011; 29: 68-72.

[58] Bestor TH. The DNA methyltransferases of mammals. Hum $\mathrm{Mol}$ Genet 2000; 9: 2395-402.

[59] Handel AE, Ebers GC,
Ramagopalan S V. Epigenetics: molecular mechanisms and implications for disease. Trends Mol Med 2010; 16: 7-16. [60] Lan J, Hua S, He X, et al. DNA methyltransferases and methyl-binding proteins of mammals. Acta Biochim Biophys Sin (Shanghai) 2010; 42: 243-52.

[61] Razin A, Riggs AD. DNA methylation and gene function. Science 1980; 210: 604-10.

[62] Tahiliani M, Koh KP, Shen Y, et al. Conversion of 5-methylcytosine to 5-hydroxymethylcytosine in mammalian DNA by MLL partner TET1. Science 2009; 324: 930-935. [63] Ito S, D’Alessio AC, Taranova O V., et al. Role of Tet proteins in 5 -mC to 5-hmC conversion, ES-cell self-renewal and inner cell mass specification. Nature 2010; 466: 1129-1133. [64] Ito S, Shen L, Dai Q, et al. Tet proteins can convert 5-methylcytosine to 5 -formylcytosine and 5-carboxylcytosine. Science; http://science.sciencemag.org/content/333/6047/1300 (2011, accessed 3 April 2017).

[65] Raiber E-A, Beraldi D, Ficz $\mathrm{G}$, et al. Genome-wide distribution of 5 -formylcytosine in embryonic stem cells is associated with transcription and depends on thymine DNA glycosylase. Genome Biol 2012; 13: R69.

[66] Song C-X, He C. Potential functional roles of DNA demethylation intermediates. Trends Biochem Sci 2013; 38: 480-484.

[67] Williams K, Christensen J, Pedersen MT, et al. TETl and hydroxymethylcytosine in transcription and DNA methylation fidelity. Nature 2011; 473: 343-348.

[68] Valinluck V, Tsai H-H, Rogstad DK, et al. Oxidative damage to methyl-CpG sequences inhibits the 
binding of the methyl-CpG binding domain (MBD) of methyl-CpG binding protein 2 (MeCP2). Nucleic Acids Res 2004; 32: 4100-4108.

[69] Ficz G, Branco MR, Seisenberger S, et al. Dynamic regulation of 5 -hydroxymethylcytosine in mouse ES cells and during differentiation. Nature 2011; 473: 398-402.

[70] Münzel M, Globisch D, Carell T. 5-Hydroxymethylcytosine, the sixth base of the genome. Angew Chemie Int Ed 2011; 50: 6460-6468.

[71] Guo JU, Su Y, Shin JH, et al. Distribution, recognition and regulation of non-CpG methylation in the adult mammalian brain. Nat Neurosci 2013; 17: 215-222.

[72] Hahn MA, Qiu R, Wu X, et al. Dynamics of 5-hydroxymethylcytosine and chromatin marks in mammalian neurogenesis. Cell Rep 2013; 3: 291-300.

[73] Jin S-G, Wu X, Li AX, et al. Genomic mapping of 5-hydroxymethylcytosine in the human brain. Nucleic Acids Res 2011; 39: 5015-5024.

[74] Mellén M, Ayata P, Dewell

$\mathrm{S}$, et al. MeCP2 binds to 5 -hmC enriched within active genes and accessible chromatin in the nervous system. Cell 2012; 151: 1417-1430.

[75] Siegmund KD, Connor CM, Campan M, et al. DNA methylation in the human cerebral cortex is dynamically regulated throughout the life span and involves differentiated neurons. PLoS One 2007; 2: e895.

[76] Tan L, Shi YG. Tet family proteins and 5-hydroxymethylcytosine in development and disease. Development 2012; 139: 1895-1902. [77] Chouliaras L, van den Hove DLA, Kenis G, et al. Histone deacetylase 2 in the mouse hippocampus: attenuation of age-related increase by caloric restriction. Curr Alzheimer Res 2013; 10: 868-76.

[78] Kriaucionis S, Heintz

$\mathrm{N}$. The nuclear DNA base 5-hydroxymethylcytosine is present in Purkinje neurons and the brain. Science 2009; 324: 929-30.

[79] Penn NW, Suwalski R,

O'Riley C, et al. The presence of

5 -hydroxymethylcytosine in animal deoxyribonucleic acid. Biochem J 1972; 126: 781-90.

[80] Lange W. Cell number and cell density in the cerebellar cortex of man and some other mammals. Cell Tissue Res 1975; 157: 115-24.

[81] Palay SL, Chan-Palay

V. Cerebellar cortex: cytology and organization. https://books.google.nl/ books?id=ZVTmCAAAQBAJ\&dq=Cerebellar+cortex:+cytology+and+organization $\& l r=\&$ source $=g b s \_n a v l i n k s \_s$ (1974, accessed 7 April 2017).

\section{[82] Chan-Palay V, Nilaver G,}

Palay SL, et al. Chemical heterogeneity in cerebellar Purkinje cells: existence and coexistence of glutamic acid decarboxylase-like and motilin-like immunoreactivities. Proc Natl Acad Sci U S A 1981; 78: 7787-91.

\section{[83] Kern JK, Jones AM. Evi-}

dence of toxicity, oxidative stress, and neuronal insult in autism. $J$ Toxicol Environ Heal Part B 2006; 9: 485-499. [84] Brock B, Basha R, DiPalma $\mathrm{K}$, et al. Co-localization and distribution of cerebral APP and SPl and its relationship to amyloidogenesis. $J$ Alzheimers Dis 2008; 13: 71-80.

\section{[85] Fukutani Y, Cairns NJ,}

Rossor MN, et al. Purkinje cell loss and astrocytosis in the cerebellum in familial and sporadic Alzheimer's disease. Neurosci Lett 1996; 214: 33-36. [86] Zhang C, Zhu Q, Hua T. Aging of cerebellar Purkinje cells. Cell
Tissue Res 2010; 341: 341-7.

[87]

Epstein CJ, Avraham KB,

Lovett M, et al. Transgenic mice with increased $\mathrm{Cu} / \mathrm{Zn}$-superoxide dismutase activity: animal model of dosage effects in Down syndrome. Proc Natl Acad Sci U S A 1987; 84: 8044-8. [88] Globisch D, Münzel M, Müller M, et al. Tissue distribution of 5 -hydroxymethylcytosine and search for active demethylation intermediates. PLoS One; 5. Epub ahead of print 2010. DOI: 10.1371/journal. pone.0015367.

[89] Franklin KBJ, Paxinos G. Paxinos and Franklin's The mouse brain in stereotaxic coordinates. 2012 . [90] Schindelin J, Arganda-Carreras I, Frise E, et al. Fiji: an opensource platform for biological-image analysis. Nat Methods 2012; 9: 676-682. [91] Miller CA, Sweatt JD, Park A, et al. Covalent modification of DNA regulates memory formation. Neuron 2007; 53: 857-69.

[92] Bhutani N, Burns DM, Blau HM. DNA demethylation dynamics. Cell 2011; 146: 866-872.

[93] Pastor WA, Pape UJ, Huang $\mathrm{Y}$, et al. Genome-wide mapping of 5 -hydroxymethylcytosine in embryonic stem cells. Nature 2011; 473: 394-397.

[94] Wu H, D’Alessio AC, Ito

$\mathrm{S}$, et al. Dual functions of Tetl in transcriptional regulation in mouse embryonic stem cells. Nature 2011; 473: 389-393.

[95] Xu Y, Wu F, Tan L, et al. Genome-wide Regulation of 5-hmC, 5-mC, and Gene Expression by Tet1 Hydroxylase in Mouse Embryonic Stem Cells. Mol Cell 2011; 42: 451-464. [96] Münzel M, Globisch D, Brückl T, et al. Quantification of the sixth DNA base hydroxymethylcyto- 
sine in the brain. Angew Chemie Int Ed 2010; 49: 5375-5377.

[97] Fraser HB, Khaitovich P, Plotkin JB, et al. Aging and gene expression in the primate brain. PLoS Biol 2005; 3: e274.

[98] Park S-K, Kim K, Page GP, et al. Gene expression profiling of aging in multiple mouse strains: identification of aging biomarkers and impact of dietary antioxidants. Aging Cell 2009; 8: 484-495.

[99] Mazin P, Xiong J, Liu X, et al. Widespread splicing changes in human brain development and aging. Mol Syst Biol 2014; 9: 633-633.

[100] Guo JU, Su Y, Zhong C, et al. Hydroxylation of 5-methylcytosine by TET1 promotes active DNA demethylation in the adult brain. Cell 2011; 145: 423-434.

[101] Valinluck V, Sowers LC. Endogenous cytosine damage products alter the site selectivity of human DNA maintenance methyltransferase DNMT1. Cancer Res 2007; 67: 946-950. [102] Jin S-G, Kadam S, Pfeifer GP. Examination of the specificity of DNA methylation profiling techniques towards 5-methylcytosine and 5-hydroxymethylcytosine. Nucleic Acids Res 2010; 38: el25-el25.

[103] Tohgi H, Utsugisawa K, Nagane Y, et al. The methylation status of cytosines in a tau gene promoter region alters with age to downregulate transcriptional activity in human cerebral cortex. Epub ahead of print 1999. DOI: 10.1016/S03043940(99)00731-4.

[104] Madrigano J, Baccarelli AA, Mittleman MA, et al. Aging and epigenetics: Longitudinal changes in gene-specific DNA methylation. Epigenetics 2012; 7: 63-70.
Dynamic expression of de novo DNA methyltransferases Dnmt3a and Dn$\mathrm{mt} 3 \mathrm{~b}$ in the central nervous system. $J$ Neurosci Res 2005; 79: 734-746.

[106] Feng J, Zhou Y, Campbell

SL, et al. Dnmtl and Dnmt3a maintain DNA methylation and regulate synaptic function in adult forebrain neurons. Nat Neurosci 2010; 13: 423-430.

[107] Wu H, Coskun V, Tao J, et al. Dnmt3a-dependent nonpromoter DNA methylation facilitates transcription of neurogenic genes. Science; http://science.sciencemag.org/ content/329/5990/444 (2010, accessed 4 April 2017).

[108] Mugatroyd C, Wu Y, Bockmühl Y, et al. The Janus face of DNA methylation in aging. Aging (Albany NY) 2010; 2: 107-110.

[109] Penner MR, Roth TL, Chawla MK, et al. Age-related changes in Arc transcription and DNA methylation within the hippocampus.

Neurobiol Aging 2011; 32: 2198-2210.

[110] Gazova I, Vlcek K, Laczó

$\mathrm{J}$, et al. Spatial navigation-a unique window into physiological and pathological aging. Front Aging Neurosci 2012; 4: 16.

[111] Obeid R, Schadt A, Dill-

mann U, et al. Methylation status

and neurodegenerative markers in Parkinson disease. Clin Chem; http://clinchem.aaccjnls.org/content/55/10/1852 (2009, accessed 4 April 2017).

[112] Hooijmans CR, Blom HJ, Oppenraaij-Emmerzaal D, et al. S-adenosylmethionine and S-adenosylhomocysteine levels in the aging brain of APP/PS1 Alzheimer mice. Neurol Sci 2009; 30: 439-445.

[113] Li Y, Liu L, Tollefsbol TO.

Glucose restriction can extend normal cell lifespan and impair precancerous cell growth through epigenetic control of hTERT and pl6 expression. FASEB $J$ 2010; 24: 1442-1453.

[114] Li Y, Tollefsbol TO. Impact on DNA methylation in cancer prevention and therapy by bioactive dietary components. Curr Med Chem 2010; 17: 2141-51.

[115] Fuso A, Nicolia V, Cavallaro RA, et al. DNA methylase and demethylase activities are modulated by one-carbon metabolism in Alzheimer's disease models. J Nutr Biochem 2011; 22: 242-251.

[116] Fuso A, Seminara L, Cavallaro RA, et al. S-adenosylmethionine/homocysteine cycle alterations modify DNA methylation status with consequent deregulation of PSI and BACE and beta-amyloid production. Mol Cell Neurosci 2005; 28: 195-204. [117] Sugden C. One-carbon metabolism in psychiatric illness. Nutr Res Rev 2006; 19: 117.

[118] Blander G, Guarente L. The Sir2 Family of Protein Deacetylases. Annu Rev Biochem 2004; 73: 417-435. [119] Cantó C, Auwerx J. Caloric restriction, SIRT1 and longevity. Trends Endocrinol Metab 2009; 20: 325-331.

[120] Chen D, Bruno J, Easlon E, et al. Tissue-specific regulation of SIRT1 by calorie restriction. Genes Dev 2008; 22: 1753-1757.

[121] Chen D, Guarente L. SIR2: a potential target for calorie restriction mimetics. Trends Mol Med 2007; 13: $64-71$.

[122] Chen D, Steele AD, Lindquist $\mathrm{S}$, et al. Increase in activity during calorie restriction requires Sirtl. Science; http://science.sciencemag.org/content/310/5754/1641 (2005, accessed 7 April 2017).

[123] Dillin A, Kelly JW. 
The Yin-Yang of Sirtuins. Science;

http://science.sciencemag.org/content/317/5837/461 (2007, accessed 7

April 2017).

[124] Bhusari SS, Dobosy JR,

$\mathrm{Fu} \mathrm{V}$, et al. Superoxide dismutase 1

knockdown induces oxidative stress

and DNA methylation loss in the pros-

tate. Epigenetics 2010; 5: 402-9.

[125] Davis CD, Uthus EO. DNA

methylation, cancer susceptibility,

and nutrient interactions. Exp Biol Med

(Maywood) 2004; 229: 988-95.

[126] Przedborski S, Jack-

son-Lewis V, Kostic V, et al. Superox-

ide dismutase, catalase, and glutathi-

one peroxidase activities in copper/

zinc-superoxide dismutase transgenic

mice. J Neurochem 1992; 58: 1760-7.

[127] Cerutti P, Ghosh R, Oya

$\mathrm{Y}$, et al. The role of the cellular antioxidant defense in oxidant carcino-

genesis. Environ Health Perspect 1994;

123-9.

[128] Rebrin I, Sohal RS. Pro-ox-

idant shift in glutathione redox state during aging. Adv Drug Deliv Rev 2008; 60: 1545-1552.

[129] Hayatsu H, Shiragami M.

Reaction of bisulfite with the 5-hydroxymethyl group in pyrimidines and in phage DNAs. Biochemistry 1979; 18: 632-7.

[130] Huang Y, Pastor WA, Shen

$\mathrm{Y}$, et al. The behaviour of 5-hy-

droxymethylcytosine in bisulfite

sequencing. PLoS One 2010; 5: e8888.

[131] Nestor C, Ruzov A, Meehan

R, et al. Enzymatic approaches and bisulfite sequencing cannot distinguish between 5-methylcytosine and 5 -hydroxymethylcytosine in DNA.

Biotechniques 2010; 48: 317-319.

[132] Miura H, Qiao H, Ohta T.

over and biosynthesis of rats elicited

Influence of aging and social isolation by novelty stress. Synapse 2002; 46 :

on changes in brain monoamine turn-

$116-124$. 

AGE-RELATED EPI-

GENETIC CHANGES IN

HIPPOCAMPAL SUBRE-

GIONS OF FOUR ANIMAL

MODELS OF ALZHEI-

\section{MER'S DISEASE}

ROY LARDENOIJE ${ }^{A, B}$, DANIËL L.A. VAN DEN HOVE ${ }^{\mathrm{B}, \mathrm{C}}$, MONIQUE HAVERMANS ${ }^{\mathrm{B}}$, ANNE VAN CASTEREN ${ }^{\mathrm{B}}$, KEVIN X. LE ${ }^{A}$, ROBERTA PALMOUR $^{\mathrm{D}, \mathrm{E}}$, CYNTHIA A. LEMERE $^{\mathrm{A}, *}$, BART P.F. RUTTEN ${ }^{\mathrm{B}, *}$

${ }^{A} A N N$ ROMNEY CENTER FOR NEUROLOGIC DISEASES, DEPARTMENT OF NEUROLOGY, BRIGHAM AND WOMEN'S HOSPITAL, HARVARD MEDICAL SCHOOL, USA BSCHOOL FOR MENTAL HEALTH AND NEUROSCIENCE (MHENS), DEPARTMENT OF PSYCHIATRY AND NEUROPSYCHOLOGY, MAASTRICHT UNIVERSITY, THE NETHERLANDS 'LABORATORY OF TRANSLATIONAL NEUROSCIENCE, DEPARTMENT OF PSYCHIATRY, PSYCHOSOMATICS AND PSYCHOTHERAPY, UNIVERSITY OF WUERZBURG, GERMANY

DBEHAVIORAL SCIENCE FOUNDATION, ST. KITTS AND NEVIS, EASTERN CARIBBEAN

EMCGILL UNIVERSITY FACULTY OF MEDICINE, MONTREAL, QUEBEC, CANADA

*THESE AUTHORS CONTRIBUTED EQUALLY TO THIS WORK. 


\section{Abstract}

Both aging and Alzheimer's disease (AD) are associated with widespread epigenetic changes, with most evidence suggesting global hypomethylation in AD. It is, however, unclear how these age-related epigenetic changes are linked to molecular aberrations as expressed in animal models of AD. Here, we investigated age-related changes of epigenetic markers of DNA methylation and hydroxymethylation in a range of animal models of $A D$, and their correlations with amyloid plaque load. Three transgenic mouse models, including the J20, APP/ PS1dE9 and 3xTg-AD models, as well as Caribbean vervets (a nontransgenic non-human primate model of $A D$ ) were investigated. In the J20 mouse model, an age-related decrease in DNA methylation was found in the dentate gyrus (DG) and a decrease in the ratio between DNA methylation and hydroxymethylation was found in the DG and cornu ammonis (CA) 3. In the 3xTg-AD mice, an age-related increase in DNA methylation was found in the DG and CA1-2. No significant agerelated alterations were found in the APP/PS1dE9 mice and non-human primate model. Hippocampal plaque load showed a negative correlation with DNA methylation in the J20 model, and a positive correlation with DNA methylation in the 3xTg-AD model. Thus, only the J20 model showed an age-related reduction in global DNA methylation, while DNA hypermethylation was observed in the 3xTg-AD model. Given these differences between animal models, future studies are needed to further elucidate the contribution of different AD-related genetic variation to agerelated epigenetic changes.

KEYWORDS: Alzheimer's disease; aging; hippocampus; DNA methylation; DNA hydroxymethylation; DNA methyltransferase; animal models 


\subsection{Introduction}

Alzheimer's disease (AD) is a complex age-related neurodegenerative disorder and the most common form of dementia [1], for which presently no effective treatment exists [2,3]. Although recent studies indicate that the widespread neurodegeneration in the AD brain may be initiated in brainstem regions [4], the development of cognitive impairment is associated with degeneration of the entorhinal cortex and hippocampus [5]. The exact molecular mechanisms underlying the neurodegeneration in $A D$ remain unclear. Nevertheless, there are two pathological hallmarks; extracellular neuritic plaques and intracellular neurofibrillary tangles, which are thought to play a pivotal role in the progression of $A D$ and that are currently the basis of a definitive postmortem diagnosis [6, 7]. These protein aggregates mainly consist of amyloid- $\beta(A \beta)$ and hyperphosphorylated tau, respectively. Genetic studies have offered important insights and have confirmed the importance of $A \beta$, especially in the development of familial forms of $A D$, by identifying mutations in the amyloid precursor protein (APP) and presenilin (PS) genes that are associated with familial AD [8].

A growing body of evidence indicates that epigenetics may play a crucial role in complex age-related neurodegenerative diseases such as $A D$ [9-11]. Epigenetic processes dynamically regulate gene expression at both the transcriptional and translational level [12]. They are thought to be able to translate environmental exposures into alterations in gene expression [13]. In particular, DNA methylation has received attention in the context of $A D$, and DNA hydroxymethylation has more recently also been increasingly studied [9, 14, 15]. Our group and others have found that with normal aging, region-specific DNA methylation and hydroxymethylation, as well as DNA methyltransferase (DNMT) $3 A$ levels rise [16-22]. In AD, however, overall DNA methylation and hydroxymethylation levels appear to be lowered [23-25]. Depending on the brain region, and likely also methodological differences (e.g. concerning tissue processing), there are, however, also conflicting reports, showing no changes in DNA methylation levels between AD patients and controls [25], or increased DNA methylation and hydroxymethylation levels [26]. Recent studies employing techniques such as Illumina's HumanMethylation450 BeadChip assay have provided further insights beyond global changes in epigenetic markers [27, 28]. These epigenome-wide association studies on homogenates of brain samples can help to identify important new candidate genes that, through altered epigenetic regulation, may play a role in the pathogenesis of AD.

Many studies investigating epigenetic changes related to AD have studied differences between postmortem brain tissue from diseased and control 
cases. To go beyond associations and elucidate the exact functional and potentially causal role of epigenetic dysregulation in the course of AD, live model systems are required. To this end, a plethora of $A D$ animal models have been established, including many transgenic rodent models that overexpress mutated human genes that have been associated with rare forms of familial $A D$ [29], but also non-human primate models that naturally develop $A \beta$ plaque pathology have been used [30,31]. While these animal models capture some of the molecular, physiological, or behavioral aspects of $A D$, none of the animal models display the full complexity of AD [32]. Most animal models have been characterized based on classical hallmarks of $A D$, such as plaque development and cognitive impairment, but there are currently no reports comparing different animal models of $A D$ on an epigenetic level. The aim of the present study was therefore to investigate age-related changes in epigenetic markers related to DNA methylation (5-methylcytosine [5-mC] and DNMT3A) and DNA hydroxymethylation (5-hydroxymethylcytosine [5$\mathrm{hmC}$ ), in three genetically different transgenic mouse models of $A D$ and a non-human primate model that naturally develops $A \beta$ plaque pathology [30, 33]. In addition, correlations between 5-mC, 5-hmC, and DNMT3A immunoreactivity (IR) and amyloid plaque load were assessed, and compared with findings in humans and other studies related to epigenetic dysregulation in AD.

\subsection{Materials and methods 5.2.1. Animal models}

For this study, 3 transgenic mouse models of AD were used, including J20 mice on a C57BL6 background [34], APP/PS1dE9 mice on a C57BL6J background [35, 36], and 3xTg-AD mice on a C57BL6 background [37]. J20 mice harbor the mutated human APP gene (APPK670N/M671L, V717F), APP/PS1dE9 mice express both mutated humanized APP and human PS1 (APPK595N/M596LPS1 deletion of exon 9), and 3xTg-AD mice express 3 mutated human genes, APP, PS1, and tau (APPK670N/ M671L, PS1M146V, TauP301L). In addition to these transgenic mouse models, archived fixed brain tissue from 12 Caribbean vervets (Chlorocebus sabaeuss; 12.2 - 32 years of age) was used (Behavioral Science Foundation, St. Kitts) [33]. See Table 1 for additional information about the used animal models. The Harvard Medical Area Standing Committee approved of the use of mice at Brigham and Women's Hospital, which is in line with all state and federal regulations. Vervet brain tissue was retrieved following protocols approved by the Behavioral Science Foundation Animal Care Committee acting under the auspices of the Canadian Council on Animal Care. 


\begin{tabular}{|c|c|c|c|c|c|c|}
\hline $\begin{array}{l}\text { Animal } \\
\text { model }\end{array}$ & Mutations & Promotor & $\begin{array}{l}\text { Start plaque } \\
\text { deposition } \\
\text { (region) }\end{array}$ & $\begin{array}{l}\text { Start } \\
\text { cognitive } \\
\text { deficits }\end{array}$ & Age & References \\
\hline J20 & $\begin{array}{c}\text { APPK670N/ } \\
\text { M671L, V717F }\end{array}$ & PDGF & $\begin{array}{c}5-7 \text { months } \\
\text { (hippocampus, } \\
\text { neocortex) }\end{array}$ & 1-2 months & $\begin{array}{l}4 \text { months }(n=4) \\
8 \text { months }(n=4) \\
16 \text { months }(n=3) \\
24 \text { months }(n=2)\end{array}$ & {$[34,38]$} \\
\hline APP/PS1dE9 & $\begin{array}{c}\text { APPK595N/ } \\
\text { M596LPS1 } \\
\text { deletion of exon } 9\end{array}$ & $m P r P$ & $\begin{array}{l}6 \text { months } \\
\text { (hippocampus, } \\
\text { cortex) }\end{array}$ & 4 months & $\begin{array}{l}6 \text { months }(n=4) \\
16 \text { months }(n=1) \\
17 \text { months }(n=1) \\
18 \text { months }(n=1) \\
23 \text { months }(n=1) \\
27 \text { months }(n=1)\end{array}$ & {$[36,39,40]$} \\
\hline $3 x T g-A D$ & $\begin{array}{l}\text { APPK670N/ } \\
\text { M671L, } \\
\text { PS1M146V, } \\
\text { TauP301L }\end{array}$ & $m$ Thy-1 & $\begin{array}{c}6 \text { months (frontal } \\
\text { cortex) }\end{array}$ & 4 months $^{*}$ & $\begin{array}{l}5 \text { months }(n=4) \\
14 \text { months }(n=2) \\
17 \text { months }(n=1) \\
27 \text { months }(n=3)\end{array}$ & {$[37,41]$} \\
\hline $\begin{array}{c}\text { Caribbean } \\
\text { vervet }\end{array}$ & NA & NA & $\begin{array}{c}15 \text { years } \\
\text { (hippocampus) } \dagger\end{array}$ & 15 years $†$ & $\begin{array}{c}12.2 \text { years }(n=1) \\
14 \text { years }(n=1) \\
14.9 \text { years }(n=1) \\
15 \text { years }(n=2) \\
16.4 \text { years }(n=1) \\
17 \text { years }(n=2) \\
19 \text { years }(n=1) \\
24 \text { years }(n=1) \\
27.4 \text { years }(n=1) \\
32 \text { years }(n=1)\end{array}$ & $\begin{array}{c}\text { This article } \\
\text { (Supplementary } \\
\text { Figure 9) }\end{array}$ \\
\hline
\end{tabular}

\subsubsection{Tissue preparation}

After anesthetization via $\mathrm{CO}_{2}$ inhalation, mice were perfused with $20 \mathrm{~mL}$ ice-cold saline. The brains were then removed and hemisected, fixed in $10 \%$ formalin or $4 \%$ paraformaldehyde for 2 to 24 hours, paraffinembedded, and further sectioned into $10 \mu$ m-thick slices. The archived non-human primate brain tissue was formalin-fixed for months to several years and was divided into nine rostrocaudal regions, paraffin-embedded and further cut into $10 \mu \mathrm{m}$-thick coronal sections.

TABLE 1. Overview of investigated animal models.

*The $3 \times \mathrm{Tg}-\mathrm{AD}$ mice used in this paper appeared to have a 2-3 month delay in Alzheimer's disease pathology compared to previously published reports, possibly due to a loss of transgene copies with successive breeding (see https://www.jax.org/strain/004807). †This was the youngest vervet with plaques and cognitive impairment, but note that while generally plaque deposition increases with age, there is no clear relationship between age, onset of plaque pathology, and cognitive decline (see Supplementary Figure 9). NA, not applicable.

\subsubsection{Immunohisto- chemistry}

10 micron-thick serial sagittal mouse brain sections or coronal vervet brain sections were used for immunohistochemistry. All steps were performed at room temperature unless specified otherwise. Sections were first deparaffinized in Histo-Clear (National Diagnostics, Atlanta, GA) and rehydrated in a series of decreasing ethanol solutions, ending with deionized water. Hydrogen peroxide $(0.3 \%)$ diluted in methanol was used to quench endogenous peroxidase for 10 minutes. To unmask antigen-binding sites, antigen retrieval was performed with BioGenex citrate buffer (BioGenex, San Ramon, CA), keeping the solution around boiling temperature for 5 minutes in the microwave. $A \beta 42$ staining on the vervet tissue required incubating the sections in $88 \%$ formic acid 
for 10 minutes. The sections were washed with deionized water for 10 minutes and incubated in blocking solution for 20 minutes. The blocking solution consisted of $10 \%$ serum dissolved in Tris-buffered saline, with serum from the same species as the secondary antibody host. The sections were subsequently incubated overnight with primary antibody, at $4^{\circ} \mathrm{C}$. The following antibodies were used: a mouse monoclonal anti5-mC antibody (1:1000 dilution for mouse sections and 1:500 for vervet sections; GenWay Biotech Inc., San Diego, CA), a rabbit polyclonal anti-5-hmC antiserum (1:10,000 dilution; Active Motif, Carlsbad, CA), a rabbit polyclonal anti-DNMT3A antibody (1:200 dilution; Santa Cruz Biotechnology, Dallas, TX), a general monoclonal IgG1 anti-A $\beta$ antibody for staining mouse sections (3A1; 1:1000 dilution, kindly provided by $\mathrm{Dr}$. Brian O`Naullain at the Ann Romney Center for Neurologic Diseases, Boston, MA), and a mouse monoclonal IgG1 anti-AB42 antibody for staining vervet sections (1:500 dilution; BioLegend, San Diego, CA). After another wash with deionized water, the slides were incubated with biotinylated secondary antibodies for 30 minutes. Secondary antibodies included a horse anti-mouse secondary antibody (Vector Laboratories, Burlingame, CA) for the 5-mC antibody, a goat anti-rabbit secondary antibody (Vector Laboratories) for the 5-hmC and DNMT3A antibodies, a goat anti-mouse secondary antibody (Vector Laboratories) for $3 A 1$, and a goat anti-mouse antibody (SouthernBiotech, Birmingham, $A L$ ) for $A \beta 42$. The VectorElite horseradish peroxidase ABC kit (Vector Laboratories), with 3,3'-diaminobenzidine tetrahydrochloride (DAB; Sigma-Aldrich, St. Louis, MO) as chromogen, was used to visualize IR. For each staining run, omission of the primary antibody was included as a negative control, which consistently showed no staining (data not shown).

\subsubsection{Analysis of 5-mC, 5-hmC and DNMT3A immunoreactivity, and plaque load}

For each staining, 3 sagittal hippocampal sections per mouse and 2 coronal hippocampal sections per vervet were examined at approximately equidistant planes. For the IR analysis of $5-\mathrm{mC}, 5-\mathrm{hmC}$, and DNMT3A stainings, images were captured from hippocampal subregions, including 4 images of the dentate gyrus (DG), 2 of the cornu ammonis (CA) 3, and 2 of the CA1-2 (Supplementary Figure 1), using the 20X objective of a BX50 brightfield microscope (Olympus, Tokyo, Japan) in conjunction with a QIcam digital camera (QImaging, Surrey, BC, Canada). The IR 
in the regions of interest (DG, CA3, and CA1-2) was analyzed in the images of the hippocampal subregions using ImageJ (version 1.48v, Wayne Rasband, National Institutes of Health, Bethesda, Maryland, USA). For each image, the mean grey value of the region of interest (ROI) was measured after delineating the ROI in the image and setting a fixed threshold for background correction. Additionally, the total ROI area and ROI area above the background threshold was determined. The grey value and area measures were then combined by multiplying the background-corrected mean grey values of the ROI with the fraction of the ROI area with values above the background threshold (i.e. the specifically stained area of the ROI), to get the integrated density. This combined measurement is a more robust representation of protein levels than intensity or area alone, as for instance a decrease in area may lead to the detection of a higher mean intensity while the actual protein levels remained unchanged.

The fraction of the hippocampal area containing plaques was determined in the sections stained for 3A1 with a BIOQUANT image analysis setup (Nashville, TN, USA), and using a fixed threshold of detection. For this analysis, the hippocampus was manually delineated using the $4 \mathrm{X}$ objective, after which plaques where automatically detected based on the fixed threshold. Before performing the final measurements, artifacts were manually removed. The sections of the vervet brains varied in plane-cut and often only a part of the hippocampus could be assessed on a single section, which made them unsuited for a BIOQUANT analysis. The plaque load in the vervets was therefore semi-quantitatively scored, with 0 for no plaques, 1 for plaques in the temporal cortex but not inside the hippocampus, 2 for 1 to 5 plaques in the hippocampus, 3 for 6 to 10 plaques in hippocampus, 4 for 11 to 100 plaques in hippocampus, and 5 for more than 100 plaques in hippocampus. All slides and images were processed blinded and in a randomized order.

\subsubsection{Statistical analysis}

To compare the relative degree of DNA methylation and hydroxymethylation between ages, the ratio of the integrated density of 5-mC and 5-hmC was calculated (i.e. 5-mC IR / 5-hmC IR). Values deviating more than two times the standard deviation from the mean were replaced by the mean plus or minus two times the standard deviation (of the uncorrected dataset). Before performing the analyses and generating plots, the data was scaled through division by the root mean square of the data per region, to allow for a better comparison between the different stainings. For each animal model, hippocampal subregion, and epigenetic marker, including the $5-\mathrm{mC}: 5-\mathrm{hmC}$ ratio, a linear regression 
model was fitted with integrated density as the outcome and age as the predictor. The data was visually inspected for abnormalities and quantile-quantile plots of the regression model residuals were analyzed to check for severe deviations from normality. The correlation between the epigenetic markers and plaque load was determined by calculating Pearson's $r$. For all significance tests, the alpha was set at 0.05 . The ImageJ measurements were collected in Microsoft Excel 2013 (Microsoft, Redmond, WA) and processed, normalized, and analyzed in R (version 3.2.2; The R Foundation, Vienna, Austria) and RStudio (version 0.99.486; The Foundation for Open Access Statistics, Boston, MA). In addition to standard $\mathrm{R}$ functions, the dplyr package was used for data handling [42], the 'rcorr' function of the Hmisc package was used to determine correlation coefficients with $p$-values [43], and the ggplot2 package was used for generating graphs [44].

\subsection{Results}

\subsubsection{Qualitative analysis of 5-mC, 5 -hmC, and DNMT3A IR}

Three main hippocampal subregions (dentate gyrus, DG; cornu ammonis 3, CA3; and CA1-2) were examined by immunohistochemical analysis as shown in Supplementary Figure 1. A few (7\%) images were excluded from the analysis due to artifacts. Visual inspection of the analyzed images indicated most cells show nuclear 5-mC, 5-hmC, and DNMT3A IR (Supplementary Figures 2-5). DNMT3A, however, also appeared to be expressed outside the nucleus, especially in the CA3. The vervet tissue also exhibited some extranuclear 5-mC and 5-hmC IR. Upon closer observation, the 5-hmC and DNMT3A signals appeared diffusely throughout the nucleus, whereas the 5-mC signal was limited to a small number of distinct punctuae. Again, the staining pattern in the vervet tissue deviated from this general pattern and appeared diffusely throughout the nucleus for 5-mC, 5-hmC, and DNMT3A.

A conservative visual comparison of the stainings at the different ages did not reveal obvious age-related changes in IR of the epigenetic markers in the J20 (Supplementary Figure 2) and APP/PS1dE9 models (Supplementary Figure 3). In the 3xTg-AD model an age-related increase of $5-\mathrm{mC}$ signal can be observed mainly in the DG, but also the CA3 and CA1-2 (Supplementary Figure 4). The vervet images did not show any consistent age-related alterations in IR (Supplementary Figure 5). 
The hippocampal DNMT3A signal was generally too variable to draw any conclusions based on visual inspection alone. Some of these observations were confirmed through a semiquantitative analysis.

\subsubsection{Semiquantitative analysis of 5-mC, 5-hmC, and DNMT3A IR}

Quantile-quantile plots of the residuals of the regression models were inspected, and although the residuals of some individual models showed deviations from normality, there were no overall indications for either right- or left-skewness of the residuals. Linear regression showed a statistically significant age-related decrease of $5-\mathrm{mC} \operatorname{IR}(\beta=-0.034, p=$ $0.036)$ and the $5-\mathrm{mC}: 5-\mathrm{hmC}$ ratio $(\beta=-0.037, p=0.018)$ in the $\mathrm{DG}$, and of the $5-\mathrm{mC}: 5-\mathrm{hmC}$ ratio in the CA3 $(\beta=-0.038, p=0.034)$, in the J20 transgenic mouse model (Figure 1). No statistically significant age-related changes of 5-mC IR were observed in APP/PS1dE9 mice (Figure 2). In contrast to the $\mathrm{J} 20$ mice, a statistically significant age-related increase of $5-\mathrm{mC}$ IR was found in the DG $(\beta=0.021, p=0.034)$ and CA1-2 ( $\beta=$ $0.019, p=0.045$ ) of the $3 x T g-A D$ model (Figure 3 ). Also, no statistically significant age-related changes of $5-\mathrm{mC}$ IR were observed in vervets (Figure 4), and no changes in 5-hmC or DNMT3A were detected in any of the tested animal models (Figures 1-4). 
J20 mice
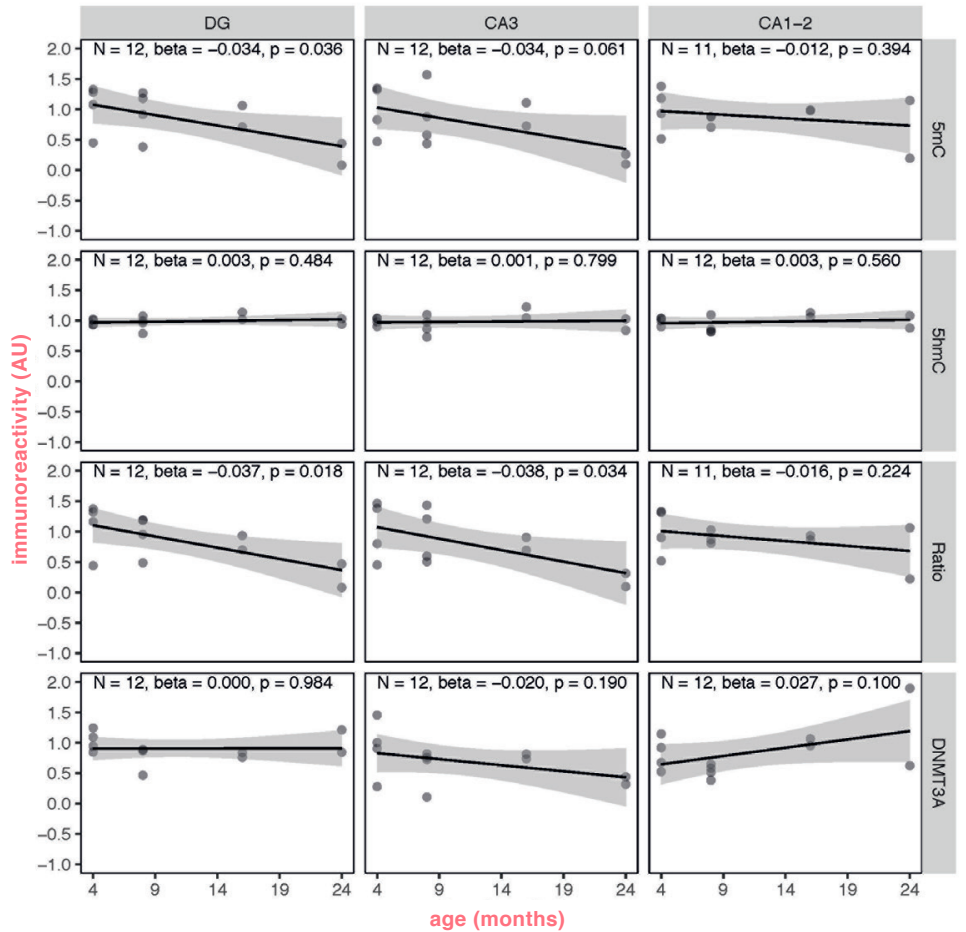

APP/PS1dE9 mice
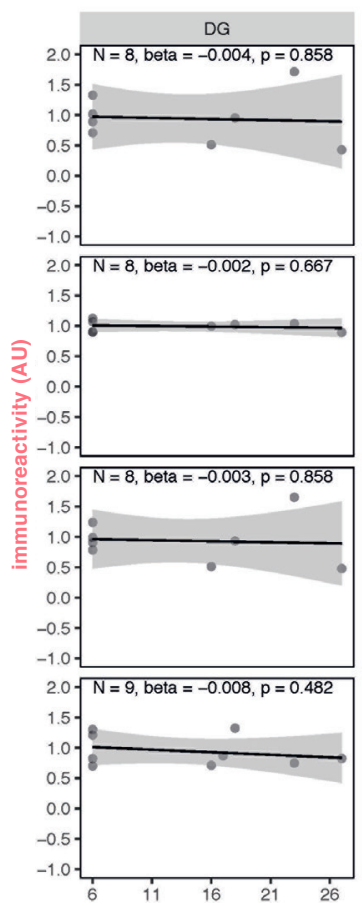

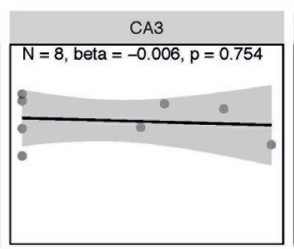

$\mathrm{N}=8$, beta $=-0.006, p=0.257$
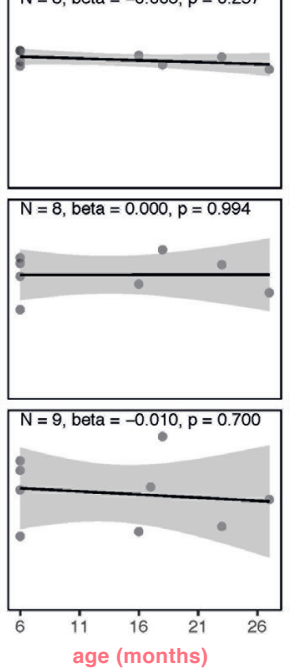

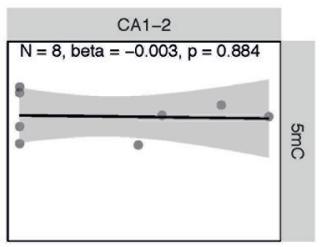

$\mathrm{N}=8$, beta $=-0.004, p=0.510$

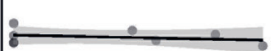

है
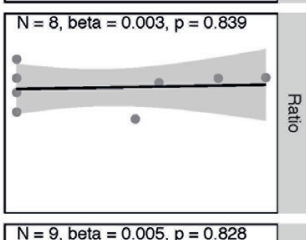

$N=9$, beta $=0.005, p=0.828$
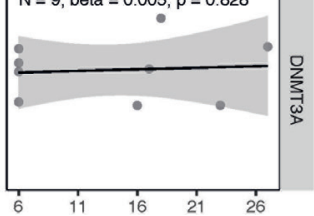

$\frac{01}{3}$

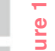

는

5-mC:5-hmC ratio in J20 mice. Shown are the background-corrected and scaled integrated density data plotted against the age of the animals, the fitted linear regression lines and the standard error (SE) of the regression lines, for the dentate gyrus (DG), cornu ammonis (CA) 3, and CAl-2 subregions of the hippocampus. A statistically significant effect of age on 5 -mC IR was found in the DG $(p=0.036)$, and on the $5-\mathrm{mC}: 5-\mathrm{hmC}$ ratio in the DG $(p=0.018)$ and CA3 $(p=0.034)$. AU, arbitrary units.

FIGURE 2. Semi-quantitative analysis results of age-related alterations in 5-methylcytosine (5-mC), 5 -hydroxymethylcytosine $(5$-hmC) and DNA methyltransferase (DNMT) $3 \mathrm{~A}$ immunoreactivity (IR), and the 5-mC:5-hmC ratio in APP/PSldE9 ¿ mice. Shown are the background-corrected and scaled integrated density data plotted against the age of the animals, the fitted linear regression lines and the standard error (SE) of the regression lines, for the dentate gyrus (DG), cornu ammonis (CA) 3, and CAl-2 subregions of the hippocampus. No statistically significant effect of age on any of the investigated epigenetic markers was found. AU, arbitrary units. 
FIGURE 3. Semi-quantitative analysis results of age-related alterations in 5 -methylcytosine $(5-\mathrm{mC})$, 5 -hydroxymethylcytosine $(5-\mathrm{hmC})$ and DNA methyltransferase (DNMT) $3 \mathrm{~A}$ immunoreactivity (IR), and the 5-mC:5-hmC ratio in 3xTg-AD mice. Shown are the background-corrected and scaled integrated density data plotted against the age of the animals, the fitted linear regression lines and the standard error (SE) of the regression lines, for the dentate gyrus (DG), cornu ammonis (CA) 3, and CAl-2 subregions of the hippocampus. A statistically significant effect of age on 5 -mC IR was found in the DG $(p=0.034)$ and CAl-2 $(p=0.045)$. AU, arbitrary units.

FIGURE 4. Semi-quantitative analysis results of age-related alterations in 5 -methylcytosine $(5-\mathrm{mC})$, 5-hydroxymethylcytosine $(5-\mathrm{hmC})$ and DNA methyltransferase (DNMT) $3 \mathrm{~A}$ immunoreactivity (IR), and the 5-mC:5-hmC ratio in Caribbean vervets. Shown are the background-corrected and scaled integrated density data plotted against the age of the animals, the fitted linear regression lines and the standard error (SE) of the regression lines, for the dentate gyrus (DG), cornu ammonis (CA) 3 , and CAl-2 subregions of the hippocampus. No statistically significant effect of age on any of the investigated epigenetic markers was found. AU, arbitrary units.
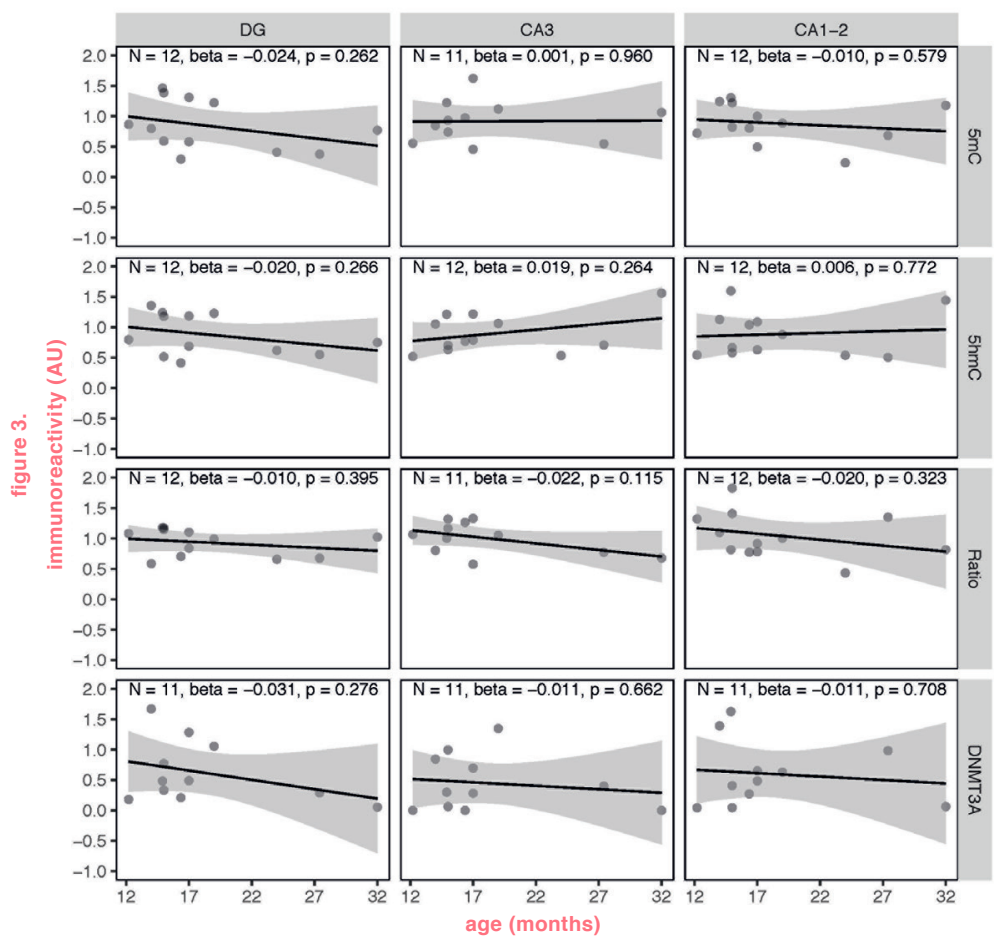

Caribbean vervets
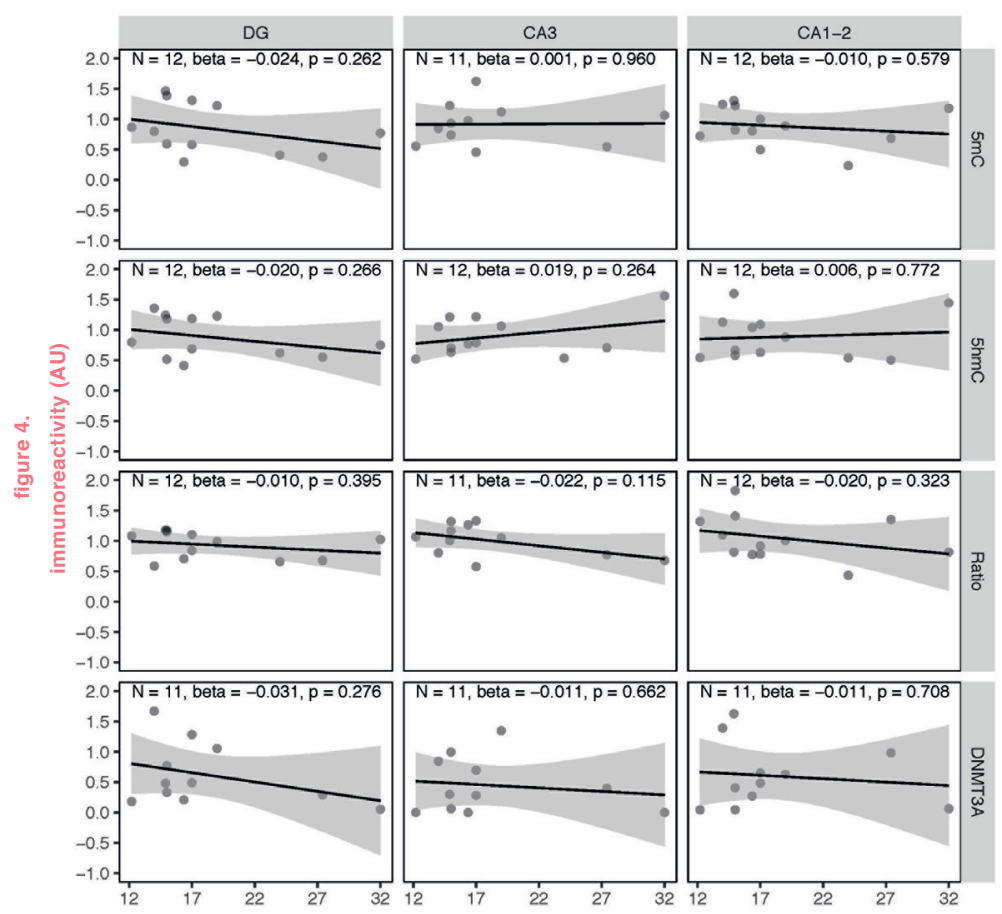

$\mathrm{N}=12$, beta $=0.019, \mathrm{p}=0.264$
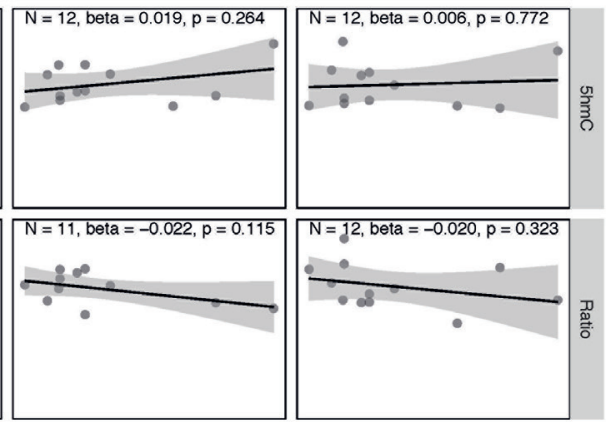

$N=11$, beta $=-0.011, p=0.662$
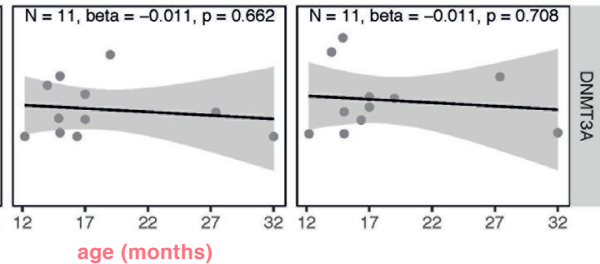


\subsubsection{Plaque load correlates with age-related changes in 5-mC IR}

As expected, all of the oldest animals of the transgenic mouse models exhibited $A \beta$ plaques (Supplementary Figures 6-8), varying between $17 \%$ and $63 \%$ of the hippocampus being covered in plaques. The regional distribution of the plaques in the different hippocampal subregions was, however, highly variable between the different mouse models. Hippocampal plaque density was the highest in the J20 model and plaques were observed within (and in vicinity of) the investigated subregions (Supplementary Figure 6). In the APP/PS1dE9 model, A plaques were present throughout the hippocampus, but the load was lower than that seen in the J20 model (Supplementary Figure 7). In the 3xTg-AD model, however, plaques were mainly located in the dorsal subiculum and deeper layers (oriens and alveus) of the hippocampus, with a generally low plaque load close to the investigated regions (Supplementary Figure 8). In the vervets, six animals (of 15, 16.4, 19, 24, 27.4 and 32 years old) had plaque pathology in temporal cortex and/or hippocampus, although not all had hippocampal plaques. Plaques were not seen in these brain regions in the other six vervets (of 12.2, 14, 14.9, 15,17 and 17 years old). The oldest animals did not necessarily have the most severe pathology (Supplementary Figure 9).

Correlation analysis between plaque load and 5-mC, 5-hmC, or DNMT3A IR revealed statistically significant inverse correlations in the J20 model between plaque load and 5-mC IR in the DG $(r=-0.60, p=0.039)$ and between plaque load and the $5-\mathrm{mC}: 5-\mathrm{hmC}$ ratio in the $\mathrm{DG}(r=-0.66$, $p=0.020)$ and CA3 $(r=-0.60, p=0.038)$ (Figure 5$)$. No statistically significant correlations between plaque load and epigenetic markers were observed in the APP/PS1dE9 model (Figure 6). In the 3xTg-AD model statistically significant positive correlations were found between plaque load and 5-mC IR in the DG $(r=0.70, p=0.026)$ and CA1-2 $(r=0.69, p=$ 0.027 ), as well as between plaque load and the $5-\mathrm{mC}: 5-\mathrm{hmC}$ ratio in the DG ( $r=0.67, p=0.034)$ (Figure 7$)$. No statistically significant correlations between plaque load and epigenetic markers were observed in the vervets (Figure 8).

\subsection{Discussion}

Age-related alterations of 5-mC, 5-hmC, and DNMT3A, three epigenetic markers previously associated with aging, age-related cognitive decline, 
FIGURE 5. Correlation analysis results between epigenetic markers 5 -methylcytosine (5-mC), 5-hydroxymethylcytosine (5-hmC) and DNA methyltransferase (DNMT) 3A immunoreactivity (IR), and the 5-mC:5-hmC ratio, and plaque load in J20 mice. Shown are the background-corrected and scaled integrated density data plotted against the scaled fraction of hippocampal area covered by plaques, for the dentate gyrus (DG), cornu ammonis (CA) 3, and CAl-2 subregions of the hippocampus. Fitted linear regression lines are shown for clarity. A statistically significant correlation with plaque load was found for 5 -mC IR in the DG $(r=-0.60, p=0.039)$, and for the 5 -mC:5-hmC ratio in the DG $(r=$ $-0.66, p=0.020)$ and CA3 $(r=-0.60, p$ $=0.038) . \mathrm{AU}$, arbitrary units.

FIGURE 6. Correlation analysis results between epigenetic markers 5 -methylcytosine (5-mC), 5-hydroxymethylcytosine $(5-\mathrm{hmC})$ and DNA methyltransferase (DNMT) $3 \mathrm{~A}$ immunoreactivity (IR), and the 5-mC:5-hmC ratio, and plaque load in APP/PSldE9 mice. Shown are the background-corrected and scaled integrated density data plotted against the scaled fraction of hippocampal area covered by plaques, for the dentate gyrus (DG), cornu ammonis (CA) 3, and CAl-2 subregions of the hippocampus. Fitted linear regression lines are shown for clarity. No statistically significant correlation was found between plaque load and any of the investigated epigenetic markers. AU, arbitrary units.

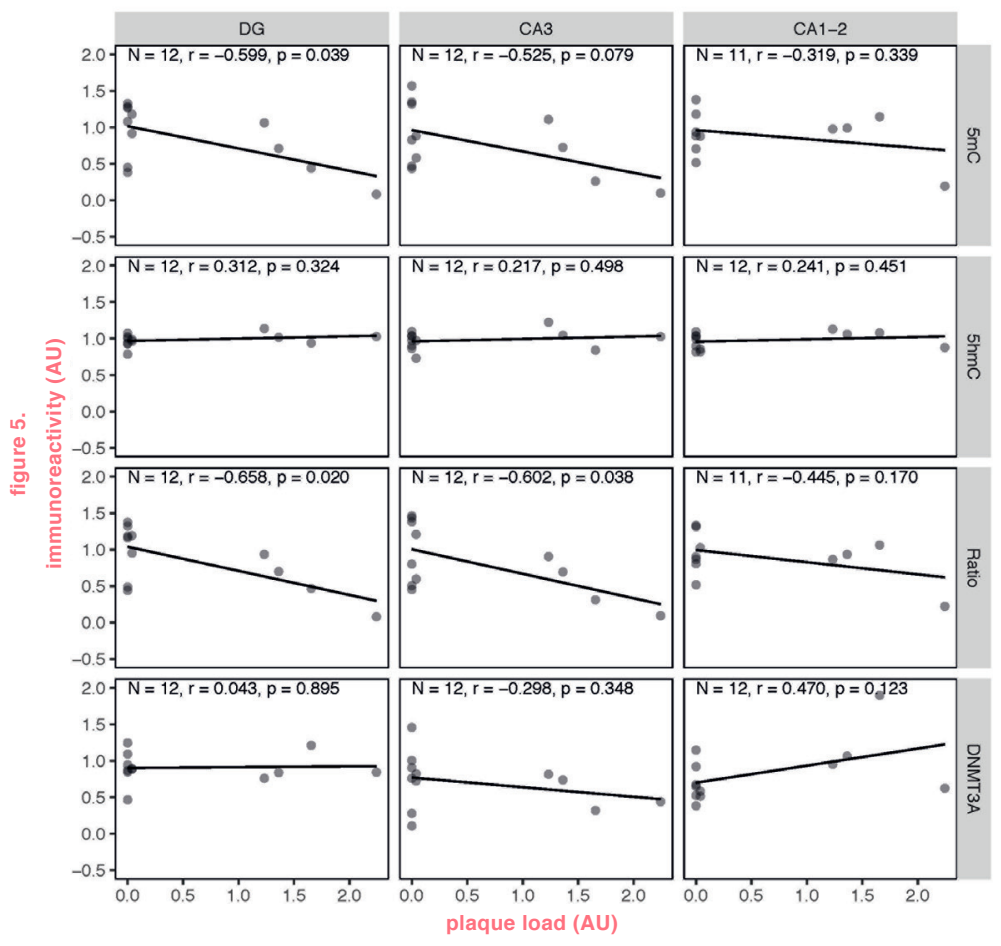

APP/PS1dE9 mice

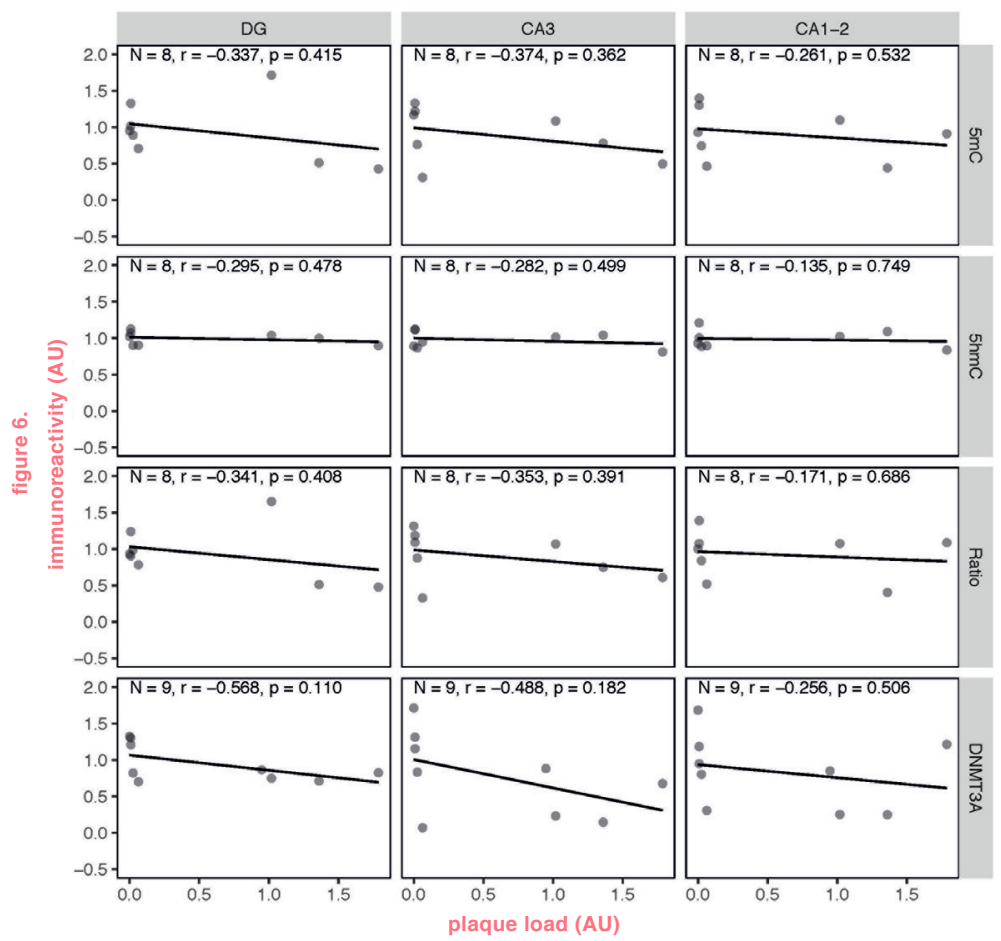



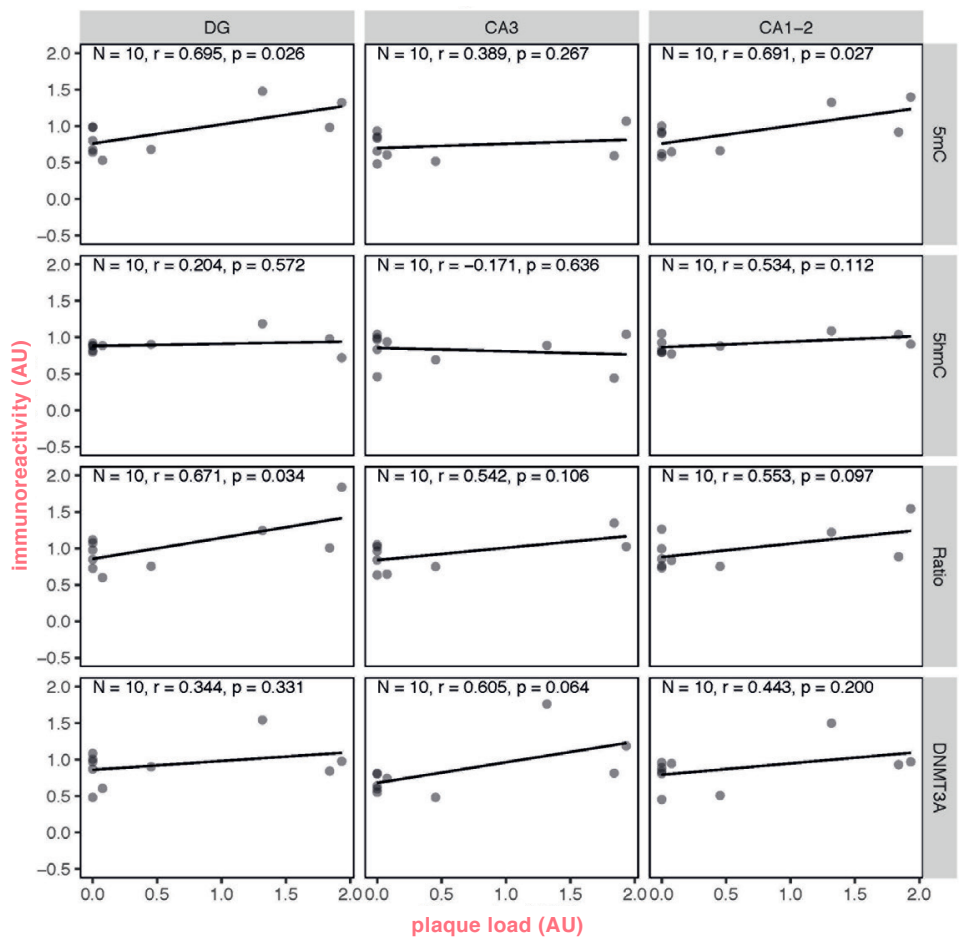

Caribbean vervets
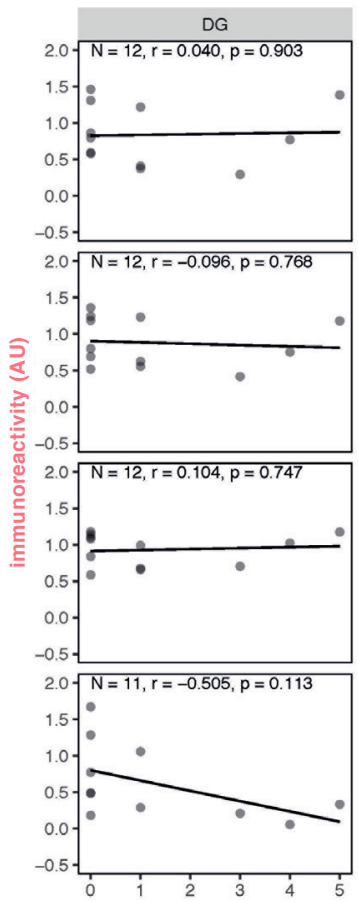
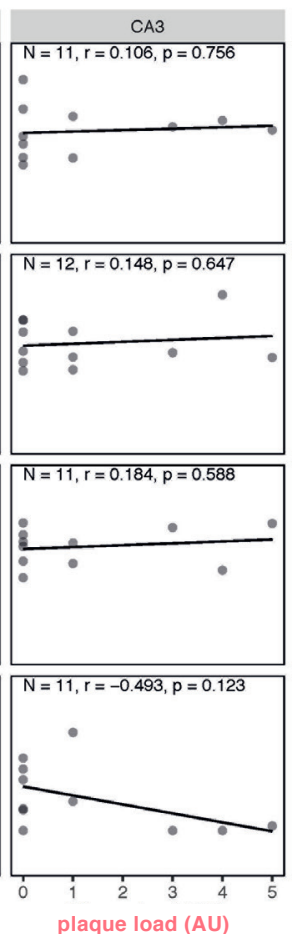
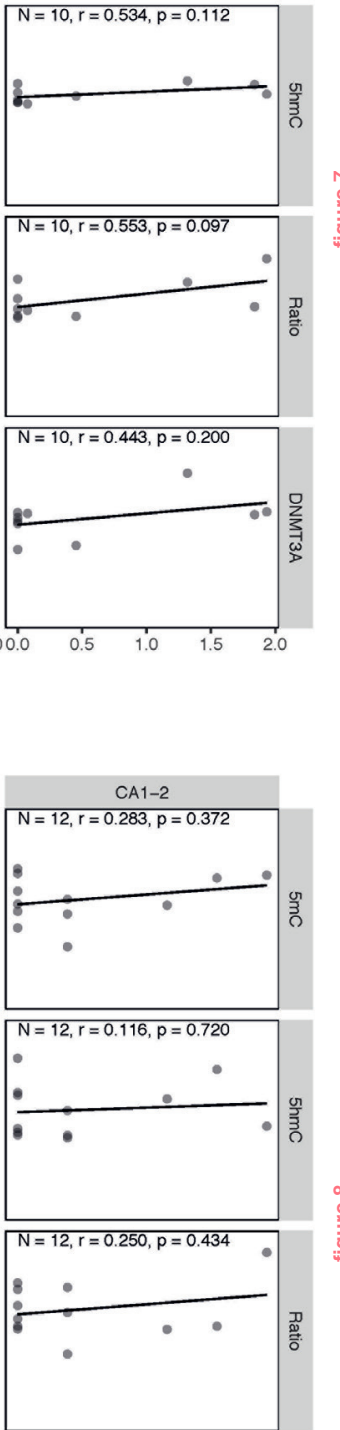

$\mathrm{N}=11, \mathrm{r}=-0.552, \mathrm{p}=0.078$

-

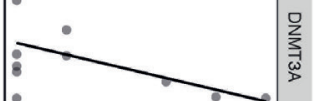

FIGURE 7. Correlation analysis results between epigenetic markers 5-methylcytosine (5-mC), 5-hydroxymethylcytosine $(5-\mathrm{hmC})$ and DNA methyltransferase (DNMT) 3A immunoreactivity (IR), and the 5-mC:5-hmC ratio, and plaque load in 3xTg-AD mice. Shown are the background-corrected and scaled integrated density data plotted against the scaled fraction of hippocampal area covered by plaques, for the dentate gyrus (DG), cornu ammonis (CA) 3, and CAl-2 subregions of the hippocampus. Fitted linear regression lines are shown for clarity. A statistically significant correlation with plaque load was found for $5-\mathrm{mC}$ IR in the DG ( $r=0.70, p=0.026)$ and CAAl-2 $(r=0.69, p=0.027)$, and for the 5 -mC:5-hmC ratio in the DG $(r=0.67$, $p=0.034)$. AU, arbitrary units.

FIGURE 8. Correlation analysis results between epigenetic markers 5-methylcytosine (5-mC), 5-hydroxymethylcytosine $(5-\mathrm{hmC})$ and DNA methyltransferase (DNMT) 3A immunoreactivity (IR), and the 5-mC:5-hmC ratio, and plaque load in Caribbean vervets. Shown are the background-corrected and scaled integrated density data plotted against the scores of hippocampal plaque load, for the dentate gyrus (DG), cornu ammonis (CA) 3, and CAl-2 subregions of the hippocampus. Fitted linear regression lines are shown for clarity. No statistically significant correlation was found between plaque load and any of the investigated epigenetic markers. AU, arbitrary units. 
and/or $A D$, were investigated in 3 transgenic mouse models of $A D$ and a non-human primate model that is known to develop $A \beta$ plaque pathology with age. Semi-quantitative analysis of 5-mC, 5-hmC, and DNMT3A IR indicated striking differences in age-related DNA methylation patterns between the different models, while none of the models showed agerelated differences in levels of DNA hydroxymethylation and DNMT3A. Generally, plaque load correlated with DNA methylation, but only in the J20 and 3xTg-AD models where age-related DNA methylation changes were detected, although in opposite directions.

\subsubsection{Age-related decrease of DNA methylation levels in the DG and CA3 of J20 mice}

In the present study, aging was associated with a decrease in 5-mC $\mathrm{IR}$ in the $\mathrm{DG}$ and a decrease of the $5-\mathrm{mC}: 5-\mathrm{hmC}$ ratio in the $\mathrm{DG}$ and CA3. Since 5-hmC seems to remain stable with age, this decrease in the $5-\mathrm{mC}: 5-\mathrm{hmC}$ ratio is likely due to a decrease in $5-\mathrm{mC}$, even though 5-mC IR alone was not significantly decreased in the CA3. Furthermore, correlation analysis between hippocampal plaque load and the investigated epigenetic markers only showed a statistically significant correlation between plaque load and 5-mC in the DG and the 5-mC:5$\mathrm{hmC}$ ratio in the DG and CA3 region; the same areas that showed agerelated alterations.

Transgenic J20 mice express human APP with both the Swedish and Indiana mutations associated with the development of familial AD [34]. It has previously been reported that these mice already show cognitive deficits starting at 1-2 months of age, but that these impairments do not seem to progress with age up to the development of plaque pathology, which starts around 6 months of age, but ramps up around 10 months of age $[34,38]$.

It is most likely that in these models based on mutations seen in familial $A D$, epigenetic alterations play a role in the progression of the disease and are instigated through other pathological processes directly related to these mutated genes. For instance, $A \beta$ has been reported to influence DNA methylation, inducing global hypomethylation [45], which is in line with the observations in the J20 model in the present study. Interestingly, the observation that the promoter of the MAPT gene, which 
has been implicated in AD, was hypomethylated in J20 mice already at 5 months of age indicates that epigenetic processes may be involved in the development of $A D$ before the occurrence of senile plaques [46]. Note, however, that the global changes in DNA methylation observed in the present study occur after the development of extracellular plaque formation.

Another study reports increased levels of histone deacetylase (HDAC) activity in J20 mice 19-20 months of age [47]. Although an increase in HDAC activity is generally linked to an increase in DNA methylation [48], this is not consistently observed in AD [9]. This inconsistency is also reflected in the present study and may implicate possible methodological differences or the existence of different pathological mechanisms with alternative effects on epigenetic profiles.

\subsubsection{Epigenetic markers remain stable with age in APP/PSldE9 mice}

In the present study, extensive plaque deposition was observed in the hippocampus of APP/PS1dE9, but no age-related epigenetic changes were detected. The APP/PS1dE9 transgenic mouse model expresses human PS1 with the deletion of exon 9 and humanized APP with the Swedish mutation [36, 49]. This mouse model is widely used and cognitive impairments have been reported for spatial working memory as early as 4 months of age, with additional impairments in reference memory, associative learning, and passive avoidance with increasing age $[38,40,50]$.

Another study in the APP/PS1dE9 mice revealed that treatment with HDAC inhibitors can ameliorate cognitive impairments, but that there are no global differences in hippocampal histone $(\mathrm{H}) 3$ and $\mathrm{H} 4$ acetylation levels [51]. Interestingly, studies in similar models with the same ADrelated genes, but different mutations, have reported different results. A study using an APP/PS1 model expressing human PS1 with the M146V mutation, found hippocampal decreases in $\mathrm{H} 4$ acetylation after fear conditioning, compared to wild-type mice [52]. Treatment with an HDAC inhibitor was able to both rescue the acetylation levels and behavioral responses in the APP/PS1 mice. Similar findings were obtained in APP/ PS1 mice with the APP KM670/671NL and PS1 L166P mutations [53], and in APP/PS1 mice with chimeric APP with the K670N/M671L mutation and the PS1 A246E mutation [54]. 
A genome-wide analysis of DNA methylation has also been performed in cortex of the APP/PS1dE9 model [55]. In this study, sites that were observed to be differentially methylated in the APP/PS1dE9 model, as compared to wild-type controls, were mainly hypermethylated. Additionally, they identified transforming growth factor $\beta 1$ and its associated signaling pathway to be mainly dysregulated. However, due to the differences in approach and brain area, these results cannot be reliably compared with the present findings.

\subsubsection{DNA methylation levels increase in the DG and $\mathrm{CAl}-2$ of $3 \times \mathrm{xT}-\mathrm{AD}$ mice}

In contrast to the J20 and APP/PS1 models, the 3xTg-AD model, expressing mutated APP, PS1, and tau, not only develops plaques, but also neurofibrillary tangles [37]. Contrary to the J20 model, the 3xTg-AD model shows an increase in DNA methylation in the DG and CA1-2 subregions. A possible explanation for this disparity between the models would be the addition of the mutated MAPT transgene, although there is evidence that tau does not cause global hypermethylation, but hypomethylation through oxidative stress and DNA damage [56]. The $3 x T g-A D$ mice are reported to develop extracellular amyloid deposits in frontal cortex by 6 months, which progressively spread throughout the brain by 12 months of age [37], and tau pathology which appears later, after about 12 to 15 months [41]. We observed a 2-3 month delay in AD pathology within our 3xTg-AD colony, possibly due to reduced transgene copies with successive breeding (see https://www.jax.org/strain/004807).

However, our findings confirm that hippocampal plaque formation occurs mainly in the deeper layers of the hippocampus and subiculum, and fewer plaques were seen in the DG, CA3, and CA1-2 subregions when compared to the J20 and APP/PS1 models. Importantly, cognitive impairments occur before the development of plaques and tangles, starting around 4 months, and correlate with intraneuronal $A \beta$ [41].

The direction of the DNA methylation changes may appear contra intuitive, but it appears that other studies investigating epigenetic changes in this triple transgenic model have made similar observations. For instance, Sanchez-Mut et al. [57] found an increase in TBXA2R, F2RL2, SPNB4, and SORBS3 methylation, with a decrease in the corresponding mRNA levels in the cortex of 3xTg-AD mice. Walker et al. [58] investigated age-related histone modification changes in neurons 
from wild-type and 3xTg-AD mice. They found that the repressive $\mathrm{H} 3$ lysine (K) 9 methylation marker increased with age in 3xTg-AD neurons, more so than in wild-type neurons. This finding of increased epigenetic repression of gene expression is confirmed by the detection of lower BDNF gene expression. Importantly, increases in H3K9 methylation were already observed at 4 months, the same age at which cognitive deficits start to emerge [58]. Another study focusing on the hippocampus of $3 x T g-A D$ mice reported an age-related loss of $\mathrm{H} 3 \mathrm{~K} 4$ trimethylation, an epigenetic marker that is associated with decreased gene expression [59]. These observations of increased epigenetic repression are in line with the present study, as DNA methylation is also generally associated with suppression of gene expression [60].

Interestingly, however, in neurons isolated from 3xTg-AD mice, Walker et al. [58] also observed increases in $\mathrm{H} 3$ and $\mathrm{H} 4$ acetylation levels with age; epigenetic markers which are associated with enhanced gene expression. As stated previously, these markers decreased in the J20 and APP/ PS1 models. A gene-specific analysis showed that the promoter region of the AD-associated BACE1 gene exhibited increased $\mathrm{H} 3$ acetylation, concomitant with increased mRNA levels, in the cortex of 3xTg-AD mice [61]. H4K12 acetylation has also been investigated as a potential biomarker in blood monocytes of 3xTg-AD mice and it was found that this marker was elevated at 10 months of age, during the development of plaque pathology, but not anymore at 20 months of age when plaque pathology is already widespread [62].

Nevertheless, treatment with HDAC inhibitors is also able to improve cognition in the 3xTg-AD mice [63, 64]. It is therefore necessary for future studies to elucidate which genes are affected by these global changes in histone acetylation, as treatment with HDAC inhibitors may have a beneficial effect independent of AD-related alterations in histone acetylation. This is exemplified by studies showing beneficial effects of HDAC inhibitors during normal aging [65].

In the present study, we do not detect any significant age-related changes in 5-hmC IR. Another immunohistochemical study, however, comparing wild-type and 3xTg-AD mice at 17 months detected an increase in cortical 5-hmC levels [66]. These differences may be due to differences in methodology, brain area investigated, and age of the studied animals, and stress the sensitive and complex nature of epigenetic processes and investigations thereof. 


\subsubsection{No age-related alter- ations of global epigenetic marks are detected in the hippocampus of Caribbean vervets}

We observed no age-related changes in the level of epigenetic marks in the hippocampus of vervets. As opposed to the transgenic mouse models, the vervets serve as a more natural model of $A D$ as some of these non-human primates develop AD-like pathology as they age, without the introduction of mutated transgenes. Interestingly, as AD in humans generally develops at advanced ages, vervets exhibit plaque pathology as early as 15 years. While their lifespan is only 15 to 20 years in the wild, it is 20 to 30 years in captivity [33]. In general, plaque deposition in vervets starts in the frontal cortex and spreads with age (C. Lemere, personal communication), sometimes approaching, by 30 years of age, $A \beta$ pathology as seen in human AD. In the J20 and 3xTg-AD models, the most drastic changes in the levels of the studied epigenetic marks were seen in the extremely old age groups. Thus, it may thus be the case that AD-pathology in the vervets was not advanced enough, especially in the hippocampus, to induce detectable changes. As the pathology in the vervets was much more variable than in the transgenic mice, the sample size may have been too small to detect significant correlations between age, $A \beta$ pathology, and epigenetic markers. Alternatively, the long-term fixation of the archived vervet brain tissue may have limited the accessibility of antigens for accurate immunohistochemical detection.

Other studies focusing on age-related epigenetic alterations in vervets are scarce, although a study investigating blood DNA methylation in relation to a high fat diet also found no significant association between age and DNA methylation levels (ages between 9.7 and 23.7 years) [67].

\subsubsection{Translational validity}

Given the discrepancy in observations regarding DNA methylation across the different animal models, it is essential to compare the results from the animal models with observations from human and related studies in order to elucidate how the genetically different models may be able to reflect the epigenetic alterations associated with AD. However, as noted previously, differences in brain regions and methodology already hamper 
comparisons between studies performed on human tissue, let alone comparisons with other species. Additionally, whereas animal models have the advantage to facilitate the study of the temporal sequence of events, human studies focusing on brain markers generally need to rely on postmortem tissue. Therefore, instead of comparing changes over time, they compare diseased with control brains. It is also important to consider that non-human primates and humans have greater genetic heterozygosity and environmental diversity than homogenous transgenic mouse models.

Targeted approaches aside, there are several studies that investigated AD-related global changes in DNA methylation and hydroxymethylation markers using human brain material. Initial studies, also by our group, have shown an AD-associated global DNA hypomethylation and hypohydroxymethylation in the entorhinal cortex and hippocampus $[23,24]$. These studies also found an AD-associated decrease in DNA methylation and hydroxymethylation in a monozygotic twin pair of which only one developed AD. Other groups, however, either did not find significant changes in global DNA methylation in the entorhinal cortex [68], or even increases in DNA methylation and hydroxymethylation in the hippocampus, middle frontal gyrus and middle temporal gyrus $[26,69]$. Although these studies do not all show the same direction of change, they observe similar alterations in DNA methylation and hydroxymethylation, whereas Condliffe et al. [25] found a decrease of only DNA hydroxymethylation in the entorhinal cortex and cerebellum. Rao et al. [70] only investigated DNA methylation and found an increase in the frontal cortex of AD patients. Using the HumanMethylation450 BeadChip assay on tissue from the dorsolateral prefrontal cortex De Jager et al. [27] reported a modest increase of the methylation value of differentially methylated loci in AD.

Since findings in the same brain region also disagree, it is unlikely that the discrepancies can be explained solely by differences in brain areas. As has been noted previously [9, 26, 68], the most likely explanation would be differences in methodology, such as tissue processing and quantification methods. To elucidate how differences in methodology can influence detected DNA methylation and hydroxymethylation levels, different procedures should be systemically tested on the same tissue, and vice versa, the same approach should be used on various tissues. For the present study, however, similar methodology was used to process all of the mouse tissues and to quantify the epigenetic markers. The different observations for the various mouse models would thus point to a genotype effect, possibly due to the expression of different transgenes and/or the use of different promoters. A genotype effect, in turn, is unlikely to explain the differences between the human studies as they generally 
study mixed samples of, based on the average age, mainly late-onset $A D$, although due to the large age-range and lack of (reported) genetic tests also familial cases may be included [26].

To get a better idea of the direction of the epigenetic changes in AD it may help to look at related studies. Observations from in vitro work related to the effect of $A \beta$ and APP mutations on DNA methylation, are more consistent and indicate there is global hypomethylation [45, 71, 72]. Additionally, work on tau indicates that it induces global heterochromatin loss, which may lead to aberrant gene expression patterns in AD [56]. A loss of heterochromatin also points towards a hypomethylated state of the DNA.

DNMTs depend on S-adenosylmethionine (SAM) as methyl donor and some studies have found striking deficiencies of SAM and S-adenosylhomocysteine (SAH), the demethylated metabolite of SAM, throughout the AD-afflicted brain and cerebrospinal fluid [73-75]. In vitro work investigating the relationship between folate, SAM, and DNA methylation indicates a SAM deficiency leads to global hypomethylation [76]. Another study found increased levels of brain SAH in AD patients and showed that SAH inhibits methyltransferases, suggesting that increased levels of SAH would also lead to DNA hypomethylation [77]. Others have indeed shown decreased methyltransferase activity in the brain of AD patients [78]. Inhibition of DNMTs could also explain alterations in DNA methylation, without changes in the levels of DNMT3A, as seen in the present study.

The direct and indirect evidence mainly points towards a hypomethylated state in AD. Of the investigated models, only the J20 mice exhibit an agerelated global DNA hypomethylation in the hippocampus and therefore seems to best capture this view. Of note, in light of previous observations that DNA methylation increases with age $[17,19]$, the lack of an increase in global DNA methylation in the old mice of the APP/PS1dE9 model could be interpreted as hypomethylation when compared to normal aging. Similarly, DNA hydroxymethylation and DNMT3A levels have been observed to increase with age [16, 18, 20, 21], therefore, the lack of an age-related change in these markers could be seen as a decrease in comparison to wild-type aged animals. This, however, should be confirmed through a direct comparison of transgenic and non-transgenic litter-mates. 


\subsubsection{Strengths, limitations, and future perspectives}

A strength of the present study is the inclusion of multiple animal models, which has provided crucial insights in how the different mouse models capture $A D$ on an epigenetic level. The inclusion of a non-transgenic non-human primate model provides an additional angle more related to sporadic $A D$, in contrast to the transgenic mouse models, which are limited to familial forms of AD. Additionally, the use of established immunohistochemistry-based techniques allows the subregion-specific qualitative and semi-quantitative analysis of epigenetic markers. Importantly, by using highly specific antibodies $5-\mathrm{mC}$ and 5 -hmC can be reliably distinguished $[18,79]$, which is not possible with most other commonly used techniques for 5-mC detection [14].

The study also has its limitations, which thus should be taken into account when interpreting the results. First, although the staining and imageanalysis procedures were identical for all the models and performed at the same time, the breeding, sacrificing and tissue processing were not done at the same time, which may have resulted in slight differences between the mouse models. The vervet tissue was differently processed and fixed for a longer time, which may affect immunoreactivity. Additionally, the vervets did not live under strict experimentally controlled conditions such as the mouse models, which may result in more variation. The exclusion of an aging wild-type mouse group may be seen as a limitation, but our group has previously reported extensive epigenetic investigations in normally aging mice [16-18, 22], which were done in a similar manner and can therefore be used to compare the current findings in relation to $A D$ with.

The borderline significance often observed in the present study suggests that it could have benefitted from larger sample sizes to increase power. However, since this explorative study depended on the availability of animals from other studies, it was not possible to increase the sample sizes. It remains an important point, which has been previously raised $[80,81]$, that most current epigenetic studies are relatively small, which may result in the large differences in results. Therefore, there is a need for large, high-powered studies that may provide more conclusive results. Nevertheless, the present study serves as an important foundation to guide future AD-related epigenetics research in animal models. 


\section{REFERENCES}

[1] Sosa-Ortiz AL, Acosta-Cas-

tillo I, Prince MJ. Epidemiology of dementias and Alzheimer's disease. Arch Med Res 2012; 43: 600-608.

[2] Raina P, Santaguida P, Ismaila A, et al. Effectiveness of cholinesterase inhibitors and memantine for treating dementia: evidence review for a clinical practice guideline. Ann Intern Med 2008; 148: 379-397.

[3] Lansdall CJ. An effective treatment for Alzheimer's disease must consider both amyloid and tau. Biosci Horizons 2014; 7: hzu002.

[4] Stratmann K, Heinsen H, Korf HW, et al. Precortical phase of Alzheimer's disease (AD)-related tau cytoskeletal pathology. Brain Pathol. Epub ahead of print 2015. DOI: 10.1111/ bpa.12289.

[5] Bartsch T, Wulff P. The hippocampus in aging and disease: From plasticity to vulnerability. Neuroscience. Epub ahead of print 2015. DOI: 10.1016/j.neuroscience.2015.07.084.

[6] Kurz A, Perneczky R. Novel insights for the treatment of Alzheimer's disease. Prog Neuropsychopharmacol Biol Psychiatry 2011; 35: 373-379.

[7] Defina PA, Moser RS, Glenn M, et al. Alzheimer's disease clinical and research update for health care practitioners. J Aging Res 2013; 2013 : 207178.

[8] Price DL, Sisodia SS.

Mutant genes in familial Alzheimer's disease and transgenic models. Annu Rev Neurosci 1998; 21: 479-505.

[9] Lardenoije R, Iatrou A, Kenis $\mathrm{G}$, et al. The epigenetics of aging and neurodegeneration. Prog Neurobiol 2015; 131: 21-64.

[10] Van den Hove DL, Kompotis K, Lardenoije R, et al. Epigenetically regulated microRNAs in Alzhei-
5.5. Conclusion

This study set out to determine age-related changes in immunohistochemically detectable markers related to DNA methylation in several widely used and genetically different animal models of $A D$, and to determine how these models reflect the epigenetic changes observed in AD. In the J20 model global DNA hypomethylation was observed in the hippocampus, while in the 3xTg-AD model global hypermethylation was observed. No alterations in DNA hydroxymethylation or DNMT3A levels were detected in these models. In APP/PS1dE9 and Caribbean vervets, no age-related global epigenetic changes were observed. Plaque load correlated with DNA methylation in those cases where age-related changes were detected, with a negative correlation in the J20 model and a positive correlation in the 3xTg-AD model. The main differences between the investigated models were thus in the age-related changes in DNA methylation. Other studies looking at DNA methylation or the effects of $A \beta$, tau, and SAM/SAH on DNA methylation report mixed results, but appear to lean towards global hypomethylation in AD. Although this would suggest the J20 model best captures the global epigenetic changes related to $A D$, it appears the different genotypes of the models differentially affect the course of age-related epigenetic changes. Exactly how mutations in the $A P P, P S 1$, and MAPT genes interact with the epigenome remains to be elucidated in future studies.

\section{Acknowledgements}

We kindly thank $\mathrm{H}$ Crehan, Q Shi, and S Chowdhury for technical assistance. Funds have been provided by the Internationale Stichting Alzheimer Onderzoek (ISAO) grants 07551 and 11532 (D.L.A.vdH.), by the ISAO grants 09552 and 13515, and the Netherlands Organization for Scientific Research (NWO), grant 916.11.086 (Veni Award) (B.P.F.R.), by an ISAO fellowship and a fellowship as part of NWO grant 022.005.019, (R.L.), and by an Anonymous Foundation and NIH/NIA R01 AG040092 (C.A.L.). Additional funds have been provided by the Joint ProgrammeNeurodegenerative Disease Research (JPND) for the EPI-AD consortium (http://www.neurodegenerationresearch.eu/wp-content/uploads/2015/10/ Factsheet_EPI-AD.pdf). The funding agencies were not involved in the study design, data collection, analysis and interpretation, writing of the report, and the decision to submit the article for publication. The authors declare no conflicts of interest. 
mer's disease. Neurobiol Aging 2014; 35: 731-745.

[11] Iatrou A, Kenis G, Rutten $\mathrm{BPF}$, et al. Epigenetic dysregulation of brainstem nuclei in the pathogenesis of Alzheimer's disease: looking in the correct place at the right time? Cellular and Molecular Life Sciences, 2016, pp. $1-15$.

[12] Choudhuri S. From

Waddington's epigenetic landscape to small noncoding RNA: some important milestones in the history of epigenetics research. Toxicol Mech Methods 2011; 21: 252-274.

[13] Liu L, Li Y, Tollefsbol TO. Gene-environment interactions and epigenetic basis of human diseases. Curr Issues Mol Biol 2008; 10: 25-36. [14] van den Hove DLA, Chouliaras L, Rutten BPF. The role of 5-hydroxymethylcytosine in aging and Alzheimer's disease: current status and prospects for future studies. Curr Alzheimer Res 2012; 9: 545-9.

[15] Chouliaras L, Rutten BPF, Kenis G, et al. Epigenetic regulation in the pathophysiology of Alzheimer's disease. Prog Neurobiol 2010; 90: $498-510$.

[16] Chouliaras L, van den Hove DLA, Kenis G, et al. Caloric restriction attenuates age-related changes of DNA methyltransferase $3 a$ in mouse hippocampus. Brain Behav Immun 2011; 25 : 616-623.

[17] Chouliaras L, van den Hove DLA, Kenis G, et al. Prevention of age-related changes in hippocampal levels of 5-methylcytidine by caloric restriction. Neurobiol Aging 2012; 33: 1672-81.

\section{[18] Chouliaras L, van den}

Hove DLA, Kenis G, et al. Age-related increase in levels of 5-hydroxymethylcytosine in mouse hippocampus is prevented by caloric restriction. Curr Alzheimer Res 2012; 9: 536-44.

[19] Hernandez DG, Nalls MA Gibbs JR, et al. Distinct DNA methylation changes highly correlated with chronological age in the human brain. Hum Mol Genet 2011; 20: 1164-1172.

\section{[20] Münzel M, Globisch D,}

Brückl T, et al. Quantification of the sixth DNA base hydroxymethylcytosine in the brain. Angew Chemie Int Ed 2010; 49: 5375-5377.

[21] Song C-X, Szulwach KE,

Fu Y, et al. Selective chemical labeling reveals the genome-wide distribution of 5-hydroxymethylcytosine. Nat

Biotechnol 2011; 29: 68-72.

[22] Lardenoije R, van den Hove

DA, Vaessen TSJ, et al. Epigenetic

modifications in mouse cerebellar

Purkinje cells: effects of aging, caloric restriction, and overexpression of superoxide dismutase 1 on 5 -methylcytosine and 5-hydroxymethylcytosine. Neurobiol Aging 2015; 36: 3079-3089.

[23] Mastroeni D, Grover A,

Delvaux E, et al. Epigenetic changes

in Alzheimer's disease: Decrements in DNA methylation. Neurobiol Aging 2010; 31: 2025-2037.

[24] Chouliaras L, Mastroeni D, Delvaux E, et al. Consistent decrease in global DNA methylation and hydroxymethylation in the hippocampus of Alzheimer's disease patients.

Neurobiol Aging 2013; 34: 2091-2099.

[25] Condliffe D, Wong A,

Troakes C, et al. Cross-region reduction in 5-hydroxymethylcytosine in Alzheimer's disease brain. Neurobiol Aging 2014; 35: 1850-1854.

[26] Coppieters N, Dieriks B V, Lill C, et al. Global changes in DNA methylation and hydroxymethylation in Alzheimer's disease human brain. Neurobiol Aging 2014; 35: 1334-1344.
G, Lunnon K, et al. Alzheimer's disease: early alterations in brain DNA methylation at $A N K 1, B I N 1, R H B D F 2$ and other loci. Nat Neurosci 2014; 17: 1156-1163.

[28] Lunnon K, Smith R, Hannon E, et al. Methylomic profiling implicates cortical deregulation of $A N K 1$ in Alzheimer's disease. Nat Neurosci 2014; 17: 1164-1170.

[29] van Goethem NP, Lardenoije R, Kompotis K, et al. Cognitive disorders: impairment, aging, and dementia. In: In Vivo Models for Drug Discovery. Wiley-VCH Verlag $\mathrm{GmbH} \&$ Co. KGaA, pp. 349-366.

[30] Frost JL, Le KX, Cynis H, et al. Pyroglutamate-3 amyloid-beta deposition in the brains of humans, non-human primates, canines, and Alzheimer disease-like transgenic mouse models. Am J Pathol 2013; 183: $369-381$.

[31] Woodruff-Pak DS. Animal models of Alzheimer's disease: therapeutic implications. J Alzheimers Dis 2008; 15: 507-521.

[32] Cavanaugh SE, Pippin JJ, Barnard ND. Animal models of Alzheimer disease: historical pitfalls and a path forward. ALTEX 2014; 31: 279-302.

[33] Lemere CA, Beierschmitt

A, Iglesias M, et al. Alzheimer's

disease abeta vaccine reduces central nervous system abeta levels in a non-human primate, the Caribbean vervet. Am J Pathol 2004; 165: 283-297. [34] Mucke L, Masliah E, Yu G-Q, et al. High-level neuronal expression of abeta 1-42 in wild-type human amyloid protein precursor transgenic mice: synaptotoxicity without plaque formation. J Neurosci 2000 ; 20: 4050-4058. 
EM, Zhang-Nunes SX, et al. Characterization of amyloid deposition in the APPswe/PSldE9 mouse model of Alzheimer disease. Neurobiol Dis 2006; 24: $516-524$.

[36] Jankowsky JL, Slunt HH, Ratovitski T, et al. Co-expression of multiple transgenes in mouse CNS: a comparison of strategies. Biomol Eng 2001; 17: 157-165.

[37] Oddo S, Caccamo A, Shepherd JD, et al. Triple-transgenic model of Alzheimer's disease with plaques and tangles: intracellular Abeta and synaptic dysfunction. Neuron 2003; 39: 409-421.

[38] Webster SJ, Bachstetter AD, Nelson PT, et al. Using mice to model Alzheimer's dementia: an overview of the clinical disease and the preclinical behavioral changes in 10 mouse models. Front Genet 2014; 5: 88.

[39] Jankowsky JL, Fadale DJ, Anderson J, et al. Mutant presenilins specifically elevate the levels of the 42 residue beta-amyloid peptide in vivo: evidence for augmentation of a 42 -specific gamma secretase. Hum $\mathrm{Mol}$ Genet 2004; 13: 159-70.

\section{[40] Park JH, Widi GA, Gimbel}

DA, et al. Subcutaneous Nogo receptor removes brain amyloid-beta and improves spatial memory in Alzheimer's transgenic mice. J Neurosci 2006; 26 : 13279-86.

[41] Billings LM, Oddo S, Green KN, et al. Intraneuronal Abeta causes the onset of early Alzheimer's disease-related cognitive deficits in transgenic mice. Neuron 2005; 45: $675-88$.

[42] Wickham H, Francois R. dplyr: A grammar of data manipulation. R package version 0.4.3. http:// CRANR-project.org/package $=$ dplyr.
Harrell FE. Hmisc: Harrell

Miscellaneous. R package version 3.17-

0. http://CRANR-project.org/package $=$ Hmisc .

[44] Wickham H. ggplot2 -

Elegant Graphics for Data Analysis. New

York: Springer-Verlag. Epub ahead of print 2009. DOI: 10.1007/978-0-387-

98141-3.

[45] Chen K-L, Wang SS-S, Yang

Y-Y, et al. The epigenetic effects of amyloid-beta(1-40) on global DNA and neprilysin genes in murine cerebral endothelial cells. Biochem Biophys Res Commun 2009; 378: 57-61.

[46] Coupland K, Kim WS, Halliday G, et al. MAPT methylation in Alzheimer's disease. Alzheimer's Dement 2014; 10: P317-P318. [47] Kuo J-W, Su K-H, Wu C-Y, et al. 18F-FAHA PET signatures of histone deacetylase activity in the transgenic mouse model of Alzheimer's disease. J Nucl Med 2012; 53: 1902-.

\section{[48] Cedar H, Bergman Y.}

Linking DNA methylation and histone modification: patterns and paradigms.

Nat Rev Genet 2009; 10: 295-304.

[49] Jankowsky JL, Fadale DJ,

Anderson J, et al. Mutant presenilins specifically elevate the levels of the 42 residue -amyloid peptide in vivo: evidence for augmentation of a 42-specific secretase. Hum Mol Genet 2003; 13: 159-170.

[50] Frost JL, Liu B, Rahfeld

J-U, et al. An anti-pyroglutamate-3 A $\beta$ vaccine reduces plaques and improves cognition in APPswe/PS1 $\triangle \mathrm{E} 9$ mice.

Neurobiol Aging 2015; 36: 3187-99.

[51] Kilgore M, Miller CA, Fass

DM, et al. Inhibitors of class 1 histone deacetylases reverse contextual memory deficits in a mouse model of Alzheimer's disease. Neuropsychophar- macology 2010; 35: 870-80.

Francis YI, Fà M, Ashraf H, et al. Dysregulation of histone acetylation in the APP/PS1 mouse model of Alzheimer's disease. J Alzheimers Dis 2009; 18: 131-9.

[53] Govindarajan N, Agis-Balboa RC, Walter J, et al. Sodium butyrate improves memory function in an Alzheimer's disease mouse model when administered at an advanced stage of disease progression. J Alzheimers Dis 2011; 26: 187-97.

[54] Wang Z, Zhang X-J, Li T, et al. Valproic acid reduces neuritic plaque formation and improves learning deficits in APP(Swe) / PSl(A246E) transgenic mice via preventing the prenatal hypoxia-induced down-regulation of neprilysin. CNS Neurosci Ther 2014; 20: 209-17.

[55] Cong L, Jia J, Qin W, et al. Genome-wide analysis of DNA methylation in an APP/PSl mouse model of Alzheimer's disease. Acta Neurol Belg 2014; 114: 195-206.

[56]

Frost B, Hemberg M, Lewis J, et al. Tau promotes neurodegeneration through global chromatin relaxation. Nat Neurosci 2014; 17: 357-66. [57] Sanchez-Mut J V., Aso E, Panayotis N, et al. DNA methylation map of mouse and human brain identifies target genes in Alzheimer's disease. Brain 2013; 136: 3018-3027. [58] Walker MP, LaFerla FM, Oddo SS, et al. Reversible epigenetic histone modifications and Bdnf expression in neurons with aging and from a mouse model of Alzheimer's disease. Age (Dordr) 2013; 35: 519-31. [59] Mastroeni D, Delvaux E, Nolz J, et al. Aberrant intracellular localization of H3k4me3 demonstrates an early epigenetic phenomenon in Alzheimer's disease. Neurobiol Aging 
2015; 36: 3121-9.

[60] Bird AP, Wolffe AP. Methylation-induced repression- belts, braces, and chromatin. Cell 1999; 99: 4.51-4.54.

[61] Marques SCF, Lemos R, Ferreiro E, et al. Epigenetic regulation of BACE1 in Alzheimer's disease patients and in transgenic mice. Neuroscience 2012; 220: 256-66.

[62] Plagg B, Ehrlich D,

Kniewallner KM, et al. Increased acetylation of histone $\mathrm{H} 4$ at lysine 12 (H4K12) in monocytes of transgenic Alzheimer's mice and in human patients. Curr Alzheimer Res 2015; 12: 752-60.

[63] Green KN, Steffan JS, Martinez-Coria H, et al. Nicotinamide restores cognition in Alzheimer's disease transgenic mice via a mechanism involving sirtuin inhibition and selective reduction of Thr231-phosphotau. J Neurosci 2008; 28: 11500-10.

[64] Sung YM, Lee T, Yoon H, et al. Mercaptoacetamide-based class II HDAC inhibitor lowers $A \beta$ levels and improves learning and memory in a mouse model of Alzheimer's disease. Exp Neurol 2013; 239: 192-201.

\section{[65] Peleg S, Sananbenesi F,}

Zovoilis A, et al. Altered histone acetylation is associated with age-dependent memory impairment in mice. Science 2010; 328: 753-6.

[66] Cadena-del-Castillo C, Valdes-Quezada C, Carmona-Aldana F, et al. Age-dependent increment of hydroxymethylation in the brain cortex in the triple-transgenic mouse model of Alzheimer's disease. J Alzheimers Dis 2014; 41: 845-54.

[67] Pheiffer C, Dias S, Muller

C, et al. Decreased global DNA methylation in the white blood cells of high fat diet fed vervet monkeys (Chloroce- bus aethiops). J Physiol Biochem 2014; 70 :

$725-733$.

[68] Lashley T, Gami P, Valiza-

deh N, et al. Alterations in global DNA methylation and hydroxymethylation are not detected in Alzheimer's disease. Neuropathol Appl Neurobiol 2015; 41: 497-506.

[69] Bradley-Whitman MA, Lovell MA. Epigenetic changes in the progression of Alzheimer's disease.

Mech Ageing Dev 2013; 134: 486-95.

[70] Rao JS, Keleshian VL, Klein

$\mathrm{S}$, et al. Epigenetic modifications in frontal cortex from Alzheimer's disease and bipolar disorder patients. Transl Psychiatry 2012; 2: el32.

[71] Sung HY, Choi EN, Ahn Jo

$\mathrm{S}$, et al. Amyloid protein-mediated differential DNA methylation status regulates gene expression in Alzheimer's disease model cell line. Biochem Biophys Res Commun 2011; 414: 700-5. [72] Hodgson N, Trivedi M, Muratore C, et al. Soluble oligomers of amyloid- $\beta$ cause changes in redox state, DNA methylation, and gene transcription by inhibiting EAAT3 mediated cysteine uptake. J Alzheimers Dis 2013; 36: 197-209.

\section{[73] Morrison LD, Smith DD,}

Kish SJ. Brain S-adenosylmethionine levels are severely decreased in Alzheimer's disease. J Neurochem 1996; 67: 1328-31.

\section{[74] Bottiglieri T, Godfrey P,}

Flynn T, et al. Cerebrospinal fluid $\mathrm{S}$-adenosylmethionine in depression and dementia: effects of treatment with parenteral and oral S-adenosylmethionine. J Neurol Neurosurg Psychiatry 1990; 53: 1096-1098.

[75] Eto K, Asada T, Arima K, et al. Brain hydrogen sulfide is severely decreased in Alzheimer's disease. Biochem Biophys Res Commun 2002; 293 :
$1485-8$.

[76] Fuso A, Seminara L, Cavallaro RA, et al. S-adenosylmethionine/homocysteine cycle alterations modify DNA methylation status with consequent deregulation of PSl and BACE and beta-amyloid production. Mol Cell Neurosci 2005; 28: 195-204.

[77] Kennedy BP, Bottiglieri T, Arning E, et al. Elevated S-adenosylhomocysteine in Alzheimer brain: influence on methyltransferases and cognitive function. J Neural Transm 2004; 111: 547-567.

[78] Goggins M, Scott JM, Weir DG. Methylation of cortical brain proteins from patients with HIV infection. Acta Neurol Scand 1999; 100: 326-31.

[79] Ficz G, Branco MR, Seisenberger S, et al. Dynamic regulation of 5 -hydroxymethylcytosine in mouse ES cells and during differentiation.

Nature 2011; 473: 398-402.

[80] Sanchez-Mut J V, Gräff J.

Epigenetic alterations in Alzheimer's disease. Front Behav Neurosci 2015; 9 : 347.

[81] Lunnon K, Mill J. Epigenetic studies in Alzheimer's disease: current findings, caveats, and considerations for future studies. Am J Med Genet B Neuropsychiatr Genet 2013; 162B: 789-99. 


\section{SUPPLEMENTARY DATA}

SUPPLEMENTARY FIGURE 1. Overview of analyzed hippocampal subregions. Shown is a representative image of the 5-hydroxymethylcytosine staining in a 6 months old APP/PSldE9 mouse, to illustrate the relative location of the analyzed dentate gyrus (DG), cornu ammonis (CA) 3, and CAl-2 subregions in the hippocampus (A). Also shown are high magnification images of these subregions (B-D, respectively), representative of those that were analyzed for the semiquantitative analysis. Scale bar represents $200 \mu \mathrm{m}$ in $\mathrm{A}$ and $75 \mu \mathrm{m}$ in $\mathrm{B}-\mathrm{D}$.

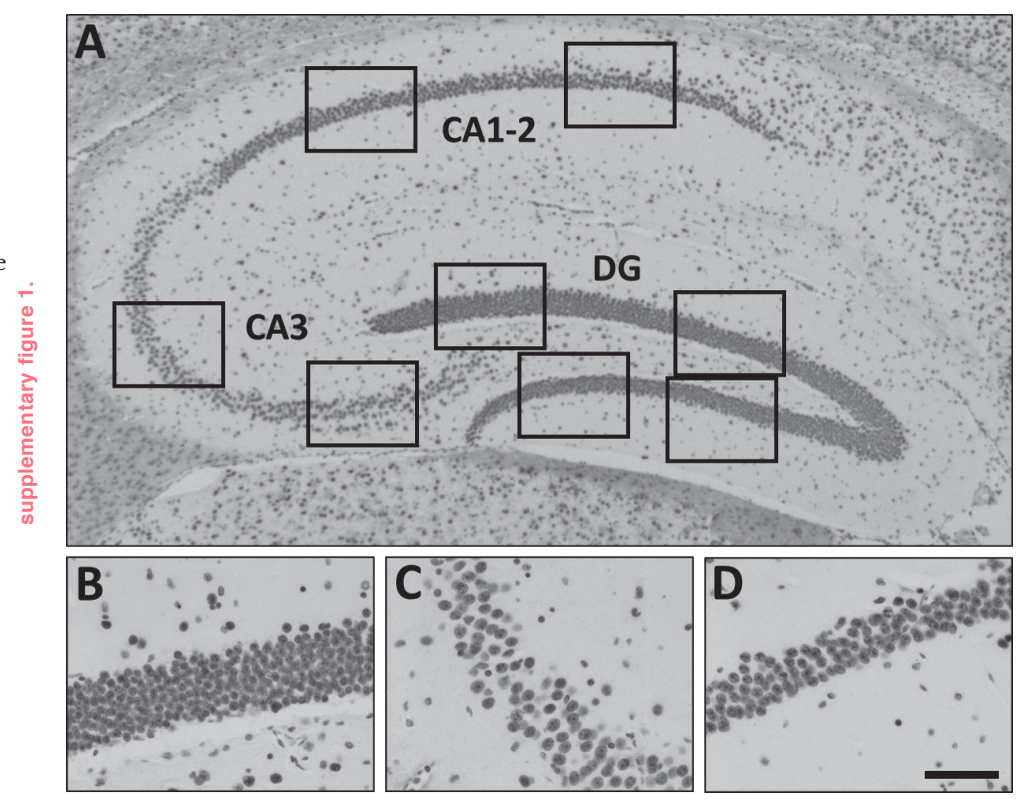




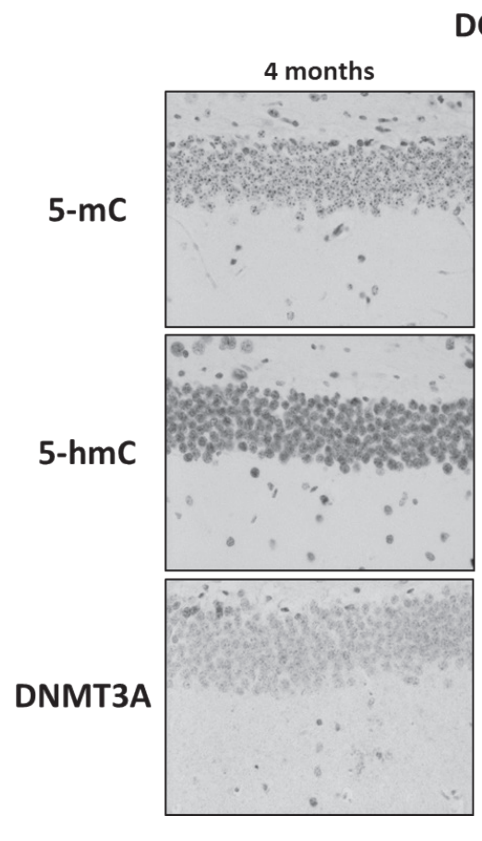

DG

CA3
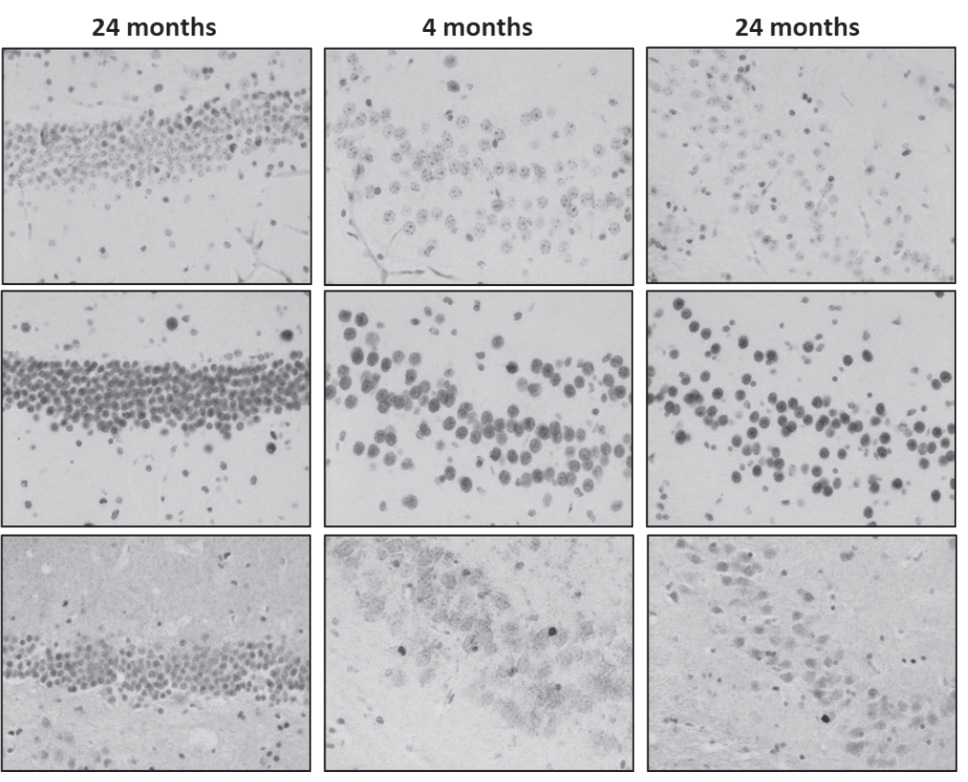

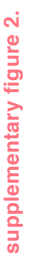

DG

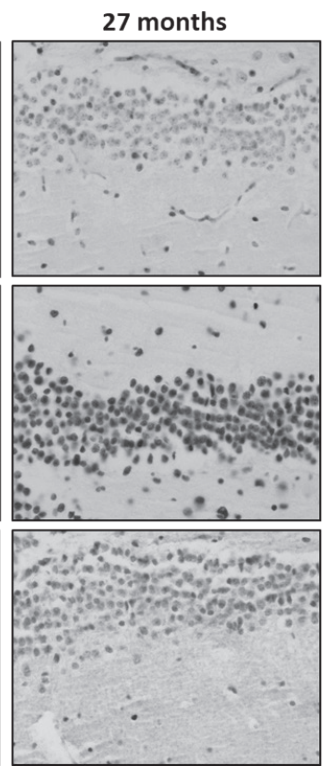

CA3
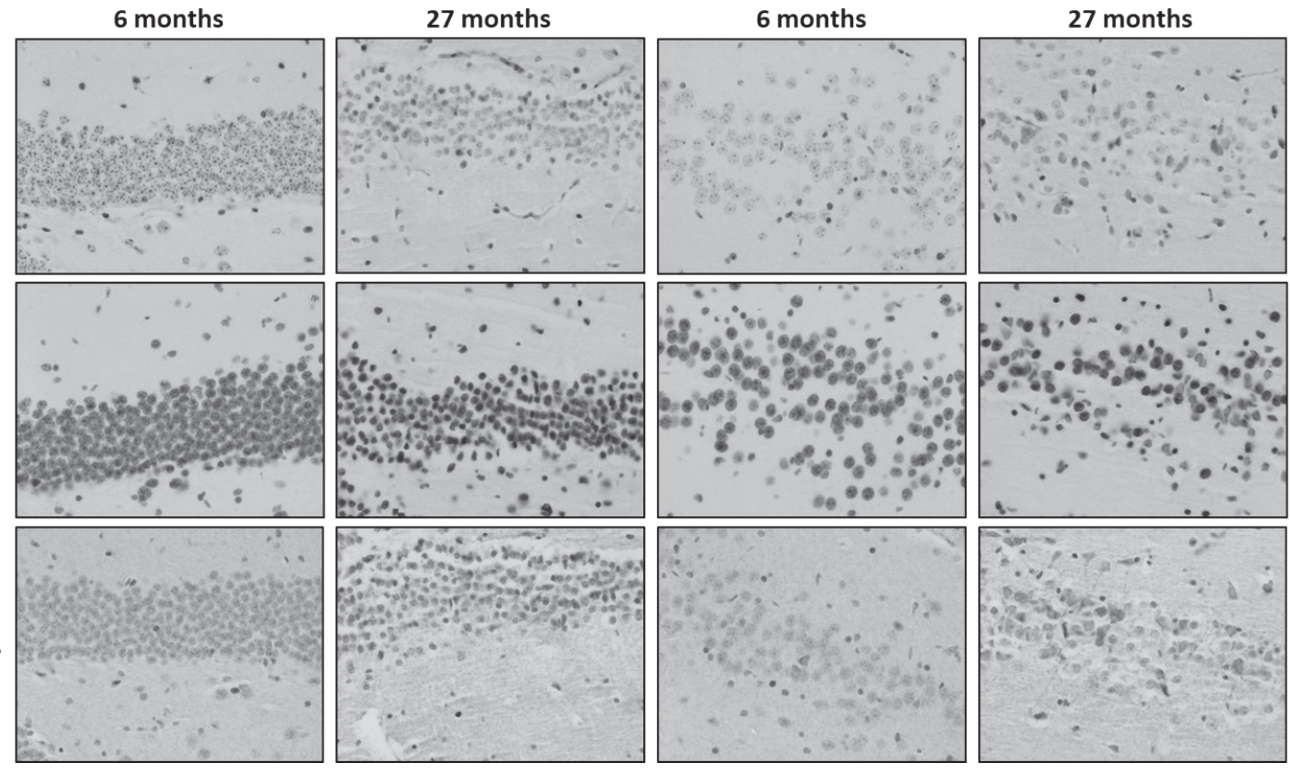


\section{CA1-2}
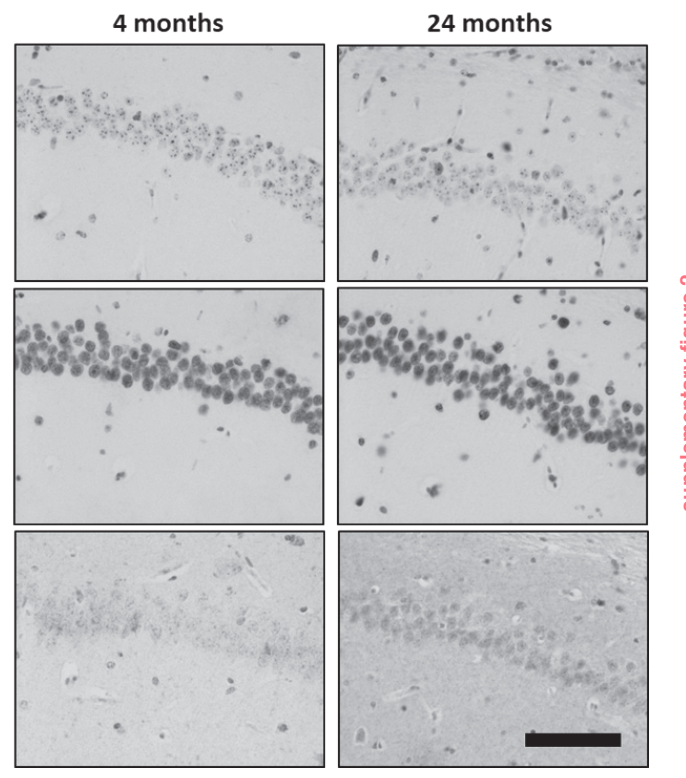

SUPPLEMENTARY FIGURE 2. Overview of the analyzed immunohistochemical stainings of epigenetic markers in the J20 transgenic mouse model. Shown are representative examples of analyzed images of the 5-methylcytosine (5-mC), 5-hydroxymethylcytosine (5-hmC), and DNA methyltransferase (DNMT) 3A stainings, in the dentate gyrus (DG), cornu ammonis (CA) 3, and CAl-2 subregions in the hippocampus, from the youngest (4 months old) and oldest (24 months) animals studied. A statistically significant age-related decrease in DG 5-mC immunoreactivity was detected after a semiquantitative analysis of the images. Scale bar represents $100 \mu \mathrm{m}$.

\section{SUPPLEMENTARY FIGURE 3. Overview}

\section{CA1-2}
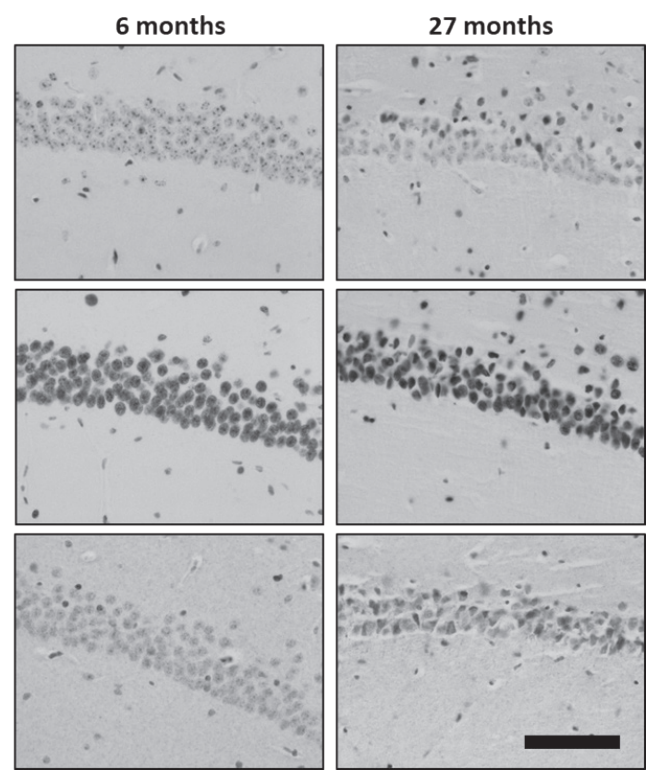

of the analyzed immunohistochemical stainings of epigenetic markers in the APP/PSldE9 transgenic mouse model. Shown are representative examples of analyzed images of the 5 -methylcytosine (5-mC), 5-hydroxymethylcytosine (5-hmC), and DNA methyltransferase (DNMT) 3A stainings, in the dentate gyrus (DG), cornu ammonis (CA) 3, and CA1-2 subregions in the hippocampus, from the youngest ( 6 months old) and oldest (27 months) animals studied. Apart from some structural changes, no clear age-related alterations in immunoreactivity can be observed, which is in line with observations from the semiquantitative analysis. Scale bar represents $100 \mu \mathrm{m}$. 
DG

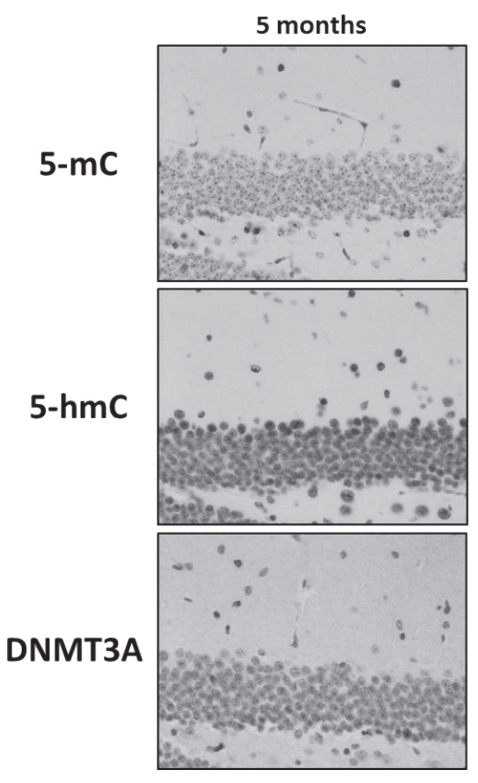

DG

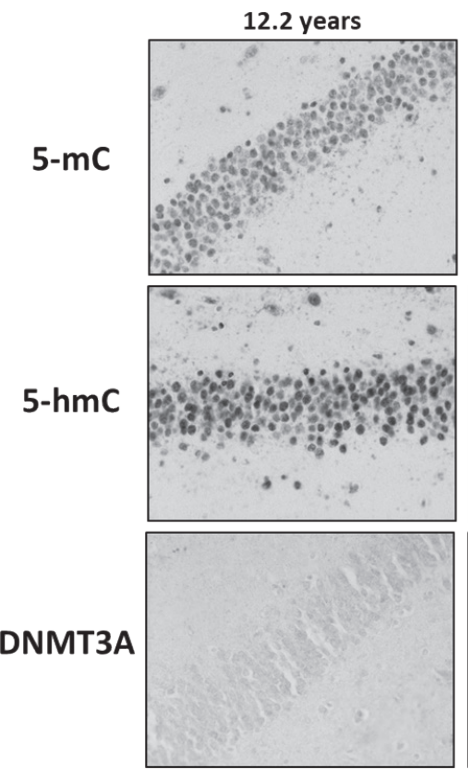

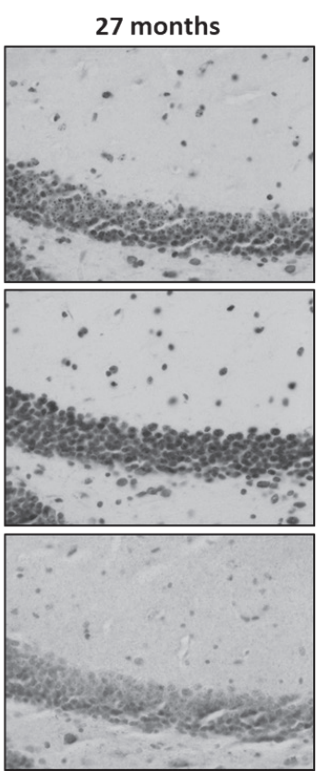

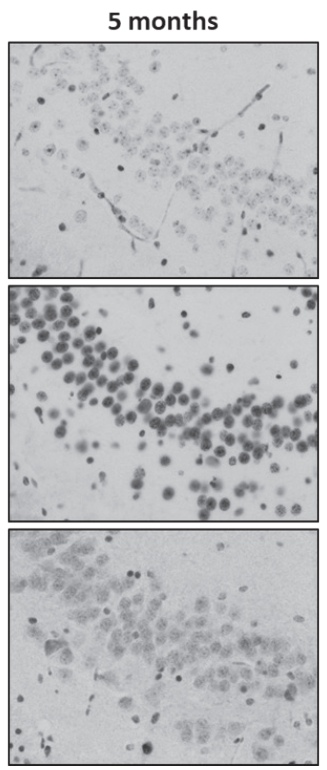

CA3

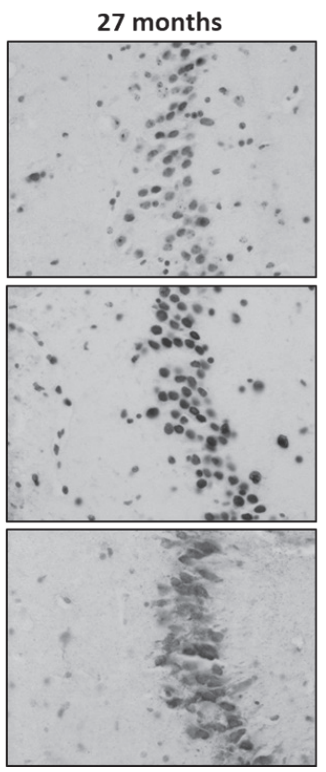

CA3
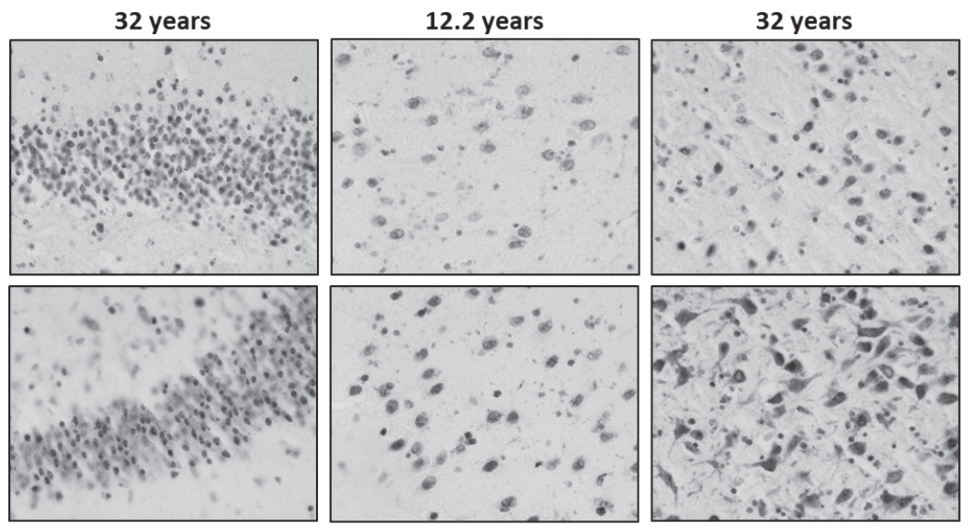

$$
\text { เก }
$$



of the analyzed immunohistochemical stainings of epigenetic markers in

\section{CA1-2}
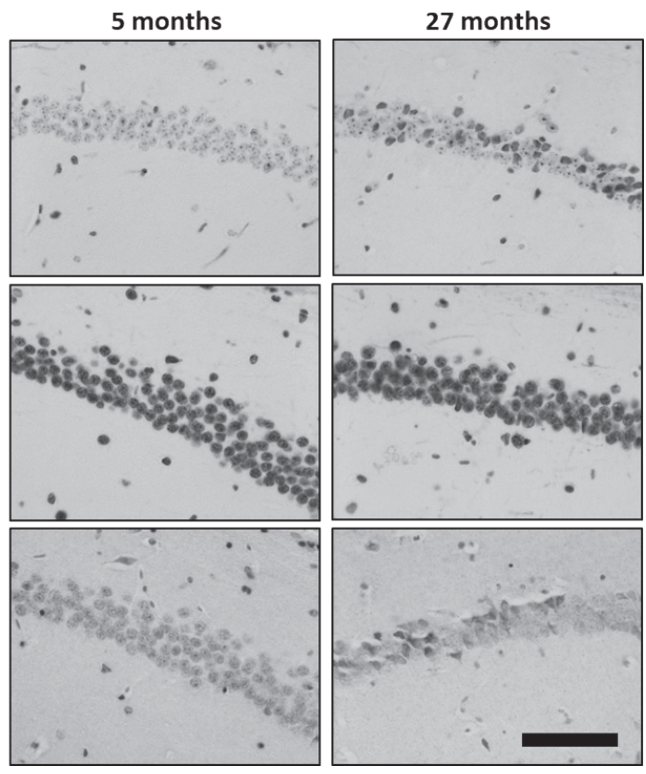

CA1-2

12.2 years
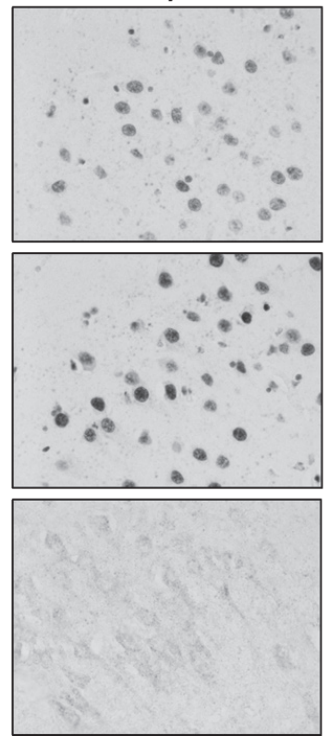

32 years
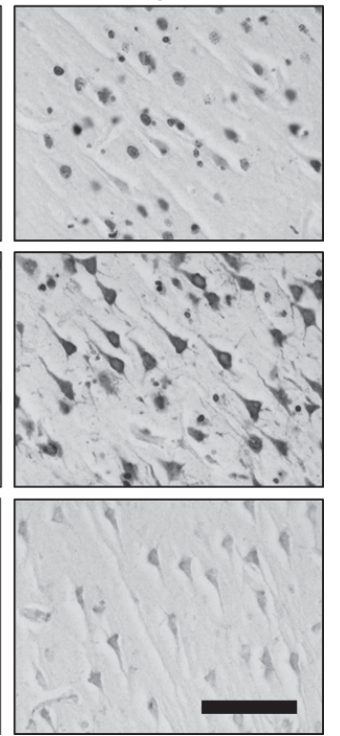

the $3 \mathrm{xTg}-\mathrm{AD}$ transgenic mouse model. Shown are representative examples of analyzed images of the 5-methylcytosine (5-mC), 5-hydroxymethylcytosine $(5-\mathrm{hmC})$, and DNA methyltransferase (DNMT) 3A stainings, in the dentate gyrus (DG), cornu ammonis (CA) 3, and CAl-2 subregions in the hippocampus, from the youngest (5 months old) and oldest (27 months) animals studied. An age-related increase in 5-mC immunoreactivity can be observed in the DG, CA3, and CAl-2 regions of this mouse model. A semiquantitative analysis, however, shows this increase is only statistically significant in the DG and CAl-2 regions. Scale bar represents $100 \mu \mathrm{m}$.

SUPPLEMENTARY FIGURE 5. Overview of the analyzed immunohistochemical stainings of epigenetic markers in the Caribbean vervets. Shown are representative examples of analyzed images of the 5 -methylcytosine $(5-\mathrm{mC})$, 5-hydroxymethylcytosine (5-hmC), and DNA methyltransferase (DNMT) 3A stainings, in the dentate gyrus (DG), cornu ammonis (CA) 3, and CAl-2 subregions in the hippocampus, from the youngest (12.2 years old) and oldest (32 years old) animals studied. From the images shown there may appear to be some age-related differences, mainly in the DG, but these did not appear consistently, as signified by a semiquantitative analysis which did not reveal any statistically significant age-related alterations in immunoreactivity of any of the tested epigenetic markers. Scale bar represents $100 \mu \mathrm{m}$. 

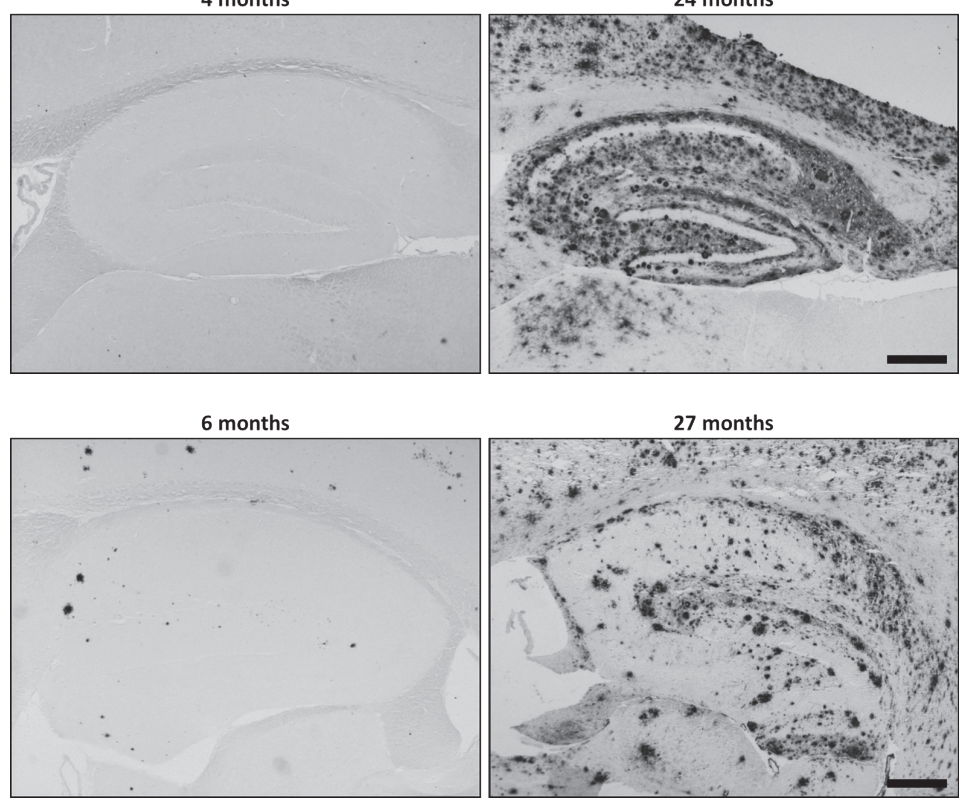

5 months
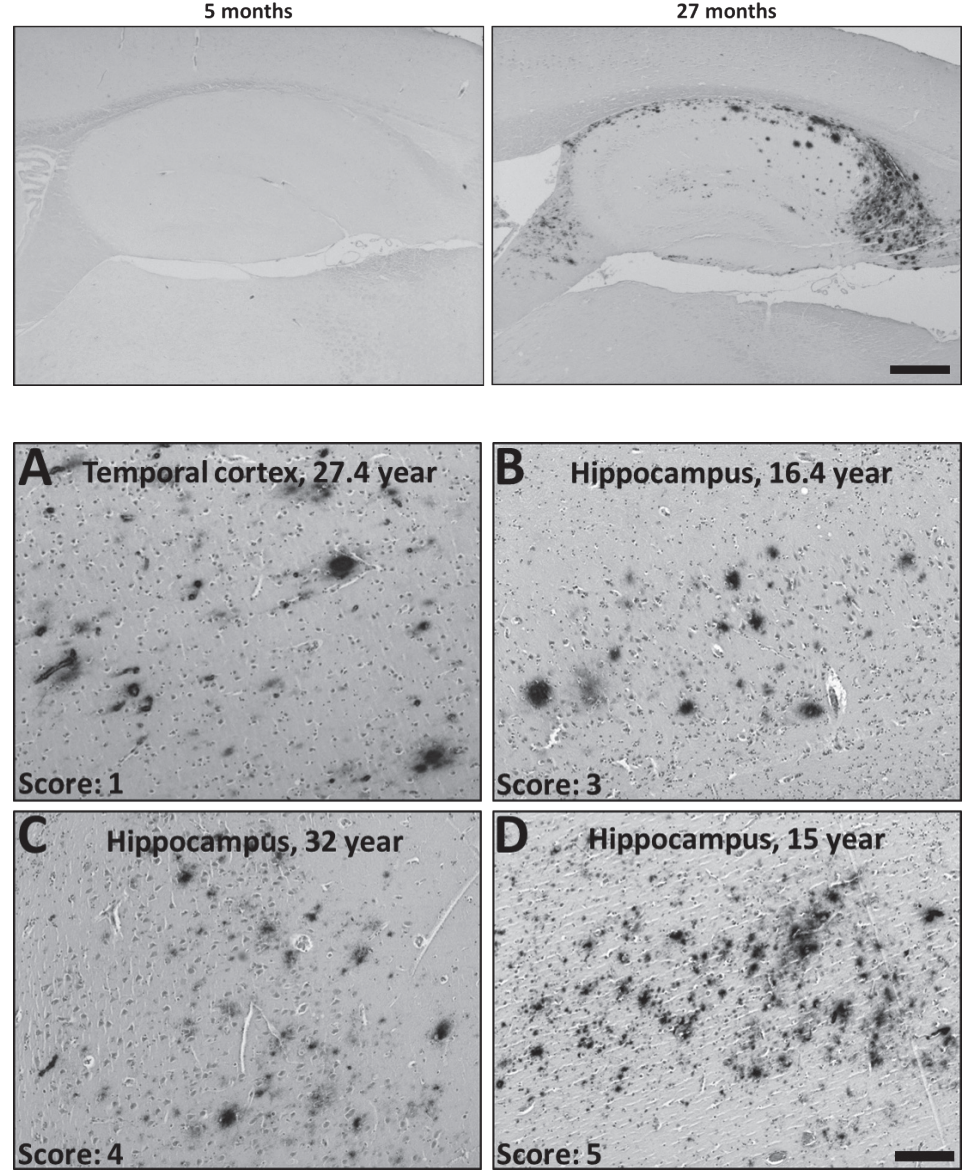

SUPPLEMENTARY FIGURE 6. Hippo-

campal 3Al plaque staining in trans-

genic J20 mice. Shown are representative images of the youngest (4 months) and oldest (24 months) animals. Note the high plaque load throughout the whole hippocampus in the oldest animals. Scale bar represents $400 \mu \mathrm{m}$.

\section{SUPPLEMENTARY FIGURE 7. Hippo-}

campal 3Al plaque staining in trans-

genic APP/PSldE9 mice. Shown are representative images of the youngest (6 months) and oldest (27 months) animals. Note the distribution of plaques throughout the whole hippocampus in the oldest animals. Amyloid deposition seems to be lower than in the J20 mice (Supplementary Figure 6). Scale bar represents $400 \mu \mathrm{m}$.

\section{SUPPLEMENTARY FIGURE 8. Hip- pocampal 3Al plaque staining in transgenic $3 x T g-A D$ mice. Shown are representative images of the youngest (5 months) and oldest (27 months) ani- mals. Note the uneven distribution of plaques throughout the hippocampus} in the oldest animals, with the highest amyloid beta immunoreactivity in the dorsal subiculum and deeper layers of the hippocampus (oriens and alveus). Scale bar represents $400 \mu \mathrm{m}$.

\section{SUPPLEMENTARY FIGURE 9. Amyloid}

beta 42 plaque staining in Caribbean vervets. The images were scored according to their plaque load; 0 for no plaques, 1 for plaques in the temporal cortex but not yet inside the hippocampus, 2 for 1 to 5 plaques in the hippocampus, 3 for 6 to 10 plaques, 4 for 11 to 100 plaques, and 5 for more than 100 plaques. Shown are representative images of the scores observed. Not all of the older vervets developed plaques 
in the hippocampus, although all vervets over 18 years of age developed plaques in the frontal cortex, prefrontal cortex and/or temporal cortex. A is from a 27.4 years old vervet, B from a 16.4 years old, C from a 32 years old, and $\mathrm{D}$ from a 15 years old. Note that $\mathrm{A}$ is an image of the temporal cortex outside the hippocampus, while B-D are images taken inside the hippocampus. Scale bar represents $200 \mu \mathrm{m}$. 

ACTIVE AMYLOID- $\beta$

IMMUNOTHERAPY

RESULTS IN EPIGENETIC

CHANGES IN THE

HIPPOCAMPUS OF AN

ALZHEIMER'S DISEASE

MOUSE MODEL

ROY LARDENOIJE ${ }^{A, B}$, DANIËL L.A. VAN DEN HOVE ${ }^{\mathrm{B}, \mathrm{C}}$, SOPHIE E. JUNG ${ }^{B}, M^{\prime}$ NIQUE HAVERMANS ${ }^{\mathrm{B}}$, BIN LIU ${ }^{A}$, BART P.F. RUTTEN ${ }^{\mathrm{B}, *}$, CYNTHIA A. LEMERE ${ }^{A, *}$

${ }^{A} A N N$ ROMNEY CENTER FOR NEUROLOGIC DISEASES,

DEPARTMENT OF NEUROLOGY, BRIGHAM AND WOMEN'S HOSPITAL, HARVARD MEDICAL SCHOOL, USA

BSCHOOL FOR MENTAL HEALTH AND NEUROSCIENCE (MHENS), DEPARTMENT OF PSYCHIATRY AND NEUROPSYCHOLOGY, MAASTRICHT UNIVERSITY, THE NETHERLANDS

'LABORATORY OF TRANSLATIONAL NEUROSCIENCE, DEPARTMENT

OF PSYCHIATRY, PSYCHOSOMATICS AND PSYCHOTHERAPY, UNIVERSITY OF WUERZBURG, GERMANY

*THESE AUTHORS CONTRIBUTED EQUALLY TO THIS WORK. 


\section{Abstract}

While evidence accumulates for a role of epigenetic modifications in the pathophysiological cascade of Alzheimer's disease (AD), amyloid- $\beta$ $(A \beta)$-targeted active immunotherapy approaches are employed to prevent the progression of AD. The impact of active immunotherapy on epigenetic markers has not been studied thus far. The current study aims to establish the relation between active immunotherapy with a MER5101based vaccine and epigenetic DNA modifications, using semi-quantitative immunohistochemistry in the hippocampus of APPswe/PS1dE9 mice.

Immunotherapy started when the mice were 10 months of age, behavioral testing occurred at 14 months of age, after which the mice were sacrificed for further analysis of their brains. Global levels of DNA methylation and hydroxymethylation were compared to previously established immunization-induced changes in AD-related neuropathology and cognition. It was found that active immunization did not affect global DNA methylation levels, but resulted in decreased DNA hydroxymethylation and DNA methyltransferase (DNMT) 3A levels. In addition, correlations with behavioral outcomes were observed for levels of DNA methylation and hydroxymethylation, but not DNMT3A, while $A \beta$ pathology and synaptic markers did not correlate with DNA methylation levels, but did correlate with DNA hydroxymethylation and levels of DNMT3A. These findings indicate that active $A \beta$ immunization has significant effects on the epigenome in APPswe/PS1dE9 mice, and that DNA methylation and hydroxymethylation may be involved in cognitive functioning.

KEYWORDS: Alzheimer's disease; amyloid- $\beta$; active immunization; epigenetics; DNA methylation; mouse model 


\subsection{Introduction}

Although the exact role of neuritic plaques and amyloid- $\beta(A \beta)$ in the pathogenesis of Alzheimer's disease (AD) remains to be elucidated, studies have shown beneficial effects of $A \beta$ immunization in various animal models [1]. The first reported study of $A \beta$ immunization involved injecting transgenic PDAPP mice intraperitoneally (i.p.) with human $A \beta 1$ 42 peptide in conjunction with Freund's adjuvant [2]. When immunized before plaque development, the PDAPP mice barely developed any plaques at all. Interestingly, even after the initial development of plaques, $A \beta$ immunization resulted in striking decreases in brain $A \beta$ levels. Later studies have corroborated these findings and found similar effects when immunizing intranasally (i.n.) with $A \beta 1-40[3,4]$ and were able to enhance antibody titers using the Escherichia coli heat-labile enterotoxin mucosal adjuvant (LT) [5].

The beneficial effects of $A \beta$ immunization are not limited to a reduction of plaques and $A \beta$ levels, but, importantly, also include reports of ameliorated behavioral and cognitive impairments in animal models of $\operatorname{AD}[6,7]$ and limited evidence for cognitive stabilization in humans [8]. The mechanisms, however, linking $A \beta$-reduction by immunization to beneficial effects on cognitive performance remain to be determined. Indeed, the relationship between soluble and aggregated forms of $A \beta$ and cognitive deficits in AD remains a hotly debated topic [9]. A possible explanation for how $A \beta$ is able to affect so many systems, including those involved in cognition, would be an interaction with the epigenetic machinery. Epigenetic mechanisms dynamically govern gene expression at both transcriptional and translational levels [10], and are thought to mediate interactions between genetic aberrations and environmental influences [11]. In particular, DNA methylation appears to be dysregulated in $A D[12,13]$, and the closely related epigenetic process of DNA hydroxymethylation has been implicated as well [14]. Long-term DNA methylation is maintained by maintenance DNA methyltransferases, whereas novel methylation modifications are imparted by de novo DNA methyltransferases [13]. DNA hydroxymethylation arises through the oxidation of DNA methylation groups by ten-eleven translocation (TET) enzymes and is particularly enriched in the brain $[15,16]$.

It has been suggested that the epigenetic alterations observed in relation to $A D$ are, at least in part, induced by $A \beta$ [17]. The exact role of $A \beta$ in the epigenetic dysregulation observed in $A D$ is, however, not well understood, nor is the impact of $A \beta$ vaccination on the epigenome. In order to explore these questions, the present study was undertaken to establish the epigenetic impact of active $A \beta$ immunization, using brain tissues from a previously published, well-characterized cohort of $A \beta$ immunized 
mice [18], and examining the relationship between several epigenetic markers (5-methylcytosine [5-mC], a marker of DNA methylation, 5-hydroxymethylcytosine [5-hmC], a marker of DNA hydroxymethylation, and de novo DNA methyltransferase [DNMT] 3A) and indicators of ADrelated pathology.

\subsection{Materials and methods 6.2.1. Animals and treatment}

For this study, the APPswe/PS1dE9 model [18] was used. APPswe/ PS1dE9 mice carry a human amyloid precursor protein (APP) transgene with the Swedish mutation (K594N/M595L) and a human presenilin (PS) 1 transgene with deletion of exon 9 (PS1dE9), co-expressed under the mouse prion protein promoter [19]. APPswe/PS1dE9 breeders (on a C57BL/6 background) were obtained from The Jackson Laboratory (Bar Harbor, ME) and crossed with DBA/2 wild-type mice to generate the APPswe/PS1dE9 mice on a B6D2F1 background that were used for the immunization experiment. Animal use was approved by the Harvard Standing Committee for Animal Use and was in line with state and federal regulations. See Table 1 for an overview of the studied animals.

\begin{tabular}{|c|c|c|c|c|c|}
\hline Model & Mutations & Age & Gender & Treatment & $\mathrm{N}$ \\
\hline \multirow{4}{*}{ APPswe/PS1dE9 } & \multirow{4}{*}{$\begin{array}{l}\text { APP K594N,M595L, } \\
\text { deletion of exon } 9\end{array}$} & \multirow{4}{*}{10 months } & \multirow{2}{*}{ Female } & Vaccine & 2 \\
\hline & & & & Vehicle & 2 \\
\hline & & & \multirow{2}{*}{ Male } & Vaccine & 3 \\
\hline & & & & Vehicle & 4 \\
\hline
\end{tabular}

The mice in this study were previously immunized with MER5101 as described [18]. Eleven 10-month-old male $(n=7)$ and female $(n=4)$ APPswe/PS1dE9 mice were randomly distributed between MER5101 immunized $(n=5)$ and vehicle treated control $(n=6)$ groups. The MER5101 vaccine was formulated in Mercia's Th2-biased adjuvant (MAS-1; Mercia Pharma, Scarsdale, NY) and consisted of multiple A $\beta 1$ 15 copies, conjugated with a 7 aa spacer to the diphtheria toxoid (DT) carrier protein. MER5101 vaccine was prepared as described previously [18]. The vehicle formulation for the control group contained sterile phosphate-buffered saline (PBS) mixed with MAS-1. Treatment consisted of five subcutaneous injections of $0.1 \mathrm{ml}$ of MER5101, containing $100 \mu \mathrm{g}$ $A \beta 1-15: D T$ conjugate, or PBS instead of antigen in case of the vehicle controls. The first two injections were administered 2 weeks apart, and the following three were given 4 weeks apart. After treatment, at 14 months old, the mice were cognitively assessed and sacrificed by $\mathrm{CO}_{2}$ inhalation and transcardial perfusion with $20 \mathrm{ml}$ PBS. Tail plasma was

TABLE 1. Overview of the animals from each treatment group available for the current study. 
sampled as previously indicated [20]. After sacrificing the animals, one brain hemisphere was processed for paraffin sectioning after a 2-hours fixation in $10 \%$ neutral-buffered formalin. The other half was snap frozen and kept at $-80^{\circ} \mathrm{C}$ for biochemical analysis. The archived tissue from these animals was used for the current study.

\subsubsection{Immunohisto- chemistry}

The paraffin embedded brains were cut in $10 \mu \mathrm{m}$-thick serial sagittal sections for immunohistochemistry (IHC). The staining procedure was performed at room temperature unless specified otherwise. After deparaffinization in Histo-Clear (National Diagnostics, Atlanta, GA), the sections were rehydrated in a graded ethanol series through to demineralized water. Endogenous peroxidase activity was quenched through submersion in a methanol solution containing $0.3 \% \mathrm{H}_{2} \mathrm{O}_{2}$ for 10 minutes. BioGenex citrate buffer (BioGenex, San Ramon, CA) was used for antigen retrieval, keeping it around the boiling point in the microwave for 5 minutes. The sections were cooled and then washed for 10 minutes in demineralized water before being placed in blocking solution for 20 minutes. Blocking solution contained $10 \%$ serum from the secondary antibody host species, dissolved in Tris-buffered saline. Primary antibody incubation took place overnight at $4^{\circ} \mathrm{C}$. Primary antibodies used included a mouse monoclonal anti-5-mC antibody (1:1000 dilution; GenWay Biotech Inc., San Diego, CA) for 5-mC detection, rabbit polyclonal anti-5-hmC anti-serum (1:10,000 dilution; Active Motif, Carlsbad, CA) for 5-hmC, a rabbit polyclonal anti-DNMT3A antibody (1:200 dilution; Santa Cruz Biotechnology, Dallas, TX) for DNMT3A, and 3A1, a general monoclonal IgG1 anti-A $\beta$ antibody (1:1000 dilution, provided by Dr. Brian O Naullain at the Ann Romney Center for Neurologic Diseases, Boston, MA), for plaques. The specificity of the $3 \mathrm{~A} 1$ antibody has previously been established [21]. After washing off the primary antibody solution, the slides were covered in secondary antibody solution for 30 minutes. Biotinylated secondary antibodies were used; horse antimouse (Vector Laboratories, Burlingame, CA) for 5-mC, goat anti-rabbit (Vector Laboratories) for 5-hmC and DNMT3A, and goat anti-mouse (Vector Laboratories) for the $3 A 1 A \beta$ antibody. For visualization, the VectorElite horseradish peroxidase ABC kit (Vector Laboratories), with 3,3'-diaminobenzidine tetrahydrochloride (DAB; Sigma-Aldrich, St. Louis, MO) as chromogen, was used. A negative control, leaving out the primary antibody, was added to each of the staining runs, and which, in all cases, displayed no signal (data not shown). 


\subsubsection{Immunoreactivity of epigenetic markers and plaque load}

Three sagittal sections at varying hippocampal planes were selected for each immunohistochemical staining. A BX50 brightfield microscope (Olympus, Tokyo, Japan) coupled to a QIcam digital camera (QImaging, Surrey, Canada) was used to take photographic images of hippocampal subregions. For the immunoreactivity (IR) analysis of 5-mC, 5-hmC and DNMT3A, 4 pictures of the dentate gyrus (DG), 2 of the cornu ammonis (CA) 3 and 2 of the CA1-2 regions were taken with the $20 \mathrm{X}$ objective (see Figure 1 for representative images). ImageJ (version $1.48 \mathrm{v}$, Wayne Rasband, National Institutes of Health, Bethesda, Maryland, USA) was used to delineate the region of interest (ROI; DG, CA3 or CA1-2) in each image, set a fixed threshold for background correction, and measure the mean grey value, the area of the ROI, and the area of the ROI with grey value above threshold. From these measurements the integrated density was determined by multiplying the background-corrected mean grey values with the fraction of the ROI area with values above the background threshold (as also described in [22]). In addition, the ratio of 5-mC to 5 -hmC integrated density values was calculated. Hippocampal plaque load was determined in 3 sagittal sections per mouse that were stained using the $3 A 1$ antibody (see Figure 2 for representative images). A BIOQUANT image analysis setup (Nashville, TN, USA) was used to measure the fraction of the hippocampal area covered by plaques. After manual delineation of the hippocampus and artifact removal, a fixed threshold was used to detect plaques and determine the area fraction of interest.

\subsubsection{Additional characterizations}

The effects of MER5101 vaccination on AD-related markers and cognition have previously been reported for the APPswe/PS1dE9 mice [18]. Some of these measurements were included in the current study to evaluate the correlation with the measured epigenetic markers. The included data concerns brain levels of $A \beta x-40$ and $A \beta x-42$ protein, as determined through enzyme-linked immunosorbent assays (ELISA), and optical density measurements of postsynaptic density protein (PSD)-95 and synaptophysin (SYP) in the CA3 and CA1 [18]. PSD-95 and SYP are markers of post- and presynaptic integrity, respectively. Moreover, behavioral data from the contextual fear conditioning (CFC) and Morris 
water maze (MWM) tests was included [18]. The MWM test data included the escape latency as a measure for spatial learning, and the annulus crossing index $(\mathrm{ACl})$, determined 2 and 24 hours after the last hidden platform trial, as a measure for spatial memory. The $\mathrm{ACl}$ is calculated as the number of crosses over the platform location in the correct quadrant, adjusted for crosses of the same location in the other quadrants [18].

\subsubsection{Data analysis}

Data were analyzed with SPSS Statistics 21 (IBM; Armonk, NY). Data were checked for normality and homoscedasticity. Due to non-normal data, the Mann-Whitney $U$ test was performed. These tests were performed to examine differences between vaccinated and control mice on the following variables: 1) fraction of the hippocampal area covered by plaques, 2) integrated density measures of the epigenetic markers 5-mC, 5-hmC, their ratio (5-mC:5-hmC), and DNMT3A. In addition, Pearson's correlation was used to correlate $A D$ markers (i.e. $A \beta x-40, A \beta x-42$, plaque covered hippocampal area fraction, SYN, and PSD-95) with epigenetic markers (5-mC, 5-hmC, 5-mC:5-hmC, and DNMT3A). All analyses were two-tailed, with alpha set at 0.05 .

\subsection{Results}

As reported previously [18], brain $A \beta x-40$ protein levels were not significantly altered in vaccinated mice, while $A \beta x-42$ protein levels were significantly reduced in vaccinated mice. Furthermore, MER5101immunized mice displayed enhanced densities of pre-synaptic SYP and post-synaptic PSD-95 in the hippocampal regions CA1 and CA3, when compared to vehicle-treated APPswe/PS1dE9 mice. Behaviorally, vaccinated animals showed an increase in freezing frequency during the CFC test and better spatial learning and memory in the MWM, when compared to the vehicle-treated mice. The following section contains a summary of the novel findings. For the full results, see Supplementary Tables 1-4.

\subsubsection{Fraction of hippocam- pal area covered by plaques}

We found a statistically significant difference between groups in the fraction of the hippocampal area covered by plaques $(U=3, p=0.028)$. Coverage by plaques in the hippocampus was greater in the control group 
$(M=0.19, S D=0.03)$ than in the treatment group $(M=0.14, S D=0.02)$

(Figure 2).

\subsubsection{Integrated density measures of epigenetic markers}

Staining and quantification of epigenetic markers in hippocampal subregions was performed (Figure 1). No differences in 5-mC levels were found between the groups (Figure 3). The 5 -hmC values were higher in the control group than in the vaccinated group across the whole hippocampus $(U=0.00, p=0.006)$, as well as in each hippocampal subregion, i.e. the DG $(U=3.00, p=0.028)$, CA3 $(U=3.00, p=0.028)$, and CA1-2 $(U=0.00, p=0.006$; Figure 4). Moreover, the DNMT3A integrated density measures were observed to be higher in the CA3 $(U=$ 2.00, $p=0.018)$ and in the hippocampus as a whole $(U=4.00, p=0.045)$ of control compared to vaccinated mice (Figure 5). Also, no difference in the $5-\mathrm{mC}: 5-\mathrm{hmC}$ ratio was found between the groups (Figure 6).

\subsubsection{Correlation analysis of $\mathrm{AD}$ markers, epigenetic markers, and behavior}

Whereas 5-mC and the 5-mC:5-hmC ratio did not correlate statistically significantly with any of the AD markers, $5-\mathrm{hmC}$ values correlated positively with hippocampal area fraction containing plaques $(r=0.643, p=0.033)$, as well as with SYP in the CA1 $(r=0.831, p=0.002)$, SYP in the CA3 ( $r=$ 0.823, $p=0.002)$, PSD-95 in the CA1 ( $r=0.692, p=0.018)$, and PSD-95 in the CA3 $(r=0.702, p=0.016)$. Note that for the densitometric analysis of SYP and PSD-95 a lower value indicates a higher density [18]. Further, DNMT3A values correlated significantly with hippocampal area fraction containing plaques $(r=0.629, p=0.038)$.

Of the behavioral outcomes, average escape latency on day 5 of the MWM correlated best with markers associated with AD pathology, showing a positive correlation with brain A $\beta x-40(r=0.694, p=0.018)$ and $A \beta x-42$ $(r=0.703, p=0.016)$, as well as with hippocampal $\mathrm{A} \beta \operatorname{load}(r=0.676, p=$ $0.022)$. CFC freezing behavior, while not correlating well with $A \beta$ directly, correlated negatively with PSD95 in the CA3 $(r=-0.709, p=0.015)$. 
FIGURE 1. Overview of the immunohistochemically stained epigenetic markers analyzed in the APP/PSIdE9 transgenic mouse model.

Shown are representative examples of analyzed images of the 5-methylcytosine $(5$-mC), 5-hydroxymethylcytosine (5-hmC), and DNA methyltransferase (DNMT) 3A staining, in the dentate gyrus (DG), cornu ammonis (CA) 3, and CAl-2 hippocampal subregions, from the vehicle-treated and vaccinated groups. Integrated density measurements showed a decrease in 5-hmC across all regions, and a decrease in DNMT3A in the CA3. Scale bar represents $100 \mu \mathrm{m}$.

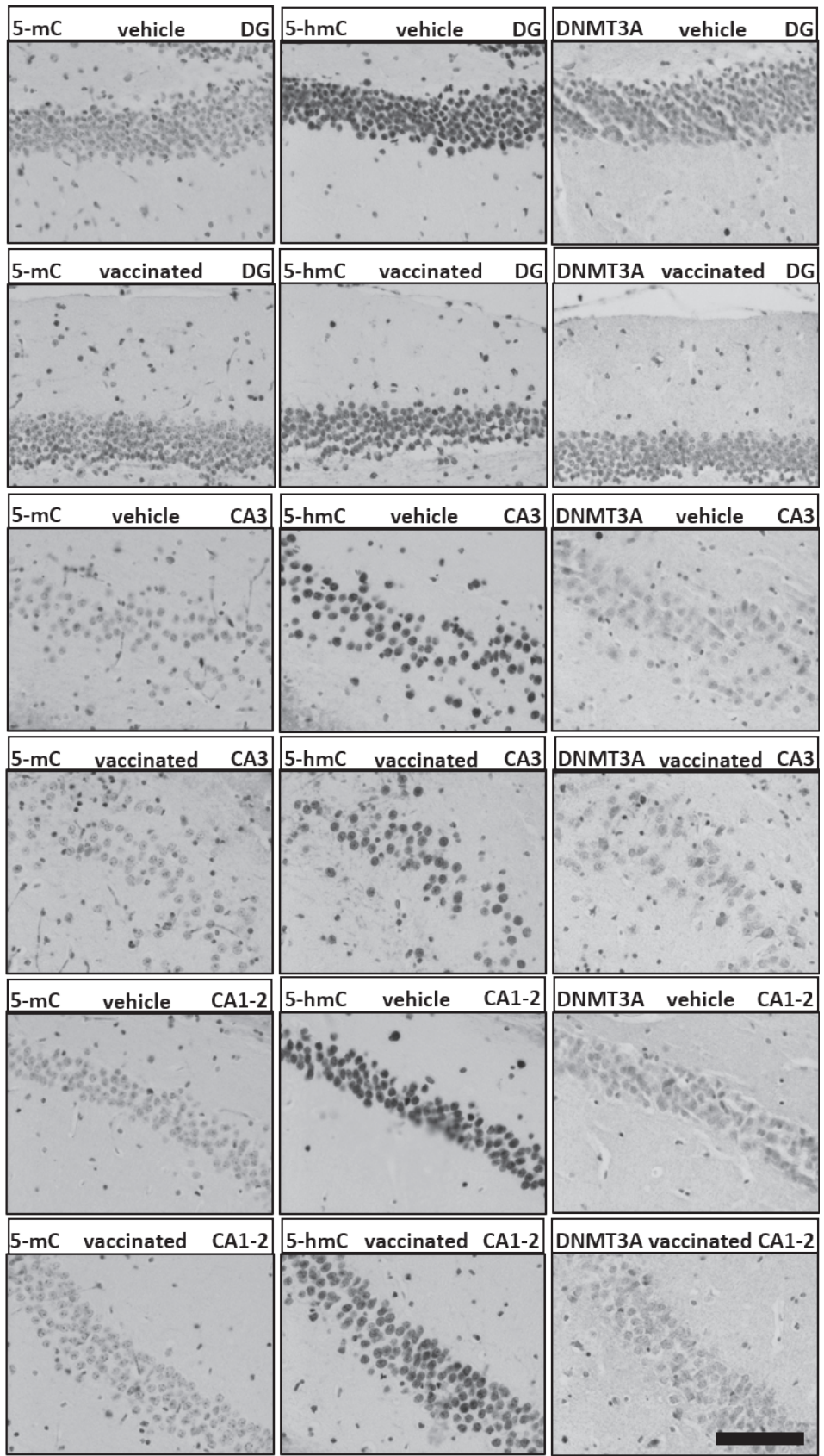

DNA methylation correlated inversely with MWM outcomes $\mathrm{ACl}$ after 2 ( $r=$ $-0.805, p=0.003)$ and 24 hours $(r=-0.670, p=0.024)$, as well as with time in the target quadrant after $2(r=-0.608, p=0.047)$ and 24 hours $(r=-0.730$, $p=0.011)$. For DNA hydroxymethylation only a statistically significant 

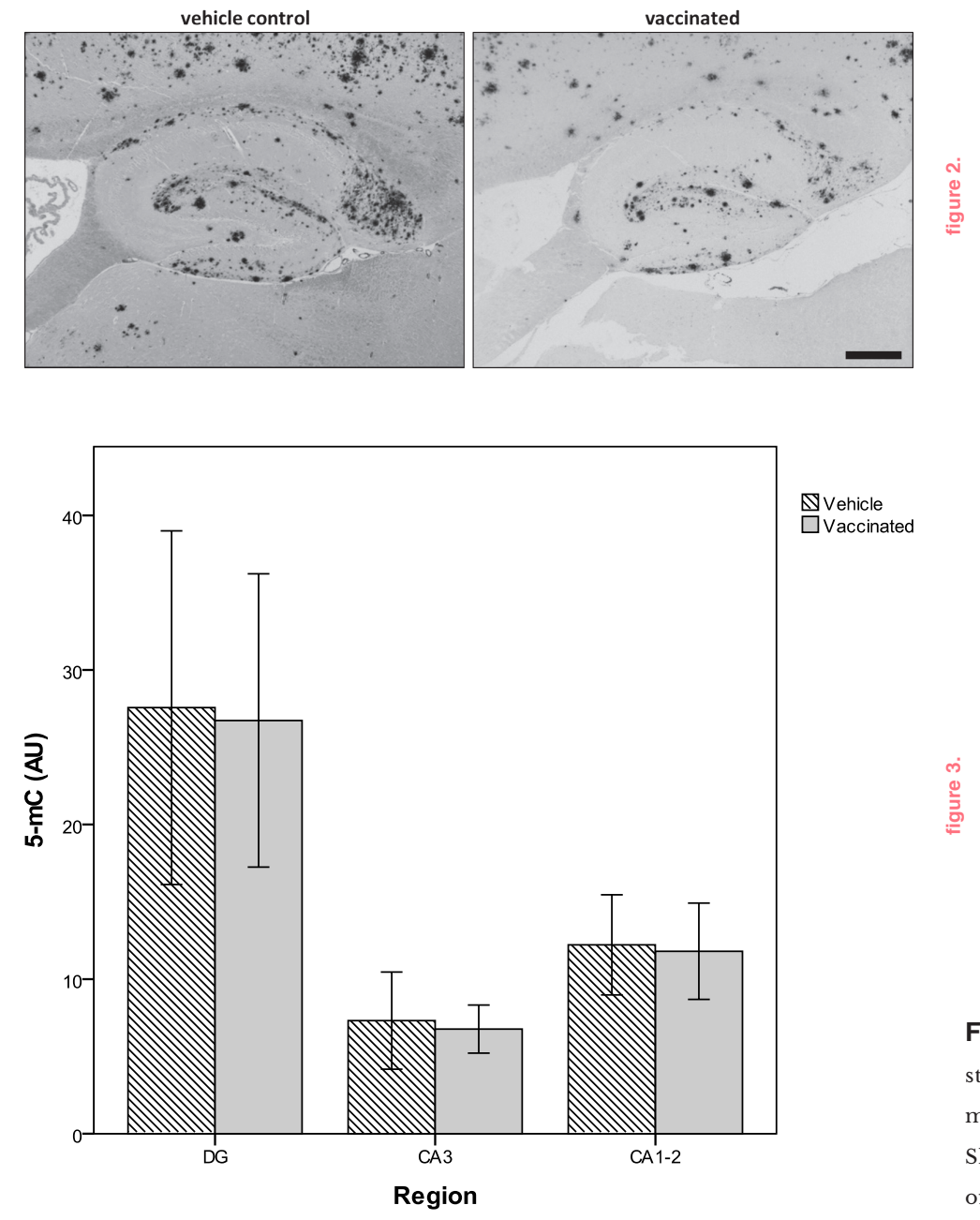

FIGURE 2. Hippocampal 3Al plaque staining in transgenic APP/PSldE9 mice.

Shown are representative images of vehicle-treated and vaccinated animals. Note the marked decrease in amyloid- $\beta$ immunoreactivity in the vaccinated animal. Scale bar rep-

inverse correlation with $\mathrm{ACl}$ after 24 hours was found ( $r=-0.656, p=0.028$ ). DNMT3A negatively correlated with $\mathrm{ACl}$ after 24 hours $(r=-0.619, p=$ 0.042 ), and positively with the average escape latency in the MWM task ( $r$ $=0.679, p=0.022)$. The $5-\mathrm{mC}: 5-\mathrm{hmC}$ ratio only inversely correlated with $\mathrm{ACl}$ after 2 hours $(r=-0.817, p=0.002)$. The $5-\mathrm{mC}: 5-\mathrm{hmC}$ ratio showed a strong correlation with 5-mC $(r=0.903, p<0.001)$, but not with $5-\mathrm{hmC}(r=$ $-0.159, p=0.640)$. Additionally, DNMT3A showed a statistically significant correlation with 5 -hmC $(r=0.714, p=0.014)$, but only a trend towards a statistically significant correlation with $5-\mathrm{mC}(r=0.594, p=0.054) .5-\mathrm{mC}$ and 5 -hmC also did not correlate with each other $(r=0.258, p=0.445)$.

FIGURE 3. Integrated density of 5 -methylcytosine $(5-\mathrm{mC})$ for the vaccinated and vehicle-treated groups in the dentate gyrus (DG), cornu ammonis (CA) 3, and CAl-2 subregions of the hippocampus.

Shown are the mean and 95\% confidence interval. No differences between the groups were detected with a Mann-Whitney U test. AU, arbitrary units. 


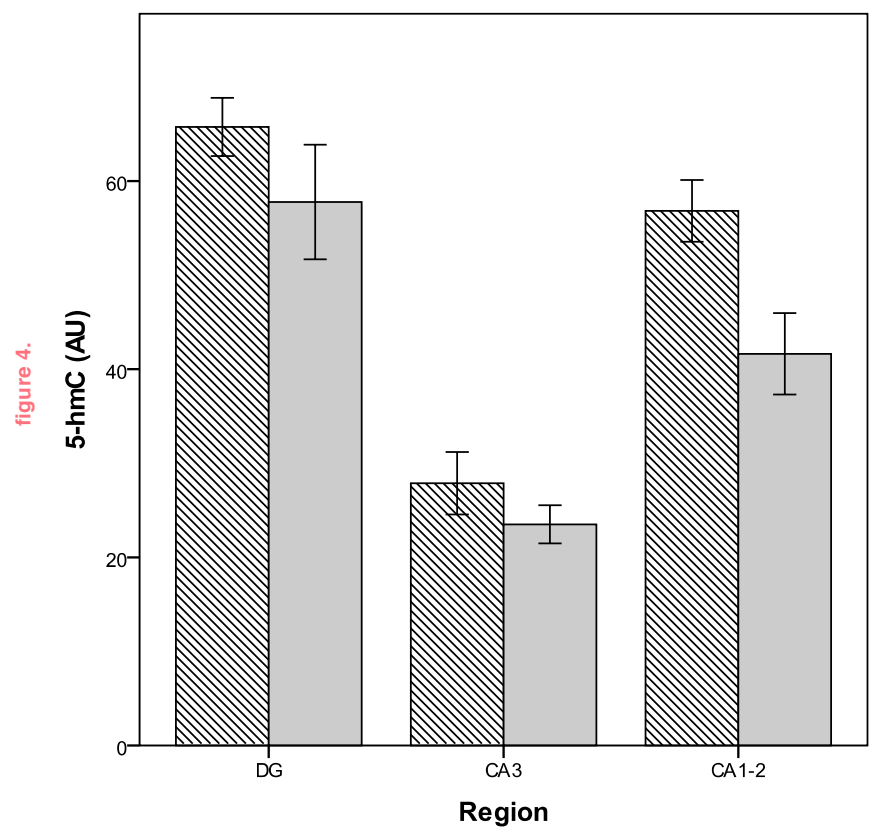

NVehicle

$\square$ Vaccinated

FIGURE 4. Integrated density of 5 -hydroxymethylcytosine $(5-\mathrm{hmC})$ for the vaccinated and vehicle-treated groups in the dentate gyrus (DG), cornu ammonis (CA) 3, and CAl-2 subregions of the hippocampus. Shown are the mean and $95 \%$ confidence interval. Mann-Whitney U tests showed that 5 -hmC levels were lowered in the vaccinated group, in all subregions. AU, arbitrary units; ${ }^{*} p<0.05 ;{ }^{* *} p<0.01$.

FIGURE 5. Integrated density of DNA methyltransferase (DNMT) 3A for the vaccinated and vehicle-treated groups in the dentate gyrus (DG), cornu ammonis (CA) 3, and CAl-2 subregions of the hippocampus. Shown are the mean and $95 \%$ con-

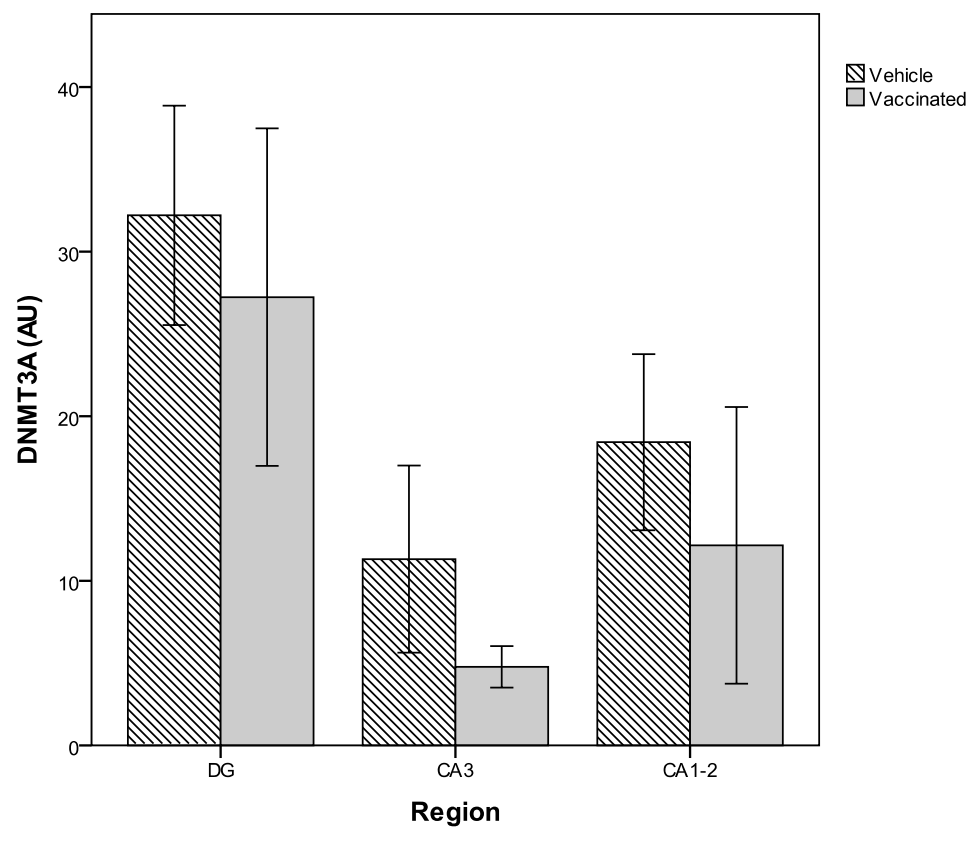

fidence interval. Mann-Whitney U tests showed that DNMT3A levels were lowered in the CA3 region of the vaccinated group. AU, arbitrary units; ${ }^{*} p<0.05$. 


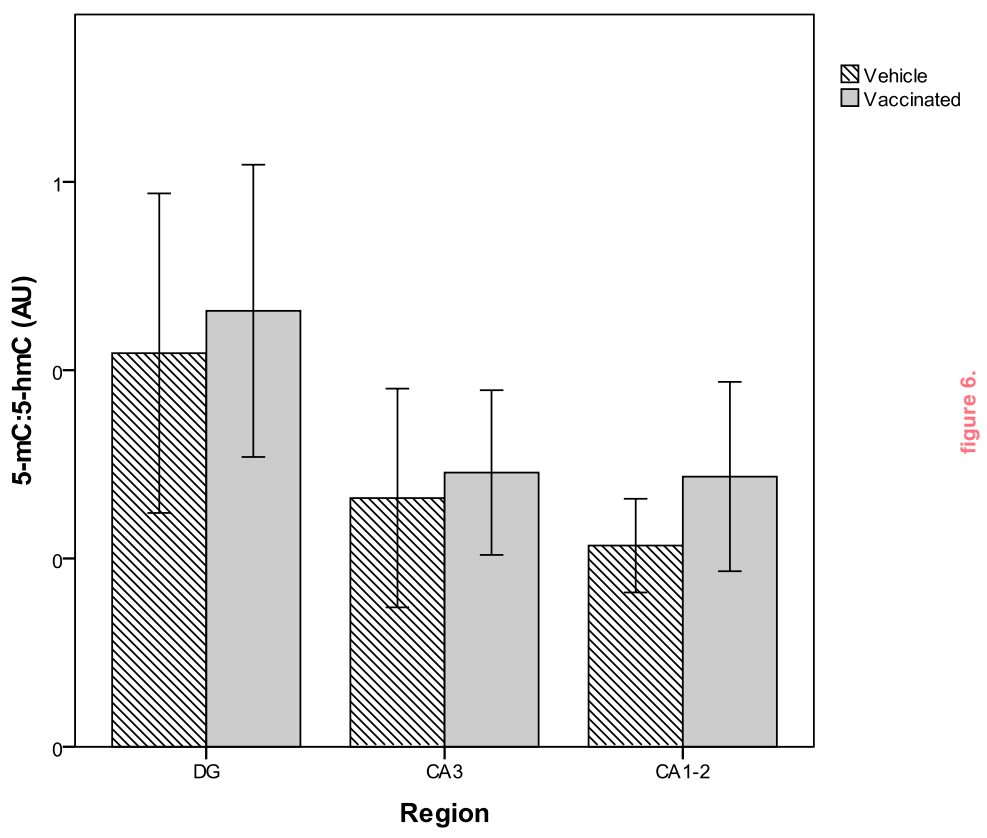

\subsection{Discussion}

Although there are many studies showing that $A D$ involves profound epigenetic changes and studies that indicate immunotherapy may be an effective means to combat this disease, this is, to our knowledge, the first study to investigate the epigenetic impact of immunotherapy in a relevant mouse model.

As previously reported [18], following chronic, active $A \beta$ immunization, APPswe/PS1dE9 mice showed decreased plaque pathology in the hippocampus. The present study indicates that these same mice show an additional decrease in general DNA hydroxymethylation and DNMT3A levels. Moreover, a positive correlation was found between $5-\mathrm{hmC}$ and DNMT3A levels, and A $\beta$ pathology. However, only 5-hmC correlated with SYP and PSD-95, thus indicating that higher levels of 5-hmC are associated with reduced synaptic integrity. On a behavioral level, escape latency in the MWM correlated best with $A \beta$ pathology, with (as expected) greater pathology associated with poorer performance, while freezing in the CFC task was the only behavioral outcome correlating with synaptic density. Interestingly, higher 5-mC, and to a lesser extent 5-hmC and DNMT3A, levels were associated with a decreased performance in the MWM test.

These observations corroborate that there is a relationship between epigenetic mechanisms and plaque pathology, and suggest that $A \beta$ may

FIGURE 6. Ratio of the 5-methylcytosine (5-mC) and 5-hydroxymethylcytosine $(5-\mathrm{hmC})$ integrated densities for the vaccinated and vehicle-treated groups in the dentate gyrus (DG), cornu ammonis (CA) 3, and CAl-2 subregions of the hippocampus.

Shown are the mean and $95 \%$ confidence interval. No differences between the groups were detected with a Mann-Whitney U test. AU, arbitrary units. 
not only be involved in inducing epigenetic changes, but may play a role in maintaining these alterations. However, the differential observations for DNA methylation and hydroxymethylation suggest that these mechanisms may play separate roles in AD. Previous studies in humans and mice show DNA methylation is involved in $\operatorname{AD}[12,13,23]$. Our findings, however, indicate this dysregulated DNA methylation may not be directly related to $A \beta$ pathology, or is not readily reversible, as global DNA methylation was not affected after lowering plaque levels. In contrast, DNA hydroxymethylation, which has previously also been implicated in $A D$, may be more directly affected by the presence of $A \beta$, as a global decrease in $5-\mathrm{hmC}$ was observed after lowering $A \beta$ levels. It is thus possible that $A \beta$ is able to increase DNA hydroxymethylation, possibly through a decrease in 5-hmC oxidation and downstream demethylation, which may result in changes in gene expression. The observation that $A \beta$ can induce global demethylation [17] is partly in line with our findings, but further investigations should elucidate whether $A \beta$ induces complete demethylation (back to cytosine), or conversion of 5-mC to $5-\mathrm{hmC}$.

Interesting in this respect is the potential role of DNMT3A, which was observed to decrease with 5 -hmC levels after lowering $A \beta$ levels, and which showed a strong correlation with 5 -hmC levels. 5 -hmC is generated through the oxidation of $5-\mathrm{mC}$ by TET enzymes, but ultimately, novel methylated cytosines are required to create more hydroxymethylated cytosines. A possible mechanism driving de novo DNA hydroxymethylation is through the combined actions of DNMT3A and TET3, but for which there is still limited evidence in zygotes [24], and much remains to be elucidated in this respect. Note that while the de novo DNA methyltransferase DNMT3A has also been attributed demethylation activity, this is proposed to involve a direct $5-\mathrm{mC}$ to $\mathrm{C}$ conversion, without a hydroxymethylated intermediate [25].

The findings of this study should be viewed in light of some limitations. Only a limited amount of animals was available for the current investigation, limiting its power. Additionally, while the mice used in this study develop $A \beta$ plaque pathology, they do not develop tau pathology or robust loss of neurons. Therefore, it is possible that the epigenetic changes we observed here may differ from those seen in mouse models with both plaques and tau pathologies and/or in humans with AD.

In summary, we confirm that active $A \beta$ immunotherapy effectively lowered $A \beta$ plaque levels, and that epigenetic markers 5-mC, 5-hmC, and DNM3A correlated with behavioral outcomes, but that mainly $5-\mathrm{hmC}$ and DNMT3A were associated with AD-related pathology. Accordingly, active immunotherapy only appeared to affect 5-hmC and DNMT3A levels. These preliminary findings raise important questions about the possibly (partially) 
independent roles of DNA methylation and hydroxymethylation in $A D$, the involvement of DNMT3A in enhancing 5-hmC levels, and the interplay between immunotherapy and epigenetic regulation, which should be addressed in future studies.

\section{Acknowledgments}

We kindly thank K Le, H Crehan, Q Shi, and S Chowdhury for technical assistance. Funds have been provided by the Internationale Stichting Alzheimer Onderzoek (ISAO) grants 07551 and 11532 (D.L.A.vdH.), by the ISAO grants 09552 and 13515, and the Netherlands Organization for Scientific Research (NWO), grant 916.11.086 (Veni Award) (B.P.F.R.), and by an ISAO fellowship and a fellowship as part of NWO grant 022.005.019, (R.L.), and by an Anonymous Foundation and NIH/NIA R01 AG040092 (C.A.L.). Additional funds have been provided by the Joint ProgrammeNeurodegenerative Disease Research (JPND) for the EPI-AD consortium (http://www.neurodegenerationresearch.eu/wp-content/uploads/2015/10/ Factsheet_EPI-AD.pdf). The funding agencies were not involved in the study design, data collection, analysis and interpretation, writing of the report, and the decision to submit the article for publication. The authors declare no conflicts of interest.

\section{REFERENCES}

[1] Agadjanyan MG, Petrovsky N, Ghochikyan A. A fresh perspective from immunologists and vaccine researchers: Active vaccination strategies to prevent and reverse Alzheimer's disease. Alzheimer's Dement 2015; 11: 1246-1259.

[2] Schenk D, Barbour R, Dunn W, et al. Immunization with amyloid-b attenuates Alzheimer disease-like pathology in the PDAPP mouse. Nature 1999; 400: 173-177.

[3] Weiner HL, Lemere CA, Maron R, et al. Nasal administration of amyloid-beta peptide decreases cerebral amyloid burden in a mouse model of Alzheimer's disease. Ann Neurol 2000; 48: 567-79.

[4] Lemere CA, Maron R, Spooner ET, et al. Nasal A.beta. treatment induces anti-A.beta. antibody production and decreases cerebral amyloid burden in PD-APP mice. Ann NY Acad Sci 2000; 920: 328-331.

[5] Lemere CA, Spooner ET, Leverone JF, et al. Intranasal immunotherapy for the treatment of Alzheimer's disease: Escherichia coli LT and LT(R192G) as mucosal adjuvants. Neurobiol Aging 2002; 23: 991-1000.

[6] Janus C, Pearson J, McLaurin J, et al. A beta peptide immunization reduces behavioural impairment and plaques in a model of Alzheimer's disease. Nature 2000; 408: 979-982.

[7] Morgan D, Diamond DM,

Gottschall PE, et al. A beta peptide vaccination prevents memory loss in an animal model of Alzheimer's disease. Nature 2000; 408: 982-985.

[8] Bayer AJ, Bullock R, Jones

RW, et al. Evaluation of the safety and immunogenicity of synthetic A beta 42 (AN1792) in patients with AD. Neurology 2005; 64: 94-101. 
Austen BM. The elusive nature and diagnostics of misfolded $A \beta$ oligomers.

Front Chem 2015; 3: 17.

[10] Choudhuri S. From

Waddington's epigenetic landscape to small noncoding RNA: some important milestones in the history of epigenetics research. Toxicol Mech Methods 2011; 21: 252-274.

[11] Liu L, Li Y, Tollefsbol TO.

Gene-environment interactions and epigenetic basis of human diseases. Curr Issues Mol Biol 2008; 10: 25-36. [12] Chouliaras L, Mastroeni D, Delvaux E, et al. Consistent decrease in global DNA methylation and hydroxymethylation in the hippocampus of Alzheimer's disease patients. Neurobiol Aging 2013; 34: 2091-2099.

[13] Lardenoije R, Iatrou A,

Kenis $\mathrm{G}$, et al. The epigenetics of aging and neurodegeneration. Prog Neurobiol 2015; 131: 21-64.

[14] Condliffe D, Wong A,

Troakes C, et al. Cross-region reduction in 5-hydroxymethylcytosine in Alzheimer's disease brain. Neurobiol Aging 2014; 35: 1850-1854.

[15] Globisch D, Münzel M, Müller M, et al. Tissue distribution of 5 -hydroxymethylcytosine and search for active demethylation intermediates. PLoS One; 5. Epub ahead of print 2010. DOI: 10.1371/journal. pone.0015367.

[16] Kriaucionis S, Heintz

$\mathrm{N}$. The nuclear DNA base 5-hydroxymethylcytosine is present in Purkinje neurons and the brain. Science 2009; 324: 929-30.

[17] Chen K-L, Wang SS-S, Yang $\mathrm{Y}-\mathrm{Y}$, et al. The epigenetic effects of amyloid-beta(1-40) on global DNA and neprilysin genes in murine cerebral endothelial cells. Biochem Biophys Res
[18] Liu B, Frost JL, Sun J, et al.

MER5101, a novel A $\beta 1-15: D T$ conjugate vaccine, generates a robust anti-A $\beta$ antibody response and attenuates $A \beta$ pathology and cognitive deficits in APPswe/PSldE9 transgenic mice. J Neurosci 2013; 33: 7027-7037.

[19] Jankowsky JL, Fadale DJ, Anderson J, et al. Mutant presenilins specifically elevate the levels of the 42 residue $\beta$-amyloid peptide in vivo: Evidence for augmentation of a 42-specific $y$ secretase. Human Molecular Genetics 2004; 13: 159-170.

[20] Maier M, Seabrook TJ, Lemere CA. Modulation of the humoral and cellular immune response in $A \beta$ immunotherapy by the adjuvants monophosphoryl lipid A (MPL), cholera toxin B subunit (CTB) and E. coli enterotoxin LT(R192G). Vaccine 2005; 23: 5149-5159.

[21] Frost JL, Liu B, Rahfeld

$\mathrm{J}-\mathrm{U}$, et al. An anti-pyroglutamate- $3 \mathrm{~A} \beta$ vaccine reduces plaques and improves cognition in APPswe/PS1 $\triangle \mathrm{E} 9$ mice. Neurobiol Aging 2015; 36: 3187-99. [22] Lardenoije R, van den Hove DA, Vaessen TSJ, et al. Epigenetic modifications in mouse cerebellar Purkinje cells: effects of aging, caloric restriction, and overexpression of superoxide dismutase 1 on 5 -methylcytosine and 5-hydroxymethylcytosine. Neurobiol Aging 2015; 36: 3079-3089. [23] Coppieters N, Dieriks B V, Lill C, et al. Global changes in DNA methylation and hydroxymethylation in Alzheimer's disease human brain. Neurobiol Aging 2014; 35: 1334-1344. [24] Amouroux R, Nashun B, Shirane K, et al. De novo DNA methylation drives 5 -hmC accumulation in mouse zygotes. Nat Cell Biol 2016; 18: 225-233.
[25] Chen C-C, Wang K-Y, Shen C-KJ. DNA 5-methylcytosine demethylation activities of the mammalian DNA methyltransferases. J Biol Chem 2013; 288: 9084-91. 


\begin{tabular}{|c|c|c|c|c|}
\hline & Variables & $\begin{array}{l}\text { Vaccinated (mean } \\
\pm \text { SD) }\end{array}$ & $\begin{array}{l}\text { Control (mean } \\
\quad \pm S D \text { ) }\end{array}$ & Mann-Whitney U \\
\hline AD marker & $\begin{array}{c}\text { Fraction of } \\
\text { hippocampal area } \\
\text { covered by plaques }\end{array}$ & $0.1447 \pm 0.0225$ & $0.1939 \pm 0.0254$ & $\begin{array}{c}U=3.00 \\
p=0.028^{*}\end{array}$ \\
\hline \multirow{16}{*}{$\begin{array}{c}\text { Epigenetic } \\
\text { markers }\end{array}$} & \multirow{4}{*}{$5-\mathrm{mC}$} & Hip: $15.10 \pm 3.05$ & Hip: $15.70 \pm 5.50$ & Hip: $U=14.00, p=0.855$ \\
\hline & & DG: $26.73 \pm 7.64$ & $D G: 27.56 \pm 10.90$ & DG: $U=14.00, p=0.855$ \\
\hline & & CA3: $6.77 \pm 1.25$ & CA3: $7.32 \pm 2.99$ & CA3: $U=15.00, p=1.00$ \\
\hline & & CA1-2: $11.80 \pm 2.51$ & CA1-2: $12.22 \pm 3.09$ & CA1-2: $U=14.00, p=0.855$ \\
\hline & \multirow{4}{*}{ 5-hmC } & Hip: $40.97 \pm 2.12$ & Hip: $50.15 \pm 2.13$ & Hip: $U=0.00, P=0.006^{\star \star}$ \\
\hline & & DG: $57.77 \pm 4.90$ & $D G: 65.75 \pm 2.95$ & DG: $U=3.00, p=0.028^{*}$ \\
\hline & & CA3: $23.51 \pm 1.63$ & CA3: $27.88 \pm 3.17$ & CA3: $U=3.00, p=0.028^{*}$ \\
\hline & & CA1-2: $41.63 \pm 3.49$ & CA1-2: $56.82 \pm 3.12$ & CA1-2: $U=0.00, p=0.006$ \\
\hline & \multirow{4}{*}{ DNMT3A } & Hip: $14.73 \pm 1.40$ & Hip: $20.65 \pm 5.37$ & Hip: $U=4.00, p=0.045^{\star}$ \\
\hline & & DG: $27.24 \pm 8.26$ & $D G: 32.21 \pm 6.35$ & $D G: U=9.00, p=0.273$ \\
\hline & & CA3: $4.78 \pm 1.01$ & CA3: $11.32 \pm 5.41$ & CA3: $U=2.00, p=0.018^{*}$ \\
\hline & & CA1-2: $12.16 \pm 6.77$ & CA1-2: $18.43 \pm 5.10$ & CA1-2: $U=6.00, p=0.100$ \\
\hline & \multirow{4}{*}{ 5-mC:5-hmC } & Hip: $0.35 \pm 0.06$ & Hip: $0.30 \pm 0.10$ & Hip: $U=10.00, p=0.361$ \\
\hline & & DG: $0.46 \pm 0.13$ & $D G: 0.42 \pm 0.16$ & DG: $U=10.00, p=0.361$ \\
\hline & & CA3: $0.29 \pm 0.07$ & CA3: $0.26 \pm 0.11$ & CA3: $U=11.00, p=0.465$ \\
\hline & & CA1-2: $0.29 \pm 0.08$ & CA1-2: $0.21 \pm 0.05$ & CA1-2: $U=5.00, p=0.068$ \\
\hline
\end{tabular}

\begin{tabular}{|c|c|c|c|c|c|c|c|}
\hline & $A \beta x-40$ & $A \beta x-42$ & $\begin{array}{c}\text { Fraction of } \\
\text { hippocampal } \\
\text { area covered } \\
\text { by plaques }\end{array}$ & SYP CA1 & $\mathrm{SY} P \mathrm{CA} 3$ & PSD-95 CA1 & PSD-95 CA3 \\
\hline \multirow{2}{*}{$5-\mathrm{mC}$} & $r=0.574$ & $r=0.173$ & $r=0.221$ & $r=-0.087$ & $r=0.036$ & $r=0.091$ & $r=0.023$ \\
\hline & $p=0.065$ & $p=0.610$ & $p=0.514$ & $p=0.799$ & $p=0.915$ & $p=0.790$ & $p=0.946$ \\
\hline \multirow{2}{*}{ 5-hmC } & $r=0.305$ & $r=0.600$ & $r=0.643$ & $r=0.831$ & $r=0.823$ & $r=0.692$ & $r=0.702$ \\
\hline & $p=0.361$ & $p=0.051$ & $p=0.033^{*}$ & $p=0.002^{\star *}$ & $p=0.002^{\star \star}$ & $p=0.018^{*}$ & $p=0.016^{\star}$ \\
\hline \multirow{2}{*}{ 5-mC:5-hmC } & $r=0.409$ & $r=-0.093$ & $r=-0.032$ & $r=-0.455$ & $r=-0.297$ & $r=-0.155$ & $r=-0.246$ \\
\hline & $p=0.212$ & $p=0.785$ & $p=0.925$ & $p=0.160$ & $p=0.376$ & $p=0.648$ & $p=0.466$ \\
\hline \multirow{2}{*}{ DNMT3A } & $r=0.594$ & $r=0.443$ & $r=0.629$ & $r=0.499$ & $r=0.439$ & $r=0.456$ & $r=0.440$ \\
\hline & $p=0.054$ & $p=0.172$ & $p=0.038^{*}$ & $p=0.118$ & $p=0.177$ & $p=0.158$ & $p=0.176$ \\
\hline
\end{tabular}

\begin{tabular}{|c|c|c|c|c|c|c|c|}
\hline & $A \beta x-40$ & $A \beta x-42$ & $\begin{array}{c}\text { Fraction of } \\
\text { hippocampal } \\
\text { area covered } \\
\text { by plaques }\end{array}$ & $\begin{array}{l}\text { SYP } \\
\text { CA1 }\end{array}$ & $\begin{array}{l}\text { SYP } \\
\text { CA3 }\end{array}$ & $\begin{array}{l}\text { PSD-95 } \\
\text { CA1 }\end{array}$ & $\begin{array}{l}\text { PSD-95 } \\
\text { CA3 }\end{array}$ \\
\hline \multirow{2}{*}{ CFC } & $r=-0.296$ & $r=-0.539$ & $r=-0.427$ & $r=-0.198$ & $r=-0.267$ & $r=-0.599$ & $r=-0.709$ \\
\hline & $p=0.376$ & $p=0.087$ & $p=0.190$ & $p=0.559$ & $p=0.428$ & $p=0.052$ & $p=0.015^{*}$ \\
\hline \multirow{2}{*}{$\begin{array}{l}\text { MWM escape } \\
\text { latency }\end{array}$} & $r=0.694$ & $r=0.703$ & $r=0.586$ & $r=0.144$ & $r=0.230$ & $r=0.300$ & $r=0.403$ \\
\hline & $p=0.018^{*}$ & $p=0.016^{*}$ & $p=0.058$ & $p=0.672$ & $p=0.496$ & $p=0.370$ & $p=0.220$ \\
\hline \multirow{2}{*}{$\begin{array}{c}\text { MWM } \\
\text { quadrant } 2 \mathrm{~h}\end{array}$} & $r=-0.279$ & $r=-0.297$ & $r=-0.152$ & $r=-0.423$ & $r=-0.335$ & $r=0.019$ & $r=-0.067$ \\
\hline & $p=0.406$ & $p=0.374$ & $p=0.655$ & $p=0.194$ & $p=0.314$ & $p=0.995$ & $p=0.845$ \\
\hline \multirow{2}{*}{$\begin{array}{c}\text { MWM } \\
\text { quadrant 24h }\end{array}$} & $r=-0.250$ & $r=-0.255$ & $r=-0.113$ & $r=-0.318$ & $r=-0.245$ & $r=-0.083$ & $r=-0.144$ \\
\hline & $p=0.459$ & $p=0.449$ & $p=0.740$ & $p=0.340$ & $p=0.468$ & $p=0.809$ & $p=0.674$ \\
\hline \multirow{2}{*}{ MWM ACl 2h } & $r=-0.271$ & $r=0.019$ & $r=-0.060$ & $r=0.315$ & $r=0.162$ & $r=-0.065$ & $r=-0.025$ \\
\hline & $p=0.420$ & $p=0.956$ & $p=0.860$ & $p=0.346$ & $p=0.634$ & $p=0.849$ & $p=0.942$ \\
\hline \multirow{2}{*}{$\begin{array}{l}\text { MWM ACl } \\
24 \mathrm{~h}\end{array}$} & $r=-0.278$ & $r=-0.352$ & $r=-0.232$ & $r=-0.430$ & $r=-0.439$ & $r=-0.377$ & $r=-0.321$ \\
\hline & $p=0.409$ & $p=0.288$ & $p=0.493$ & $p=0.187$ & $p=0.177$ & $p=0.253$ & $p=0.336$ \\
\hline
\end{tabular}




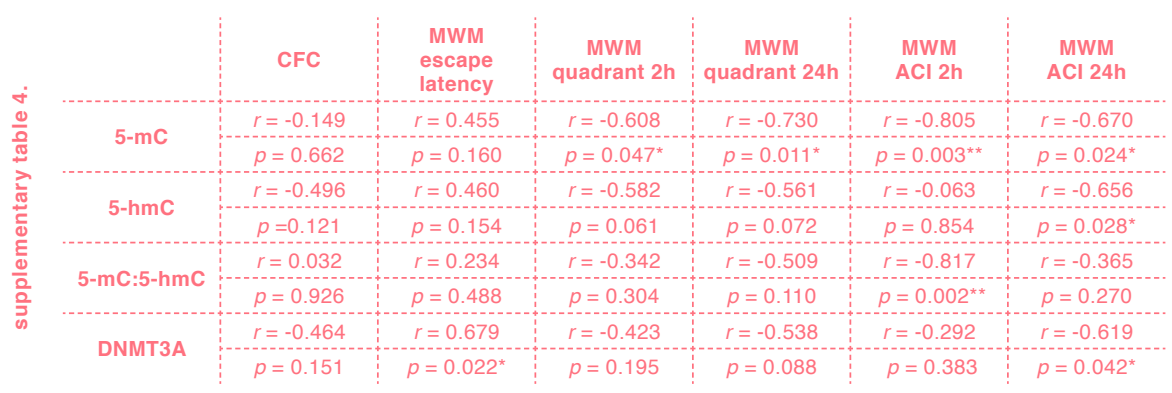

\section{SUPPLEMENTARY DATA}

SUPPLEMENTARY TABLE 1. Overview

of main results.

ABBREVIATIONS: 5-mC, 5-methylcytosine; 5-hmC, 5-hydroxymethylcytosine; AD, Alzheimer's disease; CA, cornu ammonis; DG, dentate gyrus; DNMT, DNA methyltransferase; Hip, hippocampus; SD, standard deviation; ${ }^{*} p<0.05 ;{ }^{* *} p<0.01$.

SUPPLEMENTARY TABLE 2. Correlation

Alzheimer's disease markers with epigenetic markers.

ABBREVIATIONS: 5-mC, 5-methylcytosine; 5-hmC, 5-hydroxymethylcytosine; $A \beta$, amyloid- $\beta$; CA, cornu ammonis; DNMT, DNA methyltransferase; PSD-95, postsynaptic density protein 95; $r$, Pearson's r; SYP, synaptophysin; ${ }^{*} p<0.05 ;{ }^{* *} p<0.01$.

SUPPLEMENTARY TABLE 3. Correlation behavior with Alzheimer's disease markers.

ABBREVIATIONS: A $\beta$, amyloid- $\beta$; ACI, annulus crossing index; CA, cornu ammonis; CFC, contextual fear conditioning; MWM, Morris water maze; PSD-95, postsynaptic density protein 95; $r$, Pearson's r; SYP, synaptophysin; ${ }^{*} p<0.05$.
SUPPLEMENTARY TABLE 4. Correlation epigenetic markers with behavior. ABBREVIATIONS: 5-mC, 5-methylcytosine; 5-hmC, 5-hydroxymethylcytosine; ACI, annulus crossing index; CFC, contextual fear conditioning; DNMT, DNA methyltransferase; MWM, Morris water maze; $r$, Pearson's $r ;{ }^{*} p<0.05 ;{ }^{* *} p<0.01$. 



\section{THE DNA METHYLOME AND HYDROXYMETHY- LOME IN ALZHEIMER'S DISEASE; CORTICAL DYSREGULATION OF $O X T, C 3, A N K 1$ AND RHBDF2}

ROY LARDENOIJE ${ }^{A, *}$, JANOU A.Y. ROUBROEKS ${ }^{A, B, *}$, EHSAN PISHVA $^{A, B, *}$, ARTEMIS IATROU ${ }^{A}$, ADAM R. SMITH ${ }^{\mathrm{B}}$, REBECCA G. SMITH ${ }^{B}$, LARS M.T. EIJSSEN ${ }^{A, C}$, GUNTER KENIS ${ }^{A}$, DIEGO MASTROENI ${ }^{A, D, E}$, ELAINE DELVAUX ${ }^{\mathrm{D}, \mathrm{E}}$, PAUL D. COLEMAN ${ }^{\mathrm{D}, \mathrm{E}}$, JONATHAN MILL ${ }^{B, F}$, BART P. RUTTEN ${ }^{A}$, KATIE LUNNON ${ }^{B}$, DANIËL L.A. VAN DEN HOVE ${ }^{A, G}$

ASCHOOL FOR MENTAL HEALTH AND NEUROSCIENCE (MHENS), DEPARTMENT OF PSYCHIATRY AND NEUROPSYCHOLOGY, MAASTRICHT UNIVERSITY, UNIVERSITEITSSINGEL 50, 6200 MD MAASTRICHT, THE NETHERLANDS BUNIVERSITY OF EXETER MEDICAL SCHOOL, UNIVERSITY OF EXETER, EXETER, UK.

'DEPARTMENT OF BIOINFORMATICS-BIGCAT, MAASTRICHT UNIVERSITY, PO BOX 616, 6200 MD MAASTRICHT, THE NETHERLANDS.

'L.J. ROBERTS ALZHEIMER'S DISEASE CENTER, BANNER SUN HEALTH RESEARCH INSTITUTE, 10515 W. SANTA FE DRIVE, SUN CITY, AZ 85351, USA EIODESIGN INSTITUTE, NEURODEGENERATIVE DISEASE RESEARCH CENTER, ARIZONA STATE UNIVERSITY, TEMPE, AZ, 85287, US.

FINSTITUTE OF PSYCHIATRY, KING'S COLLEGE LONDON, LONDON, UK.

GLABORATORY OF TRANSLATIONAL NEUROSCIENCE, DEPARTMENT OF PSYCHIATRY, PSYCHOSOMATICS AND PSYCHOTHERAPY, UNIVERSITY OF WUERZBURG, FUECHSLEINSTRASSE 15, 97080 WUERZBURG, GERMANY

*THESE AUTHORS CONTRIBUTED EQUALLY TO THIS WORK. 


\section{Abstract}

In the present study, we identified methylomic and hydroxymethylomic differences in middle temporal gyrus tissue from Alzheimer's disease (AD) patients and age-matched controls. Using genome-wide arrays, AD-specific cytosine, 5-methylcytosine and 5-hydroxymethylcytosine profiles were identified in numerous genes, including ANK1, RHBDF2 and $C 3$, all of which have previously been implicated in AD. Moreover, the present study, which is the first of its kind examining the methylome and hydroxymethylome in $\mathrm{AD}$, suggests that OXT harbors Braak stagedependent OXT DNA hyperhydroxymethylation, concomitant with DNA hypomethylation. 


\subsection{Introduction}

The world's leading cause of dementia, Alzheimer's disease (AD), is characterized by a progressive development of amyloid plaques and intracellular neurofibrillary tangles, followed by neuronal cell death [1, 2]. Large-scale genome-wide association studies (GWAS) have identified high effect size risk variants of genes such as $A P O E$, as well as a number of common variants with a low effect size [3]. In addition, recent studies have suggested an important role for epigenetic mechanisms in the etiology of $A D$ [4], with reports of both global and gene-specific alterations in epigenetic modifications [5-8]. 5-Methylcytosine (5-mC), reflecting DNA methylation, is the most abundant and best understood epigenetic DNA modification, but regular bisulfite (BS) conversion, a widely used procedure for quantifying DNA methylation, cannot distinguish between 5-mC and the more recently identified 5-hydroxymethylcytosine (5-hmC), reflecting DNA hydroxymethylation. As $5-\mathrm{hmC}$ is particularly enriched in the brain $[9,10]$, where it may be involved in learning and memory [11], it is crucial to investigate $5-\mathrm{mC}$ and $5-\mathrm{hmC}$ separately in the context of $A D$.

\subsection{Methods}

In the present study, we quantified both 5-mC and 5-hmC in middle temporal gyrus (MTG) tissue obtained from 46 AD patients and 36 agematched, non-demented controls (see Supplementary Table 1 for cohort demographics). The MTG shows early AD pathology [12], and differences in global levels of MTG DNA methylation and hydroxymethylation have been previously observed in association with AD progression [13]. In order to quantify the genome-wide levels of 5-mC and 5-hmC at a single base resolution, oxidative bisulfite (oxBS) and regular BS conversion of genomic DNA was performed using the TrueMethyl ${ }^{\mathrm{TM}} 24$ Kit version 2.0 of CEGX ${ }^{\mathrm{TM}}$ (Cambridge Epigenetix Limited, Cambridge, UK). OxBSand BS-converted DNA was amplified and hybridized on the Illumina HumanMethylation450 BeadChip (450K BeadChip) [14]. Although readings from the oxBS-converted DNA are directly representative of 5-mC levels, 5-hmC levels were determined by subtracting the oxBS signal from the BS signal (Figure 1), the latter of which represents the combined fraction of 5-mC and 5-hmC modified cytosines. See the Supplementary methods section for a more detailed overview of the used methods. 
DNA input

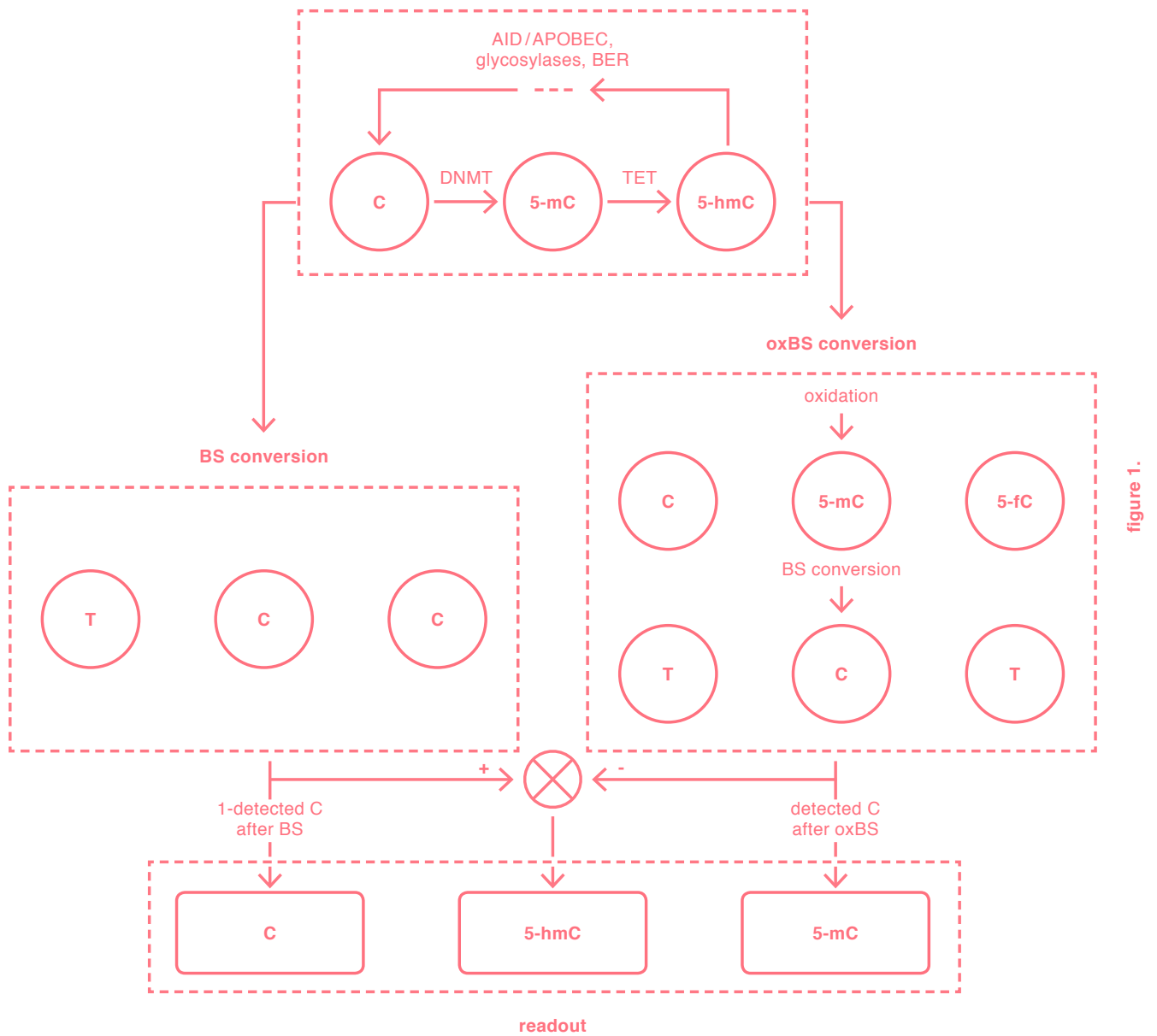

\subsection{Results and discussion}

Differentially methylated, hydroxymethylated, and unmodified cytosine positions (DMPs, DHPs, and DUPs, respectively) associated with disease diagnosis were identified (Supplementary Tables 2-4). At the nominal significance threshold ( $p=0.05$ ), 24,673 DMPs and 21,264 DUPs were identified among the 396,600 probes analyzed, while 9,261 DHPs out of a total of 178,951 probes passing the $5-\mathrm{hmC}$ detection criteria were identified (see Supplementary Methods). Several genes annotated to the 25 highest ranked DMPs, including SYNJ2 (cg05270236; $\beta=0.04$, $p=1.07 \times 10-4, \Delta=4.35 \%)$, ANK1 (cg11823178; $\beta=0.05, p=3.04 \times 10-$ $4, \Delta=5.08 \%)$ and RHBDF2 (cg12163800; $\beta=0.04, p=1.88 \times 10-4, \Delta=$ $4.32 \%)$, have been associated with AD or cognitive ability in previous DNA methylation studies [6, 15] (Supplementary Table 2). ANK1 (cg05066959; 
FIGURE 1. Overview of the proce-

dure to detect unmodified cytosines

(C), 5-methylcytosine (5-mC), and

5 -hydroxymethylcytosine $(5-\mathrm{hmC})$.

Naturally, C can be converted to

5-mC by DNA methyltransferases

(DNMTs) and 5-mC can be oxidized by ten-eleven translocation (TET)

enzymes, resulting in 5 -hmC. There are several proposed demethylation pathways through which 5-mC and 5-hmC can be converted back to C. DNA samples were split in two, one half was only treated with bisulfite (BS), which converts $\mathrm{C}$ into thymine (T). 5-mC and 5-hmC are protected against this conversion, and will be read as a $\mathrm{C}$ on the array. The detected C signal after BS conversion is thus actually the combined $5-\mathrm{mC}$ and $5-\mathrm{hmC}$ signal. As the signals are converted to fractions, with $\mathrm{C}+5-\mathrm{mC}+5-\mathrm{hmC}=1$, the fraction of $\mathrm{C}$ in the input DNA can be determined by subtracting the $\mathrm{C}$ signal after BS conversion (representing the combined 5-mC and 5-hmC fraction in the input DNA) from 1. The other half of the DNA sample was first oxidized, which converts 5 -hmC into 5 -formylcytosine (5-fC), and then treated with BS. $5-\mathrm{fC}$ is not protected against the BS conversion, so it also turns into T. C detected on the array after this oxidative BS (oxBS) conversion thus represents the fraction of $5-\mathrm{mC}$ in the input DNA.

The 5-hmC fraction in the input DNA can be determined by subtracting the fraction of 5-mC (detected $\mathrm{C}$ after oxBS) from the combined 5-mC and 5-hmC fraction (detected C after BS). This procedure results in three readout signals: unmodified C, $5-\mathrm{mC}$, and 5-hmC. Note that 5-fC, and probably also 5-carboxylcytosine (5-caC), are included in the unmodified $\mathrm{C}$ fraction. $\beta=-0.05, p=9.98 \times 10-4, \Delta=-4.94 \%)$ and RHBDF2 (cg13076843; $\beta=$ $-0.05, p=2.62 \times 10-5, \Delta=-4.57 \%, \operatorname{cg} 05810363 ; \beta=-0.04, p=9.19 \times 10-$ $5, \Delta=-4.07 \%, \operatorname{cg} 12163800 ; \beta=-0.04, p=5.12 \times 10-5, \Delta=-3.54 \%)$ were also found among the 25 highest-ranked DUPs (Supplementary Table 4). Notably, the gene OXT is annotated to the highest-ranked DMP (cg13285174; $\beta=-0.07, p=6.52 \times 10-5, \Delta=-6.95 \%$ ), as well as the fifteenth-ranked DHP (cg19592472; $\beta=0.04, p=6.35 \times 10-5, \Delta=4.39 \%$ ). The third highest-ranked disease-associated DHP is located in the gene DDR2 (cg00457087; $\beta=-0.05, p=2.63 \times 10-5, \Delta=-4.65 \%$ ), of which total DNA modifications have previously been linked to the pathogenesis of AD [16] (Supplementary Table 3). Note that no loci reached statistical significance after a false discovery rate (FDR) correction. Therefore, in addition to positions, differentially modified regions of DNA were identified, by determining spatial correlations between DMPs, DHPs or DUPs within a 500 bp window, using comb-p [17]. After Šidák correction, we identified six significant differentially methylated regions (DMRs) in OXT, ADIRF, RHBDF2, CDX1, RAP1GAP2, and FAM198B, two significant differentially hydroxymethylated regions (DHRs) in OXT and $S L C 12 A 4$, and six regions with significant differential levels of unmodified cytosine (DURs) in RHBDF2, LOC100190940, CDX1, TNK2, C3, and ANK1 (Table 1).

Using neuropathological Braak staging of the subjects, significant Braak stage-associations were observed for the majority of probes located within the DMRs, DHRs, and DURs (Supplementary Tables 5-7), demonstrating the relevance of our findings in relation to AD.

Studies using standard BS conversion only, and thus looking at the combined 5-mC and 5-hmC levels, have found strong associations between $A D$ and RHBDF2 in the prefrontal cortex (PFC) [6] and EC, as well as between $\mathrm{AD}$ and $A N K 1$ in the PFC and superior temporal gyrus [5]. ANK1 and RHBDF2 are involved in axonal compartmentalization and tumor necrosis factor signaling, respectively $[18,19]$ Distinguishing between $5-\mathrm{mC}$ and $5-\mathrm{hmC}$ signals in the current study indicates that these two genes are mainly affected by differential DNA methylation. In contrast, a region in $C 3$ only showed AD-related differences in total DNA modifications, but not 5-mC and 5-hmC separately. C3 encodes a central component of the complement system and mediates developmental synapse elimination by phagocytic microglia, and has been implicated in mediating synaptic loss during aging [20] and in the early stages of $A D$ [21]. That there are no significant AD-related changes in C3 5-mC and $5-\mathrm{hmC}$ levels when analyzed separately, and that the combined fraction of modified cytosines (BS signal) at the same time indirectly reflects the fraction of unmodified cytosine, suggests that differential levels of unmodified cytosine may be primarily relevant for the dysregulation of $\mathrm{C3}$ in $A D$. 


\begin{tabular}{|c|c|c|c|c|c|c|c|}
\hline Position & $\mathrm{n}$ & $p$-value & Šidák- $p$ & Gene & Gene feature & $\begin{array}{l}\text { Change } \\
\text { in } A D\end{array}$ & \\
\hline \multicolumn{8}{|l|}{ DMR (5-mC) } \\
\hline $\begin{array}{c}\text { chr 20: } 3052115 \text { - } \\
3052346\end{array}$ & 8 & $3.25 \mathrm{E}-13$ & 5.57E-10 & $O X T$ & TSS + utr $5+$ cds & $\downarrow$ & \\
\hline $\begin{array}{c}\text { chr 10: } 88728073- \\
88728236\end{array}$ & 4 & $1.88 \mathrm{E}-07$ & 4.58E-04 & C10orf116 & TSS + exon + utr5 & $\Downarrow$ & \\
\hline $\begin{array}{c}\text { chr 17: } 74475240- \\
74475403\end{array}$ & 5 & 5.23E-07 & 1.27E-03 & RHBDF2 & intron + cds & $\uparrow$ & \\
\hline $\begin{array}{c}\text { chr } 5: 149546331- \\
149546472\end{array}$ & 5 & $5.18 \mathrm{E}-07$ & 1.46E-03 & $C D \times 1$ & TSS + utr5 + cds & $\uparrow$ & \\
\hline $\begin{array}{c}\operatorname{chr} 17: 2951666 \text { - } \\
2951720\end{array}$ & 3 & 2.07E-06 & $1.51 \mathrm{E}-02$ & $R A P 1 G A P 2$ & intergenic & $\uparrow$ & \\
\hline $\begin{array}{c}\text { chr } 4: 159092536- \\
159092554\end{array}$ & 2 & $1.68 \mathrm{E}-06$ & 3.63E-02 & FAM198B & exon + utr5 & $\downarrow$ & \\
\hline \multicolumn{7}{|l|}{ DHR (5-hmC) } & $\overline{0}$ \\
\hline $\begin{array}{c}\text { chr 20: } 3052115- \\
3052275\end{array}$ & 7 & 4.05E-08 & 4.52E-05 & $O X T$ & TSS + exon $+u$ & $\uparrow$ & $\frac{\pi}{5}$ \\
\hline $\begin{array}{c}\text { chr } 16: 67978445- \\
67978451\end{array}$ & 2 & 1.18E-06 & $3.45 E-02$ & SLC12A4 & exon + utr3 & $\downarrow$ & \\
\hline \multicolumn{8}{|c|}{ DUR $(1-(5-m C+5-h m C))$} \\
\hline $\begin{array}{c}\text { chr 17: } 74475240- \\
74475403\end{array}$ & 5 & $1.23 \mathrm{E}-11$ & 2.99E-08 & RHBDF2 & intron + cds & $\uparrow$ & \\
\hline $\begin{array}{c}\text { chr } 12: 130554977 \\
130555092\end{array}$ & 3 & 1.97E-08 & $6.80 \mathrm{E}-05$ & LOC100190940 & intergenic & $\downarrow$ & \\
\hline $\begin{array}{c}\text { chr } 5: 149546331 \\
149546472\end{array}$ & 5 & $3.93 \mathrm{E}-07$ & 1.10E-03 & $C D \times 1$ & TSS + utr $5+$ cds & $\uparrow$ & \\
\hline $\begin{array}{c}\text { chr } 3: 195610084- \\
195610232\end{array}$ & 3 & $6.45 \mathrm{E}-07$ & 1.73E-03 & TNK2 & intron + cds & $\uparrow$ & \\
\hline $\begin{array}{c}\text { chr 19: } 6712321- \\
6712407\end{array}$ & 5 & 1.10E-06 & 5.05E-03 & C3 & cds & $\uparrow$ & \\
\hline $\begin{array}{c}\text { chr } 8: 41519308- \\
41519400\end{array}$ & 2 & $2.66 \mathrm{E}-06$ & 1.14E-02 & ANK1 & intron + cds & $\uparrow$ & \\
\hline
\end{tabular}

A novel finding in relation to $A D$ is $O X T$, the gene encoding oxytocin, a neuropeptide involved in the neuromodulation of social behavior, stress regulation, and associative learning [22]. OXT contained the fifteenth highest ranked DHP, and the highest ranked DMP, DMR and DHR. The DMR spans $231 \mathrm{bp}$, starts $223 \mathrm{bp}$ upstream from the OXT transcription start site (TSS), and overlaps with the $160 \mathrm{bp}$ long DHR that starts at the same position. All eight DMPs within the OXT DMR showed decreased levels of methylation in the MTG of AD cases, whereas four significant DHPs in the DHR showed higher levels of hydroxymethylation. Additionally, several probes within the DMR (4 probes) and DHR (3 probes; all in DMR as well) showed significant associations with Braak stages (Supplementary Tables 5 and 6). Moreover, while the 5-mC levels of all probes within the DMR were negatively correlated with OXT mRNA expression (most significant probe cg07747220; $r=-0.292, p=0.01$ ), the $5-\mathrm{hmC}$ levels of the probes in the DHR showed a positive correlation with mRNA expression (most significant probe cg01644611; $r=-0.283$, $p=0.01$; Figure 2, Supplementary Table 8). Taken together, this data suggests $O X T$ is hyperhydroxymethylated and hypomethylated. Of note,
TABLE 1. Differentially methylated, hydroxymethylated, and unmodified regions in the middle temporal gyrus associated with Alzheimer's disease. Displayed for each region is the chromosomal position, number of probes in the region (n), $p$-value and multiple testing-corrected $p$ (Šidák- $p$ ), UCSC gene name, gene feature, and directionality of modification changes in $\mathrm{AD}$ (up or down).

ABBREVIATIONS: 5-hmC, 5-hydroxymethylcytosine; 5-mC, 5-methylcytosine; AD, Alzheimer's disease; chr, chromosome; DHR, differentially hydroxymethylated region; DMR, differentially methylated region; DUR, differentially unmodified region; TSS, transcription start site; utr5, 5' untranslated region; cds, coding sequence). 
FIGURE 2. Methylation, hy-

droxymethylation, and expression of oxytocin (OXT) across Braak staging. Regressed OXT expression values and average regressed 5-methylcytosine (5-mC) and 5-hydroxymethylcytosine (5-hmC) values of 8 and 7 overlapping probes within the OXT differentially methylated region and differentially hydroxymethylated region, respectively, are shown. AU, arbitrary units. previous EWAS utilizing only BS-treated DNA could not have revealed this gene as a strong candidate for an epigenetic signature of $A D$, as the combined level of DNA modifications in OXT is not altered between AD cases and controls, and remains stable over Braak stages.

Although OXT has been linked to several forms of cognitive function, evidence implicating it in AD is very limited. A recent study did not find an association between $A D$ and OXT mRNA levels in the paraventricular nucleus and supraoptic nucleus, two hypothalamic nuclei that are considered to be the sole source of central oxytocin production [23]. Nevertheless, we found correlations between OXT mRNA expression and DNA (hydroxy)methylation levels. Possibly, MTG DNA (hydroxy) methylation signatures reflect those in hypothalamic oxytocin-expressing neurons, regulating OXT mRNA levels in their nerve endings that innervate the MTG. Evidently, the exact site-specific role of oxytocin, oxytocin receptors and their epigenetic regulation in $A D$ await further research. On a behavioral level, both oxytocin and the MTG have been implicated in facial recognition, which is impaired in AD [24-26]. However, there is currently no evidence directly linking alterations in oxytocin levels to diminished facial recognition in AD.

Concluding, the present study is the first of its kind, exploring the methylome and hydroxymethylome in parallel, shedding more light on previous observations made in relation to $A D$, but also providing compelling evidence for a role of dysregulated $O X T$ in $A D$.

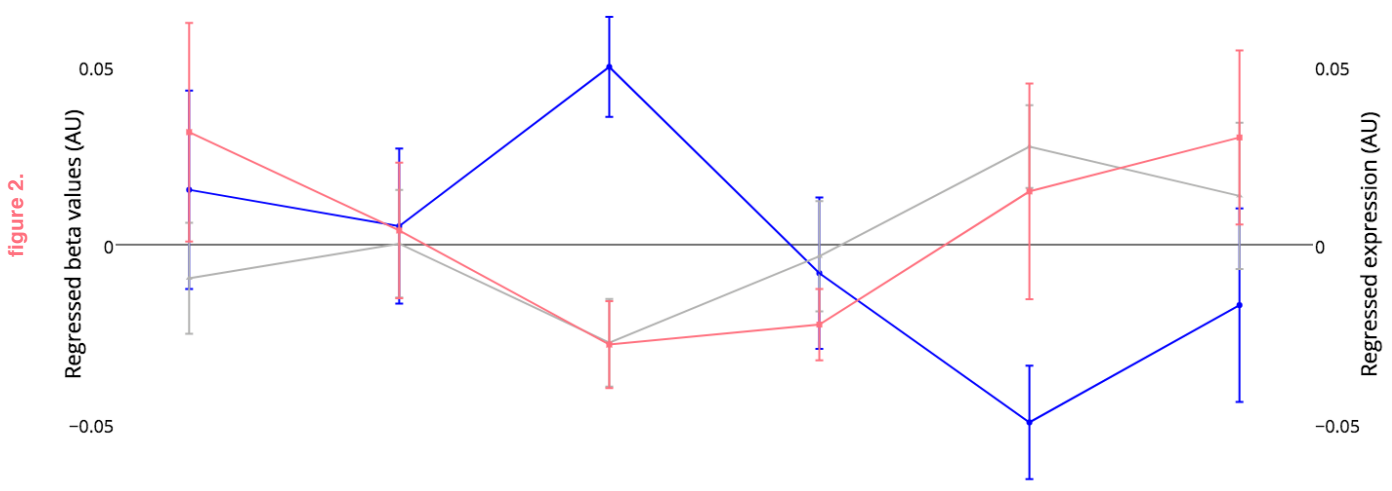




\section{REFERENCES}

[1] Yates D, McLoughlin DM.

The molecular pathology of Alzheimer's disease. Psychiatry 2008; 7: 1-5. [2] World Health Organization. Dementia: Fact Sheet. http:// www.who.int/mediacentre/factsheets/ fs362/en/ (2016, accessed 15 April 2017)

[3] Lambert J-C, Ibrahim-Verbaas CA, Harold D, et al. Meta-analysis of 74,046 individuals identifies 11 new susceptibility loci for Alzheimer's disease. Nat Genet 2013; 45: 1452-1458. [4] Lardenoije R, Iatrou A, Kenis G, et al. The epigenetics of aging and neurodegeneration. Prog Neurobiol 2015; 131: 21-64.

[5] Lunnon K, Smith R, Hannon E, et al. Methylomic profiling implicates cortical deregulation of ANKl in Alzheimer's disease. Nat Neurosci 2014; 17: 1164-1170.

[6] De Jager PL, Srivastava G, Lunnon K, et al. Alzheimer's disease: early alterations in brain DNA methylation at $A N K 1, B I N 1, R H B D F 2$ and other loci. Nat Neurosci 2014; 17: 1156-1163. [7] Chouliaras L, Mastroeni D, Delvaux E, et al. Consistent decrease in global DNA methylation and hydroxymethylation in the hippocampus of Alzheimer's disease patients. Neurobiol Aging 2013; 34: 2091-2099. [8] Zhao J, Zhu Y, Yang J, et al. A genome-wide profiling of brain DNA hydroxymethylation in Alzheimer's disease. Alzheimer's Dement. Epub ahead of print 2017. DOI: 10.1016/j. jalz.2016.10.004.

[9] Kriaucionis S, Heintz $\mathrm{N}$. The nuclear DNA base 5-hydroxymethylcytosine is present in Purkinje neurons and the brain. Science 2009; 324: 929-30.
Müller M, et al. Tissue distribution of 5-hydroxymethylcytosine and search for active demethylation intermediates. PLoS One; 5. Epub ahead of print 2010. DOI: 10.1371/journal. pone.0015367.

[11] Zhang RR, Cui QY, Murai $\mathrm{K}$, et al. Tetl regulates adult hippocampal neurogenesis and cognition. Cell Stem Cell 2013; 13: 237-24.5.

[12] Ray M, Zhang W, Liang W, et al. Analysis of Alzheimer's disease severity across brain regions by topological analysis of gene co-expression networks. BMC Syst Biol 2010; 4: 136.

[13] Coppieters N, Dragunow

M. Epigenetics in Alzheimers disease: a focus on DNA modifications. Curr

Pharm Des 2011; 17: 3398-3412.

[14] Lunnon K, Hannon E,

Smith RG, et al. Variation in 5-hydroxymethylcytosine across human cortex and cerebellum. Genome Biol 2016; 17: 27.

[15] Lopez LM, Harris SE, Luciano M, et al. Evolutionary conserved longevity genes and human cognitive abilities in elderly cohorts. Eur J Hum Genet 2012; 20: 341-347.

[16] Sung HY, Choi EN, Ahn Jo

$\mathrm{S}$, et al. Amyloid protein-mediated differential DNA methylation status regulates gene expression in Alzheimer's disease model cell line. Biochem Biophys Res Commun 2011; 414: 700-5. [17] Pedersen BS, Schwartz DA, Yang I V, et al. Comb-p: software for combining, analyzing, grouping and correcting spatially correlated P-values. Bioinformatics 2012; 28: 2986-8.

[18] Boiko T, Vakulenko M, Ewers $\mathrm{H}$, et al. Ankyrin-dependent and -independent mechanisms orchestrate axonal compartmentalization of $\mathrm{L} 1$ family members neurofascin and Ll/ neuron-glia cell adhesion molecule.
J Neurosci 2007; 27: 590-603.

[19]

Adrain C, Zettl M, Chris-

tova Y, et al. Tumor necrosis factor signaling requires $\mathrm{i}$ hhom 2 to promote trafficking and activation of TACE. Sci Reports 2012; 335: 225-228.

[20] Shi Q, Colodner KJ, Matousek SB, et al. Complement C3-deficient mice fail to display age-related hippocampal decline. J Neurosci 2015; 35: 13029-13042.

[21] Hong S, Beja-Glasser VF, Nfonoyim BM, et al. Complement and microglia mediate early synapse loss in Alzheimer mouse models. Science 2016. DOI: 10.1126/science.aad8373. Olff M, Frijling JL, Kubzansky LD, et al. The role of oxytocin in social bonding, stress regulation and mental health: An update on the moderating effects of context and interindividual differences. Psychoneuroendocrinology 2013; 38: 1883-1894.

[23] Swaab DF. Chapter II Neurobiology and neuropathology of the human hypothalamus. Handb Chem Neuroanat 1997; 13: 39-137.

[24] Gorno-Tempini M-L. Category differences in brain activation studies: where do they come from? Proc Biol Sci; 267: 1253-8.

[25] Haas BW, Filkowski MM, Cochran RN, et al. Epigenetic modification of $O X T$ and human sociability. Proc Natl Acad Sci U S A 2016; 113:

E3816-23.

[26] Hargrave R, Maddock RJ,

Stone V. Impaired recognition of facial expressions of emotion in Alzheimer's disease. J Neuropsychiatry Clin Neurosci 2002; 14: 64-71.

[27] Beach TG, Sue LI, Walker

DG, et al. The Sun Health Research Institute Brain Donation Program: Description and experience, 1987-2007. Cell Tissue Bank 2008; 9: 229-24.5. 
Davis S, Du P, Bilke S, et al. methylumi: Handle Illumina methylation data. 2016.

[29] Schalkwyk LC, Pidsley R, Wong CCY. wateRmelon: Illumina 450 methylation array normalization and metrics. R package version 1.2.2 2013 [30] Aryee MJ, Jaffe AE, Corrada-Bravo H, et al. Minfi: a flexible and comprehensive Bioconductor package for the analysis of Infinium DNA methylation microarrays. Bioinformatics 2014; 30: 1363-1369.

[31] Pidsley R, CC YW, Volta M, et al. A data-driven approach to preprocessing Illumina $450 \mathrm{~K}$ methylation array data. BMC Genomics 2013; 14: 293.

[32] Chen Y, Lemire M, Choufani S, et al. Discovery of cross-reactive probes and polymorphic CpGs in the Illumina Infinium Human Methylation450 microarray. Epigenetics 2013; 8: 203-209.

[33] Fukuzawa S, Takahashi S, Tachibana K, et al. Simple and accurate single base resolution analysis of 5-hydroxymethylcytosine by catalytic oxidative bisulfite sequencing using micelle incarcerated oxidants. Bioorg Med Chem 2016; 24: 4254-4262.

[34] Song C-X, He C. Potential functional roles of DNA demethylation intermediates. Trends Biochem Sci 2013; 38: 480-484.

[35] Slieker RC, Bos SD,

Goeman JJ, et al. Identification and systematic annotation of tissue-specific differentially methylated regions using the Illumina $450 \mathrm{k}$ array. Epigenetics Chromatin 2013; 6: 26.

[36] Phipson B, Maksimovic J, Oshlack A. missMethyl: an R package for analyzing data from Illumina's HumanMethylation450 platform. Bioinformatics 2015; 32: btv560.

\section{Supplementary methods Subjects and tissue preparation}

MTG tissue samples were obtained from 82 postmortem brains from both AD patients and neurologically normal controls provided by the Brain and Tissue Bank of the Banner Sun Health Research Institute (Sun City, Arizona, USA). Brain samples were frozen and stored at $-80^{\circ} \mathrm{C}$ after autopsy, with an average postmortem interval of 2.8 hours. Braak staging was carried out for $A D$ neurofibrillary pathology. A consensus diagnosis of $A D$ or nondemented control was reached by following National Institutes of Health (NIH) AD Center criteria [27]. Comorbidity with any other type of dementia, cerebrovascular disorders, mild cognitive impairment (MCl), and presence of non-microscopic infarcts were applied as exclusion criteria. Further information about the samples is provided in Supplementary Table 1.

DNA samples were isolated at the Banner Sun Health Research Institute. Approximately $76 \mathrm{mg}$ of frozen tissue was obtained from each of the 82 samples. The tissue was placed in a microfuge tube containing $0.5 \mathrm{ml}$ fresh lysis buffer (100 mM Tris $\mathrm{HCl}$ pH 8.5, 200 mM NaCl, 5 mM EDTA, 100 $\mu \mathrm{g} / \mathrm{ml}$ Proteinase K [Sigma-Aldrich, St. Louis, Missouri, USA], and $0.2 \%$ SDS), and then kept overnight on a heat block at $55^{\circ} \mathrm{C}$. To break up the tissue, samples were incubated for 1-2 hours in a hand-held pellet pestle mixer (Kontes), and were further homogenized the next day if needed. Next, $4 \mu \mathrm{L}$ of RNase (Qiagen, Valencia, California, USA) was added, and the samples were then kept at room temperature (RT) for 30 minutes. After incubation, phenol/chloroform/isoamyl alcohol (Sigma) was added in equal volumes and each tube was vortexed and placed on a rocking platform for 5 minutes. Samples were then centrifuged for 10 minutes at RT, at a speed of 10,000 RPM, and the aqueous phase was transferred to a new tube. Ethanol-precipitation took place overnight at $-20^{\circ} \mathrm{C}$, and pellets were resuspended in $50 \mu \mathrm{L}$ TE buffer ( $\mathrm{pH}$ 8.0). Before storing the samples at $-20^{\circ} \mathrm{C}$, they were quantified and checked for purity using spectrophotometry.

\section{Methylomic and hydroxy- methylomic profiling}

The TrueMethyl ${ }^{\mathrm{TM}} 24$ Kit version 2.0 by CEGX ${ }^{\mathrm{TM}}$ (Cambridge Epigenetix Limited, Cambridge, UK) was used for BS and oxBS conversion of genomic DNA (gDNA). All laboratory procedures were performed at 
ServiceXS (ServiceXS B.V., Leiden, the Netherlands), according to the manufacturer's instructions. Prior to the conversion, high molecular weight (HMW) gDNA was quantified using a PicoGreen assay (Invitrogen, Carlsbad, California, USA), and gel-electrophoresis was performed to assess gDNA quality. All samples were of sufficient quantity and quality. A volume of $1 \mu \mathrm{g} \mathrm{HMW} \mathrm{gDNA}$ was used per sample, which, after purification and denaturation, was split into two samples which underwent either DNA oxidation (oxBS samples) or mock DNA oxidation (BS samples). Subsequently, all samples were BS treated, and the yield of the samples was assessed by a Qubit single-stranded DNA assay (Invitrogen). An additional restriction quality control was performed for a qualitative assessment of 5-hmC oxidation and BS conversion.

From each BS/oxBS-treated DNA sample, $8 \mu \mathrm{L}$ was amplified and hybridized on the HumanMethylation450 BeadChip (Illumina, Inc., San Diego, CA, U.S.A.), and the Illumina iScan was used for imaging of the array. Sample preparation, hybridization, and washing steps for the Illumina Infinium II Methylation Assay of the BeadChip arrays were performed according to the manufacturer's protocol.

\section{Quality control and data normalization}

GenomeStudio (version 2011.1, Illumina) reports were generated using the raw data imported from Illumina iScanner. Further computational and statistical analyses were performed using the statistical programming language R (version 3.3.1; https://www.r-project.org) and RStudio (version 0.99.902). The methylumi package (version 2.20.0) [28] was used in order to import the GenomeStudio reports into the R statistical environment. The QC analysis and normalization have been conducted using the methylumi, wateRmelon (version 1.18.0) [29] and minfi (version 1.20.0) [30] packages, according to the method described by Pidsley et al. [31]. Initial tests using non-CpG single nucleotide polymorphism (SNP) probes and sex chromosome probes were performed to assess whether corresponding BS and oxBS samples were genetically identical, and whether reported sample gender was identical to predicted gender, respectively. Next, cross-hybridizing probes, probes containing a common SNP in the sequence or within 10bp of the sequence, and probes on the $X$ and $Y$ chromosomes were removed [32]. Lastly, a test to estimate the efficiency of the BS conversions was performed by calculating signal intensity percentages on control probes known to show $100 \%$ signal in BS samples (5-mC + 5-hmC signal). All 82 samples showed BS conversion percentages above the $80 \%$ threshold (average: $93 \%$ ). 
Prior to normalization, a $p$-filter was applied which removed samples having more than $1 \%$ of probes with a detection $p$-value greater than 0.05 , in addition to removing probes with a beadcount lower than 3 in more than $5 \%$ of samples, and probes having more than $1 \%$ of samples with a detection $p$-value greater than 0.05 . Although all samples passed the $p$-filter, 6,969 probes were removed due to a low beadcount or large detection $p$-value, leaving 396,600 probes for analysis.

From the wateRmelon package, thirteen different normalization strategies were tested and ranked based on three performance metrics, as described in Lunnon et al. [14] (Supplementary Table 9). Of these strategies, dasen performed consistently well, and was therefore chosen for data normalization. Following dasen normalization, two samples were dropped from the 5-mC dataset due to a skewed distribution of the global values in a density plot. The remaining 80 cases in the oxBS dataset and 82 cases in the BS dataset were used for further analyses.

\section{Data analysis}

Following normalization, two sets of beta values, from the standard BS arrays $(5-\mathrm{mC}+5-\mathrm{hmC})$ and from the oxBS arrays $(5-\mathrm{mC})$, were generated. By subtracting oxBS beta values from the BS beta values $\left(\Delta \beta_{\text {BS-oxBS }}\right)$ for each probe in each sample, 5 -hmC levels were calculated. Unmodified cytosine (UC) values were determined as $1-B S\left(1-\beta_{B S}\right)$. It should be noted that other DNA demethylation intermediates, such as 5 -formylcytosine (5-fC) and 5-carboxylcytosine (5-caC) may be represented in the BS or unmodified cytosine levels, as it is currently unclear how these intermediates respond to oxBS conversion [33]. However, these intermediates are present at very low levels, and are not enriched in brain tissue [34]. Due to technical variation, some 5-hmC values were negative. Therefore, outliers deviating more than $\pm 2 S D$ from the probe mean in the 5 -hmC dataset were determined and set to the mean $\pm 2 S D$, and subsequently a threshold of zero was applied to the mean of individual probes. Application of the threshold above zero left 178,591 probes in the 5-hmC dataset. See Supplementary Figure 1 for the UC, 5-mC, and $5-\mathrm{hmC}$ beta value distributions. Given the well-described influence of age and sex on methylation levels, these factors were regressed out from the normalized methylation and hydroxymethylation beta values. Subsequently, a linear regression analysis was performed, with DNA methylation, hydroxymethylation or the UC signal as outcome, and AD diagnosis as predictor (coded as a two-level factor; see Supplementary Figure 2 for Manhattan and QQ plots). Probes were then ordered based on a combined $p$-value and regression estimate ranking (i.e. probes with low $p$-values and high estimates ranked highest), and $p$-values were adjusted 
for multiple testing using the Benjamini-Hochberg FDR procedure. To examine the distribution of 5- $\mathrm{mC}$ and $5-\mathrm{hmC}$ across genomic regions, we annotated probes using the ENCODE annotation data, as described by Slieker et al. [35]. A Fisher's exact test was used to assess enrichment of 5-mC and 5-hmC in specific genomic regions (Supplementary Figure 3 and Supplementary Table 10).

Individual probes were annotated using the Illumina UCSC gene annotation. For identification of differentially (hydroxy)methylated and unmodified regions (DHRs/DMRs/DURs) containing spatially correlated DMPs, DHPs and DUPs (respectively), comb- $p$ was used [17]. This software examined probes above a significance threshold of 0.05 , and within 500 base pairs of each other. Obtained $p$-values are Stouffer-LiptakKechris (SLK) corrected for adjacent $p$-values, and are subsequently corrected for multiple testing using the Šidák correction.

Gene ontology (GO)-term enrichment analysis was conducted using the missMethyl package (version 1.8.0) [36], which takes into account the potential bias due to differing number of probes for each gene. Analyses were performed on the top 1000 probes, based on the combined $p$-value and estimate ranking from the $5-\mathrm{mC}, 5-\mathrm{hmC}$ and $\mathrm{UC}$ regression analyses that compared $A D$ patients and controls, while the probes included in the regression analyses were used as background lists. The top $25 \mathrm{GO}$ terms for each analysis are presented in Supplementary Tables 11-13.

\section{Expression data generation and analysis}

Raw expression data was obtained by the Banner Sun Health Research Institute (Sun City, Arizona, U.S.A.) from the same MTG samples as used for the epigenetic analyses, using the HumanHT-12 v4 BeadChip (Illumina). Total RNA extracted from frozen MTG was isolated with RNEasy Mini Kit (Qiagen) starting with at least $60 \mathrm{mg}$ of tissue. The raw data was exported from Illumina's GenomeStudio (version 2011.1) with the Expression Module (v1.9.0) for further analysis in R. Of the 82 subjects used for the epigenetic analyses, 1 case was not included on the expression array, and 3 additional cases were excluded after quality control of the data, due to extreme outlying values or failed reads, leaving 78 subjects for further analyses. Data was quantile-quantile normalized and outlier corrected. Based on the genomic position (MAPINFO), probes were matched to the DMRs, DHRs and DURs, and correlation between gene expression and corresponding probes was tested. Regions were annotated using UCSC gene annotation. Ranking is done by gene name and $p$-value (Supplementary Table 8). 


\section{Cross-region validation of DMRs}

To test the robustness of our D(h)MR and Braak association analysis, we performed a cross-regional validation using a similar dataset in an independent cohort of 96 samples from the MRC London Neurodegenerative Disease Brain Bank (http://www.kcl.ac.uk/iop/depts/ $\mathrm{cn} /$ research/MRC-London-Neurodegenerative-Diseases-Brain-Bank/ MRC-London-Neurodegenerative-Diseases-Brain-Bank.aspx), including both entorhinal cortex (EC) and cerebellum (CER) tissue from AD patients and neurologically normal controls (Supplementary Table 14). Standard $\mathrm{UC}, 5-\mathrm{mC}$ and 5-hmC levels were measured using the 450K BeadChip and processed with a similar methodology as described above. Braak stage pathology within all samples ranged from $0-\mathrm{VI}$, and all AD patients were aged 65 and over at the time of diagnosis. Cross-region validation was performed by running a Braak association analysis on MTG data on a total of 396 probes for UC and 5-mC and $2175-\mathrm{hmC}$ probes related to 11 DMR genes previously identified in our UC, $5-\mathrm{mC}$ and $5-\mathrm{hmC}$ $A D$ association analyses. Subsequently, the regression coefficients available from the MTG, EC and CER Braak association were correlated (Supplementary Table 15 and Supplementary Figure 4). 


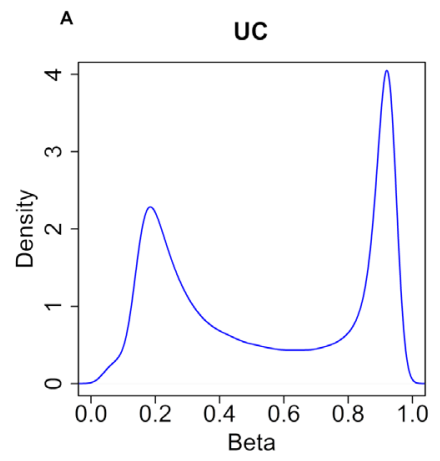

A

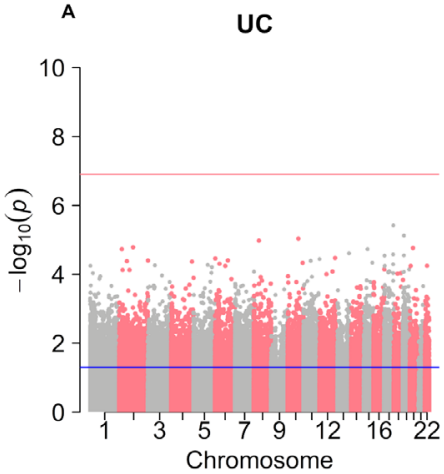

D

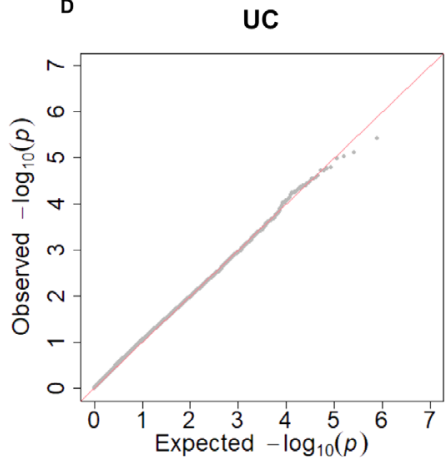

B

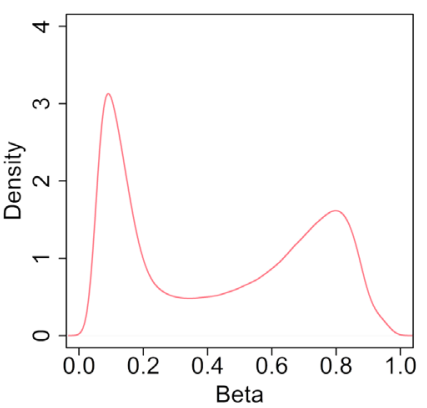

B

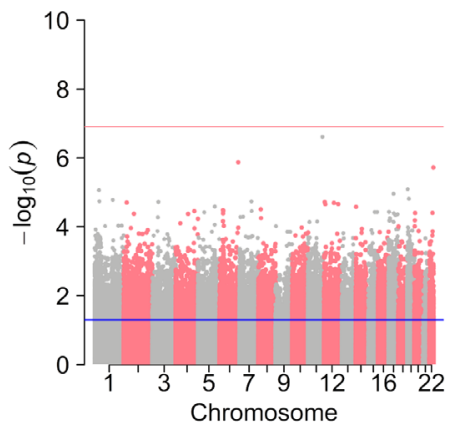

E

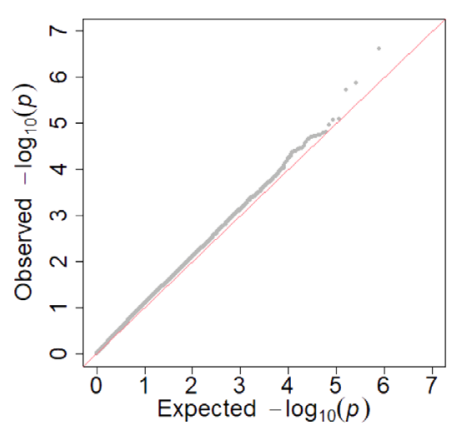

SUPPLEMENTARY DATA

SUPPLEMENTARY FIGURE 1. Density plots of normalized beta values for unmodified cytosines (UC; A), 5-methylcytosine (5-mC; B), and 5 -hydroxymethylcytosine $(5$-hmC; C). Plots include all probes used for the analysis. The UC beta values are computed by subtracting the normalized beta values obtained from bisulfite converted DNA (BS; con-

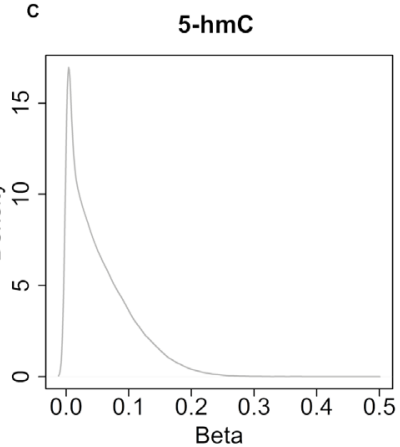

C

5-hmC

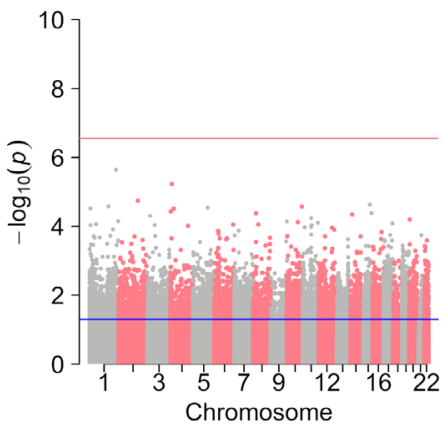

$\mathbf{F}$

5-hmC

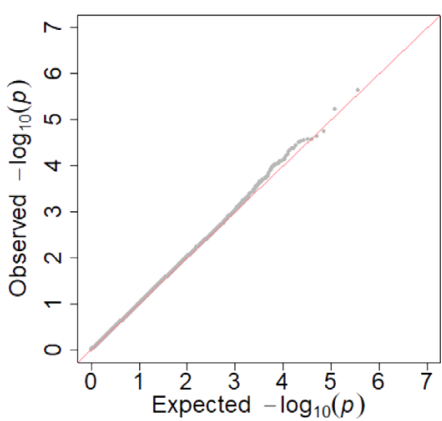

taining signals from both 5 - $\mathrm{mC}$ and 5 -hmC), from 1 . The 5 - $\mathrm{mC}$ beta values directly correspond to the normalized beta values from oxidative bisulfite converted DNA (oxBS). 5-hmC beta values are determined by subtracting the normalized oxBS beta values from the BS beta values, applying outlier correction ( $\pm 2 \mathrm{SD}$ ) and a threshold for probe means of $>0$. 
SUPPLEMENTARY FIGURE 2. Manhat-

tan and QQ plots of the unmodified

cytosine (UC; A and D, respectively),

5-methylcytosine (5-mC; B and E,

respectively), and 5-hydroxymeth-

ylcytosine (5-hmC; C and F, respec-

tively) regression analyses. The blue

line in the Manhattan plots indicates

the nominal significance threshold of

0.05 and the pink line indicates the

genome-wide significance threshold

based on the amount of probes includ-

ed in the analysis (1.261e-7 in case of

$\mathrm{UC}$ and $5-\mathrm{mC}$, and $2.800 \mathrm{e}-7$ in case of 5-hmC).

SUPPLEMENTARY FIGURE 3.A. Distri-

bution of probes across genomic re-

gions. Shown are all 5-methylcytosine

(5-mC) and unmodified cytosine (UC)

probes, all 5-hydroxymethylcytosine

(5-hmC) probes passing threshold, and the top 1000 Alzheimer's disease

(AD)-associated 5-mC, 5-hmC and

UC probes. IG, intergenic region; DP, distal promoter; PP, proximal promoter; GB, gene body; DS, downstream region; CGI, CpG island; $\mathrm{SHO}$, shore; SHE, shelf; NC, non-CGI.

\section{SUPPLEMENTARY FIGURE 3.B. Distri-}

bution of probes across functional genomic regions. Displayed are the distributions of all 5-mC and UC probes, all 5-hmC probes passing threshold, and the top $1000 \mathrm{AD}$-associated 5-mC, 5-hmC and UC probes. A3SS, alternative 3' splice site; A5SS, alternative 5 ' splice site; AFE, alternative first exon; ALE, alternative last exon; CE, cassette exon; CNE, constitutive exon; EI, exon isoforms; II, intron isoforms; IR, intron retention; MXE, mutually exclusive exon.
$25-$
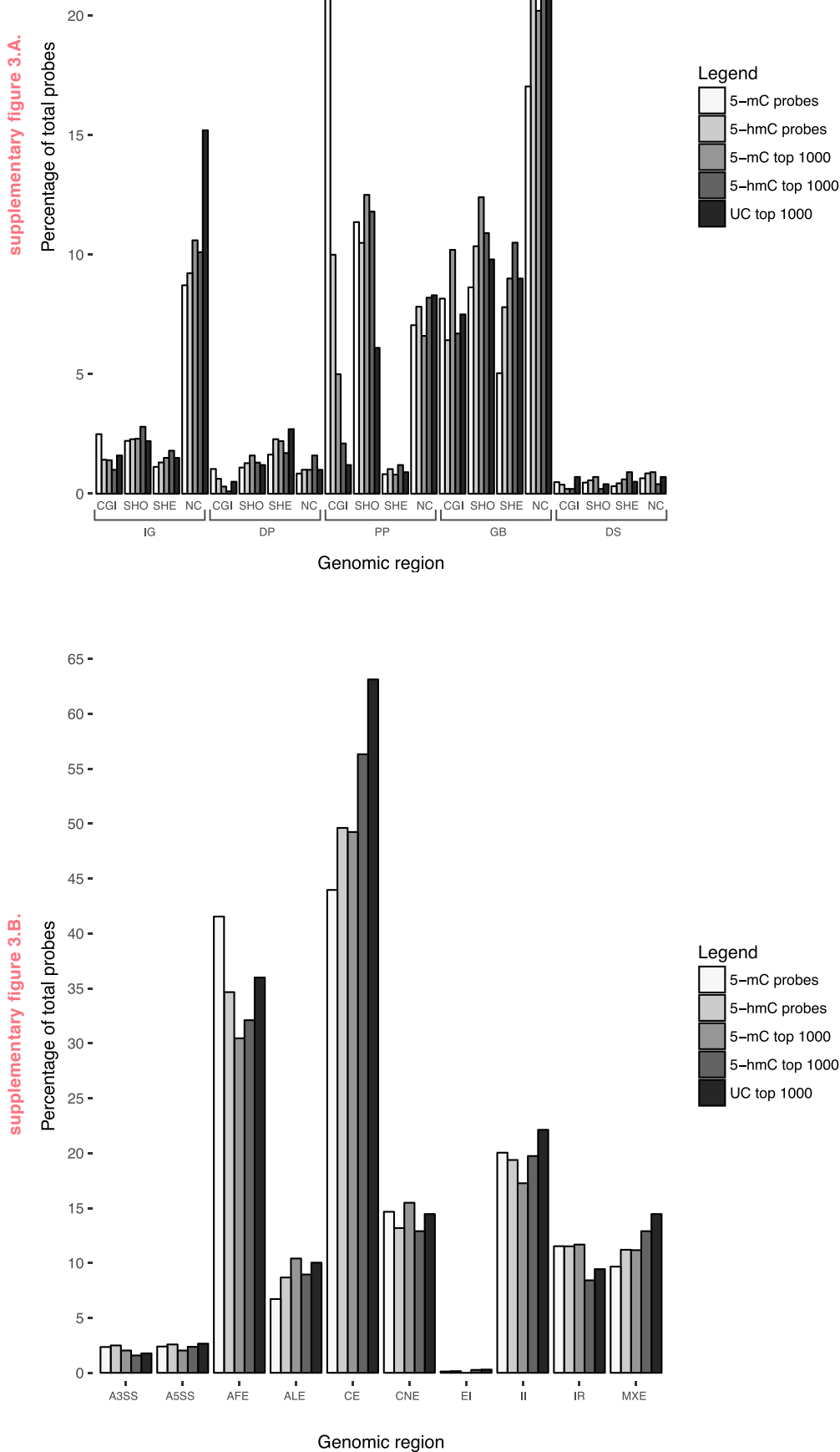

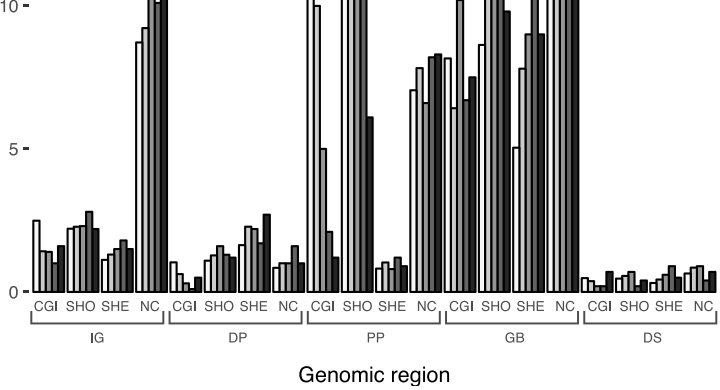

Genomic region

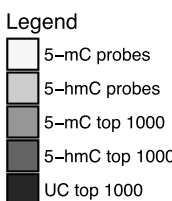

.

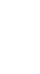

-



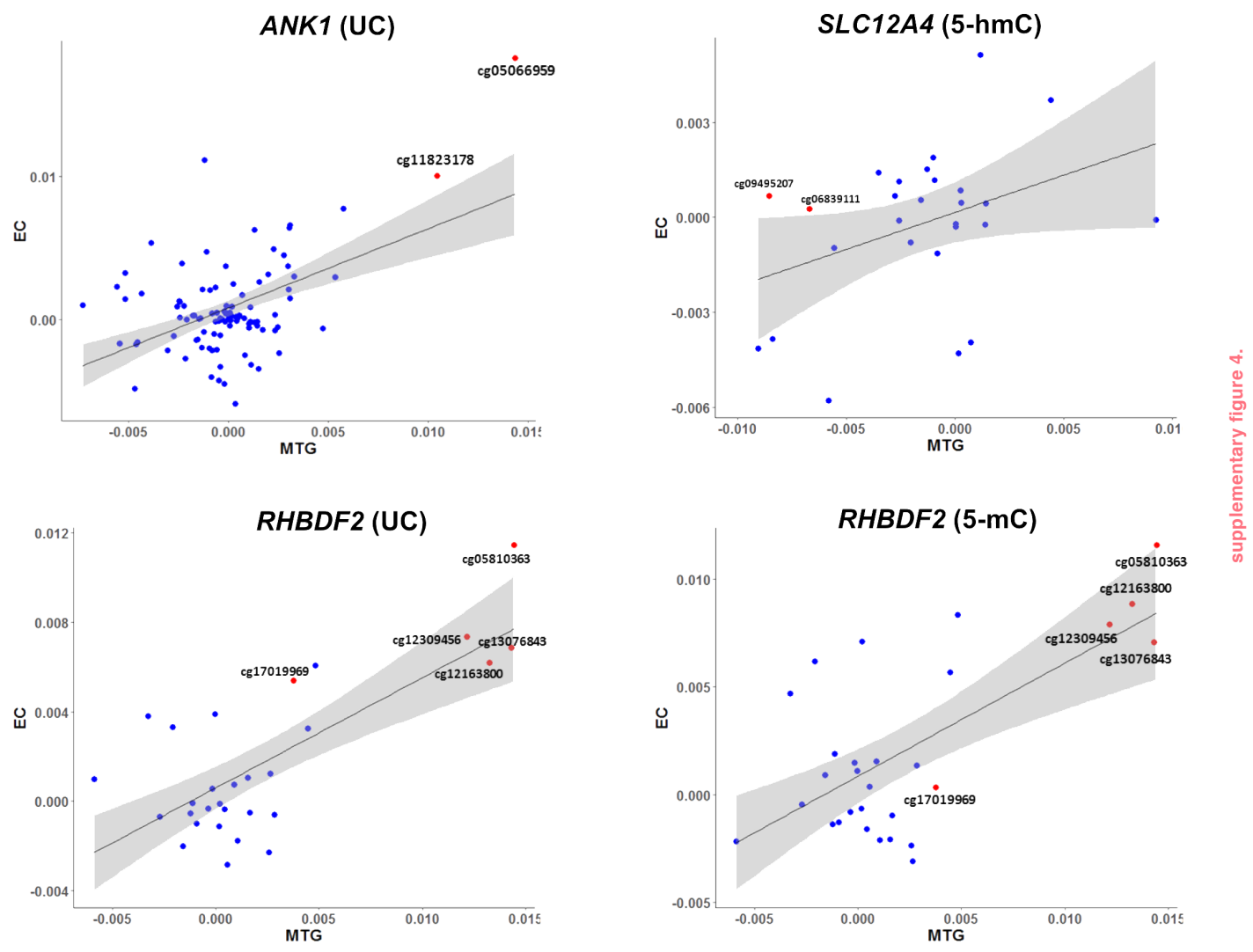

SUPPLEMENTARY FIGURE 4. The regression coefficients derived from Braak-association analysis of the middle temporal gyrus (MTG), entorhinal cortex (EC) and cerebellum (CER) showed significant correlations between (A) MTG and EC for ANK1 unmodified cytosine (UC) analysis $(r=0.490, p=2.68 \mathrm{E}-07),(\mathrm{B}) \mathrm{MTG}$ and EC for SLC12A4 5-hydroxymethylcytosine (5-hmC) analysis $(r=0.384$, $p=0.048$ ), and (C and D) MTG and EC for RHBDF2 UC and 5-methylcytosine $(5-\mathrm{mC})$ analysis $(r=0.759, p=$ $1.78 \mathrm{E}-06)$ and $(r=0.662, p=9.12 \mathrm{E}-05)$, respectively. 
SuPPLEMENTARY TABLE 1. Cohort demographics.

Middle temporal gyrus tissue obtained from the Banner Sun Health Research Institute (Sun City, Arizona, US) of Alzheimer's disease (AD patients and non-demented controls. Displayed is the number of samples in each group, and the distributions of gender, age, postmortem interval (PMI; hours), Braak stage, and total plaque and tangle load, i.e. the sum of average amyloid- $\beta$ plaque and tangle densities, respectively, in the entorhinal cortex, hippocampus, parietal lobe, temporal lobe and frontal lobe cortex).

\section{SUPPLEMENTARY TABLE 2. Top 50}

differentially methylated positions

(DMPs).

Displayed for each probe is chromosomal position (genome build 37), regression estimate for the $\mathrm{Alz}$ heimer's disease (AD) case-control analysis (Diagnosis est.), accompanying $p$-values and Benjamini-Hochberg false discovery rate (FDR) adjusted values ( $q$-value), Illumina UCSC gene annotation and gene region feature category, GREAT annotation (distance to closest transcription start site [TSS] shown in parentheses), and average 5-methylcytosine (5-mC) percentage and standard deviation per sample group. Probes are ranked based on a combined $p$-value and regression estimate score. UTR, untranslated region.

\section{SUPPLEMENTARY TABLE 3. Top 50}

differentially hydroxymethylated positions (DHPs).

Displayed for each probe is chromosomal position (genome build 37), regression estimate for the $\mathrm{Alz}-$ heimer's disease (AD) case-control

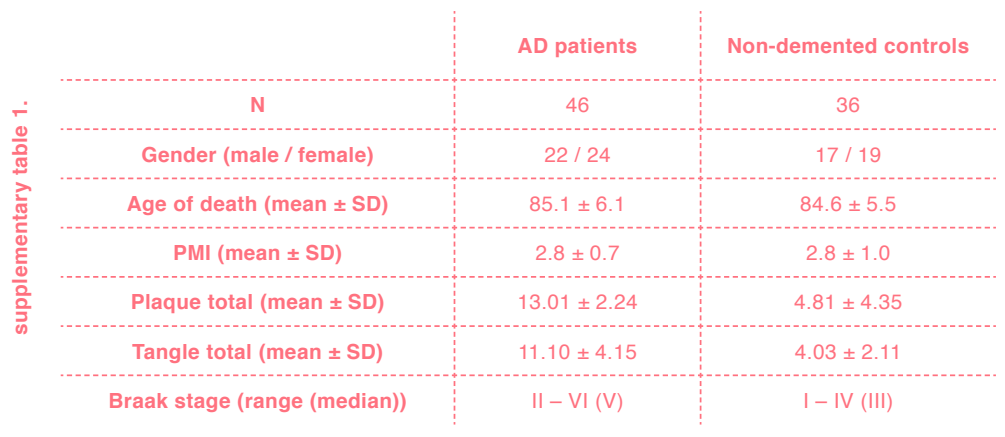

analysis (Diagnosis est.), accompanying $p$-values and Benjamini-Hochberg false discovery rate (FDR) adjusted values ( $q$-value), Illumina UCSC gene annotation and gene region feature category, GREAT annotation (distance to closest transcription start site shown in parentheses), and average 5 -hydroxymethylcytosine $(5-\mathrm{hmC})$ percentage and standard deviation per sample group. Probes are ranked based on a combined $p$-value and regression estimate score.

\section{SUPPLEMENTARY TABLE 4. Top 50}

differentially unmodified cytosine positions (DUPs).

Displayed for each probe is chromosomal position (genome build 37), regression estimate for the $\mathrm{Alz}$ heimer's disease (AD) case-control analysis (Diagnosis est.), accompanying $p$-values and Benjamini-Hochberg false discovery rate (FDR) adjusted values ( $q$-value), Illumina UCSC gene annotation and gene region feature category, GREAT annotation (distance to closest transcription start site shown in parentheses), and average unmodified cytosine (UC) percentage and standard deviation per sample group. Probes are ranked based on a combined $p$-value and regression estimate score. 


\begin{tabular}{|c|c|c|c|c|c|c|c|}
\hline Rank & Probe ID & Position & $\begin{array}{l}\text { Diagnosis } \\
\text { est. }\end{array}$ & $p$-value & $q$-value & $\begin{array}{l}\text { Illumina } \\
\text { annotation } \\
\text { (UCSC) }\end{array}$ & $\begin{array}{l}\text { Gene feature } \\
\text { (UCSC) }\end{array}$ \\
\hline 1 & $\operatorname{cg} 13285174$ & chr 20: 3052221 & -0.066611928 & $6.52 E-05$ & 0.613096979 & $O X T$ & TSS 200 \\
\hline 2 & cg05417607 & chr 17: 1373605 & 0.056371791 & 3.69E-05 & 0.503975885 & $M Y O 1 C$ & Body \\
\hline 3 & cg00539564 & chr 22: 43254081 & 0.072180813 & $1.37 \mathrm{E}-04$ & 0.683679545 & $A R F G A P 3$ & TSS1500 \\
\hline 4 & cg04819081 & chr 2: 24026544 & 0.046852736 & $1.98 \mathrm{E}-05$ & 0.503975885 & ATAD2B & Body \\
\hline 5 & cg22122808 & chr 17: 61511683 & -0.050218021 & $2.38 \mathrm{E}-04$ & 0.683679545 & CYB561 & 3'UTR \\
\hline 6 & cg05749855 & chr 16: 89472771 & 0.054927341 & $3.26 \mathrm{E}-04$ & 0.683679545 & ANKRD 11 & 5'UTR \\
\hline 7 & cg00857907 & chr 2: 202896898 & -0.044749354 & $1.26 \mathrm{E}-04$ & 0.683679545 & & \\
\hline 8 & cg08597839 & chr 1:29461008 & 0.059264985 & $3.77 \mathrm{E}-04$ & 0.683679545 & & \\
\hline 9 & cg24616382 & chr 7: 99767035 & 0.042200295 & $1.84 \mathrm{E}-05$ & 0.503975885 & GAL3ST4 & TSS1500 \\
\hline 10 & cg06463221 & chr $17: 75212862$ & 0.04265807 & 4.35E-05 & 0.507974086 & SEC14L1 & 3'UTR \\
\hline 11 & cg11823178 & chr 8: 41519399 & 0.049548502 & $3.04 \mathrm{E}-04$ & 0.683679545 & ANK1; MIR486 & Body; TSS 1500 \\
\hline 12 & cg11239720 & chr 4: 152967415 & -0.049160769 & $2.99 \mathrm{E}-04$ & 0.683679545 & & \\
\hline 13 & cg07871971 & chr 17: 40839766 & 0.041574964 & $1.10 \mathrm{E}-05$ & 0.503975885 & CNTNAP1 & Body \\
\hline 14 & cg23184226 & chr 7: 97880563 & 0.044935867 & $1.90 \mathrm{E}-04$ & 0.683679545 & TECPR1 & 5'UTR \\
\hline 15 & cg14730223 & chr 19: 18232604 & 0.044646058 & $1.85 \mathrm{E}-04$ & 0.683679545 & MAST3 & Body \\
\hline 16 & cg25775386 & chr 19: 17932001 & 0.044521783 & $1.82 \mathrm{E}-04$ & 0.683679545 & INSL3 & Body \\
\hline 17 & cg05270236 & chr 6: 158269807 & 0.042941651 & 1.07E-04 & 0.683679545 & SNX9 & Body \\
\hline 18 & cg11684897 & chr 17: 2951666 & 0.046484459 & 2.39E-04 & 0.683679545 & & \\
\hline 19 & cg14951497 & chr 2: 191875807 & 0.043417747 & $1.48 \mathrm{E}-04$ & 0.683679545 & STAT1 & 5'UTR \\
\hline 20 & cg18419358 & chr 6: 158384009 & -0.040522539 & 1.35E-06 & 0.251170502 & & \\
\hline 21 & cg12163800 & chr 17: 74475355 & 0.043215319 & $1.88 \mathrm{E}-04$ & 0.683679545 & RHBDF2 & Body \\
\hline 22 & cg03131724 & chr 11: 58731433 & 0.051079548 & $3.99 \mathrm{E}-04$ & 0.683679545 & & \\
\hline 23 & cg26733846 & chr $6: 112824384$ & 0.044549814 & $2.66 \mathrm{E}-04$ & 0.683679545 & & \\
\hline 24 & cg21597025 & chr $3: 142837564$ & 0.04427415 & $2.55 \mathrm{E}-04$ & 0.683679545 & CHST2 & TSS1500 \\
\hline 25 & cg18235088 & chr $6: 32120623$ & 0.044162472 & $2.72 \mathrm{E}-04$ & 0.683679545 & PPT2; PRRT1 & TSS1500 \\
\hline 26 & cg16908552 & chr $1: 231174153$ & 0.040553923 & $9.30 \mathrm{E}-05$ & 0.683679545 & FAM89A & Body \\
\hline 27 & cg13523576 & chr 8: 21909636 & 0.040390561 & 5.63E-05 & 0.561982879 & EPB49 & TSS 1500 \\
\hline 28 & cg08752433 & chr 12: 111016566 & 0.040773375 & $1.59 \mathrm{E}-04$ & 0.683679545 & PPTC7 & Body \\
\hline 29 & cg19592472 & chr 20: 3052274 & -0.057753516 & $5.96 \mathrm{E}-04$ & 0.707540795 & OXT & $1^{\text {st Exon; 5'UTR }}$ \\
\hline 30 & cg20580578 & $\operatorname{chr} 7: 75189143$ & 0.040671812 & 2.02E-04 & 0.683679545 & HIP1 & Body \\
\hline 31 & cg23238119 & $\operatorname{chr} 6: 74009041$ & 0.043743752 & $3.78 \mathrm{E}-04$ & 0.683679545 & C6orf147 & Body \\
\hline 32 & cg26033513 & chr 3: 195595933 & 0.044605126 & $4.11 \mathrm{E}-04$ & 0.683679545 & TNK2 & Body \\
\hline 33 & $\operatorname{cg} 10433043$ & chr 6: 152432725 & -0.039675137 & $1.50 \mathrm{E}-04$ & 0.683679545 & & \\
\hline 34 & cg26565893 & chr 17: 73501109 & 0.05019307 & $5.92 \mathrm{E}-04$ & 0.707540795 & CASKIN2 & Body \\
\hline 35 & cg06439547 & chr 1: 230779251 & 0.040185254 & $2.13 \mathrm{E}-04$ & 0.683679545 & COG2 & Body \\
\hline 36 & cg21179912 & chr 9: 2024387 & 0.038816208 & $9.70 \mathrm{E}-05$ & 0.683679545 & SMARCA2 & 5'UTR \\
\hline 37 & cg14871225 & chr 5: 139040820 & -0.03870046 & $8.75 \mathrm{E}-05$ & 0.683679545 & CXXC5 & 5'UTR \\
\hline 38 & cg10082647 & chr 12: 107348855 & 0.060028988 & 7.43E-04 & 0.707540795 & C12orf23 & TSS1500 \\
\hline 39 & cg06345462 & chr $16: 13263104$ & 0.038537598 & $1.43 \mathrm{E}-04$ & 0.683679545 & SHISA9 & Body \\
\hline 40 & cg24859236 & chr 1: 9750213 & 0.037944609 & $1.21 \mathrm{E}-04$ & 0.683679545 & PIK3CD & 5'UTR \\
\hline 41 & cg01644611 & chr 20: 3052253 & -0.047108887 & $6.32 \mathrm{E}-04$ & 0.707540795 & $O X T$ & TSS200 \\
\hline 42 & $\mathrm{cg} 00422578$ & chr 11: 119968331 & 0.036593553 & 2.47E-07 & 0.098096921 & & \\
\hline 43 & cg13725599 & chr 20: 3052262 & -0.041713301 & 4.93E-04 & 0.701395427 & $O X T$ & TSS200 \\
\hline 44 & cg06389574 & chr 2: 133834998 & 0.039731155 & $3.76 \mathrm{E}-04$ & 0.683679545 & NCKAP5 & Body \\
\hline 45 & $\operatorname{cg} 16803678$ & chr 3: 133941748 & 0.039281733 & $3.39 \mathrm{E}-04$ & 0.683679545 & $R Y K$ & Body \\
\hline 46 & $\operatorname{cg} 07881210$ & chr 17: 398090 & -0.040709357 & 4.57E-04 & 0.698683046 & & \\
\hline 47 & cg09375205 & chr 11: 63885665 & 0.038821644 & $3.26 \mathrm{E}-04$ & 0.683679545 & FLRT1; MACROD1 & Body \\
\hline 48 & $\operatorname{cg} 03783110$ & chr 17: 1463739 & 0.037571181 & 2.62E-04 & 0.683679545 & PITPNA & Body \\
\hline 49 & cg01025302 & chr 5: 3213214 & 0.036681752 & $2.09 \mathrm{E}-04$ & 0.683679545 & & \\
\hline 50 & $\mathrm{cg} 02481714$ & chr 22: 40439396 & -0.03649075 & 1.79E-04 & 0.683679545 & TNRC6B & TSS 1500 \\
\hline
\end{tabular}


Control

\begin{tabular}{|c|c|c|c|c|c|}
\hline \multicolumn{2}{|c|}{ GREAT annotation } & $\begin{array}{l}\text { Average } \\
5-\mathrm{mC}(\%)\end{array}$ & $\begin{array}{c}\text { Standard } \\
\text { deviation }(\%)\end{array}$ & $\begin{array}{l}\text { Average } \\
5 \text {-mC (\%) }\end{array}$ & $\begin{array}{c}\text { Standard } \\
\text { deviation (\%) }\end{array}$ \\
\hline OXT (-44) & & 74.61 & 6.16 & 67.66 & 9.08 \\
\hline CRK $(-14045)$ & MYO1C $(+22395)$ & 71.35 & 5.69 & 76.98 & 6.41 \\
\hline ARFGAP3 (-674) & & 59.68 & 8.12 & 67.03 & 8.08 \\
\hline ATAD2B (+123391) & & 59.05 & 5.03 & 63.83 & 4.31 \\
\hline CYB561 (+11861) & TANC2 $(+424786)$ & 30.66 & 6.37 & 25.58 & 5.59 \\
\hline ANKRD11 $(+84197)$ & ZNF778 (+188661) & 69.46 & 7.86 & 75.16 & 5.97 \\
\hline$F Z D 7(-2411)$ & & 60.53 & 4.74 & 56.04 & 5.61 \\
\hline TMEM2OOB $(-10588)$ & SRSF4 $(+47628)$ & 44.65 & 6.49 & 50.65 & 7.91 \\
\hline GAL3ST4 (-663) & & 56.29 & 4.12 & 60.57 & 4.14 \\
\hline SEPT9 $(-64629)$ & SEC14L1 $(+75858)$ & 41.00 & 3.78 & 45.34 & 4.83 \\
\hline NKX6-3 (-14525) & ANK1 $(+234880)$ & 78.23 & 5.69 & 83.31 & 6.06 \\
\hline PET112 (-285241) & FBXW7 $(+488769)$ & 51.07 & 5.14 & 46.21 & 6.32 \\
\hline CNTNAP1 $(+5135)$ & $E Z H 1(+57304)$ & 74.07 & 4.57 & 78.31 & 3.47 \\
\hline TECPR1 $(+904)$ & & 30.03 & 4.36 & 34.43 & 5.80 \\
\hline PIK3R2 (-31411) & MAST3 $(+24002)$ & 69.91 & 4.77 & 74.39 & 5.38 \\
\hline INSL3 $(+318)$ & & 70.16 & 4.67 & 74.53 & 5.41 \\
\hline SYNJ2 $(-133080)$ & SNX9 (+25514) & 58.65 & 4.78 & 63.00 & 4.61 \\
\hline OR1D5 (+15234) & RAP1GAP2 (+251935) & 33.52 & 4.75 & 38.22 & 5.88 \\
\hline STAT1 $(+3168)$ & GLS (+130261) & 54.62 & 6.00 & 59.10 & 4.07 \\
\hline SYNJ2 (-18878) & SNX9 (+139716) & 57.17 & 2.80 & 53.01 & 4.07 \\
\hline RHBDF2 (+8635) & AANAT $(+11726)$ & 79.05 & 5.52 & 83.37 & 4.37 \\
\hline FAM111B (-143224) & GLYATL1 (+20712) & 40.38 & 6.08 & 45.59 & 6.26 \\
\hline RFPL4B (+155853) & & 32.90 & 5.13 & 37.41 & 5.27 \\
\hline CHST2 (-1053) & & 62.39 & 5.70 & 66.86 & 4.83 \\
\hline PRRT1 (-904) & PPT2 (-605) & 50.45 & 5.48 & 55.01 & 5.27 \\
\hline TRIM67 (-124520) & ARV1 (+59331) & 46.12 & 5.41 & 50.31 & 3.81 \\
\hline EPB49 (-2691) & & 55.78 & 4.54 & 59.83 & 3.93 \\
\hline PPTC7 (+4497) & RAD9B $(+76562)$ & 59.16 & 4.66 & 63.23 & 4.54 \\
\hline OXT $(+9)$ & & 61.96 & 6.06 & 55.93 & 8.94 \\
\hline POM121C (-73576) & HIP1 $(+179139)$ & 59.57 & 3.79 & 63.68 & 5.31 \\
\hline KHDC1 (-36135) & DPPA5 $(+54957)$ & 20.92 & 5.50 & 25.40 & 5.15 \\
\hline MUC4 (-57090) & TNK2 $(+26498)$ & 71.94 & 6.51 & 76.51 & 4.48 \\
\hline ESR1 $(+304272)$ & SYNE1 (+525808) & 47.89 & 4.79 & 44.04 & 4.51 \\
\hline CASKIN2 (+10517) & KIAA0195 (+48446) & 68.36 & 7.36 & 73.53 & 5.52 \\
\hline COG2 $(+1050)$ & $A G T(+71084)$ & 66.23 & 5.40 & 70.29 & 3.92 \\
\hline VLDLR (-597405) & SMARCA2 (+9046) & 68.11 & 4.74 & 72.06 & 3.80 \\
\hline PSD2 (-134585) & CXXC5 $(+12520)$ & 18.91 & 4.82 & 15.01 & 3.62 \\
\hline C12orf23 (-688) & & 24.48 & 7.34 & 30.54 & 7.78 \\
\hline ERCC4 (-750909) & SHISA9 $(+267628)$ & 65.80 & 4.36 & 69.69 & 4.21 \\
\hline PIK3CD $(+38424)$ & CLSTN1 $(+134336)$ & 28.00 & 4.41 & 31.83 & 4.09 \\
\hline$O X T(-12)$ & & 84.15 & 3.88 & 79.27 & 7.61 \\
\hline PVRL1 (-368897) & TRIM29 (+40531) & 68.76 & 3.45 & 72.33 & 2.77 \\
\hline$O X T(-3)$ & & 86.27 & 3.28 & 81.92 & 6.65 \\
\hline LYPD1 (-406518) & NCKAP5 $(+491032)$ & 44.78 & 5.08 & 48.72 & 4.51 \\
\hline SLCO2A1 (-192829) & $R Y K(+27837)$ & 44.79 & 4.57 & 48.75 & 4.71 \\
\hline RPH $3 A L(-195458)$ & VPS53 $(+220005)$ & 50.14 & 4.86 & 45.90 & 5.57 \\
\hline FLRT1 $(+14304)$ & MACROD $1(+47919)$ & 78.40 & 4.92 & 82.29 & 4.47 \\
\hline INPP5K (-43558) & PITPNA $(+2370)$ & 26.04 & 4.87 & 29.85 & 3.96 \\
\hline IRX1 (-382953) & C5orf38 (+460953) & 72.06 & 4.70 & 75.73 & 3.76 \\
\hline TNRC6B (-134532) & GRAP2 (+142311) & 39.13 & 4.48 & 35.45 & 3.85 \\
\hline
\end{tabular}




\begin{tabular}{|c|c|c|c|c|c|c|c|}
\hline Rank & Probe ID & Position & $\begin{array}{l}\text { Diagnosis } \\
\text { est. }\end{array}$ & $p$-value & $q$-value & $\begin{array}{l}\text { Illumina } \\
\text { annotation } \\
\text { (UCSC) }\end{array}$ & $\begin{array}{l}\text { Gene feature } \\
\text { (UCSC) }\end{array}$ \\
\hline 1 & cg10857341 & chr 2: 161952702 & 0.060721423 & $1.82 \mathrm{E}-05$ & 0.612604396 & & \\
\hline 2 & cg01024962 & chr 14: 31389792 & 0.053715414 & $4.49 \mathrm{E}-05$ & 0.614686985 & STRN3 & Body \\
\hline 3 & cg00457087 & $\operatorname{chr} 1: 162602295$ & -0.045664223 & 2.63E-05 & 0.612604396 & DDR2 & $1^{\text {stExon; 5'UTR }}$ \\
\hline 4 & cg10310119 & chr 12: 129276152 & -0.053614088 & 1.25E-04 & 0.677914758 & & \\
\hline 5 & cg14962509 & chr 1: 36039655 & -0.073302356 & $1.92 \mathrm{E}-04$ & 0.742185585 & TFAP2E & Body \\
\hline 6 & cg05270236 & chr 6: 158269807 & -0.048035626 & $8.99 \mathrm{E}-05$ & 0.614686985 & SNX9 & Body \\
\hline 7 & cg26565893 & chr 17: 73501109 & -0.047161946 & $8.24 \mathrm{E}-05$ & 0.614686985 & CASKIN2 & Body \\
\hline 8 & cg 15418423 & chr 3: 23225524 & -0.044713764 & $4.98 \mathrm{E}-05$ & 0.614686985 & & \\
\hline 9 & cg08117728 & chr $6: 32179567$ & -0.048833262 & 1.36E-04 & 0.692660621 & $\mathrm{NOTCH} 4$ & Body \\
\hline 10 & cg24859236 & chr 1: 9750213 & -0.042085566 & $3.03 \mathrm{E}-05$ & 0.612604396 & PIK $3 C D$ & 5'UTR \\
\hline 11 & cg04197347 & chr 3: 66022776 & -0.046419228 & $1.91 \mathrm{E}-04$ & 0.742185585 & MAGI1 & Body \\
\hline 12 & cg20703928 & chr 2: 106364229 & -0.056359478 & $3.19 \mathrm{E}-04$ & 0.828359319 & NCK2 & 5'UTR \\
\hline 13 & cg07719898 & $\operatorname{chr} 1: 200274728$ & -0.04422518 & $1.22 \mathrm{E}-04$ & 0.677914758 & & \\
\hline 14 & cg08752433 & chr 12: 111016566 & -0.043912438 & $1.11 \mathrm{E}-04$ & 0.641340409 & PPTC7 & Body \\
\hline 15 & $\operatorname{cg} 19592472$ & chr 20: 3052274 & 0.042227248 & $6.35 \mathrm{E}-05$ & 0.614686985 & $O X T$ & $1^{\text {stExon; } 5 \text { 'UTR }}$ \\
\hline 16 & $\operatorname{cg} 10287485$ & $\operatorname{chr} 11: 69473145$ & -0.042090888 & $5.76 \mathrm{E}-05$ & 0.614686985 & & \\
\hline 17 & cg18804147 & chr 7: 882010 & -0.044346638 & $1.82 \mathrm{E}-04$ & 0.742185585 & UNC84A & Body \\
\hline 18 & $\operatorname{cg} 04580750$ & chr 1: 244199781 & -0.041448388 & $7.14 \mathrm{E}-05$ & 0.614686985 & & \\
\hline 19 & cg24158936 & $\operatorname{chr} 1: 177177623$ & -0.043067269 & $1.89 \mathrm{E}-04$ & 0.742185585 & FAM5B & 5'UTR \\
\hline 20 & $\operatorname{cg} 13829089$ & chr 17: 7460690 & -0.043681654 & $2.51 \mathrm{E}-04$ & 0.744217143 & TNFSF13; TNFSF12 & TSS1500; 3'UTR; Body \\
\hline 21 & cg16908552 & chr 1: 231174153 & -0.046104182 & $3.51 \mathrm{E}-04$ & 0.839277644 & FAM89A & Body \\
\hline 22 & cg22211672 & $\operatorname{chr} 15: 74610366$ & -0.039776338 & $2.34 \mathrm{E}-05$ & 0.612604396 & $C C D C 33$ & Body; TSS 1500 \\
\hline 23 & cg08410533 & chr 10: 375830 & -0.041469539 & $1.94 \mathrm{E}-04$ & 0.742185585 & $D I P 2 C$ & Body \\
\hline 24 & $\operatorname{cg} 10082647$ & chr 12: 107348855 & -0.049976039 & $5.60 \mathrm{E}-04$ & 0.849211551 & C12orf23 & TSS 1500 \\
\hline 25 & cg25449950 & chr 4: 24797174 & -0.039420471 & 3.09E-05 & 0.612604396 & SOD3 & 5'UTR; $1^{\text {stExon }}$ \\
\hline 26 & cg23987137 & chr 5: 58653624 & -0.0407906 & 2.87E-04 & 0.776384934 & $P D E 4 D$ & Body \\
\hline 27 & $\operatorname{cg} 04889800$ & chr 1: 16163555 & 0.050313798 & $7.22 \mathrm{E}-04$ & 0.873057029 & FLJ37453 & Body \\
\hline 28 & cg27365991 & chr 5: 123036376 & -0.0380364 & $2.86 \mathrm{E}-05$ & 0.612604396 & & \\
\hline 29 & cg14525270 & $\operatorname{chr} 1: 43766647$ & 0.039702108 & $2.15 \mathrm{E}-04$ & 0.743364204 & TIE1 & TSS200 \\
\hline 30 & cg00937742 & chr 2: 133429299 & -0.039427167 & $1.99 \mathrm{E}-04$ & 0.742185585 & LYPD1 & TSS 1500 \\
\hline 31 & cg10249224 & chr 1: 32828191 & -0.040004587 & $3.46 \mathrm{E}-04$ & 0.839277644 & TSSK 3 & 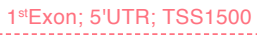 \\
\hline 32 & cg13594542 & $\operatorname{chr} 9: 138850547$ & 0.037747194 & 7.17E-05 & 0.614686985 & UBAC1 & Body \\
\hline 33 & cg01779732 & chr 15: 90015521 & -0.037431465 & 4.17E-05 & 0.614686985 & $R H C G$ & 3'UTR \\
\hline 34 & cg07655487 & $\operatorname{chr} 11: 66849820$ & -0.037710951 & $1.03 \mathrm{E}-04$ & 0.635609177 & & \\
\hline 35 & cg00682103 & $\operatorname{chr} 8: 141554380$ & 0.038203375 & $2.15 \mathrm{E}-04$ & 0.743364204 & EIF2C2 & Body \\
\hline 36 & cg00539564 & chr 22: 43254081 & -0.059919292 & $1.06 \mathrm{E}-03$ & 0.899315199 & ARFGAP3 & TSS 1500 \\
\hline 37 & cg03503988 & chr 20: 52827774 & -0.048685182 & $9.63 \mathrm{E}-04$ & 0.899315199 & PFDN4 & Body \\
\hline 38 & $\operatorname{cg} 13613245$ & $\operatorname{chr} 9: 139390036$ & -0.052895981 & $9.98 \mathrm{E}-04$ & 0.899315199 & $\mathrm{NOTCH} 1$ & 3'UTR \\
\hline 39 & cg26827533 & chr 8: 22458122 & -0.038430688 & $2.85 \mathrm{E}-04$ & 0.776384934 & C8orf58 & Body \\
\hline 40 & cg23369670 & chr 14: 104171944 & -0.037854307 & $1.98 \mathrm{E}-04$ & 0.742185585 & $X R C C 3$ & Body \\
\hline 41 & cg22023879 & $\operatorname{chr} 6: 31767600$ & -0.041759014 & $6.60 \mathrm{E}-04$ & 0.873057029 & LSM2 & Body \\
\hline 42 & cg02071305 & $\operatorname{chr} 15: 41185973$ & -0.038885457 & 3.71E-04 & 0.839277644 & VPS18 & TSS 1500 \\
\hline 43 & $\operatorname{cg} 24601055$ & chr 1: 87372153 & -0.037782265 & $2.14 \mathrm{E}-04$ & 0.743364204 & SEP15 & Body \\
\hline 44 & $\operatorname{cg} 06853339$ & $\operatorname{chr} 17: 76117687$ & 0.05693702 & $1.13 \mathrm{E}-03$ & 0.899315199 & TMC6 & Body \\
\hline 45 & cg27094173 & chr 11: 73371753 & -0.037025022 & $9.27 \mathrm{E}-05$ & 0.614686985 & PLEKHB1 & Body \\
\hline 46 & cg03402235 & $\operatorname{chr} 15: 42749336$ & -0.041794305 & $8.95 \mathrm{E}-04$ & 0.899315199 & ZFP106 & $1^{\text {st Exon }}$ \\
\hline 47 & $\operatorname{cg} 24697460$ & chr 12: 124908653 & -0.048955399 & $1.20 \mathrm{E}-03$ & 0.899315199 & NCOR2 & Body \\
\hline 48 & cg26319169 & $\operatorname{chr} 15: 65870540$ & -0.037601875 & $3.76 \mathrm{E}-04$ & 0.839277644 & PTPLAD1 & 3'UTR \\
\hline 49 & cg01783195 & $\operatorname{chr} 16: 53524545$ & 0.039006548 & $6.06 \mathrm{E}-04$ & 0.857358768 & $R B L 2$ & 3'UTR \\
\hline 50 & cg08371947 & chr 4: 8477793 & 0.035581593 & $5.92 \mathrm{E}-06$ & 0.52858505 & C4orf23 & 3'UTR \\
\hline
\end{tabular}


Control

GREAT annotation

.

\begin{tabular}{c|c}
\hline RBMS1 $(-602385)$ & TANK $(-40763)$ \\
\hline AP4S1 $(-104519)$ & COCH $(+46052)$ \\
\hline DDR2 $(+68)$ & \\
\hline SLC15A4 $(+32388)$ & TMEM132C (+524205) \\
\hline TFAP2E
\end{tabular}

TFAP2E (+685)

SYNJ2 (-133080)

CASKIN2 (+10517)

UBE2E2 (-19259)

GPSM3 (-16268)

PIK3CD $(+38424)$

MAGI1 (+1732)

FHL2 (-348549)

FAM58BP (+92073)

PPTC7 (+4497)

OXT (+9)

ORAOV1 (+17019)

GET4 (-34180)

AKT3 (-193198)

FAM5B (+36991)

SENP3 (-4618)

TRIM67 (-124520)

CYP11A1 (+49714)

ZMYND11 (+149897)

C12orf23 (-688)

SOD $3(+90)$

PDE4D (+535996)

UQCRHL (-29362)

CSNK1 G3 (+188584)

TIE1 $(+82)$

LYPD1 $(-819)$

TSSK3 $(+330)$

CAMSAP1 (-51543)

POLG (-137496)

KDM2A (-36919)

CHRAC1 (+32984)

ARFGAP3 (-674)

DOK5 (-264491)

SEC16A (-12530)

KIAA1967 (-4416)

XRCC3 (+9878)

VARS (-3889)

VPS18 (-654)

SEP15 $(+7953)$

TMC6 (+7173)

PLEKHB1 $(+13160)$

ZFP106 (+393)

NCOR2 $(+143356)$

C15orf44 (+33086)

AKTIP (+12624)

GPR78 (-104423)

\section{Average Standard}

5-hmC (\%)

SNX9 $(+25514)$

KIAAO195 (+48446)

NOTCH4 (+12276)

CLSTN1 (+134336)

NCK2 (-103974)

ZNF281 $(+104437)$

RAD9B (+76562)

CCND1 (+17273)

SUN1 (+9869)

ZNF238 (-14779)

SEC16B $(+761426)$

ARV1 $(+59331)$

CCDC33 $(+81700)$

DIP2C (+359777)

RAB3C $(+774686)$

SPEN $(-10803)$

\begin{tabular}{ccc}
-10 \\
\hdashline \\
\hdashline
\end{tabular}

UBAC1 $(+2678)$

RHCG $(+24277)$

RHOD (+25532)

EIF2C2 (+91265)

PFDN4 (+3273)

NOTCH1 (+50201)

KLC1 $(+76420)$

SH3GLB1 $(+201901)$

TNRC6C $(+117370)$

RAB6A (+100447)

ZNF664 (+450892)

PTPLAD1 (+47714)

RBL2 (+56195)

METTL19 (+35262)
1.90

2.69

3.55

13.00

10.35

21.86

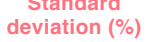

6.49

5.03

4.28

6.64

7.96

5.70

5.47

3.86

5.87

3.76

5.27

6.21

4.26

4.91

4.10

4.39

4.18

4.09

5.46

4.84

4.94

4.52

5.85

4.19

5.20

6.20

3.79

5.22

8.10

13.08

9.00

10.32

4.70

4.12

8.95

18.99

22.86

10.99

12.47

15.99

8.73

4.50

14.62

4.66

10.27

6.25

18.12

15.53

8.18

1.05

3.16
3.84

AD

Average

5-hmC (\%)

Standard

deviation (\%)

8.12

8.08

$-1.10$

7.38

2.82

17.02

6.91

2.92

5.85

$-1.50$

12.42

9.53

$-1.12$

16.71

17.38

5.48

3.31

$-0.09$

6.77

0.95

3.09

4.93

6.34

10.85

0.01

4.01

29.21

2.15

11.90

9.08

4.96

14.15

0.91

0.36

12.83

12.89

17.94

5.59

8.70

12.14

12.14

4.52

0.62

10.81

10.40

6.58

2.04

13.17

11.65

12.19

12.19
5.65

5.94

4.80

6.09

8.96

4.72

5.01

5.25

5.12

4.63

5.31

7.42

5.42

4.88

5.25

4.46

5.97

4.74

4.88

5.31

5.90

4.04

4.86

6.95

3.76

4.62

6.51

4.28

4.50

3.91

4.83

4.20

3.92

4.13

5.09

8.38

6.23

6.86

4.60

5.05

4.72

4.89

4.34

7.30

4.03

5.73

5.99

4.95

4.73

3.48 


\begin{tabular}{|c|c|c|c|c|c|c|c|}
\hline Rank & Probe ID & Position & $\begin{array}{l}\text { Diagnosis } \\
\text { est. }\end{array}$ & $p$-value & $q$-value & $\begin{array}{l}\text { Illumina } \\
\text { annotation } \\
\text { (UCSC) }\end{array}$ & $\begin{array}{l}\text { Gene feature } \\
\text { (UCSC) }\end{array}$ \\
\hline 1 & cg13076843 & chr 17: 74475294 & -0.045204925 & 2.62E-05 & 0.729466165 & RHBDF2 & Body \\
\hline 2 & cg22090150 & chr 17: 4098227 & -0.046966411 & $5.68 \mathrm{E}-05$ & 0.729466165 & ANKFY1 & Body \\
\hline 3 & cg10676327 & chr 4: 6659735 & -0.05946734 & $2.28 \mathrm{E}-04$ & 0.894099205 & & \\
\hline 4 & cg09288218 & chr 12: 130554977 & 0.03948029 & 3.33E-05 & 0.729466165 & & \\
\hline 5 & cg05810363 & chr 17: 74475270 & -0.040155937 & $9.19 \mathrm{E}-05$ & 0.81747108 & RHBDF2 & Body \\
\hline 6 & cg15363134 & chr 18: 77161214 & 0.040840506 & $1.46 \mathrm{E}-04$ & 0.894099205 & NFATC1 & Body; 5'UTR \\
\hline 7 & cg03183215 & chr 10: 1252341 & 0.039967737 & 1.14E-04 & 0.894099205 & ADARB2 & Body \\
\hline 8 & cg23689722 & $\operatorname{chr} 1: 3100956$ & 0.038762272 & $5.64 \mathrm{E}-05$ & 0.729466165 & PRDM16 & Body \\
\hline 9 & cg14150252 & chr 2: 64069583 & 0.03824478 & $4.14 \mathrm{E}-05$ & 0.729466165 & UGP2 & Body; 5'UTR \\
\hline 10 & cg23449541 & chr $21: 47855893$ & -0.040487636 & 2.20E-04 & 0.894099205 & PCNT & Body \\
\hline 11 & cg11491537 & chr 2: 132511720 & -0.04461105 & $3.94 \mathrm{E}-04$ & 0.894099205 & C2orf27A & Body \\
\hline 12 & cg25898192 & chr 15: 33418895 & 0.04452749 & $4.97 \mathrm{E}-04$ & 0.894099205 & & \\
\hline 13 & cg06674932 & chr 11: 110299342 & 0.040780155 & $4.14 \mathrm{E}-04$ & 0.894099205 & $F D \times 1$ & TSS 1500 \\
\hline 14 & $\operatorname{cg} 18102633$ & chr 19: 17487776 & -0.044204533 & $5.38 \mathrm{E}-04$ & 0.894099205 & PLVAP & $1^{\text {st }}$ Exon \\
\hline 15 & $\operatorname{cg} 12163800$ & chr 17: 74475355 & -0.03505837 & $5.12 \mathrm{E}-05$ & 0.729466165 & RHBDF2 & Body \\
\hline 16 & $\operatorname{cg} 12362517$ & $\operatorname{chr} 11: 133800685$ & 0.034839834 & $3.60 \mathrm{E}-05$ & 0.729466165 & IGSFGB & Body \\
\hline 17 & cg07799395 & chr 5: 125577565 & -0.040752803 & $5.00 \mathrm{E}-04$ & 0.894099205 & & \\
\hline 18 & cg19457506 & chr 17: 43099559 & 0.038902462 & $3.98 \mathrm{E}-04$ & 0.894099205 & & \\
\hline 19 & cg12141052 & chr 12: 66349603 & -0.039391985 & $4.40 \mathrm{E}-04$ & 0.894099205 & HMGA2 & Body \\
\hline 20 & $\operatorname{cg} 14464361$ & chr 2: 237029101 & 0.036664939 & $3.55 \mathrm{E}-04$ & 0.894099205 & AGAP1 & Body \\
\hline 21 & cg09169779 & $\operatorname{chr} 13: 20751710$ & 0.04098168 & $6.92 \mathrm{E}-04$ & 0.894099205 & & \\
\hline 22 & $\operatorname{cg} 14761019$ & chr $1: 3028485$ & 0.039329024 & $6.01 \mathrm{E}-04$ & 0.894099205 & PRDM16 & Body \\
\hline 23 & $\mathrm{cg} 05121497$ & chr 18: 60186078 & -0.033337125 & $9.22 \mathrm{E}-05$ & 0.81747108 & & \\
\hline 24 & cg23441248 & chr 15: 50140549 & -0.041251947 & 8.03E-04 & 0.894099205 & & \\
\hline 25 & cg05066959 & chr 8: 41519308 & -0.04860746 & $9.98 \mathrm{E}-04$ & 0.894099205 & ANK1; MIR486 & Body; TSS 1500 \\
\hline 26 & cg23434815 & chr 10: 75839302 & -0.041809422 & 8.36E-04 & 0.894099205 & $V C L$ & Body \\
\hline 27 & cg05417607 & chr 17: 1373605 & -0.032566779 & $2.85 \mathrm{E}-05$ & 0.729466165 & MYO1C & Body \\
\hline 28 & cg27583010 & chr 16: 30198505 & -0.042414 & $8.91 \mathrm{E}-04$ & 0.894099205 & CORO1A & Body; TSS 1500 \\
\hline 29 & cg27630153 & chr 16: 88845038 & -0.036347746 & $6.62 \mathrm{E}-04$ & 0.894099205 & FAM38A & Body \\
\hline 30 & cg03717755 & $\operatorname{chr} 6: 16136539$ & -0.034894835 & 4.83E-04 & 0.894099205 & MYLIP & Body \\
\hline 31 & cg10688297 & chr 7: 2606824 & 0.035274842 & $5.84 \mathrm{E}-04$ & 0.894099205 & IQCE & Body \\
\hline 32 & cg11823178 & chr 8: 41519399 & -0.032817675 & $1.90 \mathrm{E}-04$ & 0.894099205 & ANK1; MIR486 & Body; TSS 1500 \\
\hline 33 & cg25588787 & chr 5: 154027256 & 0.032358662 & $6.19 \mathrm{E}-05$ & 0.767470651 & & \\
\hline 34 & cg26844804 & chr 15: 102267900 & -0.03982849 & $9.42 \mathrm{E}-04$ & 0.894099205 & & \\
\hline 35 & cg21686171 & chr 1: 9504429 & -0.037272429 & $8.23 \mathrm{E}-04$ & 0.894099205 & & \\
\hline 36 & cg12309456 & chr 17: 74475402 & -0.03225529 & $1.55 \mathrm{E}-04$ & 0.894099205 & RHBDF2 & Body \\
\hline 37 & cg01434302 & chr 8: 3267208 & -0.035820147 & 7.36E-04 & 0.894099205 & CSMD1 & Body \\
\hline 38 & cg01513307 & chr 6: 108479557 & 0.031561305 & $3.95 \mathrm{E}-05$ & 0.729466165 & & \\
\hline 39 & cg07318609 & chr $10: 128585354$ & 0.032460468 & $2.58 \mathrm{E}-04$ & 0.894099205 & & \\
\hline 40 & cg01892689 & chr 8: 97657072 & 0.032411115 & $2.90 \mathrm{E}-04$ & 0.894099205 & $P G C P$ & TSS 1500 \\
\hline 41 & cg00542992 & chr 5: 141595654 & 0.033403906 & $5.88 \mathrm{E}-04$ & 0.894099205 & & \\
\hline 42 & cg02920514 & chr 3: 195610120 & -0.037030653 & $1.07 \mathrm{E}-03$ & 0.894099205 & TNK2 & Body \\
\hline 43 & cg22705835 & chr 10: 65332833 & 0.049158901 & 1.43E-03 & 0.894099205 & REEP3 & Body \\
\hline 44 & $\operatorname{cg} 17145559$ & chr 2: 114866027 & -0.034973236 & $9.24 \mathrm{E}-04$ & 0.894099205 & & \\
\hline 45 & cg01064286 & chr 1: 212777218 & -0.032117679 & $4.52 \mathrm{E}-04$ & 0.894099205 & ATF3 & 5'UTR \\
\hline 46 & cg04329433 & chr 8: 55365596 & -0.041449813 & $1.37 \mathrm{E}-03$ & 0.894099205 & & \\
\hline 47 & cg04972348 & $\operatorname{chr} 1: 1100035$ & -0.032278297 & $5.00 \mathrm{E}-04$ & 0.894099205 & & \\
\hline 48 & cg19137748 & chr 11: 69466054 & -0.031730838 & $4.01 \mathrm{E}-04$ & 0.894099205 & CCND1 & 3'UTR \\
\hline 49 & cg05802560 & chr 22: 24381773 & -0.045715242 & $1.50 \mathrm{E}-03$ & 0.894099205 & GSTT1 & Body \\
\hline 50 & cg20488756 & chr $6: 30131586$ & -0.032945715 & $7.25 \mathrm{E}-04$ & 0.894099205 & TRIM15 & $1^{\text {st Exon }}$ \\
\hline
\end{tabular}


Control

GREAT annotation

.

\begin{tabular}{c} 
RHBDF2 $(+8696)$ \\
CYB5D2 $(+51766)$ \\
\hline S100P $(-35830)$ \\
\hline TMEM132D $(-166766)$
\end{tabular}

AANAT $(+11665)$

ANKFY1 $(+69046)$

MRFAP1 (+17291)

I132D (-166766)

RHBDF2 (+8720)

NFATC1 (+889)

IDI1 (-157281)

ARHGEF16 (-270190) UGP2 $(+570)$

DIP2A (-22968)

GPR39 (-662426)

RYR3 (-184281)

FDX1 (-1318)

$P L V A P(+360)$

RHBDF2 (+8635)

SPATA19 (-85294)

ALDH7A1 (+353516)

C1QL1 (-53916)

HMGA2 (+131364)

GBX2 (+47550)

GJA3 (-16528)

ARHGEF16 (-342661)

ZCCHC2 (-4579)

ATP8B4 (+270869)

NKX6-3 (-14434)

AP3M1 (+71523)

CRK (-14045)

$S L X 1 B(-6658)$

PIEZO1 (+6333)

GMPR (-102271)

TTYH3 (-64778)

NKX6-3 (-14525)

HAND1 (-169433)

TARSL2 (-3256)

SLC25A33 (-95098)

RHBDF2 (+8588)

NONE

OSTM1 (-83617)

C10orf90 (-375345)

PGCP (-426)

NDFIP1 $(+107331)$

MUC4 (-71277)

REEP3 $(+51711)$

ACTR3 $(+218491)$

ATF3 (-4751)

SOX17 (-4898)

C1orf159 (-48300)

CCND1 (+10182)

GSTT1 $(+2510)$

TRIM10 (-2876)
FZD10 (-92026)

AANAT $(+11641)$

ADARB2 (+527328)

PRDM16 (+115215)

PCNT $(+111858)$

LOC150776 (+261335)

FMN1 (-58811)

AANAT $(+11726)$

IGSF9B $(+26194)$

DCAKD $(+29418)$

LLPH (+174929)

AGAP1 (+626369)

GJB2 (+15403)

PRDM16 (+42744)

FGF7 (+425175)

ANK1 (+234971)

VCL $(+81431)$

MYO1C (+22395)

CORO1A (+3775)

CTU2 (+72148)

MYLIP (+7223)

IQCE $(+8193)$

ANK1 (+234880)

LARP1 (-65205)

SPSB1 (+151489)

AANAT (+11773)

NR2E1 (-7657)

DOCK1 (-8668)

SPRY 4 (+108965)

TNK2 (+12311)

TTLL10 (-9250)
ORAOV1 (+24110)
GSTT2 (+59460)

Average UC

(\%)

19.98

20.55

34.46

59.15

11.88

29.04

9.79

39.60

74.54

27.68

73.62

28.94

23.94

16.19

30.35

44.26

79.91

52.43

19.25

57.60

32.35

24.99

36.18

24.51

33.09

13.96

47.50

47.50

24.53

30.15

58.17

15.80

64.60

18.79

27.80

13.63

38.86

73.07

63.68

49.92

57.33

33.04

53.78

24.59

27.37

42.50

49.33

22.02

23.00

34.63
27.69
AD Standard deviation (\%)

Average UC

(\%)

15.41

15.82

28.50

63.05

7.81

33.05

13.78

43.53

78.36

23.58

23.26

78.07

32.99

19.47

12.65

33.80

40.14

83.79

48.53

48.53

22.82

61.68

21.68

32.05

19.57

28.84

28.84

10.68

43.24

20.84

26.68

61.64

12.45

67.85

14.89

24.04

10.36

35.30

76.23

66.93

53.17

53.17

60.67

29.31

58.67

21.09

24.13

38.34

46.12

18.87

18.44

31.37
Standard deviation (\%) 3.94

4.92

6.92

4.55

3.41

5.52

5.57

4.50

4.22

4.54

5.11

5.30

4.45

5.51

2.89

4.21

4.19

3.97

4.59

5.28

5.75

4.89

3.76

4.14

6.45

5.00

3.07

6.39

3.89

3.83

4.75

3.64

3.27

2.21

4.44

3.16

4.73

3.29

3.70

4.17

4.52

5.54

7.45

3.84

4.04

5.45

3.57

3.66

3.85

4.02 


\begin{tabular}{|c|c|c|c|c|c|c|}
\hline Probe ID & Position & Braak est. & $p$-value & $\begin{array}{l}\text { Illumina } \\
\text { annotation }\end{array}$ & \multicolumn{2}{|c|}{ GREAT annotation } \\
\hline cg09573585 & chr $10: 88728128$ & -0.006016595 & 0.028384459 & C10orf116 & AGAP11 (-2369) & C10orf116 (-59) \\
\hline $\operatorname{cg} 11805311$ & chr 10: 88728073 & -0.007035095 & 0.048298223 & C10orf116 & AGAP11 (-2424) & C10orf116 (-114) \\
\hline cg26395382 & chr 10: 88728235 & -0.005149 & 0.145609597 & C10orf116 & AGAP11 (-2262) & C10orf116 (+48) \\
\hline cg01754756 & chr 10: 88728078 & -0.006316606 & 0.067157772 & C10orf116 & AGAP11 (-2419) & C10orf116 (-109) \\
\hline cg05066959 & $\operatorname{chr} 8: 41519308$ & 0.012616633 & 0.017133805 & ANK1; MIR486 & NKX6-3 (-14434) & ANK1 (+234971) \\
\hline cg11823178 & $\operatorname{chr} 8: 41519399$ & 0.013034543 & 0.005944986 & ANK1; MIR486 & NKX6-3 (-14525) & ANK1 $(+234880)$ \\
\hline cg15481294 & chr 19: 6712406 & 0.002846515 & 0.141314636 & C3 & TNFSF14 $(-41808)$ & C3 $(+8255)$ \\
\hline cg26861457 & chr 19: 6712380 & 0.003943492 & 0.082611145 & C3 & TNFSF14 (-41782) & C3 $(+8281)$ \\
\hline cg10628785 & chr 19: 6712321 & 0.001278944 & 0.49652103 & C3 & TNFSF14 $(-41723)$ & C3 $(+8340)$ \\
\hline cg25406665 & chr 19: 6712357 & 0.004362112 & 0.006920004 & $\mathrm{C3}$ & TNFSF14 (-41759) & C3 $(+8304)$ \\
\hline cg15755240 & chr 19: 6712372 & 0.002804723 & 0.175757492 & C3 & TNFSF14 (-41774) & C3 $(+8289)$ \\
\hline cg24811290 & chr 4: 159092553 & -0.006856254 & 0.019508101 & FAM198B & FAM198B (+1648) & GRIA2 (+950818) \\
\hline cg03304437 & $\operatorname{chr} 4: 159092536$ & -0.009786048 & 0.003876177 & FAM198B & FAM198B (+1665) & GRIA2 (+950801) \\
\hline cg06370094 & chr 12: 130555091 & -0.00659553 & 0.141338936 & & TMEM132D (-166880) & FZD10 (-91912) \\
\hline cg09288218 & $\operatorname{chr} 12: 130554977$ & -0.006743524 & 0.153451358 & & TMEM132D (-166766) & FZD10 (-92026) \\
\hline cg07070348 & chr 12: 130555007 & -0.002413988 & 0.561815254 & & TMEM132D (-166796) & FZD10 (-91996) \\
\hline cg07747220 & chr 20: 3052115 & -0.008173965 & 0.069260258 & $O X T$ & OXT $(-150)$ & \\
\hline cg01644611 & chr 20: 3052253 & -0.011736189 & 0.013759205 & $O X T$ & OXT $(-12)$ & \\
\hline cg13725599 & chr 20: 3052262 & -0.009424932 & 0.023323502 & $O X T$ & OXT $(-3)$ & \\
\hline cg26267561 & $\operatorname{chr} 20: 3052224$ & -0.007403822 & 0.055313901 & $O X T$ & OXT $(-41)$ & \\
\hline cg13285174 & $\operatorname{chr} 20: 3052221$ & -0.015282407 & 0.008729542 & $O X T$ & OXT $(-44)$ & \\
\hline cg19592472 & chr 20: 3052274 & -0.010865231 & 0.063920724 & $O X T$ & OXT $(+9)$ & \\
\hline cg16887334 & chr 20: 3052151 & -0.010386509 & 0.022978398 & $O X T$ & OXT (-114) & \\
\hline cg26955850 & chr 20: 3052345 & -0.001848332 & 0.565728654 & $O X T$ & OXT $(+80)$ & \\
\hline cg02046423 & chr 17: 2951689 & 0.006873988 & 0.037249269 & & OR1D5 (+15211) & RAP1GAP2 $(+251958)$ \\
\hline cg11684897 & $\operatorname{chr} 17: 2951666$ & 0.010822722 & 0.013858777 & & OR1D5 (+15234) & RAP1GAP2 (+251935) \\
\hline cg24015889 & chr 17: 2951719 & 0.002697856 & 0.481274149 & & OR1D5 $(+15181)$ & RAP1GAP2 (+251988) \\
\hline cg12163800 & $\operatorname{chr} 17: 74475355$ & 0.013257607 & 0.00082286 & RHBDF2 & RHBDF2 (+8635) & AANAT $(+11726)$ \\
\hline cg12309456 & chr 17: 74475402 & 0.012145479 & $1.56 \mathrm{E}-06$ & RHBDF2 & RHBDF2 (+8588) & AANAT $(+11773)$ \\
\hline cg05810363 & chr 17: 74475270 & 0.01445045 & 0.000161102 & RHBDF2 & RHBDF2 (+8720) & AANAT $(+11641)$ \\
\hline $\operatorname{cg} 13076843$ & chr 17: 74475294 & 0.01431989 & 0.000597182 & RHBDF2 & RHBDF2 (+8696) & AANAT $(+11665)$ \\
\hline cg17019969 & chr 17: 74475240 & 0.003757517 & 0.286766652 & RHBDF2 & RHBDF2 $(+8750)$ & AANAT $(+11611)$ \\
\hline cg06839111 & chr 16: 67978450 & 0.005045647 & 0.033641168 & SLC12A4; LCAT & LCAT $(-436)$ & \\
\hline cg09495207 & chr $16: 67978445$ & 0.004184379 & 0.059896273 & SLC12A4; LCAT & $\operatorname{LCAT}(-431)$ & \\
\hline cg20697427 & chr 3: 195610231 & 0.002414777 & 0.498219531 & TNK2 & MUC4 (-71388) & TNK2 $(+12200)$ \\
\hline cg02920514 & chr 3: 195610120 & 0.003748243 & 0.480665527 & TNK2 & MUC4 (-71277) & TNK2 (+12311) \\
\hline $\operatorname{cg} 16047223$ & chr 3: 195610084 & 0.005125395 & 0.126645191 & TNK2 & MUC4 (-71241) & TNK2 $(+12347)$ \\
\hline
\end{tabular}

$$
\text { เก }
$$

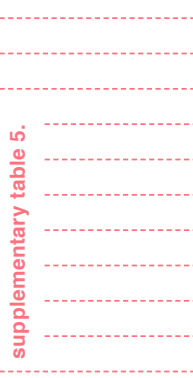

SUPPLEMENTARY TABLE 5. Association of probes in differentially methylated regions (DMRs) with Braak stage. Braak stage-association analysis of probes located in Alzheimer's disease (AD)-associated DMRs. Displayed for each probe is chromosomal position (genome build 37), regression estimate for the Braak-associated analysis (Braak est.), accompanying $p$-values, Illumina gene annotation, GREAT annotation (distance to closest tran- 
Braak stage I

Average
5-mC
$(\%)$

33.69

30.21

13.94 -

\begin{tabular}{l|l}
\hline 26.60 & 2.35
\end{tabular}

\begin{tabular}{l|l}
71.71 & 5.30
\end{tabular}

\begin{tabular}{l|l}
76.08 & 5.22 \\
\hline
\end{tabular}

\begin{tabular}{l|l}
77.03 & 2.45 \\
\hline 71.14 & 3.93
\end{tabular}

\begin{tabular}{l|l}
71.14 & 3.93 \\
\hline
\end{tabular}

65.18

81.51

79.41

20.34

26.50

\begin{tabular}{l|l}
\hline 41.26 & 4.59
\end{tabular}

i

\begin{tabular}{l|l}
31.78 & 4.69
\end{tabular}

\begin{tabular}{l|l}
79.29 & 4.71
\end{tabular}

\begin{tabular}{l|l}
84.37 & 2.82 \\
\hline 86.58 & 2.59
\end{tabular}

\begin{tabular}{l|l}
86.58 & 2.59 \\
\hline
\end{tabular}

\begin{tabular}{l|l}
67.42 & 4.07 \\
\hline 73.42 & 7.48
\end{tabular}

\begin{tabular}{l|l}
73.42 & 7.48 \\
\hline
\end{tabular}

\begin{tabular}{l|l}
61.07 & 4.38
\end{tabular}

$\begin{array}{l:l}81.38 & 7.44\end{array}$

50.63 : 4.72

$22.42 \quad 4.65$

\begin{tabular}{l|l}
29.45 & 7.09
\end{tabular}

\begin{tabular}{l|l}
30.17 & 5.09
\end{tabular}

\begin{tabular}{l|l}
76.09 & 5.59 \\
\hline
\end{tabular}

\begin{tabular}{c|c}
85.16 & 5.41 \\
\hline-80.38 & 6.64
\end{tabular}

\begin{tabular}{l|l}
80.38 & 6.64 \\
\hline
\end{tabular}

75.15

76.72

36.23

32.27

62.90

65.67

\begin{tabular}{l|l}
78.13 & 3.80
\end{tabular}

\begin{tabular}{c|c}
\hline Braak stage II \\
\hdashline Average & Standard \\
\hline $5-m C$ & deviation
\end{tabular}

$5-\mathrm{mC}$
$(\%)$

32.00

(\%)

3.56

30.07

12.45

4.40

2.89

25.12

78.93

81.39

78.25

73.07

67.30

83.26

78.82

20.44

21.18

39.41

42.92

30.40

78.35

81.03

\begin{tabular}{l|l}
84.16 & 5.04
\end{tabular}

65.87

71.80

58.90

77.09

51.62

24.67

33.72

32.57

\begin{tabular}{l|l}
82.70 & 4.29 \\
\hline
\end{tabular}

\begin{tabular}{l|l}
89.18 & 3.50 \\
\hline
\end{tabular}

\begin{tabular}{l|l}
\hline 86.15 & 3.76 \\
\hline
\end{tabular}

82.78

\begin{tabular}{l|l}
77.10 & 3.70
\end{tabular}

38.53

34.90

63.45

68.11

80.27
Braak stage III

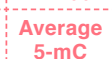

5-mC

(\%)

33.72

30.95

16.45

27.63

74.86

78.49

79.46

72.61

67.31

82.35

79.38

19.43

24.89

38.46

42.30

27.58

80.78

84.68

86.12

66.92

74.36

61.66

82.22

51.49

23.23

35.92

33.91

78.32

88.51

85.05

80.08

76.24

38.06

34.05

63.08

65.01

78.87

(\%)

4.54

5.30

2.97

3.84

5.01

6.63

6.26

5.44

3.32

3.63

5.90

6.53

3.41

4.35

4.57

4.97

3.21

5.48

4.8

3.28

3.10

4.22

6.75
Braak stage IV

Standard
deviation

(\%)

4.54

7.14

5.54

2.46

2.51

1.87

4.78

3.95

4.40

4.58

5.41

5.65
Average Standard

5-mC

(\%)

31.86

deviation

(\%)

3.38

28.89

13.21

26.03

77.20

80.15

79.28

72.84

66.61

83.61

80.14

19.18

22.96

39.29

41.22

30.47

77.83

81.51

83.84

64.26

70.45

58.27

77.67

49.22

24.38

37.45

34.60

81.04

89.81

87.34

79.26

76.29

38.96

35.61

35.61

65.38

78.22

4.38

4.95

5.22

6.84

2.13

4.15

2.01

2.01
3.05

4.57

4.57
3.44

3.76

\begin{tabular}{c}
3.76 \\
7.52 \\
\hline
\end{tabular}

7.02

6.99

7.19
-6.34

6.34

9.68

9.68

6.00

3.43

2.86

2.86

5.79

5.79

4.03

6.92

6.11
3.64
3.13

3.13

4.89

5.83

3.62
Braak stage $\mathrm{V}$

Braak stage VI

Average Standard Average Standard

5-mC

$(\%)$

29.03

deviation

$(\%)$

4.17

$5-\mathrm{mC}$

(\%)

32.64

deviation

27.32

11.89

24.30

24.30
81.29

84.79

79.73

73.27

66.7

9.33

6.66
6.92

\begin{tabular}{|c|}
66.74 \\
84.32 \\
79.81
\end{tabular}

4.84

28.97

4.84

13.06

4.14

7.42

24.1

79.43

5.68
2.70

17.39

21.56

37.25

39.25 


\begin{tabular}{|c|c|c|c|c|c|c|}
\hline \multirow{2}{*}{ Probe ID } & \multirow{2}{*}{$\begin{array}{l}\text { Position } \\
10: 88728128\end{array}$} & \multirow{2}{*}{$\begin{array}{l}\text { Braak est. } \\
0.002993664\end{array}$} & \multirow{2}{*}{$\begin{array}{c}p \text {-value } \\
0.195490293\end{array}$} & \multirow{2}{*}{$\begin{array}{c}\begin{array}{c}\text { Illumina } \\
\text { annotation } \\
\text { (UCSC) }\end{array} \\
\text { C10orf116 }\end{array}$} & \multicolumn{2}{|c|}{ GREAT annotation } \\
\hline & & & & & AGAP11 (-2369) & C10orf116 (-59) \\
\hline cg11805311 & chr 10: 88728073 & 0.000351648 & 0.918297434 & C10orf116 & AGAP11 (-2424) & C10orf116 (-114) \\
\hline cg26395382 & chr 10: 88728235 & -0.00254397 & 0.554518377 & C10orf116 & AGAP11 (-2262) & C10orf116 (+48) \\
\hline cg01754756 & chr 10: 88728078 & -0.000124756 & 0.97226127 & C10orf116 & AGAP11 $(-2419)$ & C10orf116 (-109) \\
\hline cg05066959 & chr 8: 41519308 & 0.001590789 & 0.633584577 & ANK $1 ;$ MIR486 & NKX6-3 (-14434) & ANK1 $(+234971)$ \\
\hline cg11823178 & chr 8: 41519399 & -0.002706607 & 0.342571371 & ANK1; MIR486 & NKX6-3 (-14525) & ANK1 $(+234880)$ \\
\hline cg15481294 & chr 19: 6712406 & 0.001097194 & 0.668522069 & C3 & TNFSF14 (-41808) & C3 $(+8255)$ \\
\hline cg26861457 & chr 19: 6712380 & 0.001560152 & 0.550822267 & C3 & TNFSF14 (-41782) & C3 $(+8281)$ \\
\hline cg10628785 & chr 19: 6712321 & 0.001777699 & 0.355525449 & C3 & TNFSF14 (-41723) & C3 $(+8340)$ \\
\hline cg15755240 & chr 19: 6712372 & -0.002177933 & 0.307227836 & C3 & TNFSF14 (-41774) & C3 $(+8289)$ \\
\hline cg24811290 & chr 4: 159092553 & 0.001450316 & 0.676894745 & FAM198B & FAM198B $(+1648)$ & GRIA2 (+950818) \\
\hline cg03304437 & chr 4: 159092536 & 0.002938581 & 0.424369174 & FAM198B & FAM198B $(+1665)$ & GRIA2 (+950801) \\
\hline cg07070348 & chr 12: 130555007 & -0.005354324 & 0.209154879 & & TMEM132D (-166796) & FZD10 (-91996) \\
\hline cg07747220 & chr 20: 3052115 & 0.002986068 & 0.395279658 & $O X T$ & OXT $(-150)$ & \\
\hline cg01644611 & chr 20: 3052253 & 0.006354246 & 0.06460808 & OXT & OXT $(-12)$ & \\
\hline $\operatorname{cg} 13725599$ & chr 20: 3052262 & 0.006387303 & 0.024953675 & OXT & $O X T(-3)$ & \\
\hline $\operatorname{cg} 26267561$ & chr 20: 3052224 & 0.00265964 & 0.409742344 & OXT & OXT $(-41)$ & \\
\hline $\operatorname{cg} 13285174$ & chr 20: 3052221 & 0.008335871 & 0.046156832 & OXT & OXT $(-44)$ & \\
\hline cg19592472 & chr 20: 3052274 & 0.009599409 & 0.009292317 & $O X T$ & $\operatorname{OXT}(+9)$ & \\
\hline cg16887334 & chr 20: 3052151 & 0.003805097 & 0.323232339 & $O X T$ & OXT $(-114)$ & \\
\hline cg02046423 & chr 17: 2951689 & -0.005535107 & 0.099080805 & & OR1D5 (+15211) & RAP1GAP2 $(+251958)$ \\
\hline cg24015889 & chr 17: 2951719 & 0.002035964 & 0.526532833 & & OR1D5 (+15181) & RAP1GAP2 (+251988) \\
\hline cg12163800 & chr 17: 74475355 & 0.000165722 & 0.951440963 & RHBDF2 & RHBDF2 (+8635) & AANAT $(+11726)$ \\
\hline cg05810363 & chr 17: 74475270 & 0.000790935 & 0.730915416 & RHBDF2 & RHBDF2 (+8720) & AANAT $(+11641)$ \\
\hline cg13076843 & chr 17: 74475294 & 0.004644871 & 0.104219756 & RHBDF2 & RHBDF2 (+8696) & AANAT $(+11665)$ \\
\hline cg17019969 & chr 17: 74475240 & -0.000446842 & 0.909127934 & RHBDF2 & RHBDF2 $(+8750)$ & AANAT $(+11611)$ \\
\hline cg06839111 & chr 16: 67978450 & -0.006720141 & 0.002478319 & SLC12A4; LCAT & $\operatorname{LCAT}(-436)$ & \\
\hline cg09495207 & chr 16: 67978445 & -0.008556322 & 9.93E-05 & SLC12A4; LCAT & $\operatorname{LCAT}(-431)$ & \\
\hline cg20697427 & chr 3: 195610231 & 0.002684102 & 0.426144824 & TNK2 & MUC4 (-71388) & TNK2 $(+12200)$ \\
\hline cg02920514 & chr 3: 195610120 & 0.005771686 & 0.273051915 & TNK2 & MUC4 (-71277) & TNK2 $(+12311)$ \\
\hline cg16047223 & chr 3: 195610084 & 0.001484324 & 0.60624638 & TNK2 & MUC4 (-71241) & TNK2 $(+12347)$ \\
\hline
\end{tabular}

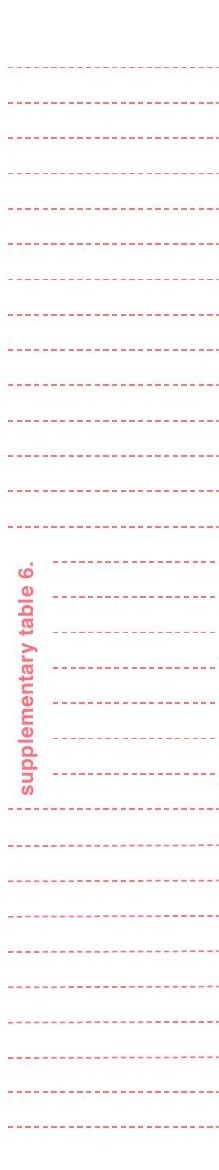


Braak stage I

Braak stage II

Braak stage III

Braak stage IV

Braak stage V

Braak stage VI

\begin{tabular}{|c|c|c|c|c|c|c|c|c|c|c|c|}
\hline $\begin{array}{c}\text { Average } \\
5-\mathrm{hmC} \\
(\%)\end{array}$ & $\begin{array}{c}\text { Standard } \\
\text { deviation } \\
(\%)\end{array}$ & $\begin{array}{c}\text { Average } \\
5-\mathrm{hmC} \\
(\%)\end{array}$ & $\begin{array}{c}\text { Standard } \\
\text { deviation } \\
(\%)\end{array}$ & $\begin{array}{c}\text { Average } \\
5-\mathrm{hmC} \\
(\%)\end{array}$ & $\begin{array}{c}\text { Standard } \\
\text { deviation } \\
(\%)\end{array}$ & $\begin{array}{c}\text { Average } \\
5-\mathrm{hmC} \\
(\%)\end{array}$ & $\begin{array}{c}\text { Standard } \\
\text { deviation } \\
(\%)\end{array}$ & $\begin{array}{c}\text { Average } \\
5-\mathrm{hmC} \\
(\%)\end{array}$ & $\begin{array}{c}\text { Standard } \\
\text { deviation } \\
(\%)\end{array}$ & $\begin{array}{c}\text { Average } \\
5-\mathrm{hmC} \\
(\%)\end{array}$ & $\begin{array}{c}\text { Standard } \\
\text { deviation } \\
(\%)\end{array}$ \\
\hline 5.71 & 1.51 & 8.07 & 3.38 & 7.06 & 3.05 & 7.68 & 2.73 & 8.91 & 3.35 & 7.63 & 2.69 \\
\hline 6.98 & 4.22 & 7.75 & 5.43 & 7.45 & 4.39 & 8.43 & 3.70 & 7.09 & 4.80 & 7.97 & 4.75 \\
\hline 16.02 & 5.28 & 19.97 & 5.44 & 16.88 & 6.31 & 17.72 & 5.12 & 15.92 & 5.41 & 17.59 & 5.62 \\
\hline 11.07 & 4.87 & 11.28 & 4.75 & 10.65 & 5.08 & 10.26 & 5.34 & 9.45 & 4.19 & 12.33 & 3.85 \\
\hline-0.75 & 2.38 & 1.19 & 2.91 & -0.33 & 5.48 & -0.07 & 3.71 & 1.81 & 4.53 & -0.05 & 4.77 \\
\hline 5.76 & 3.85 & 4.01 & 3.97 & 6.02 & 3.32 & 5.92 & 3.24 & 3.33 & 3.61 & 4.70 & 4.34 \\
\hline 1.67 & 2.99 & 0.10 & 3.75 & -0.76 & 3.28 & 0.13 & 2.48 & 0.83 & 3.56 & 0.44 & 3.89 \\
\hline 7.72 & 3.76 & 7.23 & 3.79 & 6.15 & 2.79 & 8.00 & 3.71 & 8.27 & 3.85 & 6.95 & 2.97 \\
\hline 8.71 & 0.17 & 8.41 & 3.13 & 7.55 & 2.81 & 8.41 & 2.38 & 9.35 & 2.31 & 8.55 & 2.38 \\
\hline 5.57 & 3.32 & 7.93 & 3.76 & 6.20 & 2.81 & 6.42 & 3.34 & 6.39 & 1.80 & 5.49 & 1.93 \\
\hline 17.22 & 3.28 & 14.75 & 3.78 & 16.78 & 4.67 & 15.83 & 4.35 & 17.58 & 5.38 & 16.47 & 5.17 \\
\hline 12.23 & 3.66 & 14.49 & 5.95 & 11.68 & 4.81 & 12.54 & 5.13 & 15.20 & 4.56 & 13.52 & 3.64 \\
\hline 0.69 & 6.60 & -0.39 & 6.44 & 4.38 & 5.90 & -0.29 & 6.32 & -0.31 & 4.97 & -0.22 & 4.14 \\
\hline 2.30 & 3.79 & 2.33 & 4.64 & 0.18 & 4.03 & 2.01 & 5.43 & 2.41 & 5.77 & 2.94 & 3.50 \\
\hline 4.53 & 1.83 & 8.15 & 4.56 & 4.17 & 2.68 & 5.63 & 5.02 & 9.07 & 5.44 & 7.12 & 5.20 \\
\hline 3.40 & 2.06 & 5.88 & 3.69 & 4.09 & 2.68 & 5.46 & 3.93 & 8.64 & 4.40 & 5.52 & 3.74 \\
\hline 9.41 & 3.76 & 9.63 & 5.19 & 9.37 & 3.70 & 11.06 & 4.08 & 11.05 & 4.35 & 9.38 & 5.15 \\
\hline 7.14 & 3.43 & 8.89 & 4.39 & 7.24 & 4.79 & 8.48 & 6.84 & 11.91 & 5.76 & 9.84 & 8.02 \\
\hline 12.78 & 2.18 & 15.03 & 4.75 & 13.47 & 5.04 & 14.76 & 5.04 & 19.31 & 5.03 & 15.73 & 5.23 \\
\hline 4.05 & 6.87 & 5.30 & 5.64 & 1.45 & 4.46 & 4.53 & 5.65 & 5.12 & 4.65 & 4.73 & 4.75 \\
\hline 3.80 & 4.56 & 7.14 & 4.63 & 5.42 & 4.29 & 4.10 & 3.05 & 4.64 & 5.21 & 2.48 & 4.48 \\
\hline 4.90 & 4.51 & 6.59 & 4.34 & 3.90 & 3.31 & 4.37 & 5.23 & 6.17 & 4.22 & 5.28 & 4.67 \\
\hline 2.86 & 1.79 & 3.21 & 2.78 & 5.01 & 4.00 & 5.48 & 4.14 & 4.04 & 3.86 & 3.15 & 2.92 \\
\hline 3.63 & 1.14 & 3.93 & 2.97 & 2.88 & 3.70 & 2.59 & 3.11 & 3.79 & 3.04 & 3.59 & 2.74 \\
\hline-0.46 & 2.08 & -1.09 & 2.78 & -0.48 & 3.42 & 2.77 & 4.87 & -0.02 & 3.73 & 1.45 & 2.95 \\
\hline 2.24 & 4.78 & 4.14 & 5.66 & 4.29 & 4.32 & 4.28 & 6.53 & 4.82 & 5.68 & 3.14 & 4.64 \\
\hline 2.47 & 1.66 & 2.19 & 3.48 & 2.53 & 2.11 & 2.52 & 3.27 & -0.97 & 2.67 & 0.75 & 2.32 \\
\hline 3.69 & 3.45 & 2.16 & 2.52 & 2.32 & 2.29 & 0.92 & 2.64 & -0.80 & 3.33 & -0.04 & 2.45 \\
\hline 3.20 & 2.20 & 2.06 & 4.19 & 2.91 & 4.08 & 5.49 & 5.16 & 2.67 & 4.19 & 4.13 & 4.74 \\
\hline 2.82 & 4.84 & 0.58 & 4.25 & 1.59 & 7.08 & 2.75 & 6.88 & 0.82 & 8.78 & 6.15 & 7.47 \\
\hline 6.25 & 4.24 & 4.32 & 2.96 & 4.62 & 4.39 & 6.18 & 4.36 & 4.95 & 3.06 & 6.10 & 4.09 \\
\hline
\end{tabular}




\begin{tabular}{|c|c|c|c|c|c|c|}
\hline Probe ID & Position & Braak est. & $p$-value & $\begin{array}{l}\text { Illumina } \\
\text { annotation } \\
\text { (UCSC) }\end{array}$ & \multicolumn{2}{|c|}{ GREAT annotation } \\
\hline cg09573585 & chr 10: 88728128 & 0.002955329 & 0.175284782 & C10orf116 & AGAP11 (-2369) & C10orf116 (-59) \\
\hline cg11805311 & chr 10: 88728073 & 0.006461492 & 0.037608595 & C10orf116 & AGAP11 (-2424) & C10orf116 (-114) \\
\hline cg26395382 & chr 10: 88728235 & 0.007835025 & 0.023230722 & C10orf116 & AGAP11 (-2262) & C10orf116 $(+48)$ \\
\hline cg01754756 & chr 10: 88728078 & 0.006116733 & 0.054790161 & C10orf116 & AGAP11 $(-2419)$ & C10orf116 (-109) \\
\hline cg05066959 & chr 8: 41519308 & -0.014326128 & 0.004492258 & ANK1; MIR486 & NKX6-3 (-14434) & ANK1 $(+234971)$ \\
\hline $\operatorname{cg} 11823178$ & chr 8: 41519399 & -0.010445652 & 0.000482155 & ANK1; MIR486 & NKX6-3 (-14525) & ANK1 $(+234880)$ \\
\hline cg15481294 & chr 19: 6712406 & -0.004136299 & 0.043201185 & C3 & TNFSF14 (-41808) & C3 $(+8255)$ \\
\hline cg26861457 & chr 19: 6712380 & -0.006152461 & 0.000628683 & C3 & TNFSF14 (-41782) & C3 $(+8281)$ \\
\hline cg10628785 & chr 19: 6712321 & -0.00363932 & 0.029086896 & C3 & TNFSF14 (-41723) & C3 $(+8340)$ \\
\hline cg25406665 & chr 19: 6712357 & -0.003830795 & 0.019017945 & C3 & TNFSF14 (-41759) & C3 $(+8304)$ \\
\hline cg15755240 & chr 19: 6712372 & -0.000475072 & 0.724170054 & C3 & TNFSF14 (-41774) & C3 $(+8289)$ \\
\hline cg24811290 & chr 4: 159092553 & 0.006148823 & 0.028865291 & FAM198B & FAM198B $(+1648)$ & GRIA2 (+950818) \\
\hline cg03304437 & chr 4: 159092536 & 0.007125833 & 0.023797972 & FAM198B & FAM198B $(+1665)$ & GRIA2 (+950801) \\
\hline cg06370094 & chr 12: 130555091 & 0.010645547 & 0.00069916 & & TMEM132D $(-166880)$ & FZD10 (-91912) \\
\hline cg09288218 & chr 12: 130554977 & 0.010566119 & 0.001316707 & & TMEM132D (-166766) & FZD10 (-92026) \\
\hline cg07070348 & chr 12: 130555007 & 0.007600781 & 0.017910978 & & TMEM132D (-166796) & FZD10 (-91996) \\
\hline $\operatorname{cg} 07747220$ & chr 20: 3052115 & 0.00433216 & 0.177233877 & $O X T$ & $O X T(-150)$ & \\
\hline cg01644611 & chr 20: 3052253 & 0.004740962 & 0.025082208 & $O X T$ & OXT $(-12)$ & \\
\hline cg13725599 & chr 20: 3052262 & 0.002084246 & 0.291146331 & $O X T$ & $O X T(-3)$ & \\
\hline cg26267561 & chr 20: 3052224 & 0.004746563 & 0.054074549 & OXT & OXT $(-41)$ & \\
\hline cg13285174 & chr 20: 3052221 & 0.006144414 & 0.057850503 & $O X T$ & $O X T(-44)$ & \\
\hline cg19592472 & chr 20: 3052274 & 0.000478175 & 0.892360099 & $O X T$ & $\operatorname{OXT}(+9)$ & \\
\hline $\operatorname{cg} 16887334$ & chr 20: 3052151 & 0.005530593 & 0.070362053 & $O X T$ & OXT $(-114)$ & \\
\hline cg26955850 & chr 20: 3052345 & -0.00256852 & 0.335887208 & $O X T$ & OXT $(+80)$ & \\
\hline cg02046423 & chr 17: 2951689 & -0.000626478 & 0.82804506 & & OR1D5 $(+15211)$ & RAP1GAP2 $(+251958)$ \\
\hline cg11684897 & chr 17: 2951666 & -0.003618111 & 0.29502693 & & OR1D5 (+15234) & RAP1GAP2 (+251935) \\
\hline cg24015889 & chr 17: 2951719 & -0.003588238 & 0.247758184 & & OR1D5 $(+15181)$ & RAP1GAP2 $(+251988)$ \\
\hline cg12163800 & chr 17: 74475355 & -0.013370883 & $3.59 E-06$ & RHBDF2 & RHBDF2 (+8635) & AANAT $(+11726)$ \\
\hline cg12309456 & chr 17: 74475402 & -0.012716619 & 7.33E-06 & RHBDF2 & RHBDF2 (+8588) & AANAT $(+11773)$ \\
\hline cg05810363 & chr 17: 74475270 & -0.015211785 & $9.19 \mathrm{E}-06$ & RHBDF2 & RHBDF2 $(+8720)$ & AANAT $(+11641)$ \\
\hline cg13076843 & chr 17: 74475294 & -0.019003194 & 6.50E-08 & RHBDF2 & RHBDF2 $(+8696)$ & AANAT $(+11665)$ \\
\hline cg17019969 & chr 17: 74475240 & -0.002638742 & 0.219478874 & RHBDF2 & RHBDF2 (+8750) & AANAT (+11611) \\
\hline cg06839111 & $\operatorname{chr} 16: 67978450$ & 0.001968227 & 0.440743522 & SLC12A4; LCAT & LCAT $(-436)$ & \\
\hline cg09495207 & chr 16: 67978445 & 0.004566565 & 0.059860794 & SLC12A4; LCAT & LCAT $(-431)$ & \\
\hline cg20697427 & chr 3: 195610231 & -0.005242366 & 0.029006958 & TNK2 & MUC4 (-71388) & TNK2 (+12200) \\
\hline cg02920514 & $\operatorname{chr} 3: 195610120$ & -0.010537855 & 0.006467596 & TNK2 & MUC4 (-71277) & TNK2 (+12311) \\
\hline $\operatorname{cg} 16047223$ & chr 3: 195610084 & -0.006897202 & 0.009572732 & TNK2 & MUC4 (-71241) & TNK2 (+12347) \\
\hline
\end{tabular}

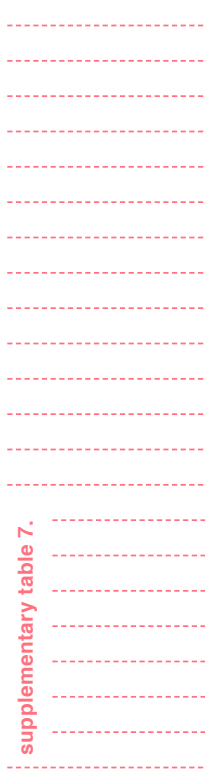

SUPPLEMENTARY TABLE 7. Association of probes in differentially unmodified regions (DURs) with Braak stage. Braak stage-association analysis of probes located in Alzheimer's disease (AD)-associated DURs. Displayed for each probe is chromosomal position (genome build 37), regression estimate for the Braak-associated analysis (Braak est.), accompanying $p$-values, Illumina gene annotation, GREAT annotation (distance to closest tran- 


\begin{tabular}{|c|c|c|c|c|c|c|c|c|c|c|c|}
\hline \multicolumn{2}{|c|}{ Braak stage I } & \multicolumn{2}{|c|}{ Braak stage II } & \multicolumn{2}{|c|}{ Braak stage III } & \multicolumn{2}{|c|}{ Braak stage IV } & \multicolumn{2}{|c|}{ Braak stage V } & \multicolumn{2}{|c|}{ Braak stage VI } \\
\hline $\begin{array}{c}\text { Average } \\
\text { UC (\%) }\end{array}$ & $\begin{array}{c}\text { Standard } \\
\text { deviation } \\
(\%)\end{array}$ & $\begin{array}{c}\text { Average } \\
\text { UC (\%) }\end{array}$ & $\begin{array}{c}\text { Standard } \\
\text { deviation } \\
(\%)\end{array}$ & $\begin{array}{l}\text { Average } \\
\text { UC (\%) }\end{array}$ & $\begin{array}{c}\text { Standard } \\
\text { deviation } \\
(\%)\end{array}$ & $\begin{array}{l}\text { Average } \\
\text { UC (\%) }\end{array}$ & $\begin{array}{l}\text { Standard } \\
\text { deviation } \\
(\%)\end{array}$ & $\begin{array}{l}\text { Average } \\
\text { UC (\%) }\end{array}$ & $\begin{array}{c}\text { Standard } \\
\text { deviation } \\
(\%)\end{array}$ & $\begin{array}{c}\text { Average } \\
\text { UC (\%) }\end{array}$ & $\begin{array}{c}\text { Standard } \\
\text { deviation } \\
(\%)\end{array}$ \\
\hline 60.60 & 2.16 & 59.77 & 2.74 & 59.47 & 2.71 & 60.51 & 2.26 & 62.02 & 3.92 & 59.82 & 2.70 \\
\hline 62.81 & 4.27 & 62.54 & 6.00 & 61.98 & 2.96 & 62.68 & 2.92 & 65.63 & 5.84 & 63.50 & 2.34 \\
\hline 70.04 & 5.16 & 67.58 & 4.69 & 66.84 & 3.37 & 69.07 & 3.71 & 72.56 & 5.73 & 69.26 & 3.30 \\
\hline 62.34 & 5.23 & 63.75 & 5.50 & 61.98 & 3.71 & 63.72 & 3.43 & 66.36 & 4.62 & 63.63 & 2.82 \\
\hline 29.04 & 6.42 & 19.88 & 6.87 & 25.02 & 5.13 & 22.87 & 6.21 & 16.90 & 5.54 & 20.22 & 7.54 \\
\hline 18.16 & 2.82 & 14.60 & 5.22 & 15.35 & 3.62 & 13.93 & 3.33 & 11.95 & 3.70 & 11.94 & 4.49 \\
\hline 21.31 & 2.26 & 21.65 & 3.93 & 21.31 & 2.39 & 20.58 & 2.74 & 19.29 & 2.74 & 20.37 & 1.80 \\
\hline 21.14 & 2.25 & 19.70 & 2.63 & 21.26 & 1.91 & 19.07 & 1.88 & 18.21 & 2.66 & 18.73 & 1.99 \\
\hline 26.11 & 0.86 & 24.67 & 2.41 & 24.90 & 2.84 & 24.78 & 1.87 & 23.82 & 2.16 & 23.60 & 1.75 \\
\hline 18.58 & 1.52 & 17.47 & 2.71 & 17.88 & 2.01 & 17.19 & 2.69 & 16.12 & 1.67 & 16.79 & 1.75 \\
\hline 15.11 & 1.94 & 13.15 & 2.26 & 14.16 & 1.65 & 13.43 & 2.01 & 13.81 & 1.44 & 13.86 & 1.51 \\
\hline 62.44 & 4.31 & 64.84 & 4.96 & 63.57 & 2.92 & 65.20 & 3.07 & 64.96 & 3.85 & 66.42 & 4.05 \\
\hline 61.26 & 4.30 & 64.51 & 5.16 & 63.76 & 3.75 & 64.50 & 3.12 & 63.11 & 3.87 & 67.61 & 4.47 \\
\hline 60.57 & 3.66 & 64.23 & 4.2 & 63.32 & 3.44 & 62.96 & 2.78 & 67.22 & 4.82 & 66.57 & 4.23 \\
\hline 58.01 & 1.87 & 61.81 & 4.41 & 58.62 & 3.97 & 61.23 & 3.03 & 64.03 & 4.58 & 63.24 & 5.10 \\
\hline 67.54 & 2.28 & 70.05 & 4.56 & 68.26 & 4.57 & 69.79 & 2.02 & 72.71 & 4.32 & 70.59 & 4.79 \\
\hline 18.41 & 5.41 & 19.33 & 3.97 & 19.42 & 2.99 & 20.22 & 5.41 & 21.17 & 4.93 & 19.80 & 4.87 \\
\hline 11.10 & 2.57 & 10.47 & 1.97 & 11.31 & 2.57 & 12.82 & 3.26 & 13.15 & 3.31 & 12.05 & 2.77 \\
\hline 10.02 & 3.20 & 9.77 & 2.55 & 9.96 & 2.23 & 10.59 & 3.22 & 10.64 & 2.57 & 10.52 & 3.38 \\
\hline 23.16 & 4.14 & 24.17 & 3.52 & 23.90 & 2.95 & 24.64 & 3.48 & 25.73 & 3.72 & 25.02 & 2.93 \\
\hline 19.44 & 5.17 & 19.31 & 4.96 & 18.55 & 3.32 & 21.01 & 4.78 & 22.30 & 4.57 & 19.93 & 5.05 \\
\hline 26.15 & 6.10 & 26.07 & 5.87 & 25.60 & 3.16 & 27.03 & 5.61 & 26.61 & 4.95 & 25.29 & 5.15 \\
\hline 14.99 & 1.78 & 17.45 & 3.10 & 16.76 & 3.89 & 17.80 & 5.25 & 19.50 & 4.11 & 17.41 & 4.61 \\
\hline 52.00 & 5.22 & 50.14 & 5.22 & 50.65 & 2.42 & 51.32 & 3.83 & 51.76 & 2.76 & 48.03 & 3.57 \\
\hline 73.78 & 5.78 & 68.04 & 4.96 & 71.30 & 2.66 & 71.52 & 3.57 & 69.31 & 2.98 & 71.03 & 3.93 \\
\hline 67.25 & 6.66 & 63.29 & 5.34 & 65.40 & 2.94 & 65.18 & 4.59 & 63.42 & 4.95 & 63.60 & 4.99 \\
\hline 64.93 & 5.24 & 60.71 & 4.00 & 62.29 & 3.34 & 61.05 & 4.52 & 59.47 & 4.05 & 62.05 & 4.27 \\
\hline 21.05 & 5.03 & 14.09 & 2.87 & 16.48 & 3.98 & 13.41 & 3.97 & 11.25 & 1.77 & 12.77 & 2.40 \\
\hline 17.58 & 5.58 & 12.06 & 3.61 & 13.64 & 3.73 & 11.60 & 3.92 & 9.34 & 2.88 & 9.79 & 1.74 \\
\hline 15.99 & 6.02 & 9.92 & 5.42 & 11.85 & 4.69 & 10.03 & 4.84 & 6.31 & 1.86 & 7.01 & 2.10 \\
\hline 25.31 & 7.33 & 18.31 & 3.70 & 19.94 & 4.46 & 17.35 & 4.94 & 14.27 & 2.81 & 13.74 & 2.97 \\
\hline 21.04 & 3.76 & 18.77 & 3.70 & 19.16 & 3.58 & 19.47 & 2.39 & 17.67 & 2.70 & 18.68 & 2.43 \\
\hline 61.30 & 2.93 & 59.12 & 2.86 & 59.46 & 2.56 & 58.52 & 3.87 & 62.26 & 3.63 & 59.37 & 2.93 \\
\hline 63.99 & 3.86 & 62.94 & 2.89 & 63.77 & 2.51 & 63.46 & 3.64 & 66.39 & 3.47 & 64.36 & 2.67 \\
\hline 33.90 & 2.09 & 34.57 & 4.68 & 33.65 & 3.23 & 32.65 & 2.52 & 32.06 & 3.75 & 32.90 & 2.83 \\
\hline 31.51 & 3.22 & 31.31 & 7.33 & 33.23 & 4.53 & 31.69 & 4.21 & 29.07 & 6.51 & 28.35 & 3.87 \\
\hline 15.62 & 2.55 & 15.42 & 5.17 & 16.05 & 2.90 & 15.53 & 3.84 & 13.24 & 4.05 & 13.45 & 2.39 \\
\hline
\end{tabular}




\begin{tabular}{|c|c|c|c|c|c|c|c|c|c|c|}
\hline \multirow{2}{*}{ Gene } & \multirow{2}{*}{ Probe ID } & \multicolumn{3}{|c|}{$5-m c$} & \multicolumn{3}{|c|}{ 5-hmC } & \multicolumn{3}{|c|}{ UC } \\
\hline & & Pearson's $r$ & $p$-value & $q$-value & Pearson's $r$ & p-value & $q$-value & Pearson's $r$ & p-value & $q$-value \\
\hline \multirow{2}{*}{ FAM198B } & cg24811290 & -0.067 & 0.567 & 0.682 & -0.070 & 0.548 & 0.680 & 0.125 & 0.276 & 0.464 \\
\hline & cg03304437 & -0.066 & 0.572 & 0.682 & -0.132 & 0.254 & 0.394 & 0.187 & 0.102 & 0.221 \\
\hline \multirow{4}{*}{$\begin{array}{c}\text { ADIRF } \\
\text { (C10orf116) }\end{array}$} & cg09573585 & -0.157 & 0.175 & 0.340 & -0.082 & 0.483 & 0.623 & 0.260 & 0.022 & 0.089 \\
\hline & cg01754756 & -0.084 & 0.471 & 0.646 & -0.133 & 0.252 & 0.394 & 0.232 & 0.041 & 0.123 \\
\hline & cg11805311 & -0.151 & 0.192 & 0.356 & -0.126 & 0.279 & 0.411 & 0.320 & 0.004 & 0.053 \\
\hline & cg26395382 & -0.116 & 0.317 & 0.498 & -0.134 & 0.249 & 0.394 & 0.292 & 0.009 & 0.066 \\
\hline \multirow{8}{*}{$O X T$} & $\mathrm{cg} 13285174$ & -0.246 & 0.033 & 0.111 & 0.230 & 0.045 & 0.169 & 0.087 & 0.451 & 0.599 \\
\hline & cg13725599 & -0.266 & 0.020 & 0.111 & 0.227 & 0.049 & 0.169 & 0.144 & 0.209 & 0.368 \\
\hline & cg19592472 & -0.245 & 0.033 & 0.111 & 0.229 & 0.046 & 0.169 & 0.109 & 0.343 & 0.512 \\
\hline & cg01644611 & -0.291 & 0.011 & 0.111 & 0.283 & 0.013 & 0.136 & 0.163 & 0.153 & 0.297 \\
\hline & cg16887334 & -0.219 & 0.058 & 0.164 & 0.193 & 0.095 & 0.268 & 0.080 & 0.486 & 0.599 \\
\hline & cg26267561 & -0.258 & 0.024 & 0.111 & 0.240 & 0.037 & 0.169 & 0.058 & 0.615 & 0.734 \\
\hline & cg07747220 & -0.292 & 0.010 & 0.111 & 0.179 & 0.122 & 0.291 & 0.173 & 0.129 & 0.266 \\
\hline & cg26955850 & -0.168 & 0.147 & 0.320 & - & - & - & 0.051 & 0.660 & 0.740 \\
\hline \multirow{3}{*}{ RAP1GAP2 } & $\operatorname{cg} 11684897$ & -0.263 & 0.022 & 0.111 & - & - & - & 0.280 & 0.013 & 0.066 \\
\hline & cg02046423 & -0.347 & 0.002 & 0.078 & 0.157 & 0.177 & 0.361 & 0.222 & 0.050 & 0.133 \\
\hline & cg24015889 & -0.190 & 0.100 & 0.246 & -0.034 & 0.768 & 0.833 & 0.276 & 0.014 & 0.066 \\
\hline \multirow{5}{*}{ RHBDF2 } & cg12163800 & 0.287 & 0.012 & 0.111 & -0.089 & 0.442 & 0.596 & -0.280 & 0.013 & 0.066 \\
\hline & cg12309456 & 0.270 & 0.019 & 0.111 & - & 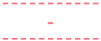 & - & -0.250 & 0.028 & 0.102 \\
\hline & $\mathrm{cg} 13076843$ & 0.138 & 0.233 & 0.401 & 0.228 & 0.048 & 0.169 & -0.303 & 0.007 & 0.064 \\
\hline & $\mathrm{cg} 05810363$ & 0.215 & 0.063 & 0.166 & 0.029 & 0.806 & 0.833 & -0.229 & 0.043 & 0.123 \\
\hline & $\operatorname{cg} 17019969$ & 0.071 & 0.543 & 0.682 & -0.015 & 0.895 & 0.895 & -0.042 & 0.717 & 0.769 \\
\hline \multirow{2}{*}{ SLC12A4 } & cg06839111 & 0.105 & 0.368 & 0.545 & -0.560 & $1.41 \mathrm{E}-07$ & 4.38E-06 & 0.450 & $3.52 \mathrm{E}-05$ & $6.51 \mathrm{E}-04$ \\
\hline & $\mathrm{cg} 09495207$ & 0.007 & 0.952 & 0.952 & -0.485 & $8.91 \mathrm{E}-06$ & $\begin{array}{l}1.38 \mathrm{E}-04 \\
\end{array}$ & 0.474 & $1.18 \mathrm{E}-05$ & $4.35 \mathrm{E}-04$ \\
\hline \multirow{3}{*}{ TNK2 } & cg02920514 & -0.031 & 0.790 & 0.846 & 0.100 & 0.388 & 0.547 & -0.082 & 0.476 & 0.599 \\
\hline & cg16047223 & 0.029 & 0.801 & 0.846 & 0.042 & 0.716 & 0.822 & -0.095 & 0.410 & 0.583 \\
\hline & cg20697427 & -0.137 & 0.239 & 0.401 & 0.237 & 0.039 & 0.169 & -0.116 & 0.310 & 0.499 \\
\hline \multirow{2}{*}{ ANK1 } & cg11823178 & -0.044 & 0.708 & 0.794 & 0.033 & 0.779 & 0.833 & 0.040 & 0.727 & 0.769 \\
\hline & cg05066959 & -0.160 & 0.169 & 0.340 & 0.169 & 0.143 & 0.317 & 0.054 & 0.642 & 0.740 \\
\hline \multirow{3}{*}{ LOC100190940 } & cg09288218 & 0.066 & 0.570 & 0.682 & - & - & - & -0.144 & 0.208 & 0.368 \\
\hline & cg06370094 & 0.170 & 0.143 & 0.320 & - & $\therefore$ & - & -0.193 & 0.091 & 0.210 \\
\hline & cg07070348 & 0.022 & 0.853 & 0.877 & 0.151 & 0.194 & 0.361 & -0.232 & 0.041 & 0.123 \\
\hline \multirow{5}{*}{ C3 } & cg25406665 & -0.088 & 0.448 & 0.637 & - & - & - & -0.085 & 0.457 & 0.599 \\
\hline & cg26861457 & -0.236 & 0.040 & 0.124 & 0.192 & 0.096 & 0.268 & 0.028 & 0.806 & 0.806 \\
\hline & cg15481294 & -0.249 & 0.030 & 0.111 & 0.149 & 0.198 & 0.361 & 0.034 & 0.767 & 0.788 \\
\hline & cg10628785 & -0.115 & 0.323 & 0.498 & 0.063 & 0.588 & 0.700 & 0.108 & 0.346 & 0.512 \\
\hline & cg15755240 & -0.056 & 0.632 & 0.731 & 0.188 & 0.104 & 0.268 & -0.216 & 0.058 & 0.143 \\
\hline
\end{tabular}

SUPPLEMENTARY TABLE 8. Correlation of DNA (hydroxy)methylation with expression.

5-methylcytosine (5-mC), 5-hydroxymethylcytosine (5-hmC), and unmodified cytosine (UC) levels of probes within Alzheimer's disease (AD)-associated differentially methylated regions (DMRs), differentially hydroxymethylated regions (DHRs), and differentially unmodified regions (DURs) were correlated with gene expression levels. Displayed per dataset 


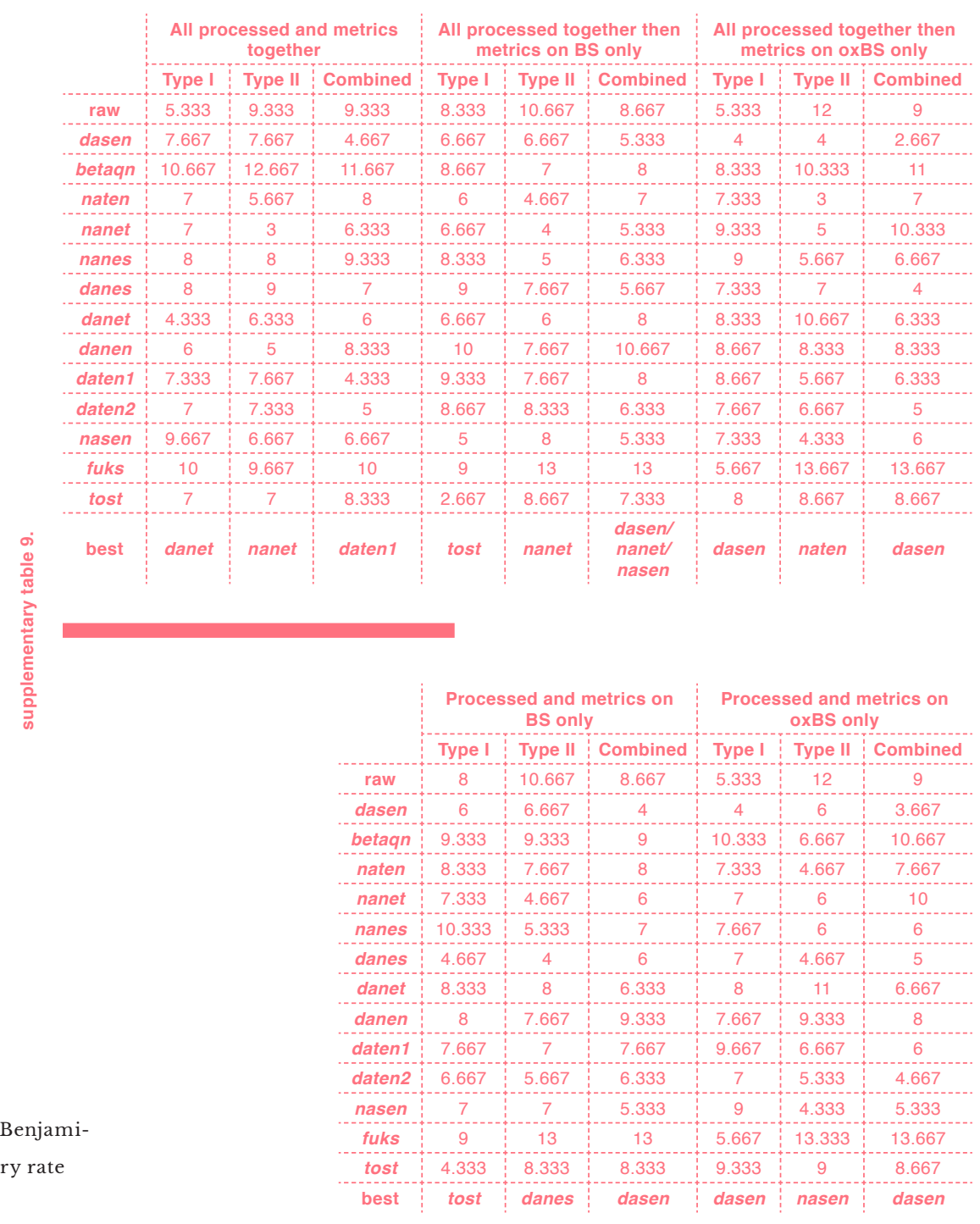

\section{Supplementary table 9. Perfor-}

mance of wateRmelon normalization

methods.

Comparison of raw data from raw

bisulfite converted (BS) and oxidative

BS (oxBS) converted DNA and 13 nor-

malization and processing methods

based on the wateRmelon $\mathrm{R}$ package.

Ranking is based on the three perfor-

mance metrics described in [31]. Type

I and Type II refer to the probe types included on Illumina's HumanMeth-

ylation450 BeadChip. 


\begin{tabular}{|c|c|c|c|c|c|}
\hline & \multirow[b]{2}{*}{ 5-mC/UC probes (\%) } & \multirow[b]{2}{*}{$\begin{array}{l}\text { Number of } \\
\text { sites }(\%)\end{array}$} & \multicolumn{3}{|c|}{ Top 1000 5-mC probes } \\
\hline & & & $\begin{array}{l}\text { Enrichment } \\
(95 \% \mathrm{Cl})\end{array}$ & $p$-value & $\begin{array}{l}\text { Number of } \\
\text { sites (\%) }\end{array}$ \\
\hline All probes & 396600 & 1000 & - & - & 1000 \\
\hline \multicolumn{6}{|c|}{$\mathrm{CpG}$ island feature } \\
\hline Island & $130971(33.0)$ & $171(17.1)$ & $0.52(0.44-0.61)$ & $<2.2 \mathrm{E}-16$ & $115(11.5)$ \\
\hline Shore & $94237(23.8)$ & $295(29.5)$ & $1.24(1.09-1.42)$ & $1.46 \mathrm{E}-03$ & $197(19.7)$ \\
\hline Shelf & $35409(8.9)$ & $141(14.1)$ & $1.58(1.31-1.89)$ & $1.41 \mathrm{E}-06$ & $146(14.6)$ \\
\hline NonCGI & $135983(34.3)$ & $393(39.3)$ & $1.15(1.02-1.29)$ & $2.28 \mathrm{E}-02$ & $542(54.2)$ \\
\hline \multicolumn{6}{|c|}{ Gene feature } \\
\hline Intergenic & $57664(14.5)$ & $158(15.8)$ & $1.09(0.91-1.29)$ & 3.31E-01 & $205(20.5)$ \\
\hline Distal promoter & $18269(4.6)$ & $51(5.1)$ & $1.11(0.82-1.47)$ & 4.52E-01 & $54(5.4)$ \\
\hline Proximal promoter & $158959(40.1)$ & $249(24.9)$ & $0.62(0.54-0.71)$ & $2.85 \mathrm{E}-12$ & $165(16.5)$ \\
\hline Gene body & $154140(38.9)$ & $518(51.8)$ & $1.33(1.20-1.48)$ & $1.82 \mathrm{E}-07$ & $553(55.3)$ \\
\hline Downstream & $7568(1.9)$ & $24(2.4)$ & $1.26(0.80-1.88)$ & $2.48 \mathrm{E}-01$ & $23(2.3)$ \\
\hline $\begin{array}{l}\text { Transcription factor } \\
\text { binding site }\end{array}$ & $33468(48.8)$ & $385(38.5)$ & $0.79(0.70-0.89)$ & $6.70 \mathrm{E}-05$ & $300(30.0)$ \\
\hline $\begin{array}{c}\text { DNase } 1 \\
\text { hypersensitivity site }\end{array}$ & $189731(47.8)$ & $449(44.9)$ & $0.94(0.84-1.05)$ & 2.73E-01 & 369 (36.9) \\
\hline
\end{tabular}

Alternative transcription events

\begin{tabular}{|c|c|c|c|c|c|}
\hline All probes & 146785 & 394 & - & - & 339 \\
\hline A3SS & $3450(2.4)$ & $8(2.0)$ & $0.96(0.41-1.90)$ & $1.00 \mathrm{E}+00$ & $8(2.4)$ \\
\hline A5SS & $3512(2.4)$ & $8(2.0)$ & $0.94(0.40-1.86)$ & $1.00 \mathrm{E}+00$ & $8(2.4)$ \\
\hline AFE & $60973(41.5)$ & $120(30.5)$ & $0.81(0.66-0.99)$ & $3.53 \mathrm{E}-02$ & $85(25.1)$ \\
\hline ALE & $9857(6.7)$ & $41(10.4)$ & $1.71(1.22-2.36)$ & $1.84 \mathrm{E}-03$ & $43(12.7)$ \\
\hline $\mathrm{CE}$ & $64532(44.0)$ & $194(49.2)$ & $1.24(1.05-1.46)$ & 1.10E-02 & $194(57.2)$ \\
\hline CNE & $21520(14.7)$ & $61(15.5)$ & $1.17(0.88-1.52)$ & $2.51 \mathrm{E}-01$ & $47(13.9)$ \\
\hline $\mathrm{El}$ & $176(0.1)$ & $0(0.0)$ & $0.00(0.00-8.76)$ & $1.00 \mathrm{E}+00$ & $0(0.0)$ \\
\hline II & $29413(20.0)$ & $68(17.3)$ & $0.95(0.73-1.23)$ & $7.54 \mathrm{E}-01$ & $63(18.6)$ \\
\hline IR & $16918(11.5)$ & $46(11.7)$ & $1.12(0.81-1.51)$ & $4.68 \mathrm{E}-01$ & $39(11.5)$ \\
\hline MXE & $14192(9.7)$ & $44(11.2)$ & $1.28(0.92-1.74)$ & 1.17E-01 & $50(14.7)$ \\
\hline
\end{tabular}

SuPPLEMENTARY TABLE 10. Structural and functional genomic annotation and enrichment analysis.

Enrichment of the 1000 highest ranked 5-methylcytosine $(5-\mathrm{mC})$, 5 -hydroxymethylcytosine $(5-\mathrm{hmC})$, and unmodified cytosine (UC) positions in specific structural and functional genomic features, calculated using Fisher's exact test. NG, non-CpG island; A3SS, alternative 3' splice site; A5SS, alternative 5' splice site; AFE, alternative first exon; ALE, alternative last exon; CE, cassette exon; CI, confidence interval; CNE, constitutive exon; EI, exon isoforms; II, intron isoforms; IR, intron retention; MXE, mutually exclusive exon. 
Top 1000 UC probes

\begin{tabular}{|c|c|c|c|c|c|}
\hline \multicolumn{3}{|c|}{ Top 1000 UC probes } & \multicolumn{3}{|c|}{ Top 1000 5-hmC probes } \\
\hline $\begin{array}{c}\text { Enrichment } \\
(95 \% \mathrm{Cl})\end{array}$ & $p$-value & 5-hmC probes (\%) & $\begin{array}{l}\text { Number of } \\
\text { sites (\%) }\end{array}$ & $\begin{array}{c}\text { Enrichment } \\
(95 \% \mathrm{Cl})\end{array}$ & $p$-value \\
\hline- & - & 178591 & 1000 & - & - \\
\hline
\end{tabular}

CpG island feature

\begin{tabular}{|c|c|c|c|c|c|}
\hline $0.35(0.28-0.42)$ & $<2.2 \mathrm{E}-16$ & 33644 (18.8) & $101(10.1)$ & $0.54(0.43-0.66)$ & $1.15 \mathrm{E}-10$ \\
\hline $0.83(0.71-0.97)$ & $1.53 \mathrm{E}-02$ & $44575(25.0)$ & $270(27.0)$ & $1.08(0.94-1.24)$ & 2.60E-01 \\
\hline $1.64(1.36-1.95)$ & $1.97 \mathrm{E}-07$ & $22956(12.9)$ & $161(16.1)$ & $1.25(1.05-1.48)$ & $9.47 \mathrm{E}-03$ \\
\hline $1.58(1.42-1.76)$ & $<2.2 \mathrm{E}-16$ & $77416(43.3)$ & $468(46.8)$ & $1.08(0.96-1.21)$ & $1.72 \mathrm{E}-01$ \\
\hline
\end{tabular}

Gene feature

\begin{tabular}{|c|c|c|c|c|c|}
\hline $1.41(1.21-1.64)$ & 1.72E-05 & 25409 (14.2) & $157(15.7)$ & $1.10(0.93-1.31)$ & $2.46 \mathrm{E}-01$ \\
\hline $1.17(0.87-1.54)$ & 0.2589 & $9258(5.2)$ & $47(4.7)$ & $0.91(0.66-1.22)$ & 5.67E-01 \\
\hline $0.41(0.35-0.49)$ & $<2.2 \mathrm{E}-16$ & 52396 (29.3) & $233(23.3)$ & $0.79(0.69-0.92)$ & 1.34E-03 \\
\hline $1.42(1.28-1.58)$ & $6.86 \mathrm{E}-11$ & $87560(49.0)$ & $546(54.6)$ & $1.11(1.00-1.24)$ & 4.46E-02 \\
\hline $1.21(0.76-1.82)$ & 0.3548 & $3968(2.2)$ & $17(1.7)$ & $0.77(0.44-1.23)$ & 3.30E-01 \\
\hline $0.61(0.54-0.70)$ & 1.847E-14 & $68710(38.5)$ & $360(36)$ & $0.94(0.83-1.06)$ & 0.2881 \\
\hline $0.77(0.68-0.87)$ & 1.603E-05 & 72283 (40.8) & $425(42.5)$ & $1.05(0.93-1.18)$ & 0.4115 \\
\hline
\end{tabular}

Alternative transcription events

\begin{tabular}{|c|c|c|c|c|c|}
\hline- & - & 66205 & 380 & - & - \\
\hline $0.88(0.38-1.74)$ & 0.8673 & $1649(2.5)$ & $6(1.6)$ & $0.66(0.24-1.45)$ & $3.99 \mathrm{E}-01$ \\
\hline $0.86(0.37-1.71)$ & 0.8677 & $1709(2.6)$ & $9(2.4)$ & $0.95(0.43-1.83)$ & $1.00 \mathrm{E}+00$ \\
\hline $0.53(0.42-0.66)$ & 2.16E-09 & 22945 (34.7) & $122(32.1)$ & $0.96(0.79-1.17)$ & 7.69E-01 \\
\hline $1.65(1.18-2.25)$ & 0.002862 & $5746(8.7)$ & $34(8.9)$ & $1.07(0.74-1.52)$ & $6.49 E-01$ \\
\hline $1.14(0.96-1.34)$ & 0.1269 & $32843(49.6)$ & $214(56.3)$ & $1.18(1.01-1.38)$ & $3.80 \mathrm{E}-02$ \\
\hline $0.83(0.60-1.11)$ & 0.2358 & $8724(13.2)$ & $49(12.9)$ & $1.02(0.74-1.36)$ & 8.81E-01 \\
\hline $0.00(0.00-8.03)$ & 1.00 & $98(0.1)$ & $1(0.3)$ & $1.85(0.05-10.58)$ & $4.20 E-01$ \\
\hline $0.81(0.61-1.05)$ & 0.1147 & $12828(19.4)$ & $75(19.7)$ & $1.06(0.82-1.35)$ & $6.16 \mathrm{E}-01$ \\
\hline $0.87(0.61-1.20)$ & 0.4379 & $7624(11.5)$ & $32(8.4)$ & $0.76(0.52-1.09)$ & 1.47E-01 \\
\hline $1.33(0.98-1.78)$ & 0.05523 & $7416(11.2)$ & $49(12.9)$ & $1.20(0.88-1.61)$ & 2.27E-01 \\
\hline
\end{tabular}

Significant results are displayed in bold. 


\begin{tabular}{|c|c|c|c|c|c|c|c|}
\hline Rank & ID & Name & Type & $\begin{array}{r}\text { Genes } \\
\text { in term }\end{array}$ & $\begin{array}{c}\text { Genes } \\
\text { in test } \\
\text { list and } \\
\text { term }\end{array}$ & $p$-value & OR \\
\hline 1 & GO:0001776 & leukocyte homeostasis & biological process & 56 & 9 & 2.04E-06 & 5.856837735 \\
\hline 2 & GO:0005089 & $\begin{array}{l}\text { Rho guanyl-nucleotide exchange } \\
\text { factor activity }\end{array}$ & molecular function & 66 & 12 & $1.05 \mathrm{E}-05$ & 4.366362966 \\
\hline 3 & GO:0001782 & B cell homeostasis & biological process & 19 & 5 & $1.42 \mathrm{E}-05$ & 10.10052838 \\
\hline 4 & GO:0048541 & Peyer's patch development & biological process & 11 & 4 & 2.05E-05 & 15.14263875 \\
\hline 5 & GO:0002260 & lymphocyte homeostasis & biological process & 45 & 7 & 2.19E-05 & 5.846940236 \\
\hline 6 & GO:0005088 & $\begin{array}{l}\text { Ras guanyl-nucleotide exchange } \\
\text { factor activity }\end{array}$ & molecular function & 111 & 16 & $2.24 \mathrm{E}-05$ & 3.327644456 \\
\hline 7 & GO:0016137 & glycoside metabolic process & biological process & 15 & 3 & $2.88 \mathrm{E}-05$ & 15.45419598 \\
\hline 8 & GO:0048537 & $\begin{array}{l}\text { mucosal-associated lymphoid } \\
\text { tissue development }\end{array}$ & biological process & 12 & 4 & 3.13E-05 & 13.40151995 \\
\hline 9 & GO:0008093 & cytoskeletal adaptor activity & molecular function & 17 & 5 & $4.59 \mathrm{E}-05$ & 8.891298139 \\
\hline 10 & GO:0051923 & sulfation & biological process & 15 & 3 & $8.15 \mathrm{E}-05$ & 12.79767839 \\
\hline 11 & GO:0045669 & $\begin{array}{l}\text { positive regulation of osteoblast } \\
\text { differentiation }\end{array}$ & biological process & 62 & 9 & 0.000154719 & 3.909317115 \\
\hline 12 & GO:0016782 & $\begin{array}{l}\text { transferase activity, transferring } \\
\text { sulfur-containing groups }\end{array}$ & molecular function & 62 & 8 & 0.000161209 & 4.192740378 \\
\hline 13 & GO:0008146 & sulfotransferase activity & molecular function & 50 & 7 & 0.000162915 & 4.6709011 \\
\hline 14 & GO:0042975 & $\begin{array}{l}\text { peroxisome proliferator activated } \\
\text { receptor binding }\end{array}$ & molecular function & 10 & 3 & 0.000179133 & 13.56004287 \\
\hline 15 & GO:0030647 & $\begin{array}{l}\text { aminoglycoside antibiotic } \\
\text { metabolic process }\end{array}$ & biological process & 10 & 2 & 0.000187903 & 20.08216287 \\
\hline 16 & GO:0050727 & $\begin{array}{l}\text { regulation of inflammatory } \\
\text { response }\end{array}$ & biological process & 225 & 17 & 0.000196101 & 2.544112361 \\
\hline 17 & GO:0004033 & $\begin{array}{l}\text { aldo-keto reductase (NADP) } \\
\text { activity }\end{array}$ & molecular function & 18 & 3 & 0.000207595 & 10.25016515 \\
\hline 18 & GO:0060457 & $\begin{array}{l}\text { negative regulation of digestive } \\
\text { system process }\end{array}$ & biological process & 10 & 3 & 0.000212253 & 13.13860491 \\
\hline 19 & GO:0046683 & response to organophosphorus & biological process & 117 & 13 & 0.000232085 & 2.949037581 \\
\hline 20 & GO:0042730 & fibrinolysis & biological process & 19 & 3 & 0.000240734 & 9.847792817 \\
\hline 21 & GO:0050829 & $\begin{array}{c}\text { defense response to Gram- } \\
\text { negative bacterium }\end{array}$ & biological process & 21 & 3 & 0.000241259 & 9.646249054 \\
\hline 22 & GO:0051000 & $\begin{array}{l}\text { positive regulation of nitric-oxide } \\
\text { synthase activity }\end{array}$ & biological process & 16 & 4 & 0.000243565 & 8.317115164 \\
\hline 23 & GO:0031904 & endosome lumen & cellular component & 12 & 3 & 0.000264042 & 11.43265297 \\
\hline 24 & GO:0050729 & $\begin{array}{l}\text { positive regulation of } \\
\text { inflammatory response }\end{array}$ & biological process & 75 & 8 & 0.000274534 & 3.846610095 \\
\hline 25 & GO:0050671 & $\begin{array}{l}\text { positive regulation of lymphocyte } \\
\text { proliferation }\end{array}$ & biological process & 101 & 10 & 0.000306663 & 3.296160951 \\
\hline
\end{tabular}

SUPPLEMENTARY TABLE 11. Gene

Ontology (GO) enrichment analysis results for the top 1000 differentially methylated positions (DMPs).

Displayed for each GO term is the ID, name, type, the number of genes in the pathway and the top 1000 (test list), $p$-value, odds ratio (OR) and genes in both the test list and GO term. Terms are ranked based on $p$-value. 
Gene names in GO term and test list

SH2B2;IL6;SPNS2;PIK3CD;STAT5A;MEF2A;BCL2;JAM3;CHST3

ABR;MCF2L;FARP1;ARHGEF10L;KALRN;PLEKHG5;ALS2CL;TRIO;SGEF;TIAM2;NGEF;ECT2L

SH2B2;SPNS2;PIK3CD;MEF2A;BCL2

CACNB4;ID2;FOXL1; STAT5A

SH2B2;SPNS2;PIK3CD;STAT5A;MEF2A;BCL2;CHST3

ABR;MCF2L;FARP1;ARHGEF10L;KALRN;PLEKHG5;DENND1A;ALS2CL;RAB3IP;TRIO;RAPGEF4;SGEF;TIAM2;RGL1;NGEF;ECT2L

TH;AKR1C4;AKR1C3

CACNB4;ID2;FOXL1;STAT5A

BAIAP2;MTSS1;GAS2L1;ANK1;NCK2

CHST4;TPST1;UST

IL6;CD276;FAM20C;DDR2;LTF;MSX2;MEF2A;CYR61;BMPR1B

WSCD1;CHST4;GAL3ST4;TPST1;UST;CHST2; TRMU;CHST3

WSCD1;CHST4;GAL3ST4;TPST1;UST;CHST2;CHST3

EP300;NFATC4;ASXL2

AKR1C4;AKR1C3

ABR;IL6;SBNO2;CD276;NLRP12;CD47;IER3;STAT5A;PDE2A;CNR1;CD28;CD59;BRD4;F12;TLR4;PTGIS;TBC1D23

ALDH3A1;AKR1C4;AKR1C3

$A B C G 5 ; A B C G 8 ; O X T$

KCNQ1;TRPV1;STAT1;SREBF1;ALDH3A1;EPB49;P2RX4;BSG;PDE2A;SLC6A4;BRAF;OXT;SLC5A5

ANXA2; F12; PLAUR

IL6;AZU1;TLR4

FCER2;NPR3; ESR $1 ; S C A R B 1$

CTSB;PRF 1 ; CTSK

IL6;NLRP12;CD47;STAT5A;PDE2A;CNR1;CD28;TLR4

IL6;CD276;STAT5A;NCK2;ICOSLG;MEF2A;BCL2;CD28;CD59;TLR4 


\begin{tabular}{|c|c|c|c|c|c|c|c|}
\hline Rank & ID & Name & Type & $\begin{array}{l}\text { Genes } \\
\text { in term }\end{array}$ & $\begin{array}{l}\text { Genes } \\
\text { in test } \\
\text { list and } \\
\text { term }\end{array}$ & $p$-value & OR \\
\hline 1 & GO:0004714 & $\begin{array}{l}\text { transmembrane receptor protein } \\
\text { tyrosine kinase activity }\end{array}$ & molecular function & 67 & 14 & $2.59 \mathrm{E}-07$ & 5.122981614 \\
\hline 2 & GO:0019199 & $\begin{array}{l}\text { transmembrane receptor protein } \\
\text { kinase activity }\end{array}$ & molecular function & 84 & 15 & $1.94 \mathrm{E}-06$ & 4.18507835 \\
\hline 3 & GO:0018108 & peptidyl-tyrosine phosphorylation & biological process & 118 & 18 & 3.32E-06 & 3.513449487 \\
\hline 4 & GO:0018212 & peptidyl-tyrosine modification & biological process & 120 & 18 & 4.83E-06 & 3.432993693 \\
\hline 5 & GO:0005925 & focal adhesion & cellular component & 123 & 18 & 1.99E-05 & 3.180778045 \\
\hline 6 & GO:0030055 & cell-substrate junction & cellular component & 134 & 19 & 2.37E-05 & 3.049805048 \\
\hline 7 & GO:0070848 & response to growth factor & biological process & 542 & 51 & 2.57E-05 & 1.965162042 \\
\hline 8 & GO:0071363 & $\begin{array}{l}\text { cellular response to growth factor } \\
\text { stimulus }\end{array}$ & biological process & 527 & 50 & $3.00 \mathrm{E}-05$ & 1.968324786 \\
\hline 9 & GO:0005924 & cell-substrate adherens junction & cellular component & 128 & 18 & $3.83 E-05$ & 3.035139208 \\
\hline 10 & GO:0016310 & phosphorylation & biological process & 753 & 61 & 3.93E-05 & 1.805512553 \\
\hline 11 & GO:0007173 & $\begin{array}{l}\text { epidermal growth factor receptor } \\
\text { signaling pathway }\end{array}$ & biological process & 190 & 22 & 4.02E-05 & 2.641330765 \\
\hline 12 & GO:0038127 & ERBB signaling pathway & biological process & 191 & 22 & 4.64E-05 & 2.619521312 \\
\hline 13 & GO:0005154 & $\begin{array}{l}\text { epidermal growth factor receptor } \\
\text { binding }\end{array}$ & molecular function & 21 & 5 & $7.29 \mathrm{E}-05$ & 7.935357156 \\
\hline 14 & GO:0031122 & $\begin{array}{l}\text { cytoplasmic microtubule } \\
\text { organization }\end{array}$ & biological process & 20 & 5 & 0.000106065 & 7.570852389 \\
\hline 15 & GO:0007167 & $\begin{array}{l}\text { enzyme linked receptor protein } \\
\text { signaling pathway }\end{array}$ & biological process & 733 & 64 & 0.000107325 & 1.748759123 \\
\hline 16 & GO:0002381 & $\begin{array}{l}\text { immunoglobulin production involved } \\
\text { in immunoglobulin mediated immune } \\
\text { response }\end{array}$ & biological process & 21 & 4 & 0.00012466 & 8.499630695 \\
\hline 17 & GO:0008093 & cytoskeletal adaptor activity & molecular function & 17 & 5 & 0.000144563 & 7.896468269 \\
\hline 18 & GO:0006468 & protein phosphorylation & biological process & 505 & 45 & 0.000156254 & 1.881144509 \\
\hline 19 & GO:0007169 & $\begin{array}{l}\text { transmembrane receptor protein } \\
\text { tyrosine kinase signaling pathway }\end{array}$ & biological process & 548 & 50 & 0.000181425 & 1.830552043 \\
\hline 20 & GO:2001222 & regulation of neuron migration & biological process & 13 & 4 & 0.00018842 & 9.508650146 \\
\hline 21 & GO:0007264 & $\begin{array}{l}\text { small GTPase mediated signal } \\
\text { transduction }\end{array}$ & biological process & 393 & 37 & 0.000225578 & 1.969856232 \\
\hline 22 & GO:0002204 & $\begin{array}{l}\text { somatic recombination of } \\
\text { immunoglobulin genes involved in } \\
\text { immune response }\end{array}$ & biological process & 16 & 3 & 0.000292117 & 10.01031101 \\
\hline 23 & GO:0002208 & $\begin{array}{c}\text { somatic diversification of } \\
\text { immunoglobulins involved in immune } \\
\text { response }\end{array}$ & biological process & 16 & 3 & 0.000292117 & 10.01031101 \\
\hline 24 & GO:0045190 & isotype switching & biological process & 16 & 3 & 0.000292117 & 10.01031101 \\
\hline 25 & GO:0060396 & $\begin{array}{l}\text { growth hormone receptor signaling } \\
\text { pathway }\end{array}$ & biological process & 24 & 5 & 0.000367005 & 6.040651405 \\
\hline
\end{tabular}


Gene names in GO term and test list

CRIM1;EPHA8;ROR1;INSR;DDR2;IGF2R;NRP2;ERBB4;EPHB3;TIE1;RYK;ERBB3;MET;PDGFRL

CRIM1;EPHA8;ROR1;INSR;DDR2;IGF2R;NRP2;ERBB4;EPHB3;BMPR1A;TIE1;RYK;ERBB3;MET;PDGFRL

CRIM1;SHC1;EPHA8;MAPK3;ROR1;INSR;DDR2;CSK;IGF2R;PXN;NRP2;ERBB4;EPHB3;RELN;TIE1;RYK;MET;PDGFRL

CRIM1;SHC1;EPHA8;MAPK3;ROR1;INSR;DDR2;CSK;IGF2R;PXN;NRP2;ERBB4;EPHB3;RELN;TIE1;RYK;MET;PDGFRL

MAPK1;LPP;MAPK3;LIMD 1;GAK;DLC1;ITGB5;IRF2;PDLIM2;ARHGAP26;PXN;SORBS3;FLNB;PARVA;ARHGEF7;TRPV4;NOX4;ADAM17

MAPK1;LPP;DST;MAPK3;LIMD1;GAK;DLC1;ITGB5;IRF2;PDLIM2;ARHGAP26;PXN;SORBS3;FLNB;PARVA;ARHGEF7;TRPV4;NOX4;ADAM17

MAPK1;ABR;MCF2L:PMEPA1;SHC1;WNT1OA;MYC;NCOR2;EIF2C2;MAPK3:PRKAR1B;VAV2;PARD3;NOTCH1;TFDP1;PRKCE;INSR;PRKCA;TRIO; SKI;AKAP13;KALRN;TRIM71;GDF15;FGF1;PDE3A;ERBB4;PIK3CD;TNRC6B;BMPR1A;TH;KLB;PLK5P;ARHGEF7;AP2A2;NDST1;RAPGEF2;LTBP2 ;NOX4;ITPR1;PCSK6;ADAM17;STAT3;MAP3K1;RELA;ERBB3;NET1;TGIF1;CDC34;PDGFRL;IBSP

MAPK1;ABR;MCF2L;PMEPA1;SHC1;WNT1OA;MYC;NCOR2;EIF2C2;MAPK3;PRKAR1B;VAV2;PARD3;NOTCH1;TFDP1;PRKCE;INSR; PRKCA;TRIO; SKI;AKAP13;KALRN;TRIM71;GDF15;FGF1;PDE3A;ERBB4;PIK3CD;TNRC6B;BMPR1A;TH;KLB;PLK5P;ARHGEF7;AP2A2;NDST1;RAPGEF2;LTBP2 ;NOX4;ITPR1;PCSK6;ADAM17;STAT3;MAP3K1;RELA;ERBB3;NET1;TGIF1;PDGFRL;IBSP

MAPK1;LPP;MAPK3;LIMD1;GAK;DLC1;ITGB5;IRF2;PDLIM2;ARHGAP26;PXN;SORBS3;FLNB;PARVA;ARHGEF7;TRPV4;NOX4;ADAM17

MAPK1;CRIM1;CTBP1;SHC1;MYC;EPHA8;MAPK3;PRKAR1B;LIMD1;DAPK2;ROR1;PFKFB3;PRKCE;INSR;MKNK2;DDR2;PRKCA;CSK;IGF2R;MAP 4K4;NDUFS6;MINK1;SPTBN1;GRK7;AKAP13;ADAM10;TSSK3;TOLLIP;KALRN;PXN;SIK3;NRP2;ERBB4;DLG2;PTPLAD1;PIK3CD;TNF;EPHB3;BMP R1A;SPHK2;ZAK;FASTK;RBM4;NDST1;MAST2;CDC42EP5;RAPGEF2;RELN;CDS1;TIE1;ULK2;NEK7;STAT3;CDC25B;MAP3K1;RYK;CCNE1;NDUF S8;MET;NDUFAF1;PDGFRL

MAPK1;SHC1;EIF2C2;MAPK3;PRKAR1B;PRKCE;PRKCA;CSK;NCK2;ADAM10;PXN;FGF1;ERBB4;PIK3CD;TNRC6B;KLB;ARHGEF7;AP2A2; ADAM12;ITPR1;ADAM17;ERBB3

MAPK1;SHC1;EIF2C2;MAPK3;PRKAR1B;PRKCE;PRKCA;CSK;NCK2;ADAM10;PXN;FGF1;ERBB4;PIK3CD;TNRC6B;KLB;ARHGEF7;AP2A2; ADAM12;ITPR1;ADAM17;ERBB3

SHC1;VAV2;ERBB4;PLSCR1;ATXN2

DST;DVL1;CHP;TRPV4;TACC2

MAPK1;ABR;MTSS1;CRIM1;MCF2L;PMEPA1;RPTOR;SHC1;MYC;NCOR2;EIF2C2;EPHA8;MAPK3;PRKAR1B;PTPRE;VAV2;PARD3;ROR1;TFDP1;P RKCE;INSR;DDR2;PLAT;PRKCA;CSK;IGF2R;TRIO;SKI;SPTBN1;AKAP13;NCK2;ADAM10;KALRN;TRIM71;PXN;GDF15;FGF1;NRP2:ERBB4;GNG7; PIK3CD;EPHB3;TNRC6B;BMPR1A;KLB;ARHGEF7;AP2A2;ADAM12;NDST1;RAPGEF2;EIF4G1;ZFP106; LTBP2;ITPR1;PCSK6;ADAM17;STAT3;MA P3K1;RELA;ERBB3;NET1;TGIF1;RHOQ;PDGFRL

\section{SWAP70;TNFSF13;AICDA;XRCC4}

\section{MTSS1;NCK2;BICD1;ARHGAP26;SORBS2}

MAPK1;CRIM1;CTBP1;SHC1;EPHA8;MAPK3;PRKAR1B;DAPK2;ROR1;PRKCE;INSR;MKNK2;DDR2;PRKCA;CSK;IGF2R;MAP4K4;MINK1;SPTBN1; GRK7;AKAP13;ADAM10;TSSK3;KALRN;PXN;SIK3;NRP2;ERBB4;PIK3CD;EPHB3;BMPR1A;ZAK;FASTK;MAST2;RELN;CDS1;TIE1;ULK2;NEK7;CD C25B;MAP3K1;RYK;CCNE1;MET;PDGFRL

MAPK1:ABR;MTSS1;CRIM1:MCF2L;RPTOR;SHC1;EIF2C2;EPHA8;MAPK3;PRKAR1B;VAV2;ROR1;PRKCE;INSR;DDR2;PLAT:PRKCA;CSK:IGF2R; TRIO;AKAP13;NCK2;ADAM10;KALRN;TRIM71;PXN;FGF1;NRP2;ERBB4;PIK3CD;EPHB3;TNRC6B;KLB;ARHGEF7;AP2A2;ADAM12;NDST1;RAPGE F2;EIF4G1;ZFP106;ITPR1;PCSK6;ADAM17;STAT3;RELA;ERBB3;NET1;RHOQ;PDGFRL

PLXNB2;RAPGEF2;RELN;STAT3

MAPK1;ARHGAP15;ABR;AGAP1;VANGL2;MCF2L;SHC1;DOCK7;MAPK3;VAV2;IQGAP2;DLC1;RASGEF1C;TRIO;AKAP13;CHP;GNA12;RASL11B;A RHGAP26;KALRN;ARHGAP23;PTPLAD1;RASGRP2;RGL1;ARL4C;ARHGEF7;DNAJC27;RAB40B;CDC42EP1;CDC42EP5;RAPGEF2;C10rf89;RAB7 $L 1 ; A R F 1 ; R H O V ; N E T 1 ; R H O Q$

SWAP70;AICDA;XRCC4

SWAP7O;AICDA;XRCC4

SWAP70;AICDA;XRCC4

MAPK 1;MAPK3;PXN;ADAM17;STAT3

ID, name, type, the number of genes

in the pathway and the top 1000 (test

list), $p$-value, odds ratio (OR) and genes

in both the test list and GO term.

Terms are ranked based on $p$-value. 


\begin{tabular}{|c|c|c|c|c|c|c|c|}
\hline Rank & ID & Name & Type & $\begin{array}{l}\text { Genes } \\
\text { in term }\end{array}$ & $\begin{array}{c}\text { Genes } \\
\text { in test } \\
\text { list and } \\
\text { term }\end{array}$ & $p$-value & OR \\
\hline 1 & GO:0042730 & fibrinolysis & biological process & 19 & 4 & $1.98 \mathrm{E}-06$ & 14.24957707 \\
\hline 2 & GO:0005938 & cell cortex & cellular component & 204 & 21 & 1.47E-05 & 2.801758255 \\
\hline 3 & GO:0051963 & regulation of synapse assembly & biological process & 35 & 7 & $3.22 \mathrm{E}-05$ & 5.955198998 \\
\hline 4 & GO:0007259 & JAK-STAT cascade & biological process & 52 & 7 & 4.30E-05 & 5.331965552 \\
\hline 5 & GO:0051015 & actin filament binding & molecular function & 81 & 11 & $5.69 \mathrm{E}-05$ & 3.776980208 \\
\hline 6 & GO:0061351 & neural precursor cell proliferation & biological process & 61 & 9 & 6.43E-05 & 4.289995618 \\
\hline 7 & GO:0032421 & stereocilium bundle & cellular component & 32 & 6 & 6.70E-05 & 6.125801749 \\
\hline 8 & GO:0008092 & cytoskeletal protein binding & molecular function & 696 & 50 & 0.000112678 & 1.820492951 \\
\hline 9 & GO:0051962 & $\begin{array}{l}\text { positive regulation of nervous } \\
\text { system development }\end{array}$ & biological process & 21 & 5 & 0.000114217 & 7.335784516 \\
\hline 10 & GO:0051965 & $\begin{array}{l}\text { positive regulation of synapse } \\
\text { assembly }\end{array}$ & biological process & 21 & 5 & 0.000114217 & 7.335784516 \\
\hline 11 & GO:0032420 & stereocilium & cellular component & 23 & 5 & 0.00011531 & 6.999602648 \\
\hline 12 & GO:0001776 & leukocyte homeostasis & biological process & 56 & 7 & 0.000119626 & 4.747572229 \\
\hline 13 & GO:0017048 & Rho GTPase binding & molecular function & 54 & 8 & 0.000137865 & 4.328550938 \\
\hline 14 & GO:0044087 & $\begin{array}{l}\text { regulation of cellular component } \\
\text { biogenesis }\end{array}$ & biological process & 393 & 31 & 0.000145058 & 2.081034444 \\
\hline 15 & GO:0003014 & renal system process & biological process & 73 & 9 & 0.000152347 & 3.874264306 \\
\hline 16 & GO:0071715 & icosanoid transport & biological process & 16 & 3 & 0.000194661 & 10.38716022 \\
\hline 17 & GO: 1901571 & fatty acid derivative transport & biological process & 16 & 3 & 0.000194661 & 10.38716022 \\
\hline 18 & GO:0015631 & tubulin binding & molecular function & 212 & 19 & 0.000200735 & 2.468580873 \\
\hline 19 & GO:0006928 & cellular component movement & biological process & 1199 & 76 & 0.000224954 & 1.601565508 \\
\hline 20 & GO:2000725 & $\begin{array}{l}\text { regulation of cardiac muscle cell } \\
\text { differentiation }\end{array}$ & biological process & 17 & 4 & 0.00025007 & 7.974004154 \\
\hline 21 & GO:0008236 & serine-type peptidase activity & molecular function & 159 & 11 & 0.000288542 & 3.031572068 \\
\hline 22 & GO:0050795 & regulation of behavior & biological process & 161 & 14 & 0.000300286 & 2.751896245 \\
\hline 23 & GO:0030866 & $\begin{array}{l}\text { cortical actin cytoskeleton } \\
\text { organization }\end{array}$ & biological process & 19 & 4 & 0.000333383 & 7.598867402 \\
\hline 24 & GO:0015629 & actin cytoskeleton & cellular component & 380 & 30 & 0.000346647 & 2.01591256 \\
\hline 25 & GO:0044089 & $\begin{array}{l}\text { positive regulation of cellular } \\
\text { component biogenesis }\end{array}$ & biological process & 24 & 5 & 0.000384951 & 6.029902321 \\
\hline
\end{tabular}


ANXA2;TMPRSS6;PLAT;KLK3

EPS8;EXOC4;CLIC5;PRKCZ;HMCN1;EPB41L2;ANXA2;ANK1;PKD1;MLPH;MYO7A;CAPZA2;NEDD9;DLG4;FMNL1;MYO10;FGF1;STIM1;AKAP12; CORO1A;SEPT14

CHRNB2;PVRL 1;NRXN2;NRXN3;ACCN1;EPHB1;OXT

PKD1;F2R;STAT3;LYN;CLCF1;PIAS1;PAR1

MYO1C;CYFIP1;MYO7A;MACF1;ADD2;FMNL1;MYO10;CORO2B;CORO1A;HTT;MAP1S

WNT3A;PCNT;FZD6;NCOR2;DAGLB;FGFR1;HMGA2;EPHB1;SIP1

EPS8;MYO1C;CLIC5;CDH23;MYO7A;TMC2

BAIAP2;EPS8;KIF26B;GAS2L1;MYO1C;SGIP1;CYFIP1;EPB41L2; TBCD;ANXA2;ENAH;CCDC64;ANK1; WIPF3;ARL8B;MLPH;MYO7A;CAPZA2;CAP N2;MACF1;KIF17;TNNI3K;CCDC88A;STMN1;SDC3;TPM1;SHROOM3;PALLD;KIAA1543;CACNA1C;ADD2;PEX14;FMNL1;KIF1B;VCL;MYO10;FRM D5;CORO2B;STIM1;CORO1A;MYLIP;KIF21B;HTT;FXYD5;TNS1;MAP1S;KLHL3;VPS41;CAV3;CAPN3

\section{NRXN2;NRXN3;ACCN1;EPHB1;OXT}

NRXN2;NRXN3;ACCN1;EPHB1;OXT

EPS8;MYO1C;CLIC5;CDH23;MYOTA

SPNS2; RIPK3:CORO1A;LYN;NKX2-3;JAM3;SKIL

EPS8;CYFIP1;CDC42EP4;CIT;AKAP13;FMNL1;CDC42BPB;DOCK9

FAM38A;EPS8;MYO1C;DNM1L;PRKCZ;TBCD;CHRNB2;ARHGEF10L;CDC42EP4;CAPZA2;MACF1;SMAD3;STMN1;RAF1;PVRL1;TPM1;NRXN2;N RXN3;ACCN1;ADD2;PEX14;MYO10;RHOQ;CORO1A;EPHB1;OXT;TACR1;NOX4;HYAL1;CAV3;CNOT6

MYO1C;CHRNB2;PTGER3;CACNA1C;F2R;OXT;TACR1;KLHL3;PAR1

ABCC2;SLCO2B1;PLA2G5

ABCC2;SLCO2B1;PLA2G5

KIF26B;GAS2L 1;SGIP1;TBCD;ARL8B;MLPH;MACF1;KIF17;CCDC88A;STMN1;KIAA1543:PEX14;KIF1B;STIM1;KIF21B:HTT:MAP1S;VPS41;CAV3

TNFSF12;EPS8;KIF26B;UNC5A;FOXP1;MYO1C;SLC7A5;WNT3A;SNX29;PCNT;PRKCZ;SPG7;SPNS2;ENAH;ANK1;NR4A2;ANKS1A;LIMD1;EFNB2; MATN2;SEMA6A;MYO7A;CAPZA2;VCAN;PDE4D;MACF1;SLC7A11;KIF17;SKI;FGFR1;TNS3;CCDC88A;KCNQ3;PLAT;RAF1;PVRL1;TPM1; KRAS; SCN3B;BOC;DLG4;NRXN3;B3GNT6;SEMA3C;CACNA1C;PEX14;MDGA1;FMNL1;KIF1B;PIK3CA;VCL;MYO10; FAT1;CDC42BPB;CORO1A; MYLIP;AP2 A2;STAT3;EPHB1;NFASC;PLXNC1;KIF21B;HTT;LYN;SLC16A3;SIP1;FN1;NKX2-3;ST8SIA2;JAM3;TNS1;MAP1S;MET;CNTNAP1; KIAA0319;BH

FOXP1;WNT3A;EFNB2;CAV3

DPP10;TMPRSS6;IMMP2L;PLAT;RHBDF2;PRSS22;MBTPS1;KLK10;CTSA;TMPRSS11E;KLK3

STRA6;WNT3A; SGIP1;CAMK1D;CHRNB2;EFNB2;PKD1;PRKD2;SMAD3;DSCAM;DLG4;LYN;JAM3;MET

EPB41L2;DLG4;FMNL1;RHOQ

BAIAP2;GAS2L1;MYO1C;CLIC5;HDAC4;SH3PXD2A;EPB41L2;DDX58;ENAH;CDC42EP4;MLPH;MYO7A;CAPZA2;MYOM2;MACF1;TPM1;PALLD;D LG4;CIT;MRPL38;ADD2; VCL;MYO10;CORO2B;RHOQ;CDC42BPB;CORO1A;DENND2A;SLC16A3;HIST4H4

NRXN2;NRXN3;ACCN1;EPHB1;OXT 


\begin{tabular}{|c|c|c|c|c|c|c|c|}
\hline & & \multicolumn{3}{|c|}{ Entorhinal cortex } & \multicolumn{3}{|c|}{ Cerebellum } \\
\hline \multicolumn{2}{|c|}{ Demographics } & UC & $5-\mathrm{mC}$ & 5-hmC & UC & $5-\mathrm{mC}$ & 5-hmC \\
\hline \multicolumn{2}{|c|}{$\mathrm{N}$} & 91 & 85 & 85 & 95 & 94 & 94 \\
\hline \multicolumn{2}{|c|}{$\begin{array}{c}\text { Gender }(\%) \mathrm{M} / \\
\mathrm{F}(\%)\end{array}$} & $\begin{array}{c}(56.0) 51 / 40 \\
(44.0)\end{array}$ & $\begin{array}{c}(56.5) 48 / 37 \\
(43.5)\end{array}$ & $\begin{array}{c}(56.5) 48 / 37 \\
(43.5)\end{array}$ & $\begin{array}{c}(56.8) 54 / 41 \\
(43.2)\end{array}$ & $\begin{array}{c}(57.4) 54 / 40 \\
(42.6)\end{array}$ & $\begin{array}{c}(57.4) 54 / 40 \\
(42.6)\end{array}$ \\
\hline \multicolumn{2}{|c|}{ Age (mean $\pm S D$ ) } & $81.2 \pm 9.5$ & $81.3 \pm 9.5$ & $81.2 \pm 9.5$ & $81.2 \pm 9.3$ & $81.2 \pm 9.3$ & $81.2 \pm 9.3$ \\
\hline \multicolumn{2}{|c|}{$\begin{array}{l}\text { Diagnosis }(\%) \\
\text { AD / control }(\%)\end{array}$} & $\begin{array}{c}(70.3) 64 / 27 \\
(29.7)\end{array}$ & $\begin{array}{c}(70.6) 60 / 25 \\
(29.4)\end{array}$ & $\begin{array}{c}(70.6) 60 / 25 \\
(29.4)\end{array}$ & $\begin{array}{c}(70.5) 67 / 28 \\
(29.5)\end{array}$ & $\begin{array}{c}(70.2) 66 / 28 \\
(29.8)\end{array}$ & $\begin{array}{c}(70.2) 66 / 28 \\
(29.8)\end{array}$ \\
\hline \multirow{7}{*}{ 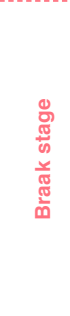 } & 0 & 8 & 7 & 7 & 8 & 8 & 8 \\
\hline & I & 3 & 3 & 3 & 3 & 3 & 3 \\
\hline & II & 11 & 10 & 10 & 12 & 12 & 12 \\
\hline & III & 6 & 6 & 6 & 6 & 6 & 6 \\
\hline & IV & 8 & 7 & 7 & 10 & 10 & 10 \\
\hline & V & 18 & 17 & 17 & 19 & 18 & 18 \\
\hline & VI & 37 & 35 & 35 & 37 & 37 & 37 \\
\hline \multicolumn{2}{|c|}{$\begin{array}{l}\text { Postmortem } \\
\text { interval (mean } \\
\quad \pm S D \text { ) }\end{array}$} & $\begin{array}{l}2539.5 \\
(1288.1)\end{array}$ & $\begin{array}{l}2490.7 \\
(1288.5)\end{array}$ & $\begin{array}{c}2490.7 \\
(1288.5)\end{array}$ & $\begin{array}{l}2576.5 \\
(1315.2)\end{array}$ & $\begin{array}{l}2581.6 \\
(1321.3)\end{array}$ & $\begin{array}{l}2581.6 \\
(1321.3)\end{array}$ \\
\hline
\end{tabular}

Entorhinal cortex and cerebellum samples from the MRC London Neurodegenerative Disease Brain Bank (London, UK). Displayed is the number of samples for each brain region and unmodified cytosine (UC), 5 -methylcytosine $(5-\mathrm{mC})$, and 5-hydroxymethylcytosine $(5-\mathrm{hmC})$ dataset, and the distributions of gender, age, Alzheimer's disease (AD) diagnosis, Braak stage, and postmortem interval (minutes). SD, standard deviation. 


\begin{tabular}{|c|c|c|c|c|c|c|c|c|}
\hline & & & \multicolumn{2}{|c|}{ UC } & \multicolumn{2}{|c|}{$5-\mathrm{mC}$} & \multicolumn{2}{|c|}{ 5-hmC } \\
\hline & & & ECest & CERest & ECest & CERest & ECest & CERest \\
\hline ANK1 & & Pearson correlation & $0.490^{\star *}$ & 0.123 & & & & \\
\hline & UC & $p$-value & $\begin{array}{c}2.682 \mathrm{E}- \\
07\end{array}$ & 0.227 & & & & \\
\hline & & \# probes & 99 & 99 & & & & \\
\hline C10orf116 & & Pearson correlation & & & 0.259 & -0.172 & & \\
\hline & $5-m C$ & $p$-value & & & 0.393 & 0.574 & & \\
\hline & & \# probes & & & 13 & 13 & & \\
\hline C3 & & Pearson correlation & -0.379 & 0.209 & & & & \\
\hline & UC & $p$-value & 0.068 & 0.328 & & & & \\
\hline & & \# probes & 24 & 24 & & & & \\
\hline$C D \times 1$ & & Pearson correlation & -0.269 & -0.330 & & & & \\
\hline & UC & $p$-value & 0.331 & 0.229 & & & & \\
\hline & & \# probes & 15 & 15 & & & & \\
\hline FAM198B & & Pearson correlation & & & $-0.653^{\star}$ & 0.293 & & \\
\hline & $5-m c$ & $p$-value & & & 0.029 & 0.381 & & \\
\hline & & \# probes & & & 11 & 11 & & \\
\hline LOC100190940 & & Pearson correlation & -0.226 & 0.432 & & & & \\
\hline & UC & $p$-value & 0.667 & 0.393 & & & & \\
\hline & & \# probes & 6 & 6 & & & & \\
\hline$O X T$ & & Pearson correlation & & & -0.259 & -0.346 & -0.159 & -0.169 \\
\hline & $\begin{array}{l}5-\mathrm{mC} \& \\
5-\mathrm{hmC}\end{array}$ & $p$-value & & & 0.470 & 0.327 & 0.707 & 0.689 \\
\hline & & \# probes & & & 10 & 10 & 8 & 8 \\
\hline RAP 1GAP2 & & Pearson correlation & & & $-0.278^{*}$ & 0.169 & & \\
\hline & $5-m C$ & $p$-value & & & 0.044 & 0.226 & & \\
\hline & & \# probes & & & 53 & 53 & & \\
\hline RHBDF2 & & Pearson correlation & $0.759^{* \star}$ & -0.215 & $0.662^{* \star}$ & -0.062 & & \\
\hline & $\begin{array}{l}\text { UC \& } \\
5-m C\end{array}$ & $p$-value & $\begin{array}{c}1.778 \mathrm{E}- \\
06\end{array}$ & 0.262 & $\begin{array}{c}9.119 \mathrm{E}- \\
05\end{array}$ & 0.748 & & \\
\hline & & \# probes & 29 & 29 & 29 & 29 & & \\
\hline SLC12A4 & & Pearson correlation & & & & & $0.384^{*}$ & -0.054 \\
\hline & 5-hmC & $p$-value & & & & & 0.048 & 0.788 \\
\hline & & \# probes & & & & & 27 & 27 \\
\hline TNK2 & & Pearson correlation & 0.249 & 0.141 & & & & \\
\hline & UC & $p$-value & 0.067 & 0.306 & & & & \\
\hline & & \# probes & 55 & 55 & & & & \\
\hline
\end{tabular}

\section{SUPPLEMENTARY TABLE 15. Cross-re-}

gional validation results.

Pearson correlations for middle

temporal gyrus (MTG) regression co-

efficients of probes in the 11 genes with

a differentially methylated region

(DMR), differentially hydroxymeth-

ylated region (DHR) and/or differen-

tially unmodified region (DUR) genes

and the regression coefficients of

the same probes from the entorhi-

nal cortex (ECest) and cerebellum

(CERest). Displayed for each gene is

the data (5-methylcytosine [5-mC],

5-hydroxymethylcytosine [5-hmC] or unmodified cytosine [UC]) in which a differentially modified region was found in the current study (indicated in bold), the corresponding Pearson correlation with the EC and CER data, the $p$-value and number of probes in the comparison. ${ }^{*} p<0.05 ;{ }^{* *} p<0.001$. 

DETECTION OF

BLOOD METHYLOMIC

MARKERS ASSOCIATED

WITH CLINICAL

FEATURES OF ALZHEI-

MER'S DISEASE;

AN EXPLORATORY

APPROACH

ROY LARDENOIJEA ${ }^{A}$ EHSAN PISHVA ${ }^{A, B}$, HEIDI JACOBS ${ }^{A}$, INEZ RAMAKERS $^{A}$, PIETER J. VISSER ${ }^{A}$, HARRY W. M. STEINBUSCH ${ }^{A}$, DANIËL L.A. VAN DEN HOVE ${ }^{A, C}$, BART P.F. RUTTEN ${ }^{A}$

ASCHOOL FOR MENTAL HEALTH AND NEUROSCIENCE (MHENS), DEPARTMENT OF PSYCHIATRY AND NEUROPSYCHOLOGY, MAASTRICHT UNIVERSITY, UNIVERSITEITSSINGEL 50, 6200 MD MAASTRICHT, THE NETHERLANDS

BUNIVERSITY OF EXETER MEDICAL SCHOOL, UNIVERSITY OF EXETER, EXETER, UK.

'LABORATORY OF TRANSLATIONAL NEUROSCIENCE, DEPARTMENT OF PSYCHIATRY, PSYCHOSOMATICS AND PSYCHOTHERAPY, UNIVERSITY OF WUERZBURG, FUECHSLEINSTRASSE 15, 97080 WUERZBURG, GERMANY 


\section{Abstract}

Alzheimer's disease (AD), the foremost cause of dementia in the elderly, is associated with an epigenetic disarray. Most previous studies have focused on the brain, which is the primary site of pathology, but has strictly limited accessibility for diagnostic purposes. The present exploratory study therefore assessed the blood methylome associated with the diagnosis of $A D$, as well as with $A D$-related phenotypes, i.e. the mini-mental state examination (MMSE) score, cerebrospinal fluid levels of amyloid- $\beta$ and phosphorylated tau, and hippocampal volume. The findings corroborate an epigenetic component in $A D$ and find the previously reported altered DNA methylation in the AD brain of $H L A-D R B 5$ to be reflected in the blood. Additional promising candidate genes that may be investigated as epigenetic blood biomarkers of $A D$ dementia are put forward, including PCDHA1, CDH13, CLSTN2, NEFL, and MAD1L1.

\section{KEYWORDS}

Alzheimer's disease; epigenetics; DNA methylation; cognition; hippocampal volume; cerebrospinal fluid 


\subsection{Introduction}

Alzheimer's disease (AD) is the primary cause of dementia and it is associated with the progressive development of protein aggregates in the brain; the extracellular amyloid plaques and the intracellular neurofibrillary tangles [1, 2]. The main constituents of these protein aggregates are amyloid- $\beta(A \beta)$ and tau, respectively. $A \beta$ and tau aggregates have been the center of focus of $A D$ research for years, especially $A \beta$ 1-42 (A 342$)$ and phosphorylated tau (ptau), generally thought to be the most toxic forms [2]. However, it remains unclear exactly how these proteins drive the progressive neurodegeneration and subsequent cognitive decline seen in AD [3]. Recent years have seen a great expansion of research on biological processes that are affected in $A D$, including the immune system [4], fat and glucose homeostasis [5, 6], and protein quality control [7]. At the center, connecting all these systems, acting as a molecular mediator or inciter, resides the epigenetic apparatus [8, 9].

Indeed, recent studies have uncovered widespread epigenetic alterations in association with $A D$, including DNA and chromatin modifications as well as non-coding RNAs [8]. Especially DNA and chromatin modifications have strong intrinsic relationships with each other [10], while the stability of DNA modifications has made them a prime target for a first wave epigenetic inquiries in postmortem tissue [11]. Understandably, most of these studies focus on the brain as the primary site of analysis. However, this limits their use for the identification of biomarkers assessible in living patients. Although the epigenome has a high variability between tissues [12], exploring the AD methylomic profile of more assessible tissues, like blood, may be more fruitful for diagnostic and prognostic purposes. Being a stable marker and with many tools available for its investigation, the methylome represents a logical first target [11].

A definite diagnosis of $A D$ depends on the postmortem identification of plaques and tangles in the brain [13]. Several diagnostic markers can be assessed in living patients and allow for the diagnosis dementia of the Alzheimer type with a certain level of certainty. Among these are levels of ptau and $A \beta 42$ in cerebrospinal fluid (CSF) [14], hippocampal atrophy [15], and measures of cognitive performance, such as the mini-mental state examination (MMSE) $[16,17]$. These markers have furthermore been shown to predict development of cognitive impairments and conversion to dementia [18].

In order to explore the feasibility of performing genome-wide analyses of DNA methylation profiles using blood samples in relation to $A D$ and $A D-$ related phenotypes, the present study examined the blood methylomic profile associated with the clinical diagnosis of $A D$, and explored within 
this profile, which genes present with altered DNA methylation levels in relation to $A D$-related cognitive, CSF, and imaging phenotypes.

\subsection{Methods \\ 8.2.1. Subjects}

Blood was collected from 6 AD patients, 11 subjects with mild cognitive impairment $(\mathrm{MCl})$, and 8 healthy controls from the EDAR cohort (Early Diagnosis of $A D$ and as marker for treatment Response, http://www. edarstudy.eu/). These subjects were selected based on the availability of blood and CSF samples and availability of magnetic resonance imaging (MRI) data, and on an equal distribution of gender. MMSE scores were also obtained for all subjects [16], CSF from most cases, but not all controls (3), and MRI scans (3 Tesla, Philips, Amsterdam, the Netherlands) only from $\mathrm{MCl}$ and $\mathrm{AD}$ subjects (Table 1). All procedures were approved by the Medical Ethics Committee of Maastricht University Medical Centre, in accordance with the declaration of Helsinki.

\subsubsection{CSF tau and amyloid beta}

CSF was obtained through a lumbar puncture and collected in $10 \mathrm{~mL}$ polypropylene tubes. Tubes were centrifuged at $2000 \mathrm{~g}$ at $4^{\circ} \mathrm{C}$, and stored within one hour after collection at $-80^{\circ} \mathrm{C}$. Ptau and $A \beta 42$ levels were determined in the CSF samples using the INNO-BIA AIzBio3 Luminex assay (Fujirebio, Gent, Belgium). The ptau:A $\beta 42$ ratio was calculated and used for subsequent analyses, as this was previously found to be a good predictor of dementia [19].

\subsubsection{Hippocampal volume}

Left and right hippocampal volumes, as well as the total intracranial volume, were determined from T1-weighted MRI scans using FreeSurfer 5.3 software [20]. Analyses were performed on the mean of the left and right hippocampal volumes, expressed as percentage of the total intracranial volume. 


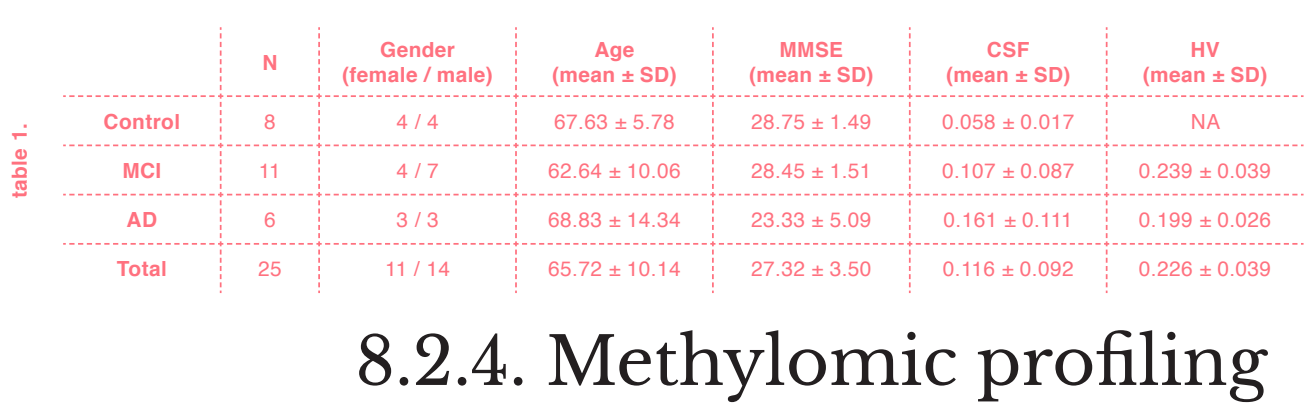

Whole blood was used for genomic DNA extraction with the AutoGen Flex Star DNA isolation system (Autogen, Holliston, MA, USA), following the manufacturer's protocol. $500 \mathrm{ng}$ genomic DNA was bisulfite (BS) treated with the EZ-96 DNA Methylation-Gold ${ }^{\text {TM }}$ Kit (Zymo Research, Irvine, CA, USA). To limit possible bias due to variable conversion efficiency, the BS conversion reaction was performed twice for each sample, after which the duplicates were pooled. Genome-wide DNA methylation levels were determined with Illumina's HumanMethylation450 BeadChip (Illumina, San Diego, CA, USA). The BS-treated DNA was amplified, fragmented, and hybridized according to the instructions of the manufacturer. The Illumina iScan was used for reading the chip.

\subsubsection{Processing of array data}

All data processing and analysis was done with the statistical programming environment R (version 3.3.2) [21] and RStudio (version 1.0.136) [22], running on a 64-bit Windows 10 pro (Microsoft, Redmond, WA, USA) machine. Raw IDAT data from the scanner was inspected for outlying samples with the MethylAid package (version 1.8.0) [23], using the default settings, and loaded into the R environment for further analysis with the minfi package (version 1.20.2) [24]. Background and dye bias correction was performed according to the noob method [25], followed by functional normalization [26], as implemented in the DNAmArray package (version 0.0 .1 ) [27]. Probes with a mean of exactly 0 , or with intensity measurements from less than 3 beads, were removed. Additionally, probes with a detection $p$-value above 0.01 (as compared to background control probes) were removed, as well as probes with a success rate below 95\%. After filtering, samples with more than $5 \%$ of their probes removed were excluded. As a simple check for sample mix-ups, the gender of the samples was determined based on $\mathrm{X}$ chromosome methylation (using the DNAmArray package), and compared with the assumed gender. Cross-hybridizing probes and probes binding polymorphic sites, as identified by Chen et al. [28], were also removed, as well as probes on the $X$ and $Y$ chromosomes. 


\subsubsection{Data analysis}

M-values were used for all analyses [29]. Data was analyzed following a two-step approach; first, probes associated with AD status were selected, followed by three separate analyses looking at the association between the AD-related probes and clinical features of $A D$, as captured by MMSE scores, CSF ptau:A 442 and hippocampal volume. Associations were tested using linear models, as implemented in the limma package (version 3.30.11) [30], using AD diagnosis, MMSE scores, CSF ptau:A 342 or hippocampal volume as predictors, and normalized M-values as outcome. Potential confounding sources of variation were investigated using the sva package [31] and were captured in surrogate variables to be included in the limma model. After fitting the model, empirical Bayes moderated test statistics were determined [32]. Bias and inflation corrected $p$-values were then calculated using the empirical null distribution, as implemented in the bacon package (version 1.2.0) [33]. All probes passing the probe filtering steps were included in the AD diagnosis-based analysis, and the probes from that analysis with a bias and inflation corrected $p$-values < 0.05 were used as input for the analyses based on MMSE scores, CSF ptau:Aß42 and hippocampal volume. Differentially methylated positions (DMPs) were identified as probes with a Benjamini-Hochberg false discovery rate (FDR) corrected $p$-value $<0.05$. Probes were annotated to genes based on proximity, using the UCSC human genome build 19 , with the DNAmArray package. In addition to DMPs, differentially methylated regions (DMRs) were determined with comb-p [34], using a seeding $p$-value of 0.05 and an extension distance of 200 base pairs. The software corrects for multiple testing according to the Šidák procedure. Comb-p analyses were done in a 64-bit virtual Kali Linux 2016.2 distribution (Offensive Security Ltd.), running in VirtualBox 5.1.18 (Oracle Corporation, Redwood City, CA, USA). DMRs were identified as regions with a corrected $p$-value below 0.05 and containing at least two probes. The presence of the AD-associated DMPs and DMRs was also investigated in the $\mathrm{MCl}$ cases, using the same approach, but using $\mathrm{MCl}$ status as predictor instead of AD.

For a subsequent Gene Ontology (GO) and Kyoto Encyclopedia of Genes and Genomes (KEGG) gene set analysis, probes were ordered based on a combined $p$-value and effect size ranking (i.e. from lowest $p$-value and highest effect size to highest $p$-value and lowest effect size). Unique gene names annotated to the top 1000 probes were used as test list and the unique gene names annotated to the complete set of probes included in the limma analysis were used as background list. The missMethyl $\mathrm{R}$ package (version 1.8.0) was used for the GO term (using the 'gometh' function) and KEGG pathway (using the 'gsameth' function) enrichment analysis, as it takes into account there are varying numbers of probes 
per gene on the array, which may lead to bias [35, 36]. Only GO terms containing between 10 and 2000 genes, and KEGG pathways with more than 10 genes (the maximum number of genes in a KEGG pathway is 1272) were considered in the gene set enrichment analysis.

\subsection{Results}

All samples passed the quality control steps and for all samples the assumed gender matched the predicted gender. See Supplementary Figure 1 for a density plot of the raw beta values. 3 principal components were used for functional normalization (see Supplementary Figure 2 for a screeplot and Supplementary Figure 3 for a density plot of the normalized beta values). After removal of cross-reacting and polymorphic site-associated probes, probes on the $\mathrm{X}$ and $\mathrm{Y}$ chromosomes, and probes not passing the performance thresholds, 396,333 probes remained. There were no global DNA methylation level differences in de $\mathrm{MCl}$ and $\mathrm{AD}$ groups when compared to the controls (controls $=48.75 \% ; \mathrm{MCl}=$ $48.66 \%, p=5.970 \mathrm{E}-01 ; \mathrm{AD}=48.61 \%, p=4.640 \mathrm{E}-01)$.

\subsubsection{Alzheimer's disease}

Using processed $\mathrm{M}$-values from the control and $\mathrm{AD}$ subjects, a surrogate variable analysis indicated the presence of 5 significant surrogate variables, which were included in the limma analysis. When applying the Gibbs sampler algorithm of the bacon package to the coefficients and standard errors from the limma analysis, a bias of -0.098 was detected and an inflation of 1.1 (Supplementary Figure 4). After FDR correction, 171 DMPs were detected $\left(p_{F D R}<0.05\right.$; Table 2; Supplementary Figure 5 ), some of which were also associated with $\mathrm{MCl}$ (Table 3). Comb-p detected a total 12 DMRs that survived the Šidák $p$-value adjustment and contained more than 1 probe (Table 4; Supplementary Figure 5). The

DMR in PRRT2 was also associated with $\mathrm{MCl}$ ( $\left.p_{\text {šidák }}=3.21 \mathrm{E}-04\right) .896$ unique genes were annotated to the top 1000 probes with the highest effect sizes and lowest $p$-values (all $p<0.05$ ). None of the terms or pathways remained statistically significantly enriched after FDR correction for the GO and KEGG gene set enrichment analysis (Supplementary Table 1 and Supplementary Table 2, respectively). There were 25,186 probes with a $p$-value below 0.05 , which were used for the subsequent MMSE, CSF marker, and hippocampal volume association analyses. 


\begin{tabular}{|c|c|c|c|c|c|c|}
\hline Probe & Gene & Region & ES & SE & $P$ & FDR \\
\hline $\operatorname{cg} 22508626$ & HLA-DRB5 & Body & -0.86 & 0.00 & 2.03E-16 & $8.03 \mathrm{E}-11$ \\
\hline cg19611616 & STK38L & 5'UTR & 5.62 & 1.00 & $1.68 \mathrm{E}-14$ & 3.32E-09 \\
\hline cg01963573 & BEND3 & 3'UTR & 0.80 & 0.00 & $1.18 \mathrm{E}-10$ & $1.35 \mathrm{E}-05$ \\
\hline cg08435945 & CERS6 & Body & 0.58 & 0.00 & 1.36E-10 & 1.35E-05 \\
\hline $\operatorname{cg} 13876921$ & LOC390705 & & 1.91 & 0.00 & $3.38 \mathrm{E}-10$ & $2.68 \mathrm{E}-05$ \\
\hline cg21926612 & PACRG & $\begin{array}{l}\text { 5'UTR; } \\
1^{\text {st }} \text { Exon; } \\
\text { TSS1500 }\end{array}$ & 1.12 & 0.00 & $6.87 \mathrm{E}-10$ & 4.54E-05 \\
\hline cg15068079 & BTNL9 & Body & 1.25 & 0.00 & $1.31 \mathrm{E}-09$ & 7.43E-05 \\
\hline cg16502980 & NEDD $4 L$ & TSS 1500 & -2.35 & 0.00 & $9.57 \mathrm{E}-09$ & 4.74E-04 \\
\hline cg13047596 & C2orf82 & $\begin{array}{c}\text { 5'UTR; } 1^{\text {st }} \\
\text { Exon }\end{array}$ & 0.74 & 0.00 & $1.46 \mathrm{E}-08$ & $6.43 \mathrm{E}-04$ \\
\hline cg23192683 & LINC00884 & & 1.01 & 0.00 & $1.93 \mathrm{E}-08$ & 7.01E-04 \\
\hline ch.6.8638596F & LOC100506207 & & -0.57 & 0.00 & $1.95 \mathrm{E}-08$ & 7.01E-04 \\
\hline cg23919678 & TIAM2 & Body & 0.58 & 0.00 & 4.34E-08 & 1.43E-03 \\
\hline cg17712928 & MAD1L1 & Body & 0.78 & 0.00 & $5.01 \mathrm{E}-08$ & $1.53 \mathrm{E}-03$ \\
\hline cg09868354 & $C D K 1$ & & 0.55 & 0.00 & 7.15E-08 & 1.92E-03 \\
\hline cg08698856 & RAB2A & Body & 0.56 & 0.00 & 7.26E-08 & 1.92E-03 \\
\hline cg14903685 & TLE3 & TSS 1500 & -0.92 & 0.00 & 8.37E-08 & 2.07E-03 \\
\hline cg11021661 & B3GNT6 & 3'UTR & 0.68 & 0.00 & $9.45 \mathrm{E}-08$ & 2.20E-03 \\
\hline cg03130180 & GPX6 & TSS200 & -0.88 & 0.00 & 1.29E-07 & $2.75 \mathrm{E}-03$ \\
\hline cg18052984 & ATF1 & 5'UTR & -0.59 & 0.00 & 1.44E-07 & 2.75E-03 \\
\hline $\operatorname{cg} 16554516$ & RASSF5 & TSS200 & -0.92 & 0.00 & 1.49E-07 & $2.75 \mathrm{E}-03$ \\
\hline cg07687332 & GPR63 & TSS 1500 & -0.75 & 0.00 & $1.50 \mathrm{E}-07$ & $2.75 \mathrm{E}-03$ \\
\hline cg03352657 & POLDIP2 & $\begin{array}{c}1^{\text {st }} \text { Exon; } \\
\text { 5'UTR; } \\
\text { TSS200 }\end{array}$ & -0.51 & 0.10 & $1.59 \mathrm{E}-07$ & $2.75 \mathrm{E}-03$ \\
\hline cg04270085 & MIR4262 & & 0.41 & 0.08 & $1.60 \mathrm{E}-07$ & 2.75E-03 \\
\hline cg02611675 & IGLL1 & & -0.43 & 0.08 & $2.51 \mathrm{E}-07$ & 4.04E-03 \\
\hline cg20054157 & SFTA1P & TSS1500 & 0.71 & 0.14 & $2.55 \mathrm{E}-07$ & 4.04E-03 \\
\hline cg17548431 & C4orf 45 & & -0.66 & 0.13 & 2.80E-07 & 4.11E-03 \\
\hline cg13421247 & CDK2AP1 & TSS1500 & 0.72 & 0.14 & 2.92E-07 & 4.11E-03 \\
\hline cg03361810 & AP $3 M 2$ & TSS 1500 & -0.54 & 0.10 & 2.92E-07 & 4.11E-03 \\
\hline cg09238162 & CREB3L2 & Body & -0.41 & 0.08 & $3.01 \mathrm{E}-07$ & 4.11E-03 \\
\hline cg24513433 & LIPG & TSS 200 & -0.83 & 0.16 & 3.30E-07 & 4.32E-03 \\
\hline cg12833168 & FBXO33 & & 0.66 & 0.13 & $3.41 \mathrm{E}-07$ & 4.32E-03 \\
\hline cg03999372 & $\operatorname{IRX} 3$ & TSS1500 & 1.03 & 0.20 & $3.49 \mathrm{E}-07$ & 4.32E-03 \\
\hline cg13406003 & RSPO3 & & 0.59 & 0.12 & 3.84E-07 & $4.58 \mathrm{E}-03$ \\
\hline cg22273042 & TPD52 & $\begin{array}{l}\text { 5'UTR; } 1^{\text {st }} \\
\text { Exon }\end{array}$ & -0.64 & 0.13 & $3.95 \mathrm{E}-07$ & $4.58 \mathrm{E}-03$ \\
\hline cg08639523 & HIST3H2A & $\begin{array}{l}\text { TSS1500; } \\
1^{\text {st }} \text { Exon; } \\
3^{\prime} \text { UTR }\end{array}$ & -0.56 & 0.11 & 4.21E-07 & $4.58 \mathrm{E}-03$ \\
\hline cg07638589 & CBFA2T3 & & 0.61 & 0.12 & 4.26E-07 & 4.58E-03 \\
\hline cg27657525 & URI1 & TSS 1500 & -0.69 & 0.14 & 4.38E-07 & 4.58E-03 \\
\hline cg12518535 & ANKRD20A19P & & -0.69 & 0.14 & 4.39E-07 & 4.58E-03 \\
\hline cg01922613 & $A B C A 4$ & Body & 0.58 & 0.11 & 4.90E-07 & 4.98E-03 \\
\hline cg11228480 & FBRSL1 & TSS1500 & -0.66 & 0.13 & $5.45 \mathrm{E}-07$ & $5.40 \mathrm{E}-03$ \\
\hline cg25533943 & MAP $3 K 14-A S 1$ & Body & -0.68 & 0.14 & 5.66E-07 & 5.47E-03 \\
\hline cg08524474 & ARHGAP22 & TSS 200 & -0.41 & 0.08 & $6.19 \mathrm{E}-07$ & $5.84 \mathrm{E}-03$ \\
\hline cg20546782 & TRPV1 & 5'UTR & 0.78 & 0.16 & $6.92 \mathrm{E}-07$ & $6.35 \mathrm{E}-03$ \\
\hline cg04571941 & TNRC18 & TSS200 & -0.50 & 0.10 & 7.19E-07 & $6.35 \mathrm{E}-03$ \\
\hline cg00546448 & SNAI3-AS1 & $\begin{array}{l}\text { Body; } \\
\text { 3'UTR }\end{array}$ & 0.67 & 0.13 & 7.26E-07 & $6.35 \mathrm{E}-03$ \\
\hline cg09306214 & TACR1 & TSS 1500 & -0.70 & 0.14 & $7.41 \mathrm{E}-07$ & 6.35E-03 \\
\hline cg17504306 & PHLDA2 & TSS 1500 & -0.56 & 0.11 & 7.53E-07 & $6.35 \mathrm{E}-03$ \\
\hline cg17720554 & SMOC2 & & 0.98 & 0.20 & 8.39E-07 & $6.93 \mathrm{E}-03$ \\
\hline
\end{tabular}

TABLE 2. DMPs from the Alzheimer's disease methylome-wide association analysis.

ABBREVIATIONS: DMPs, differentially methylated positions; ES, effect size; FDR, false discovery rate adjusted $p$-values; SE, standard error; TSS, transcription start site; UTR, untranslated region. 


\begin{tabular}{|c|c|c|c|c|c|c|}
\hline Probe & Gene & Region & ES & SE & $P$ & FDR \\
\hline cg11228717 & DERA & TSS1500 & 0.94 & 0.19 & $9.99 \mathrm{E}-07$ & 8.08E-03 \\
\hline $\operatorname{cg} 02131513$ & UBE2I & TSS1500 & -0.72 & 0.15 & 1.16E-06 & $9.23 \mathrm{E}-03$ \\
\hline cg26817121 & GPX6 & TSS1500 & 0.43 & 0.09 & 1.25E-06 & 9.70E-03 \\
\hline cg11164618 & RHBDF2 & 5'UTR & 0.58 & 0.12 & $1.63 \mathrm{E}-06$ & $1.25 \mathrm{E}-02$ \\
\hline cg03524116 & PREP & & -0.37 & 0.08 & $1.71 \mathrm{E}-06$ & $1.28 \mathrm{E}-02$ \\
\hline cg12030690 & SNCA & $\begin{array}{l}\text { TSS1500; } \\
\text { 5'UTR }\end{array}$ & 0.64 & 0.14 & 1.85E-06 & $1.34 \mathrm{E}-02$ \\
\hline cg16323293 & PACRG & Body & -0.41 & 0.09 & $1.89 \mathrm{E}-06$ & $1.34 \mathrm{E}-02$ \\
\hline cg05577437 & DLGAP2 & Body & 0.57 & 0.12 & $1.90 \mathrm{E}-06$ & 1.34E-02 \\
\hline cg07244268 & SLITRK3 & & 0.64 & 0.14 & $2.05 \mathrm{E}-06$ & 1.43E-02 \\
\hline cg01220469 & RNF26 & TSS1500 & 0.54 & 0.11 & 2.13E-06 & 1.45E-02 \\
\hline cg13688765 & GALP & TSS1500 & -0.84 & 0.18 & $2.16 \mathrm{E}-06$ & $1.45 \mathrm{E}-02$ \\
\hline cg22784047 & SMG1P2 & Body & 0.56 & 0.12 & $2.33 \mathrm{E}-06$ & $1.53 \mathrm{E}-02$ \\
\hline cg01849466 & ZFYVE21 & Body & 1.82 & 0.39 & $2.37 \mathrm{E}-06$ & $1.53 \mathrm{E}-02$ \\
\hline cg12424548 & KCNQ1 & Body & 0.52 & 0.11 & $2.42 \mathrm{E}-06$ & $1.53 \mathrm{E}-02$ \\
\hline cg05985988 & JARID2 & Body & 0.58 & 0.12 & $2.43 \mathrm{E}-06$ & 1.53E-02 \\
\hline cg15815375 & $R R B P 1$ & 3'UTR & 0.51 & 0.11 & $2.50 \mathrm{E}-06$ & $1.55 \mathrm{E}-02$ \\
\hline cg22174623 & RPL22 & Body & 0.50 & 0.11 & $2.55 \mathrm{E}-06$ & $1.56 \mathrm{E}-02$ \\
\hline cg07538944 & C7orf50 & Body & 0.57 & 0.12 & $2.62 \mathrm{E}-06$ & 1.56E-02 \\
\hline cg22077894 & TAF5L & Body & 0.60 & 0.13 & $2.64 \mathrm{E}-06$ & $1.56 \mathrm{E}-02$ \\
\hline cg05131623 & PEX5L & TSS1500 & 0.77 & 0.16 & $2.78 \mathrm{E}-06$ & $1.62 \mathrm{E}-02$ \\
\hline $\mathrm{cg} 04200362$ & RAB11FIP5 & TSS1500 & 0.39 & 0.08 & 2.93E-06 & $1.68 \mathrm{E}-02$ \\
\hline cg02869486 & FANCC & & -0.63 & 0.13 & $3.08 \mathrm{E}-06$ & $1.72 \mathrm{E}-02$ \\
\hline cg10213762 & SMOC2 & & 1.55 & 0.33 & $3.09 \mathrm{E}-06$ & $1.72 \mathrm{E}-02$ \\
\hline cg22312275 & NAALADL2 & & 0.66 & 0.14 & $3.21 \mathrm{E}-06$ & 1.73E-02 \\
\hline cg25104727 & ASTN1 & Body & -0.88 & 0.19 & $3.21 \mathrm{E}-06$ & 1.73E-02 \\
\hline cg09596260 & $A D C Y 7$ & Body & 0.78 & 0.17 & $3.22 \mathrm{E}-06$ & $1.73 \mathrm{E}-02$ \\
\hline cg06582575 & $P A C R G$ & $\begin{array}{l}\text { 5'UTR; } \\
1^{\text {st }} \text { Exon; } \\
\text { TSS1500 }\end{array}$ & 1.07 & 0.23 & $3.43 \mathrm{E}-06$ & $1.81 \mathrm{E}-02$ \\
\hline cg12762799 & CLEC4E & $\begin{array}{c}1^{\text {st }} \text { Exon; } \\
5 \text { UTR }\end{array}$ & 0.90 & 0.19 & 3.73E-06 & 1.93E-02 \\
\hline cg08045063 & POMGNT2 & TSS200 & -0.98 & 0.21 & $3.76 \mathrm{E}-06$ & $1.93 \mathrm{E}-02$ \\
\hline cg24524451 & $A D C K 2$ & TSS1500 & -0.90 & 0.2 & $3.80 \mathrm{E}-06$ & $1.93 \mathrm{E}-02$ \\
\hline cg20022862 & GPR152 & $1^{\text {st }}$ Exon & 0.75 & 0.16 & $3.98 \mathrm{E}-06$ & $1.98 \mathrm{E}-02$ \\
\hline cg17096191 & NOS1AP & TSS1500 & 1.15 & 0.25 & $4.00 \mathrm{E}-06$ & $1.98 \mathrm{E}-02$ \\
\hline $\operatorname{cg} 10441013$ & KCNJ1O & TSS200 & -0.72 & 0.16 & $4.24 \mathrm{E}-06$ & 2.07E-02 \\
\hline cg00324693 & PCED1A & TSS200 & -0.42 & 0.09 & $4.51 \mathrm{E}-06$ & 2.16E-02 \\
\hline cg10256121 & SPG & & -0.77 & 0.17 & 4.52E-06 & 2.16E-02 \\
\hline cg05588757 & ZNF514 & TSS1500 & -0.54 & 0.12 & 4.61E-06 & 2.18E-02 \\
\hline cg11855759 & USP 43 & TSS 200 & -0.42 & 0.09 & 4.73E-06 & 2.20E-02 \\
\hline cg04587220 & ARHGAP 45 & Body & 0.88 & 0.19 & 4.99E-06 & 2.28E-02 \\
\hline cg05423688 & FMNL1 & TSS200 & -0.43 & 0.09 & 5.03E-06 & 2.28E-02 \\
\hline cg13722651 & ZNF507 & 5'UTR & -0.59 & 0.13 & $5.10 \mathrm{E}-06$ & $2.28 \mathrm{E}-02$ \\
\hline cg24434232 & CLIP2 & & 0.56 & 0.12 & $5.16 \mathrm{E}-06$ & $2.28 \mathrm{E}-02$ \\
\hline cg13446235 & CENPBD1 & $\begin{array}{l}\text { Body; } \\
\text { TSS1500 }\end{array}$ & -0.44 & 0.10 & $5.18 \mathrm{E}-06$ & $2.28 \mathrm{E}-02$ \\
\hline cg11586124 & CTDSP2 & & -0.87 & 0.19 & $5.47 \mathrm{E}-06$ & 2.38E-02 \\
\hline cg02856606 & IFFO2 & $1^{\text {st }}$ Exon & -0.38 & 0.08 & $6.03 \mathrm{E}-06$ & 2.60E-02 \\
\hline cg25433267 & ZNF503-AS2 & $\begin{array}{l}\text { Body; } \\
\text { TSS200 }\end{array}$ & -0.85 & 0.19 & $6.16 \mathrm{E}-06$ & $2.62 \mathrm{E}-02$ \\
\hline cg08180070 & $E D A R$ & & 0.64 & 0.14 & $6.24 \mathrm{E}-06$ & $2.62 \mathrm{E}-02$ \\
\hline cg12598048 & CYFIP1 & $\begin{array}{l}5^{\text {UTR; }} 1^{\text {st }} \\
\text { Exon }\end{array}$ & -0.65 & 0.14 & $6.28 \mathrm{E}-06$ & 2.62E-02 \\
\hline cg13008094 & FABPG & 3'UTR & 0.61 & 0.14 & $6.41 \mathrm{E}-06$ & $2.64 \mathrm{E}-02$ \\
\hline $\operatorname{cg} 08805662$ & C2orf42 & & -0.54 & 0.12 & $6.55 \mathrm{E}-06$ & $2.68 \mathrm{E}-02$ \\
\hline
\end{tabular}




\begin{tabular}{|c|c|c|c|c|c|c|}
\hline Probe & Gene & Region & ES & SE & $P$ & FDR \\
\hline $\operatorname{cg} 17245135$ & MEF2C & Body & 0.60 & 0.13 & $6.75 \mathrm{E}-06$ & $2.71 \mathrm{E}-02$ \\
\hline cg26204328 & $\begin{array}{c}\text { C1QTNF9B- } \\
\text { AS1 }\end{array}$ & $\begin{array}{l}\text { Body; } \\
\text { 5'UTR }\end{array}$ & 0.63 & 0.14 & $6.76 \mathrm{E}-06$ & 2.71E-02 \\
\hline cg09414557 & AGXT2 & TSS200 & 0.49 & 0.11 & $6.98 \mathrm{E}-06$ & $2.76 \mathrm{E}-02$ \\
\hline cg02862748 & TBX15 & 3'UTR & 0.61 & 0.13 & $7.08 \mathrm{E}-06$ & $2.76 \mathrm{E}-02$ \\
\hline cg04510639 & JMJD6 & $\begin{array}{l}1^{\text {st }} \text { Exon; } \\
\text { TSS200; } \\
\text { 5'UTR }\end{array}$ & -0.56 & 0.13 & 7.11E-06 & $2.76 \mathrm{E}-02$ \\
\hline cg18103836 & SERPINB9P 1 & & 0.43 & 0.10 & 7.19E-06 & 2.77E-02 \\
\hline cg24877558 & FOXJ3 & TSS1500 & -0.70 & 0.16 & 7.40E-06 & 2.82E-02 \\
\hline cg20863949 & TEX261 & 3'UTR & -0.61 & 0.14 & 7.47E-06 & $2.82 \mathrm{E}-02$ \\
\hline cg12531601 & HLA-DRB5 & $\begin{array}{l}\text { TSS1500; } \\
\text { 5'UTR }\end{array}$ & -0.38 & 0.08 & 7.67E-06 & 2.87E-02 \\
\hline cg22603628 & DLGAP2 & Body & 0.33 & 0.07 & 7.77E-06 & $2.88 \mathrm{E}-02$ \\
\hline $\operatorname{cg} 10318313$ & NAP $1 L 4$ & TSS1500 & 0.57 & 0.13 & 8.07E-06 & $2.94 \mathrm{E}-02$ \\
\hline cg16457196 & $D D \times 41$ & TSS 1500 & -0.69 & 0.15 & $8.09 \mathrm{E}-06$ & 2.94E-02 \\
\hline cg19865375 & KIAA1324L & Body & 0.56 & 0.13 & $8.24 \mathrm{E}-06$ & $2.97 \mathrm{E}-02$ \\
\hline $\operatorname{cg} 03321231$ & RPH $3 A L$ & Body & 0.64 & 0.14 & $8.68 \mathrm{E}-06$ & 3.09E-02 \\
\hline cg04546186 & FOXF1 & & -0.70 & 0.16 & $8.75 \mathrm{E}-06$ & 3.09E-02 \\
\hline $\operatorname{cg} 23230176$ & GTF2I & TSS 1500 & -0.48 & 0.11 & $8.83 \mathrm{E}-06$ & $3.10 \mathrm{E}-02$ \\
\hline cg01471259 & PDPK1 & Body & 0.64 & 0.14 & $9.17 \mathrm{E}-06$ & 3.14E-02 \\
\hline cg15787377 & ITIH1 & TSS 1500 & 0.69 & 0.16 & 9.27E-06 & 3.14E-02 \\
\hline cg01383268 & SLC39A11 & TSS200 & -0.59 & 0.13 & 9.33E-06 & 3.14E-02 \\
\hline $\operatorname{cg} 18268492$ & PGAM1 & TSS200 & -0.51 & 0.12 & 9.37E-06 & 3.14E-02 \\
\hline cg06521562 & JRK & 3'UTR & 0.72 & 0.16 & $9.41 \mathrm{E}-06$ & 3.14E-02 \\
\hline cg14276515 & IFITM3 & & 0.54 & 0.12 & $9.42 \mathrm{E}-06$ & 3.14E-02 \\
\hline cg15814898 & JAK2 & $\begin{array}{l}1^{\text {st }} \text { Exon; } \\
\text { 5'UTR }\end{array}$ & -0.42 & 0.10 & $9.52 \mathrm{E}-06$ & 3.14E-02 \\
\hline cg04607442 & MIPEPP 3 & & -0.43 & 0.10 & $9.96 \mathrm{E}-06$ & 3.25E-02 \\
\hline cg14034968 & $Z I C 1$ & & -0.65 & 0.15 & $1.00 \mathrm{E}-05$ & 3.25E-02 \\
\hline cg12344004 & $P O L G$ & $\begin{array}{l}\text { 5'UTR; } 1^{\text {st }} \\
\text { Exon }\end{array}$ & -0.48 & 0.11 & 1.07E-05 & $3.46 \mathrm{E}-02$ \\
\hline $\operatorname{cg} 21786289$ & LOC100130417 & TSS200 & 0.99 & 0.23 & 1.11E-05 & $3.56 \mathrm{E}-02$ \\
\hline cg07078467 & RPTOR & Body & 0.47 & 0.11 & 1.13E-05 & $3.59 \mathrm{E}-02$ \\
\hline cg00656410 & SDF4 & Body & 0.60 & 0.14 & 1.15E-05 & 3.61E-02 \\
\hline cg03143697 & LOC202181 & TSS200 & -0.48 & 0.11 & 1.16E-05 & 3.61E-02 \\
\hline cg01453052 & TRIM65 & Body & -0.79 & 0.18 & 1.23E-05 & 3.79E-02 \\
\hline cg25289803 & $A E B P 1$ & TSS200 & -0.79 & 0.18 & 1.23E-05 & 3.79E-02 \\
\hline cg16234986 & ATP1OD & 3'UTR & -0.40 & 0.09 & 1.25E-05 & 3.81E-02 \\
\hline cg21857846 & C8orf37-AS1 & & -0.66 & 0.15 & 1.27E-05 & 3.86E-02 \\
\hline $\operatorname{cg} 06557630$ & ZNF423 & & -0.68 & 0.16 & $1.31 \mathrm{E}-05$ & $3.94 \mathrm{E}-02$ \\
\hline cg25418508 & CDR2L & & -0.59 & 0.14 & $1.32 \mathrm{E}-05$ & 3.95E-02 \\
\hline cg03187713 & $C O B L$ & Body & 0.61 & 0.14 & 1.35E-05 & $3.98 \mathrm{E}-02$ \\
\hline cg08379212 & ENTPD1-AS1 & TSS200 & -0.34 & 0.08 & 1.36E-05 & 3.99E-02 \\
\hline $\operatorname{cg} 27045062$ & EFNA3 & Body & -0.69 & 0.16 & 1.38E-05 & 4.03E-02 \\
\hline cg27514286 & AP2M1 & $\begin{array}{l}\text { 5'UTR; } 1^{\text {st }} \\
\text { Exon }\end{array}$ & -0.56 & 0.13 & $1.40 \mathrm{E}-05$ & 4.04E-02 \\
\hline cg11993118 & NFATC2 & $\begin{array}{l}\text { TSS200; } \\
\text { Body }\end{array}$ & -0.67 & 0.15 & $1.41 \mathrm{E}-05$ & 4.04E-02 \\
\hline cg09610084 & LOC286083 & & -0.81 & 0.19 & 1.45E-05 & $4.11 \mathrm{E}-02$ \\
\hline cg08985029 & TBX5 & 5'UTR & 1.15 & 0.26 & $1.46 \mathrm{E}-05$ & 4.11E-02 \\
\hline cg19465737 & SDHAP1 & & 0.63 & 0.14 & 1.47E-05 & 4.11E-02 \\
\hline cg12828819 & FAT4 & TSS1500 & -0.65 & 0.15 & 1.47E-05 & 4.11E-02 \\
\hline cg00278494 & $C P Z$ & & 0.69 & 0.16 & 1.49E-05 & 4.12E-02 \\
\hline cg03180953 & IGFALS & Body & 0.47 & 0.11 & 1.54E-05 & 4.17E-02 \\
\hline
\end{tabular}




\begin{tabular}{|c|c|c|c|c|c|c|}
\hline Probe & Gene & Region & ES & SE & $P$ & FDR \\
\hline cg04634493 & $Z M I Z 1-A S 1$ & $\begin{array}{c}\text { TSS1500; } \\
\text { TSS200 }\end{array}$ & -0.61 & 0.14 & $1.54 \mathrm{E}-05$ & 4.17E-02 \\
\hline cg09829176 & PPARA & TSS1500 & 0.59 & 0.14 & $1.54 \mathrm{E}-05$ & 4.17E-02 \\
\hline cg14495729 & PTPRN2 & Body & 0.71 & 0.16 & $1.58 \mathrm{E}-05$ & 4.26E-02 \\
\hline cg21918513 & GDNF & 5'UTR & -0.48 & 0.11 & $1.62 \mathrm{E}-05$ & 4.35E-02 \\
\hline cg06486593 & PMPCA & & 0.54 & 0.12 & 1.69E-05 & 4.48E-02 \\
\hline cg18132228 & PRTG & TSS1500 & -0.81 & 0.19 & $1.70 \mathrm{E}-05$ & 4.48E-02 \\
\hline $\operatorname{cg} 13944219$ & SREK1 & $\begin{array}{l}\text { TSS200; } \\
\text { Body }\end{array}$ & -0.44 & 0.10 & $1.71 \mathrm{E}-05$ & 4.49E-02 \\
\hline cg25736626 & MGLL & Body & -0.51 & 0.12 & $1.74 \mathrm{E}-05$ & 4.53E-02 \\
\hline cg21271026 & SLC9A3 & Body & -0.49 & 0.12 & $1.75 \mathrm{E}-05$ & 4.53E-02 \\
\hline cg00597087 & MFSD12 & $\begin{array}{c}1^{\text {st }} \text { Exon; } \\
\text { 5'UTR }\end{array}$ & -0.56 & 0.13 & $1.77 \mathrm{E}-05$ & $4.55 \mathrm{E}-02$ \\
\hline cg21777154 & C1orf21 & 5'UTR & 0.70 & 0.16 & $1.79 \mathrm{E}-05$ & $4.55 \mathrm{E}-02$ \\
\hline $\operatorname{cg} 13028789$ & ANKS1A & $\begin{array}{l}\text { TSS200; } \\
\text { TSS1500 }\end{array}$ & -0.40 & 0.09 & $1.79 \mathrm{E}-05$ & $4.55 \mathrm{E}-02$ \\
\hline cg07802917 & MYLK & TSS200 & 0.66 & 0.15 & $1.82 \mathrm{E}-05$ & 4.59E-02 \\
\hline cg10927461 & TSN & & -0.40 & 0.09 & $1.84 \mathrm{E}-05$ & 4.61E-02 \\
\hline cg24032666 & $N E F L$ & $\begin{array}{c}5^{5} \mathrm{UTR} ; 1^{\text {st }} \\
\text { Exon }\end{array}$ & 1.18 & 0.28 & $1.85 \mathrm{E}-05$ & $4.61 \mathrm{E}-02$ \\
\hline cg04678713 & $\mathrm{BDH1}$ & 3'UTR & -0.99 & 0.23 & $1.92 \mathrm{E}-05$ & $4.72 \mathrm{E}-02$ \\
\hline cg13852536 & NDUFB6 & Body & 0.97 & 0.23 & $1.94 \mathrm{E}-05$ & 4.72E-02 \\
\hline cg13808325 & SMIM20 & $\begin{array}{l}5^{\prime} \mathrm{UTR} ; 1^{\text {st }} \\
\text { Exon }\end{array}$ & -0.46 & 0.11 & 1.94E-05 & 4.72E-02 \\
\hline cg24309739 & NAF1 & TSS1500 & -1.04 & 0.24 & $1.94 \mathrm{E}-05$ & $4.72 \mathrm{E}-02$ \\
\hline cg13701768 & PAX5 & Body & 0.55 & 0.13 & $1.99 \mathrm{E}-05$ & 4.82E-02 \\
\hline cg07835232 & DENND2A & TSS1500 & 0.43 & 0.10 & $2.01 \mathrm{E}-05$ & 4.82E-02 \\
\hline cg27165920 & SYT7 & 3'UTR & 0.64 & 0.15 & $2.03 \mathrm{E}-05$ & 4.83E-02 \\
\hline cg07684809 & CAPS2 & TSS 1500 & 0.54 & 0.13 & $2.04 \mathrm{E}-05$ & 4.83E-02 \\
\hline $\operatorname{cg} 17864206$ & $S Z T 2$ & $\begin{array}{l}\text { Body; } \\
\text { TSS1500 }\end{array}$ & -0.43 & 0.10 & 2.05E-05 & 4.83E-02 \\
\hline cg08894891 & HOMER3 & Body & 0.64 & 0.15 & $2.08 \mathrm{E}-05$ & 4.87E-02 \\
\hline cg23710594 & PHC2 & TSS200 & 0.60 & 0.14 & $2.12 \mathrm{E}-05$ & 4.93E-02 \\
\hline cg07719172 & KCTD10 & Body & 1.06 & 0.25 & $2.13 \mathrm{E}-05$ & 4.93E-02 \\
\hline
\end{tabular}

TABLE 3. DMPs from the Alzheimer's disease methylome-wide association analysis also associated with MCI.

ABBREVIATIONS: DMPs, differentially methylated positions; ES, effect size; FDR, false discovery rate adjusted $p$-values; MCI, mild cognitive impairment; SE, standard error; TSS, transcription start site; UTR, untranslated region.

\begin{tabular}{|c|c|c|c|c|c|c|}
\hline Probe & Gene & Region & ES & SE & $P$ & FDR \\
\hline cg02856606 & IFFO2 & $1^{\text {st }}$ Exon & -0.42 & $\begin{array}{c}7.00 \mathrm{E}- \\
02\end{array}$ & 1.96E-08 & 4.04E-03 \\
\hline cg27657525 & URI1 & TSS1500 & -0.61 & $\begin{array}{c}1.20 \mathrm{E}- \\
01\end{array}$ & 4.60E-07 & $1.40 \mathrm{E}-02$ \\
\hline cg17712928 & MAD1L1 & Body & 0.60 & $\begin{array}{c}1.30 \mathrm{E}- \\
01\end{array}$ & $1.55 \mathrm{E}-06$ & 2.93E-02 \\
\hline cg08805662 & C2orf42 & & -0.50 & $\begin{array}{c}\begin{array}{c}1.10 \mathrm{E}- \\
01\end{array} \\
\text {. }\end{array}$ & $2.11 \mathrm{E}-06$ & 3.34E-02 \\
\hline cg22784047 & SMG1P2 & Body & 0.47 & $\begin{array}{c}1.00 \mathrm{E}- \\
01\end{array}$ & 5.85E-06 & 4.54E-02 \\
\hline cg12518535 & ANKRD20A19P & & -0.54 & $\begin{array}{c}1.20 \mathrm{E}- \\
01\end{array}$ & $6.43 \mathrm{E}-06$ & 4.54E-02 \\
\hline $\operatorname{cg} 00324693$ & PCED1A & TSS200 & -0.36 & $\begin{array}{c}8.00 \mathrm{E}- \\
02\end{array}$ & $6.76 \mathrm{E}-06$ & 4.54E-02 \\
\hline cg04510639 & JMJD6 & $\begin{array}{l}1^{\text {st }} \text { Exon; } \\
\text { TSS200; } \\
\text { 5'UTR }\end{array}$ & -0.50 & $\begin{array}{c}1.10 \mathrm{E}- \\
01\end{array}$ & 6.96E-06 & 4.54E-02 \\
\hline cg01963573 & BEND3 & 3'UTR & 0.49 & $\begin{array}{c}1.10 \mathrm{E}- \\
01\end{array}$ & 8.18E-06 & 4.94E-02 \\
\hline
\end{tabular}




\begin{tabular}{|c|c|c|c|c|c|}
\hline Position & Gene & Region & \# probes & $P$ & Šidák \\
\hline $\begin{array}{c}\text { chr } 5: 180479586- \\
180479623\end{array}$ & BTNL9 & Intron & 2 & 2.20E-08 & 2.36E-04 \\
\hline $\begin{array}{c}\text { chr16:29824542- } \\
29824600\end{array}$ & PRRT2 & CDS & 2 & $3.54 \mathrm{E}-08$ & $2.42 E-04$ \\
\hline $\begin{array}{c}\text { chr3:123603306- } \\
123603312\end{array}$ & $M Y L K$ & Intergenic & 3 & 1.01E-07 & $6.64 \mathrm{E}-03$ \\
\hline $\begin{array}{c}\text { chr1:75139347- } \\
75139365\end{array}$ & $\mathrm{ERICH} 3$ & Exon; 5'UTR & 2 & 1.33E-07 & 2.93E-03 \\
\hline $\begin{array}{c}\text { chr15:89877906- } \\
89877926\end{array}$ & POLG & $\begin{array}{c}\text { Exon; 5'UTR; } \\
\text { Intron }\end{array}$ & 2 & 1.97E-07 & 3.89E-03 \\
\hline $\begin{array}{c}\text { chr18:55710779- } \\
55710830\end{array}$ & NEDD4L & Intergenic & 2 & 2.11E-07 & 1.63E-03 \\
\hline $\begin{array}{c}\text { chr15:101419479- } \\
101419519\end{array}$ & ALDH1A3 & Intergenic & 4 & 3.14E-07 & $3.11 \mathrm{E}-03$ \\
\hline $\begin{array}{c}\text { chr1:5907446- } \\
5907509\end{array}$ & MIR4689 & Intergenic & 2 & 4.26E-07 & 2.68E-03 \\
\hline $\begin{array}{c}\text { chr16:54320670- } \\
54320675\end{array}$ & $\operatorname{IRX3}$ & Intergenic & 3 & 5.13E-07 & $3.98 E-02$ \\
\hline $\begin{array}{c}\text { chr } 4: 54966187- \\
54966248\end{array}$ & GSX2 & $\begin{array}{l}\text { TSS; Exon; } \\
\text { 5'UTR }\end{array}$ & 2 & $1.41 \mathrm{E}-06$ & 9.09E-03 \\
\hline $\begin{array}{c}\text { chr18:48405353- } \\
48405388\end{array}$ & ME2 & Intergenic & 3 & 4.27E-06 & 4.73E-02 \\
\hline $\begin{array}{c}\text { chr14:91853682- } \\
91853729\end{array}$ & CCDC88C & Intron & 2 & 5.16E-06 & 4.26E-02 \\
\hline
\end{tabular}

\subsubsection{Mini-mental state examination scores}

As expected, there was a significant difference between AD patient and control MMSE scores $(\Delta=-5.42, p=2.36 \mathrm{E}-02)$, which was not observed between the $\mathrm{MCl}$ cases and controls $(\Delta=-0.30, p=3.38 \mathrm{E}-01)$ (Supplementary Figure 6). For the MMSE association analysis of the AD-associated probes 6 surrogate variables were included in the limma analysis. A bias of -0.11 and an inflation of 1 was detected after the 'bacon' function was applied to the test statistics (Supplementary Figure 7). After bias, inflation, and FDR adjustment, 110 MMSE-associated DMPs were identified (Table 5; Supplementary Figure 8), of which cg03999372 (IRX3) and cg24032666 (NEFL) were also AD-associated DMPs, whereas $H L A-D R B 5$ contained 3 different DMPs than those found in relation to AD. 5 MMSE-associated DMRs were found (Table 6; Supplementary Figure 8), and the region in $I R X 3$ exactly matches the IRX3 DMR found in relation to AD. A total of 883 unique genes were annotated to the top 1000 ranked $\mathrm{CpG}$ sites. GO enrichment analysis identified 1 significantly enriched biological processes after FDR correction (GO:0007156, "homophilic cell adhesion via plasma membrane adhesion molecules", Table 7; Supplementary Table 3). There was some overlap between the genes in the enriched term, the genes associated with the top 1000 altered CpGs, and genes with DMPs; CDH13, PCDHA1, and CLSTN2.

TABLE 4. DMRs associated with Alzheimer's disease.

ABBREVIATIONS: CDS, coding DNA sequence; chr, chromosome; DMRs, differentially methylated regions; Šidák, Šidák-corrected $p$-values; TSS, transcription start site; UTR, untranslated region. 
Of note, a DMP in PCDHA1 was also detected in association with $\mathrm{MCl}$ $\left(E S=-0.58, p_{F D R}=4.54 E-02\right)$. The top 10 enriched KEGG pathways are shown in Supplementary Table 4, although none of them reached statistical significance after the FDR adjustment.

TABLE 5. DMPs from the MMSE score association analysis.

ABBREVIATIONS: DMPs, differentially methylated positions; ES, effect size; FDR, false discovery rate adjusted $p$-values; MMSE, mini-mental state examination; SE, standard error; TSS, transcription start site; UTR, untranslated region.

\begin{tabular}{|c|c|c|c|c|c|c|}
\hline Probe & Gene & Region & ES & SE & $P$ & FDR \\
\hline $\operatorname{cg} 27228168$ & $\operatorname{IRX} 3$ & TSS 1500 & -0.15 & 0.02 & $3.01 \mathrm{E}-12$ & $7.58 \mathrm{E}-08$ \\
\hline cg03998835 & TBX18 & & -0.14 & 0.02 & $3.41 \mathrm{E}-10$ & 4.29E-06 \\
\hline cg20188282 & GTF3C5 & $\begin{array}{c}\text { 5'UTR; }^{\text {st }} \\
\text { Exon }\end{array}$ & -0.10 & 0.02 & $7.96 \mathrm{E}-10$ & 4.81E-06 \\
\hline cg03999372 & $\operatorname{IRX} 3$ & TSS1500 & -0.09 & 0.02 & $8.50 \mathrm{E}-10$ & 4.81E-06 \\
\hline cg00767058 & ADPRHL1 & $\begin{array}{c}\text { Body; } \\
\text { TSS1500 }\end{array}$ & 0.14 & 0.02 & $9.55 \mathrm{E}-10$ & 4.81E-06 \\
\hline cg09973375 & PPID & TSS 1500 & 0.15 & 0.03 & 2.69E-09 & 1.13E-05 \\
\hline cg15125763 & SPAG16 & TSS1500 & -0.13 & 0.02 & $6.84 \mathrm{E}-09$ & 2.46E-05 \\
\hline $\operatorname{cg} 24032666$ & $N E F L$ & $\begin{array}{l}\text { 5'UTR; } 1^{\text {st }} \\
\text { Exon }\end{array}$ & -0.14 & 0.02 & $1.11 \mathrm{E}-08$ & $3.48 \mathrm{E}-05$ \\
\hline cg19073576 & F10 & & -0.24 & 0.04 & 1.27E-08 & 3.56E-05 \\
\hline cg23303311 & ZNF728 & & -0.06 & 0.01 & 2.23E-08 & $5.24 \mathrm{E}-05$ \\
\hline cg08143038 & GPR78 & & 0.12 & 0.02 & 2.29E-08 & $5.24 \mathrm{E}-05$ \\
\hline cg14942906 & DOC2GP & & 0.16 & 0.03 & $2.50 \mathrm{E}-08$ & 5.24E-05 \\
\hline cg02350039 & NHSL1 & Body & -0.09 & 0.02 & $3.26 \mathrm{E}-08$ & $6.32 \mathrm{E}-05$ \\
\hline cg04704193 & HIST1H3G & TSS 1500 & 0.15 & 0.03 & 3.83E-08 & $6.89 \mathrm{E}-05$ \\
\hline cg20992181 & FGF3 & & 0.07 & 0.01 & $5.59 \mathrm{E}-08$ & $9.39 \mathrm{E}-05$ \\
\hline cg14930075 & KCNKS & $1^{\text {st }}$ Exon & -0.19 & 0.04 & $6.89 \mathrm{E}-08$ & $1.08 \mathrm{E}-04$ \\
\hline cg01849212 & MYOF & TSS 1500 & 0.25 & 0.05 & 1.05E-07 & $1.51 \mathrm{E}-04$ \\
\hline cg20138055 & BIN3 & Body & -0.12 & 0.02 & $1.08 \mathrm{E}-07$ & $1.51 \mathrm{E}-04$ \\
\hline cg16588852 & FAM $86 E P$ & & 0.14 & 0.03 & $1.21 \mathrm{E}-07$ & $1.60 \mathrm{E}-04$ \\
\hline cg25772658 & HLA-DRB5 & Body & 0.07 & 0.01 & 1.46E-07 & 1.83E-04 \\
\hline cg06323727 & EXD3 & 5'UTR & -0.07 & 0.01 & $1.84 \mathrm{E}-07$ & 2.21E-04 \\
\hline cg10485752 & MAN1C1 & Body & -0.13 & 0.02 & 2.19E-07 & $2.51 \mathrm{E}-04$ \\
\hline cg04229851 & SORCS2 & $\begin{array}{l}\text { 3'UTR; } 1^{\text {st }} \\
\text { Exon; Body }\end{array}$ & 0.08 & 0.01 & 2.63E-07 & 2.77E-04 \\
\hline cg02833117 & $E R M N$ & $\begin{array}{l}\text { TSS1500; } \\
\text { Body }\end{array}$ & -0.08 & 0.02 & 2.75E-07 & 2.77E-04 \\
\hline cg00103448 & HOXB1 & & 0.07 & 0.01 & 2.85E-07 & 2.77E-04 \\
\hline cg05163330 & ADPRHL1 & $\begin{array}{l}\text { Body; } \\
\text { TSS1500 }\end{array}$ & 0.16 & 0.03 & $2.94 \mathrm{E}-07$ & 2.77E-04 \\
\hline cg26112170 & ADPRHL1 & $\begin{array}{c}\text { Body; } \\
\text { TSS1500 }\end{array}$ & 0.12 & 0.02 & 2.97E-07 & 2.77E-04 \\
\hline cg22830844 & TOPAZ1 & TSS200 & 0.07 & 0.01 & 3.33E-07 & $2.99 \mathrm{E}-04$ \\
\hline cg04108615 & SLC39A1 & TSS1500 & 0.11 & 0.02 & 3.84E-07 & $3.34 \mathrm{E}-04$ \\
\hline cg14656245 & GPX6 & Body & 0.06 & 0.01 & 4.22E-07 & $3.55 \mathrm{E}-04$ \\
\hline cg23161492 & ANPEP & 5'UTR & 0.10 & 0.02 & $5.77 \mathrm{E}-07$ & 4.69E-04 \\
\hline cg01000236 & CACNA1H & Body & -0.07 & 0.01 & $6.26 \mathrm{E}-07$ & 4.84E-04 \\
\hline cg16119483 & ERGIC1 & Body & 0.09 & 0.02 & $6.34 \mathrm{E}-07$ & 4.84E-04 \\
\hline cg01056174 & ANAPC1 & $\begin{array}{l}1^{\text {st }} \text { Exon; } \\
\text { 5'UTR }\end{array}$ & 0.10 & 0.02 & $6.60 \mathrm{E}-07$ & 4.87E-04 \\
\hline cg09608383 & FAM189A1 & Body & -0.12 & 0.02 & 6.77E-07 & 4.87E-04 \\
\hline cg07829001 & NPHP4 & Body & -0.10 & 0.02 & $8.31 \mathrm{E}-07$ & $5.81 \mathrm{E}-04$ \\
\hline cg08705329 & VPS25 & $\begin{array}{c}\text { 3'UTR; } \\
\text { TSS1500 }\end{array}$ & 0.08 & 0.02 & 1.43E-06 & $9.75 \mathrm{E}-04$ \\
\hline cg13424608 & PLXNA1 & Body & -0.09 & 0.02 & 1.55E-06 & $1.00 \mathrm{E}-03$ \\
\hline
\end{tabular}




\begin{tabular}{|c|c|c|c|c|c|c|}
\hline Probe & Gene & Region & ES & SE & $P$ & FDR \\
\hline cg00035847 & $M E C R$ & $\begin{array}{l}\text { Body; } \\
\text { 5'UTR }\end{array}$ & -0.15 & 0.03 & $1.55 \mathrm{E}-06$ & $1.00 \mathrm{E}-03$ \\
\hline cg24735129 & SHANK2 & & 0.10 & 0.02 & 1.62E-06 & 1.02E-03 \\
\hline cg20971147 & $K A Z N$ & & -0.09 & 0.02 & $2.09 \mathrm{E}-06$ & $1.28 \mathrm{E}-03$ \\
\hline cg02755131 & $\mathrm{CDH} 13$ & Body & 0.08 & 0.02 & $2.49 \mathrm{E}-06$ & $1.49 \mathrm{E}-03$ \\
\hline cg10089963 & NR4A2 & Body & -0.09 & 0.02 & 3.86E-06 & 2.26E-03 \\
\hline cg00089091 & DPP10 & Body & -0.08 & 0.02 & $5.08 \mathrm{E}-06$ & $2.91 \mathrm{E}-03$ \\
\hline cg25319337 & HUS1 & Body & -0.10 & 0.02 & $6.81 \mathrm{E}-06$ & 3.81E-03 \\
\hline cg03432817 & TFAP $2 B$ & & 0.08 & 0.02 & 7.54E-06 & 4.13E-03 \\
\hline cg17393016 & LINC00482 & TSS 1500 & 0.10 & 0.02 & 8.00E-06 & 4.20E-03 \\
\hline cg10342447 & TMEM97 & TSS 1500 & 0.09 & 0.02 & 8.01E-06 & 4.20E-03 \\
\hline cg08463297 & SPECC1 & & -0.11 & 0.02 & 8.57E-06 & 4.41E-03 \\
\hline cg19711879 & FAM193A & & -0.07 & 0.02 & $1.03 \mathrm{E}-05$ & 5.19E-03 \\
\hline cg14624329 & BRINP1 & & 0.08 & 0.02 & $1.05 \mathrm{E}-05$ & $5.21 \mathrm{E}-03$ \\
\hline cg10905495 & PPP $2 R 2 A$ & Body & -0.11 & 0.03 & 1.20E-05 & $5.84 \mathrm{E}-03$ \\
\hline cg12751354 & CYP24A1 & 3'UTR & -0.08 & 0.02 & $1.26 \mathrm{E}-05$ & 6.00E-03 \\
\hline cg01807426 & C7orf50 & Body & 0.06 & 0.01 & 1.42E-05 & 6.63E-03 \\
\hline cg04524088 & MIR129-1 & TSS200 & 0.09 & 0.02 & $1.74 \mathrm{E}-05$ & 7.96E-03 \\
\hline cg03043296 & CPEB1-AS1 & Body & 0.06 & 0.01 & $1.77 \mathrm{E}-05$ & 7.96E-03 \\
\hline cg10624914 & ADPRHL1 & $\begin{array}{l}\text { Body; } \\
\text { TSS200 }\end{array}$ & 0.11 & 0.03 & 1.84E-05 & $8.11 \mathrm{E}-03$ \\
\hline cg26282566 & PCDHA1 & $\begin{array}{l}\text { Body; } \\
\text { TSS200 }\end{array}$ & -0.09 & 0.02 & 1.87E-05 & $8.11 \mathrm{E}-03$ \\
\hline cg11386080 & COL21A1 & TSS200 & 0.06 & 0.01 & $1.94 \mathrm{E}-05$ & $8.29 \mathrm{E}-03$ \\
\hline cg07384357 & TPD52L2 & Body & 0.09 & 0.02 & 2.13E-05 & $8.94 \mathrm{E}-03$ \\
\hline cg08687052 & SYN3 & $\begin{array}{l}\text { Body; } \\
\text { TSS1500 }\end{array}$ & -0.08 & 0.02 & 2.30E-05 & $9.42 \mathrm{E}-03$ \\
\hline cg18220087 & LOC283177 & & -0.06 & 0.01 & 2.33E-05 & 9.42E-03 \\
\hline cg20594961 & $A D R A 2 C$ & & 0.16 & 0.04 & 2.36E-05 & $9.42 \mathrm{E}-03$ \\
\hline cg07684809 & CAPS2 & TSS1500 & -0.05 & 0.01 & $2.98 \mathrm{E}-05$ & 1.17E-02 \\
\hline cg24073777 & CASZ1 & 5'UTR & 0.09 & 0.02 & $3.16 \mathrm{E}-05$ & 1.22E-02 \\
\hline cg01029685 & PCDHA1 & $\begin{array}{l}\text { Body; } \\
\text { TSS1500 }\end{array}$ & -0.10 & 0.02 & $3.22 \mathrm{E}-05$ & $1.23 \mathrm{E}-02$ \\
\hline cg13826105 & FBXW5 & & 0.05 & 0.01 & $3.69 \mathrm{E}-05$ & 1.39E-02 \\
\hline cg25487047 & PCDHA1 & 3'UTR & 0.09 & 0.02 & $3.84 \mathrm{E}-05$ & $1.40 \mathrm{E}-02$ \\
\hline cg13267298 & C14orf180 & & -0.17 & 0.04 & 3.85E-05 & 1.40E-02 \\
\hline cg06609489 & NOL4 & TSS 1500 & -0.10 & 0.02 & 4.82E-05 & 1.72E-02 \\
\hline cg04689720 & $V L D L R$ & $\begin{array}{c}\text { TSS1500; } \\
\text { Body }\end{array}$ & -0.05 & 0.01 & 4.83E-05 & 1.72E-02 \\
\hline cg05733554 & C14orf37 & 5 UTR & -0.06 & 0.02 & $4.94 \mathrm{E}-05$ & $1.73 \mathrm{E}-02$ \\
\hline cg13921570 & $L B X 2-A S 1$ & $\begin{array}{l}\text { Body; } \\
\text { TSS200 }\end{array}$ & -0.04 & 0.01 & 5.36E-05 & $1.85 \mathrm{E}-02$ \\
\hline cg11726572 & TYMS & $\begin{array}{l}\text { Body; } \\
\text { TSS1500 }\end{array}$ & -0.06 & 0.01 & $6.01 \mathrm{E}-05$ & 2.04E-02 \\
\hline cg05726756 & HOXB1 & TSS200 & 0.07 & 0.02 & $6.16 \mathrm{E}-05$ & 2.04E-02 \\
\hline cg21577260 & SLC25A19 & TSS200 & 0.06 & 0.01 & $6.19 \mathrm{E}-05$ & 2.04E-02 \\
\hline cg08966155 & $I Q C G$ & $\begin{array}{l}\text { 5'UTR; } \\
\text { TSS1500 }\end{array}$ & 0.10 & 0.03 & $6.25 \mathrm{E}-05$ & 2.04E-02 \\
\hline cg23795893 & LOC101054525 & TSS200 & -0.10 & 0.03 & $6.75 \mathrm{E}-05$ & $2.18 \mathrm{E}-02$ \\
\hline cg04936970 & EXO5 & TSS 1500 & -0.05 & 0.01 & 7.74E-05 & 2.47E-02 \\
\hline cg09322349 & GRHL3 & TSS 1500 & -0.12 & 0.03 & 7.86E-05 & 2.47E-02 \\
\hline cg15975598 & CREB $3 L 2$ & Body & 0.04 & 0.01 & 8.69E-05 & 2.70E-02 \\
\hline cg15557878 & TSLP & TSS1500 & -0.07 & 0.02 & $9.62 \mathrm{E}-05$ & $2.96 \mathrm{E}-02$ \\
\hline cg17589866 & $A L K$ & Body & 0.15 & 0.04 & $9.95 \mathrm{E}-05$ & 3.02E-02 \\
\hline cg06866655 & SLC9A3 & Body & 0.05 & 0.01 & $1.02 \mathrm{E}-04$ & 3.06E-02 \\
\hline cg05918002 & ZNF232 & 5'UTR & 0.19 & 0.05 & $1.06 \mathrm{E}-04$ & $3.15 \mathrm{E}-02$ \\
\hline
\end{tabular}




\begin{tabular}{|c|c|c|c|c|c|c|}
\hline Probe & Gene & Region & ES & SE & $P$ & FDR \\
\hline cg25858232 & PTPRN2 & Body & 0.06 & 0.02 & 1.12E-04 & $3.29 \mathrm{E}-02$ \\
\hline cg05936555 & HLA-DRB5 & Body & -0.05 & 0.01 & 1.30E-04 & 3.73E-02 \\
\hline cg25559849 & STK32C & TSS200 & 0.10 & 0.03 & 1.32E-04 & $3.73 \mathrm{E}-02$ \\
\hline cg25671428 & CLSTN2 & Body & -0.06 & 0.02 & 1.32E-04 & $3.73 \mathrm{E}-02$ \\
\hline cg13957827 & TMA16 & TSS1500 & -0.04 & 0.01 & 1.35E-04 & $3.74 \mathrm{E}-02$ \\
\hline cg15447775 & NEDD1 & $\begin{array}{l}\text { TSS200; } \\
\text { TSS1500 }\end{array}$ & 0.05 & 0.01 & 1.35E-04 & 3.74E-02 \\
\hline cg13230208 & WDTC1 & & 0.09 & 0.02 & $1.47 \mathrm{E}-04$ & 4.01E-02 \\
\hline $\operatorname{cg} 04314247$ & $S L C 44 A 3$ & $\begin{array}{l}\text { 5'UTR; } 1^{\text {st }} \\
\text { Exon; Body }\end{array}$ & -0.06 & 0.02 & $1.54 \mathrm{E}-04$ & 4.14E-02 \\
\hline $\operatorname{cg} 16736080$ & FAM181A & $\begin{array}{l}\text { Body; } \\
\text { TSS1500 }\end{array}$ & 0.07 & 0.02 & $1.55 \mathrm{E}-04$ & 4.14E-02 \\
\hline cg01150044 & MIR124-1 & & 0.06 & 0.01 & $1.63 \mathrm{E}-04$ & 4.31E-02 \\
\hline cg12382153 & ALDH1A2 & Body & -0.09 & 0.03 & $1.70 \mathrm{E}-04$ & 4.44E-02 \\
\hline $\operatorname{cg} 09139451$ & TRAF5 & $\begin{array}{l}\text { TSS200; } \\
\text { TSS1500 }\end{array}$ & -0.04 & 0.01 & $1.71 \mathrm{E}-04$ & 4.44E-02 \\
\hline cg10801102 & DUS1L & TSS1500 & -0.05 & 0.01 & $1.74 \mathrm{E}-04$ & $4.48 \mathrm{E}-02$ \\
\hline cg20370505 & CARS2 & TSS200 & -0.05 & 0.01 & $1.77 \mathrm{E}-04$ & $4.51 \mathrm{E}-02$ \\
\hline cg11212451 & CPEB 4 & & 0.06 & 0.02 & 1.87E-04 & 4.69E-02 \\
\hline cg00986580 & DAD1 & & -0.05 & 0.01 & $1.89 \mathrm{E}-04$ & 4.69E-02 \\
\hline cg20440187 & HLA-DRB5 & Body & 0.04 & 0.01 & $1.90 \mathrm{E}-04$ & $4.69 \mathrm{E}-02$ \\
\hline cg22700328 & MIR193A & TSS1500 & -0.05 & 0.01 & $1.96 \mathrm{E}-04$ & 4.79E-02 \\
\hline cg10935723 & KCNK1O & TSS1500 & 0.12 & 0.03 & $2.00 \mathrm{E}-04$ & 4.84E-02 \\
\hline cg22448433 & UCHL3 & TSS1500 & 0.05 & 0.01 & $2.03 \mathrm{E}-04$ & 4.86E-02 \\
\hline cg24973289 & TPT1 & & -0.06 & 0.02 & 2.05E-04 & 4.86E-02 \\
\hline cg22520791 & ADAMTS2 & & -0.04 & 0.01 & 2.06E-04 & 4.86E-02 \\
\hline cg23191380 & FZD1 & TSS 200 & -0.04 & 0.01 & $2.11 \mathrm{E}-04$ & 4.93E-02 \\
\hline cg26874163 & FLJ33360 & TSS200 & 0.08 & 0.02 & $2.18 \mathrm{E}-04$ & $5.00 \mathrm{E}-02$ \\
\hline cg11519760 & DLL1 & Body & 0.08 & 0.02 & 2.18E-04 & 5.00E-02 \\
\hline
\end{tabular}

\begin{tabular}{|c|c|c|c|c|c|}
\hline Position & Gene & Region & \# probes & $P$ & Šidák \\
\hline $\begin{array}{c}\text { chr16:54320672- } \\
54320675\end{array}$ & $\operatorname{IR} \times 3$ & Intergenic & 2 & $1.52 \mathrm{E}-18$ & $1.28 \mathrm{E}-14$ \\
\hline $\begin{array}{c}\text { chr13:114103713- } \\
114103797\end{array}$ & $A D P R H L 1$ & Intron & 2 & $2.26 \mathrm{E}-14$ & $6.79 \mathrm{E}-12$ \\
\hline $\begin{array}{c}\text { chr13:45885542- } \\
45885565\end{array}$ & TPT1 & Intergenic & 2 & $2.96 \mathrm{E}-06$ & $3.23 E-03$ \\
\hline $\begin{array}{c}\text { chr2:10184444- } \\
10184458\end{array}$ & $K L F 11$ & $\begin{array}{l}\text { Exon; 5'UTR; } \\
\text { Intron }\end{array}$ & 2 & 7.39E-06 & 1.32E-02 \\
\hline $\begin{array}{c}\text { chr19:48774586- } \\
48774653\end{array}$ & ZNF114 & Exon; 5’UTR & 2 & $1.88 \mathrm{E}-05$ & 7.03E-03 \\
\hline
\end{tabular}

TABLE 6. DMRs associated with MMSE score.

ABBREVIATIONS: chr, chromosome; DMRs, differentially methylated regions; MMSE, mini-mental state examination; Šidák, Šidák-corrected $p$-values; UTR, untranslated region. 


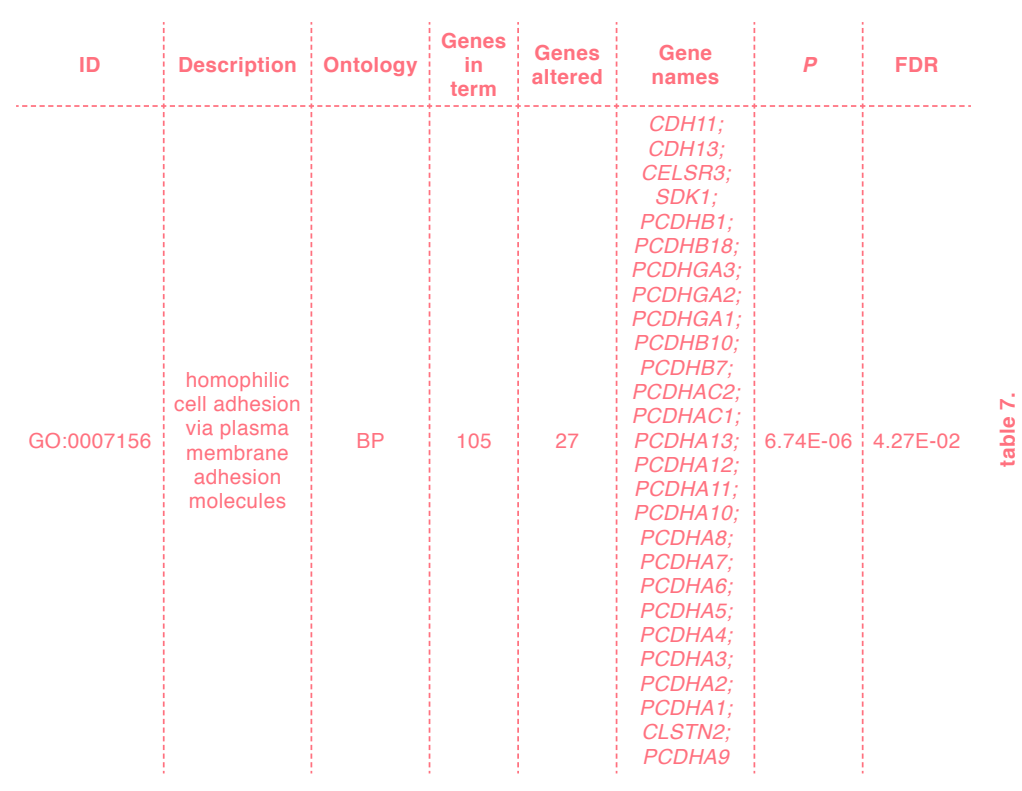

\subsubsection{Cerebrospinal fluid phosphorylated tau amyloid- $\beta$ ratio}

The CSF ptau:Aß42 ratio (Supplementary Figure 9), was increased in AD patients when compared to controls $(\Delta=0.10, p=3.58 \mathrm{E}-02)$, as expected, whereas the ratio was not statistically significantly increased in the $\mathrm{MCl}$ group, although a tendency towards an increase can be observed ( $\Delta=0.05, p=5.16 \mathrm{E}-02)$. With the ptau: $\mathrm{A} \beta 42$ ratio as predictor, 5 significant surrogate variables were detected with the 'sva' function, and which were included in the limma analysis to correct for unobserved covariates. The 'bacon' function estimated the bias and inflation of the limma test results to be 0.036 and 0.96 , respectively (Supplementary Figure 10). CSF ptau:A $\beta 42$ was associated with 12 DMPs that passed the FDR correction (Table 8; Supplementary Figure 11), including cg14930075 and cg01849212 in KCNK9 and MYOF, respectively, that were also associated with MMSE scores. There was 1 DMR associated with the ptau:A $\beta 42$ ratio (Table 9; Supplementary Figure 11). The top GO terms and KEGG pathways from the gene set (including 898 unique genes) enrichment analysis are shown in Supplementary Table 5 and Supplementary Table 6, respectively, but none of them reached statistical significance after FDR adjustment.

TABLE 7. GO term enriched by genes with altered DNA methylation in relation to MMSE score.

ABBREVIATIONS: BP, biological process; FDR, false discovery rate adjusted p-values; GO, Gene Ontology; MMSE, mini-mental state examination. 


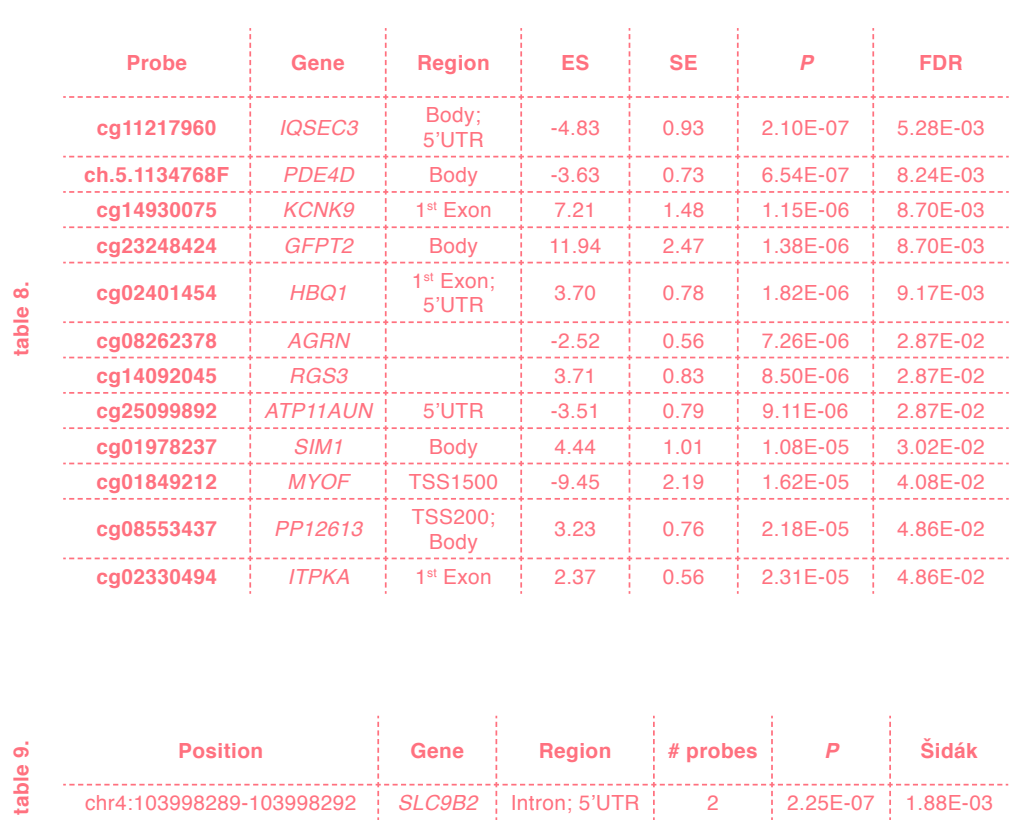

TABLE 8. DMPs from the CSF ptau:A $\beta 42$ association analysis.

ABBREVIATIONS: A $\beta 42$, amyloid- $\beta$ 1-42; CSF, cerebrospinal fluid; DMPs, differentially methylated positions; ES, effect size; FDR, false discovery rate adjusted $p$-values; ptau, phosphorylated tau; SE, standard error; TSS, transcription start site; UTR, untranslated region.

TABLE 9. DMRs associated with CSF ptau:A 342 .

ABBREVIATIONS: A $\beta 42$, amyloid- $\beta$ 1-42; chr, chromosome; CSF, cerebrospinal fluid; DMRs, differentially methylated regions; ptau, phosphorylated tau; Šidák, Šidák-corrected $p$-values; UTR, untranslated region.

\subsubsection{Hippocampal volume}

The final association analysis focused on mean hippocampal volume (Supplementary Figure 12), which was decreased in AD patients when compared to the $\mathrm{MCl}$ group ( $\Delta=-0.04, p=1.63 \mathrm{E}-02)$. The surrogate variable analysis detected 4 significant variables. After running the limma association analysis with these variables, bacon detected a bias of -0.12 and an inflation of 1.1 in the test statistics (Supplementary Figure 13). After adjustment of the test statistics, 14 DMPs were detected (Table 10; Supplementary Figure 14), including one in the TMEM232 gene that also contained one of the 2 DMRs associated with hippocampal volume (Table 11; Supplementary Figure 14). For the gene set (including 896 unique genes) enrichment analysis none of the GO terms or KEGG pathways were statistically significantly enriched after FDR correction (Supplementary Table 7 and Supplementary Table 8, respectively). 


\begin{tabular}{|c|c|c|c|c|c|c|c|}
\hline Probe & Gene & Region & ES & $\mathrm{SE}$ & $P$ & FDR & \\
\hline cg01590699 & PRLH & $1^{\text {st }}$ Exon & 10.89 & 2.05 & 1.17E-07 & $2.94 E-03$ & \\
\hline cg03704673 & GLTPD2 & $\begin{array}{l}\text { 5'UTR; } 1^{\text {st }} \\
\text { Exon }\end{array}$ & -11.19 & 2.18 & 2.70E-07 & 3.25E-03 & \\
\hline $\operatorname{cg} 02388865$ & LIN28A & $\begin{array}{l}1^{\text {st }} \text { Exon; } \\
5 \text { 'UTR }\end{array}$ & -7.67 & 1.54 & 5.83E-07 & $3.25 E-03$ & \\
\hline $\operatorname{cg} 15472170$ & PLOD1 & Body & 9.52 & 1.91 & $6.34 \mathrm{E}-07$ & $3.25 E-03$ & \\
\hline cg06583576 & ZIC4 & & -7.67 & 1.54 & 6.46E-07 & $3.25 E-03$ & \\
\hline cg11543397 & MAL2 & TSS1500 & -8.29 & 1.77 & $2.69 \mathrm{E}-06$ & $9.14 \mathrm{E}-03$ & 음 \\
\hline cg04101934 & SORBS2 & & 7.32 & 1.56 & 2.80E-06 & $9.14 \mathrm{E}-03$ & $\frac{\omega}{0}$ \\
\hline cg03030994 & KCNQ1 & Body & -5.66 & 1.21 & $2.90 \mathrm{E}-06$ & $9.14 \mathrm{E}-03$ & \\
\hline cg14549976 & CTDP1 & & -6.21 & 1.40 & $9.60 \mathrm{E}-06$ & 2.69E-02 & \\
\hline $\operatorname{cg} 02371040$ & ZNF558 & & 5.28 & 1.20 & 1.07E-05 & 2.70E-02 & \\
\hline $\operatorname{cg} 20478120$ & $B A K 1$ & $\begin{array}{l}\text { Body; } \\
\text { TSS1500 }\end{array}$ & -6.54 & 1.53 & 1.82E-05 & 3.89E-02 & \\
\hline cg15535683 & CADPS2 & & -6.73 & 1.58 & 1.91E-05 & 3.89E-02 & \\
\hline cg23217512 & UACA & & -8.82 & 2.07 & 2.01E-05 & 3.89E-02 & \\
\hline $\operatorname{cg} 11641395$ & TMEM232 & TSS200 & -14.57 & 3.43 & $2.17 \mathrm{E}-05$ & $3.90 \mathrm{E}-02$ & \\
\hline Position & Gene & Region & & bes & $P$ & Šidák & \\
\hline $\begin{array}{c}\text { chr5:110062384- } \\
110062399\end{array}$ & TMEM232 & Exon; 5'UTR & & & $2.02 E-07$ & 3.39E-04 & $\frac{0}{\frac{0}{3}}$ \\
\hline $\begin{array}{c}\text { chr6:31148370- } \\
31148525\end{array}$ & PSORS1C3 & Intergenic & & & 7.83E-06 & 1.27E-03 & \\
\hline
\end{tabular}

\subsection{Discussion}

The present study investigated the blood methylomic profile of $A D$ and established sub-profiles related to cognitive performance, CSF ptau:A $\beta 42$, and hippocampal volume. Unlike previous reports focusing on the brain [37, 38], but also blood [39], no global changes in DNA methylation were detected, although this is likely because the used array to assess gene-specific DNA methylation levels covers less than $2 \%$ of all CpGs within the human genome, with an especially low coverage of the large intergenic regions of DNA that are likely heavily methylated [40].

Previous methylome-wide association studies investigating AD-related changes in DNA methylation focusing on the brain identified various genes with altered methylation patterns related to AD pathology, including the AD-susceptibility gene $H L A-D R B 5[41,42]$. In blood, the current study identifies 2 DMPs in $H L A-D R B 5$ associated with $A D$, including the top DMP, and an additional 3 DMPs related to MMSE score. HLA-DRB5 encodes a major histocompatibility complex class II protein, the altered regulation of which supports a role for the immune system in AD [41].

TABLE 10. DMPs from the hippocampal volume association analysis. ABBREVIATIONS: DMPs, differentially methylated positions; ES, effect size; FDR, false discovery rate adjusted $p$-values; SE, standard error; TSS, transcription start site; UTR, untranslated region.

TABLE 11. DMRs associated with hippocampal volume.

ABBREVIATIONS: chr, chromosome; DMRs, differentially methylated regions; Šidák, Šidák-corrected $p$-values; UTR, untranslated region. 
An AD-associated DMR was detected in the ALDH1A3 gene, part of the gene family encoding aldehyde dehydrogenases (ALDHs). Aldehydes may be exogenous, or internally produced during various metabolic processes, and can have cytotoxic effects [43]. ALDHs play an important role in detoxifying aldehydes and their dysregulation has been implicated in $A D[43,44]$. The observed hypermethylation of probes near the ALDH1A3 transcription start site (TSS) in AD cases would support a lowered expression of aldehyde dehydrogenase family 1 member A3, which could result in the increased levels of aldehydes observed in $A D$ [45]. 3 DMPs associated with AD were identified near the promotor of PACRG, a gene linked to Parkinsonism, also a neurodegenerative condition, and which was previously reported to be abnormally methylated in relation to leukemia [46]. NEDD4L, containing a DMP and DMR associated with $A D$, encodes an E3 ubiquitin ligase, crucial for targeted lysosomal degradation, and was found to be differentially expressed in the hippocampus of $A D$ patients [47]. E3 ubiquitin ligases are thought to be important for the clearance of toxic proteins in AD [48].

POLG, encoding DNA polymerase gamma (POLG), presented with a DMP and DMR in relation to AD. POLG is crucial for the maintenance of mitochondrial DNA (mtDNA) and decreased CSF levels of POLG have been found in AD patients [14], as well as low levels of cell-free mtDNA [49]. Notably, low levels of CSF mtDNA has been proposed as a biomarker for preclinical AD [49], and as POLG is responsible for the synthesis of mtDNA, it may be fruitful to investigate the potential of the POLG DMR as an even earlier biomarker for the development of AD. Additionally, it may be interesting to note that altered methylation patterns in relation to $A D$ have also been observed for mitochondrial DNA itself [50].

Neurofilament light (NEFL), encoded by the NEFL gene, has also been investigated as a biomarker for AD progression [51], with higher levels in the CSF being associated with AD, hippocampal atrophy, and lower MMSE scores. In the current study a DMP in NEFL was found in relation to $A D$ and MMSE scores. If a relationship between NEFL methylation status and NEFL CSF levels can be established, the epigenetic marker may serve as an even earlier marker of $A D$ development than changes in NEFL levels in the CSF. As NEFL CSF levels have also been proposed as a biomarker for other neurodegenerative diseases (e.g. [52-54]), its specificity for detecting prodromal AD may be limited, and should be combined with other biomarkers. Another potential early risk factor for $A D$ may be methylation of MAD1L1, a regulator of leukocyte telomere length [55]. Shorter leukocyte telomeres is a risk factor for age-related diseases, including $A D$ [56]. Epigenetic dysregulation of MAD1L1 appeared to already exist in MCl cases, as the MAD1L1 DMP (cg17712928) was 
found to be hypermethylated in both $\mathrm{MCl}$ and $\mathrm{AD}$ cases, but more so in the AD cases. Interestingly, differential methylation of the MAD1L1 gene has previously been reported in association with schizophrenia, along with the suggestion there may be overlap in the epigenetic dysregulation seen AD and schizophrenia [57].

The IRX3 gene was associated with a DMP and DMR in relation to AD, as well as 2 DMPs and a DMR associated with MMSE score, with AD and a lower MMSE score resulting in hypermethylation. IRX3 regulation is connected to the obesity- and type 2 diabetes- associated gene FTO $[58,59]$. Both obesity and diabetes are risk factors for AD, which may be explained by a possible interaction between FTO and APOE [60], the latter of which represents a major genetic risk factor for sporadic $A D$ [61]. GFPT2, containing a DMP associated with CSF ptau:A 42 , encodes glutamine-fructose-6-phosphate transaminase (GFPT) 2, that is important for glucose energy metabolism and has been linked to diabetes mellitus $[62,63]$, and may therefore play a role in the suggested link between AD and diabetes mellitus [64-66]. Additionally, GFPT22-mediated glutamate metabolism may be disturbed in AD [67]. For other genes, such as EXO5, with an MMSE-associated DMP, TMEM232, with a DMP and DMR associated with hippocampal volume, and SLC9B2, with a DMR related to CSF ptau:A $\beta 42$, no clear relationships with $A D$ have yet been reported.

The performed GO and KEGG analyses were an attempt to identify functionally linked genes affected by altered methylation levels. Although many terms and pathways relevant for neurodegeneration could be observed amongst those that were most enriched, only one term survived FDR correction, GO:0007156 ("homophilic cell adhesion via plasma membrane adhesion molecules"), related to MMSE score. Looking at the genes associated with the top 1000 most altered CpGs in this pathway reveals a high representation of protocadherin $(\mathrm{PCDH})$ genes, including PCDHA1, which contained 3 MMSE-associated DMPs and a DMP associated with $\mathrm{MCl}$. PCDH proteins are cell-adhesion proteins located in the plasma membrane, critical for neuronal cell-cell interactions, including synaptogenesis $[68,69]$. Other genes in the enriched GO term and including a MMSE-associated DMP are CDH13 and CLSTN2. CDH13 also belongs to the cadherin family of membrane proteins, this variant being implicated in regulating neurite growth and prevention of cell death in response to oxidative stress $[70,71]$. Although not previously implicated in $A D, C D H 13$ hypermethylation has been observed in a multitude of cancer types (e.g. [72, 73]), and specific gene variants are associated with diabetes type 2 [74] and metabolic syndrome [75], but also with schizophrenia [76], attention deficit hyperactivity disorder [77], particularly in relation to working memory performance [78], and violent behavior [79], and even chronic obstructive pulmonary disease [80] and atherosclerosis 
[81]. However, its implication in such diverse pathologies may limit the usefulness of $C D H 13$ as a specific biomarker for $A D$, although its related disorders may be investigated as possible comorbidities of $A D$ to identify disease subtypes. Interestingly, genetic variation in CLSTN2, encoding calsyntenin 2, a component of the postsynaptic membrane [82], has been linked to episodic memory performance [83, 84], which is strongly affected in AD [85]. All in all, methylation of PCDHA1, CDH13, and CLSTN2 may be investigated as early markers of dementia.

The findings of the present study should be viewed in light of some limitations. One of the limitations is the relatively small sample size, which limited the power of the study, as well as the covariates that could be included. To accommodate the latter limitation, the effects of age and gender, which may influence AD diagnosis [86, 87], were individually tested and no effect was found on AD diagnosis (data not shown). Additionally, it has been suggested differences in blood cell type composition may affect the outcome of epigenetic studies in blood [88, 89]. Components of CD4+ and CD8+ T cells, B cells, monocytes, granulocytes, and natural killer cells were estimated with the 'estimateCellCounts' function of minfi $[88,89]$ and the effects on $A D$ status were investigated (data not shown). Although no significant effects were found, this may also be attributed to the low sample size, and therefore surrogate variables were included to account for potential confounders, as described in the methods section. Lastly, it should be noted that the used method to quantify DNA methylation levels cannot distinguish between DNA methylation and hydroxymethylation, a related, but functionally different epigenetic marker [90]. However, it is expected this has less impact on studies focusing on the blood methylome than those focusing on the brain, as DNA hydroxymethylation is much less prevalent than DNA methylation and is not enriched in the blood like it is in the brain [91].

Summarizing, this small exploratory study corroborates an epigenetic component in $\mathrm{AD}$ and associated measures, and shows some of the epigenetic dysregulation observed in the brain, mainly concerning $H L A-D R B 5$, is reflected in the blood methylome, in addition to some novel potential blood biomarkers of $A D$. Especially methylation of the plasma membrane triad PCDHA1, CDH13, and CLSTN2 may be further explored as early markers of $A D$ dementia, possibly complemented by the methylation status of HLA-DRB5, NEFL, and MAD1L1. 


\section{Acknowledgments}

Funds have been provided by the Internationale Stichting Alzheimer Onderzoek (ISAO) grant 11532 (D.L.A.vdH.), by the ISAO grants 09552 and 13515, and the Netherlands Organization for Scientific Research (NWO), grant 916.11.086 (Veni Award) (B.P.F.R.), and by a fellowship as part of NWO grant 022.005.019, (R.L.). The funding agencies were not involved in the study design, data collection, analysis and interpretation, writing of the report, and the decision to submit the article for publication. The authors declare no conflicts of interest.

\section{REFERENCES}

[1] Yates D, McLoughlin DM.

The molecular pathology of Alzheimer's disease. Psychiatry 2008; 7: 1-5. [2] Godyń J, Jończyk J, Panek D, et al. Therapeutic strategies for Alzheimer's disease in clinical trials. Pharmacol Reports 2015; 68: 127-138. [3] Castellani RJ, Perry G. The complexities of the pathology-pathogenesis relationship in Alzheimer disease. Biochem Pharmacol 2014; 88 : 671-676.

[4] Heppner FL, Ransohoff

RM, Becher B. Immune attack: the role of inflammation in Alzheimer disease. Nat Rev Neurosci 2015; 16 : $358-372$

[5]

Yadav RS, Tiwari NK. Lipid integration in neurodegeneration: an overview of Alzheimer's disease. $\mathrm{Mol}$ Neurobiol 2014; 50: 168-176.

[6] Chen Z, Zhong C. Decoding Alzheimer's disease from perturbed cerebral glucose metabolism: Implications for diagnostic and therapeutic strategies. Prog Neurobiol 2013; 108: 21-43.

[7] Ciechanover A, Kwon YT.

Degradation of misfolded proteins in neurodegenerative diseases: therapeutic targets and strategies. Exp $\mathrm{Mol} \mathrm{Med}$ 2015; 47: e147.

[8] Lardenoije R, Iatrou A,

Kenis G, et al. The epigenetics of aging and neurodegeneration. Prog Neurobiol 2015; 131: 21-64.

[9] Iatrou A, Kenis G, Rutten

BPF, et al. Epigenetic dysregulation of brainstem nuclei in the pathogenesis of Alzheimer's disease: looking in the correct place at the right time? Cellular and Molecular Life Sciences, 2016, pp. $1-15$.

[10] Hashimoto H, Vertino PM, Cheng X. Molecular coupling of DNA 
methylation and histone methylation.

Epigenomics 2010; 2: 657-669.

[11] Lunnon K, Mill J. Epigen-

etic studies in Alzheimer's disease:

current findings, caveats, and considerations for future studies. $\mathrm{Am} J$

Med Genet B Neuropsychiatr Genet 2013; 162B: 789-99.

[12] Slieker RC, Bos SD,

Goeman JJ, et al. Identification and systematic annotation of tissue-specific differentially methylated regions using the Illumina $450 \mathrm{k}$ array. Epigenetics Chromatin 2013; 6: 26.

[13] Defina PA, Moser RS, Glenn $\mathrm{M}$, et al. Alzheimer's disease clinical and research update for health care practitioners. J Aging Res 2013; 2013 : 207178.

\section{[14] Oláh Z, Kálmán J, Tóth}

$\mathrm{ME}$, et al. Proteomic analysis of cerebrospinal fluid in Alzheimer's disease: wanted dead or alive. J Alzheimer's Dis 2015; 44: 1303-1312.

[15] Peng G-P, Feng Z, He F-P, et al. Correlation of hippocampal volume and cognitive performances in patients with either mild cognitive impairment or Alzheimer's disease. CNS Neurosci Ther 2015; 21: 15-22. [16] Folstein MF, Folstein SE, McHugh PR. 'Mini-mental state'. A practical method for grading the cognitive state of patients for the clinician. J Psychiatr Res 1975; 12: 189-98. [17] Pradier C, Sakarovitch C, Le Duff F, et al. The mini mental state examination at the time of Alzheimer's disease and related disorders diagnosis, according to age, education, gender and place of residence: a cross-sectional study among the French National Alzheimer database. PLoS One 2014; 9: el03630.

[18] van Rossum IA, Visser PJ, van der Flier WM, et al. Predictors of time to AD diagnosis in subjects with MCI. Alzheimer's Dement 2010; 6: S99.

[19] Fagan AM, Roe CM, Xiong

C, et al. Cerebrospinal fluid tau/ $\beta$-amyloid42 ratio as a prediction of cognitive decline in nondemented older adults. Arch Neurol 2007; 64: 343.

[20] Fischl B. FreeSurfer. Neuroimage 2012; 62: 774-781.

[21] R Core Team. R: A language and environment for statistical computing. https://www.r-project.org/ (2016).

[22] RStudio Team. RStudio: Integrated Development for Rhttp:// www.rstudio.com/ (2016).

[23] van Iterson $\mathrm{M}$, Tobi $\mathrm{EW}$,

Slieker RC, et al. MethylAid: visual and interactive quality control of large Illumina $450 \mathrm{k}$ datasets. Bioinformatics 2014; 30: 3435-3437.

[24] Aryee MJ, Jaffe AE, Corrada-Bravo H, et al. Minfi: a flexible and comprehensive Bioconductor package for the analysis of Infinium DNA methylation microarrays. Bioinformatics 2014; 30: 1363-1369.

[25] Triche TJ, Weisenberger

DJ, Van Den Berg D, et al. Low-level processing of Illumina Infinium DNA Methylation BeadArrays. Nucleic Acids Res 2013; 41: e90-e90.

[26] Fortin J-P, Labbe A, Lemire M, et al. Functional normalization of $450 \mathrm{k}$ methylation array data improves replication in large cancer studies.

Genome Biol 2014; 15: 503.

[27] van Iterson M, Tobi E,

Slieker R, et al. DNAmArray. Epub ahead of print 2017. DOI: https://doi. org/10.5281/zenodo.158908.

[28] Chen Y, Lemire M, Choufani S, et al. Discovery of cross-reactive probes and polymorphic CpGs in the Illumina Infinium HumanMethylation450 microarray. Epigenetics 2013;
8: 203-209.

Du P, Zhang X, Huang C-C, et al. Comparison of Beta-value and M-value methods for quantifying methylation levels by microarray analysis. BMC Bioinformatics 2010; 11: 587.

[30] Ritchie ME, Phipson B, Wu D, et al. limma powers differential expression analyses for RNA-sequencing and microarray studies. Nucleic Acids Res 2015; 43: e47.

[31] Leek JT, Johnson WE,

Parker HS, et al. The sva package for removing batch effects and other unwanted variation in high-throughput experiments. Bioinformatics 2012; 28: 882-3.

[32] Smyth GK. Linear models and empirical Bayes methods for assessing differential expression in microarray experiments. Stat Appl Genet Mol Biol 2004; 3: 1-25.

[33] van Iterson $M$, van Zwet EW, BIOS Consortium BT, et al. Controlling bias and inflation in epigenome- and transcriptome-wide association studies using the empirical null distribution. Genome Biol 2017; 18: 19.

[34] Pedersen BS, Schwartz DA, Yang I V, et al. Comb-p: software for combining, analyzing, grouping and correcting spatially correlated $P$-values. Bioinformatics 2012; 28: 2986-8. [35] Phipson B, Maksimovic J, Oshlack A. missMethyl: an R package for analyzing data from Illumina's HumanMethylation450 platform. Bioinformatics 2015; 32: btv560.

[36] Geeleher P, Hartnett L, Egan LJ, et al. Gene-set analysis is severely biased when applied to genome-wide methylation data. Bioinformatics 2013; 29: 1851-1857.

Chouliaras L, Mastroeni D, 
Delvaux E, et al. Consistent decrease in global DNA methylation and hydroxymethylation in the hippocampus of Alzheimer's disease patients. Neurobiol Aging 2013; 34: 2091-2099. [38] Coppieters N, Dieriks B V, Lill C, et al. Global changes in DNA methylation and hydroxymethylation in Alzheimer's disease human brain. Neurobiol Aging 2014; 35: 1334-1344.

[39] Di Francesco A, Arosio B, Falconi A, et al. Global changes in DNA methylation in Alzheimer's disease peripheral blood mononuclear cells. Brain Behav Immun 2015; 45: $139-144$.

[40] Weber M, Schübeler D. Genomic patterns of DNA methylation: targets and function of an epigenetic mark. Curr Opin Cell Biol 2007; 19: 273-280.

[41] Lambert J-C, Ibrahim-Verbaas CA, Harold D, et al. Meta-analysis of 74,046 individuals identifies 11 new susceptibility loci for Alzheimer's disease. Nat Genet 2013; 45: 14.52-1458. Yu L, Chibnik LB, Srivastava GP, et al. Association of brain DNA methylation in $S O R L 1, A B C A 7$, $H L A-D R B 5, S L C 24 A 4$, and BIN1 with pathological diagnosis of Alzheimer disease. JAMA Neurol 2015; 72: 15. [43] Vasiliou V, Nebert DW. Analysis and update of the human aldehyde dehydrogenase (ALDH) gene family. Hum Genomics 2005; 2: 138-43. [44] Kamino K, Nagasaka $\mathrm{K}$, Imagawa M, et al. Deficiency in mitochondrial aldehyde dehydrogenase increases the risk for late-onset Alzheimer's disease in the Japanese population. Biochem Biophys Res Commun 2000; 273: 192-196.

[45] Chen K, Maley J, Yu PH. Potential implications of endogenous aldehydes in beta-amyloid misfolding, oligomerization and fibrillogenesis.

$J$ Neurochem 2006; 99: 1413-1424.

[46] Agirre X, Román-Gómez J,

Vázquez I, et al. Abnormal meth-

ylation of the common PARK2 and

$P A C R G$ promoter is associated with

downregulation of gene expression

in acute lymphoblastic leukemia and

chronic myeloid leukemia. Int J Cancer

2006; 118: 1945-1953.

[47] Hokama M, Oka S, Leon J, et al. Altered expression of diabetes-related genes in Alzheimer's disease brains: The Hisayama Study. Cereb Cortex 2014; 24: 2476-2488.

[48] Kumar P, Jha NK, Jha SK,

et al. Tau phosphorylation, molecular chaperones, and ubiquitin E3 ligase: clinical relevance in Alzheimer's disease. J Alzheimer's Dis 2015; 43: 341-361.

[49] Podlesniy P, Figueiro-Silva J, Llado A, et al. Low cerebrospinal fluid concentration of mitochondrial DNA in preclinical Alzheimer disease. Ann Neurol 2013; 74: 655-668.

[50] Blanch M, Mosquera JL, Ansoleaga B, et al. Altered mitochondrial DNA methylation pattern in Alzheimer disease-related pathology and in Parkinson disease. Am J Pathol 2016; 186: 385-397.

[51] Zetterberg H, Skillbäck T, Mattsson N, et al. Association of cerebrospinal fluid neurofilament light concentration with Alzheimer disease progression. JAMA Neurol 2016; 73: 60. [52] Lu C-H, Macdonald-Wallis C, Gray E, et al. Neurofilament light chain: A prognostic biomarker in amyotrophic lateral sclerosis. Neurology 2015; 84: 224.7-2257.

[53] Skillback T, Farahmand B, Bartlett JW, et al. CSF neurofilament light differs in neurodegenerative diseases and predicts severity and sur- vival. Neurology 2014; 83: 1945-1953.

[54] Abdulle S, Mellgren Å, Brew BJ, et al. CSF neurofilament protein (NFL) - a marker of active HIV-related neurodegeneration.

J Neurol 2007; 254: 1026-1032.

[55] Buxton JL, Suderman M, Pappas JJ, et al. Human leukocyte telomere length is associated with DNA methylation levels in multiple subtelomeric and imprinted loci. Sci Rep 2014; 4: 1496-1505.

[56] Guan J-Z, Guan W-P,

Maeda T, et al. The subtelomere of short telomeres is hypermethylated in Alzheimer's disease. Aging Dis 2012; 3: $164-70$.

[57] Montano C, Taub MA, Jaffe A, et al. Association of DNA methylation differences with schizophrenia in an epigenome-wide association study. JAMA Psychiatry 2016; 73: 506.

[58] Smemo S, Tena JJ, Kim

$\mathrm{K}-\mathrm{H}$, et al. Obesity-associated variants within FTO form long-range functional connections with IRX3. Nature 2014; 507: 371-375.

[59] Cedernaes J, Benedict C. Human obesity: FTO, IRX3, or both? Mol Metab 2014; 3: 505-6.

[60] Keller L, Xu W, Wang H-X, et al. The obesity related gene, FTO, interacts with $A P O E$, and is associated with Alzheimer's disease risk: a prospective cohort study. J Alzheimers Dis 2011; 23: 461-9.

[61] Liu C-C, Kanekiyo T, Xu H, et al. Apolipoprotein E and Alzheimer disease: risk, mechanisms and therapy. Nat Rev Neurol 2013; 9: 106-118.

[62] Zhang H, Jia Y, Cooper JJ, et al. Common variants in glutamine:fructose-6-phosphate amidotransferase 2 (GFPT2) gene are associated with type 2 diabetes, diabetic nephropathy, and increased GFPT2 mRNA levels. 
$J$ Clin Endocrinol Metab 2004; 89 :

$748-755$.

[63] Coomer M, Essop MF.

Differential hexosamine biosynthetic pathway gene expression with type 2 diabetes. Mol Genet Metab Reports 2014; 1: 158-169.

\section{[64] Aliev G, Shahida K, Hua}

Gan S, et al. Alzheimer disease and type 2 diabetes mellitus: the link to tyrosine hydroxylase and probable nutritional strategies. http://www. ingentaconnect.com/content/ben/ cnsnddt/2014/00000013/00000003/ art00013 (accessed 19 April 2017).

[65] de la Monte SM. Brain insulin resistance and deficiency as therapeutic targets in Alzheimer's disease. Curr Alzheimer Res 2012; 9: $35-66$.

[66] Yu R, Deochand C, Krotow A, et al. Tobacco smoke-induced brain white matter myelin dysfunction: potential co-factor role of smoking in neurodegeneration. J Alzheimer's Dis 2015; 50: 133-148.

[67] Zhang M, Schmitt-Ulms

G, Sato C, et al. Drug repositioning for Alzheimer's disease based on systematic 'omics' data mining. PLoS One 2016; 11: e0168812.

[68] Hirayama T, Yagi T. The role and expression of the protocadherin-alpha clusters in the CNS. Curr Opin Neurobiol 2006; 16: 336-342. [69] Lachman HM, Petruolo OA, Pedrosa E, et al. Analysis of protocadherin alpha gene deletion variant in bipolar disorder and schizophrenia. Psychiatr Genet 2008; 18: 110-115.

[70] Rivero O, Selten MM,

Sich S, et al. Cadherin-13, a risk gene for ADHD and comorbid disorders, impacts GABAergic function in hippocampus and cognition. Transl Psychiatry 2015; 5: e655.
[71] Joshi MB, Philippova M,

Ivanov D, et al. T-cadherin protects endothelial cells from oxidative stress-induced apoptosis. FASEB $J$ 2005; 19: 1737-9.

[72] Yang J, Niu H, Huang Y, et al. A systematic analysis of the relationship of $C D H 13$ promoter methylation and breast cancer risk and prognosis. PLoS One 2016; 11: e0149185.

[73] Ye M, Huang T, Li J, et al.

Role of CDH13 promoter methylation in the carcinogenesis, progression, and prognosis of colorectal cancer. Medicine (Baltimore) 2017; 96: e5956. [74] Nicolas A, Aubert R, Bellili-Muñoz N, et al. T-cadherin gene variants are associated with type 2 diabetes and the Fatty Liver Index in the French population. Diabetes Metab 2017; 43: 33-39.

\section{[75] Kitamoto A, Kitamoto T,}

Nakamura T, et al. CDH13 polymorphisms are associated with adiponectin levels and metabolic syndrome traits independently of visceral fat mass. J Atheroscler Thromb 2016; 23: 309-319.

[76] Børglum AD, Demontis

D, Grove J, et al. Genome-wide study of association and interaction with maternal cytomegalovirus infection suggests new schizophrenia loci. $\mathrm{Mol}$ Psychiatry 2014; 19: 325-333.

[77] Salatino-Oliveira A, Genro JP, Polanczyk G, et al. Cadherin-13 gene is associated with hyperactive/ impulsive symptoms in attention/deficit hyperactivity disorder. $\mathrm{Am} \mathrm{J} \mathrm{Med}$ Genet Part B Neuropsychiatr Genet 2015; 168: 162-169.

[78] Arias-Vásquez A, Altink

ME, Rommelse NNJ, et al. $C D H 13$ is associated with working memory performance in attention deficit/ hyperactivity disorder. Genes, Brain
Behav 2011; 10: 844-851.

[79] Tiihonen J, Rautiainen M-R, Ollila HM, et al. Genetic background of extreme violent behavior. Mol Psychiatry 2015; 20: 786-792.

[80] Yuan Y, Zhang J, Xu S, et al. Genetic variants of $C D H 13$ determine the susceptibility to chronic obstructive pulmonary disease in a Chinese population. Acta Pharmacol Sin 2016; 37: 390-397.

[81] Kostopoulos CG, Spiroglou SG, Varakis JN, et al. Adiponectin/T-cadherin and apelin/APJ expression in human arteries and periadventitial fat: implication of local adipokine signaling in atherosclerosis? Cardiovasc Pathol 2014; 23 : 131-138.

[82] Hintsch G, Zurlinden A, Meskenaite V, et al. The calsyntenins--a family of postsynaptic membrane proteins with distinct neuronal expression patterns. Mol Cell Neurosci 2002; 21: 393-409.

[83] Pantzar A, Laukka EJ, Atti $\mathrm{AR}$, et al. Interactive effects of $K I B R A$ and CLSTN2 polymorphisms on episodic memory in old-age unipolar depression. Neuropsychologia 2014; 62: 137-142.

[84] Preuschhof C, Heekeren

HR, Li S-C, et al. KIBRA and CLSTN2 polymorphisms exert interactive effects on human episodic memory. Neuropsychologia 2010; 48: 402-408.

[85] Tromp D, Dufour A,

Lithfous S, et al. Episodic memory in normal aging and Alzheimer disease: Insights from imaging and behavioral studies. Ageing Res Rev 2015; 24: 232-262.

[86] Kawas C, Gray S, Brookmeyer R, et al. Age-specific incidence rates of Alzheimer's disease: the Baltimore Longitudinal Study of Aging. 
Neurology 2000; 54: 2072-7.

[87] Podcasy JL, Epperson CN.

Considering sex and gender in Alzheimer disease and other dementias. Dialogues Clin Neurosci 2016; 18: 437-446. [88] Jaffe AE, Irizarry RA. Accounting for cellular heterogeneity is critical in epigenome-wide association studies. Genome Biol 2014; 15: R31.

[89] Houseman E, Accomando WP, Koestler DC, et al. DNA methylation arrays as surrogate measures of cell mixture distribution. $B M C$ Bioinformatics 2012; 13: 86.

[90] van den Hove DLA, Chouliaras L, Rutten BPF. The role of 5-hydroxymethylcytosine in aging and Alzheimer's disease: current status and prospects for future studies. Curr Alzheimer Res 2012; 9: 545-9.

[91] Skvortsova K, Zotenko E, Luu P-L, et al. Comprehensive evaluation of genome-wide 5-hydroxymethylcytosine profiling approaches in human DNA. Epigenetics Chromatin 2017; 10: 16 .

\section{SUPPLEMENTARY DATA}

SUPPLEMENTARY FIGURE 1. Density plot of raw beta values.

Plot includes all DNA methylation probes on the Illumina HumanMethylation450 BeadChip, plotted for each sample separately.

\section{SUPPLEMENTARY FIGURE 2. Screeplot}

for the Illumina HumanMethylation450 BeadChip assay data.

Most variance is captured by the first 3 principal components (PGs), thus 3 principal components were selected for functional normalization of the data.
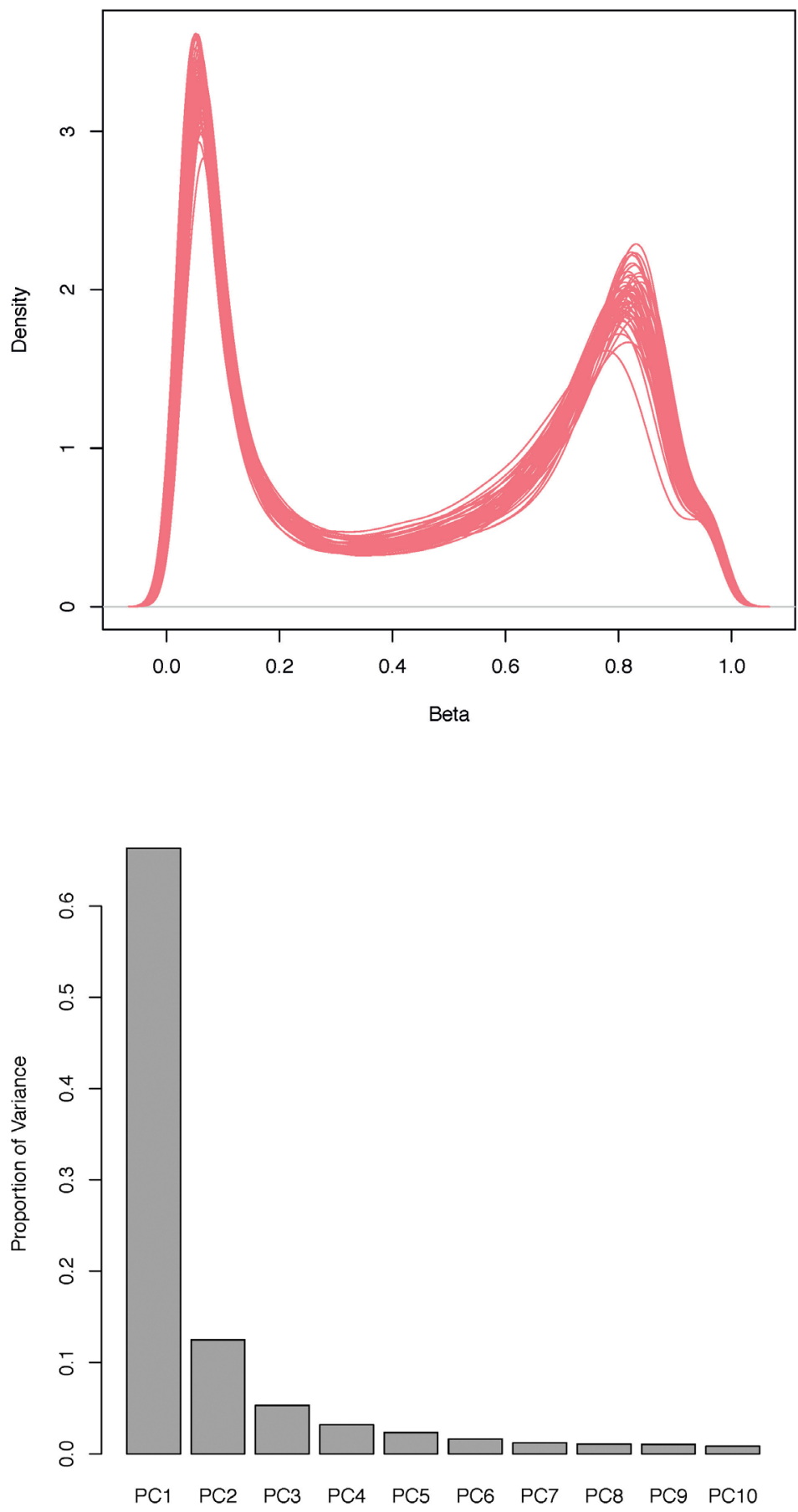
SUPPLEMENTARY FIGURE 3. Density

plot of normalized beta values. Plot includes all DNA methylation probes on the Illumina Human Methylation450 BeadChip, plotted for each sample separately.

SUPPLEMENTARY FIGURE 4. Histogram of test-statistics showing potential bias (A) and quantile-quantile (QQ) plot showing potential inflation (B) of the Alzheimer's disease methylome-wide association study results. The pink line in A represents the fit of the empirical null distribution and the black line the fit of the mixture of the empirical null distribution, the proportion of positively associated features, and the proportion of negatively associated features, both estimated as described in [33]. The estimated bias and inflation was -0.098 and 1.1 , respectively.

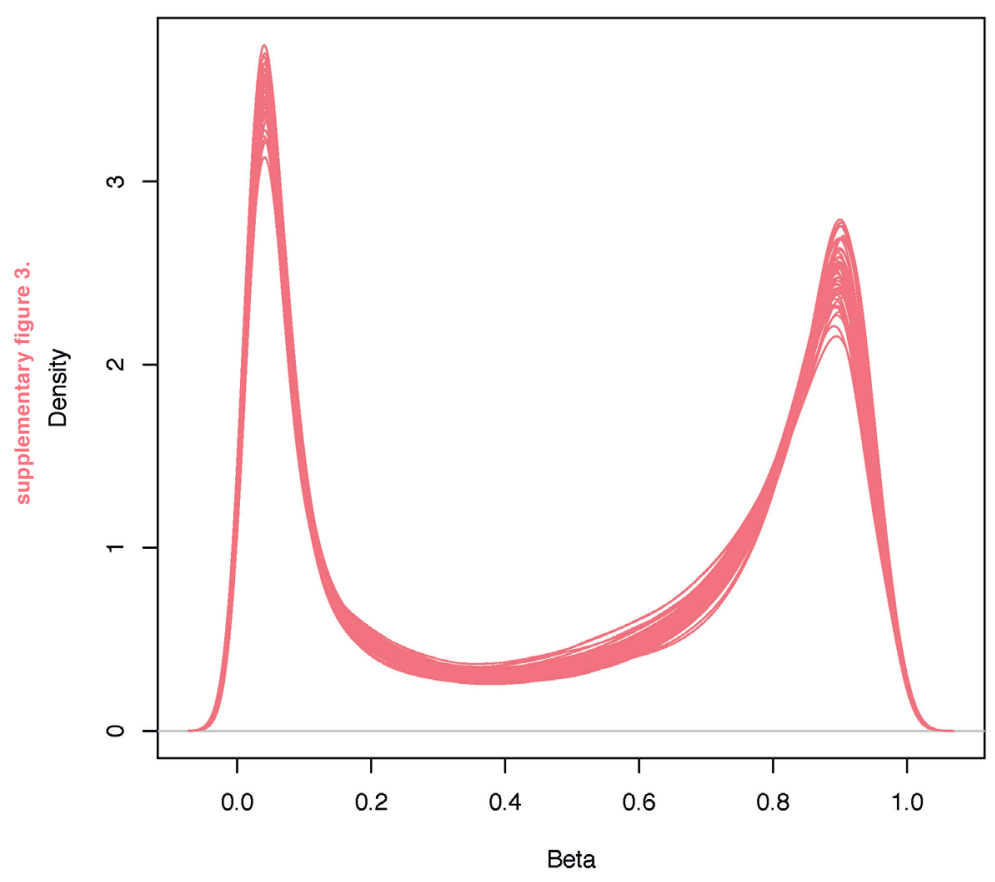

A

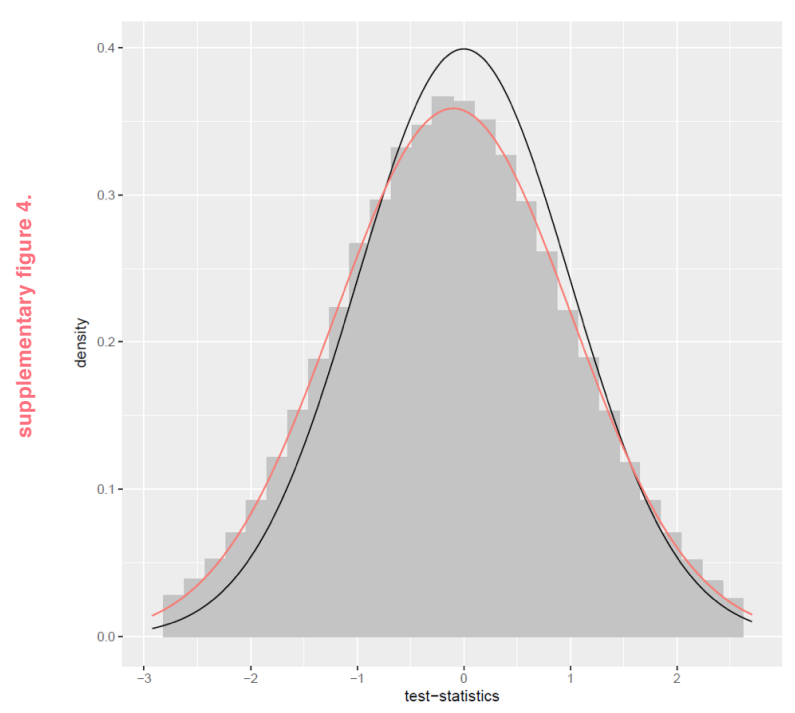

B

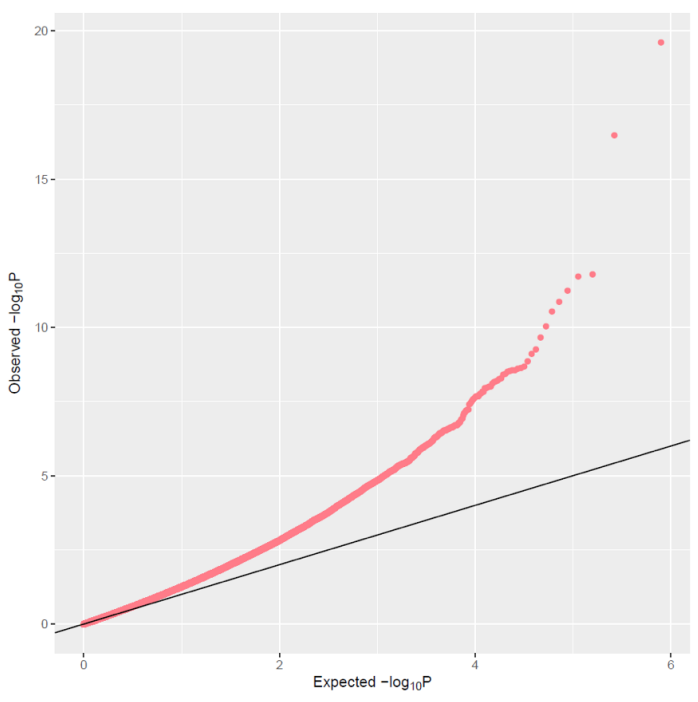



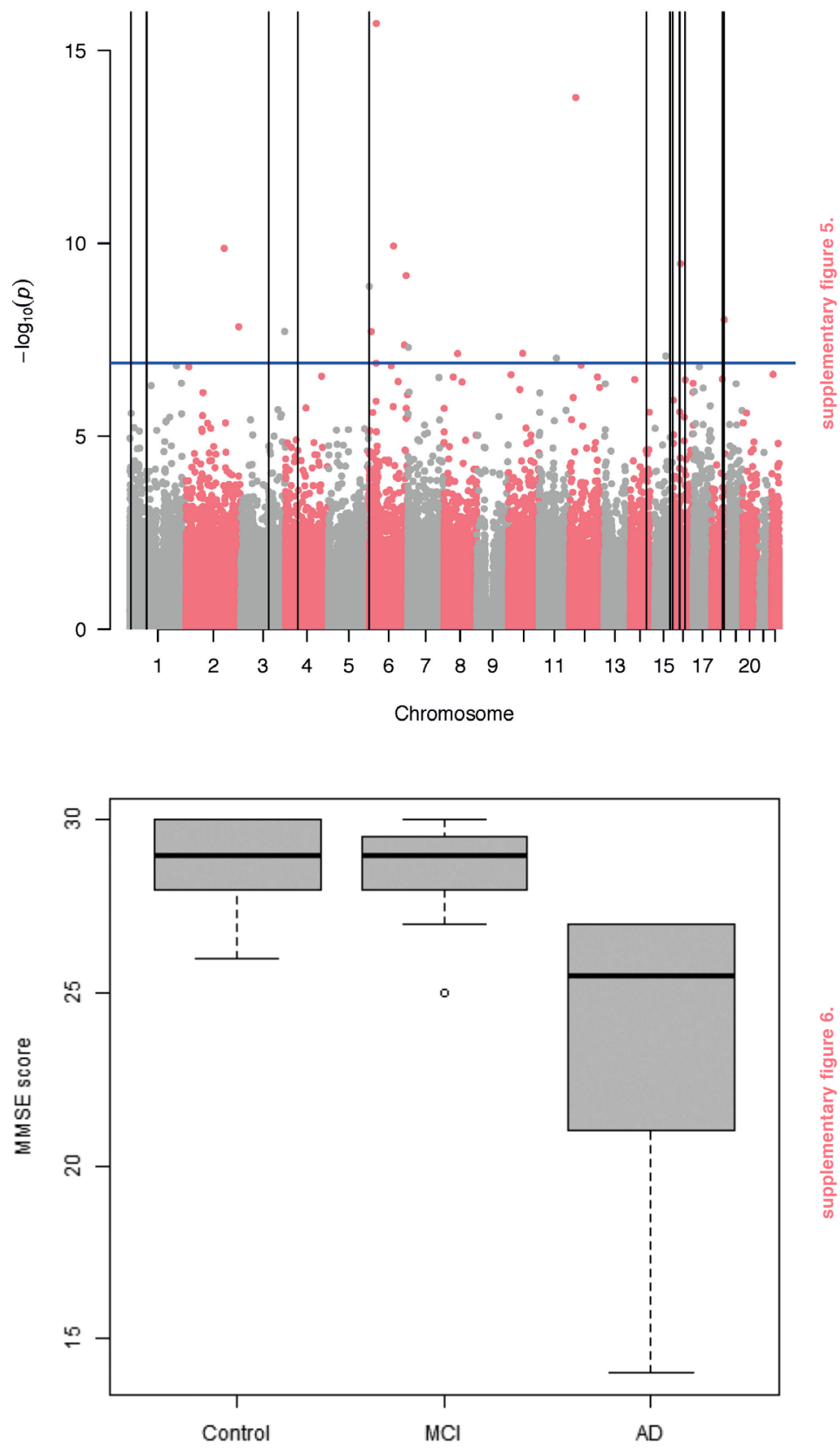

SUPPLEMENTARY FIGURE 5. Manhat-

tan plot of the Alzheimer's disease methylome-wide association study. The blue horizontal line indicates the genome-wide significance threshold (0.05 / \# probes in analysis). Vertical black lines indicate differentially

methylated regions as determined with $\operatorname{com} b$ - $p$, using a seeding $p$-value threshold of 0.05 and an extension window of 200 base pairs, and with more than two probes and a Šidák-adjusted $p$-value below 0.05 .
SUPPLEMENTARY FIGURE 6. Boxplot of mini-mental state examination (MMSE) scores for the control, mild cognitive impairment (MCI), and Alzheimer's disease (AD) groups.

SUPPLEMENTARY FIGURE 7. Histogram of test-statistics showing potential bias (A) and quantile-quantile (QQ) plot showing potential inflation (B) of the mini-mental state examination (MMSE) score association analysis in the Alzheimer's disease methylomic profile. The pink line in A represents the fit of the empirical null distribution and the black line the fit of the mixture of the empirical null distribution, the proportion of positively associated features, and the proportion of negatively associated features, both estimated as described in [33]. The estimated bias and inflation was -0.11 and 1 , respectively. 
A

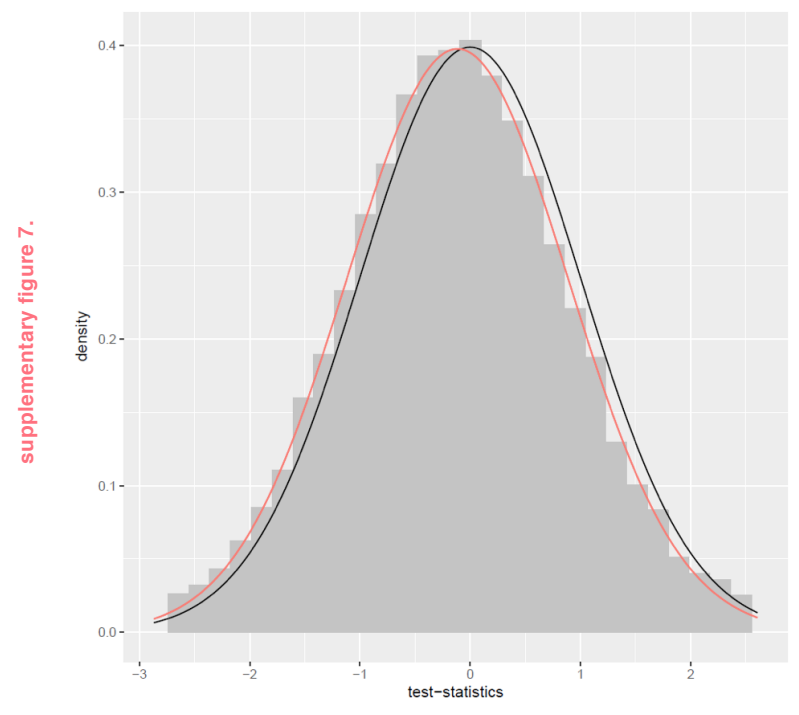

B

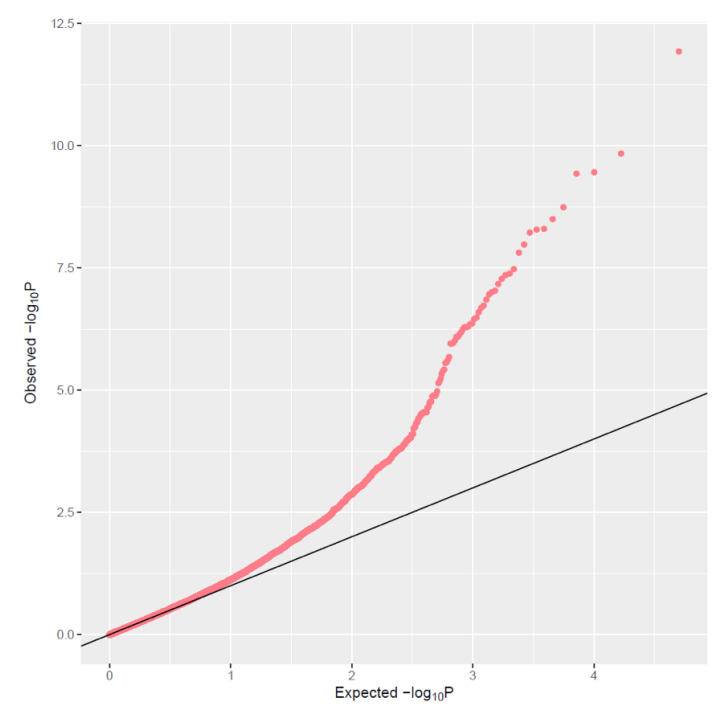

SUPPLEMENTARY FIGURE 8. Manhattan plot of the mini-mental state examination (MMSE) score association analysis in the Alzheimer's disease methylomic profile. The blue horizontal line indicates the genome-wide significance threshold (0.05 / \# probes in analysis). Vertical black lines indicate differentially methylated regions as determined with comb- $p$, using a seeding $p$-value threshold of 0.05 and an extension window of 200 base pairs, and with more than two probes and a Šidák-adjusted $p$-value below 0.05 .

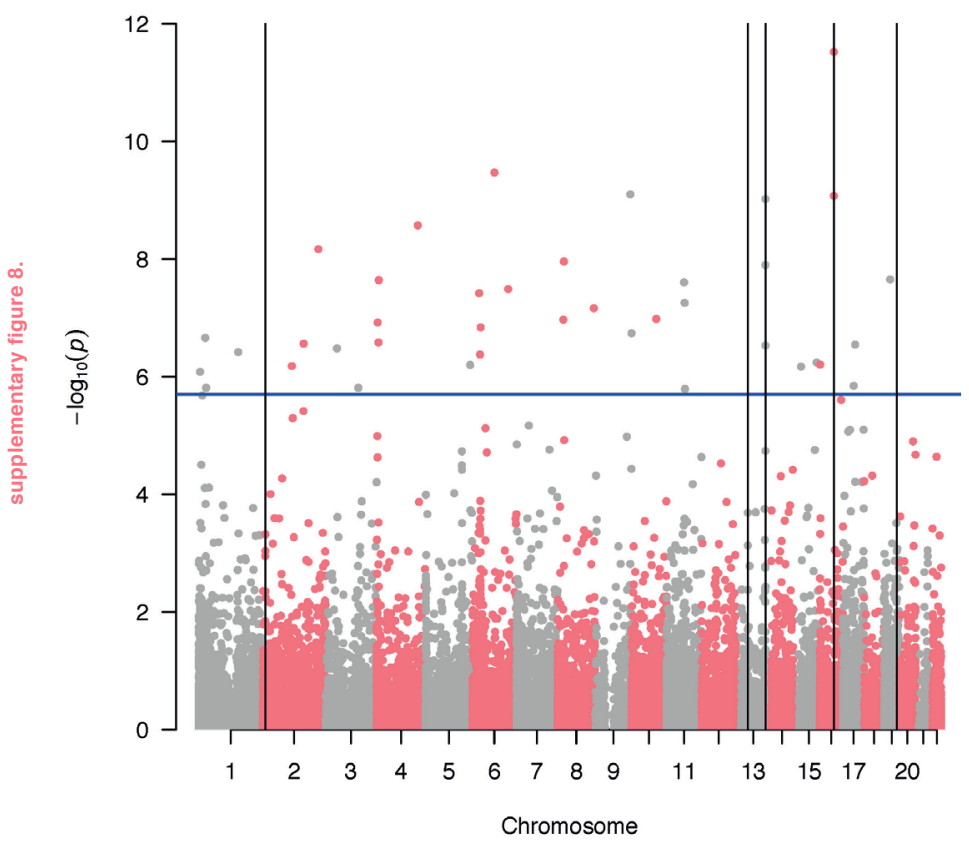




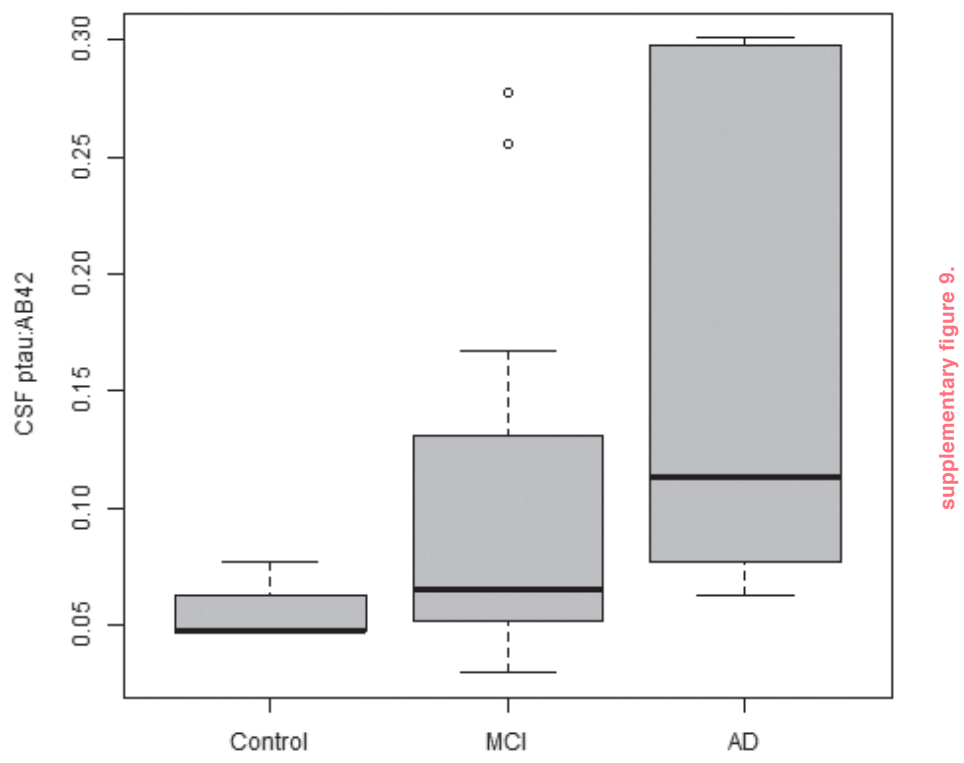

A

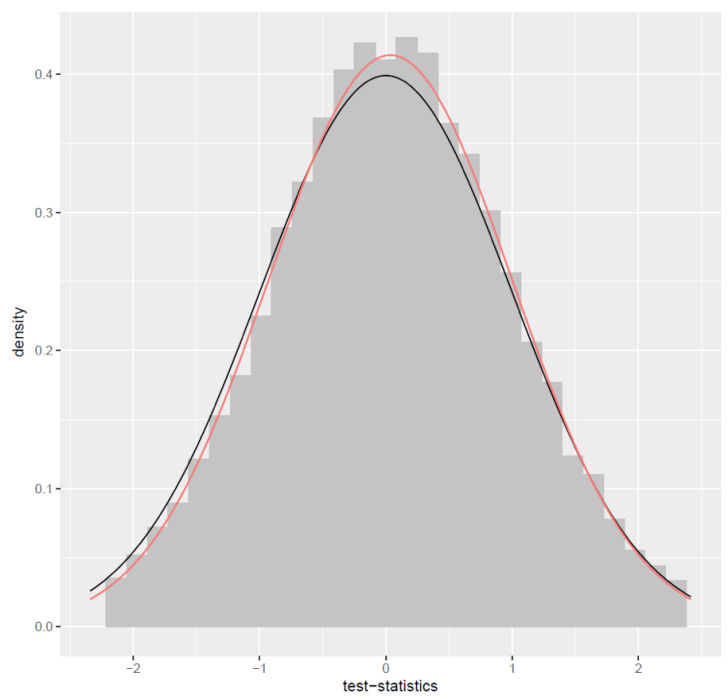

B

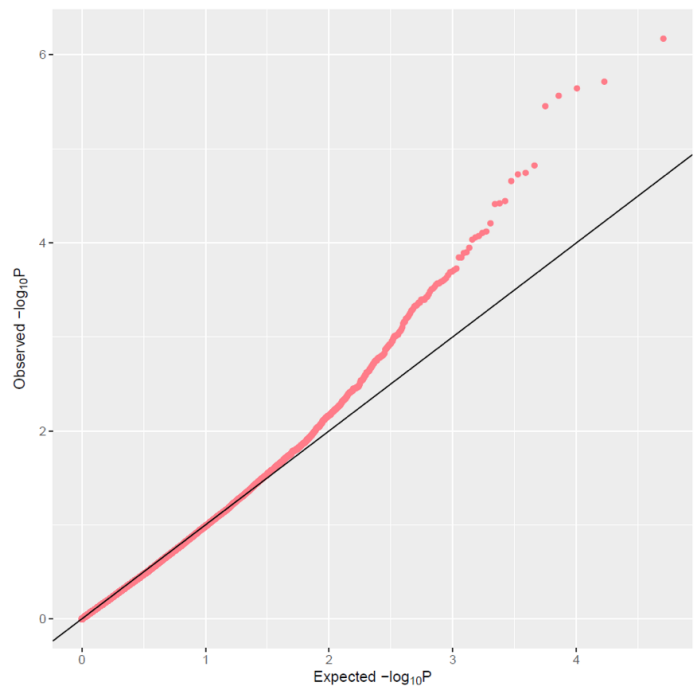

\section{SUPPLEMENTARY FIGURE 9. Boxplot} of the cerebrospinal fluid (CSF) phosphorylated tau (ptau) and amyloid- $\beta$ 1-42 (A $\beta 41$ ) ratio for the control, mild cognitive impairment (MCI), and Alzheimer's disease (AD) groups.
SUPPLEMENTARY FIGURE 10. Histogram of test-statistics showing potential bias (A) and quantile-quantile (QQ) plot showing potential inflation (B) of the cerebrospinal fluid phosphorylated tau and amyloid- $\beta$ ratio association analysis in the Alzheimer's disease methylomic profile. The pink line in A represents the fit of the empirical null distribution and the black line the fit of the mixture of the empirical null distribution, the proportion of positively associated features, and the proportion negatively associated features, both estimated as described in [33]. The estimated bias and inflation was 0.036 and 0.96 , respectively. 


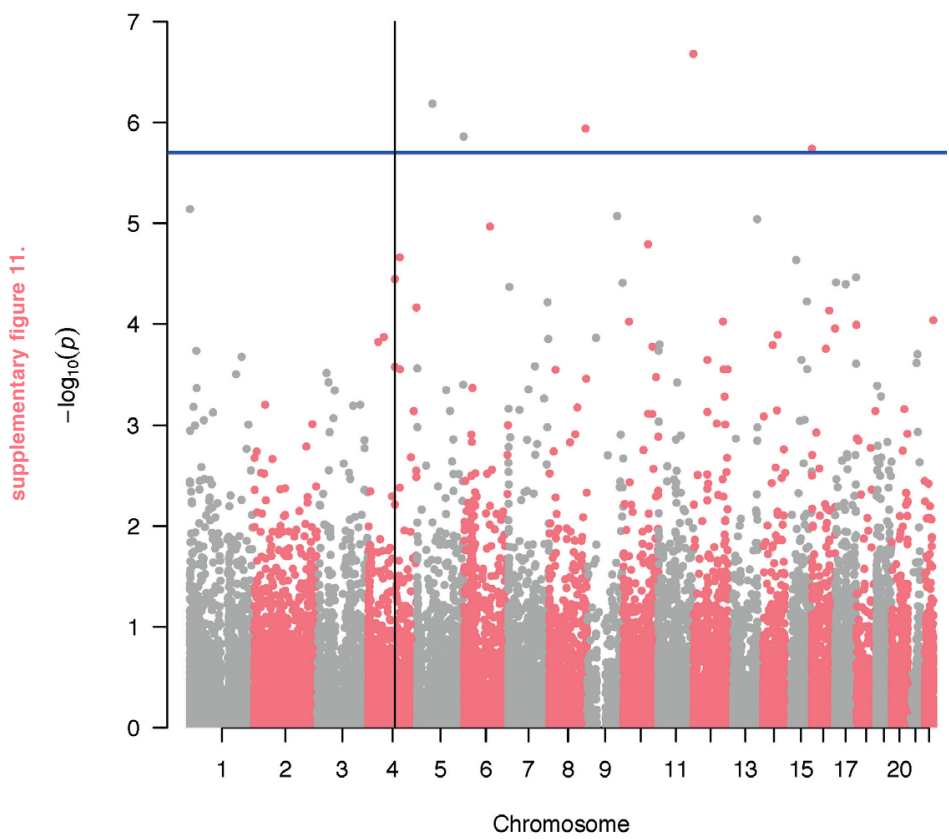

SUPPLEMENTARY FIGURE 11. Man-

hattan plot of the cerebrospinal fluid phosphorylated tau and amyloid- $\beta$ ratio association analysis in the $\mathrm{Alz}$ heimer's disease methylomic profile. The blue horizontal line indicates the genome-wide significance threshold (0.05 / \# probes in analysis). Vertical black lines indicate differentially methylated regions as determined with comb-p, using a seeding $p$-value threshold of 0.05 and an extension window of 200 base pairs, and with more than two probes and a Šidák-adjusted $p$-value below 0.05 .

\section{SUPPLEMENTARY FIGURE 12. Boxplot} of the mean hippocampal volume as percentage of the total intracranial volume for the mild cognitive impairment (MCI) and Alzheimer's disease (AD) groups.

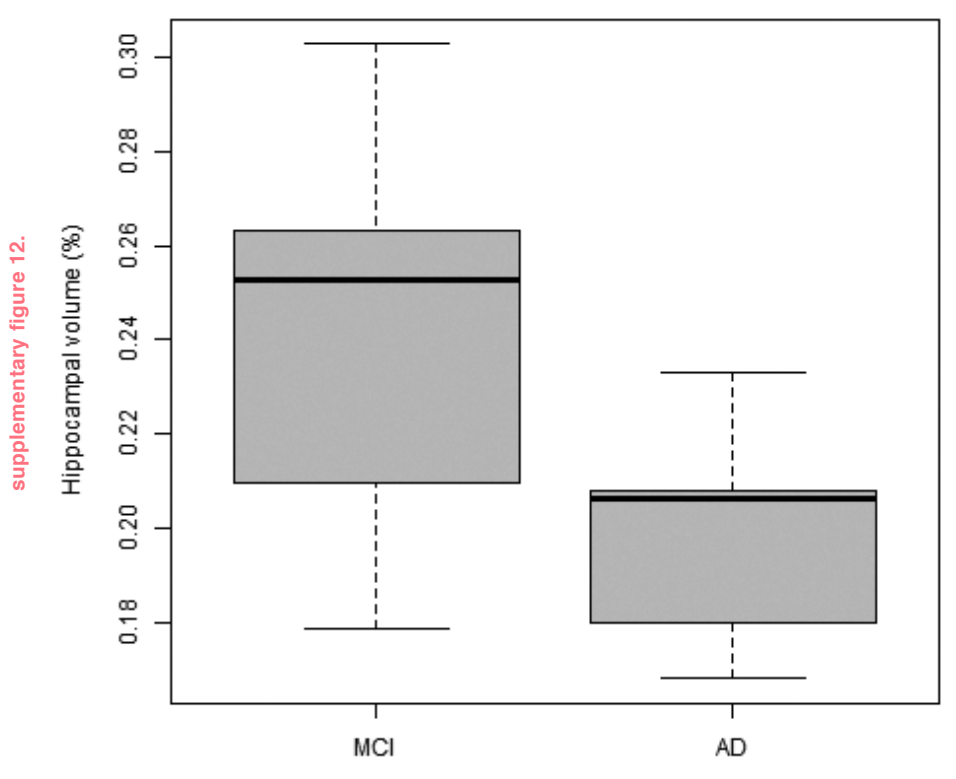



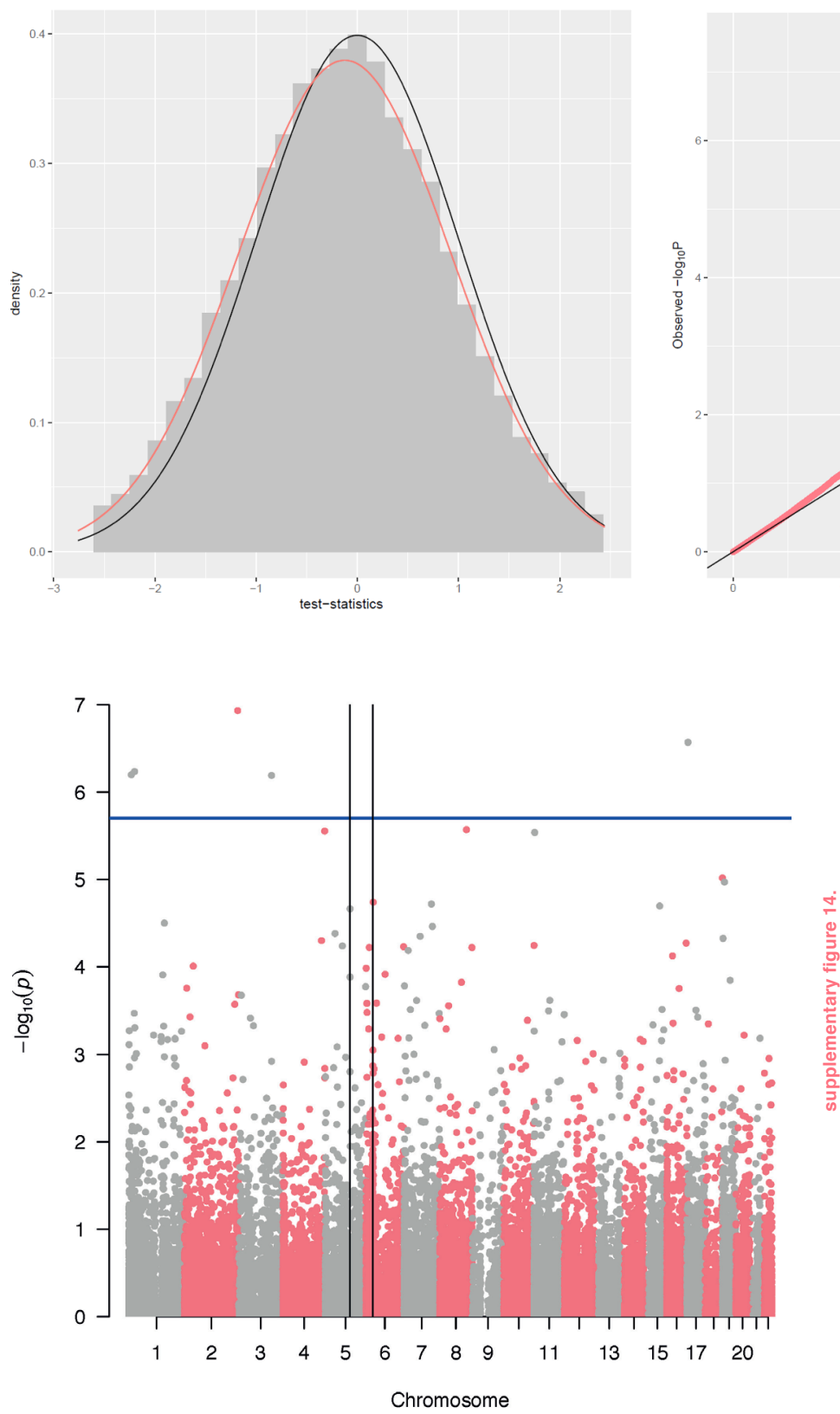

SUPPLEMENTARY FIGURE 13. Histogram of test-statistics showing potential bias (A) and quantile-quantile (QQ) plot showing potential inflation (B) of the mean hippocampal volume association analysis in the Alzheimer's disease methylomic profile.

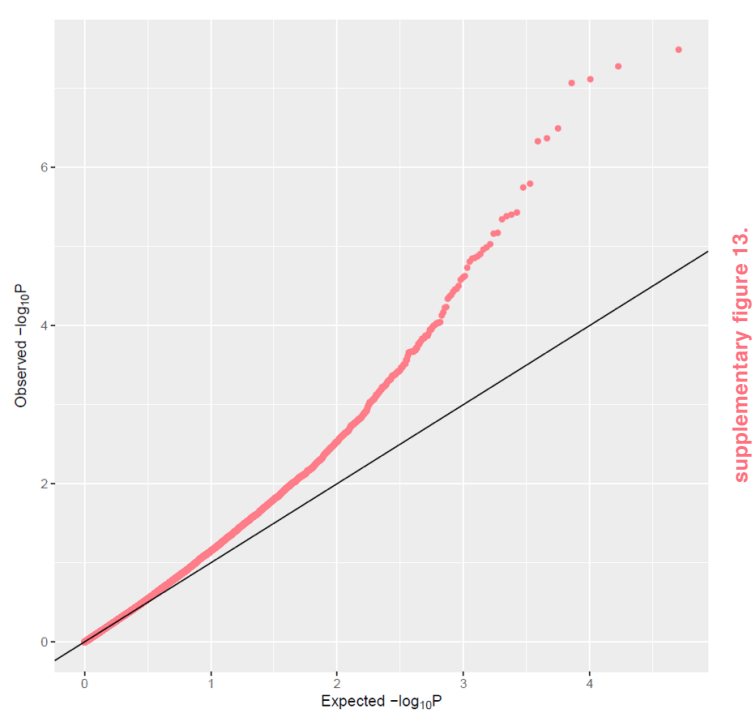

as described in [33]. The estimated bias and inflation was -0.12 and 1.1, respectively.

\section{SUPPLEMENTARY FIGURE 14. Man-}

hattan plot of the mean hippocampal volume association analysis in the Alzheimer's disease methylomic profile. The blue horizontal line indicates the genome-wide significance threshold (0.05/ \# probes in analysis). Vertical black lines indicate differentially methylated regions as determined with comb-p, using a seeding $p$-value threshold of 0.05 and an extension window of 200 base pairs, and with more than two probes and a Šidák-adjusted $p$-value below 0.05 . 


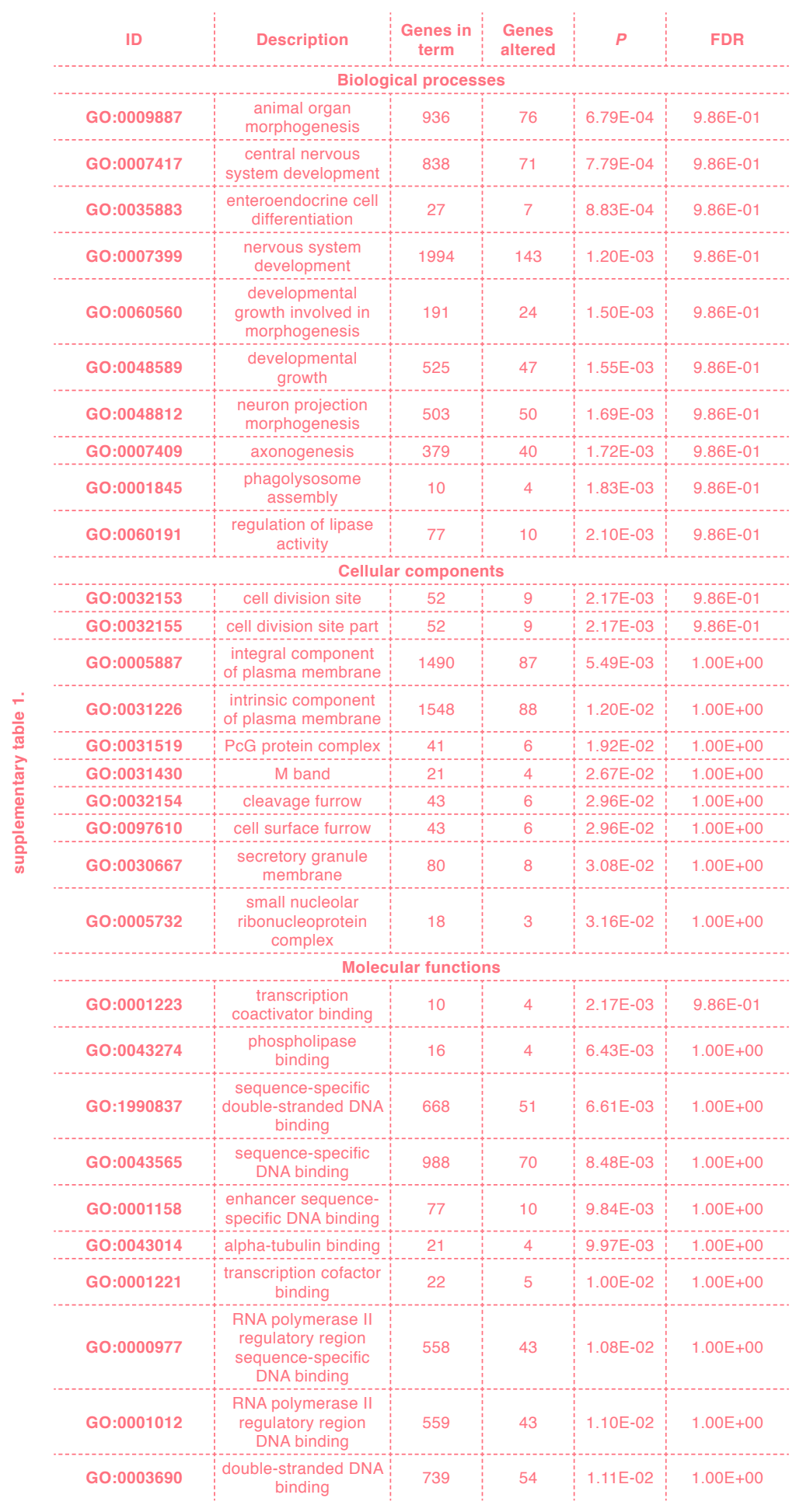

SUPPLEMENTARY TABLE 1. Top 10 GO terms for each ontology, enriched by genes with altered DNA methylation in relation to Alzheimer's disease. ABBREVIATIONS: FDR, false discovery rate adjusted $p$-values; GO, Gene Ontology. 


\begin{tabular}{|c|c|c|c|c|c|c|}
\hline ID & Description & $\begin{array}{l}\text { Genes } \\
\text { in term }\end{array}$ & $\begin{array}{l}\text { Genes } \\
\text { altered }\end{array}$ & Gene names & $P$ & FDR \\
\hline hsa04950 & $\begin{array}{l}\text { maturity onset } \\
\text { diabetes of the } \\
\text { young }\end{array}$ & 26 & 4 & $\begin{array}{l}\text { NEUROD } 1 ; \text { NKX2- } \\
2 ; \text { NKX6-1; PAX6 }\end{array}$ & 1.00E-02 & 9.13E-01 \\
\hline hsa00512 & $\begin{array}{l}\text { mucin type } \\
\text { O-glycan } \\
\text { biosynthesis }\end{array}$ & 31 & 4 & $\begin{array}{l}\text { B3GNT6; GALNT1 } \\
\text { GALNTL6; } \\
\text { GALNT9 }\end{array}$ & 1.31E-02 & $9.13 \mathrm{E}-01$ \\
\hline hsa05034 & alcoholism & 180 & 12 & $\begin{array}{c}\text { HIST3H2BB; } \\
\text { GNG4; NPY; } \\
\text { NTRK2; H2AFY2; } \\
\text { PRKACA; } \\
\text { BDNF; SLC6A3; } \\
\text { HIST1H2AJ; } \\
\text { HIST1H2BM; } \\
\text { HDAC10; } \\
\text { HIST3H2A }\end{array}$ & 2.02E-02 & $9.13 E-01$ \\
\hline hsa00130 & $\begin{array}{l}\text { ubiquinone } \\
\text { and other } \\
\text { terpenoid- } \\
\text { quinone } \\
\text { biosynthesis }\end{array}$ & 11 & 1 & VKORC1 & $2.85 E-02$ & $9.13 E-01$ \\
\hline hsa03018 & $\begin{array}{c}\text { RNA } \\
\text { degradation }\end{array}$ & 77 & 6 & $\begin{array}{l}\text { DCP1B; ENO3; } \\
\text { PABPC3; PFKL; } \\
\text { PABPC4; RQCD1 }\end{array}$ & 3.07E-02 & 9.13E-01 \\
\hline hsa04921 & $\begin{array}{l}\text { oxytocin } \\
\text { signaling } \\
\text { pathway }\end{array}$ & 153 & 13 & $\begin{array}{l}\text { MYL9; ADCY3; } \\
\text { ADCY7; EGFR; } \\
\text { GUCY1A2; } \\
\text { KCNJ12; MEF } 2 C ; \\
\text { MYLK; NFATC1; } \\
\text { NFATC2; PRKACA; } \\
\text { TRPM } 2 ; \text { CAMK } 2 B\end{array}$ & 3.94E-02 & 9.13E-01 \\
\hline hsa00600 & $\begin{array}{l}\text { sphingolipid } \\
\text { metabolism }\end{array}$ & 47 & 4 & $\begin{array}{l}\text { LASS1; LASS6; } \\
\text { UGT8; LASS4 }\end{array}$ & 4.10E-02 & 9.13E-01 \\
\hline hsa05012 & $\begin{array}{l}\text { Parkinson's } \\
\text { disease }\end{array}$ & 142 & 8 & $\begin{array}{c}\text { NDUFB6; } \\
\text { NDUFS3; PARK2; } \\
\text { PRKACA; } \\
\text { NDUFA4L2; } \\
\text { SLC6A3; SNCA; } \\
\text { UBE2G2 }\end{array}$ & 4.42E-02 & 9.13E-01 \\
\hline hsa04810 & $\begin{array}{l}\text { regulation } \\
\text { of actin } \\
\text { cytoskeleton }\end{array}$ & 212 & 16 & $\begin{array}{l}\text { MYL9; NCKAP1; } \\
\text { DIAPH1; EGFR; } \\
\text { FGFR3; FGFR2; } \\
\text { CYFIP1; FGF20; } \\
\text { MYH10; MYLK; } \\
\text { PDGFRA; PTK2; } \\
\text { VAV2; PIP5K1B; } \\
\text { ITGA8; BCAR1 }\end{array}$ & 4.43E-02 & 9.13E-01 \\
\hline hsa04350 & $\begin{array}{l}\text { TGF-beta } \\
\text { signaling } \\
\text { pathway }\end{array}$ & 84 & 7 & $\begin{array}{l}\text { E2F5; ID3; NBL1; } \\
\text { SMURF2; TFDP1; } \\
\text { TGFBR1; GDF5 }\end{array}$ & 4.80E-02 & $9.13 E-01$ \\
\hline
\end{tabular}

SUPPLEMENTARY TABLE 2. Top 10

KEGG pathways, enriched by genes with altered DNA methylation in relation to Alzheimer's disease.

ABBREVIATIONS: FDR, false discovery rate adjusted $p$-values; KEGG, Kyoto Encyclopedia of Genes and Genomes. 


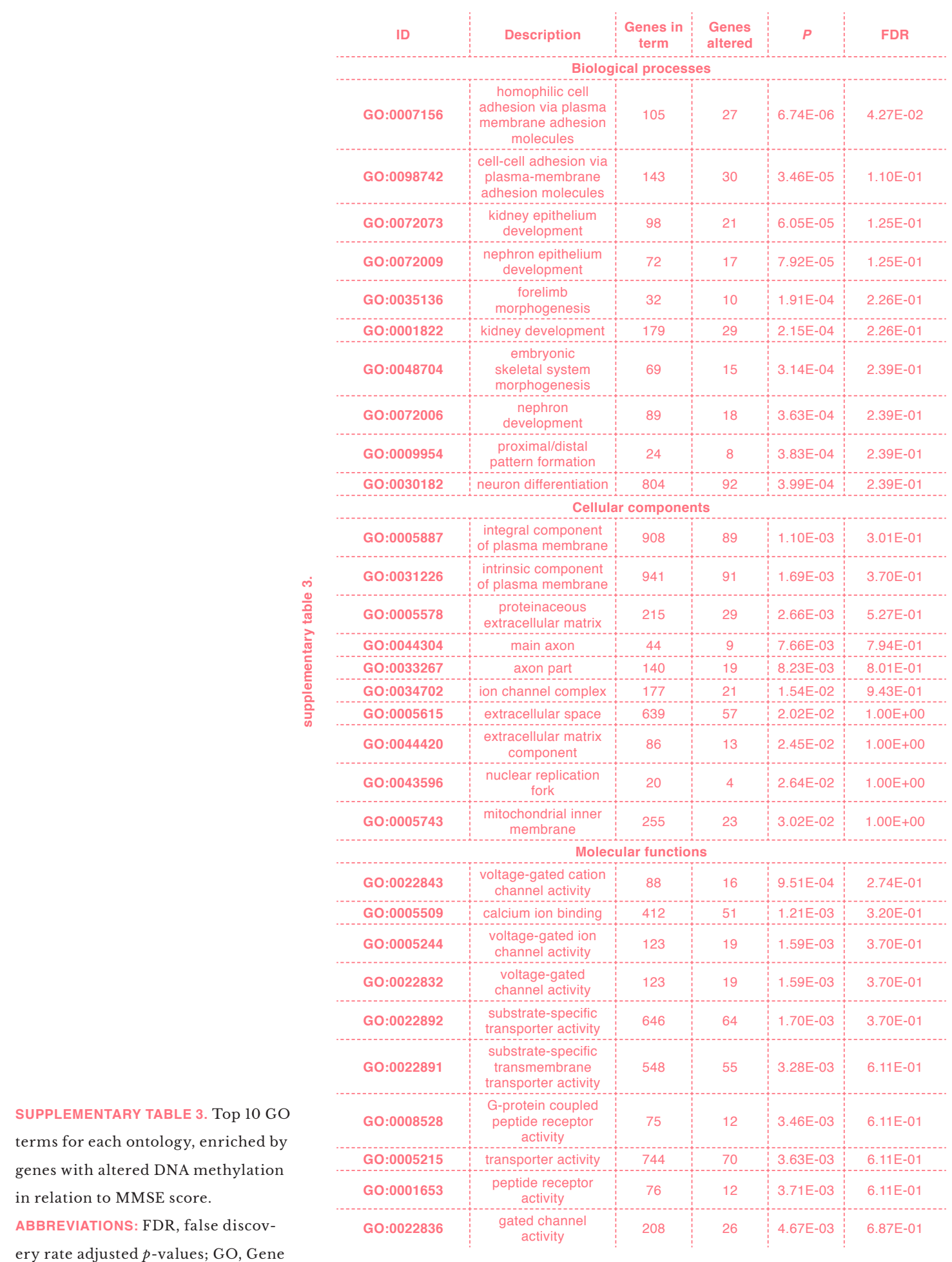




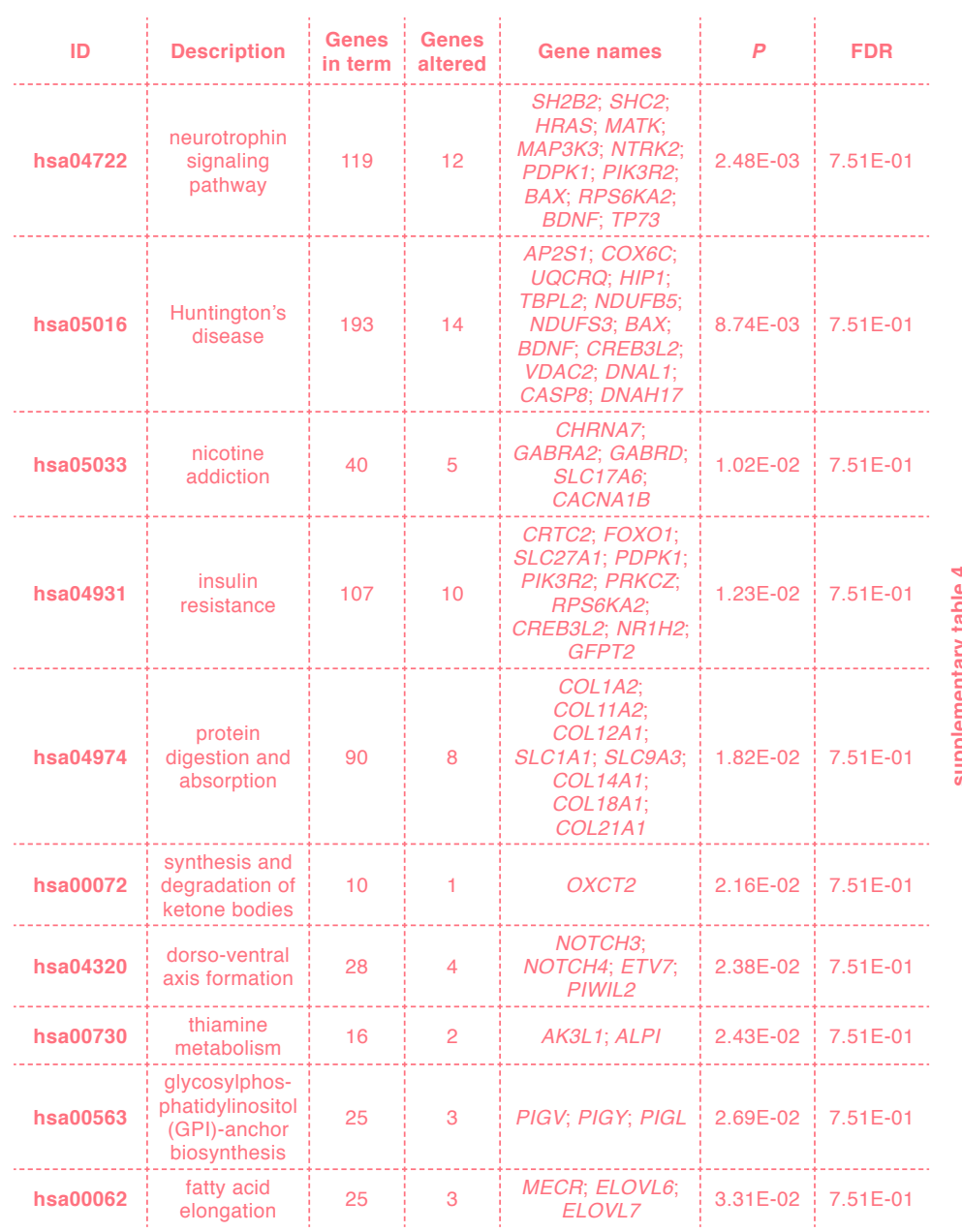

SUPPLEMENTARY TABLE 4. Top 10

KEGG pathways, enriched by genes with altered DNA methylation in relation to MMSE score.

ABBREVIATIONS: FDR, false discovery rate adjusted $p$-values; KEGG, Kyoto Encyclopedia of Genes and Genomes; MMSE, mini-mental state examination.

SupPlementary TABLE 5. Top 10 GO terms for each ontology, enriched by genes with altered DNA methylation in relation to CSF ptau:A $\beta 42$. ABBREVIATIONS: A $\beta 42$, amyloid- $\beta$ 1-42; CSF, cerebrospinal fluid; FDR, false discovery rate adjusted $p$-values; GO, Gene Ontology; ptau, phosphorylated tau. 


\begin{tabular}{|c|c|c|c|c|c|}
\hline ID & Description & $\begin{array}{c}\text { Genes in } \\
\text { term }\end{array}$ & $\begin{array}{l}\text { Genes } \\
\text { altered }\end{array}$ & $P$ & FDR \\
\hline GO:0060581 & $\begin{array}{l}\text { cell fate commitment } \\
\text { involved in pattern } \\
\text { specification }\end{array}$ & 11 & 5 & 2.53E-03 & $1.00 E+00$ \\
\hline GO:0050961 & $\begin{array}{l}\text { detection of } \\
\text { temperature stimulus } \\
\text { involved in sensory } \\
\text { perception }\end{array}$ & 11 & 5 & 3.19E-03 & $1.00 \mathrm{E}+00$ \\
\hline GO:0050965 & $\begin{array}{l}\text { detection of } \\
\text { temperature stimulus } \\
\text { involved in sensory } \\
\text { perception of pain }\end{array}$ & 11 & 5 & $3.19 E-03$ & $1.00 \mathrm{E}+00$ \\
\hline \multicolumn{6}{|c|}{ Cellular components } \\
\hline GO:0005887 & $\begin{array}{l}\text { integral component } \\
\text { of plasma membrane }\end{array}$ & 908 & 86 & 4.32E-03 & $1.00 \mathrm{E}+00$ \\
\hline GO:0031226 & $\begin{array}{l}\text { intrinsic component } \\
\text { of plasma membrane }\end{array}$ & 941 & 88 & 6.23E-03 & $1.00 \mathrm{E}+00$ \\
\hline GO:0044445 & cytosolic part & 131 & 16 & $1.52 \mathrm{E}-02$ & $1.00 E+00$ \\
\hline GO:0005581 & collagen trimer & 52 & 9 & $1.86 \mathrm{E}-02$ & $1.00 \mathrm{E}+00$ \\
\hline GO:0043197 & dendritic spine & 81 & 12 & $3.58 \mathrm{E}-02$ & 1.00E +00 \\
\hline GO:0044309 & neuron spine & 81 & 12 & $3.58 \mathrm{E}-02$ & $1.00 \mathrm{E}+00$ \\
\hline GO:0043209 & myelin sheath & 99 & 12 & $3.68 \mathrm{E}-02$ & 1.00E +00 \\
\hline GO:0097542 & ciliary tip & 29 & 5 & 4.64E-02 & $1.00 \mathrm{E}+00$ \\
\hline GO:0044459 & $\begin{array}{c}\text { plasma membrane } \\
\text { part }\end{array}$ & 1464 & 123 & 5.66E-02 & $1.00 E+00$ \\
\hline GO:0043235 & receptor complex & 198 & 22 & 5.86E-02 & $1.00 \mathrm{E}+00$ \\
\hline \multicolumn{6}{|c|}{ Molecular functions } \\
\hline GO:0005085 & $\begin{array}{l}\text { guanyl-nucleotide } \\
\text { exchange factor } \\
\text { activity }\end{array}$ & 201 & 31 & 7.07E-04 & $1.00 \mathrm{E}+00$ \\
\hline GO:0030674 & $\begin{array}{l}\text { protein binding, } \\
\text { bridging }\end{array}$ & 100 & 16 & 2.96E-03 & $1.00 \mathrm{E}+00$ \\
\hline GO:0005088 & $\begin{array}{l}\text { Ras guanyl- } \\
\text { nucleotide exchange } \\
\text { factor activity }\end{array}$ & 153 & 23 & 5.89E-03 & $1.00 \mathrm{E}+00$ \\
\hline GO:0060090 & binding, bridging & 110 & 16 & $9.48 \mathrm{E}-03$ & $1.00 \mathrm{E}+00$ \\
\hline GO:0008528 & $\begin{array}{l}\text { G-protein coupled } \\
\text { peptide receptor } \\
\text { activity }\end{array}$ & 75 & 11 & 1.01E-02 & 1.00E+00 \\
\hline GO:0001653 & $\begin{array}{l}\text { peptide receptor } \\
\text { activity }\end{array}$ & 76 & 11 & 1.06E-02 & $1.00 \mathrm{E}+00$ \\
\hline GO:0005198 & $\begin{array}{l}\text { structural molecule } \\
\text { activity }\end{array}$ & 375 & 39 & 1.32E-02 & $1.00 \mathrm{E}+00$ \\
\hline GO:0030159 & $\begin{array}{c}\text { receptor signaling } \\
\text { complex scaffold } \\
\text { activity }\end{array}$ & 19 & 5 & 1.99E-02 & $1.00 E+00$ \\
\hline GO:0016763 & $\begin{array}{c}\text { transferase activity, } \\
\text { transferring pentosyl } \\
\text { groups }\end{array}$ & 30 & 5 & 3.40E-02 & $1.00 \mathrm{E}+00$ \\
\hline GO:0001637 & $\begin{array}{l}\text { G-protein coupled } \\
\text { chemoattractant } \\
\text { receptor activity }\end{array}$ & 12 & 3 & $3.59 E-02$ & $1.00 \mathrm{E}+00$ \\
\hline
\end{tabular}




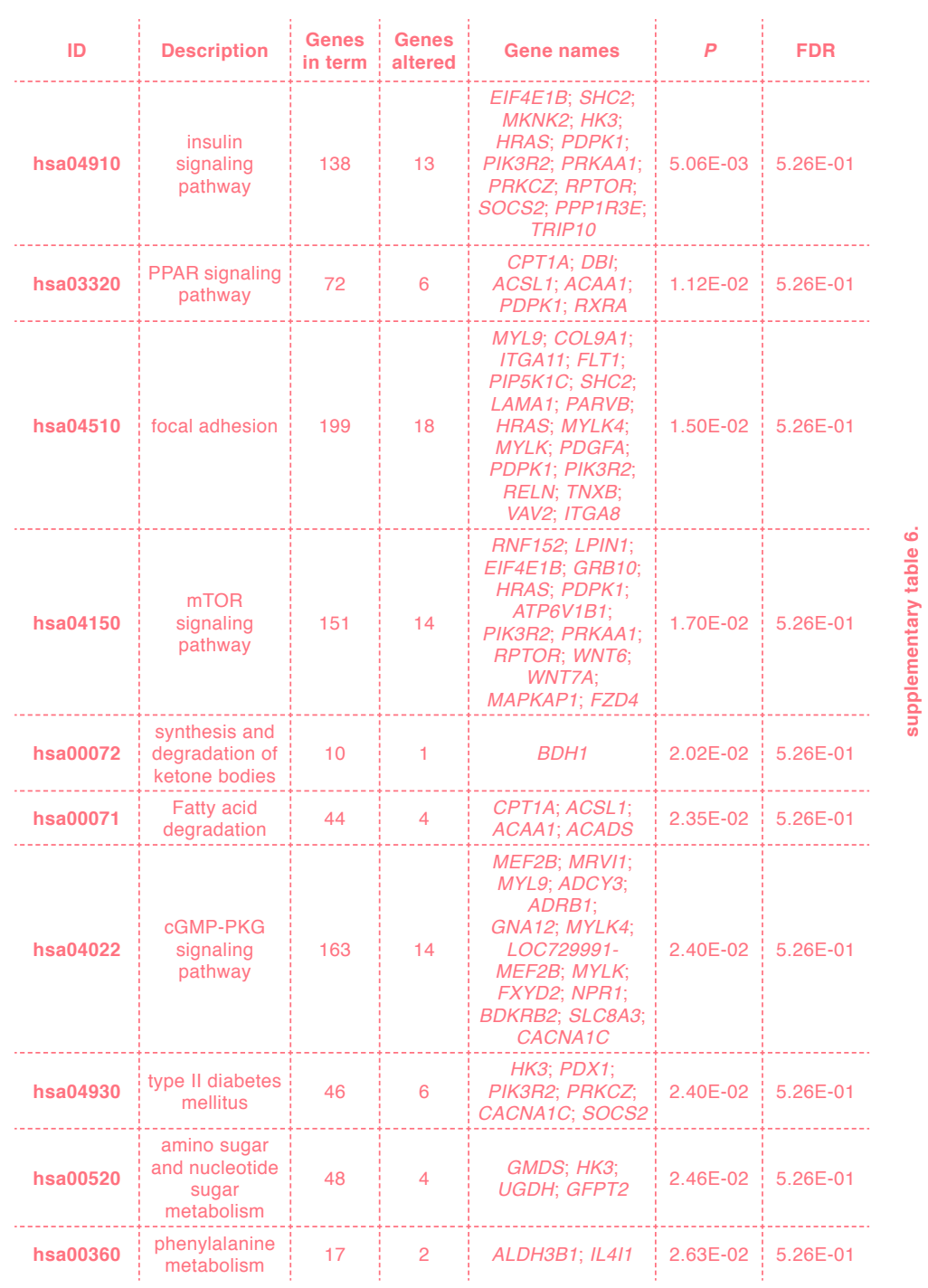

SUPPLEMENTARY TABLE 6. Top 10

KEGG pathways, enriched by genes

with altered DNA methylation in

relation to CSF ptau:A $\beta 42$.

ABBREVIATIONS: A $\beta 42$, amyloid- $\beta$

1-42; CSF, cerebrospinal fluid; FDR,

false discovery rate adjusted $p$-values;

KEGG, Kyoto Encyclopedia of Genes

and Genomes; ptau, phosphorylated

tau. 


\begin{tabular}{|c|c|c|c|c|c|c|c|}
\hline & & ID & Description & $\begin{array}{l}\text { Genes in } \\
\text { term }\end{array}$ & $\begin{array}{l}\text { Genes } \\
\text { altered }\end{array}$ & $P$ & FDR \\
\hline & & \multicolumn{6}{|c|}{ Biological processes } \\
\hline & & GO:0071875 & $\begin{array}{l}\text { adrenergic receptor } \\
\text { signaling pathway }\end{array}$ & 13 & 6 & $4.35 \mathrm{E}-04$ & 8.29E-01 \\
\hline & & GO:0050954 & $\begin{array}{l}\text { sensory perception } \\
\text { of mechanical } \\
\text { stimulus }\end{array}$ & 99 & 18 & 4.54E-04 & 8.29E-01 \\
\hline & & GO:0097435 & fibril organization & 15 & 6 & $5.62 \mathrm{E}-04$ & $8.29 \mathrm{E}-01$ \\
\hline & & GO:0002456 & $\begin{array}{l}\text { T cell mediated } \\
\text { immunity }\end{array}$ & 34 & 9 & $6.84 \mathrm{E}-04$ & 8.29E-01 \\
\hline & & GO:0032098 & regulation of appetite & 12 & 5 & $7.62 \mathrm{E}-04$ & $8.29 E-01$ \\
\hline & & GO:0007605 & $\begin{array}{l}\text { sensory perception } \\
\text { of sound }\end{array}$ & 88 & 16 & $7.86 \mathrm{E}-04$ & 8.29E-01 \\
\hline & & GO:0021545 & $\begin{array}{l}\text { cranial nerve } \\
\text { development }\end{array}$ & 33 & 9 & 1.75E-03 & $1.00 \mathrm{E}+00$ \\
\hline & & GO:1903037 & $\begin{array}{l}\text { regulation of } \\
\text { leukocyte cell-cell } \\
\text { adhesion }\end{array}$ & 154 & 22 & $2.30 \mathrm{E}-03$ & $1.00 \mathrm{E}+00$ \\
\hline & & $\mathrm{GO}: 0008306$ & associative learning & 52 & 10 & 2.38E-03 & $1.00 \mathrm{E}+00$ \\
\hline & & GO:0014850 & $\begin{array}{l}\text { response to muscle } \\
\text { activity }\end{array}$ & 14 & 5 & $2.45 \mathrm{E}-03$ & $1.00 \mathrm{E}+00$ \\
\hline & & \multicolumn{6}{|c|}{ Cellular components } \\
\hline & & GO:0005578 & $\begin{array}{c}\text { proteinaceous } \\
\text { extracellular matrix }\end{array}$ & 215 & 28 & $3.76 \mathrm{E}-03$ & $1.00 \mathrm{E}+00$ \\
\hline & & GO:0005615 & extracellular space & 639 & 56 & $1.27 E-02$ & $1.00 \mathrm{E}+00$ \\
\hline & & GO:0031082 & BLOC complex & 12 & 3 & $1.71 \mathrm{E}-02$ & $1.00 \mathrm{E}+00$ \\
\hline & & GO:0005871 & kinesin complex & 34 & 6 & $1.84 \mathrm{E}-02$ & $1.00 \mathrm{E}+00$ \\
\hline & $\stackrel{N}{0}$ & GO:0005884 & actin filament & 49 & 9 & $2.02 \mathrm{E}-02$ & $1.00 E+00$ \\
\hline & $\underset{\substack{\frac{0}{\pi} \\
\frac{\pi}{2}}}{\frac{0}{2}}$ & GO:0008076 & $\begin{array}{l}\text { voltage-gated } \\
\text { potassium channel } \\
\text { complex }\end{array}$ & 64 & 10 & $2.46 \mathrm{E}-02$ & $1.00 \mathrm{E}+00$ \\
\hline & $\stackrel{\Xi}{\Xi}$ & GO:0034705 & $\begin{array}{l}\text { potassium channel } \\
\text { complex }\end{array}$ & 65 & 10 & 2.58E-02 & $1.00 \mathrm{E}+00$ \\
\hline & 을 & GO:0031226 & $\begin{array}{l}\text { intrinsic component } \\
\text { of plasma membrane }\end{array}$ & 941 & 80 & $2.81 \mathrm{E}-02$ & $1.00 E+00$ \\
\hline & & GO:0005887 & $\begin{array}{l}\text { integral component } \\
\text { of plasma membrane }\end{array}$ & 908 & 77 & 2.90E-02 & $1.00 \mathrm{E}+00$ \\
\hline & & GO:0030864 & $\begin{array}{l}\text { cortical actin } \\
\text { cytoskeleton }\end{array}$ & 37 & 6 & $3.15 \mathrm{E}-02$ & $1.00 \mathrm{E}+00$ \\
\hline & & \multicolumn{6}{|c|}{ Molecular functions } \\
\hline & & GO:0005179 & hormone activity & 54 & 10 & $1.46 \mathrm{E}-03$ & $1.00 \mathrm{E}+00$ \\
\hline & & GO:0004888 & $\begin{array}{l}\text { transmembrane } \\
\text { signaling receptor } \\
\text { activity }\end{array}$ & 503 & 50 & 4.23E-03 & $1.00 \mathrm{E}+00$ \\
\hline & & GO:0043236 & laminin binding & 17 & 5 & $4.51 \mathrm{E}-03$ & $1.00 E+00$ \\
\hline & & GO:0005001 & $\begin{array}{l}\text { transmembrane } \\
\text { receptor protein } \\
\text { tyrosine phosphatase } \\
\text { activity }\end{array}$ & 14 & 5 & 8.08E-03 & $1.00 \mathrm{E}+00$ \\
\hline & & GO:0019198 & $\begin{array}{l}\text { transmembrane } \\
\text { receptor protein } \\
\text { phosphatase activity }\end{array}$ & 14 & 5 & 8.08E-03 & $1.00 E+00$ \\
\hline & & GO:0035064 & $\begin{array}{l}\text { methylated histone } \\
\text { binding }\end{array}$ & 31 & 7 & $9.83 E-03$ & $1.00 \mathrm{E}+00$ \\
\hline & & GO:0099600 & $\begin{array}{l}\text { transmembrane } \\
\text { receptor activity }\end{array}$ & 535 & 51 & 9.97E-03 & $1.00 \mathrm{E}+00$ \\
\hline SUPPLEMENTARY TABLE 7. Top 10 GO & & GO:0038023 & $\begin{array}{l}\text { signaling receptor } \\
\text { activity }\end{array}$ & 575 & 54 & $1.01 \mathrm{E}-02$ & $1.00 \mathrm{E}+00$ \\
\hline 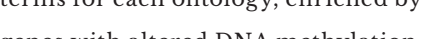 & & GO:0043394 & proteoglycan binding & 18 & 5 & $1.21 \mathrm{E}-02$ & $1.00 E+00$ \\
\hline $\begin{array}{l}\text { genes with altered DNA methylation } \\
\text { in relation to hippocampal volume. }\end{array}$ & & GO:0008066 & $\begin{array}{c}\text { glutamate receptor } \\
\text { activity }\end{array}$ & 21 & 5 & $1.34 \mathrm{E}-02$ & $1.00 \mathrm{E}+00$ \\
\hline
\end{tabular}




\begin{tabular}{|c|c|c|c|c|c|c|}
\hline ID & Description & $\begin{array}{l}\text { Genes } \\
\text { in term }\end{array}$ & $\begin{array}{l}\text { Genes } \\
\text { altered }\end{array}$ & Gene names & $P$ & FDR \\
\hline hsa05033 & $\begin{array}{l}\text { nicotine } \\
\text { addiction }\end{array}$ & 40 & 6 & $\begin{array}{c}\text { SLC17A8; } \\
\text { GABRA1; } \\
\text { GABRA5; GRIN1; } \\
\text { GRIN2A; SLC17A6 }\end{array}$ & 1.96E-03 & 5.97E-01 \\
\hline hsa04024 & $\begin{array}{l}\text { cAMP signaling } \\
\text { pathway }\end{array}$ & 198 & 16 & $\begin{array}{c}\text { ADCY3; ADRB2; } \\
\text { GLI3; GRIN1; } \\
\text { GRIN2A; NFATC1; } \\
\text { ATP1B2; NPR1; } \\
\text { ATP2B4; PDE4D; } \\
\text { GHRL; FXYD1; } \\
\text { BRAF; VAV2; } \\
\text { VIPR2; CREB5 }\end{array}$ & 8.00E-03 & 8.70E-01 \\
\hline hsa04610 & $\begin{array}{l}\text { complement } \\
\text { and } \\
\text { coagulation } \\
\text { cascades }\end{array}$ & 79 & 5 & $\begin{array}{c}C D 55 ; \text { F2RL2; } \\
C D 46 ; T H B D ; \\
\text { C1QC }\end{array}$ & 1.23E-02 & 8.70E-01 \\
\hline hsa00760 & $\begin{array}{l}\text { nicotinate and } \\
\text { nicotinamide } \\
\text { metabolism }\end{array}$ & 29 & 4 & $\begin{array}{c}\text { NT5C2; NT5C3; } \\
\text { NADSYN1; } \\
\text { NAPRT1 }\end{array}$ & 1.64E-02 & 8.70E-01 \\
\hline hsa04672 & $\begin{array}{l}\text { intestinal } \\
\text { immune } \\
\text { network for IgA } \\
\text { production }\end{array}$ & 49 & 4 & $\begin{array}{l}\text { HLA-DMB; CCL25; } \\
\text { CXCL12; TNFSF13 }\end{array}$ & 1.65E-02 & 8.70E-01 \\
\hline hsa04974 & $\begin{array}{l}\text { protein } \\
\text { digestion and } \\
\text { absorption }\end{array}$ & 90 & 8 & $\begin{array}{c}\text { COL1A1; COL5A1; } \\
\text { COL9A1; } \\
\text { SLC6A19; KCNQ1; } \\
\text { ATP1B2; SLC8A3; } \\
\text { SLC9A3 }\end{array}$ & 1.88E-02 & 8.70E-01 \\
\hline hsa04080 & $\begin{array}{l}\text { neuroactive } \\
\text { ligand-receptor } \\
\text { interaction }\end{array}$ & 278 & 16 & $\begin{array}{c}\text { ADRB2; F2RL2; } \\
\text { GABRA1; } \\
\text { GABRA5; GRIK3; } \\
\text { GRIN1; GRIN2A; } \\
\text { GRMT; GRM8; } \\
\text { MTNR1A; NMBR; } \\
\text { AVPR1A; PTGFR; } \\
\text { TACR2; TBXA2R; } \\
\text { VIPR2 }\end{array}$ & 2.93E-02 & 8.70E-01 \\
\hline hsa04970 & $\begin{array}{l}\text { salivary } \\
\text { secretion }\end{array}$ & 90 & 7 & $\begin{array}{c}\text { ADCY3; CST3; } \\
\text { ADRB } 2 ; \text { KCNMA1; } \\
\text { ATP1B2; ATP } 2 B 4 ; \\
\text { SLC4A2 }\end{array}$ & 3.38E-02 & 8.70E-01 \\
\hline hsa04940 & $\begin{array}{c}\text { type I diabetes } \\
\text { mellitus }\end{array}$ & 43 & 4 & $\begin{array}{l}\text { HLA-DMB; HLA-E; } \\
\text { ICA1; PTPRN2 }\end{array}$ & 3.78E-02 & 8.70E-01 \\
\hline hsa04120 & $\begin{array}{l}\text { ubiquitin } \\
\text { mediated } \\
\text { proteolysis }\end{array}$ & 137 & 10 & $\begin{array}{c}\text { RHOBTB2; } \\
\text { NEDD4L; RCHY1; } \\
\text { PARK2; UBR5; } \\
\text { UBE2D2; CUL3; } \\
\text { TRIP12; UBE3C; } \\
\text { CDC20 }\end{array}$ & 4.29E-02 & 8.70E-01 \\
\hline
\end{tabular}

SUPPLEMENTARY TABLE 8. Top 10

KEGG pathways, enriched by genes with altered DNA methylation in relation to hippocampal volume.

ABBREVIATIONS: FDR, false discovery rate adjusted $p$-values; KEGG, Kyoto Encyclopedia of Genes and Genomes. 




\section{IDENTIFICATION OF PRE- CLINICAL METHYLOMIC BLOOD MARKERS ASSO- CIATED WITH CONVER- SION TO ALZHEIMER'S DISEASE}

ROY LARDENOIJE ${ }^{A}$, MARKUS LEBER ${ }^{\mathrm{B}}$, EHSAN PISHVA ${ }^{\mathrm{A}, \mathrm{C}}$, BART P.F. RUTTEN $^{A}$, DANIËL L.A. VAN DEN HOVEA ${ }^{A, D}$, ALFREDO RAMIREZ ${ }^{B}$

ASCHOOL FOR MENTAL HEALTH AND NEUROSCIENCE (MHENS), DEPARTMENT OF PSYCHIATRY AND NEUROPSYCHOLOGY, MAASTRICHT UNIVERSITY, UNIVERSITEITSSINGEL 50, 6200 MD MAASTRICHT, THE NETHERLANDS ${ }^{B}$ GERMAN CENTER FOR NEURODEGENERATIVE DISEASES (DZNE), BONN, GERMANY 'UNIVERSITY OF EXETER MEDICAL SCHOOL, UNIVERSITY OF EXETER, EXETER, UK.

'LABORATORY OF TRANSLATIONAL NEUROSCIENCE, DEPARTMENT OF PSYCHIATRY, PSYCHOSOMATICS AND PSYCHOTHERAPY, UNIVERSITY OF WUERZBURG, FUECHSLEINSTRASSE 15, 97080 WUERZBURG, GERMANY 


\section{Abstract}

Previous studies have made a solid case for the entanglement of epigenetic processes in the etiopathogenesis of Alzheimer's disease (AD). However, these investigations have mainly focused on the direct comparison of AD cases and healthy controls. The present study employs a longitudinal design, including only healthy aged individuals without signs of dementia at baseline, subdivided into converters to AD dementia $(n=55)$ and non-converters $(n=44)$ at follow-up $\sim 4.5$ years later. In this sample, blood methylomic profiles of converters and nonconverters were compared at baseline to identify differences in DNA methylation associated with subsequent conversion to $A D$, before the presence of clinical symptoms. Additionally, time-related methylomic changes were compared between converters and non-converters in a group by time interaction analysis, to reveal differences in methylomic changes that may dynamically signify conversion to dementia or, alternatively, protective mechanisms preventing such a conversion. The comparison between converters and non-converters at baseline led to the identification of 35 differentially methylated positions (DMPs), including one in the AD-susceptibility gene $H L A-D R B 5,43$ differentially methylated regions (DMRs), including one located in OXT, which was recently shown to exhibit altered DNA methylation in postmortem brain samples of patients with AD. Additionally, 113 DMPs and 3 DMRs showed different time-related methylation patterns between converters and controls, also including a DMP in HLA-DRB5 and one in another human leukocyte antigen gene, $H L A-D Q A 1$. Whilst these findings provide important evidence for epigenetic involvement in the etiopathogenesis of $A D$, they furthermore are a solid basis for further investigations into the diagnostic and prognostic value of blood methylomic markers, and give preliminary insight into which genes may be involved in the stages before the development of clinical dementia, or, alternatively, resilience to conversion.

KEYWORDS: Alzheimer's disease; preclinical; epigenetics; DNA methylation; blood 


\subsection{Introduction}

Alzheimer's disease (AD) is a progressive neurodegenerative disorder with a complex, as of yet not well understood, etiology [1, 2]. A distinction can be made between early-onset familial $A D$ and the far more common late-onset sporadic variant of $A D$ [3]. While both variants have a strong genetic origin, the relationship between genetic risk factors and disease development remains elusive, particularly for the sporadic variant [4]. It is therefore thought that a combination of genetic and environmental factors is responsible not only for triggering, but also for determining the pace of disease pathogenesis. In recent years, epigenetics has risen as an important player in $A D$, although it remains to be unveiled whether its role is that of an innocent bystander or one of the main kingpins [5-7]. Nevertheless, where genetic factors can identify persons at risk for developing $A D$, epigenetic markers may offer more dynamic views on trajectories of (biological) change and therefore a more timely insight into the very early stages of $A D$, perhaps even before any clinical symptoms arise. Identifying disease-predicting biological profiles before the appearance of clinical manifestations may give potential treatments a better timeframe to successfully impede, or even halt disease progression $[8,9]$. Additionally, due the dynamic nature of epigenetics, identification of epigenetic profiles associated with the pathogenesis of the disease may also open novel therapeutic avenues [10,11].

Previous studies implicating epigenetic dysregulation on various levels in $A D$ have laid important groundwork, but focus mainly on directly comparing $A D$ patients and healthy controls, or the association of epigenetic markers with mainstay hallmarks such as amyloid beta and phosphorylated tau $[5,6,12]$. The current study uses blood samples from the AgeCoDe study, comprising a large aging cohort of elderly ( $>75$ years, $\mathrm{n}=3,327$ ) who had no signs of dementia at baseline and were monitored across the conversion to AD over time [13]. This allowed for the posthoc selection of a group of controls that did not develop dementia over the course of $\sim 4,5$ years and a group of converters that had developed clinical $A D$ at follow-up. Using this design, converters and non-converting controls could be compared at a stage before the development of clinical dementia, to gain insight into the blood methylomic profile associated with this preclinical stage. Additionally, time-related methylomic changes could be compared between converters and controls, to identify rapidly changing sites of DNA methylation that reflect the latest stages in the development of clinical AD. 


\subsection{Methods \\ 9.2.1. Subjects}

A subsample of 99 individuals aged above 75 years was selected from the German Study on Ageing, Cognition and Dementia in Primary Care Patients (AgeCoDe) cohort, based on the criteria outlined below (Table 1) [13]. Of these, 55 were converters, who showed no signs of dementia at baseline, had DNA samples available at baseline and follow-up (after 4.5 years), and were diagnosed with $A D$ dementia at follow-up. The remaining 44 subjects constitute the control group and had to adhere to the same criteria, except that they should have no signs of dementia both at baseline and follow-up. The groups were matched for age, gender, and $A P O E$ genotype. The presence of dementia was assessed in all subjects with the Structured Interview for Diagnosis of Dementia of Alzheimer Type, Multi-infarct Dementia, and Dementia of Other Etiology (SIDAM) [14], and the diagnosis of AD was based on the criteria of the National Institute of Neurological and Communicative Disorders and Stroke and the Alzheimer's Disease and Related Disorders Association (NINCDSADRDA) [15], a score of 4 or higher on the Global Deterioration Scale (GDS) [16], and a Clinical Dementia Rating (CDR) of 1 [17]. DNA samples from whole blood were used to determine the methylomic profile at baseline and follow-up with the HumanMethylation450 BeadChip (450K BeadChip; Illumina, San Diego, CA, USA).

\begin{tabular}{|c|c|c|c|}
\hline & $\mathrm{N}$ & $\begin{array}{c}\text { Gender } \\
\text { (female / male) }\end{array}$ & $\begin{array}{l}\text { Age at baseline } \\
\text { (mean } \pm \text { SD) }\end{array}$ \\
\hline Converters & 55 & $17 / 38$ & $82.33 \pm 3.52$ \\
\hline Controls & 44 & $12 / 32$ & $81.14 \pm 3.10$ \\
\hline Total & 99 & $29 / 70$ & $81.80 \pm 3.38$ \\
\hline
\end{tabular}

\subsubsection{Processing of array data}

All data processing and analysis was performed in the statistical programming environment $R$ (version 3.3.2) [18], running on SUSE Linux Enterprise Server 11 (SUSE LINUX GmbH, Nürnberg, Germany). The MethylAid package (version 1.6.2) [19] was used to detect outlying samples based on control probes on the array. Raw IDAT files were loaded into R using the minfi package (version 1.18.6) [20]. The 'pfilter' function of the wateRmelon package (version 1.17.0) [21] was used for probe filtering; probes with readings from less than 3 beads in $5 \%$ or

TABLE 1. Sample overview. ABBREVIATIONS: SD, standard deviation. 
more of the samples, and probes with a detection $p$-value above 0.05 in at least $1 \%$ of the samples were removed. Additionally, samples with more than $1 \%$ probes with a detection $p$-value greater than 0.05 were excluded. After removal of probes based on technical performance, probes overlapping SNPs (common in Europe) and repeats, crossreacting probes, and probes with mapping issues as recommended by Zhou et al. [22] were also removed. The removed set of probes ('MASK. general.EUR') was included in the DNAmArray package ('hm450. manifest.pop.GoNL'; version 0.0.2) [23]. Normalization was done according to the dasen method [24], as incorporated in the wateRmelon package. Additional quality control was performed by comparing the reported with the predicted gender (using the 'getSex.DNAmArray' function) and by visual inspection of a dendrogram after clustering the samples. For clustering, hierarchical clustering was performed with the 'hclust' function from the fastcluster package (version 1.1.22) [25]. Probes were annotated using the 'TxDb.Hsapiens.UCSC.hg19.knownGene' database, retrieved with the 'cpgInfo' function from DNAmArray and gene, with additional gene feature information added manually, as described by Price et al. [26].

\subsubsection{Data analysis}

To account for the heterogeneity of cell types in whole blood, cellular components were estimated for CD4+ and CD8+ T cells, B cells, monocytes, granulocytes, and natural killer cells using the 'estimateCellCounts' function of minfi [27, 28].

M-values were used for all subsequent analyses [29], calculated with the 'beta2m' function of lumi (version 2.27.3) [30], and probes targeting the $X$ and $Y$ chromosomes were excluded. Beta values are reported as percentages. Mean DNA methylation levels were determined for each individual by averaging the values of all probes, after which the converters and controls were compared at baseline and follow-up using linear regression.

Association analyses were performed with the limma package (version 3.28.21) [31]. To find methylation sites that (i) distinguish converters from controls at baseline and (ii) sites that show alternative time-related changes for converters and controls, a model was constructed with $\mathrm{M}$-values as outcome and a factor variable with four levels (control at baseline, converter at baseline, control at follow-up, and converter at follow-up), as main outcome. As covariates were included age, gender, chip barcode, position on the chip, and the blood cell type estimates. Correlation between samples from the same individual were determined 
with 'duplicateCorrelation' from limma and added to the 'ImFit' limma function, used for fitting the model, to account for the within-subject comparisons between baseline and follow-up. After fitting the model, contrasts were determined (i) between converters and controls at baseline, and (ii) between the differences between converters and controls at baseline and at follow-up, and empirical Bayes moderation of the test statistics was applied [39]. Results were then adjusted for bias and inflation using the empirical null distribution with the bacon package (version 1.3.5) [32]. Probes with a Benjamini-Hochberg false discovery rate (FDR) corrected $p$-value below 0.05 signified differentially methylated positions (DMPs). The changes over time of the DMPs identified in the group by time interaction analysis were also investigated with limma for the converters and non-converters separately to identify sites that only change in one group (as opposed to differences in the magnitude of change) and which may therefore signify sites particularly involved in the progression of dementia, or resilience against such progression.

The results of the association analysis were subsequently used to determine differentially methylated regions (DMRs) with comb-p [41], with a seeding $p$-value of 0.05 and an extension distance of 200 base pairs. Only regions with a corrected $p$-value below 0.05 and comprising at least two probes were termed DMRs. Comb-p corrects for multiple testing with the Šidák method. As for the DMPs from the group by time interaction analysis, the DMRs were also investigated separately for converters and non-converters.

To investigate the potential biological impact of the altered methylomic profiles, probes were ranked based on a combined $p$-value and effect size score, and used for a Gene Ontology (GO) and Kyoto Encyclopedia of Genes and Genomes (KEGG) gene set enrichment analysis. The top 1000 probes, based on the combined ranking, were used as input for the enrichment analysis, which was performed with the missMethyl package (version 1.6.2), using modified versions of the 'gometh' function for the GO analysis and 'gsameth' function for the KEGG analysis. This package was chosen because it takes into account that genes are not covered by the same amount of probes, possibly leading to bias [42, 43]. All probes included in the limma analysis were used as background list for the enrichment analyses. The results were filtered to only include GO terms with between 10 and 2000 genes, and KEGG pathways consisting of more than 10 genes (the largest KEGG pathway contains 1272 genes). 


\subsection{Results}

Out of the 198 samples (99 baseline and 99 follow-up), 28 did not pass the stringent quality control (13 converters and 15 controls) and due to the longitudinal design, the matching samples from the same individuals also had to be excluded, leaving a total of 142 samples. Supplementary Figure 1 shows a density and boxplot of the raw beta values, and Supplementary Figure 2 shows these for the normalized beta values. After processing, 430,904 probes remained for the analyses. No differences in global DNA methylation were found between converters and controls at both timepoints (controls baseline $=51.19 \%$, converters baseline $=51.25 \%, p=8.97 \mathrm{E}-01$; controls follow-up $=51.22 \%$, converters follow-up $=51.22 \%, p=9.55 \mathrm{E}-01)$.

\subsubsection{Methylomic differences before the development of dementia}

After determination of the contrast between converters to AD dementia and controls at baseline, bacon detected and adjusted for a bias of -0.042 and inflation of 0.96 (Supplementary Figure 3). Following FDR correction, 35 DMPs were identified, the top 3 of which were annotated to $H L A-D R B 5$, $T B X 2$, and $P C D H B 5$, and $A D G R D 1$ and $H L A-D R B 5$ were associated with multiple DMPs (2 and 4, respectively) (Table 2; Supplementary Figure 4). In addition to the DMPs, 43 DMRs were detected, with the top 3 DMRs located in PRRT1, OXT, and CACNA2D2 (Table 3; Supplementary Figure 4).

The top 1000 probes for the gene set enrichment analyses were annotated to 760 unique genes. After FDR correction, 68 enriched GO terms were found, with a strong representation of plasma membrane components and related processes such as transmembrane transport, but also terms related to synaptic functioning (Table 4). As for KEGG, 12 pathways were enriched, many relating to the immune system or related diseases, including diabetes mellitus type I, but also a pathway related to the glutamatergic synapse (Table 5). 


\begin{tabular}{|c|c|c|c|c|c|c|}
\hline Probe & Gene & Region & ES & SE & $P$ & FDR \\
\hline cg14531663 & HLA-DRB5 & 3'UTR & 1.14 & 0.20 & $7.48 \mathrm{E}-09$ & $2.08 \mathrm{E}-03$ \\
\hline $\operatorname{cg} 04304333$ & $T B \times 2$ & & 0.46 & 0.08 & 1.14E-08 & $2.08 \mathrm{E}-03$ \\
\hline cg13601275 & PCDHB5 & $1^{\text {st }}$ Exon & 1,00 & 0.18 & $1.45 \mathrm{E}-08$ & $2.08 \mathrm{E}-03$ \\
\hline cg06493930 & GAS2 & $\begin{array}{l}\text { 5'UTR; } 1^{\text {st }} \\
\text { Exon }\end{array}$ & -1.16 & 0.21 & 2.32E-08 & 2.12E-03 \\
\hline cg05871254 & HS3ST3B1 & Body & -0.83 & 0.15 & 2.46E-08 & 2.12E-03 \\
\hline cg19746397 & $\mathrm{LHX3}$ & Body & 0.89 & 0.16 & $3.43 E-08$ & $2.34 \mathrm{E}-03$ \\
\hline cg22670759 & CACNA2D2 & Body & 0.58 & 0.11 & $3.80 \mathrm{E}-08$ & $2.34 \mathrm{E}-03$ \\
\hline $\operatorname{cg} 21191810$ & CEP85L & TSS 1500 & 1.24 & 0.23 & $6.69 \mathrm{E}-08$ & $3.41 \mathrm{E}-03$ \\
\hline cg13782301 & HLA-DRB5 & 3'UTR & 0.73 & 0.14 & $7.12 \mathrm{E}-08$ & 3.41E-03 \\
\hline cg18114294 & KIAA1614 & & 0.51 & 0.10 & $9.34 \mathrm{E}-08$ & 4.03E-03 \\
\hline cg17272563 & HLA-DRB5 & 3'UTR & 0.61 & 0.11 & 1.16E-07 & 4.48E-03 \\
\hline cg26827033 & ADGRD1 & Body & 0.40 & 0.07 & $1.25 \mathrm{E}-07$ & $4.48 \mathrm{E}-03$ \\
\hline cg00386456 & PTH2 & Body & 0.95 & 0.18 & $2.56 \mathrm{E}-07$ & $8.49 \mathrm{E}-03$ \\
\hline cg26270027 & TECTA & Body & -0.92 & 0.18 & $3.14 \mathrm{E}-07$ & $9.11 \mathrm{E}-03$ \\
\hline cg08182160 & PDE2A & $\begin{array}{l}1^{\text {st Exon; }} \\
5 \text { 'UTR }\end{array}$ & -0.87 & 0.17 & $3.27 \mathrm{E}-07$ & $9.11 \mathrm{E}-03$ \\
\hline cg11051095 & ADGRD1 & Body & -0.58 & 0.11 & $3.38 \mathrm{E}-07$ & $9.11 \mathrm{E}-03$ \\
\hline $\mathrm{cg} 08549605$ & ADGRA1 & & -0.52 & 0.10 & $4.59 \mathrm{E}-07$ & 1.16E-02 \\
\hline $\operatorname{cg} 23264547$ & EXT2 & Body & -0.55 & 0.11 & $5.37 \mathrm{E}-07$ & 1.29E-02 \\
\hline $\operatorname{cg} 10664768$ & ZBTB46 & Body & -1.04 & 0.21 & $6.65 \mathrm{E}-07$ & $1.51 \mathrm{E}-02$ \\
\hline $\mathrm{cg} 00223593$ & ALG10 & & 0.65 & 0.13 & 8.37E-07 & $1.80 \mathrm{E}-02$ \\
\hline cg09415485 & $\mathrm{CDH} 13$ & Body & -0.83 & 0.17 & $1.23 \mathrm{E}-06$ & $2.53 \mathrm{E}-02$ \\
\hline cg14915854 & ARHGAP1 & TSS200 & -0.77 & 0.16 & $1.39 \mathrm{E}-06$ & $2.73 \mathrm{E}-02$ \\
\hline $\operatorname{cg} 25203916$ & TRIM63 & Body & -0.33 & 0.07 & $1.55 \mathrm{E}-06$ & $2.91 \mathrm{E}-02$ \\
\hline $\mathrm{cg} 07482373$ & MAPK 12 & TSS200 & -0.41 & 0.09 & $1.64 \mathrm{E}-06$ & $2.93 \mathrm{E}-02$ \\
\hline cg08530537 & DPEP3 & $\begin{array}{l}1^{\text {st }} \text { Exon; } \\
\text { 5'UTR }\end{array}$ & 1.07 & 0.22 & 1.70E-06 & 2.93E-02 \\
\hline cg21232671 & NKX2-8 & & 1.12 & 0.24 & $2.02 \mathrm{E}-06$ & 3. $26 \mathrm{E}-02$ \\
\hline cg12275981 & TLR5 & 5'UTR & 0.48 & 0.10 & $2.04 \mathrm{E}-06$ & 3.26E-02 \\
\hline $\operatorname{cg} 27545611$ & $\begin{array}{c}\text { GNG12- } \\
\text { AS1 }\end{array}$ & TSS1500 & 0.72 & 0.15 & $2.18 \mathrm{E}-06$ & 3.36E-02 \\
\hline cg03654598 & SHANK1 & Body & -0.67 & 0.14 & 2.45E-06 & 3.63E-02 \\
\hline cg04200224 & SCGN & TSS200 & 0.45 & 0.09 & $2.58 \mathrm{E}-06$ & $3.71 \mathrm{E}-02$ \\
\hline cg24029819 & PTPRN2 & Body & 0.85 & 0.18 & 2.82E-06 & 3.91E-02 \\
\hline cg18243760 & HLA-DRB5 & 3'UTR & 0.58 & 0.12 & 3.07E-06 & 3.96E-02 \\
\hline cg17100218 & SMAD2 & & 0.41 & 0.09 & 3.09E-06 & 3.96E-02 \\
\hline cg06247406 & GRIK2 & TSS200 & 0.53 & 0.11 & 3.13E-06 & 3.96E-02 \\
\hline $\operatorname{cg} 21571166$ & $\mathrm{ZIC1}$ & 3'UTR & 0.99 & 0.21 & $3.92 \mathrm{E}-06$ & $4.82 \mathrm{E}-02$ \\
\hline
\end{tabular}

TABLE 2. DMPs of the baseline methylome-wide association analysis. ABBREVIATIONS: DMPs, differentially methylated positions; ES, effect size; FDR, false discovery rate adjusted $p$-values; SE, standard error; TSS, transcription start site; UTR, untranslated region.

TABLE 3. DMRs of the baseline methylome-wide association analysis. ABBREVIATIONS: CDS, coding DNA sequence; chr, chromosome; DMRs, differentially methylated regions; Šidák, Šidák-corrected $p$-values; TSS, transcription start site; UTR, untranslated region. 


\begin{tabular}{|c|c|c|c|c|c|}
\hline Position & Gene & Region & $\begin{array}{c}\# \\
\text { probes }\end{array}$ & $P$ & Šidák \\
\hline $\begin{array}{c}\text { chr6:31846769- } \\
31847029\end{array}$ & SLC44A4 & $\begin{array}{l}\text { TSS; Exon; } \\
\text { 5'UTR }\end{array}$ & 8 & $2.30 E-12$ & 3.81E-09 \\
\hline $\begin{array}{c}\text { chr1:68517177- } \\
68517274\end{array}$ & $\begin{array}{c}\text { GNG12-AS1; } \\
\text { DIRAS3 }\end{array}$ & $\begin{array}{c}\text { Intron; Exon; } \\
\text { 5'UTR }\end{array}$ & 4 & $1.49 \mathrm{E}-11$ & $6.63 E-08$ \\
\hline $\begin{array}{c}\text { chr6: } 25652381- \\
25652408\end{array}$ & SCGN & Intergenic & 6 & $1.97 \mathrm{E}-11$ & $3.14 \mathrm{E}-07$ \\
\hline $\begin{array}{c}\text { chr5:140700449- } \\
140700639\end{array}$ & TAF7 & Intergenic & 7 & $2.75 E-10$ & $6.24 \mathrm{E}-07$ \\
\hline $\begin{array}{c}\text { chr18:45275452- } \\
45275568\end{array}$ & SMAD2 & Intergenic & 2 & $3.64 E-10$ & 1.35E-06 \\
\hline $\begin{array}{c}\text { chr12:75784855- } \\
75784885\end{array}$ & GLIPR1L2 & Exon; 5'UTR & 4 & $8.55 E-10$ & $1.23 E-05$ \\
\hline $\begin{array}{c}\text { chr6:32053600- } \\
32053749\end{array}$ & $T N X B$ & CDS & 5 & 1.51E-09 & 4.36E-06 \\
\hline $\begin{array}{c}\text { chr19:44324856- } \\
44324952\end{array}$ & LYPD5 & Intergenic & 6 & 9.76E-09 & 4.38E-05 \\
\hline $\begin{array}{c}\text { chr10:29698447- } \\
29698471\end{array}$ & SVIL-AS1 & Exon & 5 & 1.24E-08 & $2.22 \mathrm{E}-04$ \\
\hline $\begin{array}{c}\text { chr15:90039794- } \\
90039823\end{array}$ & $R H C G$ & $\begin{array}{c}\text { Exon; } 5 \text { UTR; } \\
\text { TSS }\end{array}$ & 5 & 1.63E-08 & 2.43E-04 \\
\hline $\begin{array}{c}\text { chr } 3: 170304031- \\
170304046\end{array}$ & LOC101928583 & Intron & 2 & 3.07E-08 & 8.81E-04 \\
\hline $\begin{array}{c}\text { chr6:32223076- } \\
32223115\end{array}$ & LOC101929163 & Exon & 4 & 4.86E-08 & 5.36E-04 \\
\hline $\begin{array}{c}\mathrm{chr} 1: 223316219- \\
223316273\end{array}$ & TLR5 & Intron; 5'UTR & 3 & 5.97E-08 & 4.76E-04 \\
\hline $\begin{array}{c}\text { chr2:177503592- } \\
177503640\end{array}$ & LINC01117 & Intron & 3 & 8.44E-08 & 7.57E-04 \\
\hline $\begin{array}{c}\text { chr18:13611370 } \\
13611490\end{array}$ & LDLRAD4 & $\begin{array}{l}\text { TSS; Exon; } \\
\text { 5'UTR; Intron }\end{array}$ & 5 & 8.81E-08 & $3.16 \mathrm{E}-04$ \\
\hline $\begin{array}{c}\text { chr5:68710808- } \\
68710832\end{array}$ & MARVELD2 & Intergenic & 4 & 8.83E-08 & $1.58 \mathrm{E}-03$ \\
\hline $\begin{array}{c}\text { chr17:37123669- } \\
37123910\end{array}$ & FBXO47 & Intergenic & 5 & $9.77 E-08$ & $1.75 \mathrm{E}-04$ \\
\hline $\begin{array}{c}\text { chr10:90343166- } \\
90343286\end{array}$ & RNLS & Intergenic & 6 & 1.15E-07 & 4.11E-04 \\
\hline $\begin{array}{c}\text { chr3:10806047- } \\
10806176\end{array}$ & LINC00606 & Intergenic & 4 & 1.19E-07 & $3.98 \mathrm{E}-04$ \\
\hline $\begin{array}{c}\text { chr1:212688916- } \\
212688998\end{array}$ & LINC01740 & Intergenic & 3 & $2.15 E-07$ & $1.13 \mathrm{E}-03$ \\
\hline $\begin{array}{c}\text { chr6:101846767- } \\
101846806\end{array}$ & GRIK2 & Intergenic & 5 & 2.54E-07 & 2.80E-03 \\
\hline $\begin{array}{c}\text { chr17:14201680- } \\
14201745\end{array}$ & HS3ST3B1 & Intergenic & 2 & $2.91 \mathrm{E}-07$ & $1.93 E-03$ \\
\hline $\begin{array}{c}\text { chr8:57360586- } \\
57360614\end{array}$ & LOC101929415 & Intron & 2 & 3.89E-07 & 5.97E-03 \\
\hline $\begin{array}{c}\text { chr8:110656096- } \\
110656167\end{array}$ & $S Y B U$ & Intron; 5'UTR & 2 & $3.98 \mathrm{E}-07$ & $2.42 \mathrm{E}-03$ \\
\hline $\begin{array}{c}\text { chr17:29297380- } \\
29297459\end{array}$ & RNF135 & Intergenic & 5 & 4.39E-07 & 2.39E-03 \\
\hline $\begin{array}{c}\text { chr20:30073521- } \\
30073577\end{array}$ & LINC00028 & Intergenic & 4 & 5.93E-07 & 4.55E-03 \\
\hline $\begin{array}{c}\text { chr2:177004975- } \\
177004999\end{array}$ & HOXD-AS2 & Intergenic & 2 & 6.99E-07 & $1.25 \mathrm{E}-02$ \\
\hline $\begin{array}{c}\text { chr20:36148954- } \\
36149122\end{array}$ & $B L C A P$ & $\begin{array}{l}\text { Intron; 5'UTR; } \\
\text { TSS; Exon }\end{array}$ & 10 & 8.58E-07 & $2.20 \mathrm{E}-03$ \\
\hline $\begin{array}{c}\text { chr2:242904738- } \\
242904794\end{array}$ & LINC01237 & Intron & 3 & 9.19E-07 & 7.04E-03 \\
\hline $\begin{array}{c}\text { chr7:93205237- } \\
93205296\end{array}$ & $C A L C R$ & Intergenic & 4 & $9.75 \mathrm{E}-07$ & 7.09E-03 \\
\hline $\begin{array}{c}\text { chr14:102990211- } \\
102990252\end{array}$ & ANKRD9 & Intergenic & 2 & $1.75 E-06$ & $1.82 \mathrm{E}-02$ \\
\hline $\begin{array}{c}\text { chr20:61668419- } \\
61668470\end{array}$ & LINC01749 & Intron & 2 & 2.23E-06 & 1.87E-02 \\
\hline
\end{tabular}




\begin{tabular}{|c|c|c|c|c|c|}
\hline Position & Gene & Region & $\begin{array}{c}\# \\
\text { probes }\end{array}$ & $P$ & Šidák \\
\hline $\begin{array}{c}\text { chr16:68014338- } \\
68014375\end{array}$ & $D P E P 3$ & 5'UTR; CDS & 2 & 2.30E-06 & 2.64E-02 \\
\hline $\begin{array}{c}\text { chr14:65239327- } \\
65239358\end{array}$ & SPTB & CDS & 2 & 3.23E-06 & 4.39E-02 \\
\hline $\begin{array}{c}\text { chr17:8905741- } \\
8905776\end{array}$ & NTN1 & Intergenic & 2 & 3.56E-06 & 4.29E-02 \\
\hline $\begin{array}{c}\text { chr12:132293656- } \\
132293703\end{array}$ & SFSWAP & Intergenic & 2 & 4.67E-06 & 4.19E-02 \\
\hline $\begin{array}{c}\text { chr7:102553369- } \\
102553438\end{array}$ & $\begin{array}{l}\text { FBXL13; } \\
\text { LRRC17 }\end{array}$ & $\begin{array}{c}\text { Intron; Exon; } \\
\text { 5'UTR }\end{array}$ & 3 & 8.06E-06 & 4.91E-02 \\
\hline
\end{tabular}

\begin{tabular}{|c|c|c|c|c|c|c|c|}
\hline ID & Description & $\begin{array}{l}\text { Onto- } \\
\text { logy }\end{array}$ & $\begin{array}{c}\text { Genes } \\
\text { in } \\
\text { term }\end{array}$ & $\begin{array}{l}\text { Genes } \\
\text { altered }\end{array}$ & $\begin{array}{l}\text { Genes in } \\
\text { term with } \\
\text { DMP / DMR }\end{array}$ & $P$ & FDR \\
\hline GO:0007156 & $\begin{array}{l}\text { homophilic } \\
\text { cell adhesion } \\
\text { via plasma } \\
\text { membrane } \\
\text { adhesion } \\
\text { molecules }\end{array}$ & BP & 149 & 42 & $\begin{array}{l}\text { PCDHB5; } \\
\text { CDH13 }\end{array}$ & $1.91 \mathrm{E}-21$ & $1.50 \mathrm{E}-17$ \\
\hline GO:0098742 & $\begin{array}{l}\text { cell-cell } \\
\text { adhesion } \\
\text { via plasma- } \\
\text { membrane } \\
\text { adhesion } \\
\text { molecules }\end{array}$ & BP & 214 & 43 & $\begin{array}{c}\text { PCDHB5; } \\
\text { CDH13 }\end{array}$ & $1.42 \mathrm{E}-17$ & $5.60 \mathrm{E}-14$ \\
\hline GO:0031226 & $\begin{array}{l}\text { intrinsic } \\
\text { component } \\
\text { of plasma } \\
\text { membrane }\end{array}$ & $\mathrm{CC}$ & 1557 & 98 & $\begin{array}{c}\text { HS3ST3B1; } \\
\text { TLR5; } \\
\text { SHANK1; } \\
\text { PTPRN2; } \\
\text { GRIK2; } \\
\text { RHCG; } \\
\text { CALCR; } \\
\text { SPTB }\end{array}$ & $2.32 \mathrm{E}-11$ & $4.80 \mathrm{E}-08$ \\
\hline GO:0005887 & $\begin{array}{l}\text { integral } \\
\text { component } \\
\text { of plasma } \\
\text { membrane }\end{array}$ & CC & 1498 & 95 & $\begin{array}{c}\text { HS3ST3B1; } \\
\text { TLR5; } \\
\text { SHANK1; } \\
\text { PTPRN2; } \\
\text { GRIK2; } \\
\text { RHCG; } \\
\text { CALCR }\end{array}$ & $2.44 \mathrm{E}-11$ & $4.80 \mathrm{E}-08$ \\
\hline GO:0005509 & $\begin{array}{l}\text { calcium ion } \\
\text { binding }\end{array}$ & MF & 663 & 61 & $\begin{array}{l}\text { PCDHB5; } \\
\text { CDH13; } \\
\text { SCGN }\end{array}$ & $3.05 \mathrm{E}-11$ & $4.80 \mathrm{E}-08$ \\
\hline GO:0007268 & $\begin{array}{l}\text { chemical } \\
\text { synaptic } \\
\text { transmission }\end{array}$ & $\mathrm{BP}$ & 564 & 47 & $\begin{array}{c}\text { PCDHB5; } \\
\text { CACNA2D2; } \\
\text { SHANK1; } \\
\text { PTPRN2; } \\
\text { GRIK2; OXT }\end{array}$ & $5.25 \mathrm{E}-07$ & 0.00046 \\
\hline GO:0098916 & $\begin{array}{l}\text { anterograde } \\
\text { trans-synaptic } \\
\text { signaling }\end{array}$ & $\mathrm{BP}$ & 564 & 47 & $\begin{array}{c}\text { PCDHB5; } \\
\text { CACNA2D2; } \\
\text { SHANK1; } \\
\text { PTPRN2; } \\
\text { GRIK2; OXT }\end{array}$ & $5.25 \mathrm{E}-07$ & 0.00046 \\
\hline GO:0099536 & $\begin{array}{l}\text { synaptic } \\
\text { signaling }\end{array}$ & $\mathrm{BP}$ & 564 & 47 & $\begin{array}{c}\text { PCDHB5; } \\
\text { CACNA2D2; } \\
\text { SHANK1; } \\
\text { PTPRN2; } \\
\text { GRIK2; OXT }\end{array}$ & $5.25 \mathrm{E}-07$ & 0.00046 \\
\hline GO:0099537 & $\begin{array}{l}\text { trans-synaptic } \\
\text { signaling }\end{array}$ & $\mathrm{BP}$ & 564 & 47 & $\begin{array}{c}\text { PCDHB5; } \\
\text { CACNA2D2; } \\
\text { SHANK1; } \\
\text { PTPRN2; } \\
\text { GRIK2; OXT }\end{array}$ & $5.25 \mathrm{E}-07$ & 0.00046 \\
\hline
\end{tabular}

TABLE 4. Enriched GO terms by genes displaying altered DNA methylation at baseline.

ABBREVIATIONS: DMP, differentially methylated position; DMR, differentially methylated region; FDR, false discovery rate adjusted $p$-values; 


\begin{tabular}{|c|c|c|c|c|c|c|c|}
\hline ID & Description & $\begin{array}{l}\text { Onto- } \\
\text { logy }\end{array}$ & $\begin{array}{l}\text { Genes } \\
\text { in } \\
\text { term }\end{array}$ & $\begin{array}{l}\text { Genes } \\
\text { altered }\end{array}$ & $\begin{array}{l}\text { Genes in } \\
\text { term with } \\
\text { DMP / DMR }\end{array}$ & $P$ & FDR \\
\hline GO:0034220 & $\begin{array}{c}\text { ion } \\
\text { transmembrane } \\
\text { transport }\end{array}$ & $\mathrm{BP}$ & 890 & 59 & $\begin{array}{c}\text { CACNA2D2; } \\
\text { PDE2A; } \\
\text { SHANK1; } \\
\text { GRIK2; } \\
\text { RHCG }\end{array}$ & $7.04 \mathrm{E}-07$ & 0.000555 \\
\hline GO:0098609 & $\begin{array}{l}\text { cell-cell } \\
\text { adhesion }\end{array}$ & $\mathrm{BP}$ & 1090 & 71 & $\begin{array}{l}\text { PCDHB5; } \\
\text { CDH13; } \\
\text { ARHGAP1 }\end{array}$ & 1.29E-06 & 0.000927 \\
\hline GO:0006811 & ion transport & $\mathrm{BP}$ & 1336 & 76 & $\begin{array}{c}\text { CACNA2D2; } \\
\text { PDE } 2 A ; \\
\text { SHANK1; } \\
\text { GRIK2; OXT; } \\
\text { SLC44A4; } \\
\text { RHCG }\end{array}$ & $1.61 \mathrm{E}-06$ & 0.000998 \\
\hline GO:0007267 & $\begin{array}{l}\text { cell-cell } \\
\text { signaling }\end{array}$ & $\mathrm{BP}$ & 1418 & 86 & $\begin{array}{l}\text { PCDHB5; } \\
\text { CACNA2D2; } \\
\text { SHANK1; } \\
\text { PTPRN2; } \\
\text { GRIK2; OXT }\end{array}$ & $1.64 \mathrm{E}-06$ & 0.000998 \\
\hline GO:0055085 & $\begin{array}{c}\text { transmembrane } \\
\text { transport }\end{array}$ & $\mathrm{BP}$ & 1225 & 70 & $\begin{array}{l}\text { CACNA2D2; } \\
\text { PDE2A; } \\
\text { SHANK1; } \\
\text { GRIK2; } \\
\text { SLC44A4; } \\
\text { RHCG }\end{array}$ & $4.87 E-06$ & 0.00274 \\
\hline GO:0071556 & $\begin{array}{l}\text { integral } \\
\text { component of } \\
\text { lumenal side } \\
\text { of endoplasmic } \\
\text { reticulum } \\
\text { membrane }\end{array}$ & $\mathrm{CC}$ & 26 & 8 & & $8.29 E-06$ & 0.00408 \\
\hline GO:0098553 & $\begin{array}{l}\text { lumenal side } \\
\text { of endoplasmic } \\
\text { reticulum } \\
\text { membrane }\end{array}$ & $\mathrm{CC}$ & 26 & 8 & & $8.29 \mathrm{E}-06$ & 0.00408 \\
\hline GO:0006812 & cation transport & $\mathrm{BP}$ & 901 & 55 & $\begin{array}{c}\text { CACNA2D2; } \\
\text { PDE } 2 A ; \\
\text { SLC44A4; } \\
\text { RHCG }\end{array}$ & $9.22 \mathrm{E}-06$ & 0.00428 \\
\hline GO:0008324 & $\begin{array}{l}\text { cation } \\
\text { transmembrane } \\
\text { transporter } \\
\text { activity }\end{array}$ & MF & 574 & 40 & $\begin{array}{l}\text { CACNA2D2; } \\
\text { PDE } 2 A ; \\
\text { SLC44A4; } \\
\text { RHCG }\end{array}$ & $1.07 \mathrm{E}-05$ & 0.00467 \\
\hline GO:0050877 & $\begin{array}{c}\text { neurological } \\
\text { system process }\end{array}$ & $\mathrm{BP}$ & 1129 & 57 & $\begin{array}{c}\text { TECTA; } \\
\text { SHANK1; } \\
\text { GRIK2; OXT; } \\
\text { MARVELD2 }\end{array}$ & $1.13 E-05$ & 0.00469 \\
\hline GO:0042613 & $\begin{array}{l}\text { MHC class II } \\
\text { protein complex }\end{array}$ & $\mathrm{CC}$ & 14 & 6 & & $2.05 \mathrm{E}-05$ & 0.00809 \\
\hline GO:0098655 & $\begin{array}{l}\text { cation } \\
\text { transmembrane } \\
\text { transport }\end{array}$ & $\mathrm{BP}$ & 655 & 43 & $\begin{array}{c}\text { CACNA2D2; } \\
\text { PDE2A; } \\
\text { RHCG }\end{array}$ & $3.13 E-05$ & 0.0118 \\
\hline GO:0045202 & synapse & $\mathrm{CC}$ & 678 & 51 & $\begin{array}{c}\text { PDE2A; } \\
\text { SHANK1; } \\
\text { SCGN; } \\
\text { PTPRN2; } \\
\text { GRIK2; } \\
\text { PRRT1; OXT }\end{array}$ & $3.39 \mathrm{E}-05$ & 0.0121 \\
\hline GO:0015075 & $\begin{array}{c}\text { ion } \\
\text { transmembrane } \\
\text { transporter } \\
\text { activity }\end{array}$ & MF & 759 & 47 & $\begin{array}{c}\text { CACNA2D2; } \\
\text { PDE2A; } \\
\text { GRIK2; } \\
\text { SLC44A4; } \\
\text { RHCG }\end{array}$ & $3.61 \mathrm{E}-05$ & 0.0124 \\
\hline GO:0006140 & $\begin{array}{l}\text { regulation of } \\
\text { nucleotide } \\
\text { metabolic } \\
\text { process }\end{array}$ & $\mathrm{BP}$ & 201 & 19 & $\begin{array}{l}\text { PDE2A; } \\
\text { CALCR }\end{array}$ & $4.48 E-05$ & 0.0146 \\
\hline GO:0042611 & $\begin{array}{l}\text { MHC protein } \\
\text { complex }\end{array}$ & $\mathrm{CC}$ & 23 & 7 & & 4.62E-05 & 0.0146 \\
\hline
\end{tabular}




\begin{tabular}{|c|c|c|c|c|c|c|c|}
\hline ID & Description & $\begin{array}{l}\text { Onto-1 } \\
\text { logy }\end{array}$ & $\begin{array}{l}\text { Genes } \\
\text { in } \\
\text { term }\end{array}$ & $\begin{array}{l}\text { Genes } \\
\text { altered }\end{array}$ & $\begin{array}{c}\text { Genes in } \\
\text { term with } \\
\text { DMP / DMR }\end{array}$ & $P$ & FDR \\
\hline GO:0007610 & behavior & $\mathrm{BP}$ & 518 & 39 & $\begin{array}{l}\text { SHANK1; } \\
\text { GRIK } 2 ; \text { ZIC } 1 \\
\text { OXT }\end{array}$ & 5.73E-05 & 0.0174 \\
\hline GO:0030658 & $\begin{array}{c}\text { transport vesicle } \\
\text { membrane }\end{array}$ & $\mathrm{CC}$ & 150 & 16 & $\begin{array}{l}\text { SCGN; } \\
\text { PTPRN2 }\end{array}$ & $6.32 E-05$ & 0.0185 \\
\hline GO:0007155 & cell adhesion & $\mathrm{BP}$ & 1606 & 88 & $\begin{array}{c}\text { PCDHB5; } \\
\text { TECTA; } \\
\text { CDH13; } \\
\text { ARHGAP1; } \\
\text { TNXB; LYPD5 }\end{array}$ & $7.02 E-05$ & 0.0194 \\
\hline GO:0005261 & $\begin{array}{l}\text { cation channel } \\
\text { activity }\end{array}$ & MF & 288 & 25 & $\begin{array}{l}\text { CACNA2D2; } \\
\text { PDE2A }\end{array}$ & $7.12 \mathrm{E}-05$ & 0.0194 \\
\hline GO:0003008 & system process & $\mathrm{BP}$ & 1799 & 83 & $\begin{array}{c}\text { CACNA2D2; } \\
\text { TECTA; } \\
\text { PDE } 2 A ; \\
\text { SHANK1; } \\
\text { GRIK2; OXT; } \\
\text { MARVELD2 }\end{array}$ & $7.47 \mathrm{E}-05$ & 0.0196 \\
\hline GO:0022610 & $\begin{array}{l}\text { biological } \\
\text { adhesion }\end{array}$ & $\mathrm{BP}$ & 1611 & 88 & $\begin{array}{c}\text { PCDHB5; } \\
\text { TECTA; } \\
\text { CDH13; } \\
\text { ARHGAP } 1 \\
\text { TNXB; LYPD5 }\end{array}$ & $8.00 E-05$ & 0.0203 \\
\hline GO:0022891 & $\begin{array}{l}\text { substrate- } \\
\text { specific } \\
\text { transmembrane } \\
\text { transporter } \\
\text { activity }\end{array}$ & MF & 884 & 50 & $\begin{array}{c}\text { CACNA2D2; } \\
\text { PDE } 2 A ; \\
\text { GRIK2; } \\
\text { SLC44A4; } \\
\text { RHCG }\end{array}$ & $9.68 \mathrm{E}-05$ & 0.0238 \\
\hline GO:0031644 & $\begin{array}{c}\text { regulation of } \\
\text { neurological } \\
\text { system process }\end{array}$ & $\mathrm{BP}$ & 62 & 10 & $O X T$ & $9.98 \mathrm{E}-05$ & 0.0238 \\
\hline GO:0034702 & $\begin{array}{l}\text { ion channel } \\
\text { complex }\end{array}$ & $\mathrm{CC}$ & 263 & 24 & $\begin{array}{l}\text { CACNA2D2; } \\
\text { SHANK1; } \\
\text { GRIK2 }\end{array}$ & $1.04 \mathrm{E}-04$ & 0.0241 \\
\hline GO:1900542 & $\begin{array}{l}\text { regulation } \\
\text { of purine } \\
\text { nucleotide } \\
\text { metabolic } \\
\text { process }\end{array}$ & $\mathrm{BP}$ & 195 & 18 & $\begin{array}{l}\text { PDE2A; } \\
\text { CALCR }\end{array}$ & $1.14 \mathrm{E}-04$ & 0.0256 \\
\hline GO:0022836 & $\begin{array}{c}\text { gated channel } \\
\text { activity }\end{array}$ & MF & 307 & 26 & $\begin{array}{c}\text { CACNA2D2; } \\
\text { GRIK2 }\end{array}$ & $1.25 \mathrm{E}-04$ & 0.0267 \\
\hline GO:0030001 & $\begin{array}{l}\text { metal ion } \\
\text { transport }\end{array}$ & $\mathrm{BP}$ & 753 & 46 & $\begin{array}{c}\text { CACNA2D2; } \\
\text { PDE2A }\end{array}$ & $1.28 E-04$ & 0.0267 \\
\hline GO:0099600 & $\begin{array}{l}\text { transmembrane } \\
\text { receptor activity }\end{array}$ & MF & 1171 & 51 & $\begin{array}{l}\text { TLR5; } \\
\text { PTPRN2; } \\
\text { GRIK2; } \\
\text { CALCR }\end{array}$ & $1.29 E-04$ & 0.0267 \\
\hline GO:0046873 & $\begin{array}{l}\text { metal ion } \\
\text { transmembrane } \\
\text { transporter } \\
\text { activity }\end{array}$ & MF & 399 & 30 & $\begin{array}{c}\text { CACNA2D2; } \\
\text { PDE2A }\end{array}$ & $1.41 E-04$ & 0.0281 \\
\hline GO:0030669 & $\begin{array}{l}\text { clathrin-coated } \\
\text { endocytic } \\
\text { vesicle } \\
\text { membrane }\end{array}$ & $\mathrm{CC}$ & 44 & 8 & & $1.42 E-04$ & 0.0281 \\
\hline GO:0007193 & $\begin{array}{l}\text { adenylate } \\
\text { cyclase- } \\
\text { inhibiting } \\
\text { G-protein } \\
\text { coupled } \\
\text { receptor } \\
\text { signaling } \\
\text { pathway }\end{array}$ & $\mathrm{BP}$ & 69 & 10 & & $1.48 E-04$ & 0.0284 \\
\hline GO:0022890 & $\begin{array}{l}\text { inorganic cation } \\
\text { transmembrane } \\
\text { transporter } \\
\text { activity }\end{array}$ & MF & 482 & 33 & $\begin{array}{c}\text { CACNA2D2; } \\
\text { PDE2A }\end{array}$ & $1.61 E-04$ & 0.0302 \\
\hline GO:0044057 & $\begin{array}{l}\text { regulation of } \\
\text { system process }\end{array}$ & $\mathrm{BP}$ & 474 & 34 & $\begin{array}{l}\text { CACNA2D2; } \\
\text { OXT }\end{array}$ & $1.72 E-04$ & 0.0311 \\
\hline
\end{tabular}




\begin{tabular}{|c|c|c|c|c|c|c|c|}
\hline ID & Description & $\begin{array}{l}\text { Onto- } \\
\text { logy }\end{array}$ & $\begin{array}{c}\text { Genes } \\
\text { in } \\
\text { term }\end{array}$ & $\begin{array}{l}\text { Genes } \\
\text { altered }\end{array}$ & $\begin{array}{c}\text { Genes in } \\
\text { term with } \\
\text { DMP / DMR }\end{array}$ & $P$ & FDR \\
\hline GO:0045334 & $\begin{array}{l}\text { clathrin-coated } \\
\text { endocytic } \\
\text { vesicle }\end{array}$ & CC & 59 & 9 & & $1.73 E-04$ & 0.0311 \\
\hline GO:0022838 & $\begin{array}{c}\text { substrate- } \\
\text { specific channel } \\
\text { activity }\end{array}$ & MF & 412 & 30 & $\begin{array}{c}\text { CACNA2D2; } \\
\text { PDE } 2 A ; \\
\text { GRIK2 }\end{array}$ & $1.85 \mathrm{E}-04$ & 0.0324 \\
\hline GO:0098797 & $\begin{array}{c}\text { plasma } \\
\text { membrane } \\
\text { protein complex }\end{array}$ & $\mathrm{CC}$ & 486 & 35 & $\begin{array}{l}\text { CACNA2D2; } \\
\text { SHANK1; } \\
\text { GRIK2 }\end{array}$ & $1.91 \mathrm{E}-04$ & 0.0326 \\
\hline GO:0015267 & channel activity & MF & 441 & 31 & $\begin{array}{c}\text { CACNA2D2; } \\
\text { PDE } 2 A ; \\
\text { GRIK2 }\end{array}$ & $1.94 E-04$ & 0.0326 \\
\hline GO:0022803 & $\begin{array}{c}\text { passive } \\
\text { transmembrane } \\
\text { transporter } \\
\text { activity }\end{array}$ & MF & 442 & 31 & $\begin{array}{c}\text { CACNA2D2; } \\
\text { PDE2A; } \\
\text { GRIK2 }\end{array}$ & $2.00 E-04$ & 0.0328 \\
\hline GO:0007416 & $\begin{array}{l}\text { synapse } \\
\text { assembly }\end{array}$ & $\mathrm{BP}$ & 126 & 16 & $\begin{array}{l}\text { PCDHB5; } \\
\text { OXT }\end{array}$ & $2.13 E-04$ & 0.0342 \\
\hline GO:0004888 & $\begin{array}{l}\text { transmembrane } \\
\text { signaling } \\
\text { receptor activity }\end{array}$ & MF & 1120 & 48 & $\begin{array}{l}\text { TLR5; } \\
\text { PTPRN2; } \\
\text { GRIK2; } \\
\text { CALCR }\end{array}$ & $2.17 \mathrm{E}-04$ & 0.0342 \\
\hline GO:0019233 & $\begin{array}{l}\text { sensory } \\
\text { perception of } \\
\text { pain }\end{array}$ & $\mathrm{BP}$ & 90 & 11 & $O X T$ & 2.60E-04 & 0.0392 \\
\hline GO:0022892 & $\begin{array}{l}\text { substrate- } \\
\text { specific } \\
\text { transporter } \\
\text { activity }\end{array}$ & MF & 1059 & 55 & $\begin{array}{c}\text { CACNA2D2; } \\
\text { PDE } 2 A ; \\
\text { GRIK2; } \\
\text { SLC44A4; } \\
\text { RHCG; } \\
\text { CALCR }\end{array}$ & 2.63E-04 & 0.0392 \\
\hline GO:0098662 & $\begin{array}{l}\text { inorganic cation } \\
\text { transmembrane } \\
\text { transport }\end{array}$ & $\mathrm{BP}$ & 595 & 38 & $\begin{array}{c}\text { CACNA2D2; } \\
\text { PDE2A }\end{array}$ & 2.64E-04 & 0.0392 \\
\hline GO:0044306 & $\begin{array}{l}\text { neuron } \\
\text { projection } \\
\text { terminus }\end{array}$ & $\mathrm{CC}$ & 113 & 13 & $\begin{array}{c}\text { PTPRN2; } \\
\text { GRIK2; OXT }\end{array}$ & $2.74 \mathrm{E}-04$ & 0.0401 \\
\hline GO:0051349 & $\begin{array}{l}\text { positive } \\
\text { regulation of } \\
\text { lyase activity }\end{array}$ & $\mathrm{BP}$ & 58 & 9 & $C A L C R$ & $3.01 \mathrm{E}-04$ & 0.0427 \\
\hline GO:0044708 & $\begin{array}{c}\text { single-organism } \\
\text { behavior }\end{array}$ & $\mathrm{BP}$ & 372 & 29 & $\begin{array}{l}\text { SHANK1; } \\
\text { GRIK2; ZIC } 1 ; \\
\text { OXT }\end{array}$ & $3.05 E-04$ & 0.0427 \\
\hline GO:1903522 & $\begin{array}{l}\text { regulation of } \\
\text { blood circulation }\end{array}$ & $\mathrm{BP}$ & 277 & 23 & $\begin{array}{l}\text { CACNA2D2; } \\
\text { OXT }\end{array}$ & $3.09 \mathrm{E}-04$ & 0.0427 \\
\hline GO:0031281 & $\begin{array}{c}\text { positive } \\
\text { regulation of } \\
\text { cyclase activity }\end{array}$ & $\mathrm{BP}$ & 58 & 9 & $C A L C R$ & $3.21 \mathrm{E}-04$ & 0.0429 \\
\hline GO:0005216 & $\begin{array}{l}\text { ion channel } \\
\text { activity }\end{array}$ & MF & 398 & 29 & $\begin{array}{c}\text { CACNA2D2; } \\
\text { PDE2A; } \\
\text { GRIK2 }\end{array}$ & $3.21 \mathrm{E}-04$ & 0.0429 \\
\hline GO:0012507 & $\begin{array}{c}\text { ER to Golgi } \\
\text { transport vesicle } \\
\text { membrane }\end{array}$ & $\mathrm{CC}$ & 52 & 8 & & $3.58 \mathrm{E}-04$ & 0.0468 \\
\hline GO:0016339 & $\begin{array}{l}\text { calcium- } \\
\text { dependent cell- } \\
\text { cell adhesion } \\
\text { via plasma } \\
\text { membrane } \\
\text { cell adhesion } \\
\text { molecules }\end{array}$ & $\mathrm{BP}$ & 28 & 6 & $\begin{array}{l}\text { PCDHB5; } \\
\text { CDH13 }\end{array}$ & $3.64 \mathrm{E}-04$ & 0.0468 \\
\hline GO:0022857 & $\begin{array}{c}\text { transmembrane } \\
\text { transporter } \\
\text { activity }\end{array}$ & MF & 956 & 51 & $\begin{array}{c}\text { CACNA2D2; } \\
\text { PDE } 2 A ; \\
\text { GRIK2; } \\
\text { SLC44A4; } \\
\text { RHCG }\end{array}$ & $3.68 \mathrm{E}-04$ & 0.0468 \\
\hline GO:0042391 & $\begin{array}{l}\text { regulation of } \\
\text { membrane } \\
\text { potential }\end{array}$ & $\mathrm{BP}$ & 331 & 26 & $\begin{array}{l}\text { SHANK1; } \\
\text { GRIK2 }\end{array}$ & $3.78 \mathrm{E}-04$ & 0.0471 \\
\hline
\end{tabular}




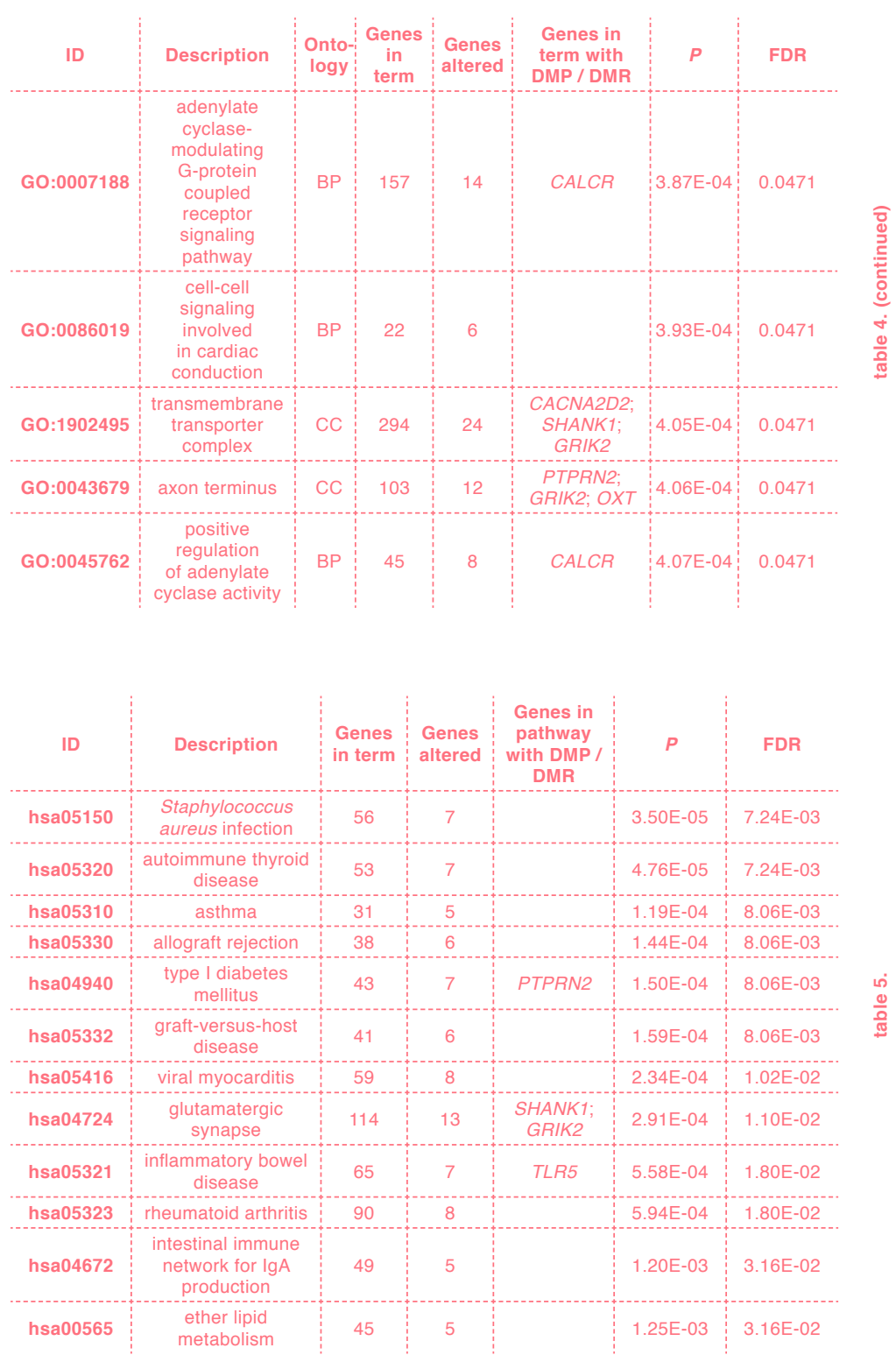

\subsubsection{Methylomic differ- ences during conversion to dementia}

Test statistics from the timepoint by group interaction analysis had an estimated bias of 0.16 and an estimated inflation of 0.89 (Supplementary

TABLE 5. KEGG pathways enriched by genes displaying altered DNA methylation at baseline.

ABBREVIATIONS: FDR, false discovery rate adjusted $p$-values; KEGG, Kyoto Encyclopedia of Genes and Genomes. 
Figure 5). 113 DMPs were identified, with one in $A T P 11 B$, one in $D G K D$, and one in $A T G 2 B$ comprising the top 3 (Table 6; Supplementary Figure 6), and 3 DMRs, in GLB1L3, TAF3, and DOC2GP (Table 7; Supplementary Figure 6). Of the positions showing different longitudinal methylation patterns for converters and controls, 3 in ADGRD1, ZBTB46, and $A R H G A P 1$ also showed up as DMPs at baseline. Additionally, $H L A$ $D R B 5$ contained 4 DMPs at the baseline comparison and an additional, different DMP was detected in the group by time interaction analysis. The 113 DMPs from the group by time interaction analysis were analyzed for methylation differences between baseline and follow-up for the converters and non-converters separately. For the converters, 4 out of the 113 positions showed significant changes in methylation, interestingly all of which were hypermethylated at follow-up (Table 8). Additionally, the DMR in DOC2GP from the group by time interaction analysis was also detected for the converters separately $\left(p_{\text {Šidák }}=4.80 \mathrm{E}-03\right)$. The non-converters presented with significantly altered methylation at 35 out of the 113 positions (Table 9). There was no overlap between the DMPs observed for the converters and non-converters.

None of the GO terms or KEGG pathways were significantly enriched after FDR correction for the group by time interaction analysis (Supplementary Table 1; Supplementary Table 2). However, it is interesting to note that there was overlap between the GO terms significantly enriched at baseline and those enriched with an uncorrected $p$-value $<0.05$ in the interaction analysis; GO:0007267 ('cell-cell signaling'), GO:0007416 ('synapse assembly'), and GO:0045202 ('synapse').

TABLE 6. DMPs of the group by time interaction methylome-wide association analysis.

ABBREVIATIONS: DMPs, differentially methylated positions; ES, effect size; FDR, false discovery rate adjusted $p$-values; SE, standard error; TSS, transcription start site; UTR, untranslated region.

\begin{tabular}{|c|c|c|c|c|c|c|}
\hline Probe & Gene & Region & ES & SE & $P$ & FDR \\
\hline $\operatorname{cg} 19820921$ & ATP11B & Body & -0.77 & 0.13 & $9.15 \mathrm{E}-09$ & $3.94 \mathrm{E}-03$ \\
\hline $\operatorname{cg} 26215113$ & $D G K D$ & Body & 0.44 & 0.08 & 3.70E-08 & $7.63 \mathrm{E}-03$ \\
\hline $\operatorname{cg} 04173048$ & ATG2B & & 0.43 & 0.08 & $5.83 \mathrm{E}-08$ & $7.63 \mathrm{E}-03$ \\
\hline $\operatorname{cg} 26827033$ & $A D G R D 1$ & Body & -0.42 & 0.08 & $7.08 \mathrm{E}-08$ & $7.63 \mathrm{E}-03$ \\
\hline cg07934604 & LINC00616 & & 0.37 & 0.07 & $1.81 \mathrm{E}-07$ & $1.26 \mathrm{E}-02$ \\
\hline cg10540754 & FAM49A & 5'UTR & 0.42 & 0.08 & $2.06 \mathrm{E}-07$ & $1.26 \mathrm{E}-02$ \\
\hline $\mathrm{cg03049303}$ & C10orf76 & TSS1500 & 0.43 & 0.08 & $2.40 \mathrm{E}-07$ & 1.26E-02 \\
\hline $\operatorname{cg} 21984711$ & $B C L 2$ & 3'UTR & -0.64 & 0.12 & $2.49 \mathrm{E}-07$ & $1.26 \mathrm{E}-02$ \\
\hline cg19282889 & KLHL25 & TSS1500 & -0.43 & 0.08 & $2.64 \mathrm{E}-07$ & $1.26 \mathrm{E}-02$ \\
\hline $\operatorname{cg} 10664768$ & ZBTB46 & Body & 1.10 & 0.22 & $3.80 \mathrm{E}-07$ & $1.56 \mathrm{E}-02$ \\
\hline ch.11.720675R & NAT1O & Body & -0.57 & 0.11 & $4.22 \mathrm{E}-07$ & $1.56 \mathrm{E}-02$ \\
\hline cg26854588 & WIPF2 & & 0.43 & 0.08 & $4.34 \mathrm{E}-07$ & $1.56 \mathrm{E}-02$ \\
\hline ch.11.2495959R & ARHGEF12 & Body & -1.00 & 0.20 & $6.65 \mathrm{E}-07$ & $1.79 \mathrm{E}-02$ \\
\hline $\operatorname{cg} 11642909$ & TRIM59 & & -0.34 & 0.07 & 7.37E-07 & $1.79 \mathrm{E}-02$ \\
\hline cg26577348 & NCOA3 & TSS1500 & 0.32 & 0.06 & $7.89 \mathrm{E}-07$ & $1.79 \mathrm{E}-02$ \\
\hline $\mathrm{cg} 00087005$ & CTBP2 & 5'UTR & 0.36 & 0.07 & $7.96 \mathrm{E}-07$ & $1.79 \mathrm{E}-02$ \\
\hline cg20576094 & $A D A M 21 P 1$ & TSS1500 & 0.47 & 0.09 & $8.05 E-07$ & $1.79 \mathrm{E}-02$ \\
\hline $\operatorname{cg} 13126206$ & LOC286059 & & 0.95 & 0.19 & $8.40 \mathrm{E}-07$ & $1.79 \mathrm{E}-02$ \\
\hline $\operatorname{cg} 19536127$ & C2orf61 & TSS1500 & -0.44 & 0.09 & $8.66 \mathrm{E}-07$ & $1.79 \mathrm{E}-02$ \\
\hline
\end{tabular}




\begin{tabular}{|c|c|c|c|c|c|c|}
\hline Probe & Gene & Region & ES & SE & $P$ & FDR \\
\hline cg16838132 & MIEF1 & 3'UTR & -0.84 & 0.17 & $9.02 \mathrm{E}-07$ & $1.79 \mathrm{E}-02$ \\
\hline cg03101580 & TOLLIP & Body & 0.41 & 0.08 & $9.23 \mathrm{E}-07$ & $1.79 \mathrm{E}-02$ \\
\hline cg24628076 & $A R A P 3$ & Body & -0.38 & 0.08 & $9.44 \mathrm{E}-07$ & $1.79 \mathrm{E}-02$ \\
\hline cg04337538 & MYCN & & -0.52 & 0.11 & $9.69 \mathrm{E}-07$ & 1.79E-02 \\
\hline cg03047400 & ZFYVE1 & & -0.81 & 0.17 & $9.99 \mathrm{E}-07$ & 1.79E-02 \\
\hline cg27487704 & CYLD & & 0.32 & 0.07 & $1.25 \mathrm{E}-06$ & $2.15 \mathrm{E}-02$ \\
\hline cg26207102 & TEAD1 & 5'UTR & -0.67 & 0.14 & $1.38 \mathrm{E}-06$ & 2.27E-02 \\
\hline cg25554183 & $C O B L$ & Body & -0.57 & 0.12 & $1.42 \mathrm{E}-06$ & 2.27E-02 \\
\hline cg04905421 & IFT140 & Body & -0.38 & 0.08 & $1.55 \mathrm{E}-06$ & 2.36E-02 \\
\hline cg00005861 & SMNDC1 & & -0.45 & 0.09 & $1.63 \mathrm{E}-06$ & $2.36 \mathrm{E}-02$ \\
\hline cg12501870 & ZNF704 & & 0.54 & 0.11 & $1.64 \mathrm{E}-06$ & 2.36E-02 \\
\hline cg18575438 & $C C D C 170$ & & 0.51 & 0.11 & $2.13 \mathrm{E}-06$ & 2.89E-02 \\
\hline cg25750404 & VAMP1 & Body & -0.27 & 0.06 & $2.19 \mathrm{E}-06$ & 2.89E-02 \\
\hline cg07937999 & LINC00645 & & -0.51 & 0.11 & $2.26 \mathrm{E}-06$ & $2.89 \mathrm{E}-02$ \\
\hline $\mathrm{cg} 06111454$ & NFATC4 & Body & 0.85 & 0.18 & $2.29 \mathrm{E}-06$ & $2.89 \mathrm{E}-02$ \\
\hline cg14088090 & TMEM125 & Body & -0.35 & 0.07 & 2.39E-06 & 2.89E-02 \\
\hline cg05194545 & ARHGAP 12 & 3'UTR & 0.64 & 0.14 & $2.42 \mathrm{E}-06$ & $2.89 \mathrm{E}-02$ \\
\hline cg09510874 & WNK1 & $1^{\text {st }}$ Exon & -0.42 & 0.09 & $2.59 \mathrm{E}-06$ & $3.01 \mathrm{E}-02$ \\
\hline cg09506183 & ASB3 & $\begin{array}{l}\text { Body; } \\
\text { TSS1500 }\end{array}$ & -0.35 & 0.08 & $2.91 \mathrm{E}-06$ & 3.26E-02 \\
\hline cg10960920 & $C P Q$ & Body & -0.51 & 0.11 & $2.95 \mathrm{E}-06$ & $3.26 \mathrm{E}-02$ \\
\hline cg14337324 & PACRG & Body & -0.33 & 0.07 & $3.09 \mathrm{E}-06$ & $3.31 \mathrm{E}-02$ \\
\hline cg07794500 & $E M B P 1$ & Body & 0.36 & 0.08 & $3.37 \mathrm{E}-06$ & 3.31E-02 \\
\hline cg16475558 & GNAO1 & Body & -0.44 & 0.09 & $3.44 \mathrm{E}-06$ & 3.31E-02 \\
\hline cg14915854 & $A R H G A P 1$ & TSS200 & 0.77 & 0.17 & $3.53 \mathrm{E}-06$ & $3.31 \mathrm{E}-02$ \\
\hline cg01768433 & ADGRV1 & Body & 0.61 & 0.13 & $3.59 \mathrm{E}-06$ & 3. $31 \mathrm{E}-02$ \\
\hline cg13654276 & GRAMD $1 C$ & TSS1500 & -0.43 & 0.09 & 3.60E-06 & 3.31E-02 \\
\hline cg18388802 & ARVCF & TSS200 & 0.43 & 0.09 & $3.66 \mathrm{E}-06$ & 3.31E-02 \\
\hline cg21104351 & GLB1L3 & Body & -0.29 & 0.06 & $3.75 \mathrm{E}-06$ & 3.31E-02 \\
\hline cg24251448 & $A G O 2$ & Body & 0.56 & 0.12 & $3.83 \mathrm{E}-06$ & 3.31E-02 \\
\hline cg22703724 & ETS2 & TSS1500 & 0.72 & 0.16 & $3.95 \mathrm{E}-06$ & 3.31E-02 \\
\hline cg23817292 & CCT8 & Body & -0.83 & 0.18 & $4.04 \mathrm{E}-06$ & 3.31E-02 \\
\hline cg27614376 & $P L C L 2$ & & -0.69 & 0.15 & $4.05 \mathrm{E}-06$ & 3.31E-02 \\
\hline cg15946545 & MRPS 31 & $1^{\text {st }}$ Exon & 1.30 & 0.28 & $4.12 \mathrm{E}-06$ & 3.31E-02 \\
\hline $\operatorname{cg} 10456710$ & SNAP23 & $\begin{array}{l}5^{5} \text { UTR; } 1^{\text {st }} \\
\text { Exon }\end{array}$ & -0.74 & 0.16 & 4.18E-06 & 3.31E-02 \\
\hline cg05289055 & SOST & & 0.36 & 0.08 & $4.22 \mathrm{E}-06$ & $3.31 \mathrm{E}-02$ \\
\hline cg26554699 & WFIKKN2 & Body & 0.28 & 0.06 & 4.22E-06 & 3.31E-02 \\
\hline cg15582126 & BTBD3 & & -0.56 & 0.12 & 4.37E-06 & 3.36E-02 \\
\hline $\operatorname{cg} 10981736$ & СТВP1 & $\begin{array}{l}\text { TSS1500; } \\
\text { 5'UTR; } 1^{\text {st }} \\
\text { Exon }\end{array}$ & -0.39 & 0.09 & $4.52 \mathrm{E}-06$ & $3.42 \mathrm{E}-02$ \\
\hline cg10527300 & COL $4 A 1$ & Body & -0.44 & 0.10 & $4.65 \mathrm{E}-06$ & 3.44E-02 \\
\hline cg07608496 & $\begin{array}{c}\text { MCM } 3 A P- \\
A S 1\end{array}$ & $\begin{array}{l}\text { Body; } \\
\text { TSS1500 }\end{array}$ & -0.50 & 0.11 & $4.71 \mathrm{E}-06$ & 3.44E-02 \\
\hline cg26414731 & PSMF1 & TSS200 & -0.61 & 0.13 & $5.32 \mathrm{E}-06$ & $3.75 \mathrm{E}-02$ \\
\hline cg11631610 & DOCK & Body & 0.37 & 0.08 & $5.43 \mathrm{E}-06$ & $3.75 \mathrm{E}-02$ \\
\hline cg05879527 & DLGAP3 & Body & 0.41 & 0.09 & $5.54 \mathrm{E}-06$ & 3.75E-02 \\
\hline cg06151171 & NCOA1 & & -0.83 & 0.18 & 5.67E-06 & 3.75E-02 \\
\hline cg23844090 & TMEM 42 & Body & 1.47 & 0.32 & $5.74 \mathrm{E}-06$ & 3.75E-02 \\
\hline cg26824807 & GPX6 & $\begin{array}{c}5^{\text {UUTR; }} 1^{\text {st }} \\
\text { Exon }\end{array}$ & -0.31 & 0.07 & 5.78E-06 & $3.75 \mathrm{E}-02$ \\
\hline cg08124722 & CCL7 & Body & -0.40 & 0.09 & $6.00 \mathrm{E}-06$ & 3.75E-02 \\
\hline cg12764704 & PCNX2 & & -0.27 & 0.06 & $6.03 \mathrm{E}-06$ & $3.75 \mathrm{E}-02$ \\
\hline cg00734760 & RCC1L & TSS200 & 0.36 & 0.08 & $6.05 \mathrm{E}-06$ & 3.75E-02 \\
\hline ch.16.1667936F & PSMD & & -0.50 & 0.11 & $6.12 \mathrm{E}-06$ & 3.75E-02 \\
\hline cg05794244 & $\mathrm{RNH1}$ & $\begin{array}{c}\text { TSS } 1500 \\
\text { TSS } 200\end{array}$ & -0.28 & 0.06 & $6.12 \mathrm{E}-06$ & $3.75 \mathrm{E}-02$ \\
\hline cg09027493 & $T C P 11$ & TSS1500 & 0.28 & 0.06 & $6.21 \mathrm{E}-06$ & $3.75 \mathrm{E}-02$ \\
\hline cg00875096 & SLC39A10 & 5'UTR & 0.35 & 0.08 & $6.26 \mathrm{E}-06$ & 3.75E-02 \\
\hline cg05028306 & $\angle A P 3$ & TSS1500 & 0.37 & 0.08 & $6.84 \mathrm{E}-06$ & $4.04 \mathrm{E}-02$ \\
\hline
\end{tabular}




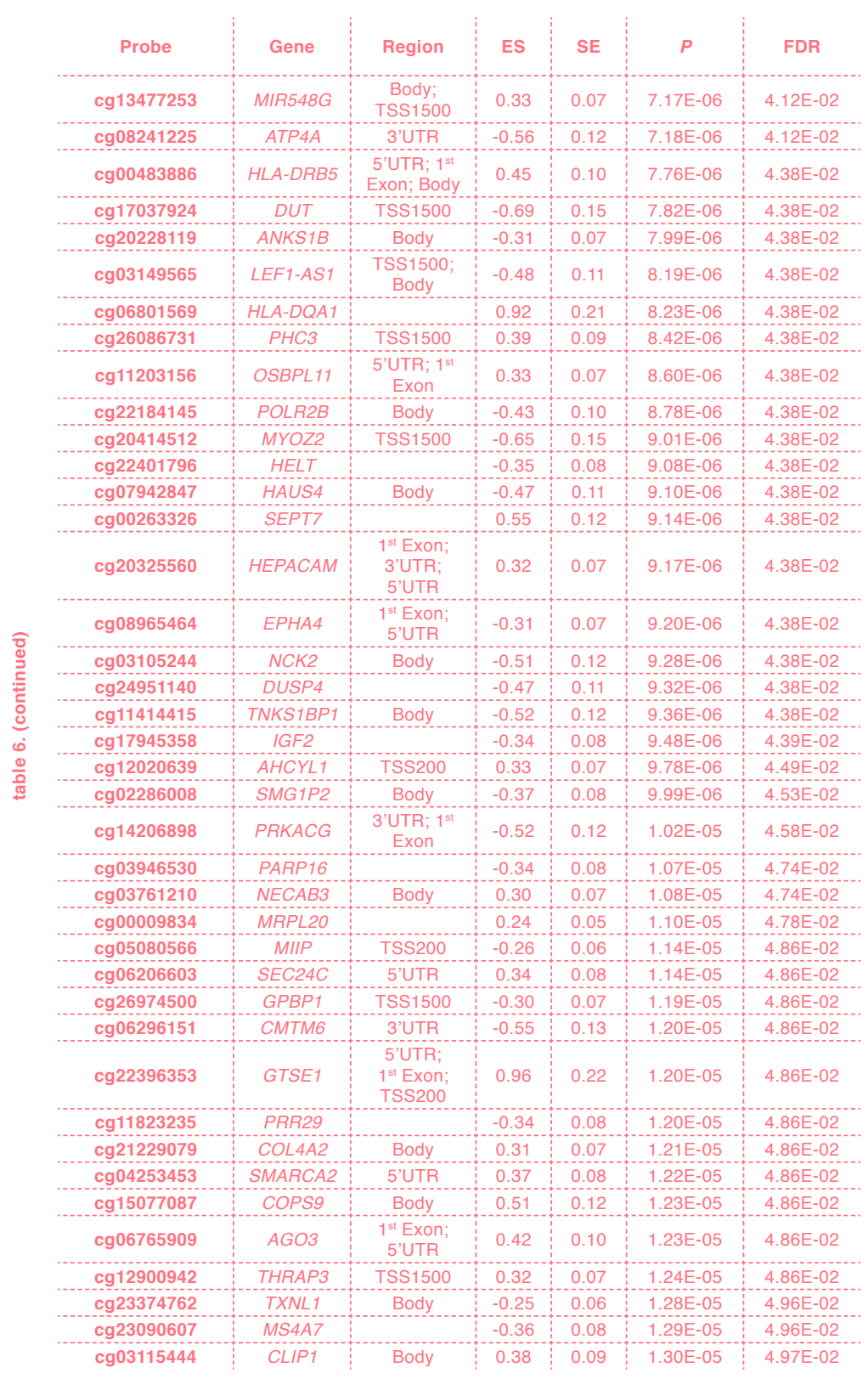

TABLE 7. DMRs of the group by time interaction methylome-wide association analysis.

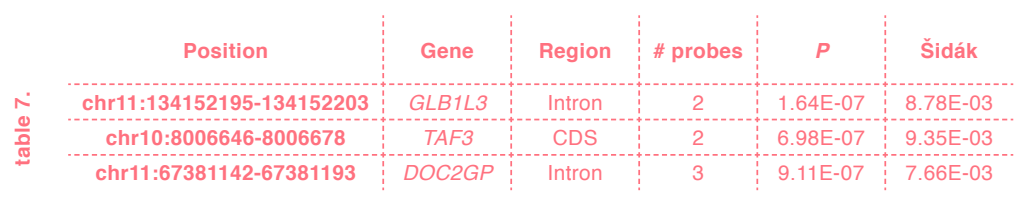

ABBREVIATIONS: CDS, coding DNA sequence; chr, chromosome;

DMR, differentially methylated region; Šidák, Šidák-corrected $p$-values. 


\begin{tabular}{|c|c|c|c|c|c|c|}
\hline Probe & Gene & Region & ES & SE & $P$ & FDR \\
\hline cg26215113 & $D G K D$ & Body & 0.48 & 0.11 & 2.89E-05 & 3.26E-03 \\
\hline cg00009834 & MRPL20 & & 0.28 & 0.08 & $3.60 \mathrm{E}-04$ & $1.94 \mathrm{E}-02$ \\
\hline cg05194545 & ARHGAP12 & 3'UTR & 0.67 & 0.19 & $5.15 \mathrm{E}-04$ & $1.94 \mathrm{E}-02$ \\
\hline cg07934604 & LINC00616 & & 0.34 & 0.10 & 7.27E-04 & 2.06E-02 \\
\hline
\end{tabular}

\begin{tabular}{|c|c|c|c|c|c|c|}
\hline Probe & Gene & Region & ES & SE & $P$ & FDR \\
\hline $\operatorname{cg} 13654276$ & GRAMD 1C & TSS 1500 & 0.64 & 0.14 & 4.11E-06 & 4.64E-04 \\
\hline cg00005861 & SMNDC1 & & 0.58 & 0.14 & 4.07E-05 & 2.30E-03 \\
\hline $\operatorname{cg} 14915854$ & ARHGAP1 & TSS200 & -0.97 & 0.25 & $9.29 \mathrm{E}-05$ & $2.91 \mathrm{E}-03$ \\
\hline $\operatorname{cg} 16838132$ & MIEF1 & 3'UTR & 0.98 & 0.26 & 1.19E-04 & $2.91 \mathrm{E}-03$ \\
\hline $\operatorname{cg} 10664768$ & ZBTB46 & Body & -1.24 & 0.32 & $1.29 \mathrm{E}-04$ & 2.91E-03 \\
\hline cg17945358 & IGF2 & & 0.43 & 0.12 & 2.30E-04 & 4.33E-03 \\
\hline cg23374762 & TXNL1 & Body & 0.30 & 0.09 & 4.54E-04 & 7.33E-03 \\
\hline cg20325560 & HEPACAM & $\begin{array}{l}1^{\text {st }} \text { Exon; } \\
\text { 3'UTR; } \\
\text { 5'UTR }\end{array}$ & -0.37 & 0.11 & $6.56 \mathrm{E}-04$ & $9.24 \mathrm{E}-03$ \\
\hline cg25750404 & VAMP1 & Body & 0.29 & 0.09 & 7.77E-04 & $9.24 \mathrm{E}-03$ \\
\hline cg25554183 & COBL & Body & 0.58 & 0.18 & $1.03 \mathrm{E}-03$ & 9.24E-03 \\
\hline cg03101580 & TOLLIP & Body & -0.41 & 0.12 & $1.04 \mathrm{E}-03$ & $9.24 \mathrm{E}-03$ \\
\hline cg27487704 & CYLD & & -0.33 & 0.10 & 1.06E-03 & $9.24 \mathrm{E}-03$ \\
\hline $\operatorname{cg} 16475558$ & GNAO1 & Body & 0.46 & 0.14 & 1.06E-03 & $9.24 \mathrm{E}-03$ \\
\hline cg04905421 & IFT140 & Body & 0.38 & 0.12 & $1.51 \mathrm{E}-03$ & 1.22E-02 \\
\hline $\operatorname{cg} 26827033$ & $A D G R D 1$ & Body & 0.37 & 0.12 & $1.62 \mathrm{E}-03$ & 1.22E-02 \\
\hline cg24251448 & AGO2 & Body & -0.56 & 0.18 & $1.73 \mathrm{E}-03$ & 1.22E-02 \\
\hline cg00734760 & WBSCR16 & TSS200 & -0.36 & 0.12 & $2.15 \mathrm{E}-03$ & 1.43E-02 \\
\hline cg26974500 & GPBP1 & TSS1500 & 0.30 & 0.10 & 3.36E-03 & 2.05E-02 \\
\hline cg10540754 & FAM49A & 5'UTR & -0.35 & 0.12 & $3.69 \mathrm{E}-03$ & $2.05 \mathrm{E}-02$ \\
\hline cg14337324 & PACRG & Body & 0.31 & 0.11 & $3.70 \mathrm{E}-03$ & 2.05E-02 \\
\hline cg12764704 & PCNX2 & & 0.25 & 0.09 & $3.90 \mathrm{E}-03$ & 2.05E-02 \\
\hline cg19820921 & ATP11B & Body & 0.58 & 0.20 & $3.99 \mathrm{E}-03$ & 2.05E-02 \\
\hline cg21104351 & GLB1L3 & Body & 0.26 & 0.09 & 4.67E-03 & 2.29E-02 \\
\hline $\operatorname{cg} 11203156$ & OSBPL11 & $\begin{array}{l}5^{\prime} U T R ; 1^{\text {st }} \\
\text { Exon }\end{array}$ & -0.31 & 0.11 & 5.19E-03 & 2.44E-02 \\
\hline cg14206898 & PRKACG & $\begin{array}{l}\text { 3'UTR; } 1^{\text {st }} \\
\text { Exon }\end{array}$ & 0.48 & 0.17 & 5.73E-03 & 2.59E-02 \\
\hline cg03049303 & C10orf76 & TSS 1500 & -0.34 & 0.13 & $6.57 \mathrm{E}-03$ & 2.86E-02 \\
\hline $\operatorname{cg} 13477253$ & MIR548G & $\begin{array}{l}\text { Body; } \\
\text { TSS1500 }\end{array}$ & -0.29 & 0.11 & 7.30E-03 & 3.06E-02 \\
\hline cg10960920 & $C P Q$ & Body & 0.43 & 0.16 & $7.71 \mathrm{E}-03$ & $3.11 \mathrm{E}-02$ \\
\hline cg11642909 & TRIM59 & & 0.27 & 0.10 & $8.40 \mathrm{E}-03$ & 3.20E-02 \\
\hline cg12020639 & AHCYL1 & TSS200 & -0.29 & 0.11 & $8.49 \mathrm{E}-03$ & 3.20E-02 \\
\hline cg27614376 & PLCL2 & & 0.59 & 0.23 & $9.15 \mathrm{E}-03$ & 3.34E-02 \\
\hline ch.16.1667936F & PSMD7 & & 0.42 & 0.16 & $1.03 \mathrm{E}-02$ & $3.59 \mathrm{E}-02$ \\
\hline cg00483886 & $H L A-D R B 5$ & $\begin{array}{l}\text { 5'UTR; } 1^{\text {st }} \\
\text { Exon; Body }\end{array}$ & -0.38 & 0.15 & 1.06E-02 & 3.59E-02 \\
\hline cg26554699 & WFIKKN2 & Body & -0.23 & 0.09 & $1.08 \mathrm{E}-02$ & $3.59 \mathrm{E}-02$ \\
\hline $\operatorname{cg} 20228119$ & ANKS1B & Body & 0.25 & 0.10 & 1.39E-02 & 4.50E-02 \\
\hline
\end{tabular}

TABLE 8. DMPs of the group by time interaction methylome-wide association analysis also observed to change over time for converters specifically. ABBREVIATIONS: DMPs, differentially methylated positions; ES, effect size; FDR, false discovery rate adjusted $p$-values; SE, standard error; UTR, untranslated region.

TABLE 9. DMPs of the group by time interaction methylome-wide association analysis also observed to change over time for non-converters specifically.

ABBREVIATIONS: DMPs, differentially methylated positions; ES, effect size; FDR, false discovery rate adjusted $p$-values; SE, standard error; TSS, transcription start site; UTR, untranslated region. 


\subsection{Discussion}

The present work aimed to identify methylomic markers associated with conversion to AD dementia at a preclinical stage, as well as dynamic markers showing different time-related changes in converters and non-converters. A methylome-wide association study at baseline and a group by time interaction analysis revealed several DMPs and DMRs, associated with known $A D$ risk genes, genes part of pathways previously implicated in $A D$, as well as novel candidate genes that merit further investigations.

Past investigations into blood DNA methylation in relation to $A D$ is limited, mainly focusing on the direct comparison of $A D$ cases and healthy controls [33-35]. Di Francesco et al. [33] focused on global DNA methylation levels, and observed these to be increased in late-onset $A D$ patients. Although we did not detect differences in global methylation between converters and controls, these outcomes cannot really be compared due to the difference in methodology. The luminometric methylation assay used by Di Francesco et al. is geared towards the global quantification of DNA methylation, whereas the beadchip array used for the present work covers only a minor fraction of all possible methylation sites, but does so at a single-site resolution [36].

Tannorella et al. [37] performed a candidate-based comparison of blood DNA methylation between $A D$ cases and healthy controls in six genes (PSEN1, BACE1, DNMT1, DNMT3A, DNMT3B, and MTHFR), related to $A D$ pathology or DNA methylation itself, but did not find a difference in methylation levels between groups. This is in line with the current findings, as no DMPs or DMRs associated with conversion to AD were found in these genes. It may be interesting to point out that even when looking at the nominal $p$-values of all the probes (188) in these six genes, only 2 probes, both in MTHFR, had a $p$-value below 0.05 when comparing converters with controls at baseline, which would not survive FDR correction for 188 probes. Similarly, while looking at the group by time interaction analysis there were more probes with a nominal $p$-value below 0.05 (2 in PSEN1, 2 in BACE1, 1 in DNMT1, 5 in DNMT3A, 3 in DNMT3B, and 2 in MTHFR), none of these survive FDR correction for 188 probes.

Lunnon et al. [34], also utilizing the 450K BeadChip, identify several DMPs related to clinical AD in blood, but with minimal overlap with the present study. Both studies find a DMP in a COP9 signalosome subunit gene, although they find it in COPS8, and we in COPS9. In either case, COP9 signalosome dysfunction may be involved in dendritic spine deterioration [38], one of the early hallmarks of $A D$ [39]. Of note, the main focus of Lunnon et al. [34] is the brain, where they find strong 
evidence for the epigenetic dysregulation of $A N K 1$, but they do not find evidence for altered DNA methylation of $A N K 1$ in the blood. We also did not detect any DMPs or DMRs in ANK1 surviving multiple testing correction, but there were some sites in ANK1 and ANK2 within the

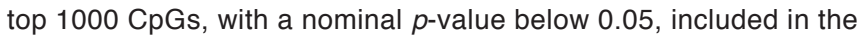
gene set enrichment analysis and occurring in various enriched terms, including GO:0045202 ("synapse"), GO:0007267 ("cell-cell signaling"), GO:0055085 ("transmembrane transport"), GO:1903522 ("regulation of blood circulation"), and GO:0042391 ("regulation of membrane potential”).

Adding to the evidence of a disturbed immune system in AD, we found a DMP at baseline in TRIM63, and a DMP in TRIM59 showing differential changes in methylation between controls and converters. These genes encode tripartite motif-containing proteins important for innate immunity [40]. While different TRIM variants, Lunnon et al. [34] find a DMP in TRIM26 in blood of AD cases, and another study by Niikura et al. [41] implicates TRIM11 in AD.

Several members of the solute-carrier $(S L C)$ gene superfamily have been detected in relation to AD. SLC genes encode all manner of membrane transporters, responsible for the transmembrane transport of a wide array of compounds, including amino acids, peptides, sugars, ions, lipids, vitamins, and neurotransmitters [42]. Before the development of clinical AD, we observed a DMR in SLC44A4, considered to be a choline transporter [43], and in the group by time interaction analysis a DMP in SLC39A10, a zinc transporter in the blood-brain barrier [44], was found. Additionally, many of the GO terms found to be enriched in converters at baseline included differentially methylated SLC genes, including GO:0099536 ("synaptic signaling"), GO:0007267 ("cell-cell signaling"), GO:0055085 ("transmembrane transport"), GO:0050877 ("neurological system process"), GO:1903522 ("regulation of blood circulation"), and GO:0043679 ("axon terminus"), as well as the KEGG pathway hsa04724 ("Glutamatergic synapse") (Table 4 and Table 5). Comparing AD cases with controls, Lunnon et al. [34] found SLC15C4 and SLCO3A1 to be differentially methylated, and differentially expression in blood. Moreover, a SNP in SLC24H4 has been identified as a risk factor for AD [45].

Interestingly, SLC44A4 is located within the human leukocyte antigen $(H L A)$ super-locus and may contain an enhancer region that is also associated with HLA-DRB5 [46, 47]. HLA-DRB5 is one of the major genes with differential methylation found in the current study, with 4 DMPs at baseline, and 1 DMP with a different time-related pattern for converters and controls. Differential methylation of HLA-DRB5 was also observed in the AD brain [48] and a SNP in this gene has been established as a susceptibility factor for AD [49]. Additional support for a role of $H L A$ 
genes in AD [50] comes from the identification of a DMP in HLA-DQA1 in the group by time interaction analysis, and from multiple enriched $\mathrm{GO}$ terms at baseline including HLA genes, such as GO:0098609 ("cell-cell adhesion"), GO:0050877 (“neurological system process"), GO:0042611 ("MHC protein complex"), GO:0004888 ("transmembrane signaling receptor activity"), and in all but 2 out of the 12 enriched KEGG pathways (Table 4 and Table 5).

$O X T$ is another gene associated with altered epigenetic regulation in $A D$, previously identified in the superior temporal gyrus [51] and by our group in the middle temporal gyrus (MTG) (Chapter 7), whereas now a blood DMR associated with conversion to AD was observed. Although the possible role of $O X T$ in the etiopathogenesis of $A D$ remains unknown, the identification of a DMR in this gene, identical to the one identified in the MTG, before the development of clinical symptoms indicates it may serve as a valuable early diagnostic marker.

It is thought that the first steps in the etiopathogenesis of AD start years, possibly decades before any clinical symptoms can be observed [6,52]. The present study captures the methylomic changes associated with the final stages of disease progression, just before the development of clinical symptoms. Especially the group by time interaction analysis is, to our knowledge, a first attempt to map which genes, as far as can be detected in the blood, show altered methylation levels during the onset of dementia. The results indicate that over a period of about 4.5 years many genes show a different methylation pattern in converters and non-converting healthy controls. With a very high mean age of 81.80 , both the control and converter groups fall within the extreme risk group for the development of AD [53], allowing for two, likely overlapping, interpretations of the methylomic differences between controls. They may reflect pathological changes leading up to the manifestation of dementia in the converters, or, in contrast, may represent protective mechanisms in the control group, making them resilient to the development of dementia. Both interpretations may be worthwhile for future investigations, as it may prove to be more difficult to halt disease progress, than to bolster innate mechanisms that delay the onset of clinical symptoms. Especially those DMPs also identified in the separate converter and non-converter comparisons between baseline and follow-up may be of particular interest for future studies into markers for conversion to dementia or resilience to such a conversion.

Even though the post-hoc selection design of the current study allowed for the inclusion of a sizeable group of converters, it remains a relatively small sample size for a genome-wide approach. However, to lack of similar studies focusing on the preclinical blood methylome of $A D$ 
precluded a targeted approach. Additionally, the design could have benefitted from the inclusion of clinical data at multiple timepoints to match the changes in methylation to changes in cognition, or other clinical hallmarks of $A D$. DNA from the blood originates from a heterogenous population of cell types, the proportions of which change during aging and may also be affected by disease, and each with their distinct epigenetic profile $[27,54,55]$. It has therefore been noted that disease- or agerelated epigenetic changes in the blood may be explained by shifts in the white blood cell composition [27, 35]. The current study adjusts for these changes through the inclusion of covariates representing the most prominent white blood cell types into the model [28], even though this cannot guarantee changes in blood cell type composition had absolutely no effect on the observed methylomic changes. It should also be kept in mind that even though the $450 \mathrm{~K}$ BeadChip covers most known genes, it actually only covers only $1.5-2 \%$ of all $\mathrm{CpG}$ sites, and practically none of the $\mathrm{CpA}, \mathrm{CpT}$, and $\mathrm{CpC}$ sites [36], meaning that it cannot be considered as truly 'genome-wide'.

Taken together, the present work offers a unique window into the methylomic profile of $A D$ before the onset of clinical symptoms, and the critical methylomic changes that have occurred after the onset of symptoms. Thereby the results provide a compelling basis for the use of blood methylomic markers for the pre-clinical diagnosis and prognosis of $A D$, and provides candidate sites that can be investigated in this respect, as well as potential markers of resilience to $A D$ dementia.

\section{Acknowledgments}

Funds have been provided by the Internationale Stichting Alzheimer Onderzoek (ISAO) grant 11532 (D.L.A.vdH.), by the ISAO grants 09552 and 13515, and the Netherlands Organization for Scientific Research (NWO), grant 916.11.086 (Veni Award) (B.P.F.R.), and by a fellowship as part of NWO grant 022.005.019, (R.L.). The funding agencies were not involved in the study design, data collection, analysis and interpretation, writing of the report, and the decision to submit the article for publication. The authors declare no conflicts of interest.

\section{REFERENCES}

[1] Yates D, McLoughlin DM. The molecular pathology of Alzheimer's disease. Psychiatry 2008; 7: 1-5. [2] Godyń J, Jończyk J, Panek D, et al. Therapeutic strategies for Alzheimer's disease in clinical trials. Pharmacol Reports 2015; 68: 127-138.

[3] Tanzi RE. The genetics of Alzheimer disease. Cold Spring Harb Perspect Med; 2. Epub ahead of print 2012. DOI: 10.1101/cshperspect. a006296.

[4] Robinson M, Lee BY, Hane FT. Recent progress in Alzheimer's disease research, part 2: genetics and epidemiology. J Alzheimers Dis 2017; 57: 317-330.

[5] Lardenoije R, Iatrou A, Kenis $\mathrm{G}$, et al. The epigenetics of aging and neurodegeneration. Prog Neurobiol 2015; 131: 21-64.

[6] Iatrou A, Kenis G, Rutten BPF, et al. Epigenetic dysregulation of brainstem nuclei in the pathogenesis of Alzheimer's disease: looking in the correct place at the right time? Cellular and Molecular Life Sciences, 2016, pp. $1-15$.

[7] van den Hove D LA, Kenis G, Rutten BP. Epigenetic dysregulation in Alzheimer's disease: cause or consequence? Epigenomics 2014; 6 : $9-11$.

[8] St-Amour I, Cicchetti F, Calon F. Immunotherapies in Alzheimer's disease: Too much, too little, too late or off-target? Acta Neuropathol 2016; 131: 481-504.

[9] Sperling R, Mormino E, Johnson K. The evolution of preclinical Alzheimer's disease: implications for prevention trials. Neuron 2014; 84: $608-622$.

[10] Guidotti A, Auta J, Davis

JM, et al. Toward the identification of 
peripheral epigenetic biomarkers of schizophrenia. J Neurogenet 2014; 28 : 41-52.

[11] Cacabelos R, Torrellas C.

Epigenetic drug discovery for Alzheimer's disease. Expert Opin Drug Discov 2014; 9: 1059-1086.

[12] Wen K-X, Miliç J, El-

Khodor B, et al. The role of DNA methylation and histone modifications in neurodegenerative diseases: a systematic review. PLoS One 2016; 11: e0167201.

[13] Luck T, Riedel-Heller SG, Luppa M, et al. Risk factors for incident mild cognitive impairment - results from the German Study on Ageing, Cognition and Dementia in Primary Care Patients (AgeCoDe). Acta Psychiatr Scand 2010; 121: 260-272.

[14] Zaudig M, Hiller W, Geiselmann B, et al. SIDAM -

Handbuch, strukturiertes Interview für die Diagnose einer Demenz vom Alzheimer-Typ, der Multiinfarkt-(oder vaskulären) Demenz und Demenzen anderer Ätiologie nach DSM III R, DSM IV and ICD 10http:// pubman.mpdl.mpg.de/pubman/faces/ viewItemFullPage.jsp?itemId=escidoc\%3Al629566\%3Al\&view $=$ EXPORT (1995, accessed 20 April 2017).

[15] McKhann G, Drachman D, Folstein M, et al. Clinical diagnosis of Alzheimer's disease: report of the NINCDS-ADRDA Work Group under the auspices of Department of Health and Human Services Task Force on Alzheimer's Disease. Neurology 1984; 34: 939-44.

[16] Reisberg B, Ferris SH, de Leon MJ, et al. The Global Deterioration Scale for assessment of primary degenerative dementia. Am J Psychiatry 1982; 139: 1136-1139. ziger WL, et al. A new clinical scale for the staging of dementia. Br J Psychiatry 1982; 140: 566-72.

[18] R Core Team. R: A language and environment for statistical computing.https://www.r-project.org/ (2016).

[19] van Iterson M, Tobi EW,

Slieker RC, et al. MethylAid: visual and interactive quality control of large Illumina $450 \mathrm{k}$ datasets. Bioinformatics 2014; 30: 3435-3437.

[20] Aryee MJ, Jaffe AE, Corrada-Bravo H, et al. Minfi: a flexible and comprehensive Bioconductor package for the analysis of Infinium DNA methylation microarrays. Bioinformatics 2014; 30: 1363-1369.

[21] Pidsley R, CC YW, Volta M, et al. A data-driven approach to preprocessing Illumina $450 \mathrm{~K}$ methylation array data. BMC Genomics 2013; 14: 293.

[22] Zhou W, Laird PW, Shen

H. Comprehensive characterization, annotation and innovative use of Infinium DNA methylation BeadChip probes. Nucleic Acids Res 2016; 15 . gkw967.

[23] van Iterson M, Tobi E, Slieker R, et al. DNAmArray. Epub ahead of print 2017. DOI: https://doi. org/10.5281/zenodo.158908.

[24] Fortin J-P, Labbe A, Lemire M, et al. Functional normalization of $450 \mathrm{k}$ methylation array data improves replication in large cancer studies.

Genome Biol 2014; 15: 503.

[25] Müllner D. fastcluster: Fast hierarchical, agglomerative clustering routines for R and Python. J Stat Softw 2013; 53: 1-18.

[26] Price ME, Cotton AM, Lam LL, et al. Additional annotation enhances potential for biologically-rele- vant analysis of the Illumina Infinium HumanMethylation450 BeadChip array. Epigenetics Chromatin 2013; 6: 4. Jaffe AE, Irizarry RA. Accounting for cellular heterogeneity is critical in epigenome-wide association studies. Genome Biol 2014; 15: R31.

[28] Houseman E, Accomando WP, Koestler DC, et al. DNA methylation arrays as surrogate measures of cell mixture distribution. $B M C$ Bioinformatics 2012; 13: 86.

[29] Du P, Zhang X, Huang C-C, et al. Comparison of Beta-value and M-value methods for quantifying methylation levels by microarray analysis. BMC Bioinformatics 2010; 11: 587.

[30] Du P, Kibbe WA, Lin SM. lumi: a pipeline for processing Illumina microarray. Bioinformatics 2008; 24: 1547-1548.

[31] Ritchie ME, Phipson B, Wu D, et al. limma powers differential expression analyses for RNA-sequencing and microarray studies. Nucleic Acids Res 2015; 43: e47.

[32] van Iterson $M$, van Zwet EW, BIOS Consortium BT, et al. Controlling bias and inflation in epigenome- and transcriptome-wide association studies using the empirical null distribution. Genome Biol 2017; 18: 19.

\section{[33] Di Francesco A, Arosio}

B, Falconi A, et al. Global changes in DNA methylation in Alzheimer's disease peripheral blood mononuclear cells. Brain Behav Immun 2015; 45: 139-144.

[34] Lunnon K, Smith R, Hannon E, et al. Methylomic profiling implicates cortical deregulation of $A N K 1$ in Alzheimer's disease. Nat Neurosci 2014; 17: 1164-1170.

Li H, Guo Z, Guo Y, et al. 
Common DNA methylation alterations of Alzheimer's disease and aging in peripheral whole blood. Oncotarget 2016; 7: 19089-98.

[36] Clark C, Palta P, Joyce CJ, et al. A comparison of the whole genome approach of MeDIP-seq to the targeted approach of the Infinium $\mathrm{Hu}$ manMethylation450 BeadChip( $\left({ }^{\circledR}\right)$ for methylome profiling. PLoS One 2012; 7: e50233.

[37] Tannorella P, Stoccoro A,

Tognoni G, et al. Methylation analysis of multiple genes in blood DNA of Alzheimer's disease and healthy individuals. Epub ahead of print 2015. DOI: 10.1016/j.neulet.2015.06.009.

[38] Djagaeva I, Doronkin S.

Dual regulation of dendritic morphogenesis in Drosophila by the COP9 signalosome. PLoS One 2009; 4: e7577. [39] Knobloch M, Mansuy IM. Dendritic spine loss and synaptic alterations in Alzheimer's disease. $\mathrm{Mol}$ Neurobiol 2008; 37: 73-82.

[40] Ozato K, Shin D-M, Chang $\mathrm{T}-\mathrm{H}$, et al. TRIM family proteins and their emerging roles in innate immunity. Nat Rev Immunol 2008; 8: 849-60. [41] Niikura T, Hashimoto Y, Tajima H, et al. A tripartite motif protein TRIM11 binds and destabilizes Humanin, a neuroprotective peptide against Alzheimer's disease-relevant insults. Eur J Neurosci 2003; 17: 1150-8. [42] He L, Vasiliou K, Nebert DW. Analysis and update of the human solute carrier (SLC) gene superfamily. Hum Genomics 2009; 3: 195-206. Mattie M, Raitano A, Morrison K, et al. The discovery and preclinical development of ASG-5ME, an antibody-drug conjugate targeting SLC44A4-positive epithelial tumors including pancreatic and prostate cancer. Mol Cancer Ther 2016; 15:

2679-2687.

[44] Huntley MA, Bien-Ly N,

Daneman R, et al. Dissecting gene expression at the blood-brain barrier.

Front Neurosci 2014; 8: 355.

[45] Karch CM, Goate AM.

Alzheimer's disease risk genes and mechanisms of disease pathogenesis. Biol Psychiatry 2015; 77: 43-51.

[46] Shiina T, Hosomichi K,

Inoko $\mathrm{H}$, et al. The HLA genomic loci map: expression, interaction, diversity and disease. J Hum Genet 2009; 54: $15-39$.

[47] GeneCards Human Gene Database. SLC44A4. http://www. genecards.org/cgi-bin/carddisp. pl?gene=SLC44A4 (2017, accessed 30 April 2017).

[48] Yu L, Chibnik LB, Srivastava GP, et al. Association of brain DNA methylation in SORL1, $A B C A 7$, HLA-DRB5, SLC24A4, and BIN1 with pathological diagnosis of Alzheimer disease. JAMA Neurol 2015; 72: 15.

[49] Lambert J-C, Ibrahim-Verbaas CA, Harold D, et al. Meta-analysis of 74,046 individuals identifies 11 new susceptibility loci for Alzheimer's disease. Nat Genet 2013; 45: 1452-1458.

[50] Steele NZR, Carr JS,

Bonham LW, et al. Fine-mapping of the human leukocyte antigen locus as a risk factor for Alzheimer disease: A case-control study. PLoS Med 2017; 14: el002272.

[51] Watson CT, Roussos P, Garg P, et al. Genome-wide DNA methylation profiling in the superior temporal gyrus reveals epigenetic signatures associated with Alzheimer's disease. Genome Med 2016; 8: 5.

[52] Lahiri DK, Maloney B. The

'LEARn' (Latent Early-life Associated Regulation) model integrates environmental risk factors and the developmental basis of Alzheimer's disease, and proposes remedial steps. Exp Gerontol 2010; 45: 291-296.

[53] $\mathrm{Xu}$ W, Ferrari C, Wang H-X. Epidemiology of Alzheimer's Disease. In: Understanding Alzheimer's Disease. InTech. Epub ahead of print 27 February 2013. DOI: 10.5772/54398. [54] Reynolds LM, Taylor JR, Ding J, et al. Age-related variations in the methylome associated with gene expression in human monocytes and T cells. Nat Commun 2014; 5: 5366.

[55] Shad KF, Aghazadeh Y, Ahmad S, et al. Peripheral markers of Alzheimer's disease: Surveillance of white blood cells. Synapse 2013; 67: 541-543. 
A

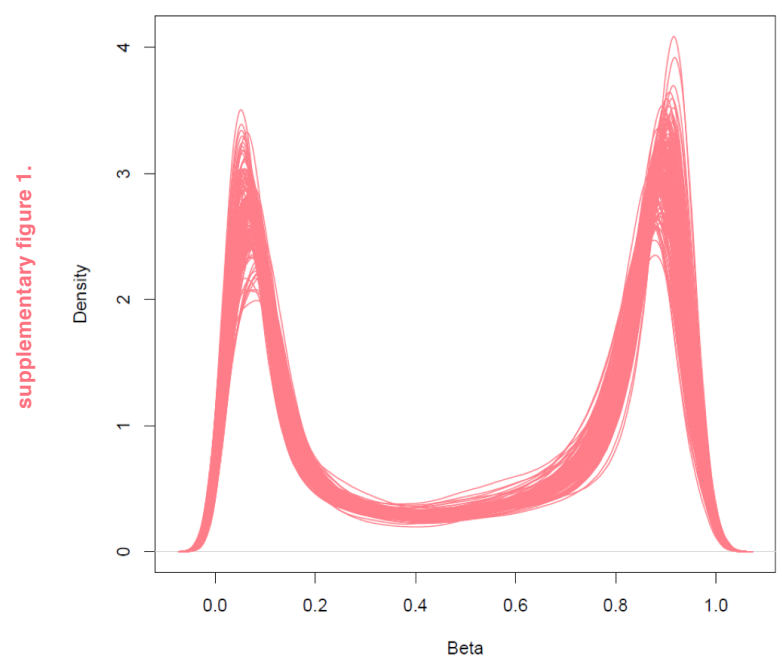

A

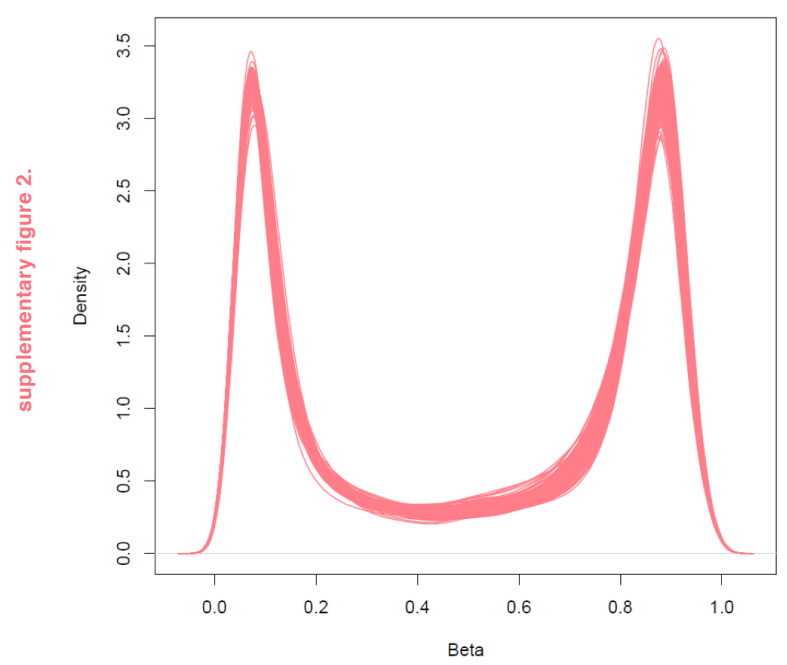

B

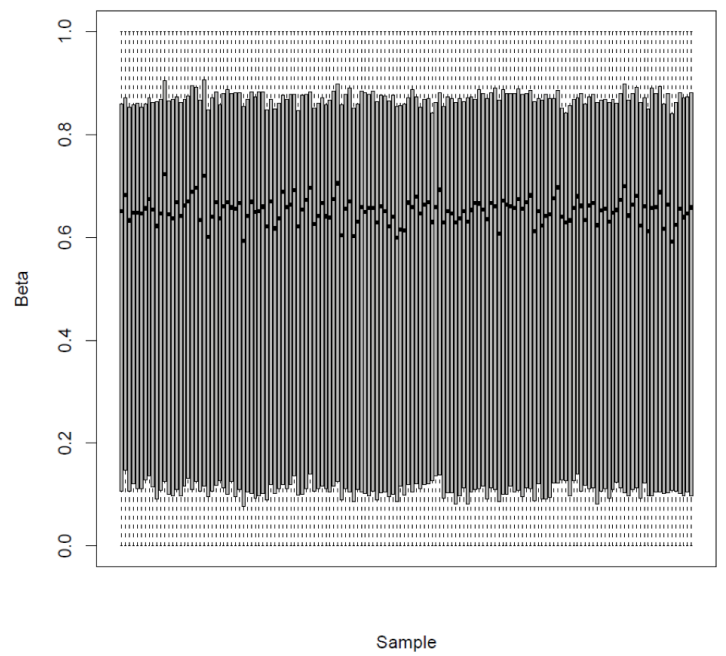

B

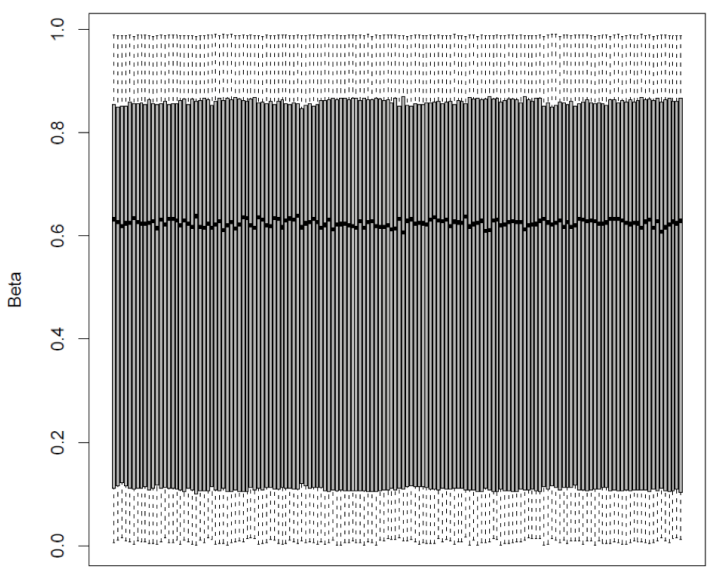

Sample

\section{SUPPLEMENTARY DATA}

SUPPLEMENTARY FIGURE 1. Density

plot (A) and boxplot (B) of raw beta

values. Plots include all DNA methyla-

tion probes on the Illumina Human-

Methylation450 BeadChip, plotted for each sample separately.
SUPPLEMENTARY FIGURE 2. Density

plot (A) and boxplot (B) of normal-

ized beta values. Plots include DNA

methylation probes passing the

filtering steps, plotted for each sample

separately. 
A
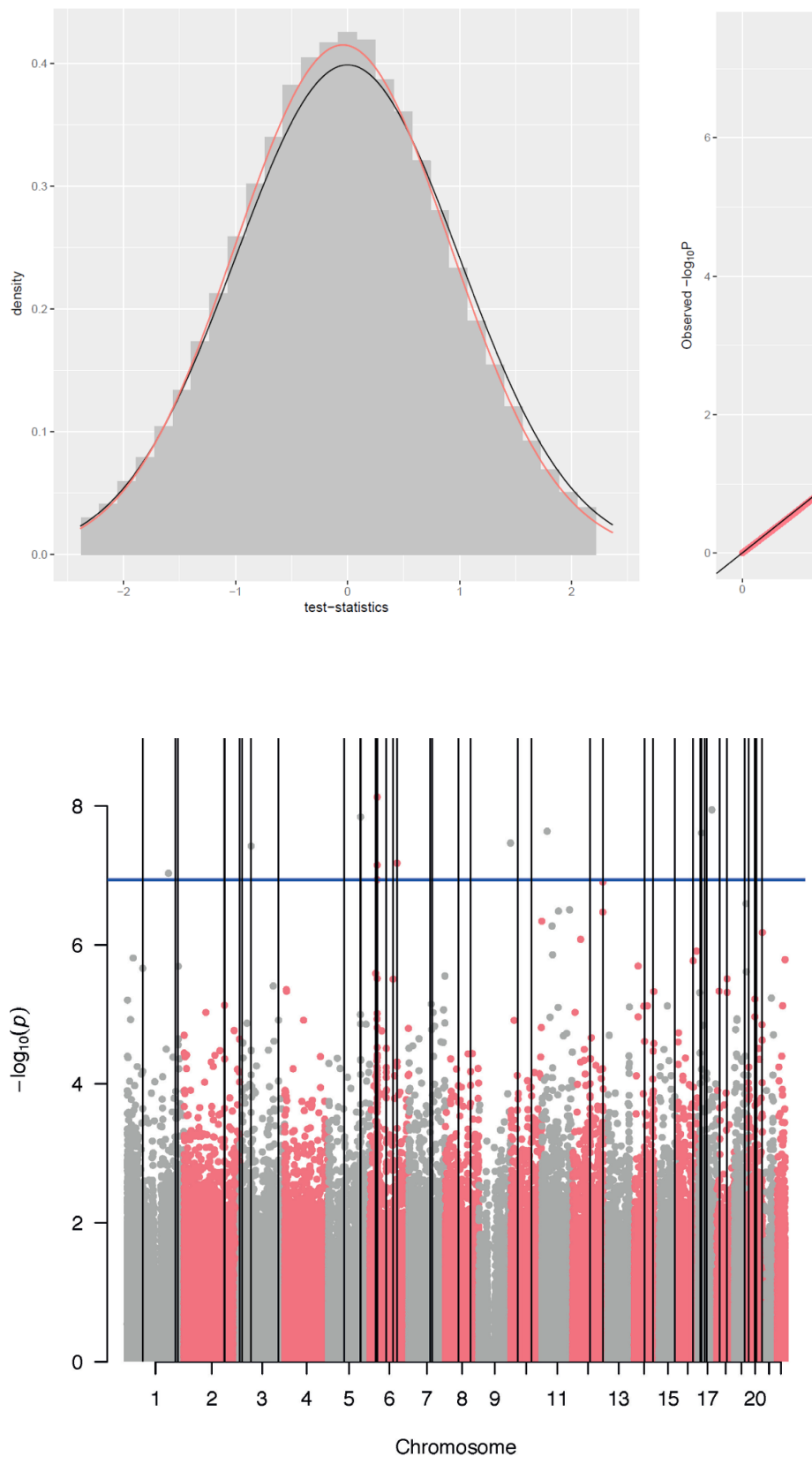

\section{SUPPLEMENTARY FIGURE 3. Histogram}

of test-statistics showing potential bias (A) and quantile-quantile (QQ) plot showing potential inflation (B) of the methylome-wide association study results at baseline. The pink line in A

\section{B}

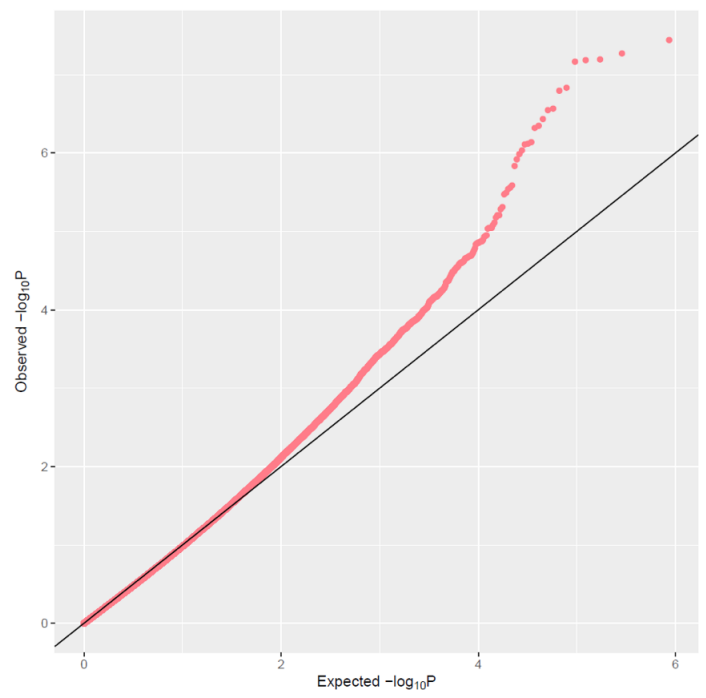

लं features, both estimated as described in [32]. The estimated bias and inflation was -0.042 and 0.96 , respectively.

SUPPLEMENTARY FIGURE 4. Manhattan plot of the methylome-wide association study at baseline. The blue horizontal line indicates the genome-wide significance threshold (0.05 / \# probes in analysis). Vertical black lines indicate differentially methylated regions as determined with comb-p, using a seeding $p$-value threshold of 0.05 and an extension window of 200 base pairs, and with more than two probes and a Šidák-adjusted $p$-value below 0.05 . 
A

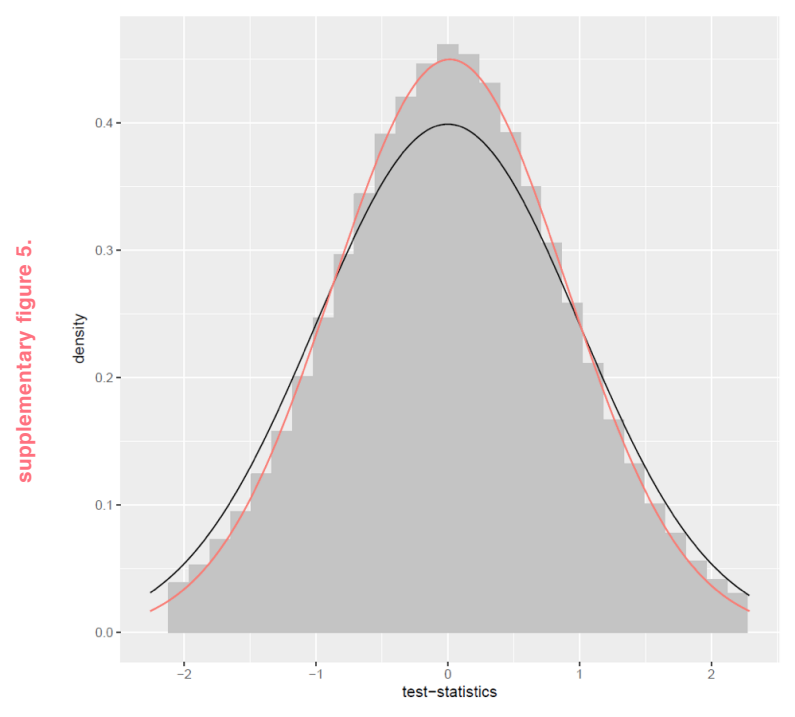

B

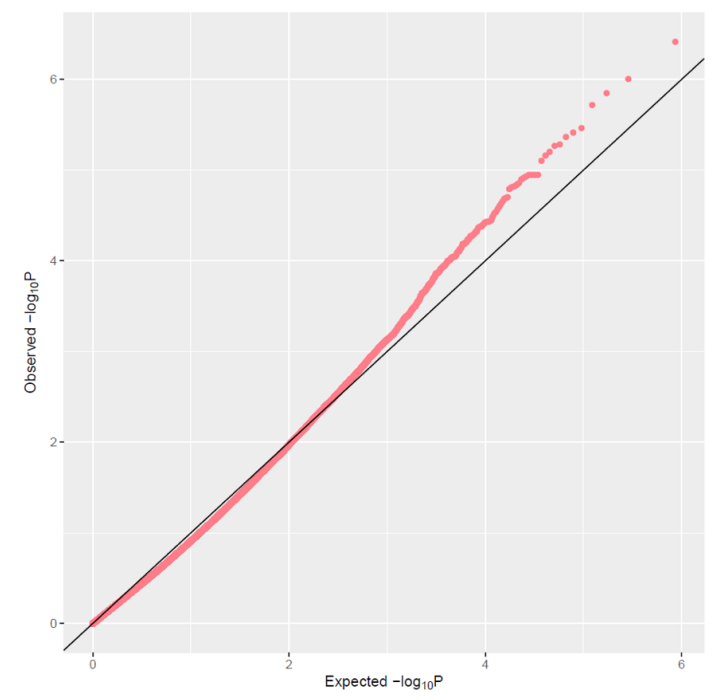

SUPPLEMENTARY FIGURE 5. Histogram of test-statistics showing potential bias (A) and quantile-quantile (QQ) plot showing potential inflation (B) of the group by time methylome-wide association analysis. The pink line in A represents the fit of the empirical null distribution and the black line the fit of the mixture of the empirical null distribution, the proportion of positively associated features, and the proportion of negatively associated features, both estimated as described in [32]. The estimated bias and inflation was 0.16 and 0.89 , respectively.

SUPPLEMENTARY FIGURE 6. Manhattan plot of the group by time interaction methylome-wide association analysis. The blue horizontal line indicates the genome-wide significance threshold (0.05 / \# probes in analysis). Vertical black lines indicate differentially methylated regions as determined with comb-p, using a seeding $p$-value threshold of 0.05 and an extension

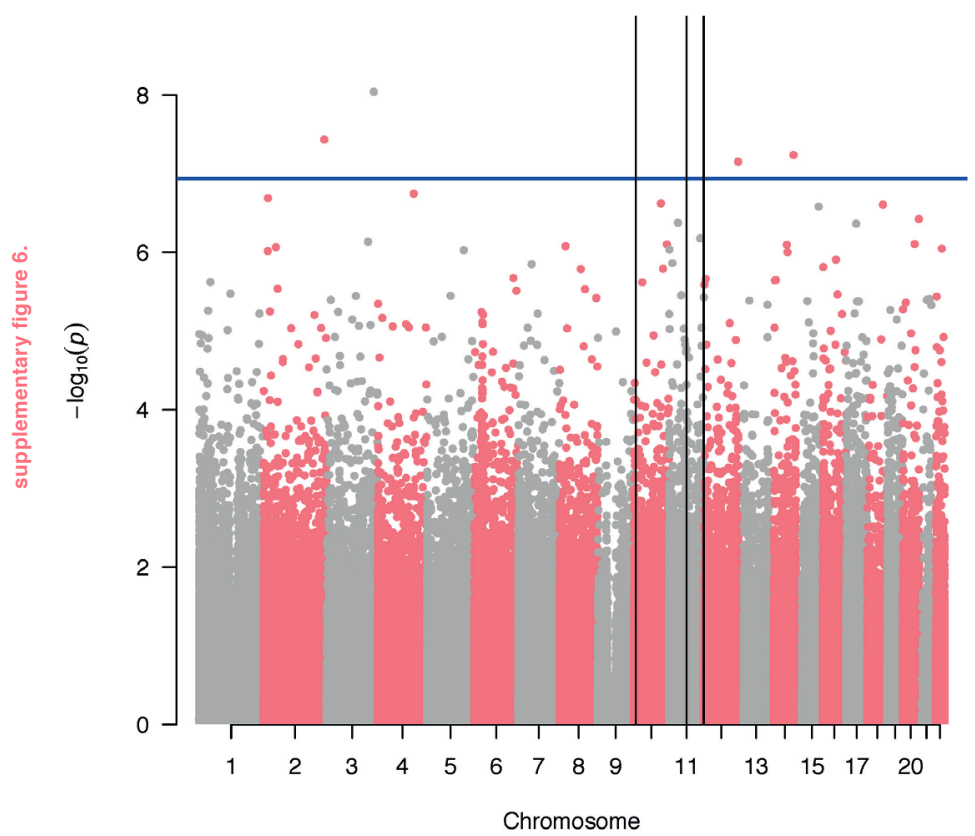

window of 200 base pairs, and with more than two probes and a Šidák-adjusted $p$-value below 0.05 . 



\section{GENERAL DISCUSSION}




\subsection{Summary of key findings}

This thesis commences with a broad exposition of the current status of epigenetics research in relation to aging and neurodegeneration in Chapter 2. Starting with a description of the various layers of epigenetic regulation of transcription and translation, from DNA and chromatin modifications, to non-coding RNAs, followed by a discussion of the epigenetic dysregulation observed in aging and neurodegenerative diseases Alzheimer's disease (AD), Parkinson's disease, and Huntington's disease. From this it followed that epigenetic dysregulation in neurodegeneration may be the result of other pathological processes, such as the proposed interaction between amyloid- $\beta(A \beta)$ and DNA methylation in $A D$, leading to an exacerbation of pathology. Alternatively, epigenetic cascades leading to pathology may be set in motion in response to environmental factors, such as the apparent effect of insufficient vitamin B12 and B6, folate, and S-adenosylmethionine intake on the development of dementia. The potential of epigeneticsbased therapies was also discussed, including the off-label use of existing drugs that impact epigenetic regulation, usually on a global level, and the development of novel, more targeted and disease-specific approaches. Such therapies may directly target epigenetic dysregulation observed in relation to a disease, or may be aimed at improving symptoms associated with the disease, for instance in order to enhance cognitive function in AD. The perspective ended with a critical appraisal of the limitations of current epigenetics studies and supplied suggestions for future research.

As animal models are crucial for validation and for investigation of the functional impact of disease-related factors in a strictly regulated experimental setting, and as half of the studies included within this thesis rely on animal models, Chapter 3 provides a concise overview of animal models used for studying cognitive impairment as seen in neurodegenerative diseases such as AD. In addition to the animal models used in the three chapters following Chapter 3, pharmacological models and models used for other neurodegenerative diseases were also described.

In contrast to the later AD-centered studies, the study in Chapter 4 focused on epigenetic changes associated with healthy aging, and how these may be influenced to prolong the lifespan. Specifically, global levels of DNA methylation and hydroxymethylation were measured in the nuclei of mouse cerebellar Purkinje cells and analyzed with respect to age, caloric restriction, and endogenous antioxidant overexpression. It was found that both DNA methylation and hydroxymethylation increase with age in Purkinje cells, and that caloric restriction, but not overexpression of antioxidant, was able to prevent these age-related epigenetic changes. Although caloric restriction was associated with an increased lifespan, it 
remains to be established exactly what the role of epigenetic alterations is in prolonging the lifespan.

Chapter 5 moved from normal aging to aging in animal models of $A D$, looking at age-related changes of DNA methylation and hydroxymethylation, and DNA methyltransferase 3A (DNMT3A) in the hippocampus. Animals with different genetic mutations related to $A D$ pathology were included to investigate the differences in age-related epigenetic changes; a transgenic mouse model expressing human mutant $A P P(\mathrm{~J} 20)$, a transgenic mouse model expressing both human mutant $A P P$ and $P S 1$ (APP/PS1dE9), a transgenic mouse model expressing mutant $A P P, P S 1$ and $M A P T$ (3xTg-AD), and a non-human primate model (Caribbean vervets), naturally exhibiting sporadic AD-like pathology with aging. A great discrepancy was observed between the different models, with the J20 model showing a decrease of global DNA methylation levels in the hippocampus, the 3xTg-AD model displaying an age-related increase in DNA methylation levels, and the APP/PS1dE9 and vervets showing no significant age-related changes. Accordingly, a negative correlation between DNA methylation and plaque load was observed in the J20 model, while this correlation was positive in the 3xTgAD model. No significant age-related change in DNA hydroxymethylation and DNMT3A were observed in any of the models. This study showed that the selection of an animal model for epigenetic studies may have a profound effect on the outcome, although it remains to be elucidated what the exact relationship is between the different genetic mutations and the observed epigenetic differences. Additionally, due the discrepancy in observations regarding global levels of epigenetic markers in AD it may be more fruitful to use targeted approaches, focusing on specific AD-related genomic regions.

The dynamic relationship between $A \beta$ pathology and markers of DNA methylation and hydroxymethylation, as well as DNMT3A, was investigated and covered in Chapter 6 . Making use of hippocampal tissue from APP/PS1dE9 mice that underwent active anti-A $\beta$ immunotherapy, the epigenetic impact of lowering $A \beta$ levels at an advanced disease stage could be studied. It was found that global DNA hydroxymethylation and DNMT3A levels were decreased in the vaccinated group, while global DNA methylation levels remained the same. Interestingly, DNA methylation and hydroxymethylation, but not DNMT3A, correlated with behavioral outcomes related to cognition. Additionally, $A \beta$ pathology and markers of synaptic integrity correlated with DNA hydroxymethylation and DNMT3A levels, but not DNA methylation. This study showed that lowering $A \beta$ levels by anti-A $\beta$ immunotherapy has a profound effect on epigenetic markers, and that DNA methylation and hydroxymethylation levels may be an indicator of cognitive performance in the transgenic APP/PS1dE9 model of AD. 
For Chapter 7 the methylomic and hydroxymethylomic profiles associated with $A D$ were investigated using a genome-wide microarray with tissue from the human middle temporal gyrus, one of the brain regions affected by $A D$ pathology. Using bisulfite (BS)- and oxidative bisulfite (oxBS)treated DNA, the combined DNA methylation and hydroxymethylation signal, and the isolated DNA methylation signal could be determined, respectively. Subtracting the signal from the oxBS-treated DNA from the BS-treated DNA signal yielded the DNA hydroxymethylation signal. Changes in levels of unmodified cytosines could also be determined by subtracting the beta values of the BS converted DNA from 1. This novel approach, separating the different DNA modifications, led to the detection of genes previously associated with $\mathrm{AD}$, such as RHBDF2, ANK1, and C3, but also $O X T$, which has only very recently been associated with $A D$. Inspection of $O X T$ methylation and hydroxymethylation as a function of Braak stage indicated progressive hyperhydroxymethylation and hypomethylation with advancing Braak stages. This study illustrates the importance of separating DNA methylation and hydroxymethylation signals when studying the epigenome in the brain where DNA hydroxymethylation is enriched.

The explorative study described in Chapter 8 assessed the blood methylomic profile of $A D$. Additionally, it looked at the overlap between the AD methylomic profile and that observed in individuals with mild cognitive impairment $(\mathrm{MCl})$, and the association of the AD methylomic profile with other measures of clinical $A D$ dementia, including cognitive performance, $A \beta$ and phosphorylated tau in the cerebrospinal fluid, and hippocampal volume. The identified blood methylomic profile of AD, comprising various genes with differentially methylated positions (DMPs) and regions (DMRs), showed limited overlap with previously reported methylomic profiles of the AD brain, including HLA-DRB5. Nonetheless, multiple affected genes which may play a role in $A D$ etiopathogenesis were identified, such as PCDHA1, CDH13, CLSTN2, NEFL, and MAD1L1, some of which already showed differential methylation at the $\mathrm{MCl}$ stage or also showed associations with other markers of clinical AD dementia. These markers may therefore be investigated as early markers of $A D$ dementia and could represent dysregulated pathways part of the etiopathogenesis of AD.

Chapter 9 presents a seminal study exploring the pre-clinical AD blood methylome in a sample of individuals without signs of dementia at baseline, but part of which had converted to AD dementia at follow-up 4.5 years later. Comparing the baseline methylome of converters and non-converters led to the identification of multiple genes with DMPs and DMRs associated with conversion to AD dementia, including HLA-DRB5 and $O X T$, candidates from previous investigations of the AD methylomic 
profile in the brain. In addition, using the methylome at baseline and follow-up, a group by time interaction analysis was performed to identify positions and regions showing differences in time-related alterations in methylation levels between converters and non-converters. The candidates from this analysis were subsequently analyzed for age-related changes in converters and non-converters separately, to establish in which group they were mainly affected. Epigenetic regulation of the resulting set of genes may be used as an indication for conversion to clinical dementia, or resilience to such a conversion.

\subsection{Limitations}

The most important limitations encountered in epigenetics studies are described in Chapter 2, and the studies presented in this thesis are also subjected to some of these limitations. One of the most critical limitations may be the small to moderate sample size of most of the studies, limiting their power to detect significant changes, in particular at the level of DMPs. This is exemplified by some of the $p$-values reported in Chapters 4-6 being close to the 0.05 threshold. These findings would greatly benefit from a replication study followed by a meta-analysis. Also the results of Chapter 7 should be interpreted with caution, as none of the identified DMPs survived correction for multiple testing. Generally, as it may be difficult to gather large samples, as well as the funds to perform epigenome-wide analyses on them, it is crucial for the field to collaborate and merge assets, perform replication studies, and attempt metaanalyses of existing observations.

A limitation inherent to epigenetic studies is their interpretability regarding causality. When comparing a diseased group with a healthy group and epigenetic differences are detected, it is impossible to say whether the epigenetic dysregulation caused the pathology, or whether it is a consequence of it, or an epiphenomenon. However, as, for instance, environmental exposures and $A \beta$ can both have an effect on epigenetic regulation, the observed epigenetic changes in relation to $A D$ are likely a combination of early, potentially causal changes, and changes that are caused by other pathological hallmarks of the disease. Disentangling these processes will require highly regulated longitudinal in vitro and animal model studies, involving, for example, in vitro and in vivo epigenetic editing.

Another difficulty of epigenetics research is the dynamic nature of epigenetic regulation, meaning that experimental procedures themselves may induce epigenetic changes. Thus, while it is crucial to determine the behavioral impact of epigenetic alterations, it should be taken into 
account that, for instance, behavioral testing in animal models may be stressful and induce epigenetic changes [1], or repeated testing may induce epigenetic processes related to learning and memory [2].

It is also important to be aware of the extent of the epigenetic regulatory machinery and the limitations of the tools used to investigate it. For instance, the studies described in Chapters 4-6 use immunohistochemistry for the detection of epigenetic markers. This allows for the study of these markers in a highly localized manner, focusing on a small sub-region or even a specific cell-type as in Chapter 4. However, this method, especially when using 3,3'-diaminobenzidine tetrahydrochloride (DAB) as chromogen, cannot be used for absolute quantification of the targets, as there is no strictly linear relationship between staining intensity and antigen levels. Additionally, while in principle the immunohistochemical detection of DNA methylation and hydroxymethylation targets the whole genome, it obviously cannot distinguish between genes or genomic regions, something which can be achieved through sequencing techniques.

To investigate single $\mathrm{CpG}$ resolution changes in DNA methylation the Illumina HumanMethylation450 BeadChip (450K BeadChip) was used in Chapters 7-9. While overcoming some of the limitations of the immunohistochemical approach, it comes with its own set of restrictions. The probes of the $450 \mathrm{~K}$ BeadChip are spersed over the genome, covering most of the known genes, but just a small fraction of all $\mathrm{CpGs}$ in the genome, let alone $\mathrm{CpA}, \mathrm{CpT}$, and $\mathrm{CpC}$ sites [3]. This array thus cannot be truly considered 'genome-wide', and its use to determine global DNA methylation levels (to compare with immunohistochemical observations) is thus also limited due to its sparse and biased coverage of the genome. Another important consideration when using the $450 \mathrm{~K}$ BeadChip is the need for BS conversion of the input DNA. This procedure does not distinguish between DNA methylation and hydroxymethylation [4], which particularly hampers the interpretation of observations in the brain, which is enriched in DNA hydroxymethylation [5]. This limitation can be overcome through the use of oxBS conversion, which results in the 'true' DNA methylation signal, as opposed to the combined DNA methylation and hydroxymethylation signal gained from only BS treated DNA [6], as was applied in Chapter 7. However, the more recently discovered DNA formylation and carboxylation modifications are not taken into account using the oxBS approach, which actually converts 5-hydroxymethylcytosine (5-hmC) to 5-formylcytosine (5-fC) [6, 7]. Due to the low presence of 5-fC and 5-carboxylcytosine (5-caC), even when compared to $5-\mathrm{hmC}$, they are not likely to have a strong impact on DNA methylation and hydroxymethylation readings [7]. A possibly larger limitation of the indirect approach of determining DNA hydroxymethylation 
levels used in Chapter 7 is its vulnerability to noise, which is already relatively high due to the much higher abundance of DNA methylation in relation to DNA hydroxymethylation, but becomes even higher due to the subtraction procedure to obtain $5-\mathrm{hmC}$ levels [6]. This is exemplified by the large amount of negative $5-\mathrm{hmC}$ beta values observed in Chapter 7 , which required the implementation of strict detection thresholds.

While the work in this thesis focused on epigenetic DNA modifications, it is important to keep in mind the biological relevance of the findings. Global changes in epigenetic markers detected by immunohistochemistry are likely to reflect significant changes in cellular physiology, although it cannot be said which pathways are affected. On the other hand, significant changes in DNA methylation at a single CpG site may not result in a change in gene expression and may not have a subsequent functional outcome. Therefore, the studies in Chapters 7-9 are complemented by gene set enrichment analyses, identifying sets of genes with altered epigenetic regulation, which are more likely to have biological relevance. Nevertheless, such studies would benefit from secondary measures, such as gene expression.

Cell-type and tissue specific epigenetic profiles may limit the use of material not extracted directly from the main site of pathology, and which may explain the limited overlap between the AD methylomic profiles determined from the brain and those from the blood [8, 9]. This becomes particularly important for longitudinal studies, where age-related changes in epigenetic markers could be explained by a shift in the cell-type composition of tissues with age [10], and therefore this was corrected for in the study of Chapter 9.

\subsection{Future perspectives}

One of the main limitations of genome-wide studies in the epigenetics field remains sample size, which makes studies often underpowered and limits the reproducibility of findings. Tissue banks, ideally also recording antemortem phenotypic data, may constitute an important source to increase sample sizes or of replication cohorts for simple designs. Nevertheless, as it may not be possible to gather large samples in practice, other approaches may be employed to strengthen observations. For instance, multiple tissues can be investigated, independent cohorts can be used to replicate findings, and alternative techniques can be employed to reproduce observations [9, 11]. Additionally, the detection of changes in epigenetic regulation should be followed-up by investigating changes in transcription and protein levels, and may be complemented by other 'omics' data $[12,13]$. As for diagnostic purposes it may be 
necessary to investigate tissues, such as blood, when the main site of pathology is not readily accessible, such as the brain in case of $A D$, it is important to determine how representative the investigated tissue is for pathological processes occurring at the site mainly affected by disease. It should also be noted that, while more expensive and more difficult to process, the field may greatly benefit from truly genome-wide sequencing approaches, as opposed to microarray techniques, which remain limited and biased in their coverage of the genome [3]. Furthermore, the use of tissue homogenates may dilute effects due to the inclusion of cell-types not relevant for the investigation, which may be overcome by advances in single-cell sequencing techniques [14].

Even though the research covered within this thesis covers a range of species, tissues, and techniques, and even looking at the broader perspective offered by Chapter 2, the current status of our understanding of the epigenetics of $A D$ and aging barely scratches the surface of its full complexity. Thus, while solutions can be offered to address the specific limitations mentioned above, a more radical shift in approach may be needed to truly evolve the field. With advances in microarray and sequencing techniques of recent years, the generation and sharing of big 'omics' datasets has become easier and easier, but the field seems to lag behind in how to deal with these datasets. For instance, harnessing the computational power of the current era and advances in computational modelling and machine learning, it may be possible to generate predictive models based on epigenetic profiles that may aid in the diagnosis and prognosis of $A D$ [15]. This may be crucial for the implementation of personalized medicine, which can benefit the development of effective treatment strategies for complex multifaceted diseases such as AD [16].

The World Health Organization (WHO) stated that early diagnosis and the identification of modifiable risk factors is pivotal for the optimal management of AD [17]. With the suspected gene-environment interactions at the bedrock of sporadic $\operatorname{AD}[18,19]$, and epigenetics as mediator [20], it may be necessary to apply a systems biology approach to fully grasp the etiopathogenesis of $A D$ and identify novel therapeutic targets [21]. Furthermore, as AD etiopathogenesis is thought to start long before the onset of clinical symptoms, longitudinal study designs such as employed in Chapter 9, but spanning a larger timeframe and including more follow-ups, are crucial to elucidate the early pre-clinical stages of $A D$, to disentangle healthy aging from pathological aging, and distinguish between epigenetic cascades driving disease progression from epigenetic epiphenomena [22].

\section{REFERENCES}

[1] Ryan J, Chaudieu I, Ancelin M-L, et al. Biological underpinnings of trauma and post-traumatic stress disorder: focusing on genetics and epigenetics. Epigenomics 2016; 8: 1553-1569.

[2] Molfese DL. Advancing neuroscience through epigenetics: molecular mechanisms of learning and memory. Dev Neuropsychol 2011; 36: 810-827.

[3] Clark C, Palta P, Joyce CJ, et al. A comparison of the whole genome approach of MeDIP-seq to the targeted approach of the Infinium $\mathrm{Hu}$ manMethylation450 BeadChip(®) for methylome profiling. PLoS One 2012; 7: e50233.

[4] van den Hove DLA, Chou-

liaras L, Rutten BPF. The role of 5-hydroxymethylcytosine in aging and Alzheimer's disease: current status and prospects for future studies. Curr Alzheimer Res 2012; 9: 545-9.

[5] Skvortsova K, Zotenko E, Luu P-L, et al. Comprehensive evaluation of genome-wide 5-hydroxymethylcytosine profiling approaches in human DNA. Epigenetics Chromatin 2017; 10 : 16.

[6] Booth MJ, Ost TWB, Beraldi D, et al. Oxidative bisulfite sequencing of 5-methylcytosine and 5 -hydroxymethylcytosine. Nat Protoc 2013; 8: 1841-1851.

[7] Ito S, Shen L, Dai Q, et al. Tet proteins can convert 5 -methylcytosine to 5 -formylcytosine and 5-carboxylcytosine. Science; http://science.sciencemag.org/content/333/6047/1300 (2011, accessed 3 April 2017).

[8] Davies MN, Volta M, Pidsley R, et al. Functional annotation of the human brain methylome identifies 
tissue-specific epigenetic variation across brain and blood. Genome Biol 2012; 13: R43.

[9] Lunnon K, Smith R, Han-

non E, et al. Methylomic profiling implicates cortical deregulation of $A N K 1$ in Alzheimer's disease. Nat Neurosci 2014; 17: 1164-1170.

[10] Li H, Guo Z, Guo Y, et al.

Common DNA methylation alterations of Alzheimer's disease and aging in peripheral whole blood. Oncotarget 2016; 7: 19089-98.

[11] De Jager PL, Srivastava

G, Lunnon K, et al. Alzheimer's disease: early alterations in brain DNA methylation at $A N K 1, B I N 1, R H B D F 2$ and other loci. Nat Neurosci 2014; 17: 1156-1163.

[12] Lunnon K, Mill J. Epigen-

etic studies in Alzheimer's disease: current findings, caveats, and considerations for future studies. $\mathrm{Am} \mathrm{J}$

Med Genet B Neuropsychiatr Genet 2013; 162B: 789-99.

[13] Dong HK, Gim J-A, Yeo SH, et al. Integrated late onset Alzheimer's disease (LOAD) susceptibility genes: Cholesterol metabolism and trafficking perspectives. Gene 2017; 597: 10-16. [14] Grün D, van Oudenaarden A. Design and analysis of single-cell sequencing experiments. Cell 2015; 163: 799-810.

[15] Goldstein BA, Navar AM, Carter RE. Moving beyond regression techniques in cardiovascular risk prediction: applying machine learning to address analytic challenges. Eur Heart $J$ 2016; ehw302.

[16] Tebani A, Afonso C, Marret $\mathrm{S}$, et al. Omics-based strategies in precision medicine: toward a paradigm shift in inborn errors of metabolism investigations. Int J Mol Sci 2016; 17: 1555.
[17] World Health Organization. Dementia. WHO Media Centre. http://www.who.int/mediacentre/ factsheets/fs362/en/ (2016, accessed 29 July 2016).

[18] Lahiri DK, Maloney B, Zawia NH. The LEARn model: an epigenetic explanation for idiopathic neurobiological diseases. Mol Psychiatry 2009; 14: 992-1003.

[19] Iatrou A, Kenis G, Rutten BPF, et al. Epigenetic dysregulation of brainstem nuclei in the pathogenesis of Alzheimer's disease: looking in the correct place at the right time? Cellular and Molecular Life Sciences, 2016, pp. $1-15$.

[20] Vineis P, Chatziioannou A, Cunliffe VT, et al. Epigenetic memory in response to environmental stressors. FASEB J 2017; fj.201601059RR. [21] Parikshak NN, Gandal MJ, Geschwind DH. Systems biology and gene networks in neurodevelopmental and neurodegenerative disorders. Nat Rev Genet 2015; 16: 441-458.

[22] van den Hove D LA, Kenis G, Rutten BP. Epigenetic dysregulation in Alzheimer's disease: cause or consequence? Epigenomics 2014; 6: 9-11. 



\section{SUMMARY}


The work in this thesis explores the involvement of epigenetics in aging and Alzheimer's disease (AD), using various techniques, animal models, and tissues.

CHAPTER 2 presents a thorough overview of epigenetic regulation and its involvement in aging and neurodegeneration. It covers the various layers of the epigenetic apparatus, including the mechanisms operating at the DNA level, the chromatin level, and the RNA level. Following this is a critical literature review of investigations implicating epigenetic mechanisms in aging and the neurodegenerative diseases $A D$, Parkinson's disease, and Huntington's disease. From this review, it followed that epigenetic processes may be disturbed due to genetic variation, environmental exposures, or due to interactions with diseaserelated pathology, in which case establishing causality remains a major challenge of the field. The implication of epigenetic processes in these neurodegenerative diseases provides novel therapeutic options that target these processes. While epigenetics-based treatments targeting specific disease-associated genes are still in their infancy, there are also investigations into the off-label use of existing drugs that have a widespread impact on epigenetic regulation. Furthermore, there are therapies being developed that aim to counteract certain symptoms, such as cognitive decline, but that do not necessarily target disease-related pathways, while other therapies directly aim to counter pathological cascades. Finally, the limitations of the field were discussed, such as the problem of causality in epigenetics research, and the challenge of replicating findings, complemented with suggestions for future studies, including the utilization of novel techniques and integration of multiple data modalities.

In CHAPTER 3 a selection of the most used rodent models to study cognitive impairments are described. Various modes of achieving cognitive impairment are covered, including pharmacological models, models based on aging, and transgenic disease models, among which transgenic models of $A D$.

The first experimental part of this thesis starts off with an investigation into epigenetic changes associated with normal aging in CHAPTER 4. For this study, global levels of DNA methylation and hydroxymethylation, two pivotal epigenetic markers, were determined at two ages in the nuclei of mouse cerebellar Purkinje cells. These cells are known for their vulnerability to age-related deterioration. Additionally, the impact on these epigenetic changes of caloric restriction and endogenous antioxidant overexpression, two strategies previously reported to extend the lifespan, were studied. It was observed that both global DNA methylation and hydroxymethylation increased with age in Purkinje cells, that caloric 
restriction was able to reduce this age-related increase, but that antioxidant overexpression did not have a significant effect on age-related epigenetic changes. These observations suggest that the life-prolonging effects of caloric restriction may be mediated through epigenetic processes, the exact nature of which remains to be established.

CHAPTER 5 covers a similar study as the previous chapter, but instead of healthy aging focuses on age-related epigenetic changes in models of $A D$. This study included three transgenic mouse models, expressing human mutant genes APP and PS1, associated with familial AD, or related to $A D$ pathology in case of the tangle-inducing mutant $M A P T$ gene. One model only expressed mutant $A P P$ (J20), another both APP and PS1 (APP/PS1dE9), and the third model expressed mutant APP, $P S 1$ and MAPT (3xTg-AD). Additionally, Caribbean vervets, a non-human primate model which may naturally develop early AD-like pathology with age, were included in the study. Global levels of DNA methylation and hydroxymethylation, as well as DNA methyltransferase (DNMT) $3 \mathrm{~A}$, responsible for de novo DNA methylation, were investigated in the hippocampus, a brain region related to memory heavily afflicted by AD. Curiously, the models showed divergent age-related changes in global DNA methylation levels; the J20 model exhibited decreases, the APP/PS1dE9 and vervets showed no changes, whereas the 3xTg-AD presented increases. Global DNA hydroxymethylation and DNMT3A levels, however, did not change significantly between ages in any of the models. Correlation analyses with plaque load, which, as expected, increased with age, detected a negative correlation with DNA methylation in the J20 model, and a positive correlation with DNA methylation in the 3xTg-AD model, while no significant correlations were observed for the other epigenetic markers. These results indicate that, on a global level, AD-related pathology mainly appears to be associated with DNA methylation, but that the choice of animal model may profoundly affect this association. It may thus be more insightful for future studies into the relationship between genetic mutations and epigenetic dysregulation to focus on specific disease-related genes and pathways to complement the observations on a global level.

For CHAPTER 6 , the impact of lowering amyloid- $\beta(A \beta)$ levels through active immunotherapy on epigenetic DNA modifications was investigated. Global levels of DNA methylation, DNA hydroxymethylation and DNMT3A were determined in the hippocampus of APP/PS1dE9 mice, which were distributed between a vaccinated and a vehicle control group. Anti-A $\beta$ immunotherapy was associated with lower levels of DNA hydroxymethylation and DNMT3A. Looking at correlations between the epigenetic markers and previously established markers of $A D$ pathology, including behavioral outcomes related to cognition, indicated 
that DNA hydroxymethylation and DNMT3A levels correlated with A $\beta$ pathology and synaptic integrity. Interestingly, both DNA methylation and hydroxymethylation, both not DNMT3A levels, correlated with the behavioral scores. These results offer a first glimpse into the epigenetic component of anti-A $\beta$ immunotherapy, although it remains to be elucidated whether the epigenetic differences stem from lowering $A \beta$ level or from immunologic processes associated with active immunization.

The second experimental part of this thesis focuses on human $A D$ patients, and switches from global immunohistochemical enquiries to a genome-wide, and site-specific, microarray approach. For CHAPTER 7 the methylome and hydroxymethylome were interrogated in the middle-temporal gyrus, a brain region affected by AD pathology. A novel approach integrating readings from bisulfite (BS)- and oxidative bisulfite (oxBS)-treated DNA was used to determine differences in the proportion of methylated, hydroxymethylated, and unmodified signals between AD patients and controls for each of the over 450,000 investigated $\mathrm{CpG}$ sites. Correlations between nearby $\mathrm{CpG}$ sites were also investigated to determine differentially methylated regions associated with $A D$. This implicated several genes known to be associated with $\mathrm{AD}$, including $R H B D F 2, A N K 1$, and $C 3$, but also $O X T$, which was only very recently found in relation to $A D$. Interestingly, OXT showed hyperhydroxymethylation and hypomethylation with increasing Braak stages. These findings underpin the importance of separating the DNA methylation and hydroxymethylation signals when studying the epigenome of the brain, which is highly enriched in DNA hydroxymethylation.

CHAPTER 8 can be seen as a more clinically oriented extension of the previous chapter, exploring the methylomic profile of $A D$ in blood. For this study, the blood methylome was determined in AD patients, individuals with mild cognitive impairment $(\mathrm{MCl})$, and a group of healthy controls. Sites associated with $A D$ were also investigated in relation to other determinants of clinical AD dementia, such as mini-mental state examination (MMSE) scores, $A \beta$ and phosphorylated tau in the cerebrospinal fluid, and hippocampal volume. Several differentially methylated positions (DMPs) and regions (DMRs) were observed in relation to $\mathrm{AD}$, including some in $\mathrm{AD}$ risk gene $H L A-D R B 5$, which was previously observed to also exhibit altered DNA methylation in the brain of AD patients. Implicated genes such as PCDHA1, CDH13, CLSTN2, $N E F L$, and MAD1L1, which already presented with altered methylation profiles in the $\mathrm{MCl}$ group or showed associations with other markers of $A D$ dementia, may reflect disturbed pathways in the etiopathogenesis of $A D$ and could be further investigated as early blood markers of $A D$ dementia. 
For CHAPTER 9 the pre-clinical methylome associated with conversion to $A D$ dementia was determined. Using samples from a large aging cohort including only healthy subjects at baseline and monitoring their conversion to $\mathrm{AD}$ dementia, it was possible to compare the methylome of individuals converting to AD dementia after an interval of 4.5 years, with that of controls still cognitively sound after 4.5 years, at a stage both groups did not yet show signs of dementia. This comparison led to the identification of multiple DMPs and DMRs, including the AD risk gene $H L A-D R B 5$ and, once more, $O X T$, which may represent prime candidates for the pre-clinical diagnosis of AD. Additionally, to gain insight into the differences in dynamic epigenetic regulation between converters and nonconverters, a group by time interaction analysis was performed. DMPs from this analysis were further investigated for time-related alterations in converters and non-converters separately to identify in which group they were significantly altered. Differences in methylation of top genes, such as DGKD, MRPL20, and ARHGAP12 from this analysis associated with converters may signify vulnerability to convert to $A D$ dementia. Conversely, methylation levels of top genes associated with nonconverters, including GRAMD1C, SMNDC1, and ARHGAP1 may reflect resilience to $A D$ dementia. 

SAMENVATTING 
Het werk in dit proefschrift verkent de rol van epigenetica in het verouderingsproces en de ziekte van Alzheimer (ZvA), en maakt hierbij gebruik van verschillende technieken, diermodellen en weefsels.

HOOFDSTUK 2 presenteert een gedetailleerd overzicht van epigenetische regulatie en de rol hiervan bij veroudering en neurodegeneratie. Het hoofdstuk omvat de verschillende lagen van het epigenetische systeem, inclusief de mechanismen die actief zijn op het DNA niveau, het chromatine niveau en het RNA niveau. Dit overzicht wordt gevolgd door een kritische evaluatie van de huidige literatuur omtrent de implicatie van epigenetische processen in de neurodegeneratieve ziektes van Alzheimer, Parkinson en Huntington. Hieruit blijkt dat epigenetische processen kunnen worden verstoord door genetische factoren en omgevingsfactoren, almede door interacties met ziekte-gerelateerde pathologie, waardoor causaliteit een van de grote uitdagingen van het onderzoeksveld is. De implicatie van epigenetische processen in deze ziektes kan leiden tot de ontwikkeling van nieuwe behandelingsstrategieën, die op deze processen inspelen. Momenteel staat de ontwikkeling van epigenetische behandelingen nog in de kinderschoenen, al wordt er ook onderzoek gedaan naar bestaande medicijnen die invloed kunnen hebben op epigenetische regulatie. Nieuwe behandelingen proberen symptomen zoals cognitieve achteruitgang tegen te gaan, of om direct pathologische cascades te onderbreken en zo ziekte te voorkomen. Als laatste worden de beperkingen van het huidige epigenetica onderzoek aan de tand gevoeld en worden suggesties gegeven voor toekomstige studies in dit veld. Bijvoorbeeld het gebruik van nieuwe technieken en de integratie van verschillende datatypes, zoals genetische en expressie data om de epigenetische data te complementeren.

In HOOFDSTUK 3 wordt een selectie van de meest gebruikte knaagdiermodellen voor cognitieve stoornissen beschreven. Verschillende modellen worden behandeld, waaronder farmacologische modellen, modellen gebaseerd op natuurlijke veroudering en transgene modellen, waaronder modellen voor ZvA.

HOOFDSTUK 4, het eerste onderzoek in dit proefschrift, focust op epigenetische veranderingen tijdens normale veroudering. Voor deze studie werd het globale niveau van DNA methylatie en hydroxymethylatie, twee belangrijke epigenetische indicatoren, bepaald in de kernen van cerebellaire Purkinje cellen van twee lijftijdsgroepen muizen. Deze cellen staan bekent om hun gevoeligheid voor verouderingsprocessen. Ook was de invloed van calorische restrictie en overexpressie van endogene antioxidanten, twee strategieën waarvan men denkt dat deze de levensduur kunnen verlengen, op deze epigenetische 
veranderingen onderzocht. Hieruit bleek dat globale DNA methylatie en hydroxymethylatie toeneemt met veroudering in Purkinje cellen, dat calorische restrictie deze toename tegengaat en dat antioxidant overexpressie geen significante invloed heeft op de geobserveerde epigenetische veranderingen. Deze observaties suggereren dat de levensduur-verlengende effecten van calorische restrictie gemedieerd worden door epigenetische processen. Het onderliggende mechanisme van deze mediatie zal nog verduidelijkt moeten worden in toekomstig onderzoek.

HOOFDSTUK 5 betreft een soortgelijk onderzoek als het vorige hoofdstuk, maar bestudeerd verouderings-gerelateerde epigenetische veranderingen in diermodellen van ZvA. In deze studie werd gebruik gemaakt van drie transgene muismodellen met transgenen die geassocieerd zijn met de familiale vorm van ZvA (APP en PS1) of pathologische kenmerken van ZvA (MAPT). Het eerste model (J20) was alleen gebaseerd op een gemuteerde variant van het APP gen, het tweede model (APP/PS1dE9) op mutanten van APP alsmede PS1 en het derde model (3xTgAD) bracht drie gemuteerde genen tot expressie; APP, $P S 1$ en MAPT. Ook werd een non-humaan primaat model bestudeerd, de Caribische vervet, die van nature pathologie kan ontwikkelen die lijkt op de vroege stadia van ZvA. Het globale niveau van DNA methylatie en hydroxymethylatie, maar ook DNA methyltransferase (DNMT) 3A, het enzym verantwoordelijk voor de novo DNA methylatie, werd op verschillende leeftijden in de hippocampus bepaald. De hippocampus speelt een belangrijke rol bij het geheugen en is ernstig aangetast bij ZvA. Opmerkelijk was de observatie dat de modellen verschillende ouderdoms-gerelateerde veranderingen in DNA methylatie lieten zien; in het $\mathrm{J} 20$ model ging het methylatie niveau omlaag, bij de APP/PS1dE9 muizen en de vervetten was er geen verandering en bij de 3xTgAD muizen ging de methylatie juist omhoog. Daarentegen lieten DNA hydroxymethylatie en DNMT3A geen significante veranderingen zien bij veroudering in alle modellen. De aanwezigheid van amylö̈de plaques nam, zoals verwacht, toe met veroudering en deze liet een negatieve correlatie zien met DNA methylatie in het J20 model en een positieve correlatie in het 3xTg-AD model, maar correleerde niet met de andere epigenetische indicatoren. Deze resultaten lijken erop te wijzen dat, op een globaal niveau, Alzheimer pathologie vooral geassocieerd is met DNA methylatie, maar dat het gekozen diermodel een grote invloed kan hebben op deze associatie. Voor toekomstige onderzoeken naar de relatie tussen genetische mutaties en epigenetische misregulatie kan het dus gunstig zijn als globale metingen gecombineerd worden met relevante gen-specifieke observaties. 
Voor HOOFDSTUK 6 werd de impact van een vermindering van bètaamyloïd $(A \beta)$ door middel van actieve immunotherapie op epigenetische DNA modificaties onderzocht. De globale aanwezigheid van DNA methylatie, DNA hydroxymethylatie en DNMT3A werd bepaald in de hippocampus van APP/PS1dE9 muizen, verdeeld over een gevaccineerde en een controle groep. Anti-A $\beta$ immunotherapie werd geassocieerd met een vermindering van DNA hydroxymethylatie en DNMT3A. Met behulp van bestaande gegevens met betrekking tot Alzheimer pathologie en cognitie, werd een correlatie gevonden met $A \beta$ pathologie en synaptische integriteit voor DNA hydroxymethylatie en DNMT3A. Verder correleerde DNA methylatie en hydroxymethylatie, maar niet DNMT3A, met gedragsscores. Deze resultaten bieden een eerst blik op de epigenetische component van anti-A $\beta$ immunotherapie, al is vervolgonderzoek nodig om uit te wijzen of de epigenetische veranderingen een gevolg zijn een vermindering van $A \beta$, of van immunologische processen die onderdeel uitmaken van de actieve immunisatie.

Het overige experimentele deel van dit proefschrift verplaatst de focus van modellen van ZvA naar menselijke patiënten en van globale immunohistochemische epigenetische metingen naar genoomwijde, plaats specifieke, microarray bepalingen. Voor HOOFDSTUK 7 was het methyloom en hydroxymethyloom van de gyrus temporalis medius bepaald, een hersengebied aangetast door ZvA. Een nieuwe methode die metingen van bisulfiet (BS)- en oxidatief BS-behandeld DNA integreert werd gebruikt om verschillen in de proporties gemethyleerd, gehydroxymethyleerd en ongemodificeerd signaal te bepalen tussen patiënten met ZvA en controles voor meer dan $450.000 \mathrm{CpG}$ plekken in het genoom. Correlaties tussen nabijgelegen CpG plekken werd ook onderzocht voor de bepaling van differentieel gemethyleerde regio's (DGRs) geassocieerd met ZvA. Dit leidde tot de identificatie van enkele genen die geassocieerd zijn met ZvA, waaronder RHBDF2, ANK1 en C3, maar ook OXT, waarvan afwijkende epigenetische regulatie nog maar zeer recentelijk geïmpliceerd is in ZvA. OXT laat hypomethylatie en hyperhydroxymethylatie zien met toenemende Braak stadia. Deze bevindingen onderstrepen het belang van het scheiden van DNA methylatie en hydroxymethylatie signalen bij het bestuderen van het epigenoom van het brein, welke sterk verrijkt is met hydroxymethylatie.

HOOFDSTUK 8 kan gezien worden als een wat meer klinisch georiënteerde extensie van het vorige hoofdstuk, waarvoor het methylomische profiel van ZvA werd verkent in het bloed. Voor deze studie werd het methyloom van bloed bepaald voor patiënten met ZvA, personen met een milde cognitieve beperking (MCB) en gezonde controles. Posities in het methyloom die geassocieerd werden met 
ZvA, werden vervolgens verder onderzocht in relatie tot indicatoren van Alzheimer dementie, zoals mini-mental state examination (MMSE) scores, $A \beta$ en gefosforyleerd tau in de cerebrospinale vloeistof en hippocampaal volume. Verscheidene genen met differentieel gemethyleerde posities (DGPs) en/of DGRs werden gedetecteerd in verband met ZvA, waaronder het risico gen voor ZvA HLA-DRB5, waarvan in een andere studie de methylatie status in het brein van patiënten met ZvA ook anders was bevonden ten opzicht van gezonde controles. Andere genen zoals PCDHA1, CDH13, CLSTN2, NEFL en MAD1L1 toonden al differentiële methylatie in de MCB groep, of werden naast ZvA ook geassocieerd met andere indicatoren van dementie. Deze genen reflecteren mogelijk verstoorde processen die onderdeel zijn van de pathogenese van ZvA en die, na verder onderzoek, gebruikt zouden kunnen worden als vroege indicatoren van ZvA in het bloed.

Voor HOOFDSTUK 9 werd het preklinische methylomisch profiel bepaald, dat geassocieerd is met conversie naar Alzheimer dementie. Door gebruik te maken van DNA, geïsoleerd uit bloed van een groot verouderingscohort waarbij gezonde personen werden geincludeerd en waarvan conversie naar Alzheimer dementie werd bijgehouden, was het mogelijk om individuen te vergelijken die na 4,5 jaar Alzheimer dementie hadden ontwikkeld, met individuen die binnen deze tijd geen dementie ontwikkelden. Deze vergelijking leidde tot de identificatie van enkele DGPs en DGRs, waaronder ook weer HLA-DRB5 en OXT, die mogelijk gebruikt kunnen worden voor de preklinische diagnose van ZvA. Om inzicht te verkrijgen in verschillen in de dynamische epigenetische regulatie tussen geconverteerde en niet geconverteerde personen, was een interactie analyse uitgevoerd voor groep en tijd. De DGPs uit deze analyse waren vervolgens verder onderzocht voor veranderingen tussen de twee tijdpunten voor de geconverteerde en niet geconverteerde groep apart om te bepalen voor welke groep de verandering over tijd significant waren. Verschillen in methylatie in de geconverteerde groep, in genen zoals DGKD, MRPL20 en ARHGAP12, kunnen een teken zijn van conversie naar Alzheimer dementie. Anderzijds, in de niet geconverteerde groep, zouden verschillen in de methylatie van genen zoals GRAMD1C, SMNDC1 en ARHGAP1, juist een indicatie kunnen zijn van bescherming tegen conversie naar Alzheimer dementie. 

VALORIZATION 
As society becomes more critical about what science feeds it, it is a logical requirement to dedicate a chapter to the societal impact of my research. However, before I present my view, let us first take a look at what 'non-scientists' think the impact of my research is. When someone asks me what I do, I usually just say "I do research into aging and Alzheimer's disease". For this chapter, I asked a few people what they think the societal impact of my research is (admittedly, they have not much to go on):

"Immense. You are able to scientifically show how many people get Alzheimer's in these times with many elderly. More years to live; live longer at home, economic target, save money on healthcare; people are able to live longer independently, etc."

"I have no knowledge of the actual content of your research, but I myself have no urge to further extend the lifespan, something many strive for. Your research seems part of this and I think it may eventually contribute to this in the future."

"I think it has a positive influence, as our careers get longer these days. Additionally, the quality of life can, especially at advanced ages, be drastically improved for many people when aging can be slowed and Alzheimer's can be detected/treated earlier."

"The research will lead to a better understanding of Alzheimer's disease, and because of this a better treatment can be provided or the symptoms can be managed better."

"If you succeed, you will profoundly increase the quality of life for the elderly, but especially for those close to the otherwise affected individuals."

Now, how do the findings of this thesis compare with these steep expectations? In short, unfortunately there still is no cure for Alzheimer's disease and our life expectancy remains the same. On the one hand, it should be recognized that, especially the first chapters of this thesis involving animal research, are very fundamental and exploratory in nature. The research presented in this thesis will likely not have a direct impact on society in the short run, but mainly offers insights important for other scientists and is able to guide future studies. On the other hand, the research covered in CHAPTER 4 offered new insights into caloric restriction, which is known to prolong life in rodents. As a life-long reduction in calorie intake may not sound attractive to many people to extend their lifespan a bit, my research and that of others in our group, investigated the mechanisms behind the effects of caloric restriction so 
that these processes may in the (far) future be targeted through e.g. pharmaceutical intervention instead of reducing calorie intake. Although most responses above do not address aging directly, promoting healthy aging, or even 'curing' aging would have the largest impact on society imaginable (only one responder seems to realize this!).

The research of CHAPTER 5 may be the furthest from society. Comparing different animal models, its main conclusions are about how to tackle future studies in the field of epigenetics ${ }^{1}$ and Alzheimer's disease. Useful for fellow researchers, but not so much for society at large. Or is it? It is easy to diminish the usefulness of a single study when viewed separately, but it may play a pivotal role in directing future research into more fruitful avenues. As the study in CHAPTER 5 indicates, current animal models do not capture Alzheimer's disease very well, and findings are not easily translated to the human situation. Indeed, while Alzheimer's disease has been cured over and again in animal models (see e.g. [1] for an overview), it should not be forgotten the end goal is to treat human patients. Thus, although animal models are extremely important for fundamental research (see e.g. [2]), it needs to be complemented with human-oriented studies to ensure the clinical validity of the observations.

Starting from CHAPTER 7, the research focuses on humans and gets closer to society. In this chapter, we offer novel insights into epigenetic dysregulation associated with Alzheimer's disease, comparing brain tissue from patients and healthy controls. While for this type of study replication is crucial, the affected markers identified can be further investigated as potential diagnostic markers or treatment targets in future studies. Note that many of the findings of epigenome-wide association studies (i.e. covering the epigenetic regulation of most known genes), such as those described in Chapters 7 through 9 , are often not easily replicated, making the selection of viable targets for mechanistic and functional follow-up studies a major challenge. Nevertheless, expanding our knowledge of the disease is already a merit on itself, as a greater understanding is crucial for the development of effective treatment strategies, whereas a lack of knowledge may explain why there currently

${ }^{1}$ Wonder what epigenetics is? In short, epigenetic regulation supervises gene expression; it dynamically determines which genes are expressed (and to which extent) and which are not in response to your diet, physical activity and other environmental factors. For a more detailed, but still accessible explanation of epigenetics see: http:// www.whatisepigenetics.com/what-isepigenetics/. is no cure for Alzheimer's disease.

The final scientific efforts described in this thesis, in CHAPTERS 8 AND 9, are similar in nature to CHAPTER 7, but focus on the blood instead of the brain. While it may seem strange at first, to investigate the blood in relation to a neurodegenerative disease, this actually makes the findings much more relevant for clinical purposes in the shorter run. Brain tissue can in most cases only be obtained postmortem, but blood can be easily obtained from patients, and importantly, possible future patients. Even though blood markers may thus indirectly represent 
what happens in the brain, they can be directly used for diagnostic and prognostic applications. In fact, this is what my future research will focus on ; using machine learning to make predictions about the development of Alzheimer's disease based on blood markers and identify novel candidates for therapy, such as oxytocin. Even though the identification of Alzheimer's disease at a pre-clinical stage has currently, without the availability of an effective treatment, not much clinical use, it may be fundamental for the development of such treatments, as it is thought past clinical trials have focused on a disease stage where damage to the brain is too advanced to reverse [3]. Furthermore, to fully grasp the etiology of complex diseases like Alzheimer's disease it will be necessary for future research to embrace advances in computer modelling and systems biology to integrate genomic, epigenomic, transcriptomic, proteomic, and other data modalities. This will also allow for a better informed development of treatment strategies.

Looking again at what others think the impact my research has on society, it seems many people ${ }^{2}$ think of what the impact could be. This is indeed what is traditionally described in, say, a grant proposal, as this is perhaps what people want to hear. Therefore, I decided to write about the toneddown, in my view more realistic impact my research may have on society, as you see above. Notably, apart from the societal impact of the research performed by $\mathrm{PhD}$ students, it is in my opinion extremely important to look at the societal impact of successful PhD students themselves (i.e. the most important outcome of a $\mathrm{PhD}$ project is not the research, but the researcher). While I cannot speak for all $\mathrm{PhD}$ students, going through the $\mathrm{PhD}$ trajectory has allowed me to explore a field of research in depth, identify gaps and weaknesses in the current body of knowledge and related approaches. During the latter part of my project, I have expanded my capabilities in my specific field of study beyond the strong foundation provided by my supervisors to meet the requirements to advance this field. I now feel ready and confident to design my own studies, write my own grant proposals, and supervise my own team. In short, now, I am ready to make a real impact on society.

\footnotetext{
${ }^{2}$ Please forgive me the crude extrapolation of 5 people's opinion to that of many.
}

\section{REFERENCES}

[1] Franco R, Cedazo-

Minguez A. Successful therapies for Alzheimer's disease: why so many in animal models and none in humans? Front Pharmacol 2014; 5: 146.

[2] Granholm A-C. Why do we need to use animal models to study cognition and aging? Neuropsychopharmacology 2010; 35: 1621-2.

[3] Sperling RA, Jack $\mathrm{CR}$, Aisen PS. Testing the right target and right drug at the right stage. Sci Transl Med 2011; 3(111): $111 \mathrm{~cm} 33$. 




\section{GURRICULUM VITAE}


Roy Lardenoije was born on the 11th of April 1990 in Leiderdorp, the Netherlands. He grew up and went to school in Leiden. In 2006, he got his first chance to work in a laboratory, during a high school internship at PharmaCell BV in Maastricht, where he studied the differentiation of muscle stem cells. He obtained his high school diploma cum laude (VWO, Nature \& Health profile) in 2008 at the Marecollege in Leiden. After a country-wide search for a suitable Bachelor program that could satisfy his broad interests, he moved to Maastricht and entered University College Maastricht (UCM). There, he sculpted his own curriculum, blending molecular biology, computer science, psychology, philosophy, and with towards the end, a focus on neuroscience. This cumulated in a study on the functional organization of the visual cortex for his bachelor thesis, supervised by Dr. Vincent van de Ven at the Cognitive Neuroscience department of Maastricht University. Shortly after this, in 2011, he was granted his cum laude Bachelor of Science degree. After another arduous search for a Master program, it was the friendly and welcoming interview with Dr. Daniël van den Hove and Dr. Jos Prickaerts for the Fundamental Neuroscience specialization of the Cognitive and Clinical Neuroscience Research Master that convinced him to stay in Maastricht. For his Master thesis Roy investigated the epigenetics of aging under supervision of Dr. Daniël van den Hove and Dr. Bart Rutten, and he obtained his Master of Science degree cum laude in 2013. For his thesis, he received the Studentprize 2013 for the best Master thesis of the Faculty of Psychology and Neuroscience. He was able to continue the research of his Master internship as a research and education assistant with a Kootstra Talent Fellowship, until he obtained an NWO Graduate School of Translational Neuroscience Fellowship in 2014 to fund a PhD position under the supervision of Prof. dr. Bart Rutten, Prof. dr. Harry Steinbusch, and Dr. Daniël van den Hove. Apart from his work in Maastricht, Roy visited the lab of Dr. Cynthia Lemere at Harvard Medical School in Boston (USA) for half a year. He also shortly visited the group of Dr. Alfredo Ramirez, at both the University of Bonn and the University of Cologne. Additionally, he presented his research at numerous national and international conferences, including the Society for Neuroscience Annual Meeting and the Dutch Neuroscience Meeting. In addition to research, Roy has also taken on the roles of internship coordinator and meeting organizer for the Translational Neuropsychiatry / Neuroepigenetics group and was a PhD representative of the Neuroscience division and member of the educational committee of the School for Mental Health and Neuroscience (MHeNS). During his PhD, Roy has also been tutor and practical supervisor in several Bachelor and Master courses at Maastricht University and has supervised many Bachelor and Master students during their internship. Next to teaching others, Roy also kept on expanding his own skills, taking courses and workshops in statistics, bioinformatics, and programming to evolve his research. Since 2016 Roy also operates as a 
freelance programmer and writer, including a collaboration with graphic designer Eric Lardenoije as Brave Monkeys. Currently, Roy is a postdoc fellow in the group of Dr. Daniël van den Hove and Prof. dr. Bart Rutten, further expanding his work on the epigenetics of aging and Alzheimer's disease through the implementation of machine learning and systems biology approaches. 



\section{LIST OF PUBLICATIONS}




\section{Published}

Pishva E, Kenis G, Hannon E, Viechtbauer W, Jeffries A, Lardenoije R, Sienaert P, van OS J, Stek ML, Rutten BPF. Genome-wide meta-analysis of DNA methylation changes associated with antidepressant effects of Electroconvulsive Therapy. Brain Stimulation 2017; 10:388.

Bey K, Wolfsgruber S, Karaca I, Wagner H, Lardenoije R, Becker J, Milz E, Kornhuber J, Peters O, Frölich L, Hüll M, Rüther E, Wiltfang J, RiedelHeller S, Scherer M, Jessen F, Maier W, van den Hove DLA, Rutten BPF, Wagner M, Ramirez A. No association of the variant rs11887120 in DNMT3A with cognitive decline in individuals with mild cognitive impairment. Epigenomics 2016; 8(5):593-8.

Lardenoije R, van den Hove DLA, Vaessen TSJ, latrou A, Meuwissen KPV, van Hagen BTJ, Kenis G, Steinbusch HWM, Schmitz C, Rutten BPF. Epigenetic modifications in mouse cerebellar Purkinje cells: effects of aging, caloric restriction, and overexpression of superoxide dismutase 1 on 5-methylcytosine and 5-hydroxymethylcytosine. Neurobiology of Aging 2015; 36:3079-89.

Lardenoije R, latrou A, Kenis G, Kompotis K, Steinbusch HWM, Mastroeni DF, Coleman P, Lemere CA, Hof PR, van den Hove DLA, Rutten BPF. The epigenetics of aging and neurodegeneration. Progress in Neurobiology 2015; 131:21-64.

Fitzsimons CP, van Bodegraven E, Lardenoije R, Kompotis K, Kenis G, Chouliaras L, Biojone C, Joca S, Steinbusch HWM, Lunnon K, Mastroeni DF, Mill J, Coleman P, van den Hove DLA, Rutten BPF. Epigenetic regulation of neural stem cells: Implications for Alzheimer's disease.

Molecular Neurodegeneration 2014; 9:25.

Van den Hove DLA, Kompotis K, Lardenoije R, Kenis G, Mill J, Steinbusch HW, Lesch KP, Fitzsimons CP, De Strooper B, Rutten BPF. Epigenetically regulated microRNAs in Alzheimer's disease. Neurobiology of Aging 2014; 35(4):731-45.

Lardenoije R*, Van Goethem N*, Kompotis K, Rutten BPF, Prickaerts J, Steinbusch HWM. Cognitive disorders: aging, impairment and dementia cognitive deficit models, In: In Vivo Models for Drug Discovery, WileyVCH Verlag. 2014: 349-366. 
Lardenoije R*, Brasnjevic I*, Schmitz C, Van Der Kolk N, Dickstein DL, Takahashi H, Hof PR, Steinbusch HWM, Rutten BPF. Region-specific neuron and synapse loss in the hippocampus of APPSL/PS1 knock-in mice. Translational Neuroscience 2013; 4:8-19.

\section{Submitted and in preparation}

Lardenoije R, van den Hove D, Havermans M, van Casteren A, Le K, Palmour R, Lemere CA, Rutten BPF. Age-related epigenetic changes in hippocampal subregions of four animal models of Alzheimer's disease. Submitted.

Pishva E, Kenis G, Viechtbauer W, Arts B, Jeffries A, Hannon E, Lardenoije R, Burrage J, Murphy T, van den Hove DLA, Sienaert P, Bouckaert F, Mill J, Stek M, van Os J, Dols A, Rutten BPF. Longitudinal changes in genome-wide DNA methylation associated with electroconvulsive therapy. Submitted.

Chouliaras L, Lardenoije R, Kenis G, Mastroeni DF, Hof PR, van Os J, Steinbusch HWM, van Leeuwen FW, Rutten BPF, van den Hove DLA. Age-related disturbances in DNA methylation and hydroxymethylation in the hippocampus of transgenic APPswe/PS1 $\triangle \mathrm{E} 9$ mice. Submitted.

Lardenoije R, van den Hove DLA, Jung SE, Havermans M, Liu B, Rutten $\mathrm{BPF}^{*}$, Lemere $\mathrm{CA}^{*}$. Active amyloid- $\beta$ immunotherapy results in epigenetic changes in the hippocampus of an Alzheimer's disease mouse model. In preparation.

Lardenoije $\mathrm{R}^{\star}$, Roubroeks JAY*, Pishva $E^{\star}$, latrou A, Smith $A R$, Smith $R G$, Eijssen LMT, Kenis G, Mastroeni DF, Delvaux E, Coleman PD, Mill J, Rutten BPF, Lunnon K, van den Hove DLA. The DNA methylome and hydroxymethylome in Alzheimer's disease; Cortical dysregulation of OXT, C3, ANK1 and RHBDF2. In preparation.

Lardenoije R, Pishva E, Jacobs H, Ramakers I, Visser PJ, Steinbusch HWM, van den Hove DLA, Rutten BPF. Detection of blood methylomic markers associated with clinical features of Alzheimer's disease; An exploratory approach. In preparation.

Lardenoije R, Leber M, Pishva E, Rutten BPF, van den Hove DLA, Ramirez A. Identification of preclinical methylomic blood markers associated with conversion to Alzheimer's disease. In preparation. 

ACKNOWLEDGMENTS 
First and foremost I would like to thank my promotion team, Prof. dr. Bart Rutten, Prof. dr. Harry Steinbusch, Dr. Daniël van den Hove, and Dr. Cynthia Lemere, who made it possible for me to embark on the wondrous journey that led to this thesis.

Daniël, I first met you for the Fundamental Neuroscience interview. I had gone through interviews for two other Master programs, but there the people felt indifferent, like I was being interrogated. However, the interview with you and Jos was very welcoming and it was more like we were having a friendly chat. I believe this is characteristic for you, and what makes you such an amazing supervisor; I always had the feeling I could walk into your office and discuss anything, even things not related to my research. You treat the people under your supervision with interest and respect and not just as workhorses and also give them the opportunity to grow in the direction they want. Having talked with many other PhD students about their supervision, I truly believe you are one of the best daily supervisors around and that I was very lucky to end up under your care.

Bart, I got introduced to you by Daniël as my second supervisor for my Master internship because I wanted to do aging research. During this first meeting you asked me the classic question of what I wanted to do next, after my Master, and further in the future. A normal question at the time, but I like how you always think about 'the next thing'. It seems every time I talk to you, you have a new exciting opportunity to be explored and I greatly enjoy the challenges this provides. You always seem to have a cheerful spark in your eyes and have an infectious optimistic attitude towards science. While the meetings with you at the beginning of my $\mathrm{PhD}$ were a bit overwhelming, leaving me with so many new things to delve into, I have learned to be more selective so I can better appreciate the opportunities you have to offer. Together with Daniël, I think you two strike an excellent balance between realism and idealism; daring to explore novel fields, but without getting lost.

Dear Cindy, I am extremely grateful that you gave me the opportunity to work with you and your wonderful group. It was my first time going so far from home for an extended period of time, and if not for your warm and welcoming personality and the amazing people in your group I would not remember my time in Boston as one of the greatest highlights of my $\mathrm{PhD}$ and as a pivotal milestone in my personal development. I not only got to learn a different approach to research, but also learned a lot about American culture such as by experiencing an absolutely delightful Christmas dinner at your home, witnessing a baby shower at work, and you even organized a surprise birthday party in your office! 
Harry, although we did not meet that often during my time as a PhD student, when we did you always had some valuable input for my research. Even though my specific field of study was not your specialization, you were still able to always provide quick and useful feedback on my work.

Apart from my promotion team, I also would like to thank another very important team, the wonderful technician team, Hellen, Marjan, and Denise. Hellen greatly helped me to enhance my skills in the lab at the start of my $\mathrm{PhD}$ and was always ready to share her expertise when setting up new experiments. Marjan and Denise have always been friendly and helpful, even when I needed some last-minute materials. Without them, many experiments would not have run so smoothly as they did!

Barbie, a special thank you goes out to you, as you are a special technician from a different world (the lab upstairs truly feels like a different world). You have thought me some of the most elementary basics, such as running a gel, without which my lab work would likely have been doomed. You act with a certain pragmatism which makes it easy to work with you and to get things done.

Rachelle, we started around the same time at the department and ever since your assistance has been invaluable. Whether it was making a simple copy, planning a meeting, or making sure I didn't go broke in Boston, I could always rely on you to help out with a smile!

Kostas, although we technically were rivals during our Master internship, vying for a PhD position, it never felt that way. Although we stayed many evenings and weekends at the lab, there was always some time for fun; from deep technical discussions to just watching some anime in the MPR. Thanks for being the funniest IHC instructor I ever met, your instructions lay at the foundation of this thesis!

Leo, as my predecessor, your work forms the pillars upon which my thesis is build. You have always greatly helped me when I had questions, even though you did not work in Maastricht anymore. The few times we met were a pleasure, so much so that I believe we spend the majority of this time in bars.

Julie, as I am myself not the most socially skilled person, your kind and caring nature were integral for my integration in the broader PhD student community in Maastricht. You were always ready to help out when needed and it was always a delight chatting with you in the lab or the office. 
Ehsan! Hoi. One of the few veterans remaining in the group from before I started, you have been an inspiring mentor, as well as a great friend. You were introduced to me as 'the freezer guy' who knows where stuff is, and I think we have both grown a lot since then. I greatly enjoy our discussions and highly value your thoughts on subjects that go beyond the expertise of my supervisors. Your modesty and realistic view of science is refreshing and your cooking is enrapturing. Be sure to let me know when you start your own group!

Jo, the first impression I got of you was as a strict tutor for FN. However, when I started as a PhD student I quickly learned you were the true embodiment of "work hard, party harder"! Bringing life to the department, while also delivering inspiring work, you are truly a living legend and the department will never be the same without you.

Yara, you had your setbacks at work, but you did not let this influence your life outside the office and you were always fun to be around. You were the only true match for Jo in the partying department, and I will never forget the crazy trip to Munich for the Startbierfest that you organized!

Nicole, while we have not worked a lot together, your grant-obtaining skills are truly legendary and reading them has thought me a lot for writing my own proposals. Your cheerfulness and dedication to science is very inspiring, and you are great to chat with to boot!

Mark, you offered some well-needed male presence in our femaledominated office and it was great to have a sparring partner also going trough the trials of becoming an R master. You also introduced the department to CrossFit and avocados, leading to some fascinating discussions!

Marion, you are one of the nicest persons I ever met and your cheerfulness and enthusiasm always brightened up the room. It was great to have an even greater geek as myself in the group, to share some of life's delights only understood by a select few. I also have you to thank for introducing me to the glorious raclette tradition, those tasty movie nights at your place were truly something to look forward to!

Magda, I really appreciate your directness and honesty and enjoyed our critical discussions. You were a nice fresh breath in a lab where most people accepted the status quo. You introduced me to some great German beers, a great treat in the Netherlands! And I hope to try some more; maybe when we watch Black Sheep? 
Lars, you were a great help to get me started with some of my bioinformatic analyses. However, your true expertise shines as a fellow whisky connoisseur. Did you already catch a Kelpie?

Laurence, you were the first postdoc joining our group since I started as a PhD and it was really nice to have a 'heavyweight' researcher in the lab to discuss and do experiments with. As the seniors focus more on the planning aspect of research, I think your more pragmatic approach of not only planning but also executing research really pushed things forward. Additionally, I must say being a tutor in your course at the Maastricht Science Programme was one of the best tutoring experiences I had.

Gunter "The Big G" Kenis, while not officially part of my promotion team, you are a crucial member of the triad at the head of our TNP group. We worked more together at the start of my $\mathrm{PhD}$, when I still worked on miR-137, and later I had the honor of disturbing the peace in your office while meeting with Ehsan. Nonetheless, I have gotten to know you as a scientific mastermind, with a great sense of humor to match.

Koen, you were one of my first interns and I hope that elective didn't scar you as me eating a banana did. While our scientific collaboration was of a short duration, we had many memorable moments, such as in Indonesia and Berlin, where I thoroughly got to enjoy your great sense of humor. And how do you manage to always look good in a picture!?

Britt, I think you were actually my first intern, definitely the first to do lab work, so you thought me possibly more about supervising a student than I thought you about immunohistochemistry. After that, you became a great colleague, especially our time in San Diego with the great performance of Artemis was unforgettable.

I also would like to thank all the other wonderful people (past and present) at our department and beyond, for many memorable occasions during my time as a PhD student in Maastricht. Especially the great group of guys, Ehsan, Jo, Koen, Maarten, and Pim, for the epic Berlin experience. Gerard for some excellent beer, whisky and coffee experiences. Gisela, for your great enthusiasm, and fun conversations in and outside the lab. Nick, it was a pleasure working on the book chapter together, and it is always fun talking to you. Simone for some hilariously fun moments in Braga. Marina for the fantastic Spanish flair you bring to the department, as well as your hospitality and the great events you host, bringing the department closer on a social level together with Artemis. The same can be said about Shannon, organizer of the best Christmas dinners. Shengua, for the fun we had organizing the lab day out. Carolin, although we never really worked together, I feel we have a certain bond, going 
through the same trials and getting the same fellowships. An, I hope you have by now memorized all the distilleries on Islay. Your dedication to learn Dutch is amazing, goed zo! Fred, even though we don't talk often, it always ends with a laugh.

I also owe thanks to many of the senior scientists at our department. Especially, Jos, for being the perfect FN coordinator, not only teaching prospective scientists to work hard, but also to party harder. Mario for helping with my microscope image analyses. Fred van Leeuwen for the insightful discussions.

Obviously, I would not have been able to achieve so much during my PhD without the help of some wonderful students. Bhavana, you thought me more than you can imagine, but unfortunately I was not able to instill some sense of responsibility in you. Thomas, you showed a critical mind and a great dedication to the project, showing a greater independence and responsibility than expected of an intern. I hope you finish all your studies and find a way to combine them in your future career. Nathalie, thanks to your efficient and independent work we were able to run many PCR experiments, but alas, they did not make it into this thesis even though these experiments were crucial to move my project forward. Kirsten, you had barely set a foot in the lab before you started, but your cautious approach to the lab work resulted in brilliant gels, which were fundamental for the LR-PCR experiments, which sadly, did not make it into this thesis. Büsra, you had a bit of a rough start, but it was a delight to have you visit our lab and I think you really grew as a scientist during your stay. Anne and Sophie, you really helped me out by daring to take on an internship with long distance supervision while I was in Boston! Sophie, as your report stood out in quality, I am happy you could further help me with turning the data you generated into a chapter of this thesis. Sabine, you were a great help to make sense of my first EWAS results. Monique, when I came back from Boston you were a godsend for the quick processing and analysis of the immunohistochemistry image data, while also assisting with other projects, quickly learning new techniques. I dare you to give more of your own input during your future endeavors, as you have a lot of potential! Renzo, even though the results were not earth-shattering, you achieved something amazing for your elective; setting up and running your own little study, with only minimal guidance. I look forward to see what you can achieve during a full PhD project! Floris, you also did your own pilot study and delivered great work considering the not so ideal state of the tissue you had to work with. Narek, you were my last intern during my $\mathrm{PhD}$ period and it was a breeze. Despite the sketchy content of your internship, you quickly mastered the laboratory techniques, especially admirable considering you never took notes. It was great getting to know you and I hope you have become a Pokémon Master by now! 
Special thanks go out to Bert Smeets, Jo Vanoevelen and Wanwisa Dijkstra, who were pivotal in setting up the next-generation sequencing study, which unfortunately had to be cancelled. Especially Wanwisa thought me a lot about LR-PCR, kept answering my incessant stream of questions, and went above and beyond to get the experiment to work.

Apart from Cindy, it was also thanks to her amazing group that my time in Boston was such a success. Particularly, Helen, your welcoming and fun personality made me immediately feel accepted in the group, and it was extremely kind of you and Qiaoqiao to scout my room before I arrived in Boston! And that Boston Beer Summit we went to really made me fall in love with the contemporary US brewing styles. Kevin and Emmett, it was great working with you and exploring the lunch options that the American cuisine has to offer was delightful, especially that "All the Way" hotdog/ hamburger monster from Tasty Burger was a delicious challenge, and that sushi place Kevin took us remains the stuff of dreams! Saba, your cheerful demeanor and our chats made those countless hours behind the microscope much more enjoyable than they had the right to be. Finally, I would like to thank Allison Roper for helping me through the bureaucratic maze required to work in the US, without you I would likely not have been able to even enter the country!

Frank, having you as a roommate at the beginning of my stay in Boston was hilarious (you know what time it is!), and we had some great moments with other Dutchies Nadine, Nienke and Anouk, and your labmate Gea. I still haven't found a place here that can compete with that Thai in Boston!

Alfredo, your collaboration with our group gave me an exciting new project to work on the end of my $\mathrm{PhD}$, and our collaboration will likely continue into the future. Thanks for showing me Bonn and I must agree a couple of beers form the perfect foundation for invigorating scientific discourse. Markus, working with you in Cologne and your continued support was invaluable for finishing Chapter 9 of this thesis, and thanks for curing my fear of the terminal.

Artemis. You have probably been the most important person in making my time as a PhD student so memorable. Without you I would probably have had a very sober life outside the lab. I think if someone else had been my roommate it might have been painfully awkward, but your wonderfully sociable character, open mind, and great intellect has made you a precious friend. I really miss our culinary exploits, late night chats, the marvelous gatherings at our place, and we still have to finish Gears of War 3... But also at work you have always been a reliable friend, when I needed your expert opinion or an ever important coffee break. I could think of no other person better suited to be my paranymph! 
Janou, I also got to know you as my intern, and you immediately struck me as very kind, charming and hardworking. It was delightful to work with you on the same project, but we also had lots of fun outside the $R$ environment, such as during the epic quiz nights. As fellow whisky lovers, you and Artemis are the perfect paranymphs!

My brother and good friend Eric, thank you for your amazing dedication to this project and for turning the physical thesis into something truly unique! Be sure to check him out at ericlardenoije.com if you also want an exceptional thesis or have any other undertaking that requires some designer flair.

Mom and dad, you are at the genesis of this thesis. Your views on life and how you raised me have always inspired me to think about what I am doing and to make sure I enjoy it; to always explore beyond perceived boundaries. Whatever choices I made and will make in the future, I feel I can always rely on your love and support; truly, what else can a son wish from his parents? I brewing buddy perhaps?

Sophie, my other (better) half, and only person that not only 'knows me longer than today', but also knows me today. You have been instrumental in the completion of this thesis. You are the one it is always worth for to take a break. Especially during that final push to complete this thesis, you were there to pull me out of the thesis haze when needed. Now as a PhD veteran, I hope to be of service to you in your journey as a PhD student, especially for the extracurricular activities! 


ABBREVIATIONS 
12SOD-CD: 12-months-old SOD1 mice on the CD

12SOD-CR: 12-months-old SOD1 mice on the CR diet

12WT-CD: 12-months-old WT mice on the CD

12WT-CR: 12-months-old WT mice on the GR diet

24SOD-CD: 24-months-old SODl mice on the $\mathrm{CD}$

24SOD-CR: 24-months-old SODl mice on the CR diet

24WT-CD: 24-months-old WT mice on the $\mathrm{CD}$

24WT-CR: 24-months-old WT mice on the GR diet

3NP: 3-nitropropionic acid

3R-tau: tau with 3 microtubule-bind-

ing repeats

3xTg-AD mice: triple transgenic mouse model of $\mathrm{AD}$ expressing human mutant APPK670N/M671L, PS1M146V, and TauP301L

$450 \mathrm{~K}$ BeadChip: Illumina Infinium $\mathrm{Hu}-$ manMethylation 450K BeadChip 4PBA: 4-phenylbutyrate 4E-BP: eukaryotic translation initiation factor $4 \mathrm{E}$-binding protein 4R-tau: tau with 4 microtubule-binding repeats

5-caC: 5-carboxylcytosine

5-fC: 5-formylcytosine

5-hmc: 5-hydroxymethylcytosine

5-hmU: 5-hydroxymethyluracil

5HT: serotonin

5HTR3: type 3 serotonin receptor

5-mc: 5-methylcytosine

5xFAD mice: transgenic mouse model overexpressing mutant human APP (695) with the Swedish (K670N and M671L), Florida (I716V), and London (V717I) mutations, and mutant human PSl with the M146L and L286V fAD mutations

7-mG: 7-methylguanine

A: acetyl modification / adenosine (depending on context)

A3SS: alternative 3' splice site

A5SS: alternative 5' splice site

$A \beta$ : amyloid $-\beta$

Aß42: A $\beta$ 1-42

ABCA1: ATP binding cassette subfami-

ly A member 1

ac: acetylation (as in H3K9ac)

ACh: acetylcholine

$\mathrm{ACI}$ : annulus crossing index

AD: Alzheimer's disease

ADAM: a disintegrin and metalloprote-

ases domain

ADAR: adenosine deaminases that act

on RNA

ADP: adenosine diphosphate

AFE: alternative first exon

AgeCoDe: German Study on Ageing,

Cognition and Dementia in Primary

Care Patients

AGK2: 2-cyano-3-(5-(2,5-dichlo-

rophenyl)-2-furanyl)-N-5-quino-

linyl-2-propenamide

AICDA: activation-induced cytidine

deaminase

ALDH: aldehyde dehydrogenase

ALE: alternative last exon

ALKBH: alkylation repair homolog

Alu: Arthrobacter luteus elements

AMPA: alpha-amino-3-hy-

droxy-5-methyl-4-isoxazolepropionic

acid

ANOVA: analysis of variance

antagomirs: anti-miRNAs conjugated

to cholesterol molecules

APOBEC: apolipoprotein B mRNA

editing enzyme, catalytic polypep-

tide-like protein

APOE: apolipoprotein E

APP: amyloid- $\beta$ precursor protein

APP23 mice: transgenic mouse model

with a 7-fold overexpression of

APPswe

APP/PS1 mice: transgenic mouse

model expressing mutant human AP-

PK670N/M671L and PS1M146V
APP/PS1-21 mice: transgenic mice overexpressing APPswe and human PS1 with the L166P mutation, line 21 APP/PS1dE9 mice: transgenic mice overexpressing APPswe and PSldE9 APPind: human APP with the V717F Indiana mutation APPswe: human APP isoform 695 with the double KM670/671NL Swedish mutation

a-Syn: a-synuclein

ATC: anterior temporal cortex

ATP: adenosine triphosphate

AU: arbitrary units

BACE: $\beta$-secretase

BACE1-AS: BACE1-antisense

BAF: Brgl/hBrm associated factor

BAG2: B-cell chronic lymphocytic

leukemia/lymphoma 2-associated athanogene 2

BBB: blood-brain barrier

BC200: brain cytoplasmic RNA 200

BDNF: brain-derived neurotrophic

factor

BER: base excision repair

BP: biological process

BS: bisulfite

BSeq: bisulfite sequencing

C: cytosine

C57BL/6 mice: C.57 black 6 inbred

mouse strain

CA: cornu ammonis

CAG: cytosine-adenine-guanine

cAMP: cyclic adenosine monophos-

phate

caspase: cysteine-dependent aspar-

tate-directed protease

CBP: cAMP response element-binding protein binding protein

CD: control diet

Cdk5cKO mice: cyclin-dependent

kinase 5 conditional knock-out mouse model

CDR: Clinical Dementia Rating

CDS: coding DNA sequence

CE: cassette exon 
CEC: cerebral endothelial cell

CiEC: circulating endothelial cells

C. elegans: Caenorhabditis elegans

CER: cerebellum

CFC: contextual fear conditioning

CGI: CpG island

CHD: chromodomain, helicase, DNA

binding

ChIP: chromatin immunoprecipitation

ChIP-Seq: chromatin immunoprecipi-

tation sequencing

chr: chromosome

CHRM1: muscarinic acetylcholine

receptor 1

$\mathrm{Cl}$ : confidence interval

CK-p25 mice: transgenic mouse model

overexpressing p25 under control of

an inducible calcium/calmodulin-de-

pendent protein kinase II a promoter

CLU: clusterin

CNE: constitutive exon

CNS: central nervous system

Co-IP: protein complex immunopre-

cipitation

cox: cyclooxygenase

CpG: cytosine-phosphate-guanine

CR: caloric restriction

CREB: CAMP response element-bind-

ing protein

CREBBP: CREB binding protein

CSF: cerebrospinal fluid

DAB: 3,3'-diamino benzidine tetrahy-

drochloride

DAC: 5-aza-2'-deoxycytidine (decit-

abine)

DAF-16/FOXO: dauer 16/forkhead box O

DAPI: 4',6-diamidino-2-phenylindole

dihydrochloride

DG: dentate gyrus

DHbE: dihydro-beta-erythroidine

DHP: differentially hydroxymethylat-

ed position

DHR: differentially hydroxymethylat-

ed region

Dicer cKO mice: conditional Dicerl

knock-out mouse model
DMP: differentially methylated position

DMR: differentially methylated region

DNAm age: DNA methylation age

DNMT: DNA methyltransferase

DNMT3L: DNMT3-like

DP: differentiation regulated tran-

scription factor protein (chapter 2)

DP: distal promotor (chapter 7)

DS: downstream region

dsRNA: small double-stranded RNA

DT: diphtheria toxoid

DUP: position with differentially un-

modified cytosine levels

DUR: region with differentially un-

modified cytosine levels

E1A: adenovirus early region $1 \mathrm{~A}$

E2F1: E2F transcription factor 1

EC: entorhinal cortex

ECA: enzyme complementation assay

ECD: electrochemical detection

EDAR: Early Diagnosis of AD and as

marker for treatment Response

El: exon isoforms

EID1: EP300 interacting inhibitor of

differentiation 1

ELISA: enzyme-linked immunosor-

bent assay

EP300: ElA-binding protein P300

ERK: extracellular signal-regulated

kinase

eRNA: enhancer RNA

ES: effect size

EWAS: epigenome-wide association

studies

EZH: enhancer of zeste homolog

(Drosophila)

f6A: N6-formyladenosine

fAD: familial AD

FAIRE: formaldehyde-assisted isola-

tion of regulation

FC: frontal cortex

FDA: Food and Drug Administration

FDR: false discovery rate

FTD: frontotemporal dementia

FTLD: frontotemporal lobar degen-

eration
FTO: fat mass and obesity-associated protein

fPD: familial PD

FRM-0334: Forum Pharmaceuticals compound 0334

GABA: gamma-aminobutyric acid

GABRA: $Y$-aminobutyric acid receptor subunit alpha

GAD-3: gastrulation defective 3

GADD45: growth arrest and DNA

damage 45

GB: gene body

GCF: granulocyte chemotactic factor

GCN: general control of amino acid

synthesis

gDNA: genomic DNA

GDNF: glial cell line-derived neuro-

trophic factor

GDS: Global Deterioration Scale

GFAP: glial fibrillary acidic protein

GFPT: glutamine-fructose-6-phos-

phate transaminase

GluR: glutamate receptor

GO: Gene Ontology

GR: glucocorticoid receptor

GSK: glycogen synthase kinase

GWAS: genome-wide association

studies

GWES: genome-wide epistasis study

$\mathrm{H}$ : histone protein, always followed by

a number (for example, H3 in H3K4)

H2AX: histone protein H2A member X hAD-MSC: human adipose tissue-de-

rived MSC

HAEC: human aortic endothelial cells HAT: histone acetyltransferase

HCAEC: human coronary artery endothelial cells

Hcy: homocysteine

HD: Huntington's disease

HDAC: histone deacetylase

HDACI: HDAC inhibitor

HdhQ150/Q7 mice: transgenic mouse

model expressing one mutant HTT

copy with 150 CAG repeats and a nor-

mal HTT copy with 7 CAG repeats 
HEK293 cells: human embryonic kidney 293 cell line

HeLa cells: cell line established from cervical cancer cells

Hip: hippocampus

His: histidine

HKDM: histone lysine demethylase HKMT: histone lysine methyltransferase

HLA: human leukocyte antigen hm6A: N6-hydroxymethyladenosine HMW: high molecular weight hnRNP: heterogeneous nuclear ribonuclear protein HPLC: high-performance liquid chromatography

HRDM: histone arginine demethylase HSP: heat shock protein

HTS: high-throughput sequencing

HTT: huntingtin

hUCB-MSC: human umbilical cord

blood-derived MSC

HuR: human antigen $\mathrm{R}$

HUVEC: human umbilical vein endo-

thelial cells

HV: hippocampal volume

I: inosine

IBA1: ionized calcium-binding adapter molecule 1

ICC: immunocytochemistry

IF: immunofluorescence

IG: intergenic region

IGF: insulin-like growth factor

IHC: immunohistochemistry

II: intron isoforms

IKK: inhibitor of kappaB kinase

i.n.: intranasally

INO: inositol requiring 80

INPC: induced neural progenitor-like cell

i.p.: intraperitoneally

iPSC: induced pluripotent stem cell

IR: immunoreactivity

IR: intron retention (chapter 7)

IRAK: interleukin-1 receptor-associated kinase
ISH: in situ hybridization

ISWI: imitation SWI

J20 mice: transgenic mouse model

expressing mutant human APP (AP-

PK670N/M671L, V717F)

JMJD: jumonji domain containing

$\mathrm{K}$ : lysine (as in H3K9ac)

KAT: lysine acetyltransferase

KEGG: Kyoto Encyclopedia of Genes

and Genomes

KI: knock-in

L-DOPA: L-3,4-dihydroxy-phenylal-

anine

lincRNA: large intergenic non-coding

RNA

LINE-1: long interspersed element 1

IncRNA: long ncRNA

LRRK2: leucine-rich repeat kinase 2

LT: Escherichia coli heat-labile en-

terotoxin

M: methyl modification

m1A: N1-methyladenosine

m1G: N1-methylguanine

m6A: N6-methyladenosine

MALDI-TOF : matrix assisted laser

desorption/ionization time-of-flight

MAP: microtubule-associated protein

MAPK: mitogen-activated protein

kinase

MAPT: microtubule-associated protein

tau

MAS-1: Mercia's Th2-biased adjuvant

MAT: methionine adenosyltransferase

MBP: methyl-CpG-binding domain

protein

$\mathrm{MCl}$ : mild cognitive impairment

me: methylation (as in H3K4me3)

MeCP: methyl CpG-binding protein

MeDIP-Seq: methylated DNA immuno-

precipitation sequencing

MedFG: medial frontal gyrus

MetH: methionine synthase

METTL: m6A methyltransferase-like

protein

MFG: middle frontal gyrus

miR: microRNA
miRNA: microRNA

MLA: methyllycaconitine

MMSE: mini-mental state examination

MPP+: 1-methyl-4-phenyl-pyridinium

ion

MPTP: 1-methyl-4-phenyl-1,2,3,6-tet-

rahydropyridine

MRI: magnetic resonance imaging

mRNA: messenger RNA

MSC: mesenchymal stem cells

MSK: mitogen- and stress-activated

protein kinase

MS-PCR: methylation-specific PCR

mtDNA: mitochondrial DNA

mtDNMT: mitochondrial DNMT

MTG: middle temporal gyrus

MTHF: methylenetetrahydrofolate

MTHFR: MTHF reductase

MWM: Morris water maze

MXE: mutually exclusive exon

N171-82Q mice: transgenic mouse mod-

el expressing the first 171 amino acids

of HTT with 82 CAG repeats

N2a: Neuro-2a

NA: not applicable

nAChR: ionotropic nicotinic ACh

receptor

NAD: nicotine adenine dinucleotide Nano-LC: nano liquid chromatography

NAT: natural antisense transcript

NB: northern blot

NC: non-CGI

ncRNA: non-coding RNA

NDM29: neuroblastoma differentiation

marker 29

NEFL: neurofilament light

NeuN: Neuronal nuclei

NF-KB: nuclear transcription factor

kappa B

NFT: neurofibrillary tangle

NGF: nerve growth factor

NIH: National Institutes of Health

NINCDS-ADRDA: National Institute of

Neurological and Communicative

Disorders and Stroke and the Alzhei-

mer's Disease and Related Disorders 
Association

NMDA: N-methyl-D-aspartic acid

NS: not specified

NS20Y cells: mouse cholinergic neuro-

blastoma cell line

NSE: neuron-specific enolase

NuRD: nucleosome remodeling and

histone deacetylase

OR: odds ratio

oxBS: oxidative BS

p: phosphorylation (as in H3S10p)

P300: ElA-binding protein P300

PAR: promoter-associated RNA

PARP: poly[ADP]-ribose polymerase

PASR: promoter-associated short RNA

P-bodies: processing bodies

PBS: phosphate-buffered saline

PC: principal component

PC12 cells: rat pheochromocyto-

ma-derived cell line

PCAF: P300/CBP-associated factor

PCDH: protocadherin

PcG: Polycomb-group

PCP: phencyclidine

PCR: polymerase chain reaction

PD: Parkinson's disease

PDAPP mice

transgenic mice overexpressing AP-

Pind

PFC: prefrontal cortex

PGC1-a: peroxisome proliferator re-

ceptor gamma coactivator-1 alpha

piRNA: piwi-interacting RNA

PKCঠ: protein kinase C $\bar{c}$

PMI: postmortem interval

POLG: DNA polymerase gamma

PP: proximal promoter

PP2A: protein phosphatase 2A

PPT: protein phosphatase

PRC1: polycomb repressive complex member Bmil

PRC2: polycomb repressive complex member EZH2

pre-miRNA: precursor miRNA

PRMT: protein arginine methyltrans-

ferase
PROMPTS: promoter upstream tran-

scripts

PS: presenilin

PS1dE9: human PS1 deleted in exon 9 mutation

PSD: postsynaptic density protein

ptau: phosphorylated tau

PTBP: polypyrimidine tract binding

protein

$Q Q$ plot: quantile-quantile plot

r: Pearson's correlation coefficient

$\mathrm{R}$ : arginine (as in H3R2me)

R6/2 mice: transgenic mice overex-

pressing exon 1 of human HTT with

an expanded CAG repeat length

RAN: Ras-related nuclear protein

Ras: rat sarcoma

rasiRNA: repeat-associated small inter-

fering RNA

RFLP: restriction fragment length

polymorphism

RISC: RNA-induced silencing complex

RNAi: RNA interference

RNAP: RNA polymerase

ROI: region of interest

ROS: reactive oxygen species

RRBS: reduced representation bisulfite sequencing

IRNA: ribosomal RNA

RT: room temperature

RT-PCR: real-time PGR

S: serine (as in $\mathrm{H} 3 \mathrm{~S} 10 \mathrm{p}$ )

SAD: sporadic AD

SAH: S-adenosylhomocysteine

SAHA: suberoylanilide hydroxamic

acid

SAHF: senescence-associated heter-

ochromatin foci

SAHH: SAH hydrolase

SAM: S-adenosylmethionine

SAMP8 mice: senescence-accelerated

prone mouse model

SAT-a: satellite- $a$

SB: sodium butyrate

SB: Southern blot (in table)

scaRNA: small Cajal body-specific
RNA

SD: standard deviation

SE: standard error

SET: Drosophila Su(var)3-9 and en-

hancer of zeste proteins

SETDB1: SET domain bifurcated 1

SFG: superior frontal gyrus

SHE: shelf

SHMT: serine hydroxymethyltrans-

ferase

SHO: shore

SH-SY5Y cells: human neuroblastoma

cell line

shRNA: short hairpin RNA

SIDAM: Structured Interview for Diag-

nosis of Dementia of Alzheimer

Type, Multi-infarct Dementia, and

Dementia of Other Etiology

siRNA: small interfering RNA

SIRT: sirtuin

SI-SD: Stereo Investigator Confocal

Spinning Disk

SK-N-SH, SK-N-BE: human neuroblas-

toma cell lines

SLC: solute-carrier

SLK: Stouffer-Liptak-Kechris

smRNA: small modulatory RNA

SN: substantia nigra

sncRNA: small ncRNAs

snoRNA: small nucleolar RNA

SNP: single nucleotide polymorphism

snRNA: small nuclear RNA

SOD: copper-zinc superoxide dis-

mutase

SOD1 mice: transgenic mouse model

with 7 copies of the human SODl gene

SP: specificity factor

SPD: sporadic PD

SPF: specified pathogen free

spliRNA: splice junction-associated

RNA

SPT: serine palmitoyltransferase

ss-siRNA: single-stranded siRNA

STC: superior temporal cortex

SUV39H: suppressor of variegation 3-9

homologue 
SWI/SNF: switching defective/sucrose

nonfermenting

SYP: synaptophysin

T: thymine

Th: threonine

TACE: TNF-a converting enzyme

TDG: thymine DNA glycosylase

TDP: TAR DNA-binding protein

TE: transposable element

TET: ten-eleven translocation

Tg19959 mice: transgenic mice over-

expressing a combination of APPswe and APPind

Tg2576 mice: transgenic mice overexpressing APPswe

THF: tetrahydrofolate

TIP60: human immunodeficiency

virus type 1 transactivating protein

interactive protein

tiRNA: transcription initiation RNA

TNF-a: tumor necrosis factor alpha

TOF MS: time-of-flight mass spec-

trometry

tRNA: transfer RNA

TSA: trichostatin A

TSS: transcription start site

TSSa-RNA: TSS-associated RNA

$\mathrm{U:} \mathrm{uracil}$

U373 cells: human glioblastoma astro-

cytoma-derived cell line

UC: unmodified cytosine

UTR: untranslated region

VPA: valproate/valproic acid

WB: western blot

WHO: World Health Organization

WT: wild-type

WTAP: Wilm's tumor-associated

protein

Y: tyrosine

YAC128 mice: transgenic mouse model

expressing mutant human HTT with

128 CAG repeats

YTHDF: YTH domain family 





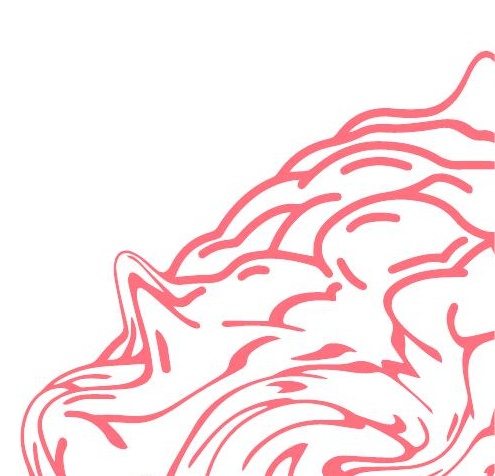


VENTURE 
INTO 
THE 
EPIGENETICS 
OF 
AGING

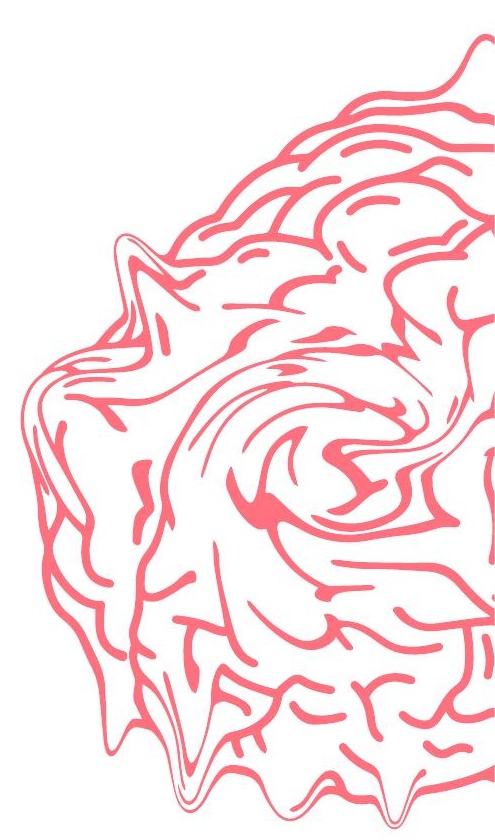




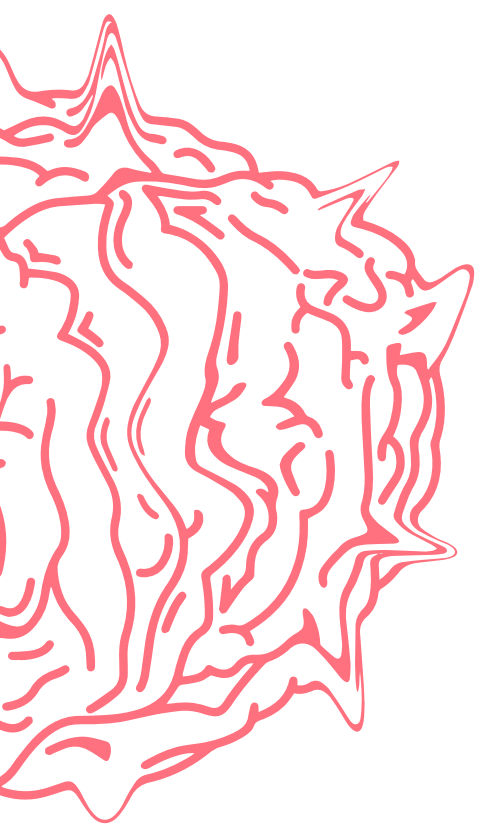

AND 
ALZHEIMER'S 
DISEASE 
ㄴ. 
